FHWA/IN/JTRP-2008/9

Final Report

REAL-TIME ARTERIAL TRAFFIC SIGNAL PERFORMANCE MEASURES

Christopher M. Day

Edward J. Smaglik

Darcy M. Bullock

James R. Sturdevant

August 2008 
Final Report

FHWA/IN/JTRP-2008/9

\title{
REAL-TIME ARTERIAL TRAFFIC SIGNAL PERFORMANCE MEASURES
}

\author{
By \\ Christopher M. Day \\ Graduate Research Assistant \\ Purdue University \\ Edward J. Smaglik \\ Assistant Professor \\ Northern Arizona University \\ Darcy M. Bullock, P.E. \\ Professor \\ Purdue University \\ James R. Sturdevant, P.E. \\ Indiana Department of Transportation \\ Joint Transportation Research Program \\ Project No. C-36-17YYY \\ File No. 8-4-77 \\ SPR-3089 \\ Conducted in cooperation with the \\ Indiana Department of Transportation \\ and the \\ U.S. Department of Transportation \\ Federal Highway Administration
}

The contents of this report reflect the views of the authors, who are responsible for the facts and accuracy of the data presented herein. The contents do not necessarily reflect the official views or policies of the Indiana Department of Transportation or the Federal Highway Administration at the time of publication. This report does not constitute a standard, specification, or regulation.

\author{
Purdue University \\ West Lafayette, IN 47907 \\ August 2008
}


TECHNICAL REPORT STANDARD TITLE PAGE

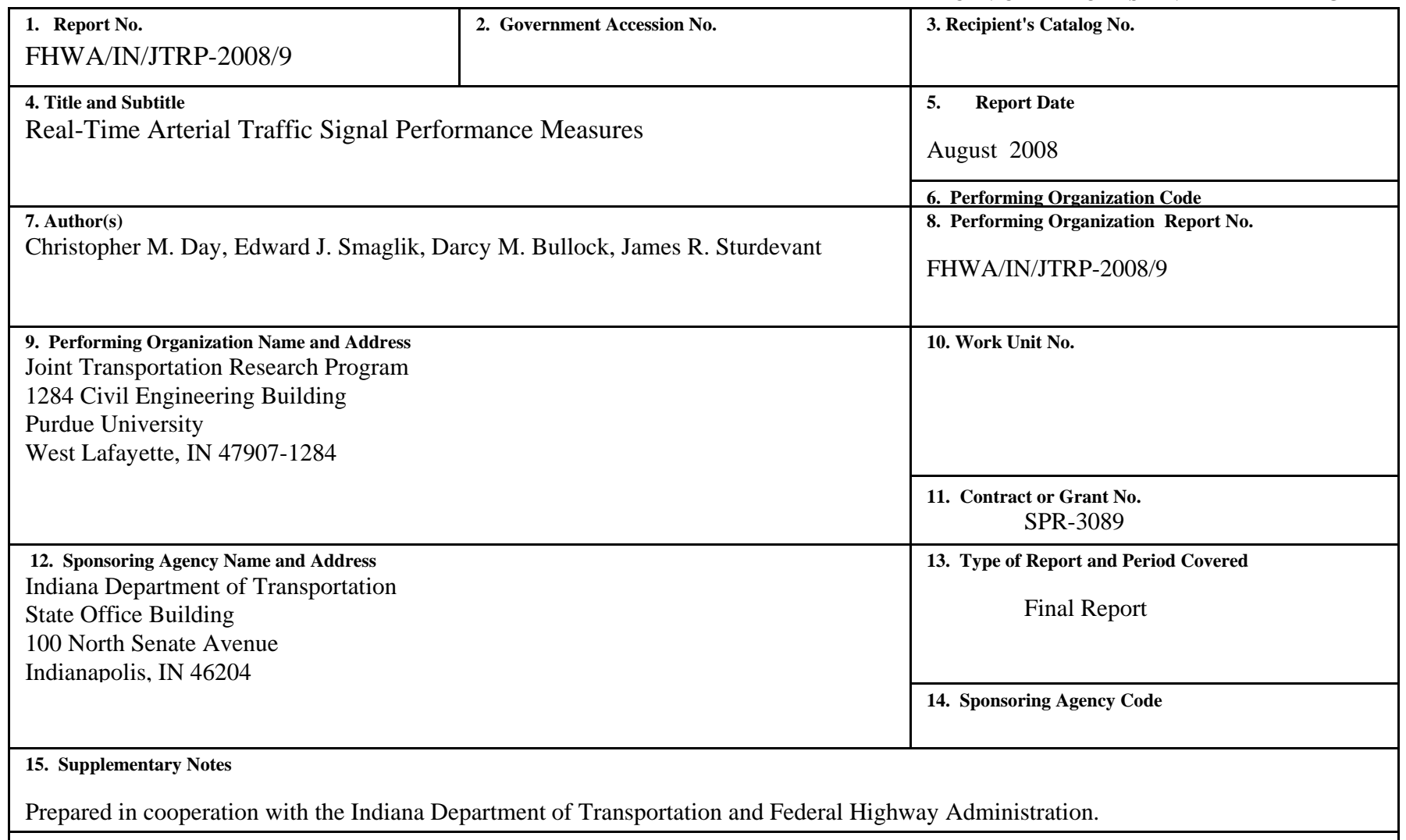

\section{Abstract}

Increasing demand for highway system capacity compels transportation agencies to extract as much capacity as possible from existing systems. Traffic signals represent a major component of highway systems. Improvement of traffic signal operation requires accurate performance measures. Despite recent improvements in computing technology, traffic signal controllers are currently not able to capture useful performance measures on a cycle-by-cycle basis. This report presents a set of performance measures that could be collected on a cycle-by-cycle basis by a traffic signal controller given the capability to log phase indications and detector actuations. The performance measures investigated here include the volume-to-capacity ratio and arrival type defined by the Highway Capacity Manual. The effectiveness of these performance measures in evaluating operation at a traffic signal is demonstrated in two comparative case studies. The first study investigates the impact of actuating a portion of the coordinated phases at a coordinated arterial intersection. The second study investigates the results of retiming a traffic signal on a coordinated arterial.

\begin{tabular}{|c|c|c|c|c|}
\hline \multicolumn{2}{|c|}{$\begin{array}{l}\text { 17. Key Words } \\
\text { Traffic Signals, Traffic Signal Performance Measures, } \\
\text { Traffic Signal Timing, Traffic Signal Monitoring, Signal } \\
\text { Coordination. }\end{array}$} & \multicolumn{3}{|c|}{$\begin{array}{l}\text { 18. Distribution Statement } \\
\text { No restrictions. This document is available to the public through the } \\
\text { National Technical Information Service, Springfield, VA } 22161\end{array}$} \\
\hline 19. Security Classif. (of this report) & \multicolumn{2}{|c|}{ 20. Security Classif. (of this page) } & 21. No. of $\mathrm{Pa}$ & 22. Price \\
\hline Unclassified & \multicolumn{2}{|c|}{ Unclassified } & 24 & \\
\hline
\end{tabular}


Page

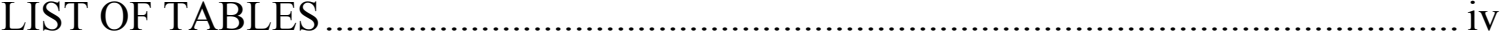

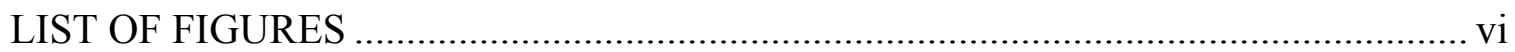

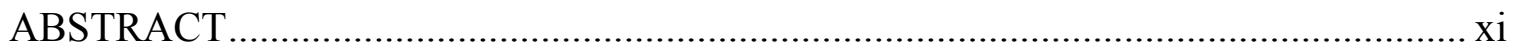

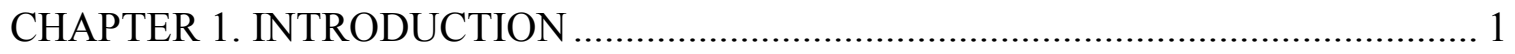

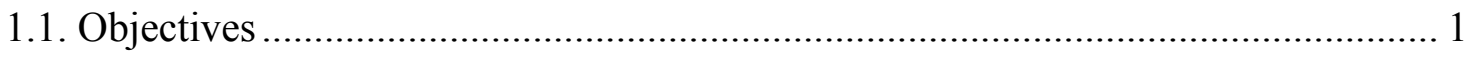

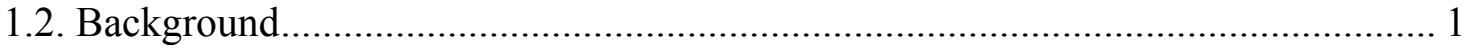

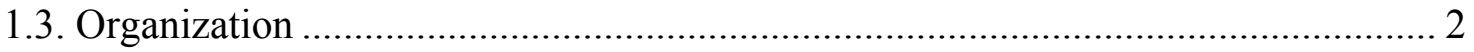

CHAPTER 2. REVIEW OF SIGNAL OPERATION CONCEPTS .................................... 4

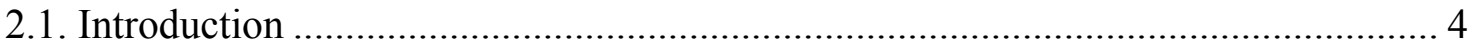

2.2. Cycle and Phase ............................................................................................ 4

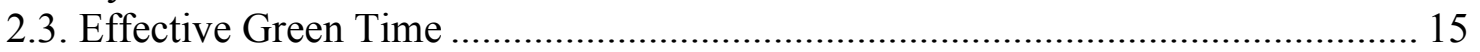

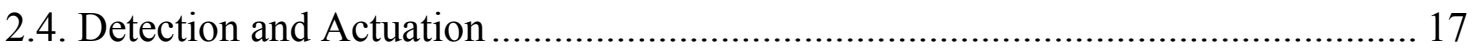

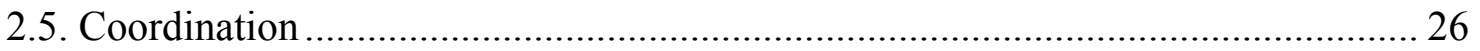

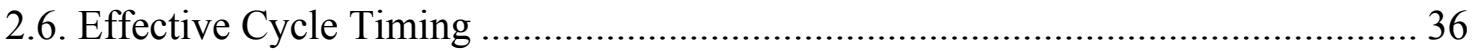

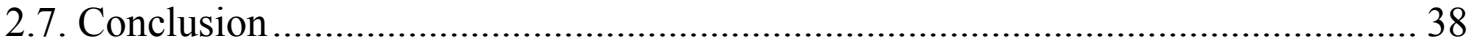

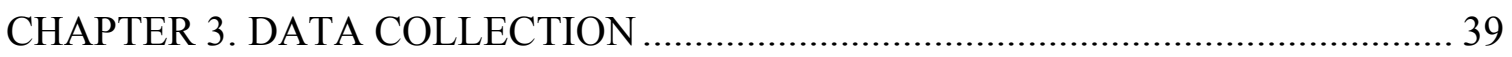

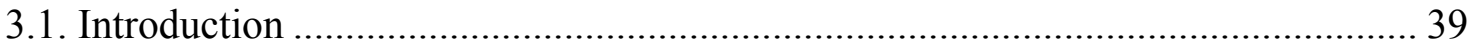

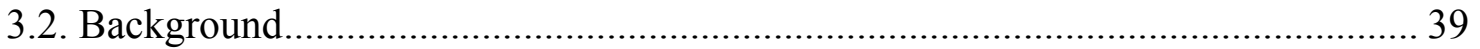

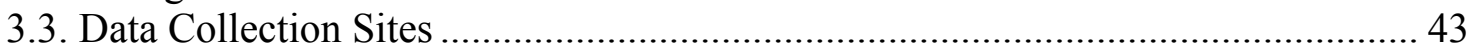

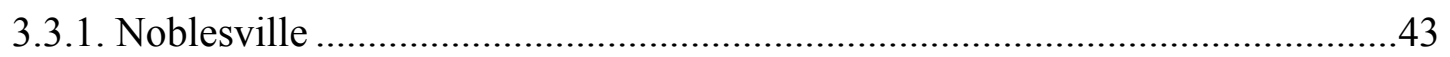

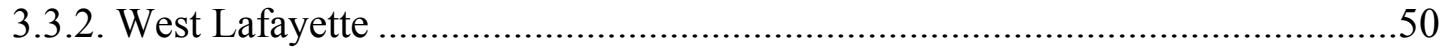

3.4. Data Collection Process............................................................................. 55

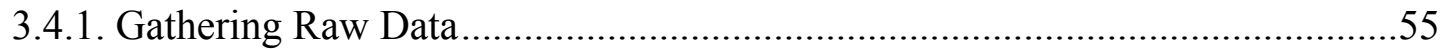

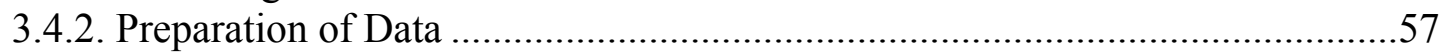

CHAPTER 4. CALCULATION OF PERFORMANCE MEASURES ............................... 66

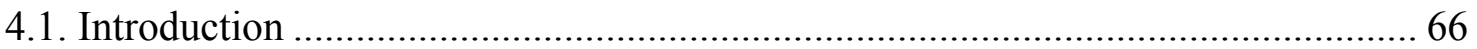

4.2. Quantities Describing the State of the Intersection ................................................. 67

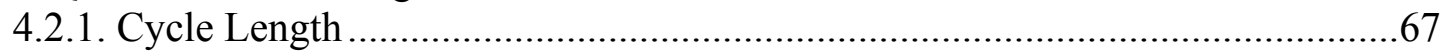

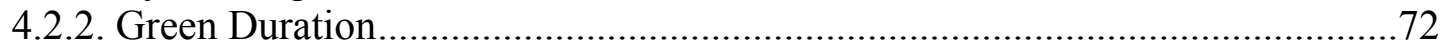

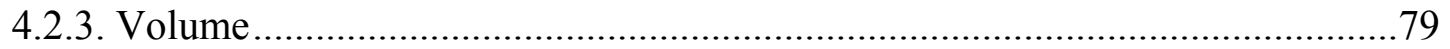




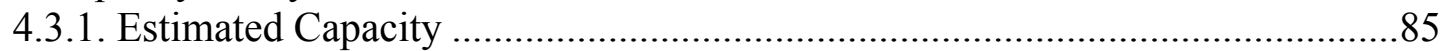

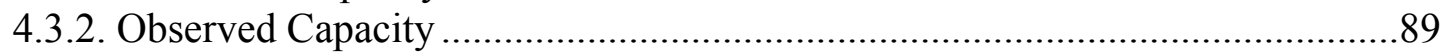

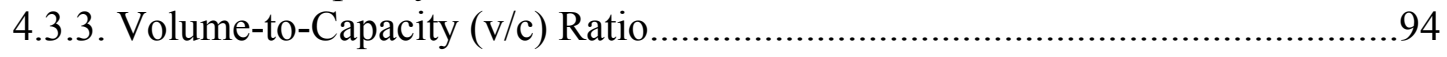

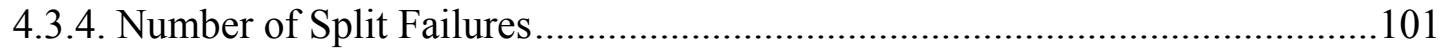

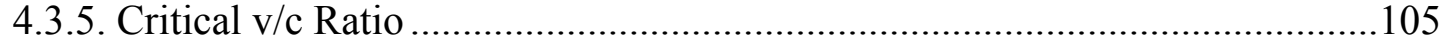

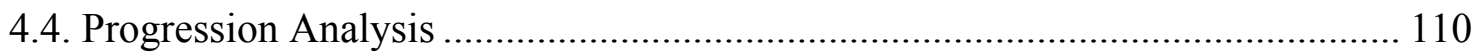

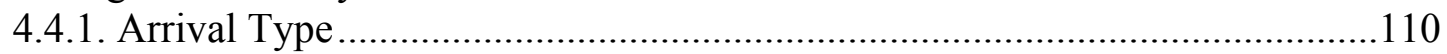

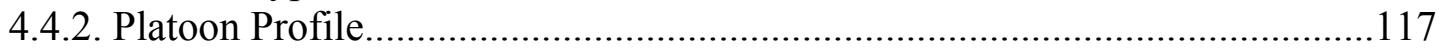

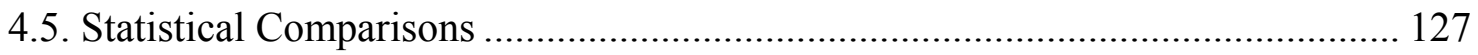

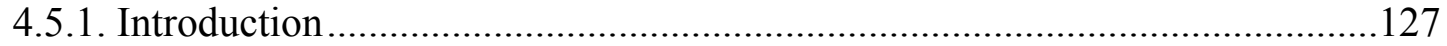

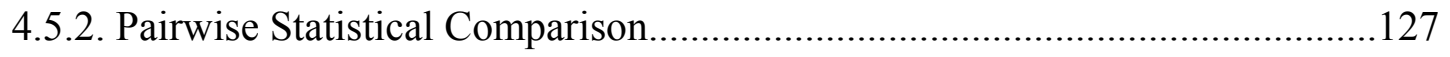

4.5.3. Aggregate Statistical Comparison......................................................... 128

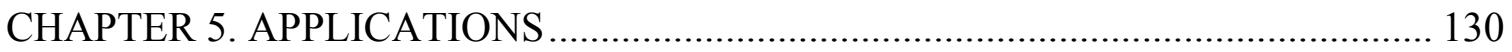

5.1. Introduction ........................................................................................... 130

5.2. Implementation of Actuated Coordinated Phases at Noblesville ....................... 130

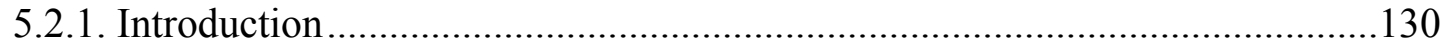

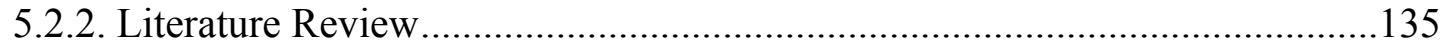

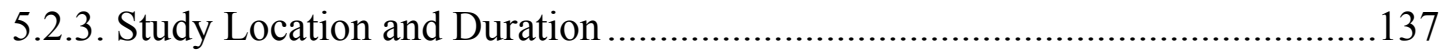

5.2.4. Experiment Design and Expectations .........................................................139

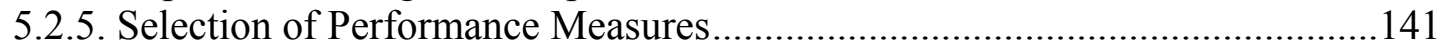

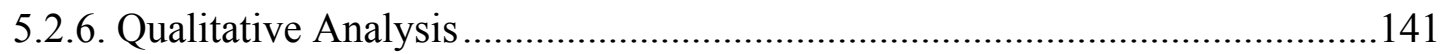

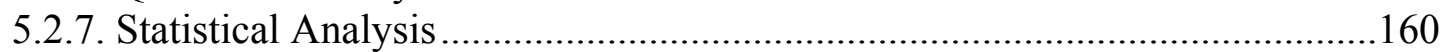

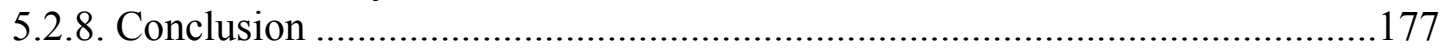

5.3. Operational Impacts of Noblesville Signal Retiming..................................... 178

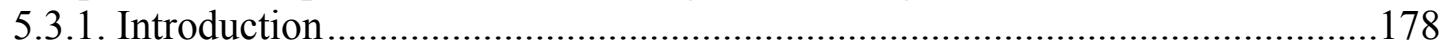

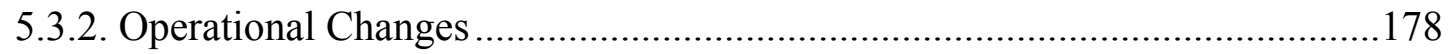

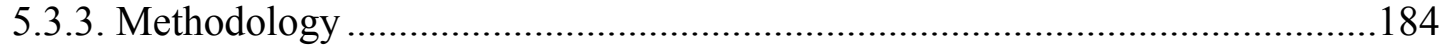

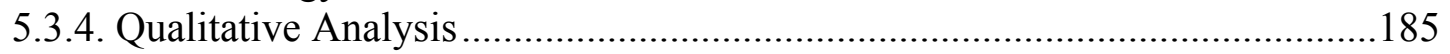

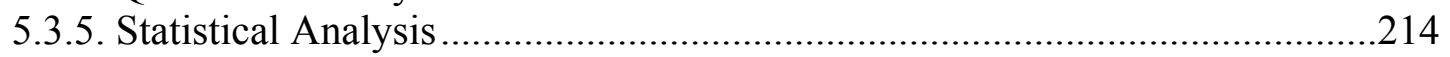

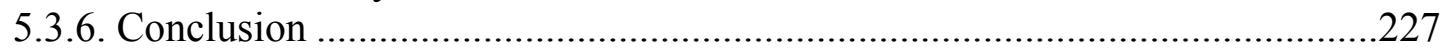

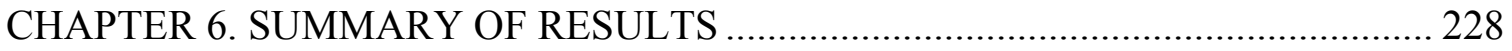

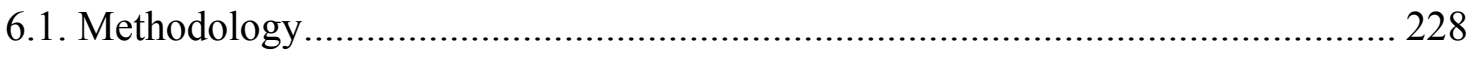

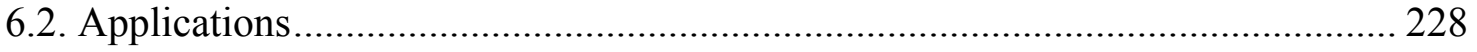

6.3. Moving Toward Real-Time Data Collection................................................. 228

6.4. Directions For Future Research ................................................................... 229

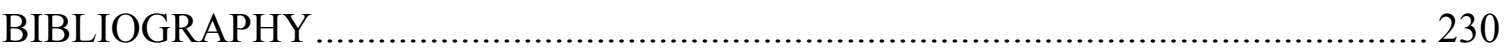




\section{LIST OF TABLES}

Table

Page

Table 2.1 Calculation of the duration of green indications from splits by cycle length, showing increasing proportion of green time with increasing cycle length...... 12

Table 2.2 TOD plan showing cycle length and proportional splits for weekday operation at the instrumented intersection at Noblesville, IN . ................................................. 14

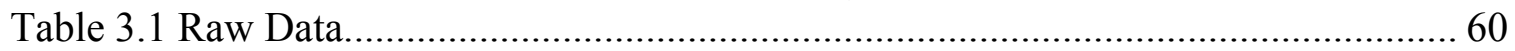

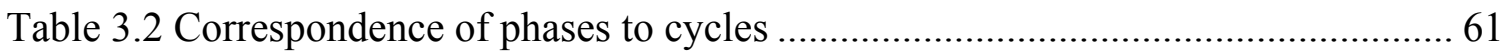

Table 4.1 Calculation of cycle length at Noblesville on March 14, 2007...................... 70

Table 4.2 Example calculation of green duration from logged events, based on data for

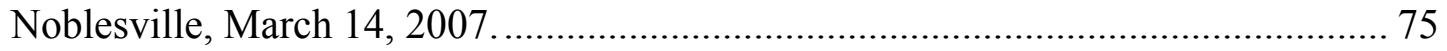

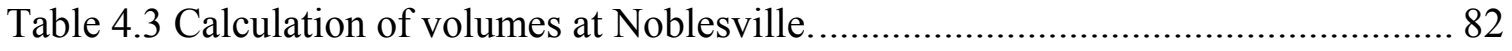

Table 4.4 Calculation of estimated capacities for Noblesville. ................................... 88

Table 4.5 Calculations of observed capacity at Noblesville....................................... 91

Table 4.6 Calculation of $\mathrm{v} / \mathrm{c}$ ratio at Noblesville. ....................................................... 97

Table 4.7 Counts of split failures and average v/c ratio values at Noblesville on March

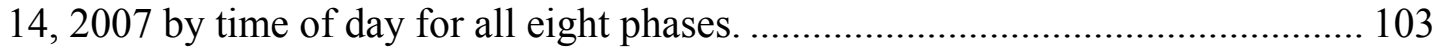

Table 4.8 Sample calculation of $\mathrm{X}_{\mathrm{C}}$ on a cycle-by-cycle basis................................... 107

Table 4.9 Sample calculation of $\mathrm{X}_{\mathrm{C}}$ on a cycle-by-cycle basis.................................. 108

Table 4.10 Definition of arrival type (5) based on platoon ratio, including equations used

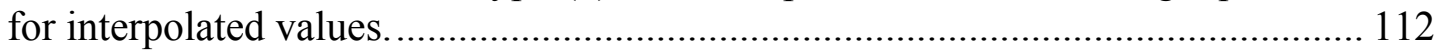

Table 4.11 Calculation of Arrival Type at Noblesville.............................................. 114

Table 4.12 Calculation of vehicle arrival distribution at Noblesville on Wednesday, March 14, 2007, 6:00 - 9:00 ................................................................... 120

Table 4.13 Calculations of green profile at Noblesville on Wednesday, March 14, 2007, 6:00- 9:00.

Table 5.1 Number of split failures reported for Wednesday, March 7, 2007 (without actuated coordinated phases) and for Wednesday, March 28, 2007 (with actuated coordinated phases) by phase and by TOD plan time period.....

Table 5.2 Difference in means (significant in boldface) for the pairwise comparison of operation on March 7 (without actuated coordinated phases) and March 28, 2007 (with actuated coordinated phases) at Noblesville, IN.

Table 5.3 Statistical t-values (significant in boldface) for the pairwise comparison of operation on March 7 (without actuated coordinated phases) and March 28, 2007 (with actuated coordinated phases) at Noblesville, IN.

Table 5.4 Statistical P-values (significant in boldface) for the pairwise comparison of operation on March 7 (without actuated coordinated phases) and March 28, 2007 (with actuated coordinated phases) at Noblesville, IN.

Table 5.5 Difference in means (significant in boldface) for the 15-weekday aggregate comparison of operation with and without actuated coordinated phases at Noblesville, IN. 
Table

Table 5.6 Statistical t-values (significant in boldface) for the 15-weekday aggregate comparison of operation with and without actuated coordinated phases at Noblesville, IN. 168

Table 5.7 Statistical P-values (significant in boldface) for the 15-weekday aggregate comparison of operation with and without actuated coordinated phases at Noblesville, IN. 169

Table 5.8 Mean values of v/c Ratio for all eight phases at Noblesville, IN by time of day, aggregated over 15 weekdays of operation with and without actuated-coordinated phases. 170

Table 5.9 Number of split failures reported for 15 weekdays without actuated coordinated phases, and 15 weekdays with actuated coordinated phases, by phase and by TOD plan time period.....

171

Table 5.10 Percentage of cycles having split failures in all 15 weekdays without actuated coordinated phases, and 15 weekdays with actuated coordinated phases, by phase and by TOD plan time period.

Table 5.11 Observed splits. 183

Table 5.12 Percentage of cycles ending in split failure at Noblesville, tabulated by phase and by TOD plan time period, averaged over 15 days before retiming and 15 days after retiming. 203

Table 5.13 Difference in means (significant in boldface) for the pairwise comparison of operation on June 19, 2006 (before changes) and March 12, 2007 (after changes) at Noblesville, IN. 218

Table 5.14 Statistical t-values (significant in boldface) for the pairwise comparison of operation on June 19, 2006 (before changes) and March 12, 2007 (after changes) at Noblesville, IN.

Table 5.15 Statistical P-values (significant in boldface) for the pairwise comparison of operation on June 19, 2006 (before changes) and March 12, 2007 (after changes) at Noblesville, IN.

Table 5.16 Difference in means (significant in boldface) for the pairwise comparison of operation on June 28, 2006 (before changes) and March 21, 2007 (after changes) at Noblesville, IN.

Table 5.17 Statistical t-values (significant in boldface) for the pairwise comparison of operation on June 28, 2006 (before changes) and March 21, 2007 (after changes) at Noblesville, IN.

Table 5.18 Statistical P-values (significant in boldface) for the pairwise comparison of operation on June 28, 2006 (before changes) and March 21, 2007 (after changes) at Noblesville, IN.

Table 5.19 Difference in means (significant in boldface) for the 15-weekday aggregate comparison of operation before and after operational changes at Noblesville, IN. . 224

Table 5.20 Statistical t-values (significant in boldface) for the 15-weekday aggregate comparison of operation before and after operational changes at Noblesville, IN. . 225

Table 5.21 Statistical P-values (significant in boldface) for the 15-weekday aggregate comparison of operation before and after operational changes at Noblesville, IN. . 226 


\section{LIST OF FIGURES}

Figure

Page

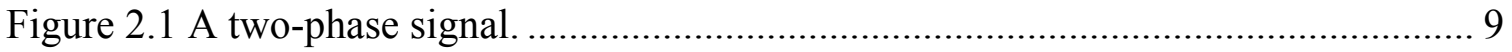

Figure 2.2 Cycle length and splits for a two-phase controller.................................... 10

Figure 2.3 Dual-ring eight phase controller configuration. ...................................... 11

Figure 2.4 Basic divisions of time by traffic signals. ................................................ 13

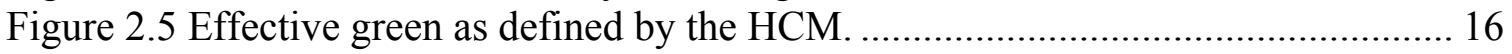

Figure 2.6 Picture of a loop detector installation......................................................... 21

Figure 2.7 (a) Typical appearance of loop detectors in field. (b) Basic electrical diagram for loops. (c) Detector output signals operating in presence and count detection

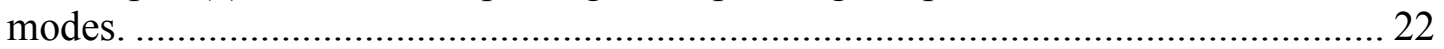

Figure 2.8 A comparison of presence and count detection modes. .............................. 23

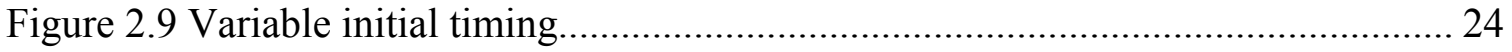

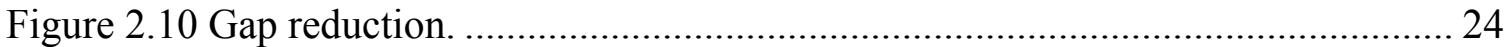

Figure 2.11 Comparison of phase termination logic by the green extension and gap

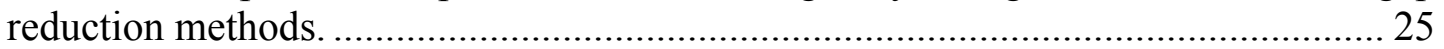

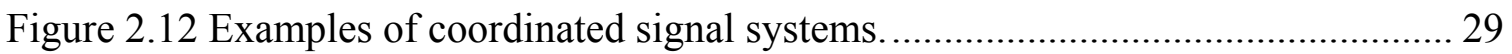

Figure 2.13 Conceptual comparison of coordinated operation to free operation. ............ 30

Figure 2.14 Spillback of queues into an adjacent intersection................................... 31

Figure 2.15 A time-space diagram showing a simple example of signal coordination.... 32

Figure 2.16 Issues with coordination with actuated phases......................................... 33

Figure 2.17 An overhead view of platoons becoming more disperse as distance increases

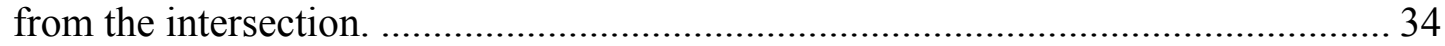

Figure 2.18 Mismatches of platoon dispersion and duration of green for a coordinated

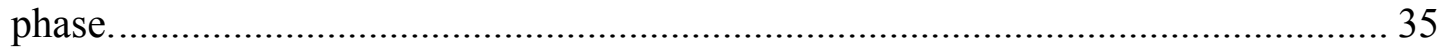

Figure 2.19 Cycles as they are effectively handled by a signal controller, and with variations in signal timing due to skipped phases and early terminations. ................ 37

Figure 3.1 Example graph of detector occupancy, binned in 15-minute intervals.......... 41

Figure 3.2 Example graph of count data, binned in 15-minute intervals....................... 42

Figure 3.3 Example graph of count data, with cycle-by-cycle values plotted................. 42

Figure 3.4 An aerial photo of the Noblesville testbed, showing surrounding land use

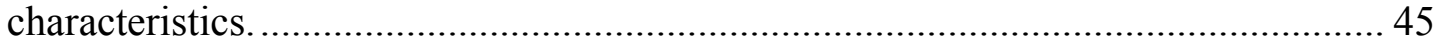

Figure 3.5 Locations of the nearest major intersections from the Noblesville site, placement of advance detectors, and approximate distances to nearby cities. Arrows indicate AADT for each segment................................................................. 46

Figure 3.6 Schematic of the Noblesville testbed, showing the placement of loop detectors.

47

Figure 3.7 Phases at the Noblesville testbed............................................................. 48

Figure 3.8 A map of distances between signalized intersections along SR 37, illustrating opportunities for signal coordination. Arrows indicate AADT along each major segment. 
Figure

Page

Figure 3.9 An aerial photo of Northwestern and Stadium, showing surrounding land use

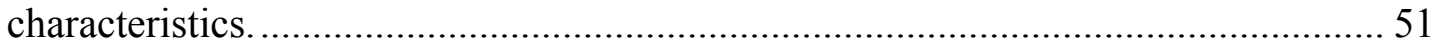

Figure 3.10 Adjacent major intersections from Northwestern and Stadium in West

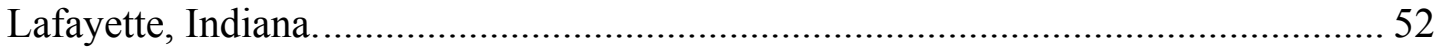

Figure 3.11 Schematic of the West Lafayette testbed, showing the placement of loop

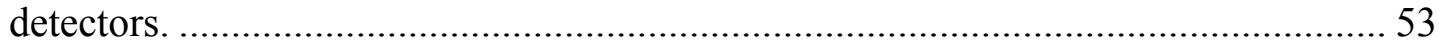

Figure 3.12 Phases at Northwestern and Stadium. ................................................... 54

Figure 3.13 Block diagram showing the data collection and reduction process from start

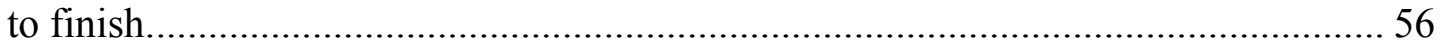

Figure 3.14 Trace of red indications for 8 phases at Noblesville on March 14, 2007, from $10: 55$ to $11: 10$. 62

Figure 3.15 Detail of phase state traces showing impact of clock drift. Phase 1 and phase 2 events are recorded by different clocks........................................................... 63

Figure 3.16 Matching of phases to cycles in data collected at Noblesville on Wednesday,

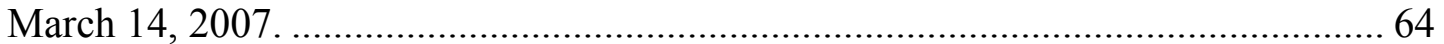

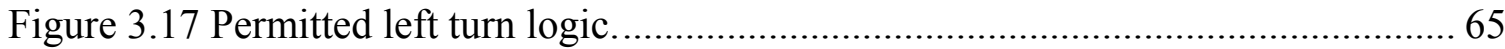

Figure 4.1 Measurement of cycle length from the reference phase red indication and its meaning in relation to the ring diagram. In (a), phase 2 is the reference phase; in (b),

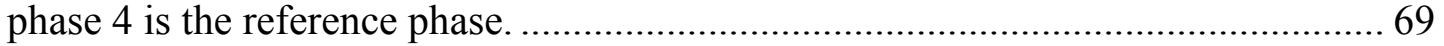

Figure 4.2 Graph of cycle length at Noblesville on Wednesday, March 14, 2007, with TOD plan changeovers marked by dashed lines............................................... 71

Figure 4.3 Measurement of green duration from phase events..................................... 74

Figure 4.4 Graph of green duration at Noblesville for a 24-hour period......................... 76

Figure 4.5 Graph of green duration at Noblesville on Wednesday, March 14, 2007 ,

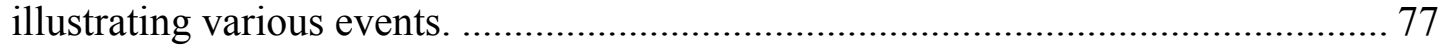

Figure 4.6 Distribution of green times for phases 2 and 5 during the midday time period at Noblesville on Wednesday, March 14, 2007. ................................................... 78

Figure 4.7 Vehicle counting based on phase state and cycle state events. ..................... 81

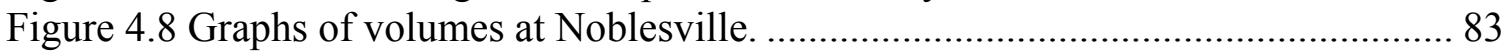

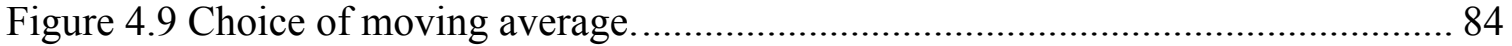

Figure 4.10 Graphs of observed vs. estimated capacity at Noblesville. ......................... 92

Figure 4.11 A comparison of estimated capacity to observed capacity for ring 1 (phases 1-4) for data collected at Noblesville at 10:55:37 on March 14, 2007.................... 93

Figure 4.12 Graphs of Volume-to-Capacity Ratio for Noblesville................................. 98

Figure 4.13 A graph of v/c ratio for phase 5 at Noblesville on Wednesday, March 14, 2007, showing stratification patterns. ..................................................................... 99

Figure 4.14 Camera views of traffic conditions for phase 5 at Noblesville on March 14,

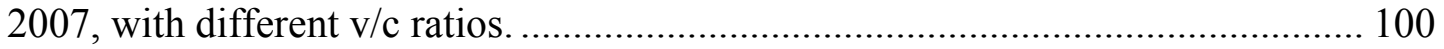

Figure 4.15 Graph of v/c ratio at Noblesville for phase 1, Wednesday, March 14, 2007, with five split failures indicated.................................................................... 102

Figure 4.16 Average v/c ratio of a TOD plan period versus the proportion of phases within the same TOD plan period reporting split failures..................................... 104

Figure 4.17 Possible critical paths through an eight-phase, dual ring controlled intersection. 106 
Figure

Page

Figure 4.18 An example plot of critical v/c ratio at the Noblesville test intersection on Wednesday, March 14, 2007............................................................................. 109

Figure 4.19 Interpolated versus integer arrival type plotted against platoon ratio........ 113

Figure 4.20 Graphs of Arrival Type at Noblesville. ................................................. 115

Figure 4.21 Arrival type calculated for phase 6 at West Lafayette on August 13, 2007.116

Figure 4.22 Graph of time in cycle for phase 2 arrivals over time of day at Noblesville on

Wednesday, March 14, 2007, with green windows represented by the shaded regions.

Figure 4.23 Graph of vehicle arrival distributions at Noblesville. .............................. 122

Figure 4.24 Graphs of cycle profiles at Noblesville on Wednesday, March 14, 2007... 124

Figure 4.25 Synthesis of cycle profiles and arrival distribution graphs, for data collected

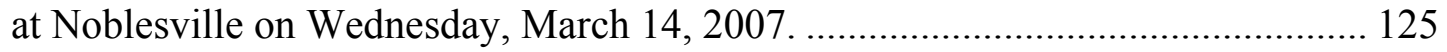

Figure 4.26 Interpretation of platoon profile graphs.............................................. 126

Figure 4.27 An illustration of the data aggregation methodology for statistical

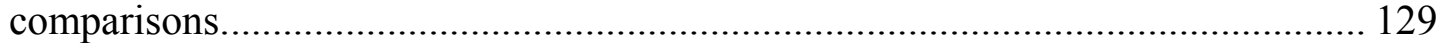

Figure 5.1 Actuated and non-actuated coordinated phases in a typical 8-phase dual ring operating scheme with coordinated phases 2 and 6 ........................................... 134

Figure 5.2 Calendar showing dates of data collection used in the study of fully actuated-

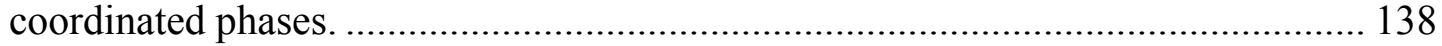

Figure 5.3 Experimental expectations of implementing actuated coordinated phases at the

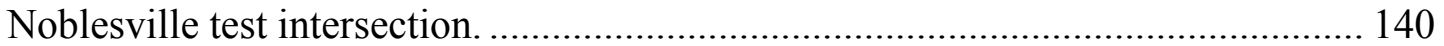

Figure 5.4 Equivalent hourly volumes by phase (20 pt. average), for Wednesday, March 7, 2007 (with non-actuated coordinated phases) and Wednesday, March 28, 2007 (with actuated coordinated phases).

Figure 5.5 Green time histograms for all 8 phases for cycles. Arrows indicate changes in mean values.

Figure 5.6 Plots of green duration by phase (20 pt. average), for Wednesday, March 7 (with non-actuated coordinated phases) and Wednesday, March 28, 2007 (with actuated coordinated phases).

Figure 5.7 Plots of capacity by phase (20 pt. average), for Wednesday, March 7, 2007 (with non-actuated coordinated phases) and Wednesday, March 28, 2007 (with

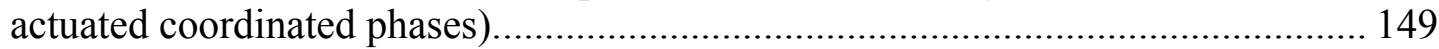

Figure 5.8 Cycle-by-cycle calculations of volume-to-capacity ratio............................ 150

Figure 5.9 Graphs of cumulative frequency of v/c ratios. Arrows indicate change in average value of $\mathrm{v} / \mathrm{c}$ ratio.

Figure 5.10 Total number of split failures by phase at Noblesville on Wednesday, March 7, 2008 (Before, without actuated coordinated phases) and Wednesday, March 28, 2007 (After, with actuated coordinated phases).

Figure 5.11 Total number of split failures for all eight phases at Noblesville on Wednesday, March 7, 2008 (Before, without actuated coordinated phases) and Wednesday, March 28, 2007 (After, with actuated coordinated phases)................ 154

Figure 5.12 Comparison of critical v/c ratio between Wednesday, March 7, 2007 (without actuated coordinated phases) and Wednesday, March 28, 2007 (with actuated coordinated phases). 155 
Figure

Figure 5.13 Distribution of critical path for calculation of critical v/c ratio at Noblesville, showing the prevalence of phase pairs $(5,6)$ and $(3,4)$. 156

Figure 5.14 Arrival type by time of day, for (a) phase 2 and (b) phase 6 for Wednesday, March 7 (with non-actuated coordinated phases) and Wednesday, March 28, 2007 (with actuated coordinated phases).

Figure 5.15 Platoon profile charts for Phase 2 at Noblesville on Wednesday, March 7 and Wednesday, March 28, 2007. 158

Figure 5.16 Platoon profile charts for Phase 6 at Noblesville on Wednesday, March 7 and Wednesday, March 28, 2007.

Figure 5.17 Total number of split failures by phase at Noblesville in all 15 weekdays without actuated coordinated phases (Before), and 15 weekdays with actuated coordinated phases (After). 172

Figure 5.18 Total number of split failures in all eight phases at Noblesville in all 15 weekdays without actuated coordinated phases (Before), and 15 weekdays with actuated coordinated phases (After). 173

Figure 5.19 Percentage of all cycles by phase at Noblesville having split failures in all 15 weekdays without actuated coordinated phases (Before), and 15 weekdays with actuated coordinated phases (After)......

Figure 5.20 Percentage of all cycles in all eight phases at Noblesville having split failures in all 15 weekdays without actuated coordinated phases (Before), and 15 weekdays with actuated coordinated phases (After). 176

Figure 5.21 Ring diagrams showing operation at the Noblesville test intersection (a) with conventional phasing and (b) with lead/lag phasing......................................... 181

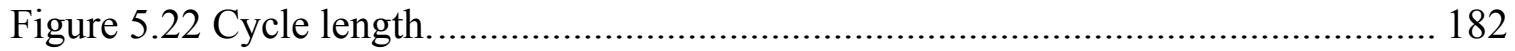

Figure 5.23 Cycle-by-cycle volumes (20 point average) at Noblesville for Monday, June 19, 2006 and Monday, March 12, 2007, with the midday (11:00-13:00) and early evening (19:00-22:00) time periods highlighted. 192

Figure 5.24 Cycle-by-cycle volumes (20 point average) at Noblesville for Wednesday, June 28, 2006 and Wednesday, March 21, 2007, with the AM (6:00-9:00) and PM (15:00-19:00) peak periods highlighted. 193

Figure 5.25 Green time distributions at Noblesville for Monday, June 19, 2006 (before retiming) and Monday, March 12, 2006 (after retiming). 194

Figure 5.26 Green time distributions at Noblesville for Wednesday, June 28, 2006 (before retiming) and Wednesday, March 21, 2006 (after retiming). ............................... 195

Figure 5.27 Cumulative green time for phase 2 at Noblesville during evening hours for Monday, June 19, 2006 (before retiming) and Monday, March 12, 2007 (after retiming). 196

Figure 5.28 Cumulative green time for phase 5 at Noblesville during evening hours for Monday, June 19, 2006 (before retiming) and Monday, March 12, 2007 (after retiming).

Figure 5.29 Cumulative green time for phase 2 at Noblesville during the AM peak period for Wednesday, June 28, 2006 (before retiming) and Wednesday, March 21, 2007 (after retiming). 
Figure

Page

Figure 5.30 Cycle-by-cycle observed capacity (20 point average) at Noblesville for Monday, June 19, 2006 and Monday, March 12, 2007, with the midday (11:0013:00) and early evening (19:00-22:00) time periods highlighted.

Figure 5.31 Cycle-by-cycle observed capacity (20 point average) at Noblesville for Wednesday, June 28, 2006 and Wednesday, March 21, 2007, with the AM (6:009:00) and PM (15:00-19:00) peak periods highlighted. 200

Figure 5.32 Plots of v/c ratio cumulative frequencies at Noblesville for Monday, June 19, 2006 (before retiming) and Monday, March 12, 2006 (after retiming).................. 201

Figure 5.33 Plots of v/c ratio cumulative frequencies at Noblesville for Wednesday, June 28, 2006 (before retiming) and Wednesday, March 21, 2006 (after retiming)....... 202

Figure 5.34 Percentage of cycles at the Noblesville test intersection ending in split failure, shown by phase and by TOD plan time period, averaged over 15 days before retiming and 15 days after retiming. 204

Figure 5.35 Percentage of cycles ending in split failure for coordinated phases 2 and 6 at Noblesville, averaged over 15 days before retiming and 15 days after retiming.... 205 Figure 5.36 Percentage of cycles ending in split failure for non-coordinated phases 1,3 , $4,5,7$, and 8 at Noblesville, averaged over 15 days before retiming and 15 days after retiming. 206

Figure 5.37 Percentage of cycles ending in split failure for all eight phases at Noblesville, averaged over 15 days before retiming and 15 days after retiming. ....................... 207

Figure 5.38 Arrival Type (20 point average) at Noblesville, for Monday, June 19, 2006 (before retiming) and Monday, March 12, 2007 (after retiming), with the 11:0013:00 midday and 19:00-22:00 early evening time periods highlighted................ 208

Figure 5.39 Arrival Type (20 point average) at Noblesville, for Wednesday, June 28, 2006 (before retiming) and Wednesday, March 21, 2007 (after retiming), with the 6:00-9:00 AM and 15:00-19:00 PM peak periods highlighted. 209

Figure 5.40 Platoon profiles for phases 2 and 6 at Noblesville, before and after retiming, during the 11:00-13:00 midday time period. 210

Figure 5.41 Platoon profiles for phases 2 and 6 at Noblesville, before and after retiming, during the 15:00-19:00 early evening time period.......................................... 211

Figure 5.42 Platoon profiles for phases 2 and 6 at Noblesville, before and after retiming, during the 6:00-9:00 AM peak period. 212

Figure 5.43 Platoon profiles for phases 2 and 6 at Noblesville, before and after retiming, during the 15:00-19:00 PM peak period 213 


\section{CHAPTER 1. INTRODUCTION}

\subsection{Objectives}

This study focuses on the collection of performance measures at a traffic signal. This work focuses primarily on measures that can be extracted and logged in real time by an automatic traffic signal controller using information about detector actuations and phase information. This study has two objectives:

- to serve as part of the general effort toward improving data collection at traffic signals by investigating methods that could be applied feasibly within the existing technology; and

- to investigate a body of performance measures that could be calculated on a cycle-by-cycle basis using events recorded in real time, and interpreted by a traffic engineer to evaluate the performance of the signal.

\subsection{Background}

Traffic signals emerged rapidly during the early part of the twentieth century, transforming from manually operated semaphores to electromechanically timed, trafficactuated, electric lights within the space of less than twenty years (1). The impetus for the rapid development of traffic signals was the sudden availability of affordable automobiles to the public. Through the years, traffic signal technology has continued to improve, particularly with the advent of microprocessor technology in the 1950s and 1960s that enabled computer control of signals. The number of road vehicles and the amount of vehicle miles traveled have risen moderately in the US in recent decades and continue to increase disproportionately to the amount of urban road miles. Between 1980 and 2000, urban vehicle miles traveled increased by $85 \%$ whereas the number of urban road miles increased by only $35 \%$ (2). These trends are driving an increasing demand for capacity in the current road system in the US. Elsewhere, similar trends are taking place in developing economies where travel by automobile is gaining market share against other modes. 
Traffic signals are a major component of road systems globally. Improving the operation of signals is a relatively inexpensive way to extract additional capacity from existing infrastructure. For example, retiming signals has been shown to cost approximately $\$ 3,000$ per intersection. In return, travel time reductions between 8 and $25 \%$ are reported, resulting in benefit-cost ratios of $40: 1$ or more (3). A considerable portion of the cost of retiming procedures is incurred due to the time-consuming nature of data collection.

The 2007 National Traffic Signal Report Card (4) analyzes various aspects of traffic signal operation and assigns a letter grade based on a scoring system. The overall score for 2007 was "D," reflecting that there is a lot of potential for improving traffic signal systems nationwide. In particular, the category of "traffic monitoring and data collection" was assigned a score of "F." Overall, there is a widening gap between signal settings and traffic conditions caused by a lack of adequate data collection. The availability of highly accurate information is essential to provide excellent traffic signal operation.

The work presented in this report is aimed at narrowing the gap between actual traffic conditions and the information available to the traffic engineer. A set of performance measures was extracted from data collected in real time at instrumented intersections, using methods that for could be replicated given a $\log$ of intersection events, for which the infrastructure to generate already exists in many signal systems.

\subsection{Organization}

In Chapter 2, fundamental traffic signal operation concepts are reviewed. Definitions are given for basic elements of signal operation schemes. The concepts of actuation and coordination are introduced, and various issues pertaining to these are discussed. This material is presented to characterize the technical environment of this study, forming a basis for the succeeding chapters.

Chapter 3 discusses data collection methods. A brief review of current data collection techniques is given. The data collection sites used in this study are described. The process by which data were collected for this study is outlined.

Chapter 4 presents how performance measures may be calculated using data collected by the methods presented in Chapter 3. Various measures are presented relating to capacity and progression analysis. For each performance measure, example 
calculations and graphs are given. The statistical methods used for comparison of beforeand-after cases in this report are described.

Chapter 5 presents two before-and-after studies showing applications of the performance measures presented in Chapter 4. In the first study, the effects are investigated of implementing fully actuated coordinated signal operation. The second study is more general, studying various impacts of numerous operational changes at an intersection.

Finally, Chapter 6 concludes this report with a summary of the material presented herein. 


\section{CHAPTER 2. REVIEW OF SIGNAL OPERATION CONCEPTS}

\subsection{Introduction}

This chapter reviews general concepts related to traffic signal operation, and describes current technology used in practice. The concepts include basic physical and temporal components of a traffic signal system; coordination between signals in a network; vehicle detection; and basic actuated signal operation. The remainder of this report will build on the concepts conveyed in this chapter.

\subsection{Cycle and Phase}

Traffic signal operation is the art and science of controlling the flow at a signalized intersection by managing the allocation of capacity through the intersection among the set of possible movements through the intersection for which there is demand. Access through the intersection is managed by controlling the length of time which the red, green, and yellow indications are shown to different movements. The goal of the traffic engineer is to provide the best possible service to all vehicles attempting to traverse the intersection. At many signals, there exists strong demand for multiple conflicting movements, which makes the task of the traffic engineer more challenging.

A traffic signal manages capacity by distributing green time among various phases. Each phase represents a movement or set of movements based on the configuration of lanes at each approach of an intersection. The most basic operating scheme is a two-phase controller, as shown in Figure 2.1. Figure 2.1(a) shows the intersection of two two-way streets, while Figure 2.1(b) shows the ring diagram for this type of operation. This diagram represents the division of time between the two phases. It is called a "ring" because the pattern repeats itself perpetually. The arrows in the diagram correspond to vehicle movements, while the dashed lines indicate pedestrian movement. In the two-phase scheme, all movements made by vehicles on each street must be made within a phase corresponding to the street. All movements made from the north-south street are made in phase A, while all movements made from the east-west street are made in phase B. The through movements (both vehicle and pedestrian) have the right of way, 
while turning movements are permitted. Left-turning vehicles must yield to opposing through traffic. Most early traffic signals operated in this manner.

The amount of time that it takes for each of the two phases to be served is called the cycle length, and the proportion of the cycle length that is taken up by each phase is called the split of that phase. The relationships between cycle length, split, and the signal indications for each phase are shown in Figure 2.2. The relative size of the splits are adjusted by the traffic engineer based on expectations of the demand (i.e., volumes) for each set of movements. Each phase goes through three indications: green, yellow, and red. The red indication can be divided into two intervals. The all-red clearance interval immediately precedes yellow, and is included to promote safety by permitting vehicles that entered the intersection during yellow to exit before allowing the next set of movements to proceed. Each phase must also be red at times when the other phase is being served.

The two-phase signal configuration is still used when turning movements are minimal or not allowed due to heavy traffic. Left turns in particular are difficult for an intersection such as the one in Figure 2.1 to handle. A northbound left turning vehicle yielding to southbound through traffic prevents northbound through vehicles from clearing the intersection. If southbound volume is high, the left turner will probably have to make the turn during yellow or in the red, if the southbound vehicles take advantage of the yellow. Turning lanes help alleviate these problems. A number of phase configurations exist for intersections with turn lanes, but the most widely used configuration is the dual-ring eight phase configuration, which is shown in Figure 2.3.

A typical assignment of phases in a dual-ring eight phase controlled intersection is shown in Figure 2.3(a). This figure shows an intersection with four two-way approaches, each having channelized left and right turn lanes. The numbers represent the phase numbers for each movement. Typically, right turns share phases with adjacent through movements. Right turns can also be made with the left turns for the cross street. These are referred to as overlaps, indicated by the letters. For example, the northbound right turn has the green indication during phase 2 . This movement is not protected, as it must yield to pedestrians in the crosswalk. It could also move during phases 3 and 7 , as the movements do not conflict. This can be made possible by allowing right turns on red (RTOR) or by the use of a right-turn arrow assigned to overlap A. Pedestrian movements are indicated by the dashed lines inside of the crosswalks. Pedestrian phases are numbered according to the through movement that they cross to the right of. Usually, through movements on the major street correspond to phases 2 and 6; in other words, phase 2 need not be northbound. 
A typical ring diagram is shown in Figure 2.3(b), which explains the nomenclature "dual ring." The eight phases are separated into two rings (1234 and 5678) that operate concurrently. A phase cannot run at the same time as another in its ring, but they can run with some phases in the other ring. Barriers are shown by thick black lines in the figure; no phase may operate at the same time as one on the opposite side of a barrier. Phase 1, for example, may run at the same time as either 5 or 6 ; but it cannot run with 2, which is in the same ring; and it cannot run at the same time as phases $3,4,7$, or 8 , which are on the other side of the barrier.

There are three ways to pair phases within the sets delineated by barriers. Each of these pairs corresponds to a specific relationship between phases:

- Conflicting movements are those on the same side of a barrier which cannot run at the same time; they are adjacent in the ring diagram. These pairs are $(1,2),(3,4)$, $(5,6)$, and $(7,8)$.

- Complementary movements have a symmetric partner on the opposite side of the intersection. These movements do not conflict with each other (with the exception of intersections where the geometry causes movements to share a limited space). They typically run at the same time. These phase pairs are $(1,5),(2,6),(3,7)$, and $(4,8)$.

- Adjacent movements share a particular approach. These movements do not conflict with each other. These pairs are $(1,6),(2,5),(3,7)$, and $(4,8)$.

Table 2.1 shows the calculation of durations of the green indication for an eight phase intersection based on percentage splits and known clearance intervals, based on cycle lengths of 60,90 , and $120 \mathrm{~s}$. The split-based estimation of the green indication for phase $i$ is found by

$$
\hat{G}_{i}=\left(\frac{S_{\%}}{100} \times C\right)-\left(Y_{i}+R_{i}\right),
$$

where $S_{\%}$ is the split in percent; $C$ is the cycle length; $Y_{i}$ is the length of the yellow indication; and $R_{i}$ is the length of the red clearance interval. Because $Y_{i}$ and $R_{i}$ are constants, the amount of time in which the signal is green for any movement increases as $C$ increases. This implies that as we increase the cycle length, we also improve the efficiency of the intersection. The tradeoff is that with longer cycle lengths, the waiting time for vehicles increases. 
Splits are chosen based on the relative demand for movements at an intersection. Figure 2.4(a) shows a ring diagram scaled to the split proportions given in Table 2.1. Note that the divisions between phases within a barrier are staggered across the two rings. Phase 1, for example, runs concurrently with phases 5 and 6 . This implies that the traffic engineer expects phase 1 to have more demand than phase 5. Figure 2.4(b) shows the division of phase 2 into green, yellow, and all-red clearance intervals. The total duration of the split is found from multiplying the split proportion by the cycle length. The estimated green duration is found after subtracting the clearance interval from the total split in seconds.

Clearance intervals are based on intersection geometry; the length of the interval is intended to reflect the amount of time for vehicles to safely exit the intersection before allowing the next movement to proceed. The yellow interval corresponds to the amount of time that the yellow indication is given to a phase, which alerts drivers that they must prepare to stop or finish exiting the intersection. The red interval is a buffer that allows vehicles to safely complete maneuvers before allowing the same space to be used by the next group. The total clearance interval is found by (7)

$$
Y_{i}+R_{i}=T_{r}+\frac{V}{2 a+64.6 G}+\frac{w+L}{V},
$$

where $T_{r}$ is the average perception-reaction time of drivers in s (typically $1 \mathrm{~s}$ ), $V$ is the approach speed in fps; $a$ is the design acceleration rate (typically $10 \mathrm{ft} / \mathrm{s}^{2}$ ); $G$ is the decimal grade; $w$ is the intersection width in $\mathrm{ft}$; and $L$ is vehicle length in $\mathrm{ft}$ (typically 20 $\mathrm{ft}$ is used).

The amount of yellow time is given by

$$
Y_{i}=T_{r}+\frac{V}{2 a+64.6 G}
$$

while the amount of all red clearance time is found by

$$
R_{i}=\frac{w+L}{V}
$$

At many intersections, relative demand for movements at an intersection varies by time of day and by day of the week. For example, traffic volumes during the morning peak hour often mirror volumes during the evening peak hour, and weekend traffic 
patterns can be considerably different from weekday patterns. Because of this, traffic signal controllers are programmed to run different cycle lengths and split proportions at different times of day on different days of the week. This is referred to as a time of day (TOD) plan. An example TOD plan is shown in Table 2.2. This is the TOD plan used at the test intersection in Noblesville, Indiana. Cycle length and splits can be seen to vary from one time period to the next. For example, the southbound movement carried by phase 6 is given a 37\% split in the 6:00-9:00 time period, in comparison to splits as low as $25 \%$ during other times. The term "Free" appears during times of day when the signal operates in free mode. Cycle length is not fixed during those times, and each phase in the cycle is served when vehicles arrive. That process, called actuation, is discussed in Section 2.4 . 


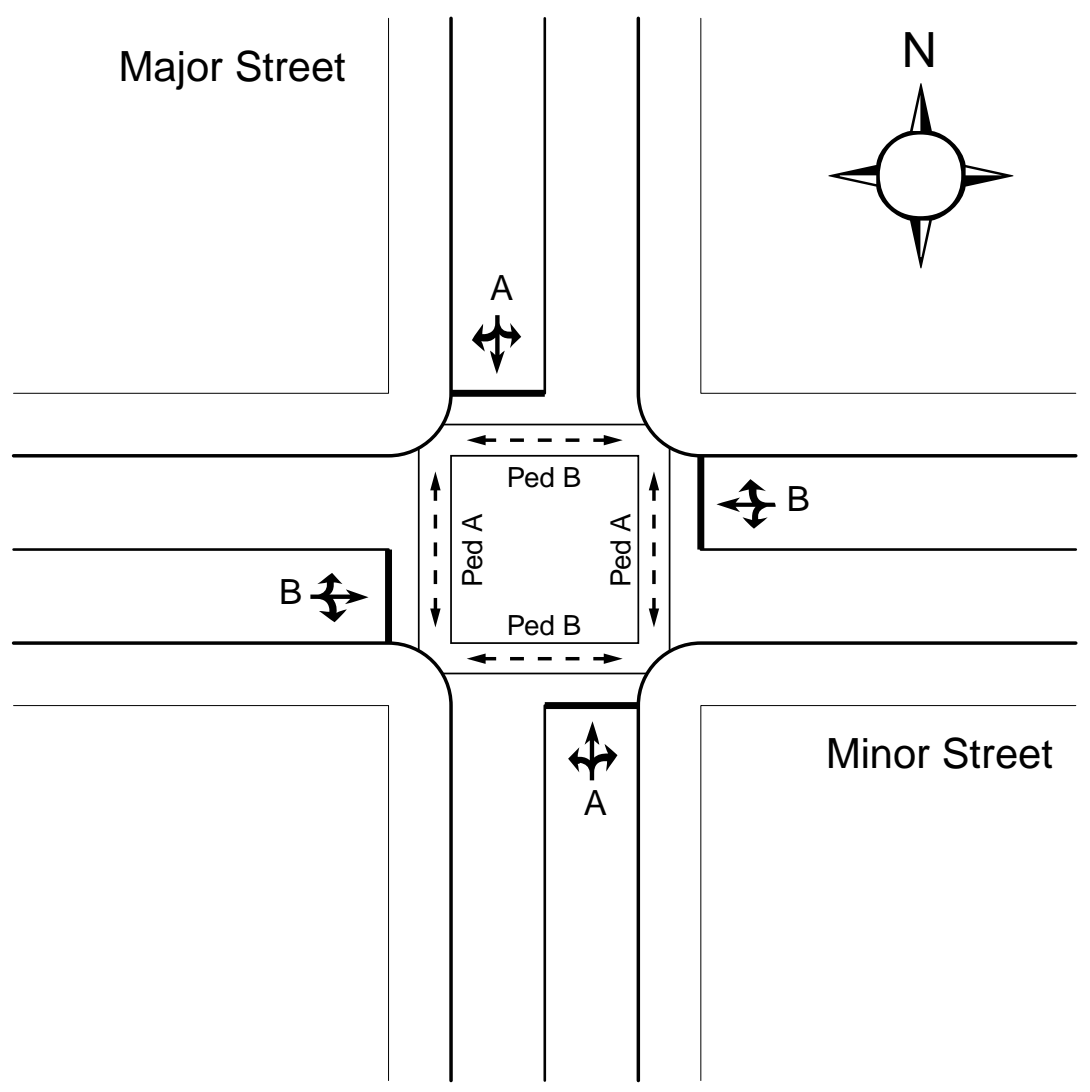

(a) Intersection layout with a two-phase signal.

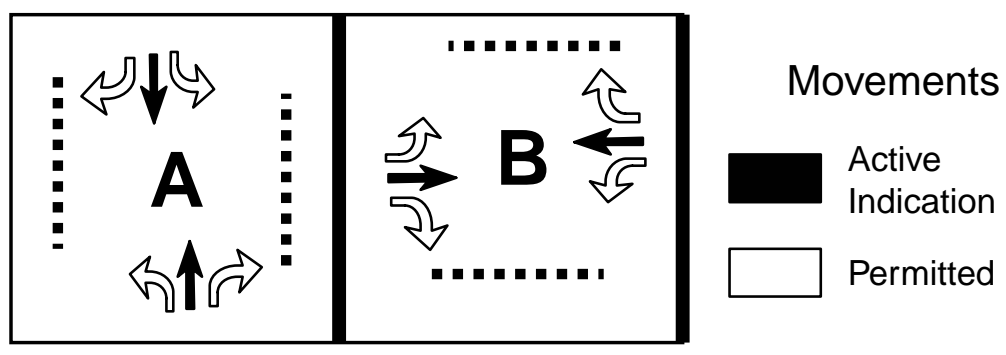

(b) Diagram of phases in a two-phase signal.

Figure 2.1 A two-phase signal. 


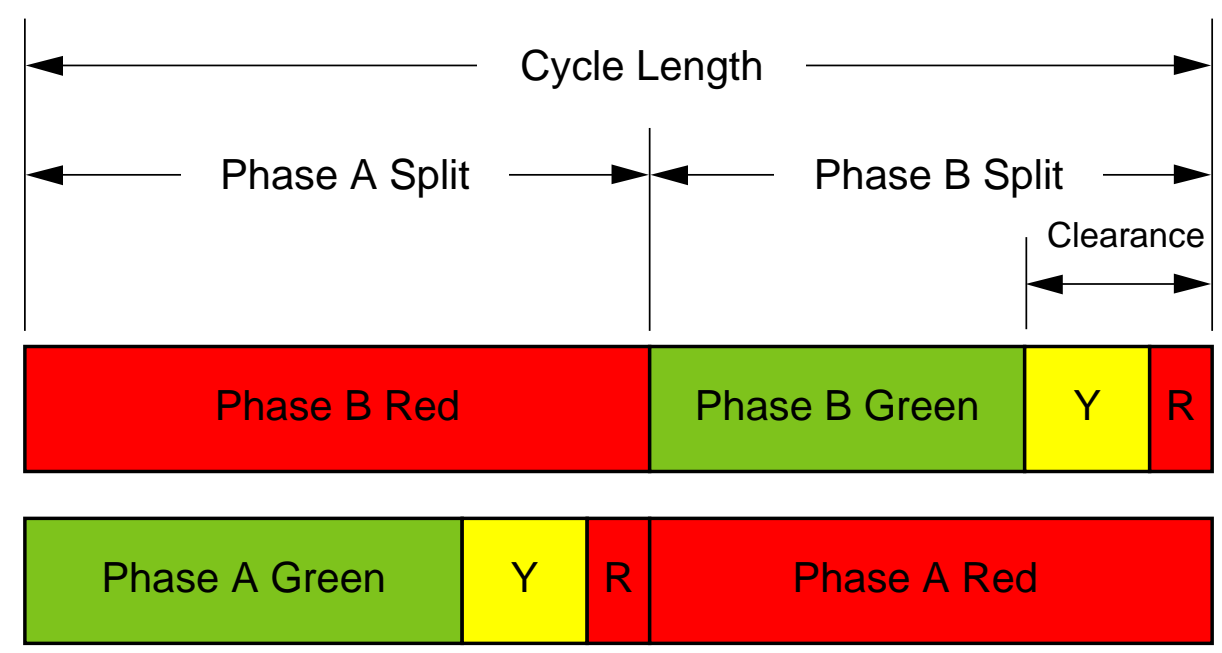

Figure 2.2 Cycle length and splits for a two-phase controller. 


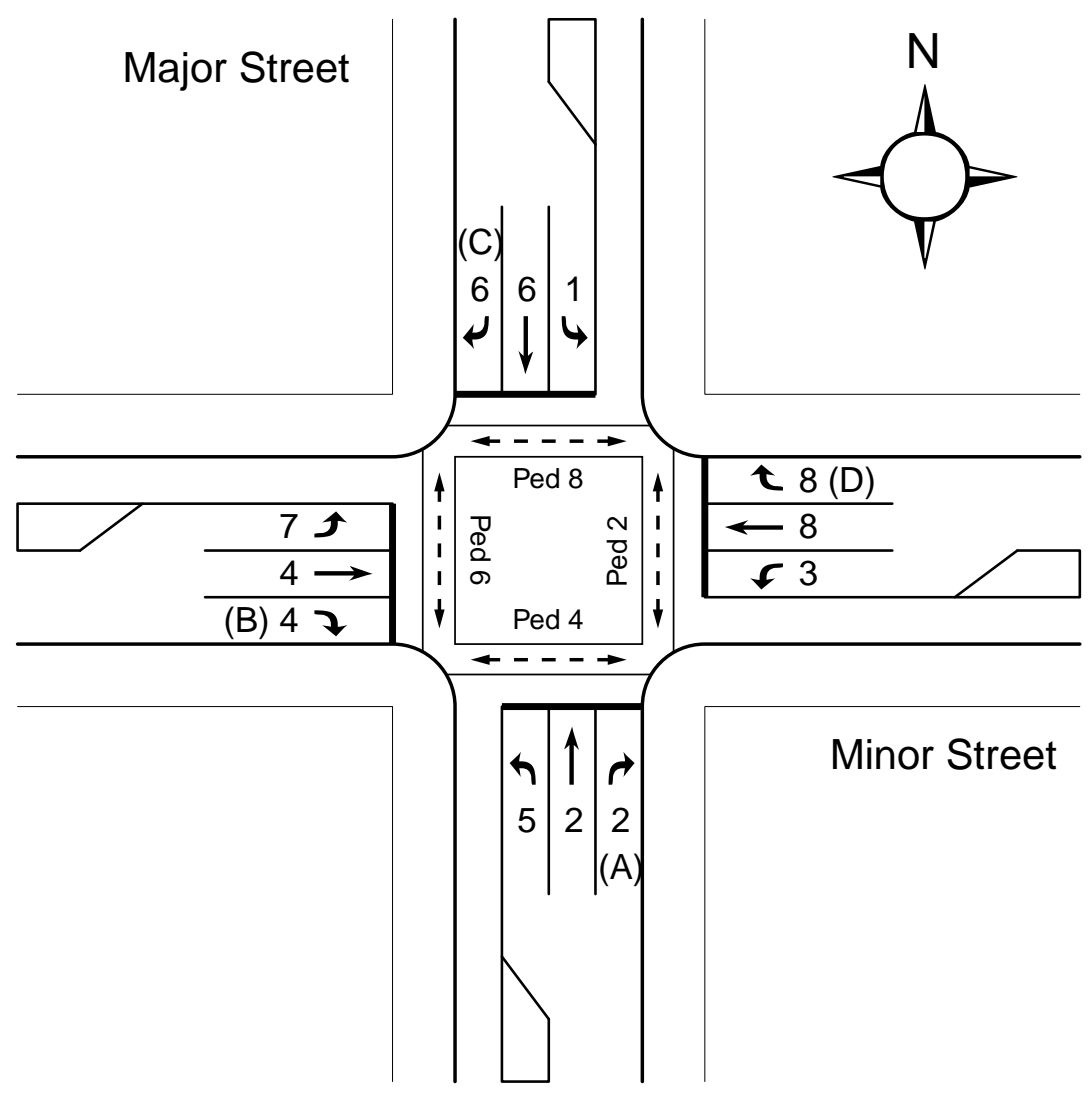

(a) Intersection layout with a dual-ring, eight phase controller

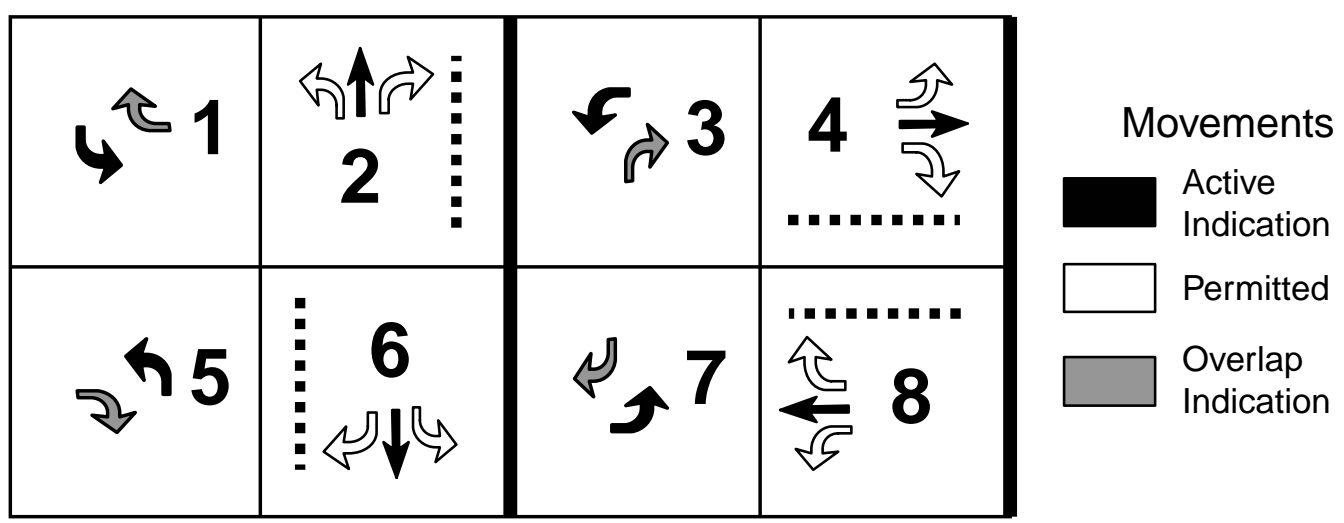

(b) Ring diagram for a dual-ring eight phase controller.

Figure 2.3 Dual-ring eight phase controller configuration. 
Table 2.1 Calculation of the duration of green indications from splits by cycle length, showing increasing proportion of green time with increasing cycle length.

\begin{tabular}{|c|c|c|c|c|c|c|}
\hline \multirow{2}{*}{ Phase } & \multicolumn{2}{|c|}{ Clearance Interval (s) } & \multirow{2}{*}{ Split (\%) } & \multirow{2}{*}{$\begin{array}{l}\text { Green (s) } \\
\text { for } C=60\end{array}$} & \multirow{2}{*}{$\begin{array}{c}\text { Green (s) } \\
\text { for } C=90\end{array}$} & \multirow{2}{*}{$\begin{array}{c}\text { Green (s) for } \\
\mathrm{C}=120\end{array}$} \\
\hline & Yellow & Red & & & & \\
\hline 1 & 2 & 1.5 & 15 & 5.5 & 10 & 14.5 \\
\hline 2 & 3 & 1.2 & 40 & 19.8 & 31.8 & 43.8 \\
\hline 3 & 2 & 1.5 & 15 & 5.5 & 10 & 14.5 \\
\hline 4 & 3 & 1.2 & 30 & 13.8 & 22.8 & 31.8 \\
\hline 5 & 2 & 1.5 & 10 & 2.5 & 5.5 & 8.5 \\
\hline 6 & 3 & 1.2 & 45 & 22.8 & 36.3 & 49.8 \\
\hline 7 & 2 & 1.5 & 20 & 8.5 & 14.5 & 20.5 \\
\hline 8 & 3 & 1.2 & 25 & 10.8 & 18.3 & 25.8 \\
\hline
\end{tabular}

\begin{tabular}{|r|r|r|r|}
\hline Total Green, phases $1,2,3,4(\mathrm{~s})$ & 44.6 & 74.6 & 104.6 \\
\hline Total Y + R, phases $1,2,3,4(\mathrm{~s})$ & 15.4 & 15.4 & 15.4 \\
\hline Total Green, phases $1,2,3,4(\%$ of Cycle $)$ & 74 & 83 & 87 \\
\hline
\end{tabular}

\begin{tabular}{|r|r|r|r|}
\hline Total Green, phases 5,6,7,8 (s) & 44.6 & 74.6 & 104.6 \\
\hline Total Y + R, phases 5, 6, 7,8 (s) & 15.4 & 15.4 & 15.4 \\
\hline Total Green, phases 5,6,7,8 (\% of Cycle) & 74 & 83 & 87 \\
\hline
\end{tabular}




\begin{tabular}{|c|c|c|c|}
\hline $\mathbf{1}$ & $\mathbf{2}$ & $\mathbf{3}$ & \multicolumn{1}{|c|}{$\mathbf{4}$} \\
$15 \%$ & $40 \%$ & $15 \%$ & $30 \%$ \\
\hline $\mathbf{5}$ & $\mathbf{6}$ & $\mathbf{7}$ & $\mathbf{8}$ \\
$10 \%$ & $45 \%$ & $20 \%$ & $25 \%$ \\
\hline
\end{tabular}

(a) Division of cycle into splits.

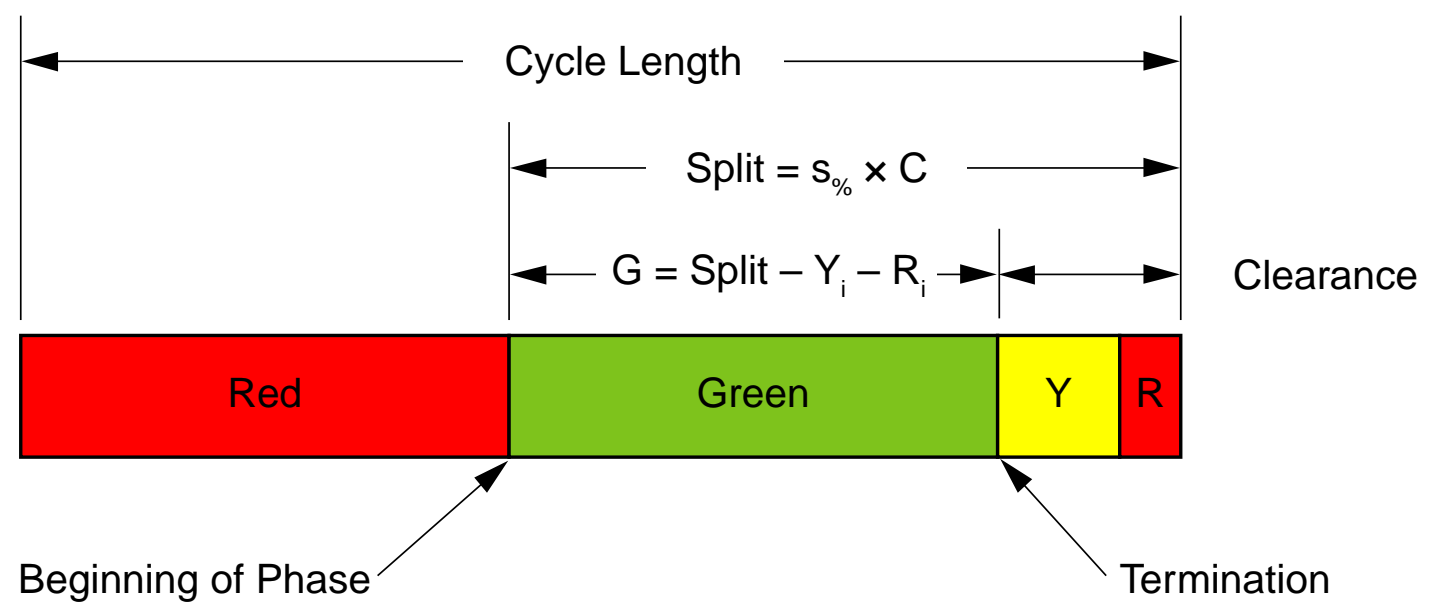

(b) Phase indications shown in the context of the split and cycle length.

Figure 2.4 Basic divisions of time by traffic signals. 
Table 2.2 TOD plan showing cycle length and proportional splits for weekday operation at the instrumented intersection at Noblesville, IN.

\begin{tabular}{|r|r|c|c|c|c|c|c|c|c|}
\cline { 3 - 11 } \multicolumn{2}{c|}{} & \multicolumn{4}{c|}{ Splits, Ring 1 } & \multicolumn{4}{c|}{ Splits, Ring 2 } \\
\hline Begin Time & C (s) & 1 & 2 & 3 & 4 & 5 & 6 & 7 & 8 \\
\hline $0: 00$ & Free & Free & Free & Free & Free & Free & Free & Free & Free \\
\hline $6: 00$ & 116 & 0.12 & 0.41 & 0.22 & 0.25 & 0.16 & 0.37 & 0.11 & 0.36 \\
\hline $9: 00$ & 96 & 0.14 & 0.37 & 0.15 & 0.34 & 0.22 & 0.29 & 0.14 & 0.35 \\
\hline $11: 00$ & 102 & 0.13 & 0.41 & 0.16 & 0.30 & 0.25 & 0.29 & 0.13 & 0.33 \\
\hline $13: 00$ & 104 & 0.13 & 0.33 & 0.14 & 0.40 & 0.21 & 0.25 & 0.13 & 0.41 \\
\hline $15: 00$ & 116 & 0.12 & 0.38 & 0.14 & 0.36 & 0.20 & 0.30 & 0.12 & 0.38 \\
\hline $19: 00$ & 104 & 0.13 & 0.33 & 0.14 & 0.40 & 0.21 & 0.25 & 0.13 & 0.41 \\
\hline $22: 00$ & Free & Free & Free & Free & Free & Free & Free & Free & Free \\
\hline
\end{tabular}




\subsection{Effective Green Time}

Motorist utilization of the green time provided by the signal is described in Chapter 10 of the Highway Capacity Manual (HCM). The phase indications given at an intersection regulate traffic flows, but there is a delay between indication changes and activity changes at the intersection. For example, at the start of green, it takes a few seconds for motorists to react to the green indication, and for the vehicles to start to move. At the end of green, motorists use a portion of the clearance interval for movement, effectively extending the green.

Figure 2.5 shows a comparison of the effective green to the controller green. A typical graph of the flow rate is shown over the phase indications. The time in which the movement corresponding to this phase could move is equal to the green indication plus the yellow and red clearance interval $(\mathrm{Y}+\mathrm{R})$. At the beginning of the green indication, the flow rate is zero; it takes a certain amount of time to achieve the saturation flow rate. Finally, at the end of the cycle, after the yellow indication is given, flow rates drop off, taking a certain amount of time to do so. The remaining portion of the clearance interval is lost.

The total lost time $t_{L}$ for phase $i$ is equal to

$t_{L}=I_{1}+I_{2}=I_{1}+\left(Y_{i}+R_{i}\right)-e$

Equation 2.5

where $I_{1}$ is the start-up lost time, $I_{2}$ is the clearance lost time, $Y_{i}$ is the length of the yellow indication, $R_{i}$ is the length of the all-red clearance interval, and $e$ is the amount of the total clearance interval that is used by vehicles. All of these times are given in seconds. The effective green is given by

$g_{i}=G_{i}+\left(Y_{i}+R_{i}\right)-t_{L}$

Equation 2.6

where $G_{i}$ is the length of the green indication. The HCM suggests using a value of the lost time equal to the clearance interval. This is based on the assumptions that $I_{1}=2 \mathrm{~s}$ and $e=2$ s. However, the HCM also suggests that in congested conditions, $e$ is sometimes longer than 2 s. For real-time performance monitoring, we are primarily interested in performance during congested conditions, so we use a value of total lost time $t_{L}$ equal to $2 \mathrm{~s}$ in Equation 2.6. 


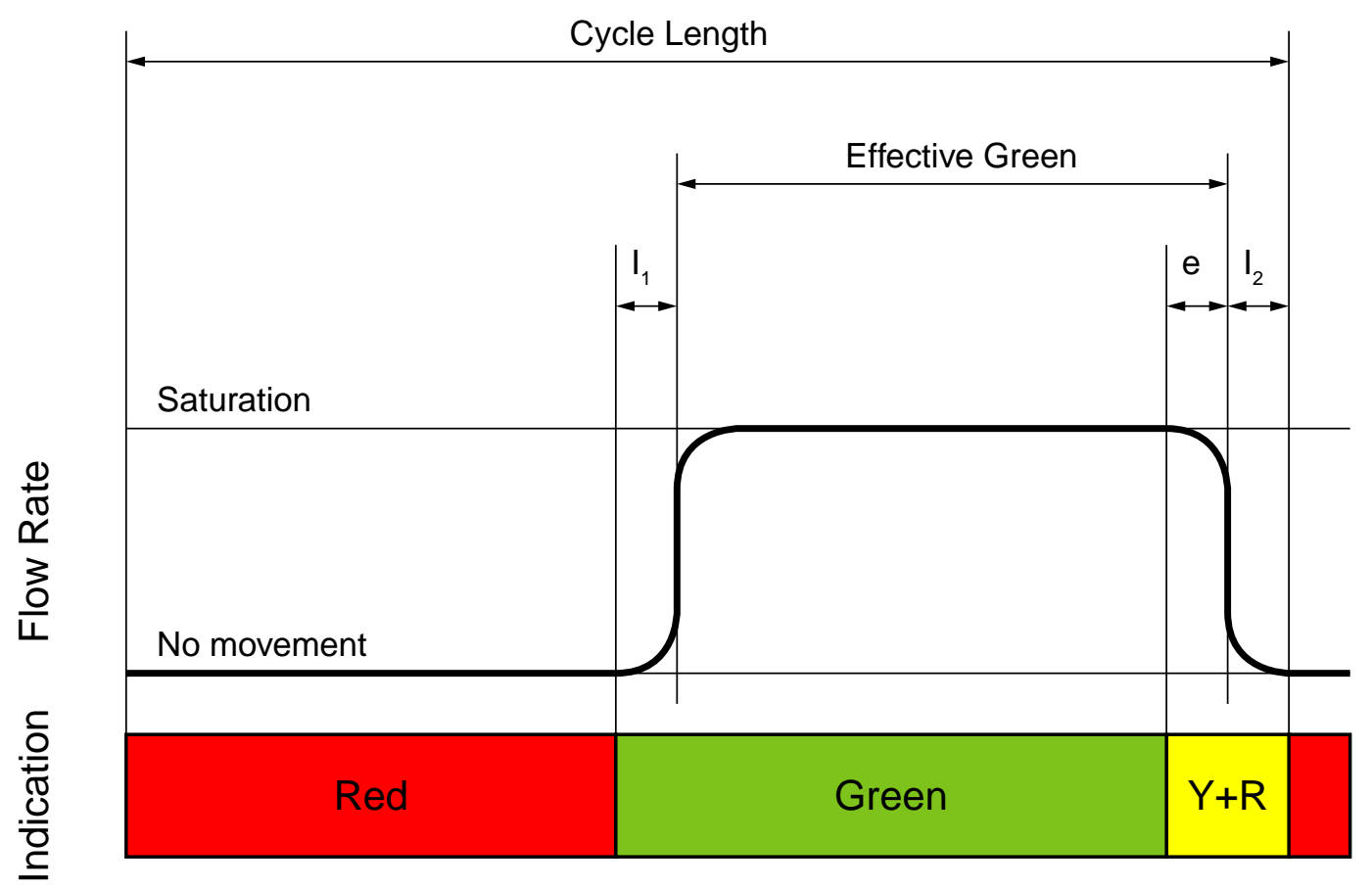

Figure 2.5 Effective green as defined by the HCM. 


\subsection{Detection and Actuation}

Detectors are the eyes and ears of a signal controller. A signal controller may adjust the distribution of green time by interpreting the information about demand gathered by detectors. In this section, we describe how this is accomplished, as well as the limitations of those methods.

There are a number of technologies for vehicle detection, but this work will focused entirely on inductive loop detectors, as these are the most widely used at present. An inductive loop detector consists of a loop of wire placed in the pavement. A typical installation is shown in Figure 2.6. The ends of the wire are connected via conduit to a signal amplifier in the traffic cabinet. An oscillating voltage is placed on the loop. When a metal object passes over the loop, a small portion of the oscillation energy is absorbed by the metal object by the principle of electrical induction. This is sensed as a very small change in the oscillation voltage. Inside of the cabinet, the voltage is monitored by an "amplifier," which not only amplifies the signal in order to detect the small changes in voltage, but also returns the information about whether a vehicle has been detected to the other equipment in the cabinet.

Figure 2.7(a) shows a typical detection zone at the stop bar of an intersection. Multiple detectors are connected in series, as shown in Figure 2.7(b), to produce a wide detection zone. It is advantageous to use a wide detection zone close to a stop bar, since vehicles stop at different distances from the stop bar itself. It is common to create a detection zone extending forward of the stop bar, as illustrated by Figure 2.7(a). Detectors are also placed rather far from the stop bar in order to detect vehicles as they arrive at the intersection. These are called advance or setback detectors.

Two ways in which the amplifier may report vehicle detections are illustrated in Figure 2.7(c). The "presence" mode of the amplifier sends a high output signal as long as there is an object within the detection zone. The "count" mode sends only a short pulse when the object first arrives. Presence mode allows the calculation of detector occupancy, which is a commonly used performance measure for analyzing signal operation. However, overlapping vehicles at a detector make presence data ineffective for determining vehicle counts. This is shown in Figure 2.8. The analog signal shows the loop voltage; each passing vehicle causes the voltage to decrease by a certain amount. Overlapping vehicles are individually counted when count mode is used. We may identify each arriving vehicle using the leading edge of the pulse. However, if the same logic is applied to presence mode output, only two "vehicles" are detected. 
Actuation is the name given to the process by which the signal grants the green indication to a phase based on the presence of vehicles waiting for the associated movement. An arriving vehicle that is detected places a "call" for a particular phase, which is then serviced by the controller as soon as possible. Signal controllers can also place calls internally. The logic involved in carrying out this process is the topic of the remainder of this section. The amount of time that each movement must wait for its turn is a consequence of the chosen controller logic.

There are three basic types of signal operation based on the amount of detection that is used:

- Pretimed signals do not use detection at all, but provide green to movements based on fixed splits. The splits are adjusted according to predicted traffic volumes. Cycle lengths are constant.

- Semi-actuated signals have detection only on the minor street and in the left turn lanes of the major street. When vehicles arrive at any of the access points for these movements, the related phases are served as soon as possible, in the order of the ring diagram, phases without calls are skipped. The green indication defaults to the major through movement once the signal is finished serving the minor streets. This is required, since without detectors that movement cannot be actuated. Cycle lengths may or may not be constant.

- Fully-actuated signals have detection on all approaches, including the major movement. Each phase is served after vehicles arrive for it. Because there are detectors on the main streets, it is not required to automatically return the green to these movements. Cycle lengths may or may not be constant.

The amount of green time used by a phase is determined by the signal controller according to various rules. Fixed-time phases must begin and end at fixed points in time. This is usually done at actuated signals to facilitate coordination with other signals in the network; this is discussed in the next section. Actuated phases begin after a call has been placed for them. Each phase has a minimum and maximum green time set by the traffic engineer in addition to the splits. Signal controllers will not violate minimum green times. The rules for terminating a phase may require termination sooner than the maximum green based on the need to serve other phases in a fixed cycle length. The point in time when a phase must terminate for this reason is called a force-off point. A phase may be forced off before it achieves its maximum time, depending on the status of the other phases. This is discussed more in the following section. 
When setback detectors are used, it is possible to count the number of vehicles that arrive during the red indication for a phase. Once that count exceeds the number of vehicles that could be served during the minimum green, additional green time may be provided for each additional arrival. This is called variable initial timing. This is illustrated in Figure 2.9.

Termination of an actuated phase occurs because the green extends to its maximum time or force-off point ("max out"), or because demand for the movement drops off, detected by a period of detector inactivity ("gap out"). Max out logic is straightforward; there are two methods of measuring gaps.

- In the gap reduction method, the phase is terminated once a period of detector inactivity is measured to be longer than the allowable gap. The initial allowable gap is fairly long, because speeds at the beginning of green are expected to be small. After an amount of time called the "time before reduction," the allowable gap is decreased by the controller to a minimum value. This reflects an expectation of increasing speed as the green continues. The reduction of the allowable gap time is shown in Figure 2.10.

- The green extension method causes the green to be extended by a fixed amount with each vehicle arrival. The phase is terminated if another vehicle does not arrive before the extension expires.

Figure 2.11(a) compares the two types of gap out logic. The upper portion of the graph shows the allowable gap as described by Figure 2.10, with the measured gap superimposed. The controller begins measuring gaps as soon as the initial green ends. Once a gap is measured to be greater than the allowable gap, the phase terminates by the gap reduction method [marked by "Gap Out (GR)"]. The lower portion of the graph shows the green extension method. Every vehicle arrival extends the green by a fixed amount of time, starting from that vehicle's time of arrival. Two vehicles were served during the initial green period. Vehicle A produces a green extension that expires before the initial green has ended. Vehicle $\mathrm{B}$ causes the green to extend beyond the initial green; Vehicle $\mathrm{C}$ arrives in that time and extends the phase further. Finally, after Vehicle D, no more vehicles arrive during the extension period and the phase terminates [marked by "Gap Out (GE)"]. Vehicle E, arriving after the end of green in both cases, must wait for the next cycle. In this example, the gap reduction method results in a longer green time than the green extension method, but this is not generally true. 
Figure 2.11(b) shows the termination of the phase when it maxes out. In this example, there were enough vehicle arrivals to prevent either green extension or gap reduction to cause the phase to terminate.

A phase may be prevented from gapping out if simultaneous gap out logic is in place. It is usually desirable to have two phases that end on a barrier to terminate at the same time. In other words, the barrier is crossed in both rings simultaneously. For example, in the typical eight-phase ring diagram shown in Figure 2.3(b), suppose that phases 2 and 6 begin at the same time. If phase 6 terminates early while phase 2 is still serving a large number of vehicles, then additional phase 6 arrivals are obliged to wait till the next cycle, even though there is no reason why they could not proceed. With simultaneous gap out logic, the green for phase 6 is held until phase 2 terminates.

A similar concept is that of dual entry. Under dual entry, whenever a phase is given the green indication, the controller is required to give the green to a non-conflicting phase in the opposite ring. This could either be the complementary (i.e., geometrically opposing) or adjacent phase. For example, if phase 5 is called, the controller would need to call either phase 1 or 2 to satisfy dual entry. Under single entry, phase 5 alone would be called.

At most intersections, each approach has one detection zone, regardless of the number of lanes within the zone. That is, detection in not differentiated between lanes. It has been shown that lane-by-lane detection is superior to this form of detection (9). With lane-by-lane detection, the phase gaps out after each of the two lanes gaps out separately. Without this distinction, the phase tends to be extended for a long time, even the flow rate drops off, because a low flow rate across two lanes appears as a high flow rate when the two lanes are combined. This is mentioned here because the intersections in this study operate under lane-by-lane detection. 


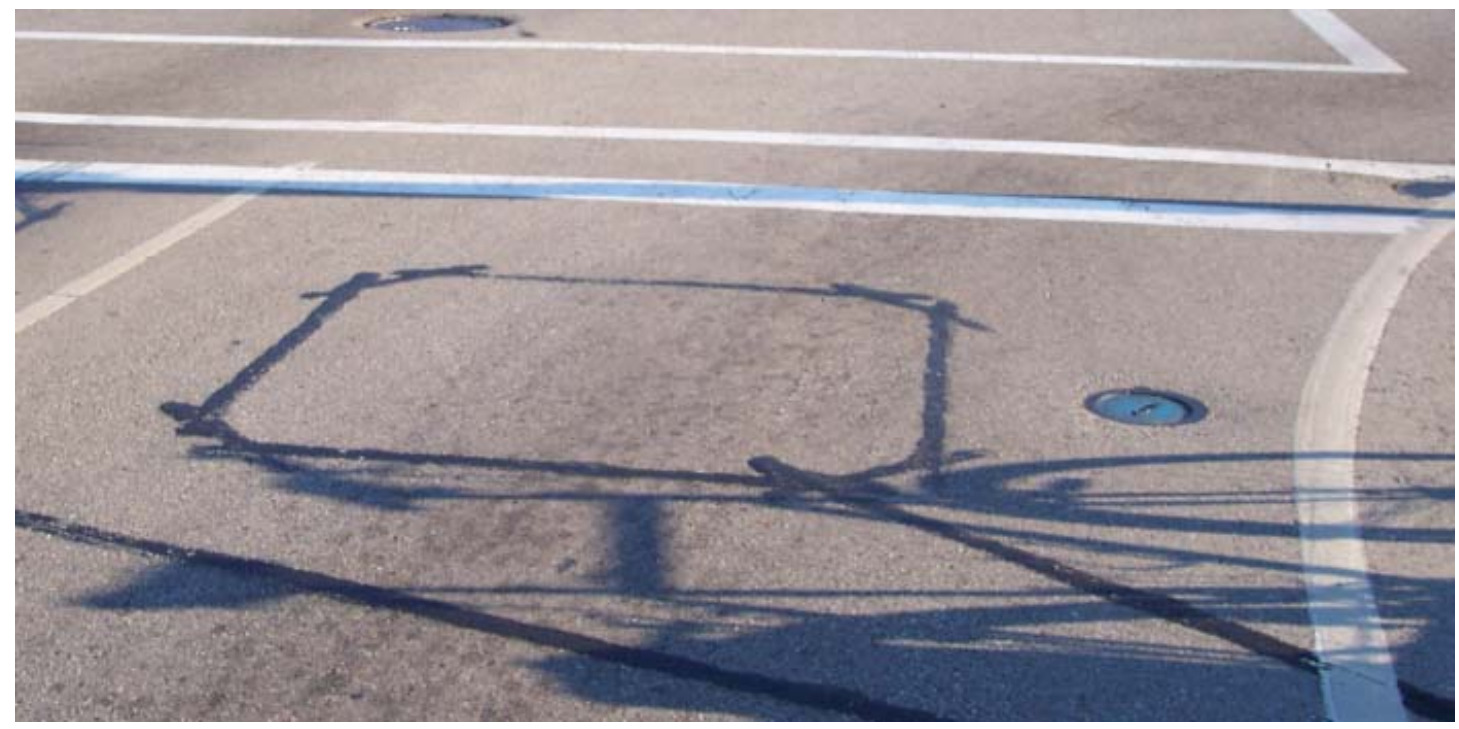

Figure 2.6 Picture of a loop detector installation. 
(a)

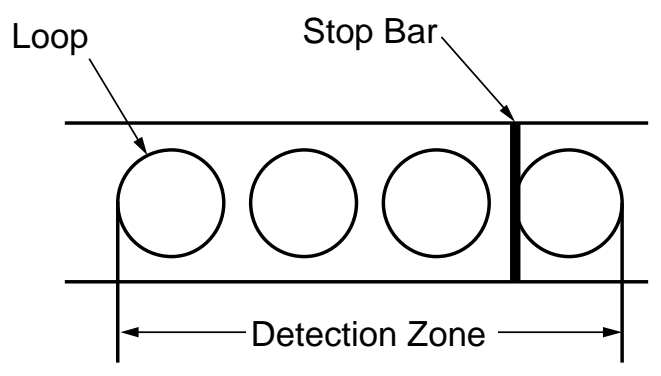

(c)

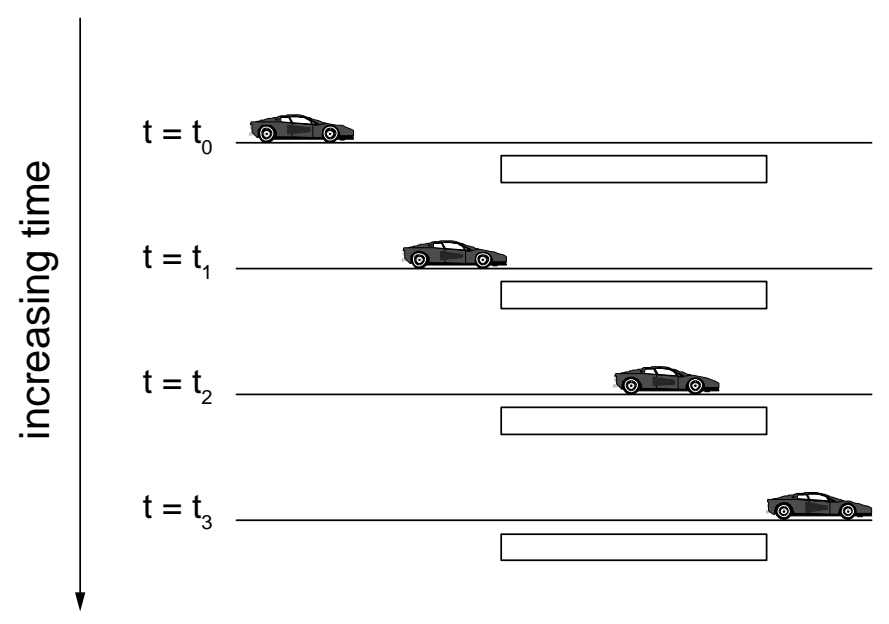

(b)
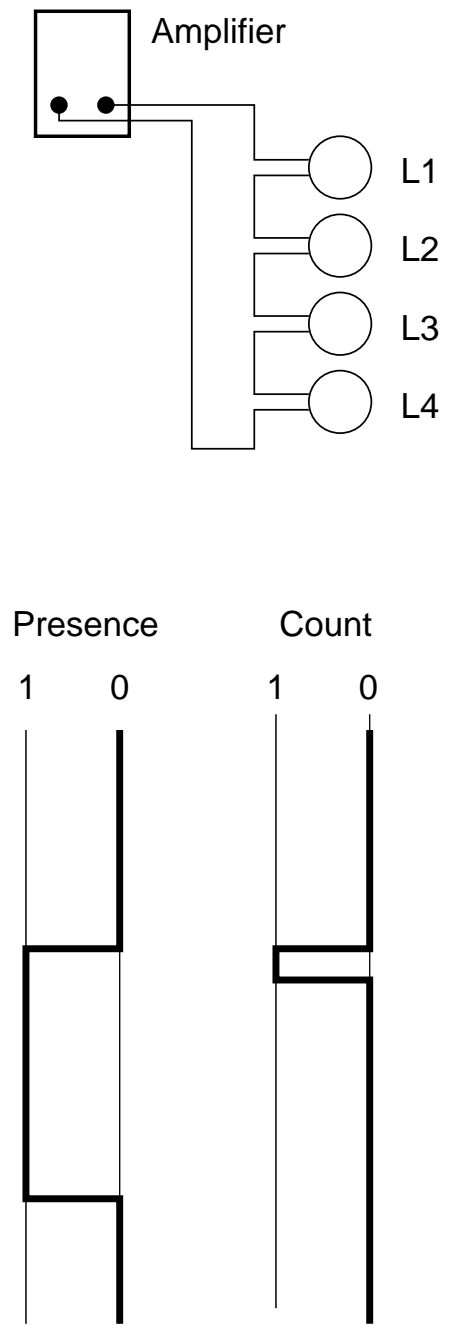

Figure 2.7 (a) Typical appearance of loop detectors in field. (b) Basic electrical diagram for loops. (c) Detector output signals operating in presence and count detection modes. 


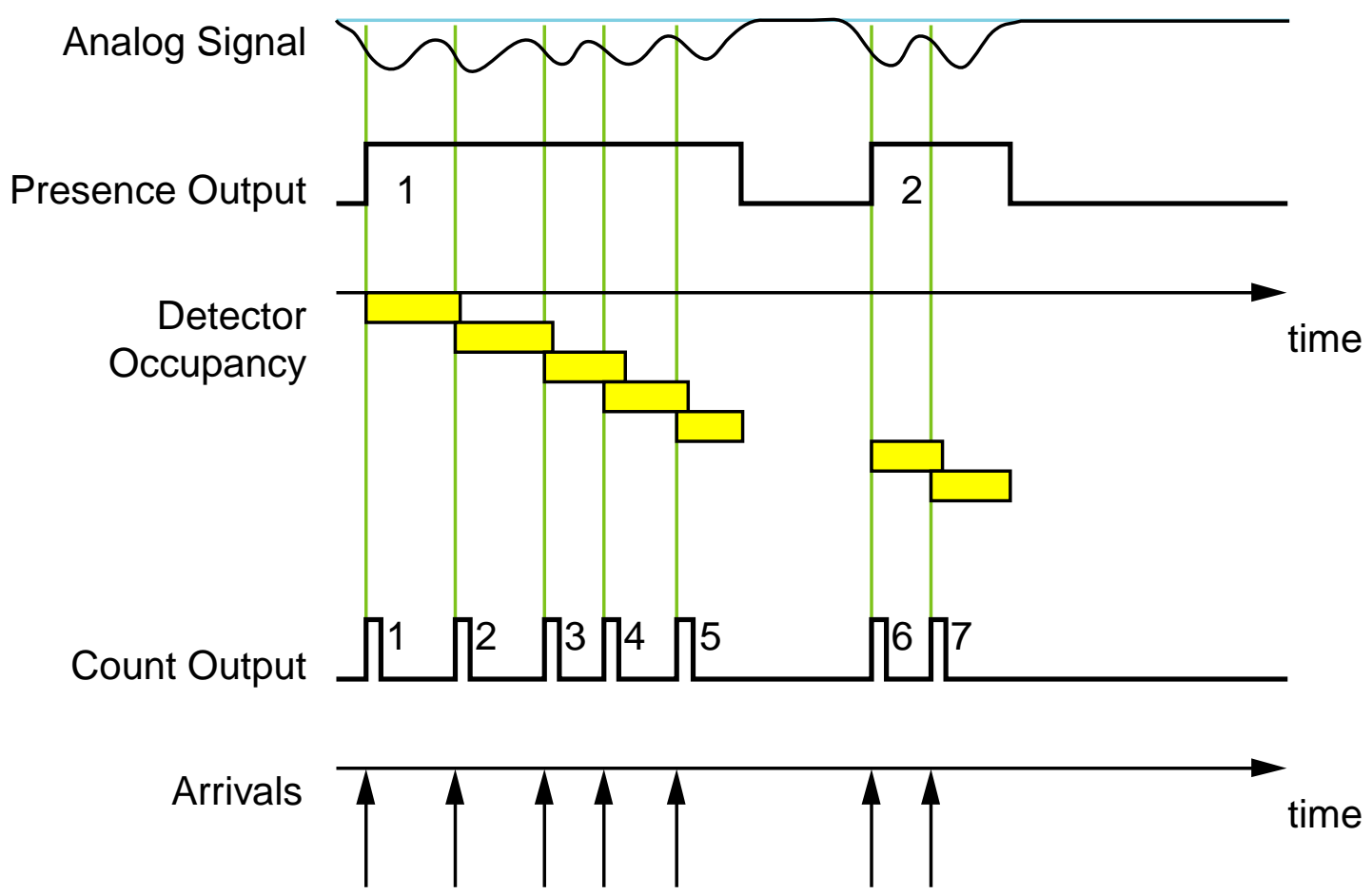

Figure 2.8 A comparison of presence and count detection modes. 


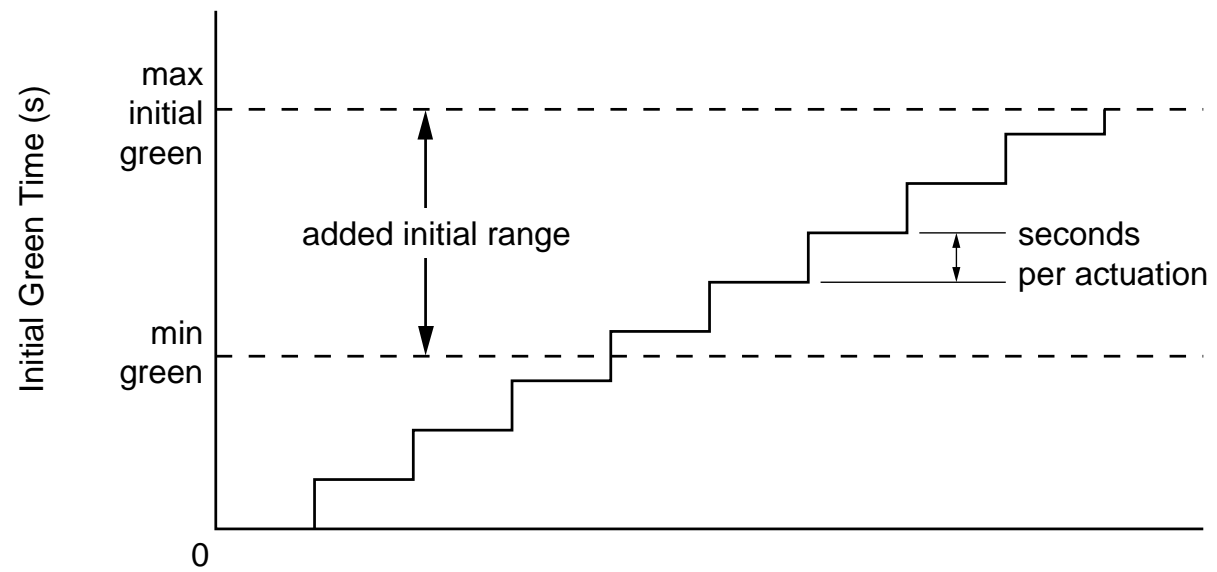

Number of Vehicle Actuations

Figure 2.9 Variable initial timing.

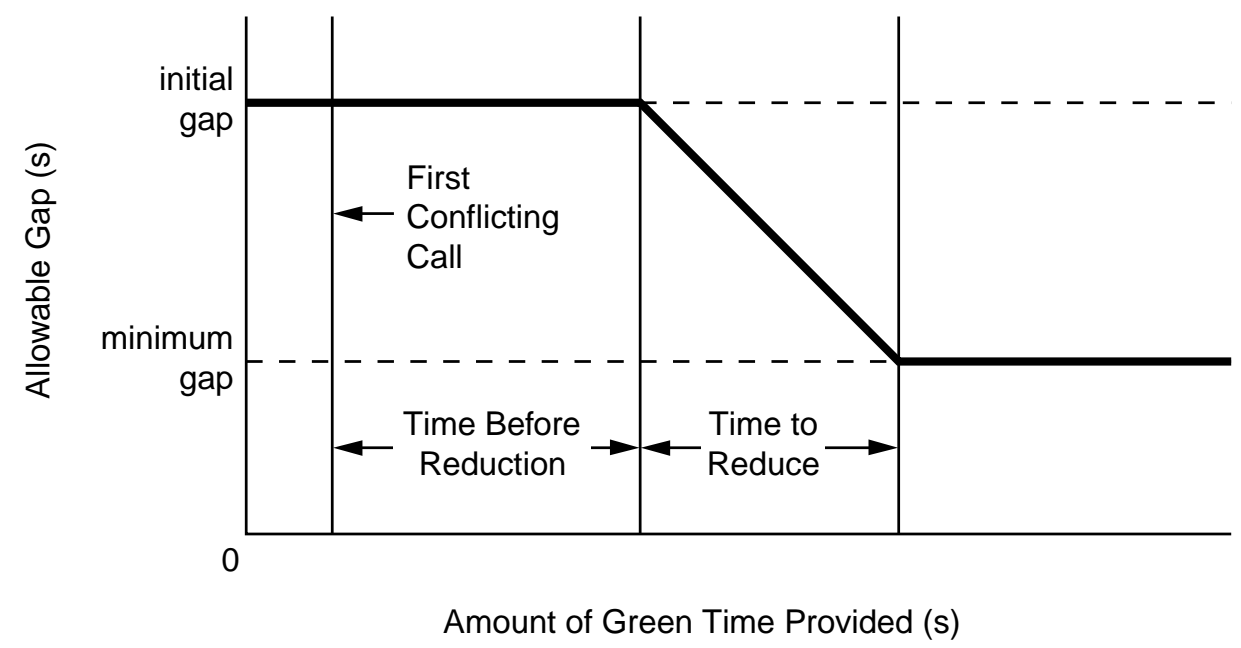

Figure 2.10 Gap reduction. 


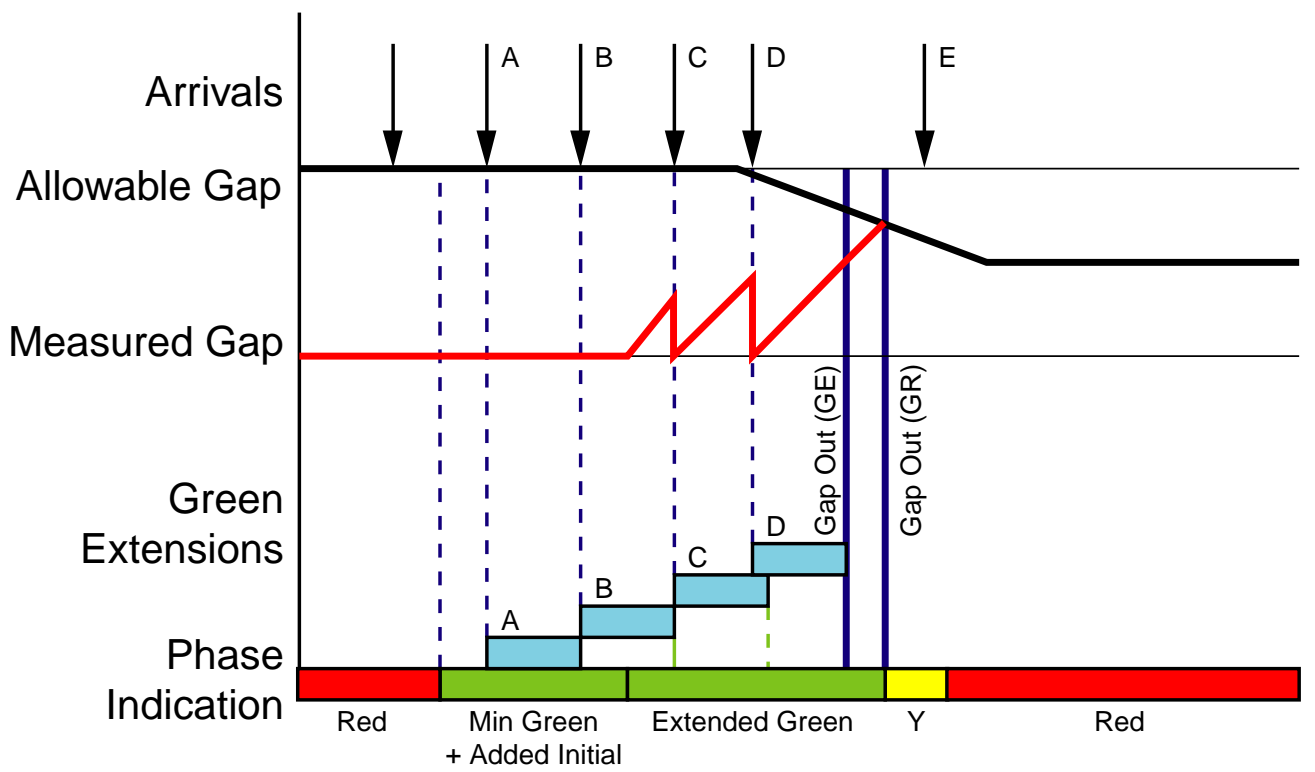

(a) Gap out conditions for green extension (GE) and gap reduction (GR).

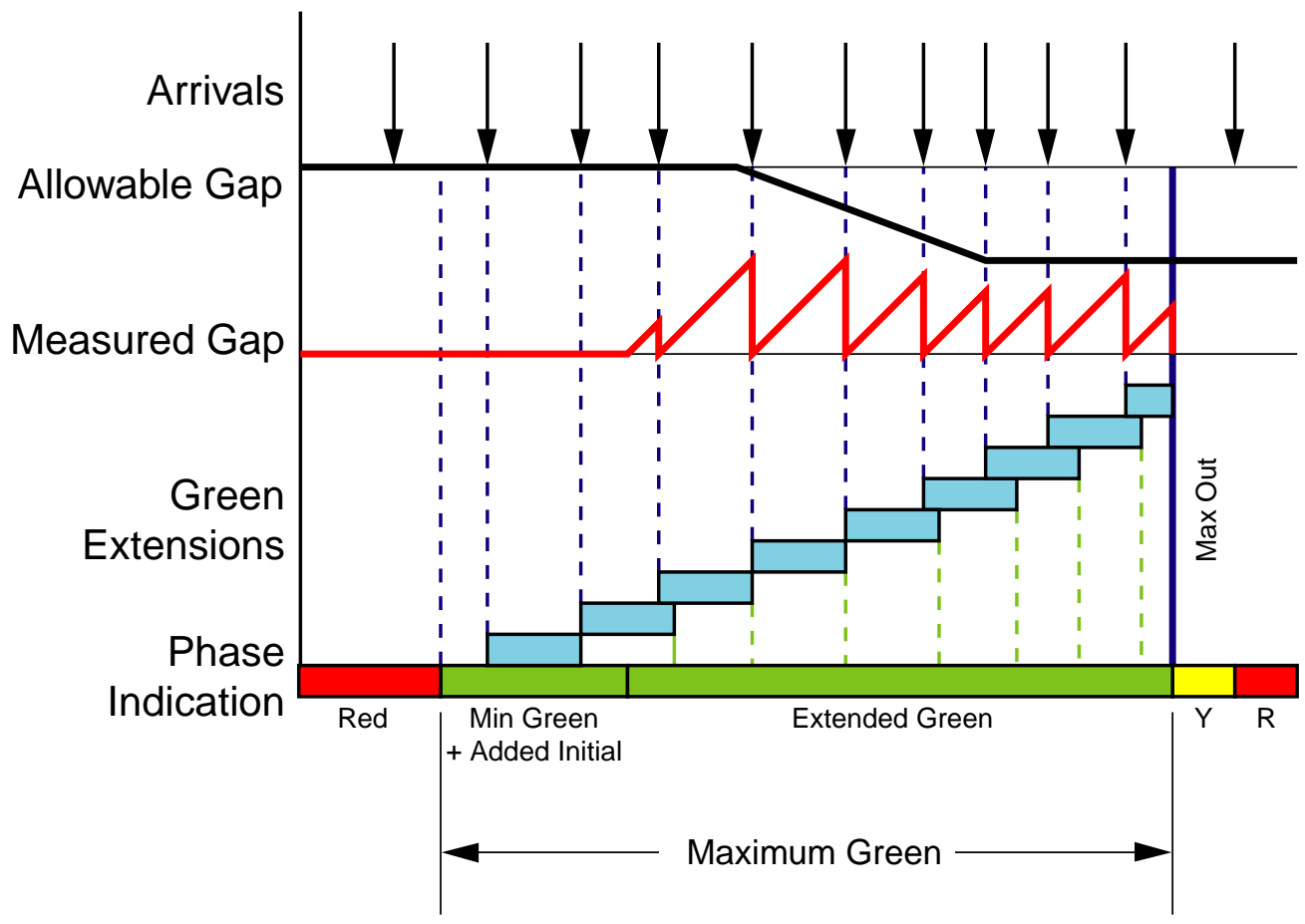

(b) Maxing out with both green extension and gap reduction logic.

Figure 2.11 Comparison of phase termination logic by the green extension and gap reduction methods. 


\subsection{Coordination}

In the preceding sections, signal operation was discussed for a single, isolated intersection. When signals are closely spaced, the release of vehicles from one into the approach of another has consequences for operation at the downstream intersection. In an ideal case, a vehicle traveling through a series of closely spaced intersections will be able to pass through the green indication for each one in succession. The way that this is done is to coordinate the green indications between signals, offsetting the start of green of the second signal in series by the amount of time it would take vehicles leaving the first to arrive at a typical speed.

Figure 2.12(a) shows a picture of a typical coordinated network in a central business district (CBD). This is perhaps the most familiar illustration of a coordinated network. The close spacing of signals and fast progression between them makes the coordination immediately apparent. However, higher speed roads with more dispersed intersections are also coordinated. Additionally, coordinated systems can incorporate actuated signals, not only the pretimed signals that are commonly used in CBDs. An example of a coordinated arterial network is shown in Figure 2.12(b).

The advantage of coordinating signals is illustrated conceptually in Figure 2.13. This shows a diagram of an arterial with four intersections with side streets. Consider one vehicle moving from left to right. The number at each intersection indicates a hypothetical probability that traffic moving along the arterial will have to stop. Figure 2.13(a) shows a situation where all four signals operate in free mode. In this example, we assume that the state of each signal is completely random and that there is a $25 \%$ chance that the signal will be red for the arterial at any given time. Assuming a Bernoulli process, there is a $68 \%$ chance of having to stop at least once, and a $21 \%$ chance of having to stop twice. Figure 2.13(b) shows a hypothetical ideally coordinated case. Here, we assume that will be a $50 \%$ chance of receiving a red signal at the first intersection; however, once the vehicle is released from the first intersection, there will be virtually no chance of needing to stop at the last four intersections as long as it is able to maintain the design speed. Coordination compels the system to behave in a more deterministic way. Of course, real systems are never free of random effects.

Another reason for using coordination is illustrated by Figure 2.14, which shows the spillback of a queue into an upstream signal. Notice that there is hardly any traffic at all on the cross street where traffic is halted. The truck in the middle of the intersection onto which the queue is spilling is obliged to change lanes in order to avoid blocking the intersection. Not only is the cross street blocked in this case, but southbound left turns are 
also prevented from moving. This illustrates the importance of good coordination in traffic systems.

Figure 2.15 shows a time space diagram of an arterial passing through a coordinated system of three intersections. The horizontal axis represents time, and the vertical axis represents distance. The red squares represent times when the signal is green for the major road. The distance between each intersection (from one stop bar to the next) is $800 \mathrm{ft}$. Using a design speed of $27 \mathrm{mph}$ (40 fps), it is found that it takes $20 \mathrm{~s}$ for a vehicle that passes through First Street on green to arrive at Second Street. This value is used to offset the beginning of green for the signal at Second Street. Two vehicle trajectories are indicated by the letters. Vehicle A drives at the design speed, and is able to clear all of the intersections without stopping. Vehicle B, on the other hand, moves somewhat slower than Vehicle A and is forced to stop.

The above example was very simple, and not uncommon for CBD areas with closely spaced intersections and pretimed signals. Urban arterial systems are complicated by the need to coordinate roads in two directions, coordination requirements of minor streets with their upstream signals, and by the presence of actuated phases in the coordinated system.

Figure 2.16 presents a more detailed time space diagram, including details about the phases of two signals in the system. Only the upper ring is shown for sake of simplicity. Phase 2 is the green phase for vehicles moving upward in the diagram. Figure 2.16(a) shows the meaning of various elements of the ring diagrams. The reference point (or yield point) is used to set the timing of one signal against the others. The end of green for the coordinated phase is used. The offset is the amount of time between the two reference points of two intersections, and is calculated by finding the amount of time it would take a vehicle moving at the design speed to traverse the link between the two intersections, as demonstrated in the previous example. The force-offs are the points in time when each non-coordinated phase must terminate in order to guarantee that the coordinated phase receives the green indication at an appropriate time to serve the platoon of vehicles released upstream.

When phases terminate early, or are skipped, then the green is passed along ultimately to the coordinated phase, causing the situation shown in Figure 2.16(b). This can cause problems with progression, as the downstream signal does not reach green in time to serve the platoon that was released by the upstream signal. This is called the "early return to green" problem, which may be observed at actuated signals in coordinated systems. Solutions to this problem would involve the optimization and adjustment of offsets (10), as shown in Figure 2.16(c). Of course, even the best offsets 
will encounter this problem occasionally due to stochastic variation in demand for the minor streets crossing the arterial. The early return problem illustrates the need accurate real-time information on which operation decisions could be based, not only as part of the basic signal settings but also by controllers in real time.

A final aspect of signal coordination that will be mentioned is the influence that the distance between signals has on progression between them. Figure 2.17 shows an illustration of how vehicle platoons evolve upon release from queue. In Figure 2.17(a), at $t=t_{0}$, vehicles achieve their maximum density while waiting in queue. At $t=t_{1}$, shown in Figure 2.17(b), the platoon disperses yet remains tight within short distances of the intersection. This is the prevailing condition in CBDs, where intersections are closely spaced. However, along arterials, longer distances allow platoons additional time to disperse further. At $t=t_{2}$, shown in Figure 2.17(c), vehicles typically form a staggered distribution across lanes; and finally, given enough time, vehicles become randomly distributed along the arterial, as shown in Figure 2.17(d).

Distance clearly influences the amount of green time needed by coordinated phases to facilitate progression. To prevent excessive stops on the major street, the size of the green "window" should be sufficient to serve a reasonable proportion of arriving vehicles. Figure 2.18(a) shows the case where a dispersed platoon arrives at an intersection with a short green phase; half of the arriving vehicles must stop. To avoid this situation in practice, longer cycle lengths are typically used, with large splits for the coordinated phases. However, a general application of this policy can lead to situations as in Figure 2.18(b), where a tight platoon is served by a long green phase. There is a significant amount of "wasted" green time following the service of the coordinated platoon, where vehicles may stand waiting for crossing movements stand waiting. When demand for the minor road is high, excessive queueing may occur on the minor approaches. 


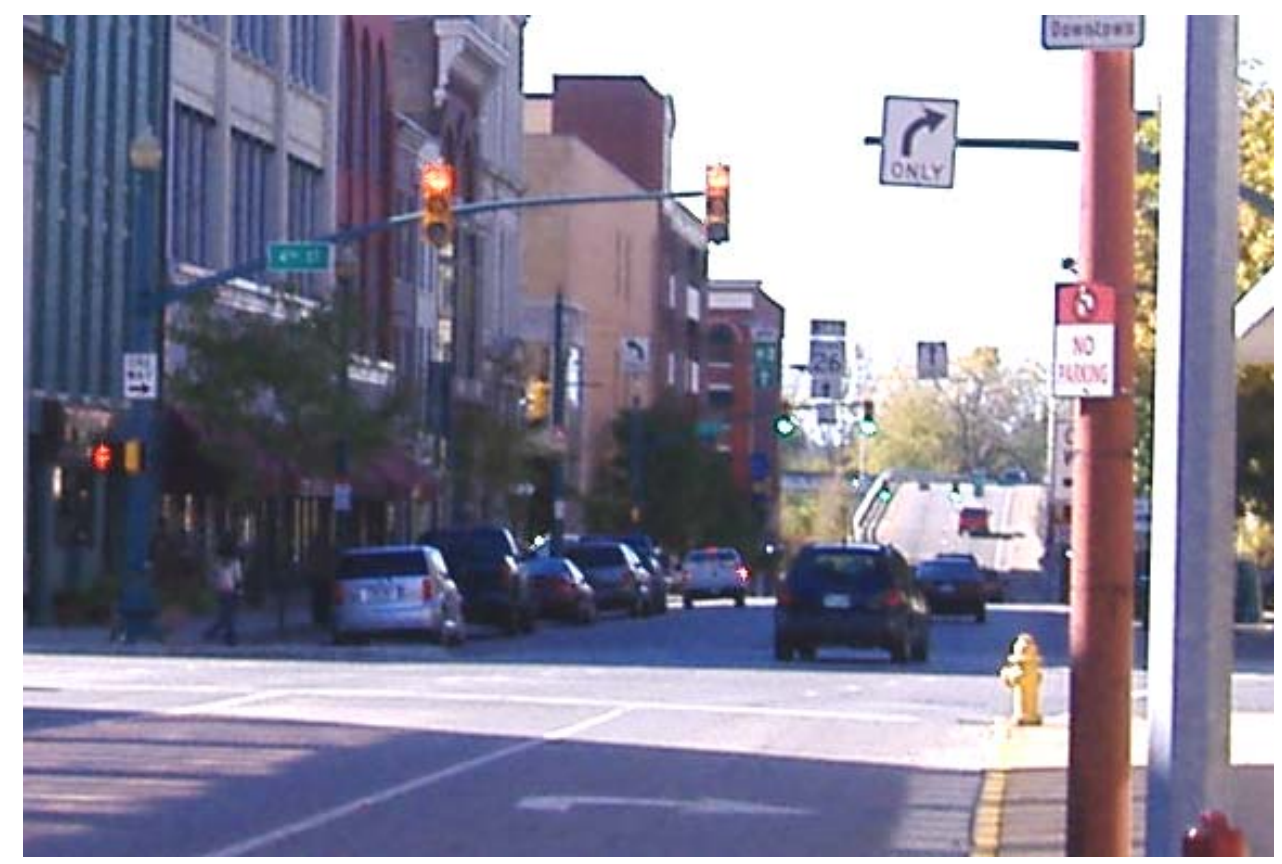

(a) Columbia Street, in the CBD of Lafayette, IN.

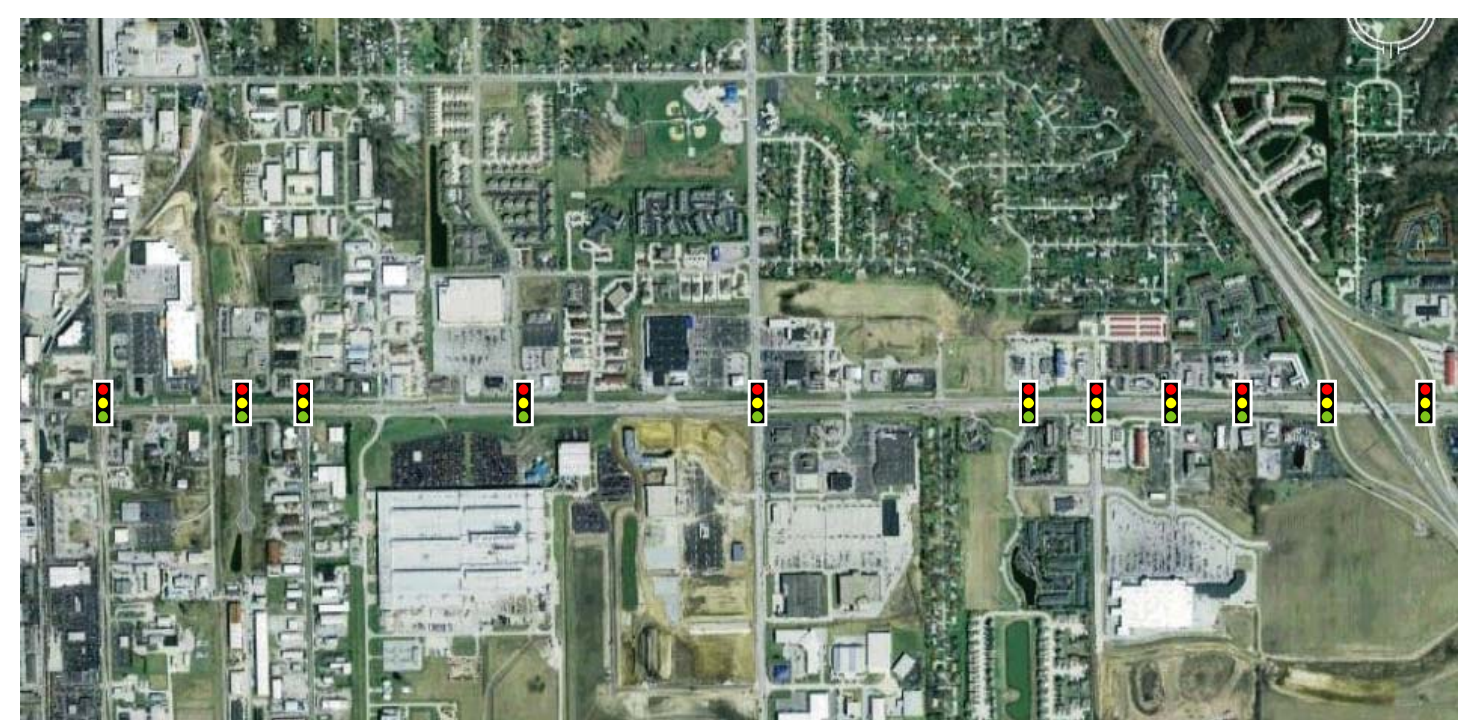

(b) Aerial view of Indiana SR 26 in Lafayette, IN, an example of a high-speed coordinated arterial system, with signal locations along SR 26 indicated.

Figure 2.12 Examples of coordinated signal systems. 


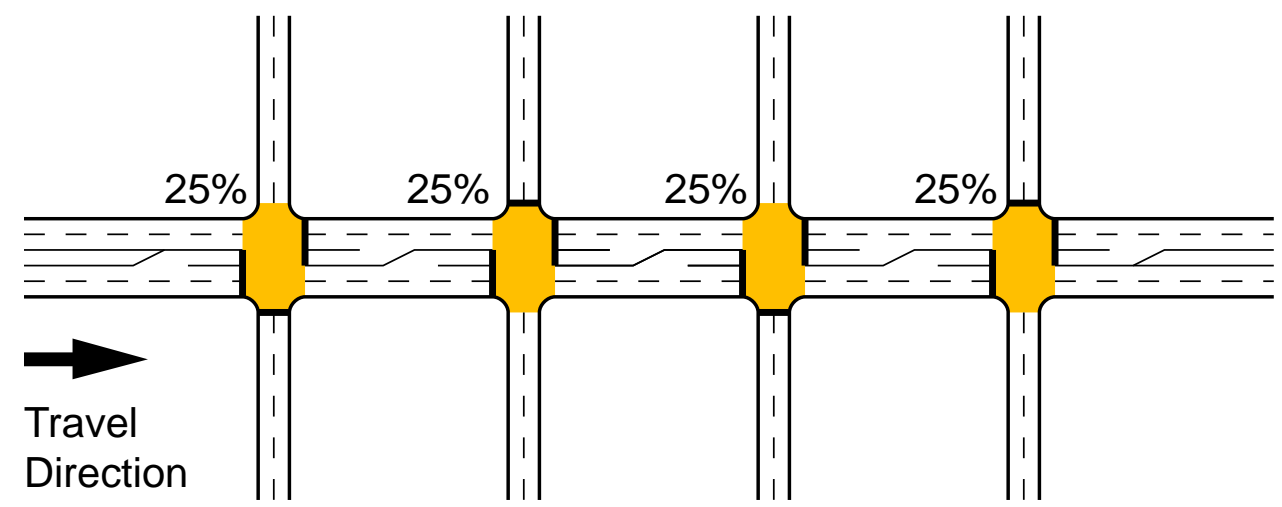

(a) Probability of red for the major road under free operation.

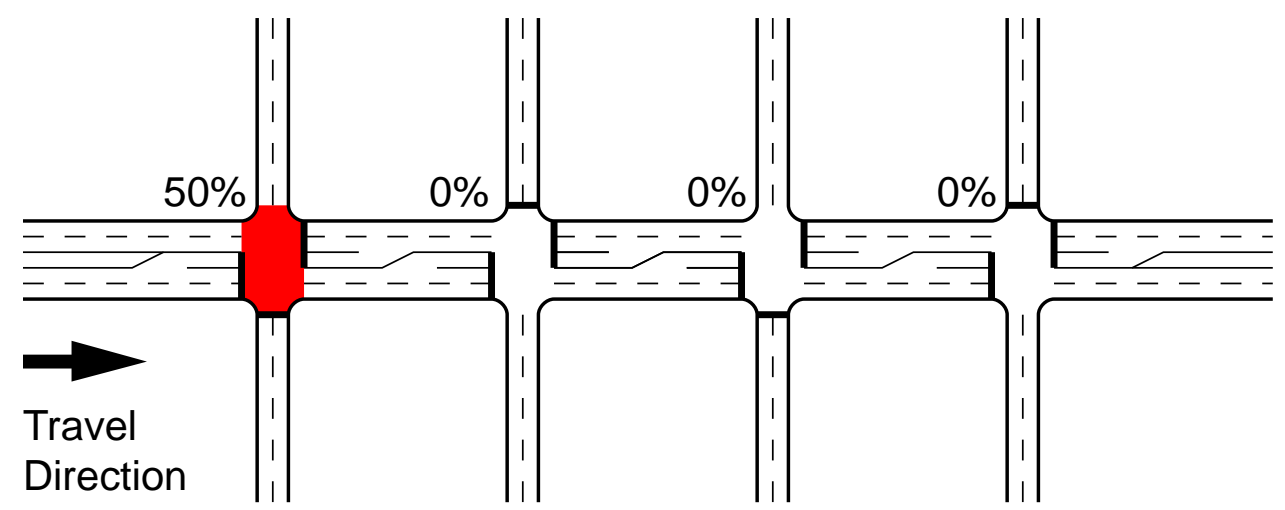

(b) Probability of red for the major road under coordinated operation.

Figure 2.13 Conceptual comparison of coordinated operation to free operation. 


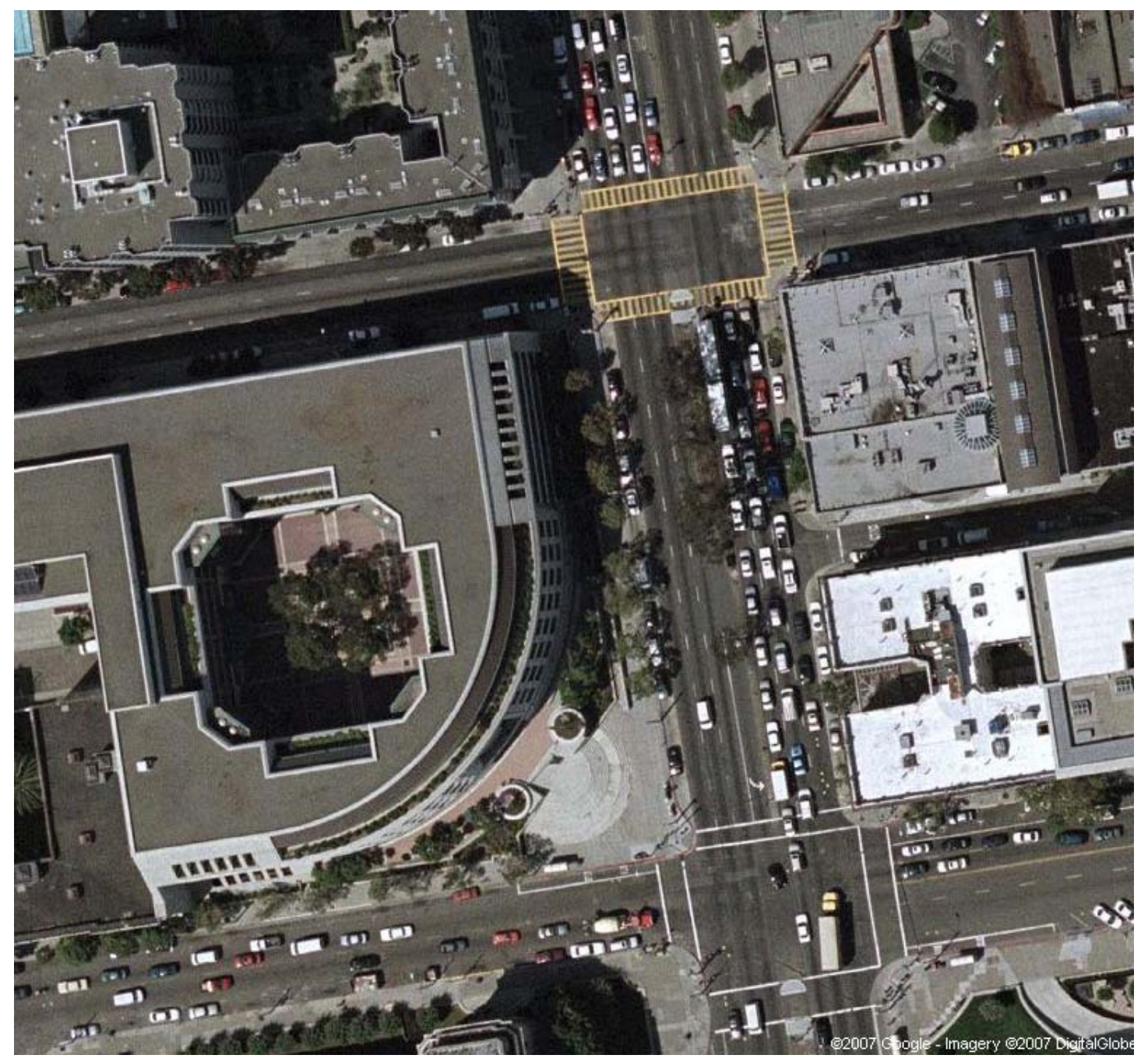

Figure 2.14 Spillback of queues into an adjacent intersection. 


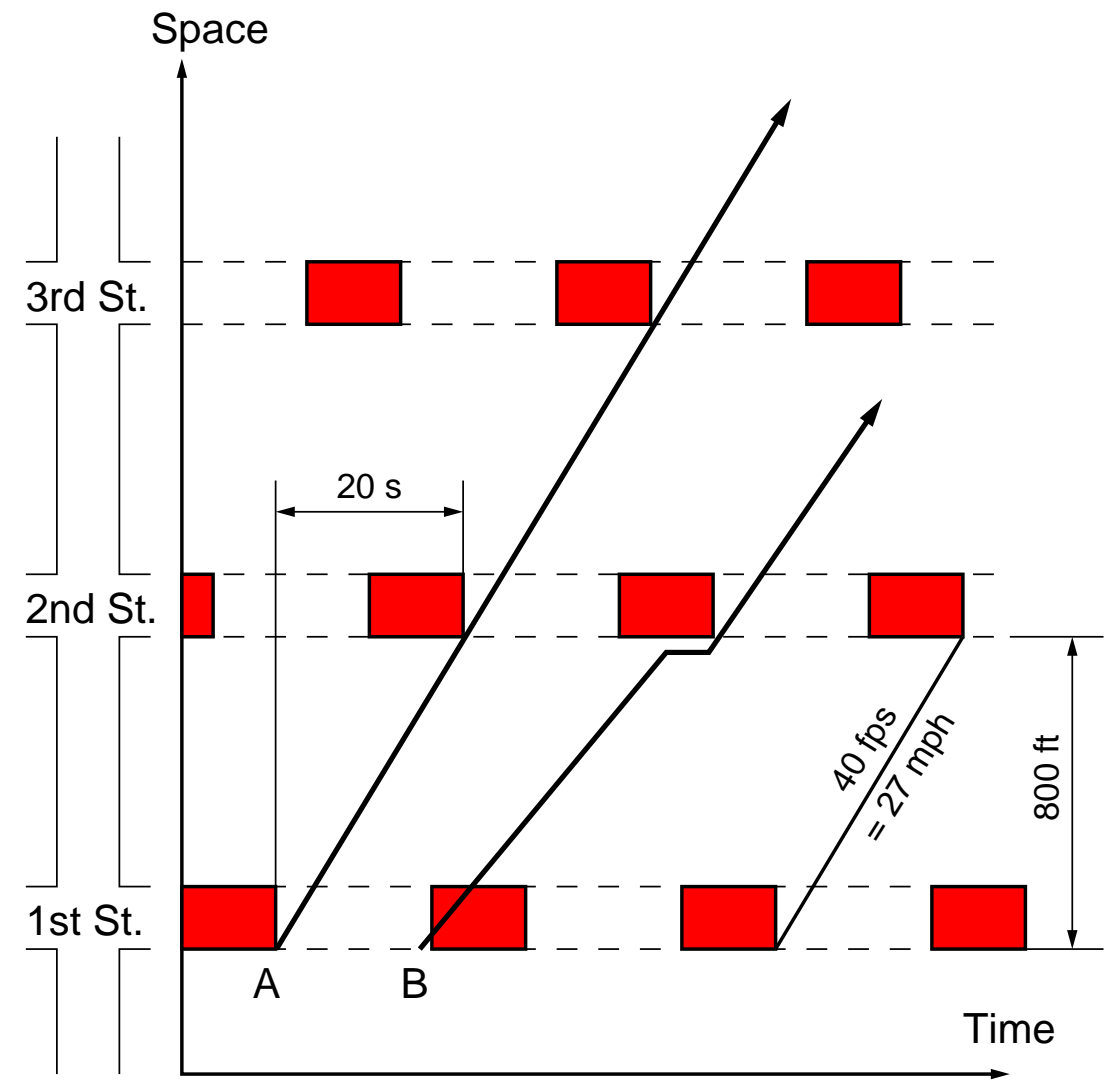

Figure 2.15 A time-space diagram showing a simple example of signal coordination. 


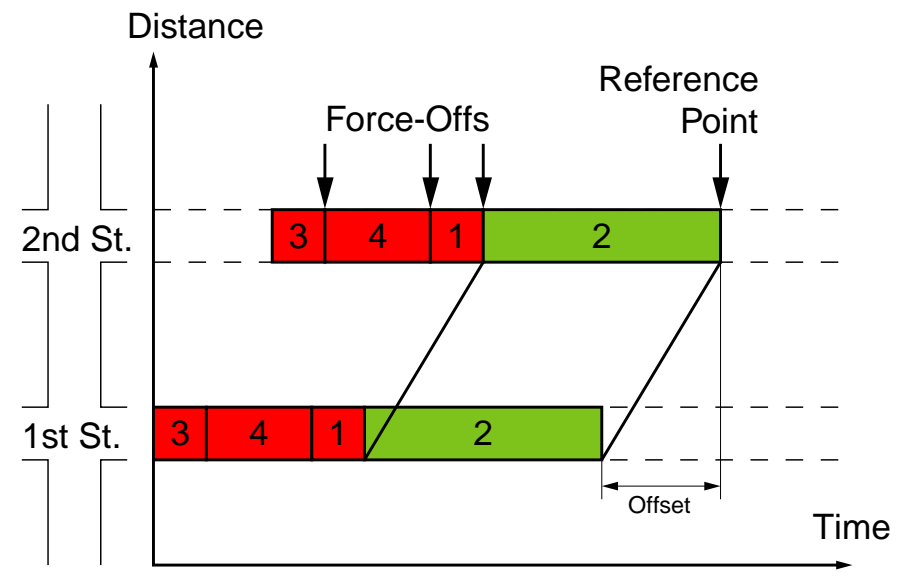

(a) Phase diagram in coordinated system.

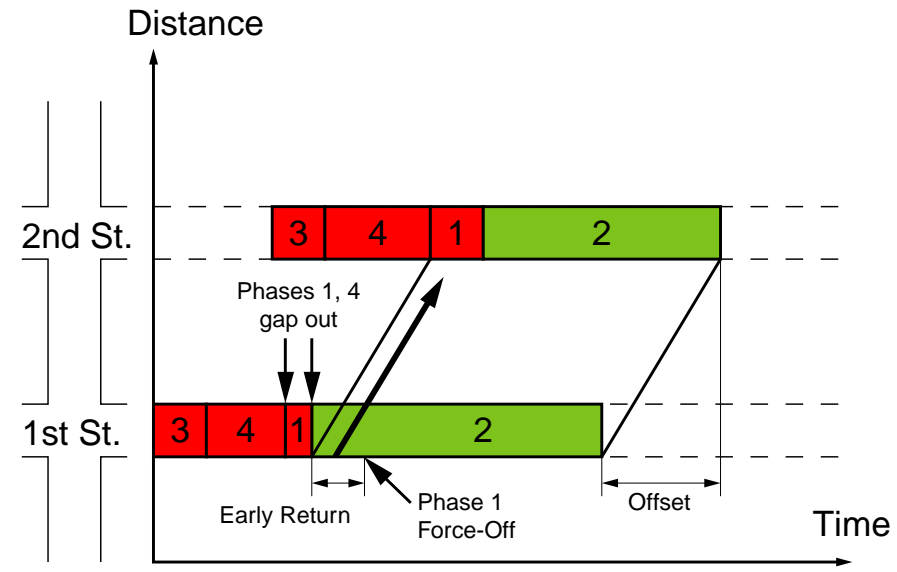

(b) Early return to green for phase 2 in upstream intersection.

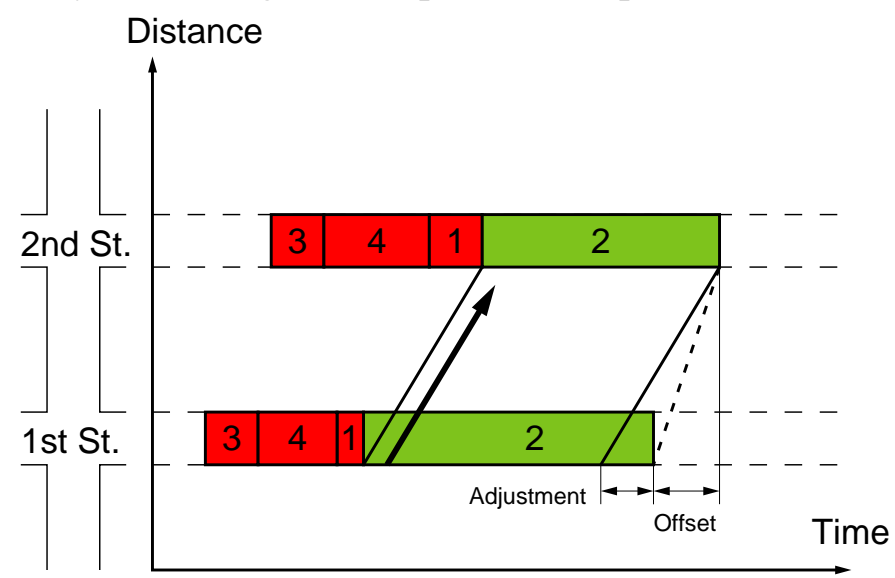

(c) Adjustment of offset to deal with the early return problem.

Figure 2.16 Issues with coordination with actuated phases. 
(a) $t=t_{0}$

- - - $\frac{\square \square \square \square}{\square \square \square \square}$

(b) $\mathrm{t}=\mathrm{t}_{1}$

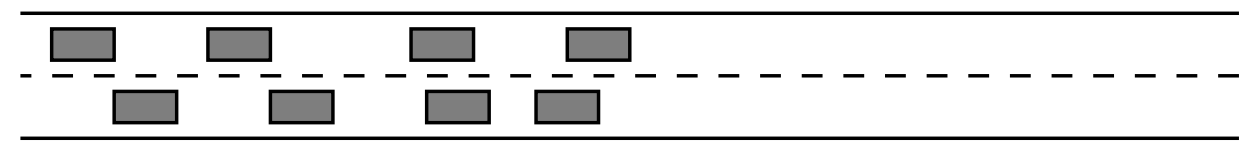

(c) $\mathrm{t}=\mathrm{t}_{2}$

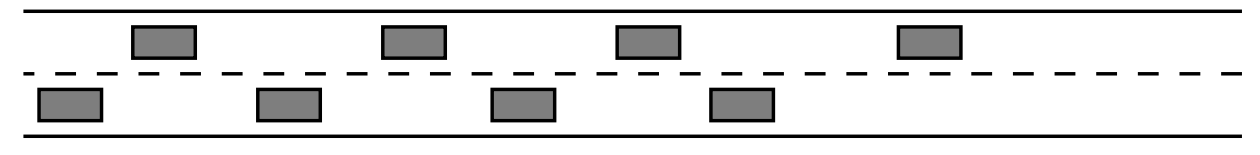

(d) $t=t_{3}$

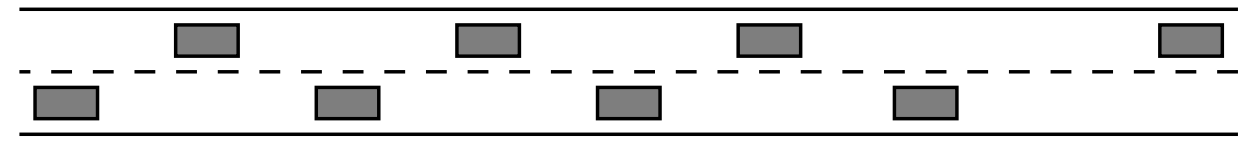

Figure 2.17 An overhead view of platoons becoming more disperse as distance increases from the intersection. 


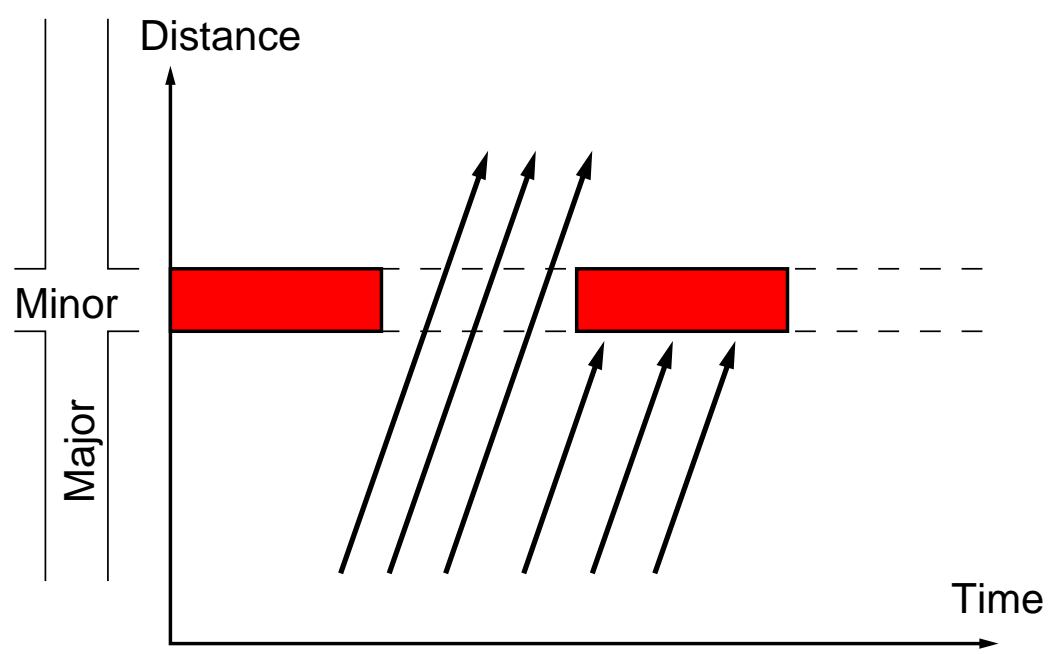

(a) A dispersed platoon served by a short green phase.

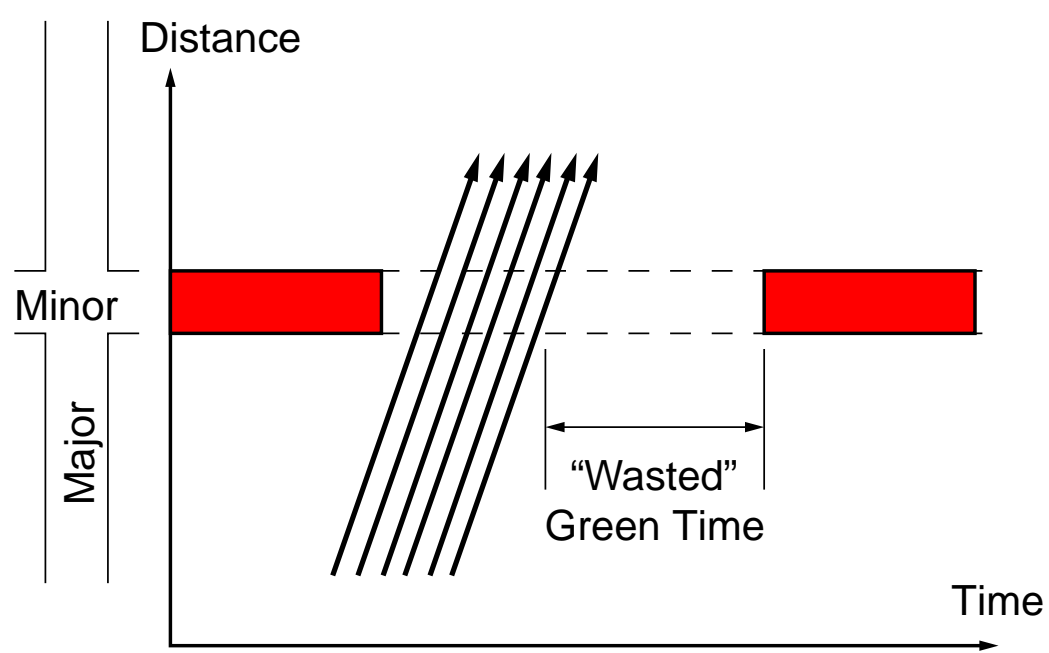

(b) A tight platoon served by a long green phase.

Figure 2.18 Mismatches of platoon dispersion and duration of green for a coordinated phase. 


\subsection{Effective Cycle Timing}

The ring diagram in Figure 2.3 is useful for visualizing the division of time between movements at an intersection. However, the signal controller does not necessarily time the cycle according to this diagram. Instead, cycle length is typically measured relative to the yield point. If phases 2 and 6 are coordinated movements, then this would be the point where the barrier is crossed between phases 2 and 3 (or 6 and 7). The resulting "effective" ring diagram is shown in Figure 2.19(a). This represents the order that the controller serves phases relative to the way it counts time in the cycle. The upper ring has the phase order 3, 4, 1, 2 rather than 1, 2, 3, 4, as we may expect from Figure 2.3. Likewise, the lower ring has the phase order 7, 8, 5, 6. The through movements for the major street, phases 2 and 6 , are served last. The reason for this is has to do with the early termination of preceding phases. In the case of phase 2 , if phases 3,4 , or 1 are skipped or terminate early, phase 2 inherits the green time that they give up. This scheme enables the major street, which is likely to be coordinated, to be allocated excess green time. Phases 2 and 6 will not terminate until they reach the yield point.

Figure 2.19(b) shows an example of the actual phasing that may take place at an intersection. The dashed lines show where the boundaries between phases would have fallen if they each timed according to their splits. This would be the situation at a pretimed controller or at an actuated controller where every phase maxes out. When phases early in the cycle are skipped or terminate early, they pass the additional green time to those that follow. However, the endpoints of the cycle length are fixed. The result is that phases 2 and 6 , which typically come last, increase their share of the cycle relative to the other phases. Figure 2.19(c) shows the case when cycle length is not fixed. This represents free operation. Phases are served according to demand for each movement, with minimum and maximum green times determining the length of each phase and of the cycle, rather than splits. 


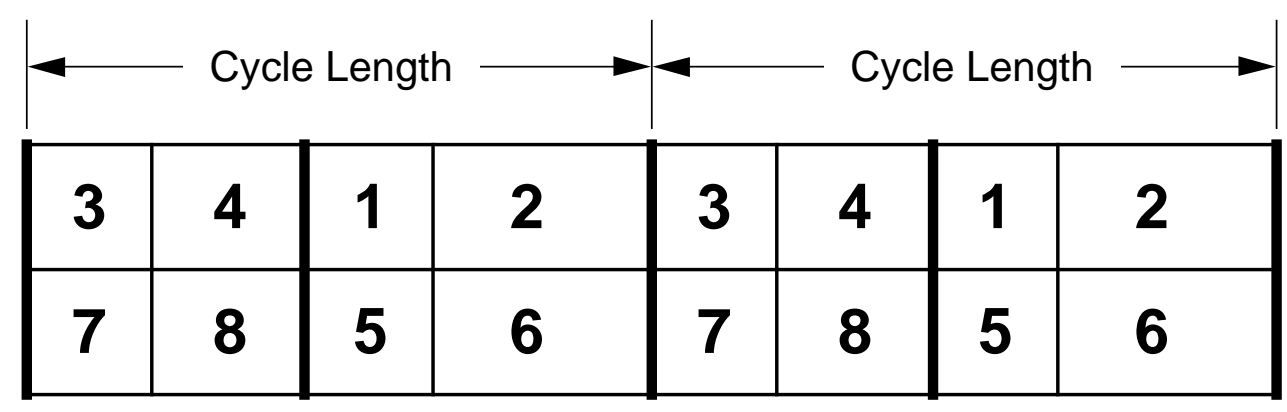

(a) Two effective cycles with every phase running its split time.

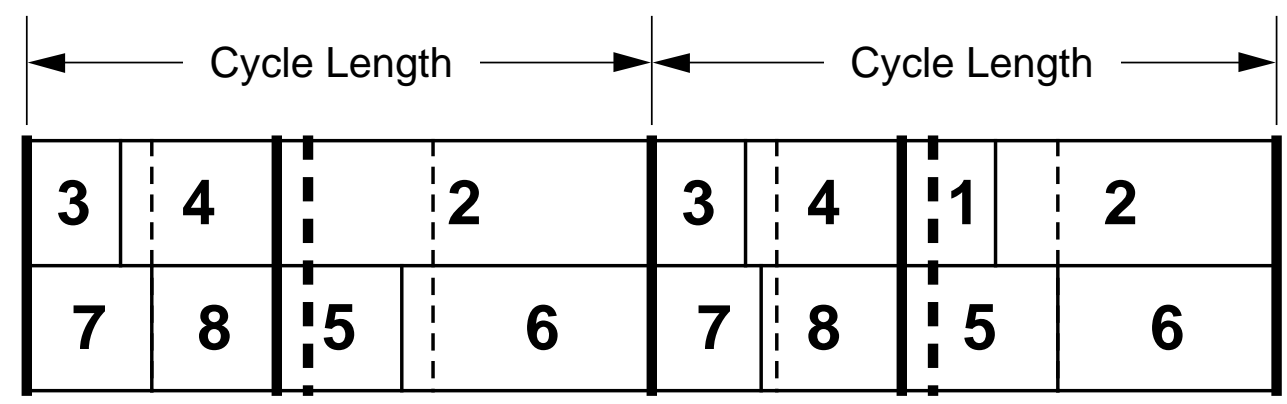

(b) Two cycles with variations in vehicle demand.

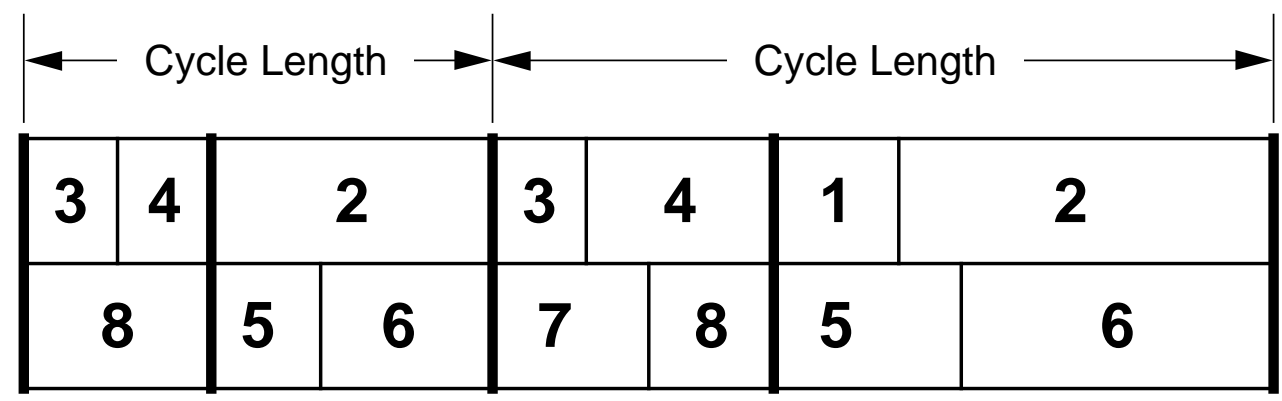

(c) Two cycles under free operation, with varying cycle length.

Figure 2.19 Cycles as they are effectively handled by a signal controller, and with variations in signal timing due to skipped phases and early terminations. 


\subsection{Conclusion}

This chapter described the basic elements and terminology of traffic signal operation. Decisions to change controller settings rely on accurate performance measures; signal system performance would generally be improved with the availability of inexpensively obtained performance measures. Currently, performance measures are typically measured by visual inspection, and the effectiveness of operational changes relies on the experience of the traffic engineer and observation time. Accurate performance measures would improve the quality of operational changes, while an automated data collection process would allow the engineer to rapidly analyze operation through 24 hours of the day and 7 days of the week.

The use of vehicle actuation to manage intersection capacity represents the scope wherein vehicle detectors are currently employed. Within these detectors also lies the potential for data collection. Each detector produces an electrical signal representing an event occurring at the intersection. A major component of the infrastructure for data collection is thus already available at many intersections and plays an essential role in operations. However, the means of collecting the data is not widely available, despite the use of solid state electronics in modern signal controllers and the advancement of computing technology in the past thirty years. One reason for this is the requirement for traffic devices to withstand harsh weather conditions; however, as technology improves, the ability of more advanced equipment to satisfy this requirement seems likely become more readily available at declining costs.

The succeeding chapters of this report will describe how a $\log$ of events at an intersection can be converted into performance measures in a manner that could be performed by a solid state computing device in a traffic cabinet. 


\section{CHAPTER 3. DATA COLLECTION}

\subsection{Introduction}

This chapter discusses data collection at traffic signals. A brief review of previous work is given. The concept of the instrumented intersection is introduced, study locations are presented, and the methods of collecting and interpreting raw data are reviewed.

\subsection{Background}

Various methods of data collection have been developed in the past to characterize the operation of traffic signals. Currently, the most common method for evaluating signal operation is that described by the Highway Capacity Manual (HCM) (5). The HCM method is intended for data collected by a human observer. The inputs for the HCM model include 15-minute traffic counts, signal operation parameters, and information about traffic characteristics that are expressed in numerous adjustment factors. The HCM describes signal operation using models that are based on the collective experience of signal systems researchers. However, the resulting performance measures are more predicative of what conditions should be given the inputs, rather than a record of what conditions actually were.

The desirability of real-time traffic information that can be obtained from loop detectors at signalized intersections has been expressed by various researchers (11) and agencies (12). In 2005, the Texas Transportation Institute (TTI) published a study (13) describing a prototype system for data collection in which a black box was placed in the signal cabinet to log data and calculate various performance measures. The TTI system automatically produced a set of measures that could be calculated within the bounds of current loop detection technology.

Despite being motivated by the 2007 national traffic signal report card score of "D", and the data collection category score of "F" (4), highway transportation agencies are not likely to include expensive data collection black boxes in traffic signal cabinets. The relatively small market size of traffic signal components does not encourage commercial production of a data collection unit, particularly if few agencies express an interest in purchasing the unit. However, if an equivalent module were added to traffic 
signal controllers, data collection capability could be realized more easily. It would be added to a device for which there is an already existing market because of its requirement. The implementation of controller-based data collection would require a demonstration of the feasibility and effectiveness of that data collection method. This report endeavors to accomplish that demonstration. Additionally, to make controllerbased data collection a reality, agencies would need write the capability into specifications in order to encourage manufacturers to begin adding this capability to controllers; and it would also need to be demonstrated that adding such capability does not excessively burden manufacturers. Those points are outside the scope of the present work.

Two kinds of data are currently available from traffic signal controllers making use of loop detectors. These are vehicle counts and rates of detector occupancy, available typically in static bins of 5, 10, and 15 minutes. It is not currently within the capability of traffic signal controllers to tabulate this information on a cycle-by-cycle basis.

An example graph of occupancy data is shown in Figure 3.1. This is a 24-hour plot of detector occupancy, with 15 minute bins. Plots of occupancy for 12 detectors are shown. From this graph, it is apparent that there is a great deal of activity between the hours of 05:00 and 22:00. Some detectors show peaks occurring at certain times of day, but others simply report high occupancy levels throughout the entire day. Occupancy does not reveal any information about traffic volumes; The critical lanes at an intersection will have high occupancy rates, regardless of volume. It is not possible to determine the effectiveness of coordination, or whether demand for a particular movement is adequately served.

An example of count data is shown in Figure 3.2. Here, vehicle counts are shown for a two-lane through movement at an intersection. 15 minute bins are used. As with occupancy, only a limited amount of information is available from this graph. For example, it is clear that demand peaks in the 16:00-18:00 time period. However, the performance of the phase that serves this movement can only be revealed by additional observation. Using the HCM method, the 15-minute counts can be used to generate the level of service. However, this is an ex post facto measurement, neither allowing us to observe signal operation in real time nor allowing us to see the cycle-by-cycle variations.

Even if controllers were able to tabulate data in cycle-by-cycle bins, this alone does not provide enough information to describe the quality of operation. Figure 3.3 shows the same data as Figure 3.2, except in cycle-by-cycle bins. The resolution of the data has greatly increased. However, we are not much closer to revealing information about the signal operation. We cannot determine whether the phase is adequately served 
by green, unless the green time is also known; the quality of progression cannot be determined without information about whether each arrival occurred on red or green; and so on. Adding cycle-by-cycle binning in itself is only the first step toward characterizing signal operation. Chapter 4 will focus on refining cycle-by-cycle data into useful information.

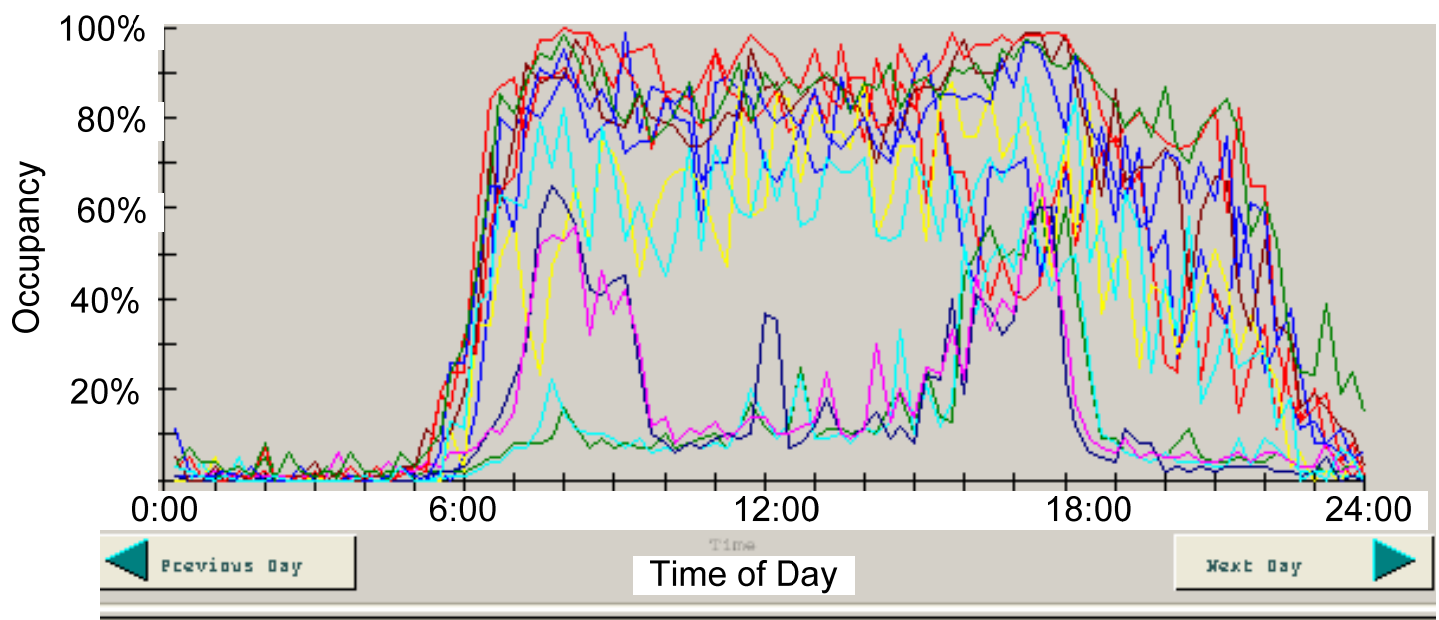

4/12/2006 Zone 11 Intersection 6 Detector 1 Occupancy Data

4/12/2006 Zone 11 Intersection 6 Detector 2 Occupancy Data

4/12/2006 Zone 11 Intersection 6 Detector 3 Occupancy Data

4/12/2006 Zone 11 Intersection 6 Detector 4 Occupancy Data

4/12/2006 Zone 11 Intersection 6 Detector 5 Occupancy Data

4/12/2006 Zone 11 Intersection 6 Detector 6 Occupancy Data

4/12/2006 Zone 11 Intersection 6 Detector 7 Occupancy Data

4/12/2006 Zone 11 Intersection 6 Detector 8 Occupancy Data

4/12/2006 Zone 11 Intersection 6 Detector 9 Occupancy Data

4/12/2006 Zone 11 Intersection 6 Detector 10 Occupancy Data

4/12/2006 Zone 11 Intersection 6 Detector 11 Occupancy Data

4/12/2006 Zone 11 Intersection 6 Detector 12 Occupancy Data

Figure 3.1 Example graph of detector occupancy, binned in 15-minute intervals. 


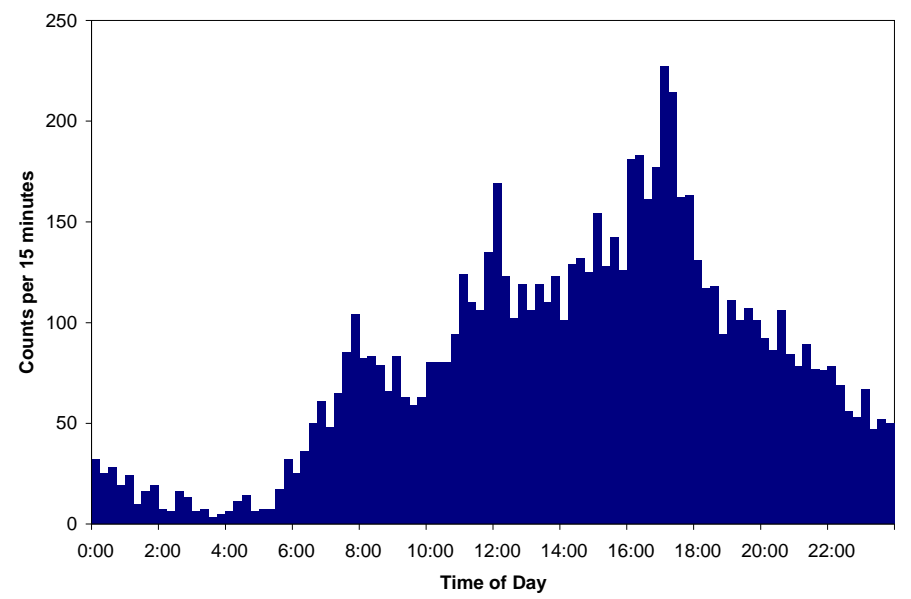

Figure 3.2 Example graph of count data, binned in 15-minute intervals.

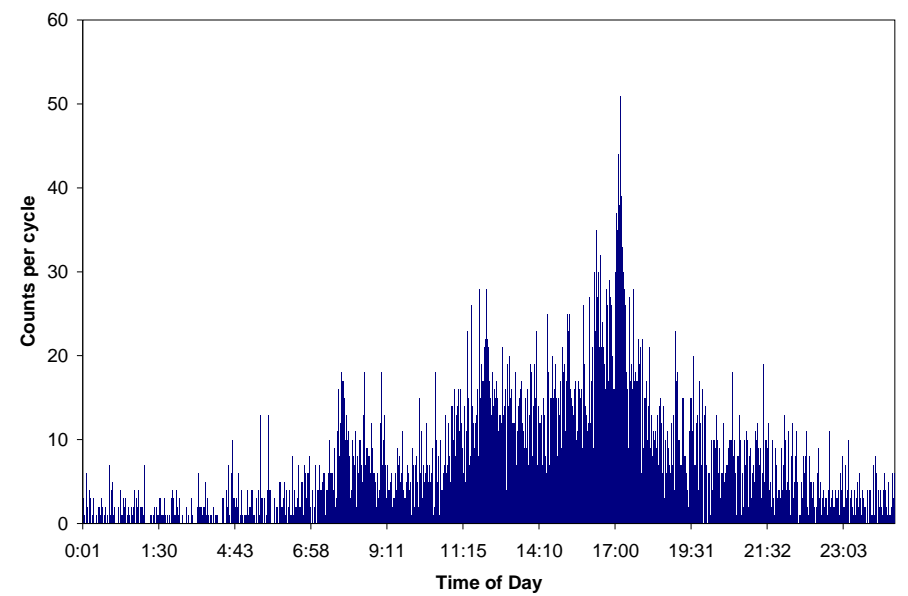

Figure 3.3 Example graph of count data, with cycle-by-cycle values plotted. 


\subsection{Data Collection Sites}

In this research, two instrumented intersections belonging to the Indiana Department of Transportation (INDOT) were utilized for data collection: the intersection of S.R. 32/38 and S.R. 37 in Noblesville, Indiana; and the intersection of US 231 and Stadium Ave. in West Lafayette, Indiana. In this section, we will describe characteristics of these intersections.

\subsubsection{Noblesville}

S.R. 37 is a two-lane divided arterial highway north of Indianapolis, Indiana. It is an important corridor connecting Indianapolis to its northeastern suburbs. To the east of Noblesville, S.R. 37 intersects the multiplexed S.R. 32/38, which is an two-lane east-west highway. It expands to multiple lanes in the vicinity of the intersection with S.R. 37. S.R. 32 is a minor arterial that cuts across central Indiana, connecting several medium-sized cities. S.R. 38 follows a more circuitous route and serves smaller towns in the region.

The 2004 annual average daily traffic (AADT) at the intersection is as follows: S.R. 37 serves $32,160 \mathrm{veh} /$ day along the segment to the south of the intersection and $21,810 \mathrm{veh} /$ day to the north. S.R. $32 / 38$ serves $18,870 \mathrm{veh} /$ day on the segment to the east, and $15,470 \mathrm{veh} /$ day to the west. The asymmetry of the counts implies a large number of turning movements. The northbound left turn is the strongest of these, with many vehicles making a turn onto S.R. 32/38 heading toward Noblesville.

Figure 3.4 is an aerial photo of the intersection. The abutting properties are mostly dedicated to retail operations and farming. There are some access points along S.R. 32/38 rather close to the intersection, but access to S.R. 37 is relatively limited. None of the approaches have sidewalks, and the intersection does not have pedestrian indications. All of the left turn movements, as well as the eastbound, northbound, and southbound right turns, have dedicated lanes.

Note the short distance from the intersection to the west. This is Cumberland Rd., and the length of the eastbound approach is only $405 \mathrm{ft}(123 \mathrm{~m})$. Distances to all of the nearest neighboring intersections are given in Figure 3.5. Beyond the intersection with Cumberland, S.R. 32/38 leads to Noblesville after $1 \mathrm{mi}(1.6 \mathrm{~km})$. To the south, it is 2400 $\mathrm{ft}(732 \mathrm{~m})$ to the intersection with Pleasant St.; to the east, the nearest major intersection is where S.R. 32 and 38 diverge, which is $7600 \mathrm{ft}$ (2316 m) away; and to the north, the distance is $4750 \mathrm{ft}(1448 \mathrm{~m})$ to $191^{\text {st }} \mathrm{St}$. The AADTs are also shown in Figure 3.5. 
Figure 3.6 is a schematic drawing of the intersection showing the location of loop detectors at the intersection. As can be seen in the figure, every lane of every approach is outfitted with detectors. The northbound and southbound approaches have advance (or "setback") detectors. Both sets of advance detectors are $405 \mathrm{ft}(123 \mathrm{~m})$ from the intersection. The detector names are a result of standard INDOT nomenclature of lanes and detectors. The innermost lane of a road is lane "A," with lane being the one to its right, and lane $\mathrm{C}$ being the one to the right of lane $\mathrm{B}$. Left turn lanes are designated by $\mathrm{L}$. Detectors are numbered in order of their distance from the stop bar. Each approach has a set of three cameras that are used for recording video. Lane-by-lane detection was used on the through movements on all four approaches for all of the studies contained in this paper.

The assignment of phases to movements is shown in Figure 3.7(a). Phases 2 and 6 represent northbound and southbound respectively, which are the major movements at this intersection. Phase 2 is coordinated with signals to the south of the intersection along S.R. 37. During the day, this signal operates in semi-actuated mode, with the phase pairs $2 / 6$ and $4 / 8$ being called in every cycle, with all left turn phases being run when there is a call for them. At night (between 22:00 and 6:00), the signal operates in fully-actuated, free mode. Dual entry is used at this intersection, meaning that whenever a phase is called, its complementary phase is also given the green indication.

Figure 3.7(b) shows a ring diagram that defines signal controller operation at Noblesville. As can be seen here, left turns for the northbound and southbound movements are only allowed during the protected phases. Note the lead/lag scheme, whereby phase 5 follows phase 6 . The northbound left turn lags the southbound through movement. This operating plan was adopted to better serve the high demand for phase 5 . In Chapter 5, we will compare lead/lag operation to conventional operation and highlight the benefits.

A map of the S.R. 37 corridor is shown in Figure 3.8. AADTs are indicated by the arrows. The segment between Greenfield Ave. and S.R. 32/38 is coordinated (i.e., the intersection with $191^{\text {st }}$ St. is not coordinated). Thus, vehicles arriving on the northbound approach (Phase 2) tend to arrive in platoons, while vehicles arriving on the southbound approach (Phase 6) are randomly dispersed. The segment south of $146^{\text {th }}$ St. is also coordinated. If additional intersections are constructed between $146^{\text {th }}$ St. and Greenfield Ave., it would likely be necessary to coordinate all of the signals along S.R. 37 from I-69 to S.R. 32/38. The location of the interchange with I-69 is shown in Figure 3.8. 


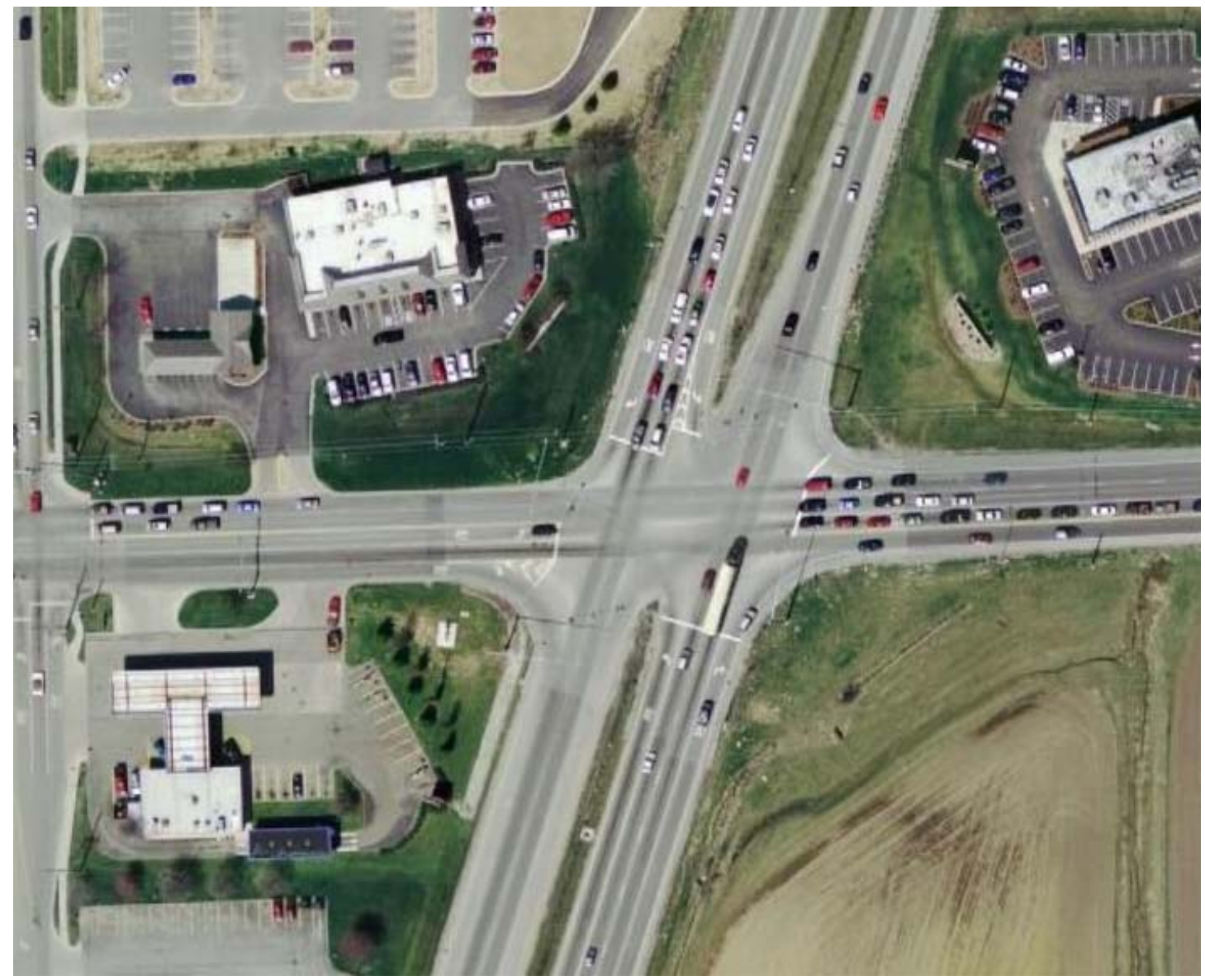

Figure 3.4 An aerial photo of the Noblesville testbed, showing surrounding land use characteristics. 


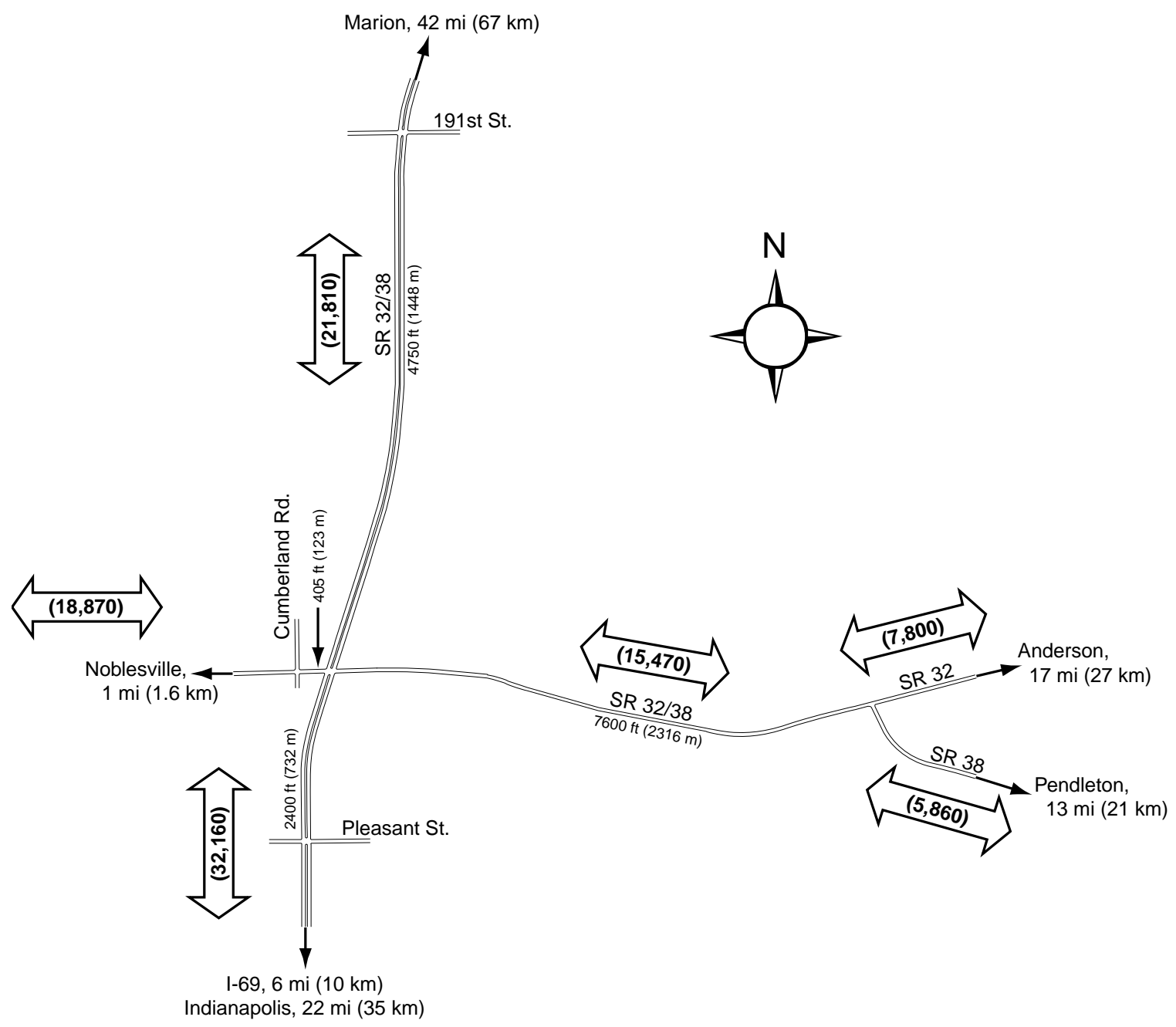

Figure 3.5 Locations of the nearest major intersections from the Noblesville site, placement of advance detectors, and approximate distances to nearby cities. Arrows indicate AADT for each segment. 


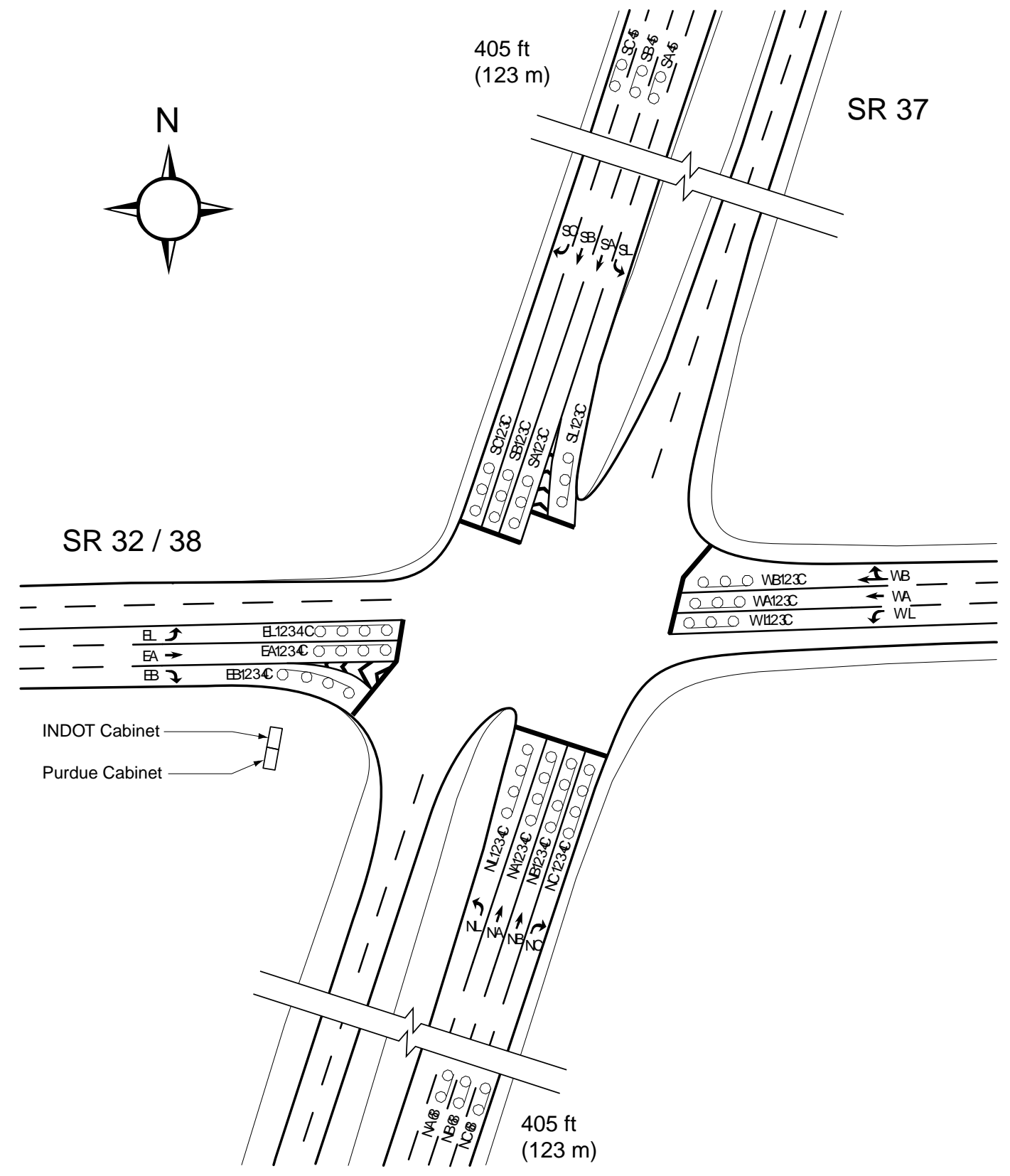

Figure 3.6 Schematic of the Noblesville testbed, showing the placement of loop detectors. 


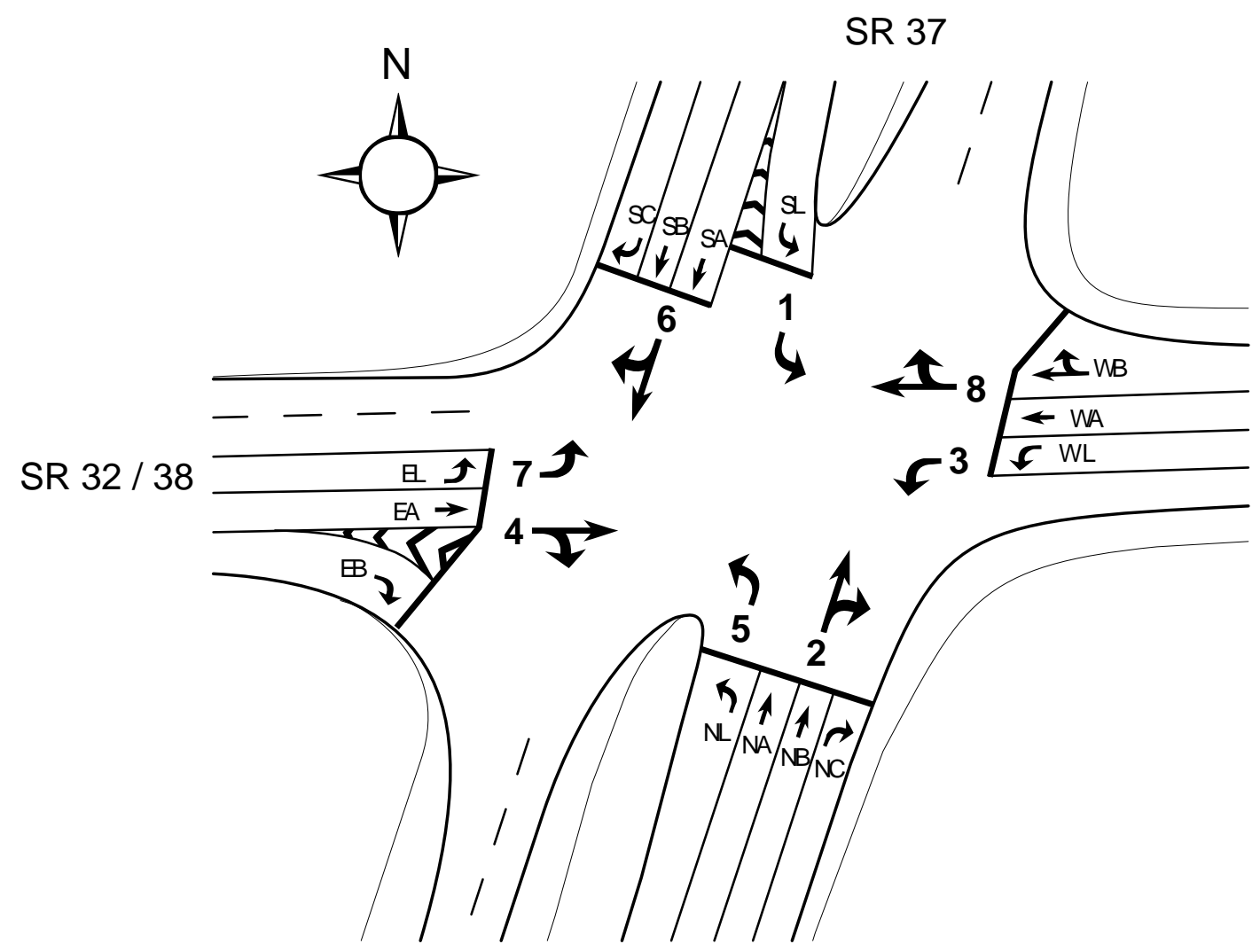

(a) Plan view showing phasing.

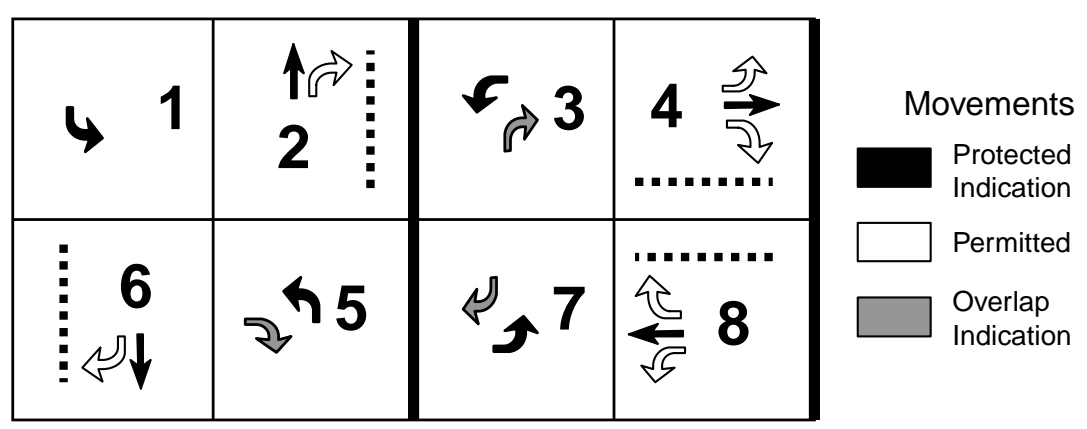

(b) Ring diagram.

Figure 3.7 Phases at the Noblesville testbed. 


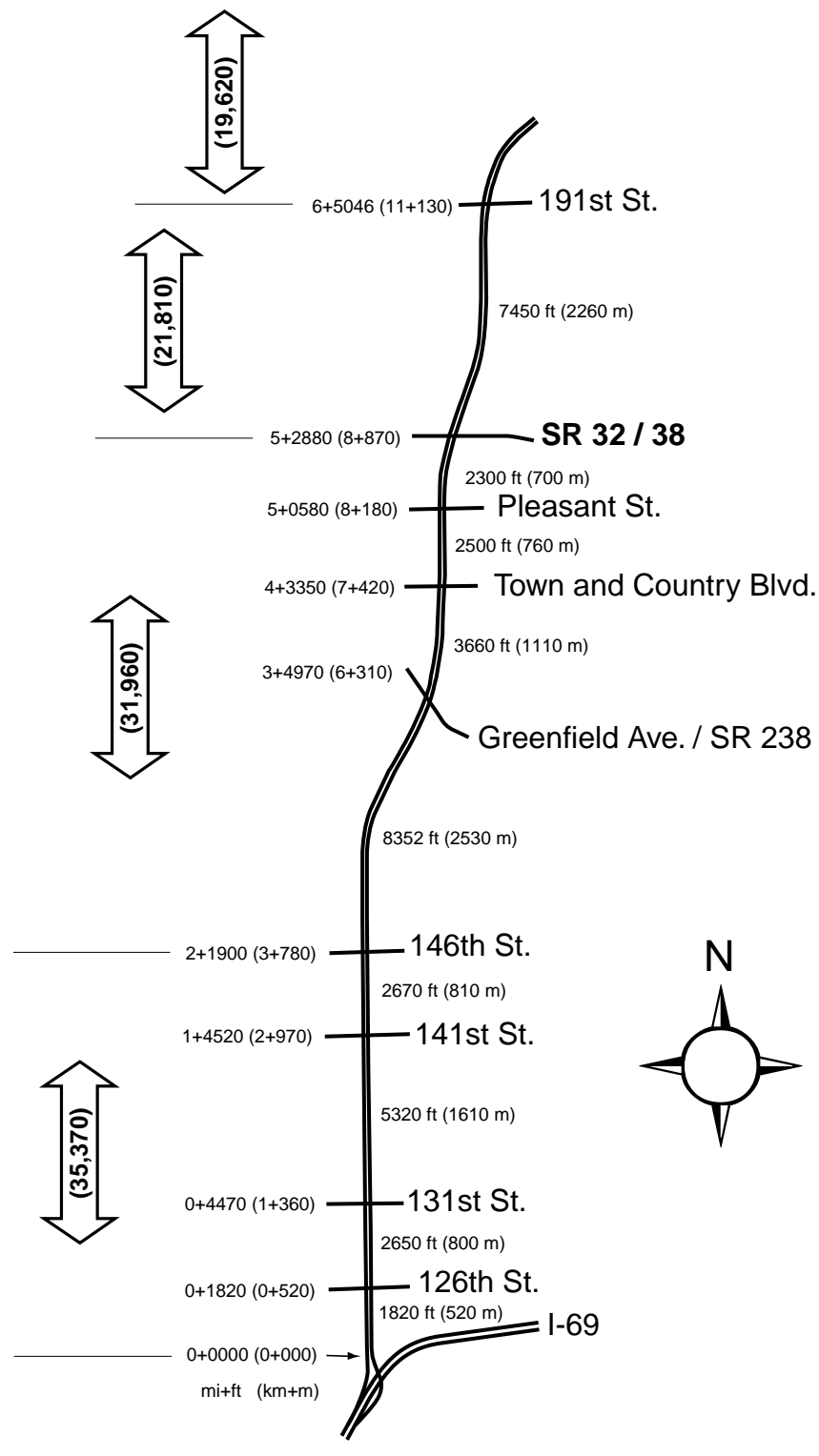

Figure 3.8 A map of distances between signalized intersections along SR 37, illustrating opportunities for signal coordination. Arrows indicate AADT along each major segment. 


\subsubsection{West Lafayette}

Northwestern Ave. (US 231) is an arterial highway serving west central Indiana, connecting the cities of Lafayette and West Lafayette to surrounding communities. The intersection of Northwestern Ave. and Stadium Ave. in West Lafayette, Indiana is situated on the loop that wraps around the Purdue University campus. As a consequence, it encounters a modest amount of pedestrian traffic in addition to vehicular traffic. Additionally, several bus routes pass through this intersection. These characteristics put contrast this intersection with the suburban intersection at Noblesville.

An aerial photo of the site is given in Figure 3.9. The Purdue University campus can be seen in the southwest (lower left hand) quadrant of the intersection. Pedestrian facilities exist on both sides of each approach. There are numerous vehicle access points near to the intersection. All left turn movements as well as the southbound and eastbound right turn movements have dedicated lanes. The southbound right turn is not subject to control by the signal. Vehicles making this movement yield to any other vehicles that are moving into the westbound lane exiting the intersection. Pedestrians crossing the path of vehicles making the southbound right turn are not protected by a walk signal.

Figure 3.10 shows the location of the nearest intersections from Northwestern and Stadium. Stadium and University lies $597 \mathrm{ft}(179 \mathrm{~m})$ to the east. All of the other intersections are far enough to avoid interaction. None of the neighboring signalized intersections are coordinated with Northwestern and Stadium. The termination of Stadium at Grant is unsignalized, with vehicles on Grant having the right of way. This reflects the status of Stadium as a local road along that segment. The segment of Northwestern to the south of the intersection features a wide median that facilitates pedestrian crossing at all points along the roadway. Pedestrian usage of the signal reflects only of a portion of all pedestrian traffic in the vicinity.

In Figure 3.11, we see a schematic of the intersection that shows the location of loop detectors. Every movement is outfitted with detectors, including the detached southbound right turn. This intersection was also outfitted with various cameras for the purpose of observing traffic movements. Only the northbound direction has advance detectors. These are relatively close from the stop bar; in peak periods, queues can form over the advance detectors. The southbound detectors SL4C, SA4C, and SB4C could ostensibly be considered as advance detectors, but only during time periods when queues do not form over them.

Figure 3.12(a) shows the assignment of phases at Northwestern and Stadium. This follows the same scheme as the Noblesville intersection, with phases 2 and 6 being the 
through movements of the major road. However, unlike Noblesville, there are pedestrian movements at this intersection. Ped 6 and ped 8 both cross to the pedestrian island and cross the southbound right turning movement outside of the signalized intersection. The ring diagram is shown in Figure 3.12(b), showing that there are permitted left turns for every left turning movement, and that the eastbound right turn movement has an overlap with the northbound left turn.

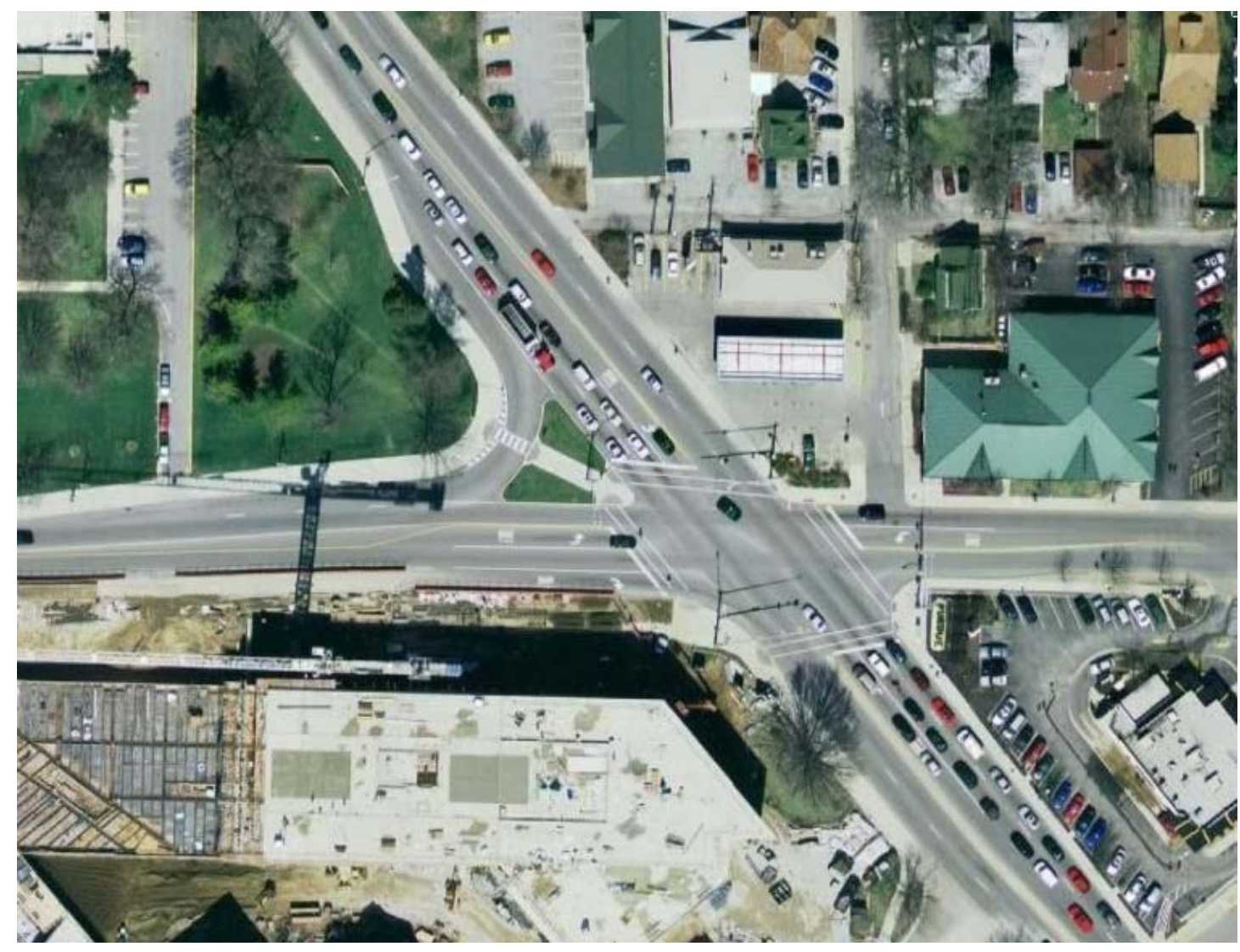

Figure 3.9 An aerial photo of Northwestern and Stadium, showing surrounding land use characteristics. 


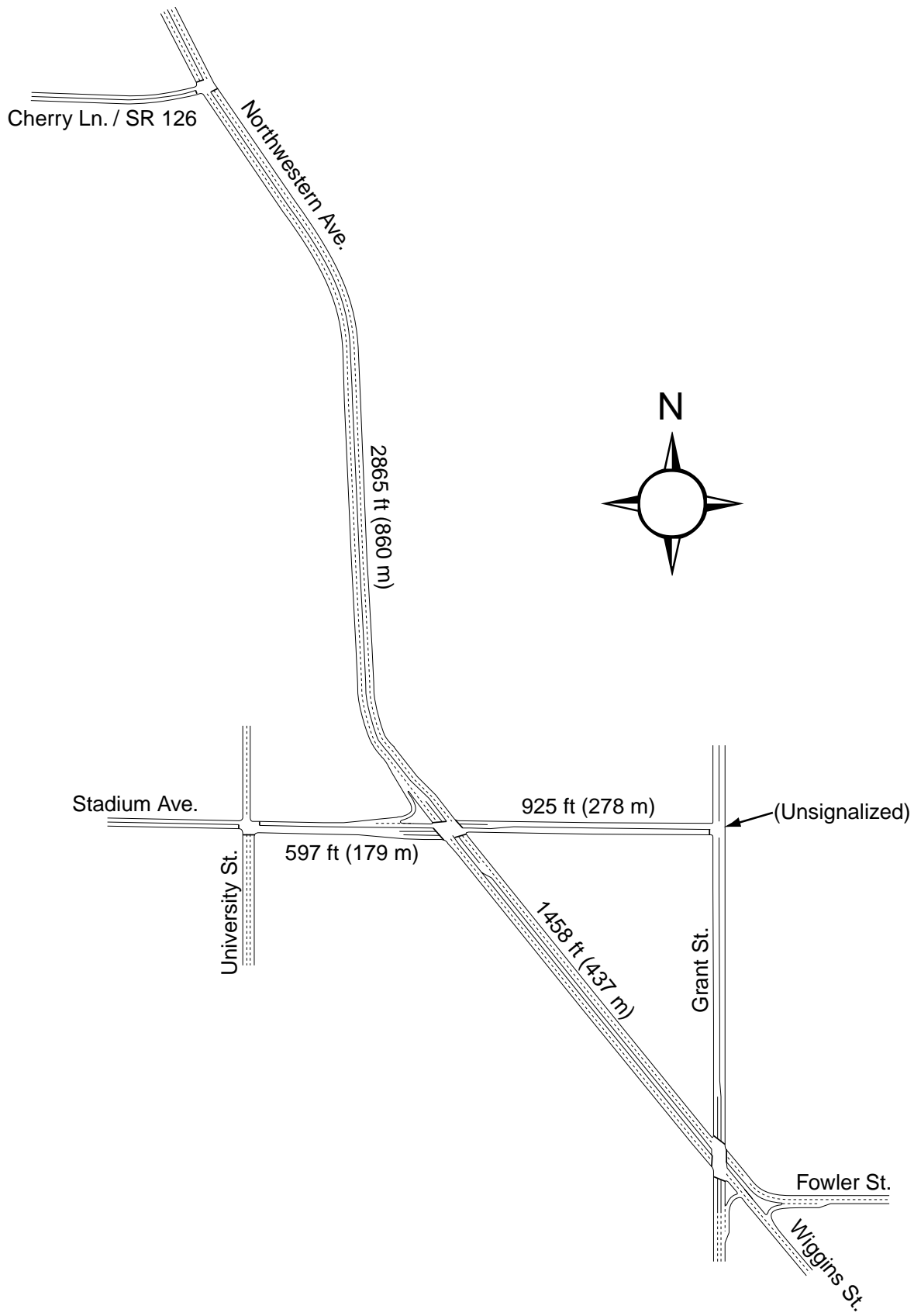

Figure 3.10 Adjacent major intersections from Northwestern and Stadium in West Lafayette, Indiana. 


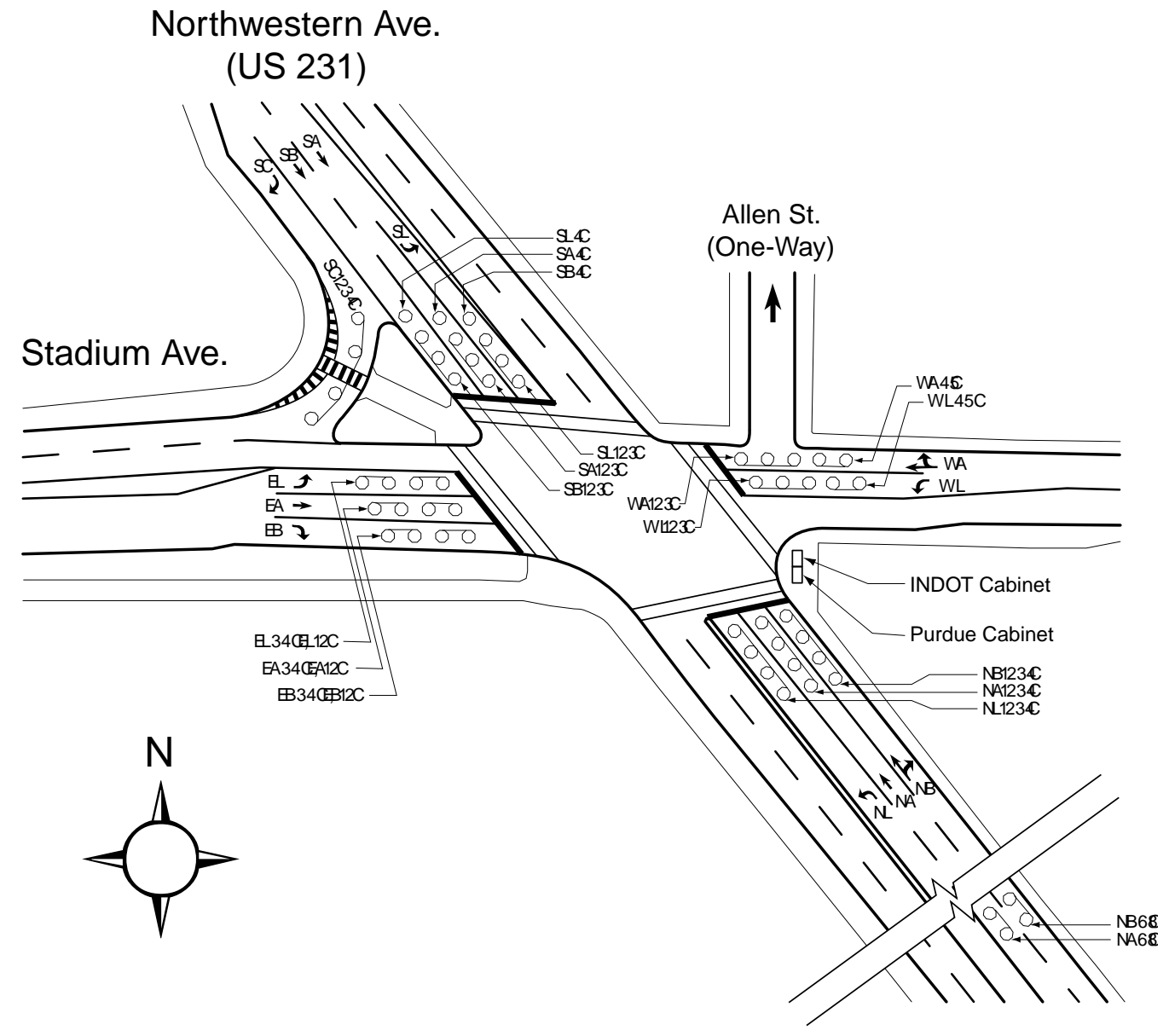

Figure 3.11 Schematic of the West Lafayette testbed, showing the placement of loop detectors. 


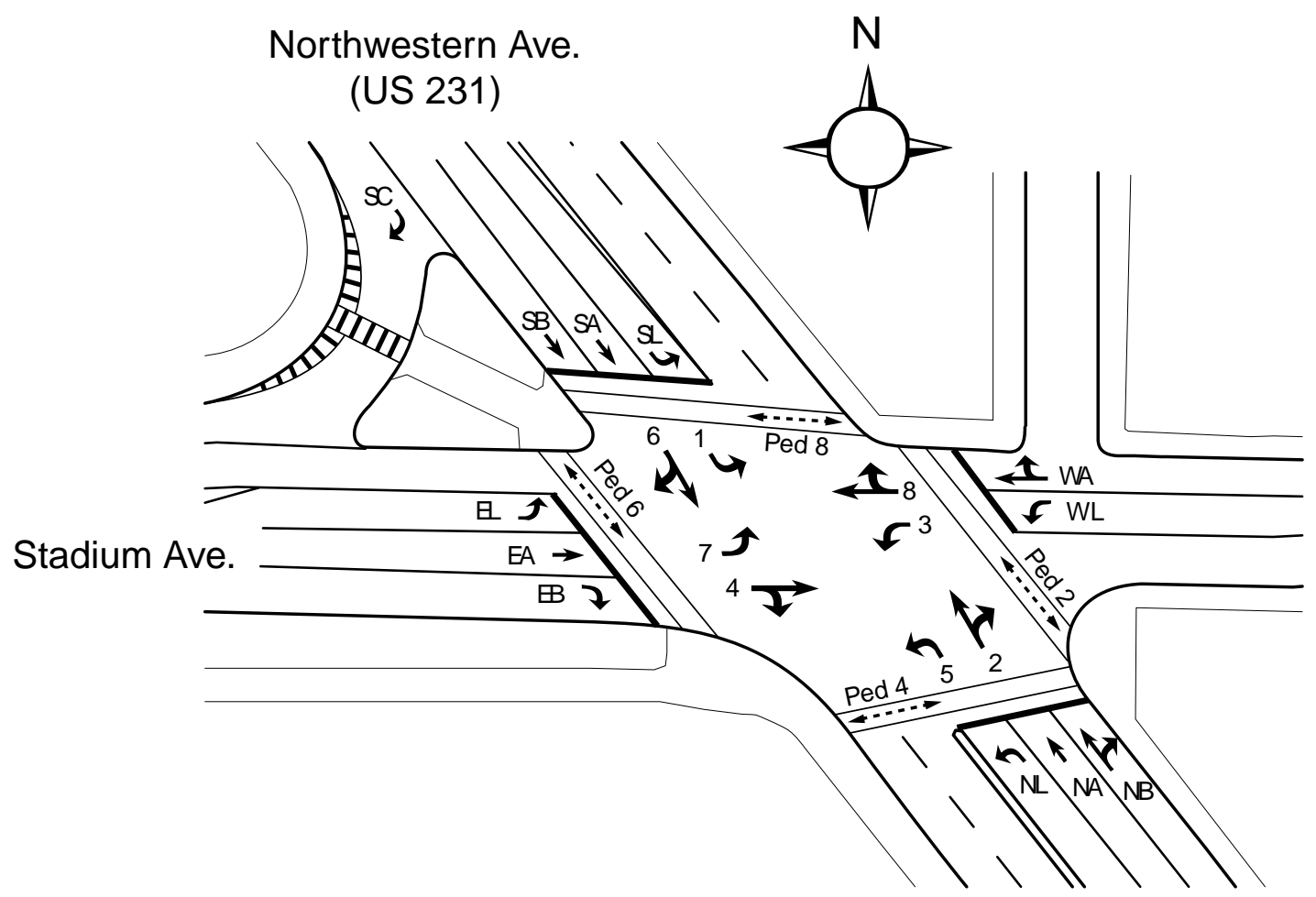

(a) Plan view showing phasing.

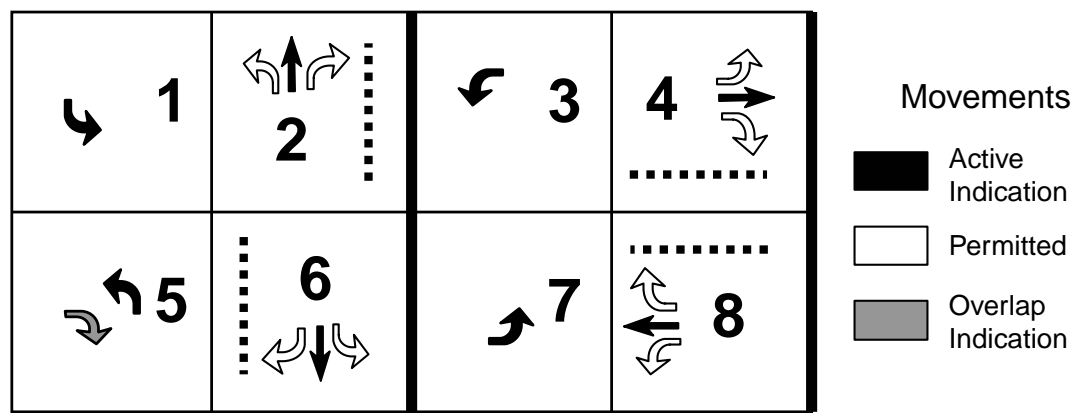

(b) Ring diagram.

Figure 3.12 Phases at Northwestern and Stadium. 


\subsection{Data Collection Process}

The objective of this section is to comprehensively describe the data collection process. The traffic data that was collected in this study was dependent on what data was available at the test intersection. The types of data were limited to phase information and vehicle arrivals. These inputs represent what would be available to a signal controller at a typical intersection. In the following chapter, the methods used to convert raw data into performance measures will be presented.

\subsubsection{Gathering Raw Data}

Inside the research cabinets at both intersections, digital signals representing detector states and phase indications were available as 24 -volt digital signals. Pulse mode detection was used for vehicle arrivals. The data was logged using Autoscope Solo Pro cameras, which offered a feature by which the states of digital signals could be recorded over time. Each camera was capable of recording up to eight digital inputs. These inputs were recorded in a text file on a computer using a data collection program provided by Autoscope. These data in the text files were later translated into performance measures using a series of Excel macros. A block diagram of the process is shown in Figure 3.13.

A groundtruthing procedure was carried out to verify that events were logged correctly. This was accomplished by watching the video and confirming that the order of logged events were matched by events recorded in the video as the change in overlay appearance. 


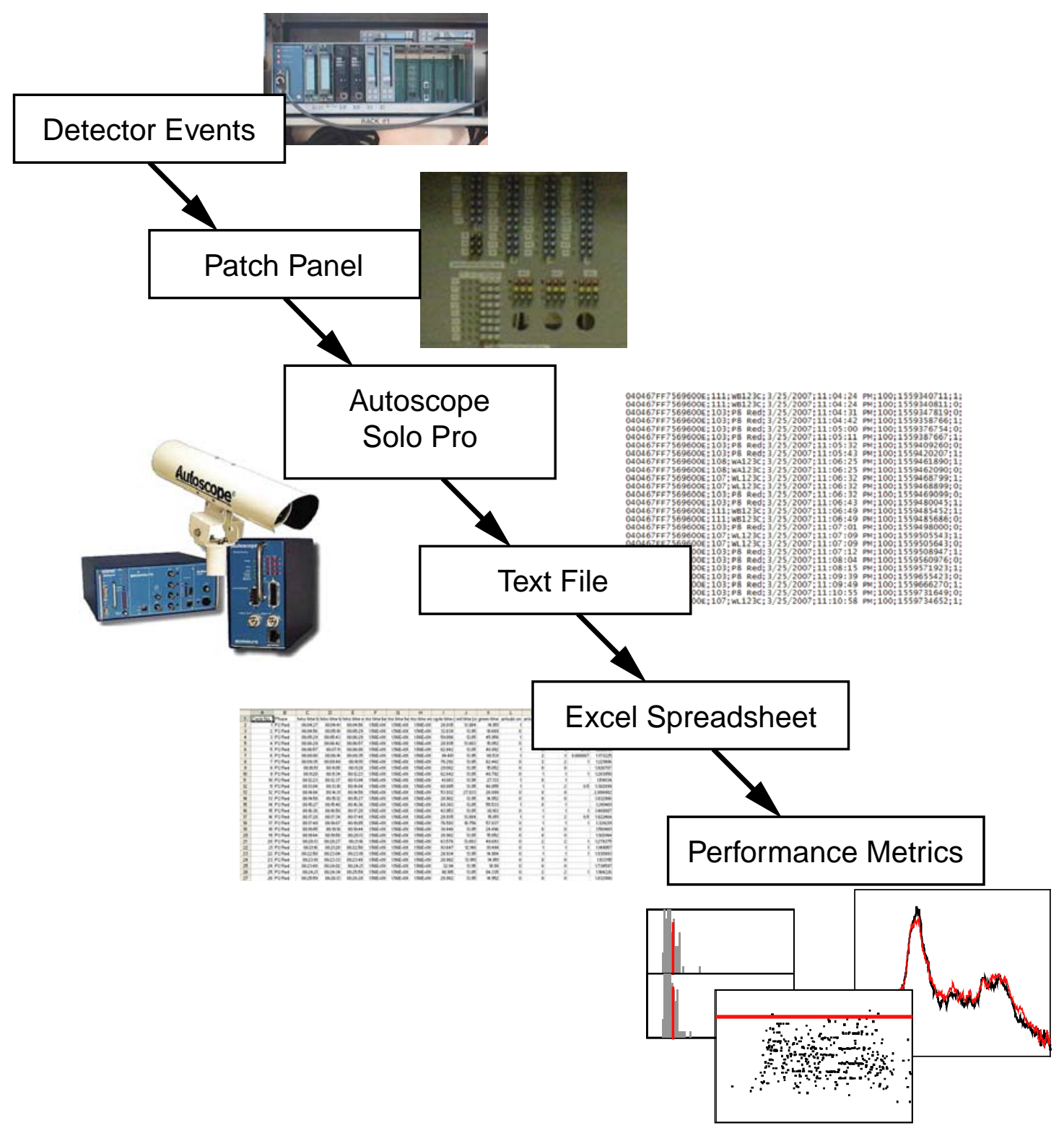

Figure 3.13 Block diagram showing the data collection and reduction process from start to finish. 


\subsubsection{Preparation of Data}

Traffic events were classified into categories of data based on the state information required to build a picture of the intersection performance.

- Vehicle phase data was captured by recording information about the red indication. The points in time when the red ball was shown for a particular intersection were recorded, which included the time when this indication came on (the "high" state, represented as "1") and the time when this indication was removed (the "low" state, "0"). Pedestrian phase data was similarly recorded, except that the length both of the walk and pedestrian clearance intervals were desired.

- For vehicle arrivals, only the detector turn-on time was needed. Therefore, only the high states were included. Because pulse mode detection was used, the width of the high state did not contain any relevant information. Each vehicle arrival was identified at a single point in time.

One Autoscope camera was used for each approach, which included two phases (the left turn and the through movement). Because of the limitation of the number of inputs to eight, it was not possible to record the states of all phase color indications for two phases in addition to all of the detector actuations. Only the red indication was included; the times when the red signal was "off" implied when the green was "on." Using the length of the yellow interval, it was possible to calculate the duration of the green indication. This is described in the next chapter. It was not desirable to use multiple cameras for a single approach, because of clock drift between cameras that would have recorded event times for phase changes and vehicle arrivals on slightly different clocks.

Table 3.1(a) provides an example of the appearance of the raw data as it appears in the event logs. After parsing the data into column format and eliminating unnecessary items, five objects were defined for each item of data, as shown in Table 3.1(b).

- The name of a data item was used to identify a particular phase indication or detector to which the item of data pertained.

- The date was also included. Data was logged and analyzed in 24-hour blocks.

- The time was recorded in h:m:s (hour, minute, second) format. This time was used to match events between different cameras. 
- The time was also recorded in ms. This number varied from one Autoscope camera to the next, and different cameras experienced unpredictable clock drift, making it infeasible to use multiple Autoscope cameras for interdependent numbers. The ms time was used to match vehicle arrivals to the phases that corresponded with relevant vehicle movements. This number was stored as a long integer, the overflow of which was a source of time discontinuity requiring surgical repair of the data.

- The state recorded whether a phase indication was turning on or off, or whether a detector was turning on or off.

Table 3.2 shows a list of cycles and times in which the green indication for eight phases began, taken from data collected at Noblesville on March 14, 2007. The abbreviation "NC" refers to a phase not being called for that particular cycle. Each approach phase pair $(1,6),(2,5),(3,8)$, and $(4,7)$ shared a common clock. Cycle times were based on the same clock as phase 2. Figure 3.14 shows a trace in time of the red indication for eight phases at Noblesville. The transitions from high to low correspond to the times listed in Table 3.2. Each low state of the red indication indicates a time period in which the phase received the green ball (or arrow, in the case of left turns). The beginning of a cycle is indicated by the vertical dashed line. Following the shapes of the lines in one ring through one cycle, it can be seen that phases 3 and 7 come first, followed by phases 4 and 8 , then 1 and 6 , followed by 2 and 5 . This is expected, following the discussion in Section 2.6 about the effective order of phases in a cycle. Note the lagging left turn that takes place at Noblesville (i.e., phase 5 follows phase 6). Also notice that the cycle boundaries coincide with the beginning of the high state for the red indication of phase 2. The method by which cycle lengths and green indications were found are described in more detail in the following chapter.

Some overlap between succeeding cycles is visible in Figure 3.14 as a consequence of clock drift. This is examined in more detail in Figure 3.15, which contains a magnification of the traces of phase 1 and 2 within the minute of 10:58. The end of green for phase 1 is reported as occurring about 1 second after than the beginning of green for phase 2. Of course, phases 1 and 2 are conflicting movements, so if this had actually happened it would have been a potentially catastrophic occurrence. This figure demonstrates the limitations imposed by the clock drift in comparing data between cameras. It was possible to match phases to cycles; the precision of measurements was on the order of seconds. Figure 3.16 illustrates the mapping of phases to cycles for the ring containing phases 1, 2, 3, and 4 . The beginning of green (BOG) times, implied by the end 
of the red indication, are matched to the intervals defined by boundaries using a lookup function.

This clock drift did not affect the analysis of vehicle movement, because no measurements pertaining to analysis were made between phases occurring on different cameras. Event times for vehicle movement analysis was contained on one clock, and was based on the more accurate ms time.

The green indications shown for left turn movements $(1,3,5$, and 7) reflect the protected portion (i.e., during the green arrow). Permitted left turns could be made during the "red" for those phases (except for phases 1 and 5 at Noblesville, where they were not allowed); in this study, permitted left turns were not included in analysis; only protected left turns were counted. A simple logic function was used to determined whether an arrival was protected. An arrival at a left turn lane was protected if either of the two conditions held:

- The corresponding left turn phase was green at the time of arrival; this meant that the vehicle performed the left turn during the protected phase.

- All of the phases on the same side of the barrier were red at the time of arrival. This meant that the vehicle arrived during a time in which it could not have made a permitted left turn, and would therefore move in the next protected phase.

This is illustrated in Figure 3.17, which shows the red phase traces for phases 3, 4, 7 , and 8 , and arriving vehicles on phase 7 , which would be the eastbound left turn at a typical intersection. Vehicle A arrives during the green arrow for phase 7, and is therefore a protected left turn. Vehicle B arrives at a time when phase 7 is red, but phase 8 is still green. There is therefore still a chance that this vehicle can perform a permitted left turn and exit the intersection. There is also a chance that it could be trapped by high volumes of the opposing through movement and forced to wait until the next cycle. In this work it is assumed that all permitted turns carry out their movements. Finally, Vehicle $\mathrm{C}$ arrives at a time in which phases on the other side of the barrier are being served, so there is no opportunity for it to carry out a permitted left turn. Vehicle $\mathrm{C}$ is counted for next green indication of phase 7 .

The translation of phase and detector events into performance measures is the topic of the following chapter. 
Table 3.1 Raw Data.

(a) A sample of raw data.

\begin{tabular}{|c|c|}
\hline Line & Data \\
\hline 1 & 040468FFDC65620E;107;EL1234C;3/14/2007;3:11:27 AM;100;1683325101;1; \\
\hline 2 & 040468FFDC65620E;107;EL1234C;3/14/2007;3:11:27 AM;100;1683325301;0; \\
\hline 3 & 040468FFDC65620E;103;P4 Red;3/14/2007;3:11:39 AM;100;1683337449;0; \\
\hline 4 & 040468FFDC65620E;104;P7 Red;3/14/2007;3:11:39 AM;100;16833374449;0; \\
\hline 5 & 040468FFDC65620E;106;P7 Green;3/14/2007;3:11:39 AM;100;1683337449;1; \\
\hline 6 & 040468FFDC65620E;105;P7 Yellow;3/14/2007;3:11:46 AM;100;1683344458;1; \\
\hline 7 & 040468FFDC65620E;106;P7 Green;3/14/2007;3:11:46 AM;100;1683344458;0; \\
\hline 8 & 040468FFDC65620E;104;P7 Red;3/14/2007;3:11:49 AM;100;1683347662;1; \\
\hline 9 & 040468FFDC65620E;105;P7 Yellow;3/14/2007;3:11:49 AM;100;1683347662;0; \\
\hline 10 & 040468FFDC65620E;103;P4 Red;3/14/2007;3:11:50 AM;100;1683348396;1; \\
\hline 11 & 040468FFDC65620E;108;EA1234C;3/14/2007;3:12:23 AM;100;1683381202;1; \\
\hline 12 & 040468FFDC65620E;108;EA1234C;3/14/2007;3:12:23 AM;100;1683381302;0; \\
\hline 13 & 040468FFDC65620E;103;P4 Red;3/14/2007;3:12:26 AM;100;1683384439;0; \\
\hline 14 & 040468FFDC65620E;103;P4 Red;3/14/2007;3:12:37 AM;100;1683395352;1; \\
\hline 15 & 040468FFDC65620E;108;EA1234C;3/14/2007;3:13:22 AM;100;1683440340;1; \\
\hline
\end{tabular}

(b) Relevant items parsed from the above data.

\begin{tabular}{|r|l|c|c|c|r|}
\hline line & \multicolumn{1}{|c|}{ name } & date & h:m:s time & ms time & state \\
\hline 1 & EL1234C & $3 / 14 / 2007$ & $3: 11: 27$ AM & 1683325101 & 1 \\
\hline 2 & EL1234C & $3 / 14 / 2007$ & $3: 11: 27 \mathrm{AM}$ & 1683325301 & 0 \\
\hline 3 & P4 Red & $3 / 14 / 2007$ & $3: 11: 39 \mathrm{AM}$ & 1683337449 & 0 \\
\hline 4 & P7 Red & $3 / 14 / 2007$ & $3: 11: 39 \mathrm{AM}$ & 1683337449 & 0 \\
\hline 5 & P7 Green & $3 / 14 / 2007$ & $3: 11: 39 \mathrm{AM}$ & 1683337449 & 1 \\
\hline 6 & P7 Yellow & $3 / 14 / 2007$ & $3: 11: 46 \mathrm{AM}$ & 1683344458 & 1 \\
\hline 7 & P7 Green & $3 / 14 / 2007$ & $3: 11: 46 \mathrm{AM}$ & 1683344458 & 0 \\
\hline 8 & P7 Red & $3 / 14 / 2007$ & $3: 11: 49 \mathrm{AM}$ & 1683347662 & 1 \\
\hline 9 & P7 Yellow & $3 / 14 / 2007$ & $3: 11: 49 \mathrm{AM}$ & 1683347662 & 0 \\
\hline 10 & P4 Red & $3 / 14 / 2007$ & $3: 11: 50 \mathrm{AM}$ & 1683348396 & 1 \\
\hline 11 & EA1234C & $3 / 14 / 2007$ & $3: 12: 23 \mathrm{AM}$ & 1683381202 & 1 \\
\hline 12 & EA1234C & $3 / 14 / 2007$ & $3: 12: 23 \mathrm{AM}$ & 1683381302 & 0 \\
\hline 13 & P4 Red & $3 / 14 / 2007$ & $3: 12: 26 \mathrm{AM}$ & 1683384439 & 0 \\
\hline 14 & P4 Red & $3 / 14 / 2007$ & $3: 12: 37 \mathrm{AM}$ & 1683395352 & 1 \\
\hline 15 & EA1234C & $3 / 14 / 2007$ & $3: 13: 22 \mathrm{AM}$ & 1683440340 & 1 \\
\hline
\end{tabular}


Table 3.2 Correspondence of phases to cycles

\begin{tabular}{|c|c|c|c|c|c|c|c|c|}
\hline \multirow{2}{*}{$\begin{array}{c}\text { Cycle } \\
\begin{array}{c}\text { Begin } \\
\text { Time }\end{array}\end{array}$} & \multicolumn{7}{|c|}{ Phase Beginning of Green Indication } \\
\cline { 2 - 9 } & 1 & 2 & 3 & 4 & 5 & 6 & 7 & 8 \\
\hline $10: 54: 21$ & $10: 55: 00$ & $10: 55: 13$ & NC & $10: 54: 41$ & $10: 55: 37$ & $10: 55: 00$ & NC & $10: 54: 36$ \\
\hline $10: 55: 57$ & $10: 56: 30$ & $10: 56: 38$ & $10: 54: 24$ & $10: 56: 03$ & $10: 57: 13$ & $10: 56: 30$ & $10: 54: 27$ & $10: 56: 00$ \\
\hline $10: 57: 33$ & NC & $10: 58: 32$ & $10: 57: 36$ & $10: 57: 53$ & $10: 58: 49$ & $10: 58: 24$ & $10: 57: 39$ & $10: 57: 48$ \\
\hline $10: 59: 09$ & $10: 58: 24$ & $10: 59: 46$ & $10: 59: 12$ & $10: 59: 34$ & $11: 00: 41$ & $10: 59: 49$ & NC & $10: 59: 27$ \\
\hline $11: 01: 10$ & $11: 01: 49$ & $11: 01: 57$ & $11: 01: 14$ & $11: 01: 36$ & $11: 02: 43$ & $11: 01: 49$ & NC & $11: 01: 14$ \\
\hline $11: 03: 09$ & $11: 04: 00$ & $11: 04: 10$ & $11: 03: 12$ & $11: 03: 31$ & $11: 04: 35$ & $11: 04: 00$ & NC & $11: 03: 12$ \\
\hline $11: 04: 51$ & NC & $11: 05: 51$ & $11: 04: 54$ & $11: 05: 13$ & $11: 06: 09$ & $11: 05: 42$ & $10: 59: 15$ & $11: 04: 54$ \\
\hline $11: 06: 33$ & $11: 05: 42$ & $11: 07: 06$ & $11: 06: 36$ & $11: 06: 56$ & $11: 07: 51$ & $11: 07: 09$ & NC & $11: 06: 50$ \\
\hline $11: 08: 15$ & $11: 08: 53$ & $11: 09: 05$ & $11: 08: 18$ & $11: 08: 38$ & $11: 09: 33$ & $11: 08: 53$ & $11: 06: 39$ & $11: 08: 18$ \\
\hline $11: 09: 57$ & $11: 10: 37$ & $11: 10: 46$ & $11: 10: 00$ & $11: 10: 20$ & $11: 11: 15$ & $11: 10: 37$ & $11: 10: 03$ & $11: 10: 14$ \\
\hline
\end{tabular}




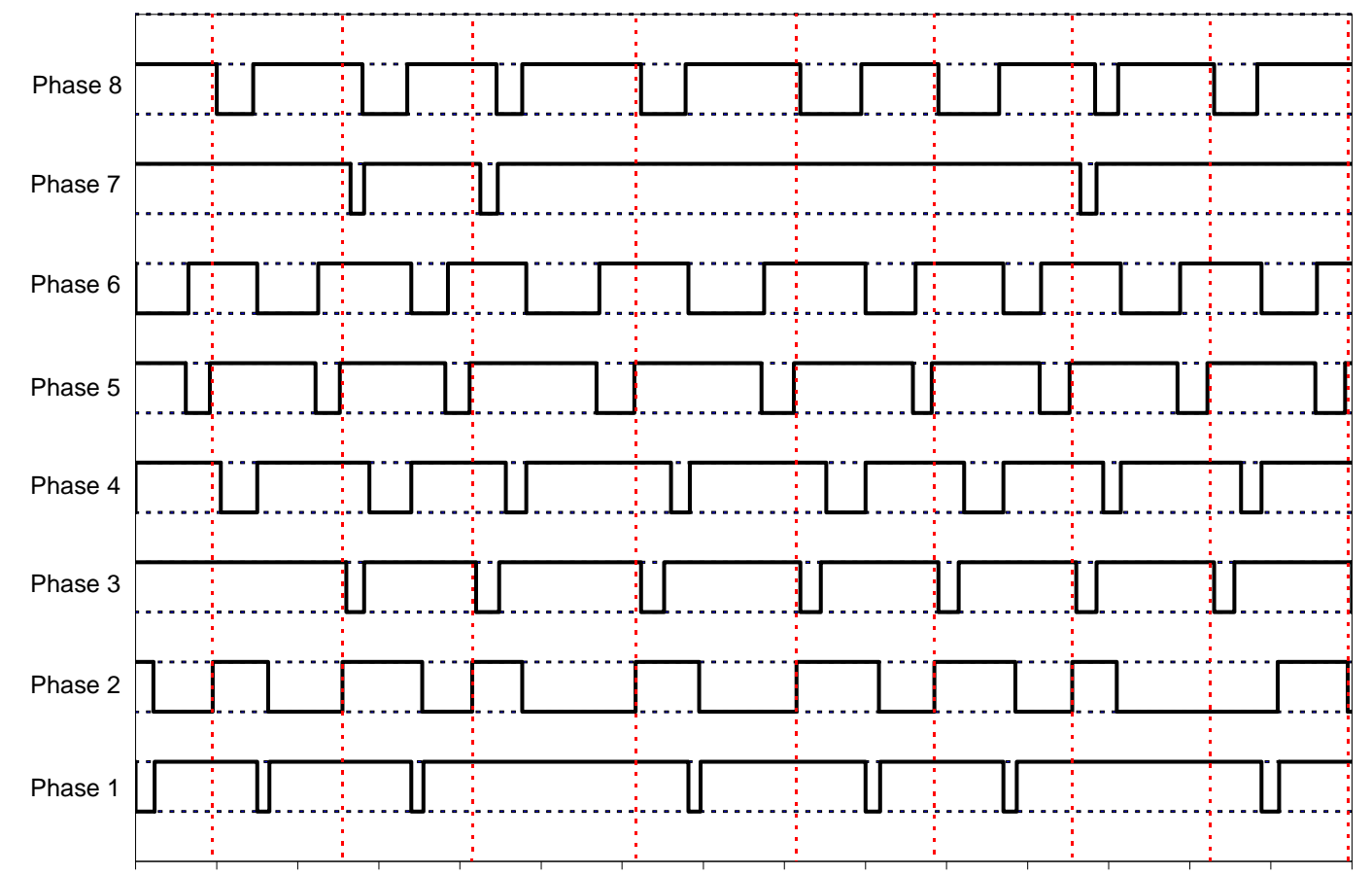

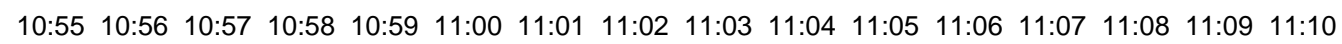

Figure 3.14 Trace of red indications for 8 phases at Noblesville on March 14, 2007, from 10:55 to $11: 10$. 


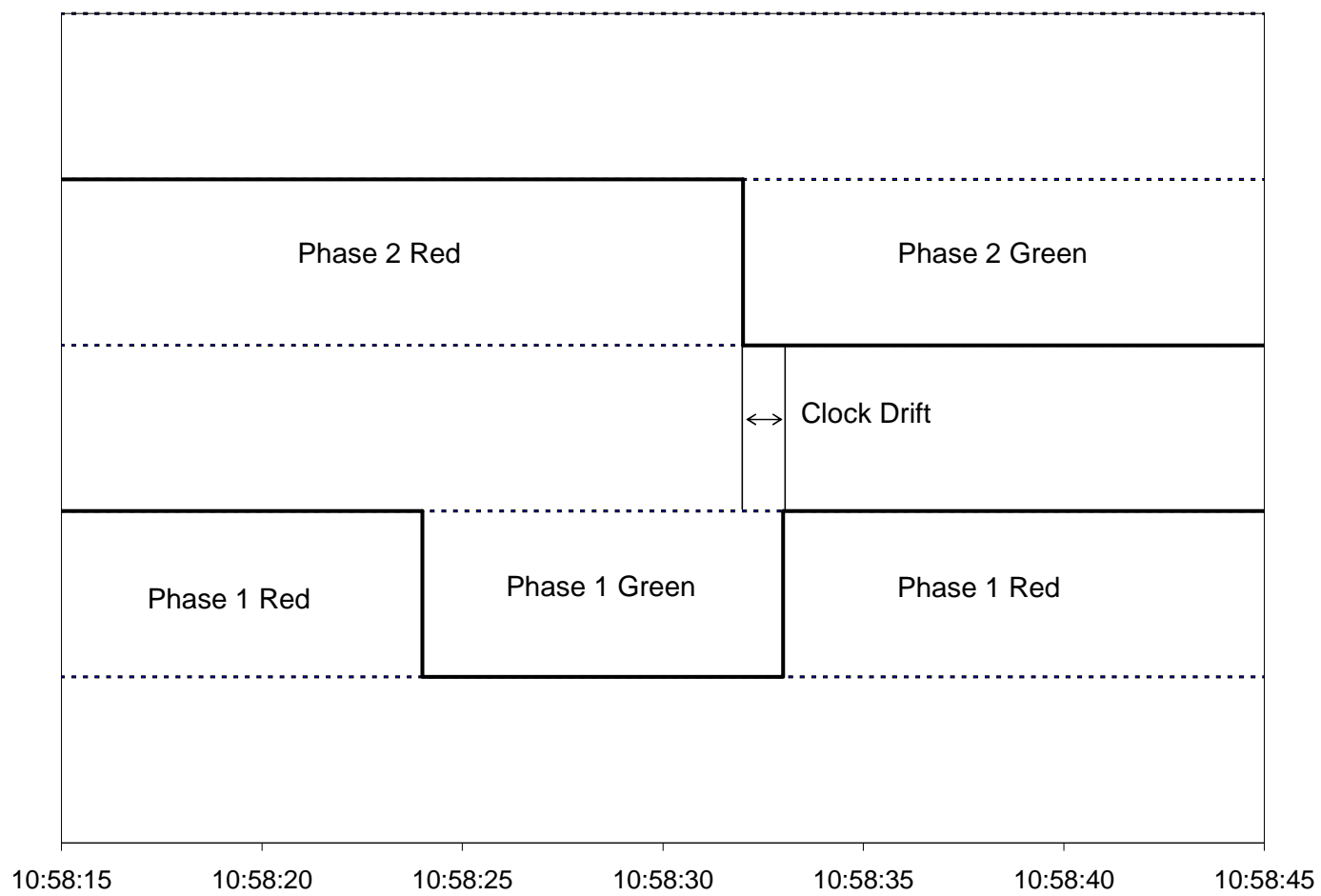

Figure 3.15 Detail of phase state traces showing impact of clock drift. Phase 1 and phase 2 events are recorded by different clocks. 


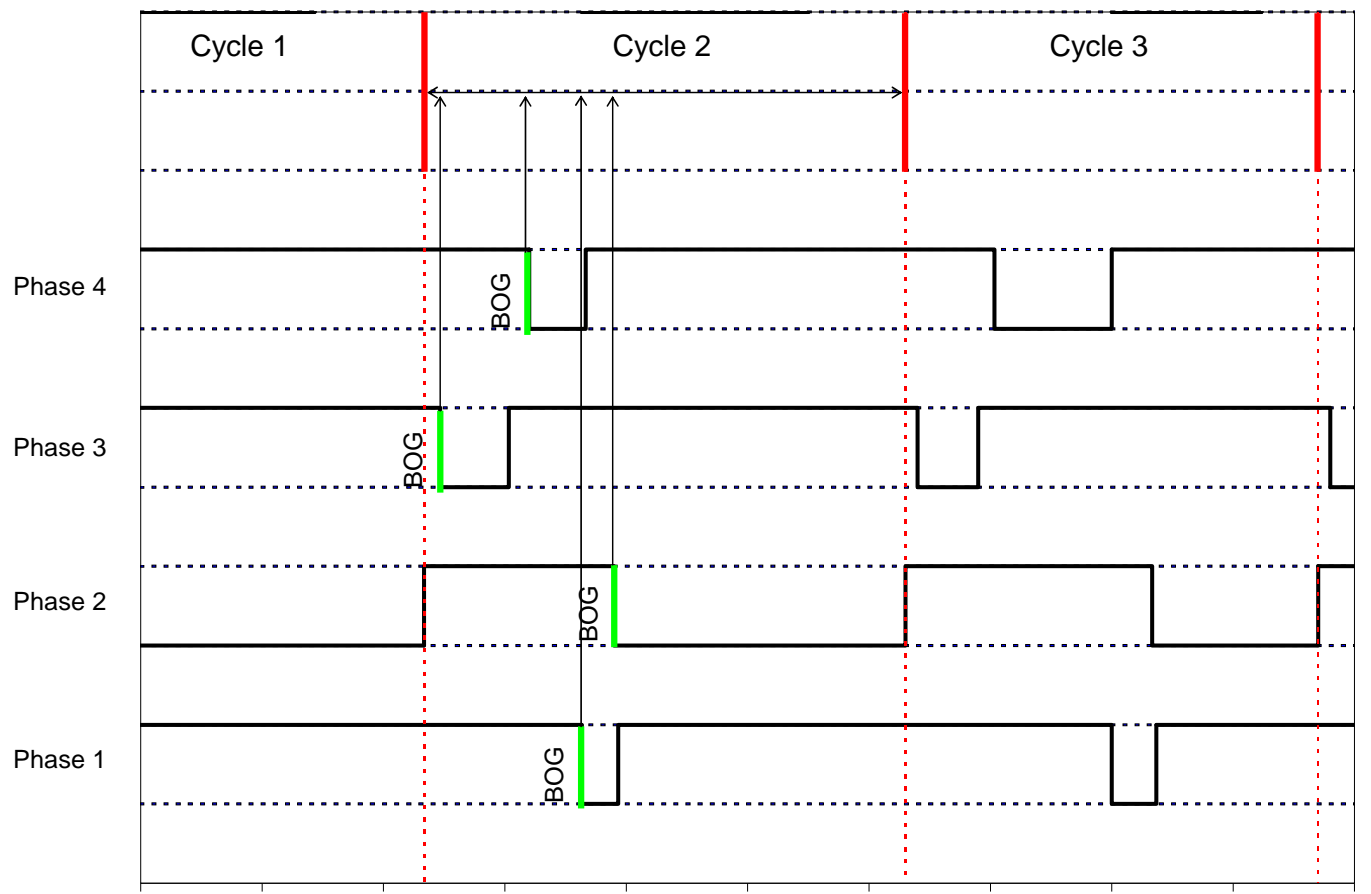

11:00:00 11:00:30 11:01:00 11:01:30 11:02:00 11:02:30 11:03:00 11:03:30 11:04:00 11:04:30 11:05:00

Figure 3.16 Matching of phases to cycles in data collected at Noblesville on Wednesday, March 14, 2007. 


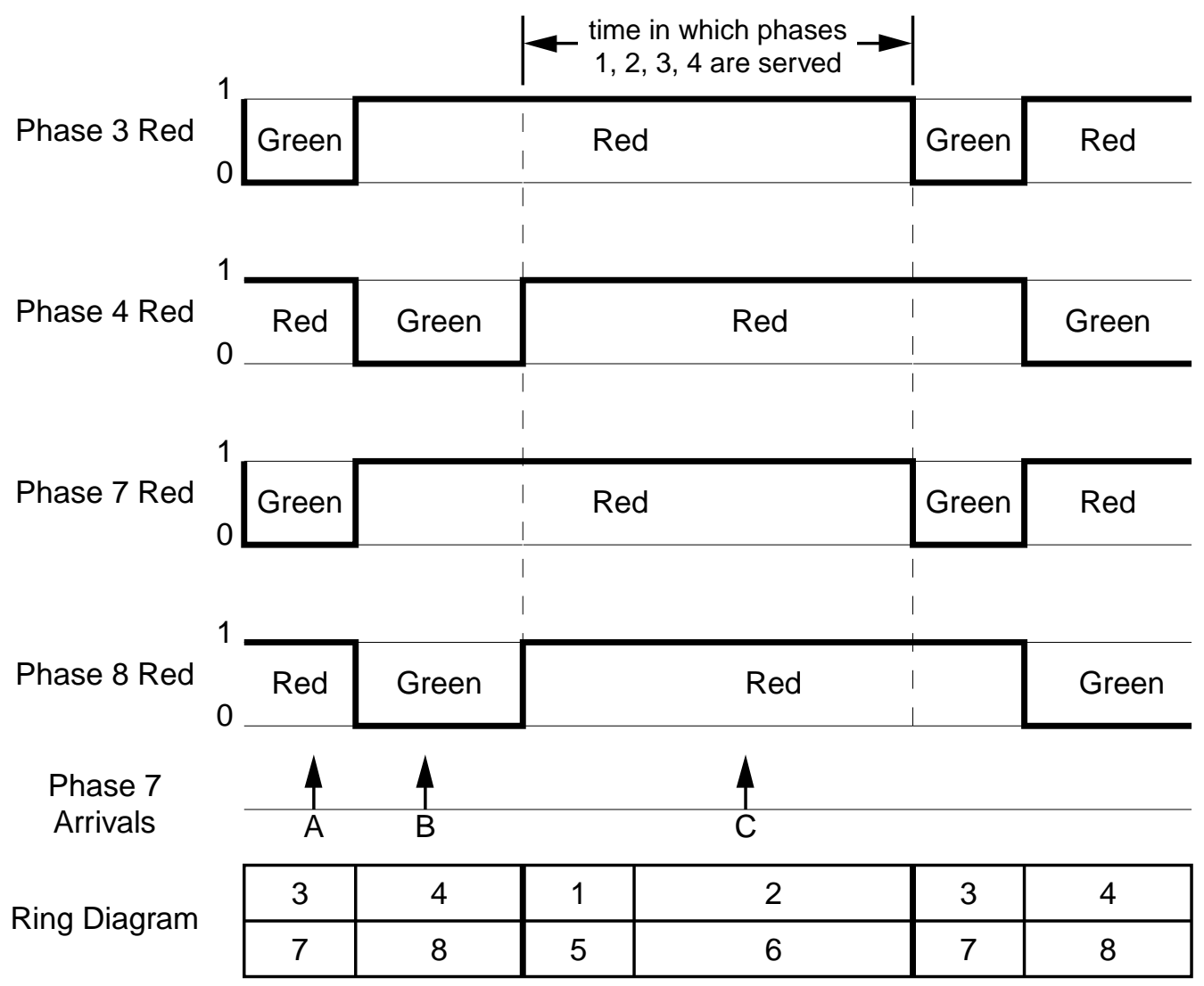

Figure 3.17 Permitted left turn logic. 


\section{CHAPTER 4. CALCULATION OF PERFORMANCE MEASURES}

\subsection{Introduction}

In Chapter 3, we discussed the collection of data, and the processing of that data into useful form. In this chapter, we present the mechanics of converting this raw data into various quantities, including performance measures and other quantities needed to obtain those performance measures. All of the analysis in this work was carried out in Excel using Visual Basic macros, but the formulas and methods could be recreated in other formats for use on various platforms. Ultimately, performance measures might be calculated by a device inside of a traffic signal cabinet; or possibly, with improvements in technology, by signal controllers themselves as a standard feature.

The quantities discussed in this chapter are divided into three categories. The first category includes quantities that describe the state of the intersection, and are necessary to calculate all of the performance measures. These include:

- cycle length,

- green duration, and

- volume.

The second category is a set of derived quantities that measure the performance of the intersection with regard to vehicular traffic, using the concept of intersection capacity as the basis for analysis. This group comprises

- $\quad$ service flow rate,

- estimated capacity,

- observed capacity,

- volume-to-capacity (v/c) Ratio,

- number of split failures, and

- $\quad$ critical $\mathrm{v} / \mathrm{c}$ ratio. 
The third category quantifies the intersection's performance in coordination with vehicle progression along an arterial corridor. The performance measures presented here are

- percent of arrivals on green

- arrival type, and

- platoon profile.

\subsection{Quantities Describing the State of the Intersection}

\subsubsection{Cycle Length}

Cycle length is the amount of time it takes a traffic signal to serve every phase for which there is demand. Where signal coordination is required, cycle length is usually kept constant to maintain flow patterns, with green indications synchronized for coordinated movements. Under free actuated operation, each phase is served as vehicles place calls for them, and cycle length varies from cycle to cycle. During low volume times of day, it is a common practice to recall the green to the major through movements. These phases may retain the green for several minutes or longer, when no calls occur for conflicting movements. Because the signal remains at a particular position in the ring diagram for an extended period of time, very long cycle lengths are calculated.

Whether cycle length is constant or is allowed to vary, it is desirable to measure it in order to substantiate what occurs in the field. Cycle length is measured by calculating the amount of time that passes between similar points in the ring diagram. We refer to the chosen point as the deterministic point. This point occurs in every cycle, and serves as the cutoff between one cycle and the next. In this paper, we have used the end of green for the leading phase as the deterministic point.

At Noblesville, Phase 2 was typically the coordinated reference phase in the data presented in this work, with Phase 4 occasionally taking the role during certain time periods in data from 2006. At West Lafayette, the controller was operated in free mode and set to recall phases 2 and 6 . Either phase could have been used as the reference phase.

Cycle length was found by measuring the amount of time passing between successive end of green times for the leading phase. The method of calculation is illustrated in Figure 4.1. The equation for cycle length (in seconds) would be

$C=\alpha\left(T_{2}-T_{1}\right)$,

Equation 4.1 
where $T_{1}$ is the point in time marking the beginning of the cycle, $T_{2}$ is the point in time marking the beginning of the next cycle, and $\alpha$ is a constant converting the native units of time to seconds. In the data collected for this work, time was recorded in milliseconds, so a value of 0.001 was used for $\alpha$.

Table 4.1 shows example calculations of cycle length at Noblesville on Wednesday, March 14, 2007. For example, a cycle beginning at 10:55:57 and ending at 10:59:09 had 95,982 ms between the two timestamps, from which we calculate a cycle length of $96 \mathrm{~s}$. Of particular note in this selection of cycles is the changeover from one time of day (TOD) plan to the next. Cycles in the 9:00-11:00 plan have $C=96 \mathrm{~s}$, while those the 11:00 - 13:00 plan have $C=102 \mathrm{~s}$.

Note that the two cycles close to the changeover point have unusually large cycle lengths. Typically, cycles near to the TOD plan boundaries report larger or smaller values of $C$. When cycles change from one reference phase to another, there is an opportunity for erroneous values to be calculated by the spreadsheet analysis tool because of clock drift between phases. However, in the example shown here, phase 2 was the leading phase in both plans, so the reported cycle lengths reflect the actual controller behavior.

In Figure 4.2, we see a plot of cycle length over 24 hours on March 14, 2007. Each plateau represents a constant cycle length being applied across a particular time period. Spikes can be seen at each boundary. Before 6:00 and after 22:00, the controller operated in free mode. Wide variation between cycles can be seen in the trace of cycle length through those periods. 


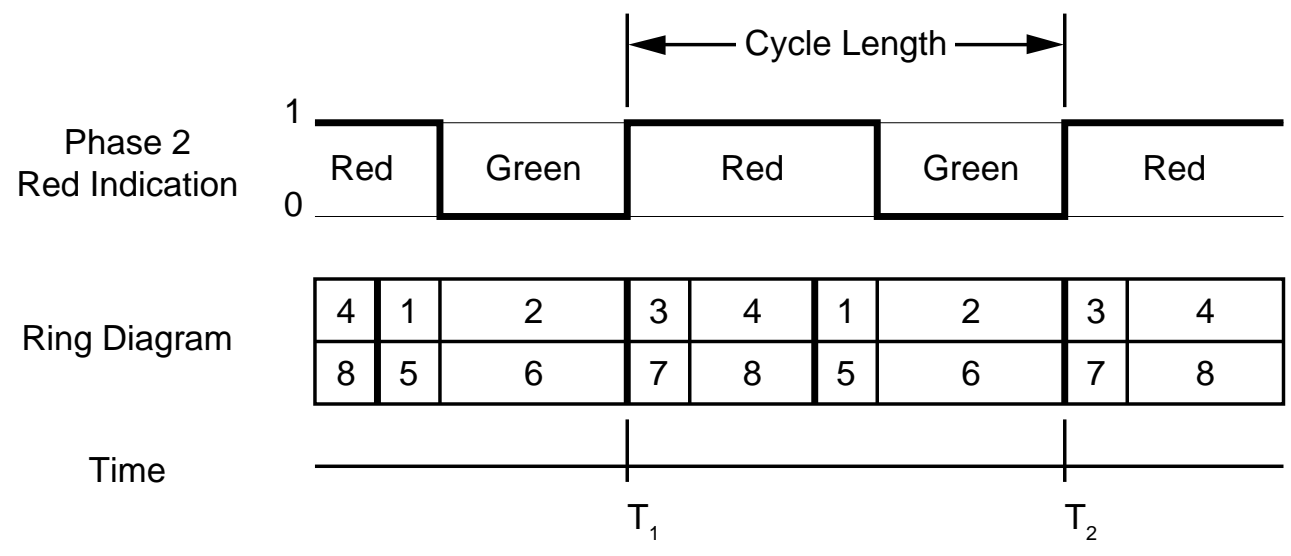

(a) Phase 2 Reference

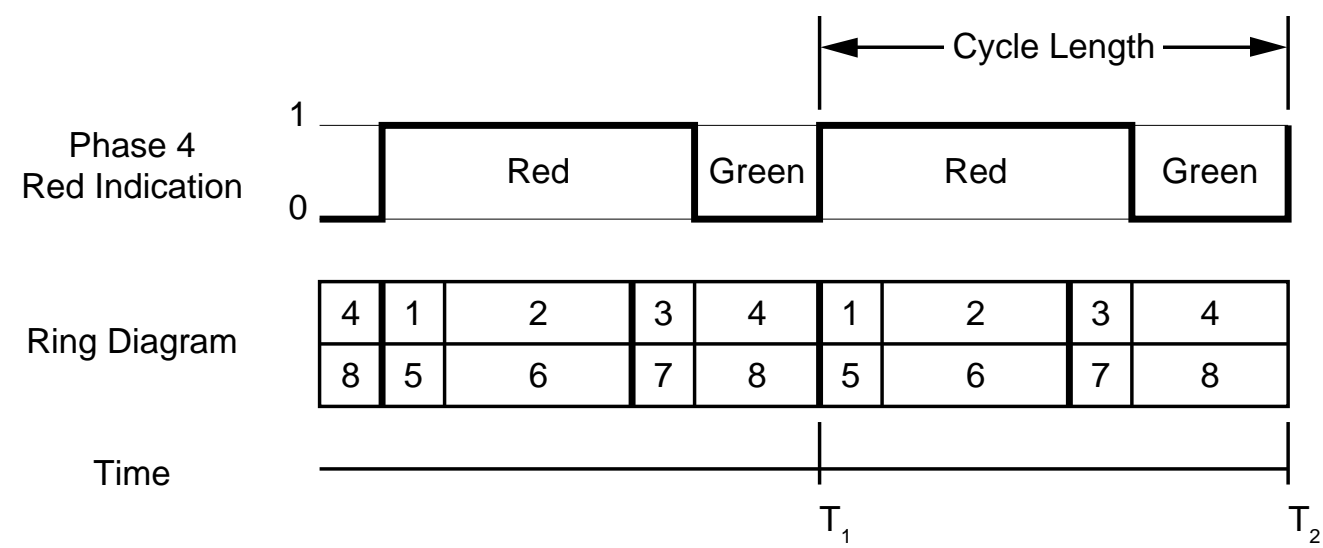

(b) Phase 4 Reference

Figure 4.1 Measurement of cycle length from the reference phase red indication and its meaning in relation to the ring diagram. In (a), phase 2 is the reference phase; in (b), phase 4 is the reference phase. 
Table 4.1 Calculation of cycle length at Noblesville on March 14, 2007.

\begin{tabular}{|l|l|l|r|r|r|r|}
\hline \multicolumn{2}{|c|}{ Beginning of Cycle } & \multicolumn{2}{|c|}{ End of Cycle } & \multicolumn{1}{|c|}{} \\
\hline $\mathrm{h}: \mathrm{m}: \mathrm{s}$ & $\mathrm{T}_{1}(\mathrm{~ms})$ & $\mathrm{h}: \mathrm{m}: \mathrm{s}$ & $\mathrm{T}_{2}(\mathrm{~ms})$ & $\mathrm{T}_{2}-\mathrm{T}_{1}(\mathrm{~ms})$ & $\alpha$ & $\mathrm{C}(\mathrm{s})$ \\
\hline $10: 52: 45$ & 561392304 & $10: 54: 21$ & 561488354 & 96050 & 1000 & 96.1 \\
\hline $10: 54: 21$ & 561488354 & $10: 55: 57$ & 561584336 & 95982 & 1000 & 96.0 \\
\hline $10: 55: 57$ & 561584336 & $10: 57: 33$ & 561680318 & 95982 & 1000 & 96.0 \\
\hline $10: 57: 33$ & 561680318 & $10: 59: 09$ & 561776401 & 96083 & 1000 & 96.1 \\
\hline $10: 59: 09$ & 561776401 & $11: 01: 10$ & 561897881 & 121480 & 1000 & 121.5 \\
\hline $11: 01: 10$ & 561897881 & $11: 03: 09$ & 562016624 & 118743 & 1000 & 118.7 \\
\hline $11: 03: 09$ & 562016624 & $11: 04: 51$ & 562118647 & 102023 & 1000 & 102.0 \\
\hline $11: 04: 51$ & 562118647 & $11: 06: 33$ & 562220636 & 101989 & 1000 & 102.0 \\
\hline $11: 06: 33$ & 562220636 & $11: 08: 15$ & 562322726 & 102090 & 1000 & 102.1 \\
\hline $11: 08: 15$ & 562322726 & $11: 09: 57$ & 562424749 & 102023 & 1000 & 102.0 \\
\hline
\end{tabular}




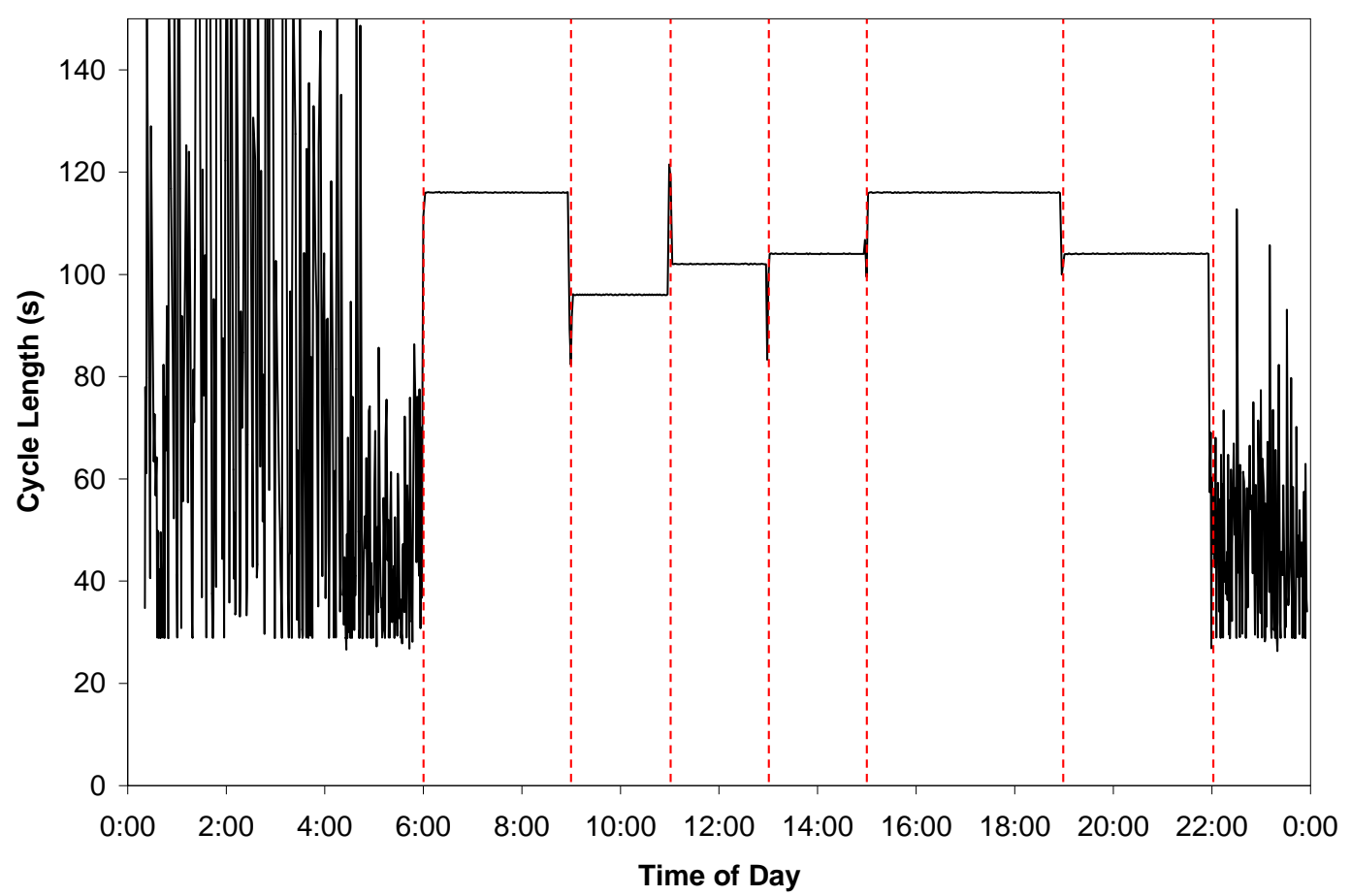

Figure 4.2 Graph of cycle length at Noblesville on Wednesday, March 14, 2007, with TOD plan changeovers marked by dashed lines. 


\subsubsection{Green Duration}

Green duration is the amount of time in which the green indication is given to a particular phase. This quantity is important in the calculation of the capacity provided to each phase. Green duration is measured by calculating the amount of time between the beginning and the ending of the green indication. In this work, this was done by two steps.

- The first step was to determine the amount of time during each cycle in which the red indication was off. This was done by identifying the point in time when the red ball was turned off, and the next point when it came back on. The amount of time passing between was equal to the total green and yellow time.

- The second step was to subtract the amount of yellow time, leaving the green time. The amount of yellow time was known from the controller settings. This process is illustrated in Figure 4.3.

The amount of green time (in seconds) for phase $i$ is found from

$G_{i}=\alpha\left(t_{R}-t_{G}\right)-Y_{i}$,

Equation 4.2

where $G_{i}$ denotes the controller green time for phase $i, t_{\mathrm{G}}$ is the point in time when the green indication was given to phase $i ; t_{R}$ is the subsequent point in time when the red indication was given; $\alpha$ is the same as in Equation 4.1; and $Y_{i}$ is the amount of yellow time for this phase. Yellow times are known from the controller settings.

The effective green, being that portion of the green indication that is actually used, is given by the following equation (5):

$g_{i}=G_{i}+Y_{i}-t_{L}$

Equation 4.3

where $g_{i}$ represents the effective green time for phase $i, G_{i}$ and $Y_{i}$ are the actual controller green and yellow times for phase $i$, and $t_{L}$ is the lost time (given by Equation 2.5) for the phase. In this work, we assume $t_{L}$ to be equal to $2 \mathrm{~s}$, the start-up lost time. This assumption implies that vehicles utilize virtually all of the clearance interval for movement. 
Table 4.2 shows sample calculations of green duration at Noblesville on March 14, 2007 for phase 2 and phase 5. A phase 2 green indication beginning (marked by a red indication ending) at 9:55:39 and ending (marked by a red indication beginning) at 9:56:45 has $65,346 \mathrm{~ms}$ passing between the two events, from which we calculate $G_{2}+Y_{2}$ $=65.3 \mathrm{~s}$. Subtracting $t_{L}=2 \mathrm{~s}$ from this value, we obtain the effective green $g_{2}=63.3 \mathrm{~s}$. Note that phase 2 varies between cycles, while phase 5 does not. Variation in phase 2 is due to early termination of the phase, as well as the absorption of green time from early termination of preceding phases. Phase 5 is not able to acquire any additional green time, and is such a high demand movement that it rarely gaps out.

Figure 4.4 shows graphs of phase 2 and phase 5 green times over 24 hours on March 14, 2007. Variations in phase 2 can clearly be seen in this graph. Minimum green times are revealed in the horizontal lines drawn out by points in each TOD period. During low volume free periods, the phenomenon whereby the phase dwells in phase 2 results in very long green times, which is revealed in tall spikes in the trace of the 20-phase moving average. The plot for phase 5 is considerably different. Phase 5 maxes out in nearly every cycle from 6:00 to 22:00, with a few exceptions standing out sporadically. Plateaus in the graph correspond to changing force-off points which are determined in each time period by a combination of the cycle length and the phase 5 split.

Figure 4.5 shows a plot of green time for phases 1 and 5 between 10:00 and 12:00. As in Figure 4.4, green time for phase 5 is consistent between cycles, with the exception of one cycle around 10:10 in which phase 5 was not called, and another cycle around 11:30 in which phase 5 terminated early. Phase 1 is a low volume movement, and seems to be skipped about every other cycle. Green times for phase 5 cycles close to the transition between TOD plan time periods are different from the other cycles.

Figure 4.6 contains a distribution of green times for phases 2 and 5 for Wednesday, March 14, 2007 between 9:00 and 11:00. The size of each bin for these distributions is $1 \mathrm{~s}$. The dashed red lines indicate the controller split times. Phase 2's distribution features a spike below the split, which shows that for a large proportion of cycles, phase 2 obtains green time equal to what is expected for its split. The spread of the distribution to the right of the split shows where phase 2 has acquired additional green time due to early termination of preceding phases. In Figure 4.6(b), Phase 5's green distribution lies just below the split line. $100 \%$ of phases had green times within 16-17 s. This represents the termination of phase 5 green as it reaches the force-off point. There is no distribution forward of the split line because this phase cannot capture any additional green time from preceding phases. This is consistent with Figure 4.4(b). 
Because of the lead/lag phasing at Noblesville that was in place on March 14, 2007, phase 5 comes after phase 6, and any green time that is given up by phases 7 and 8 is consumed by phase 6 . In Chapter 5, we will investigate the impacts of using lead/lag operation as opposed to conventional phasing at this intersection.

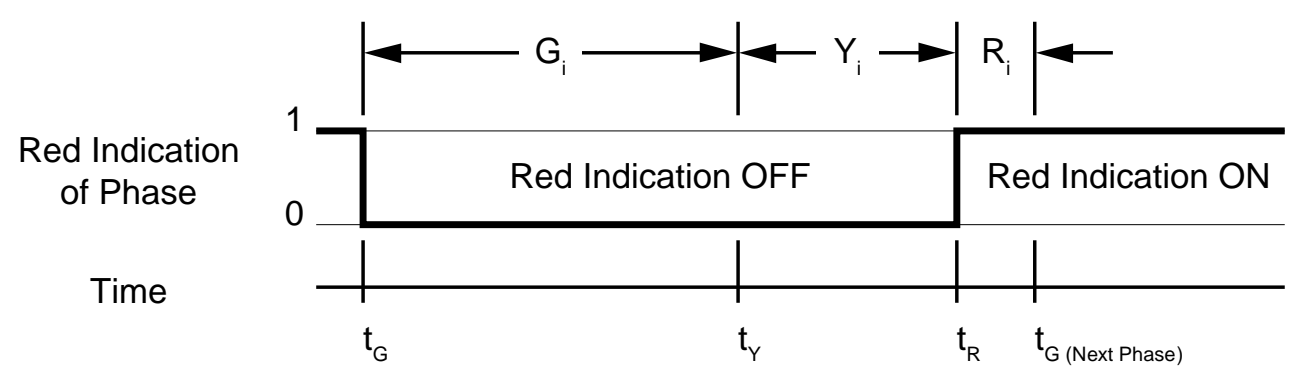

Figure 4.3 Measurement of green duration from phase events. 
Table 4.2 Example calculation of green duration from logged events, based on data for Noblesville, March 14, 2007.

(a) Phase 2

\begin{tabular}{|c|c|c|c|c|c|c|c|c|}
\hline \multicolumn{2}{|c|}{ Begin Green Indication } & \multicolumn{2}{|c|}{ End Green Indication } & \multirow[b]{2}{*}{$\begin{array}{c}t_{R}-t_{G} \\
(m s)\end{array}$} & \multirow[b]{2}{*}{$\alpha$} & \multirow[b]{2}{*}{$\mathrm{G}_{2}(\mathrm{~s})$} & \multirow[b]{2}{*}{$t_{L}(s)$} & \multirow[b]{2}{*}{$\mathrm{g}_{2}(\mathrm{~s})$} \\
\hline h:m:s & $\mathrm{t}_{\mathrm{G}}(\mathrm{ms})$ & $\mathrm{h}: \mathrm{m}: \mathrm{s}$ & $\mathrm{t}_{\mathrm{R}}(\mathrm{ms})$ & & & & & \\
\hline $09: 52: 45$ & 557791363 & 09:53:33 & 557839354 & 47991 & 1000 & 48.0 & 2.0 & 46.0 \\
\hline $09: 54: 27$ & 557893353 & 09:55:09 & 557935436 & 42083 & 1000 & 42.1 & 2.0 & 40.1 \\
\hline $09: 55: 39$ & 557966040 & $09: 56: 45$ & 558031386 & 65346 & 1000 & 65.3 & 2.0 & 63.3 \\
\hline $09: 57: 45$ & 558091425 & 09:58:21 & 558127368 & 35943 & 1000 & 35.9 & 2.0 & 33.9 \\
\hline 09:59:21 & 558187407 & 09:59:57 & 558223450 & 36043 & 1000 & 36.0 & 2.0 & 34.0 \\
\hline 10:00:49 & 558275246 & 10:01:33 & 558319533 & 44287 & 1000 & 44.3 & 2.0 & 42.3 \\
\hline $10: 02: 19$ & 558365722 & 10:03:09 & 558415515 & 49793 & 1000 & 49.8 & 2.0 & 47.8 \\
\hline $10: 03: 53$ & 558459569 & $10: 04: 45$ & 558511598 & 52029 & 1000 & 52.0 & 2.0 & 50.0 \\
\hline $10: 05: 21$ & 558547508 & 10:06:21 & 558607547 & 60039 & 1000 & 60.0 & 2.0 & 58.0 \\
\hline 10:07:09 & 558655538 & $10: 07: 57$ & 558703629 & 48091 & 1000 & 48.1 & 2.0 & 46.1 \\
\hline
\end{tabular}

(b) Phase 5

\begin{tabular}{|c|c|c|c|c|c|c|c|c|}
\hline \multicolumn{2}{|c|}{ Begin Green Indication } & \multicolumn{2}{|c|}{ End Green Indication } & \multirow[b]{2}{*}{$\begin{array}{c}t_{R}-t_{G} \\
(m s)\end{array}$} & \multirow[b]{2}{*}{$\alpha$} & \multirow[b]{2}{*}{$\mathrm{G}_{5}(\mathrm{~s})$} & \multirow[b]{2}{*}{$t_{L}(s)$} & \multirow[b]{2}{*}{$\mathrm{g}_{5}(\mathrm{~s})$} \\
\hline h:m:s & $\mathrm{t}_{\mathrm{G}}(\mathrm{ms})$ & h:m:s & $\mathrm{t}_{\mathrm{R}}(\mathrm{ms})$ & & & & & \\
\hline $09: 53: 13$ & 557819263 & 09:53:31 & 557837652 & 18389 & 1000 & 18.4 & 2.0 & 16.4 \\
\hline 09:54:49 & 557915346 & 09:55:07 & 557933601 & 18255 & 1000 & 18.3 & 2.0 & 16.3 \\
\hline 09:56:25 & 558011328 & 09:56:43 & 558029583 & 18255 & 1000 & 18.3 & 2.0 & 16.3 \\
\hline 09:58:01 & 558107310 & 09:58:19 & 558125666 & 18356 & 1000 & 18.4 & 2.0 & 16.4 \\
\hline 09:59:37 & 558203360 & $09: 59: 55$ & 558221648 & 18288 & 1000 & 18.3 & 2.0 & 16.3 \\
\hline 10:01:13 & 558299342 & $10: 01: 31$ & 558317731 & 18389 & 1000 & 18.4 & 2.0 & 16.4 \\
\hline 10:02:49 & 558395424 & 10:03:07 & 558413813 & 18389 & 1000 & 18.4 & 2.0 & 16.4 \\
\hline 10:04:25 & 558491507 & 10:04:43 & 558509762 & 18255 & 1000 & 18.3 & 2.0 & 16.3 \\
\hline $10: 07: 37$ & 558683572 & $10: 07: 55$ & 558701827 & 18255 & 1000 & 18.3 & 2.0 & 16.3 \\
\hline 10:09:13 & 558779521 & 10:09:31 & 558797910 & 18389 & 1000 & 18.4 & 2.0 & 16.4 \\
\hline
\end{tabular}




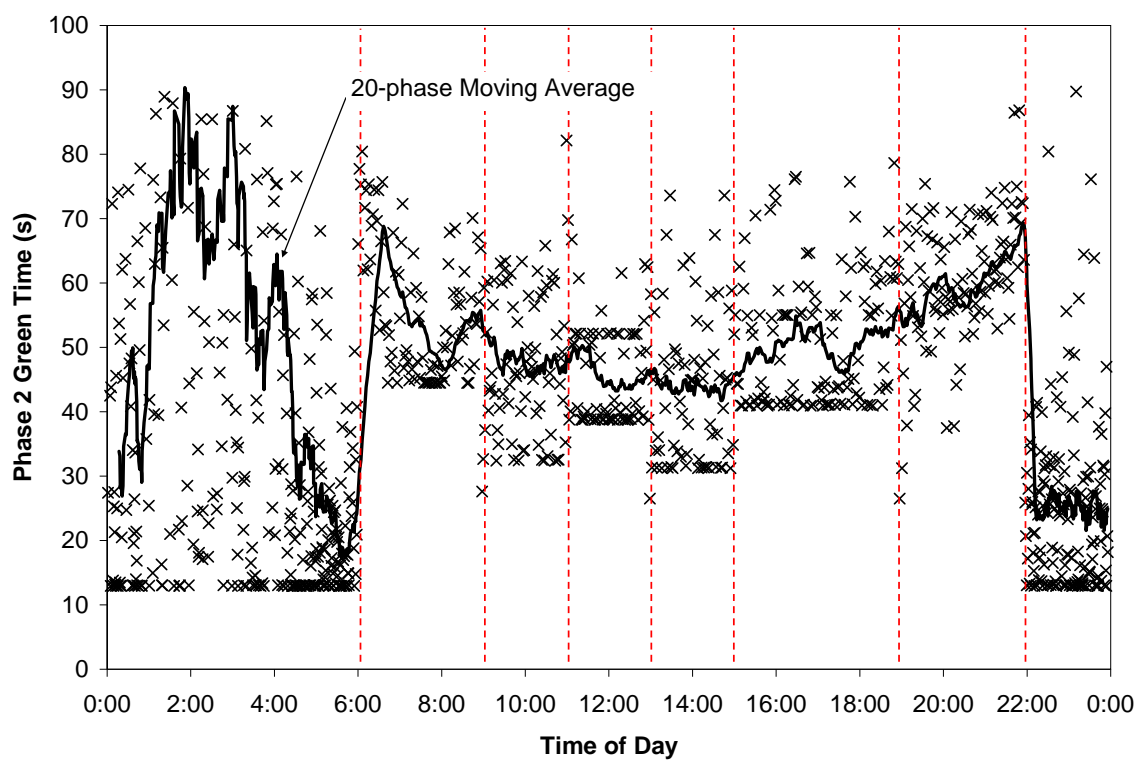

(a) Phase 2, Wednesday, March 14, 2007

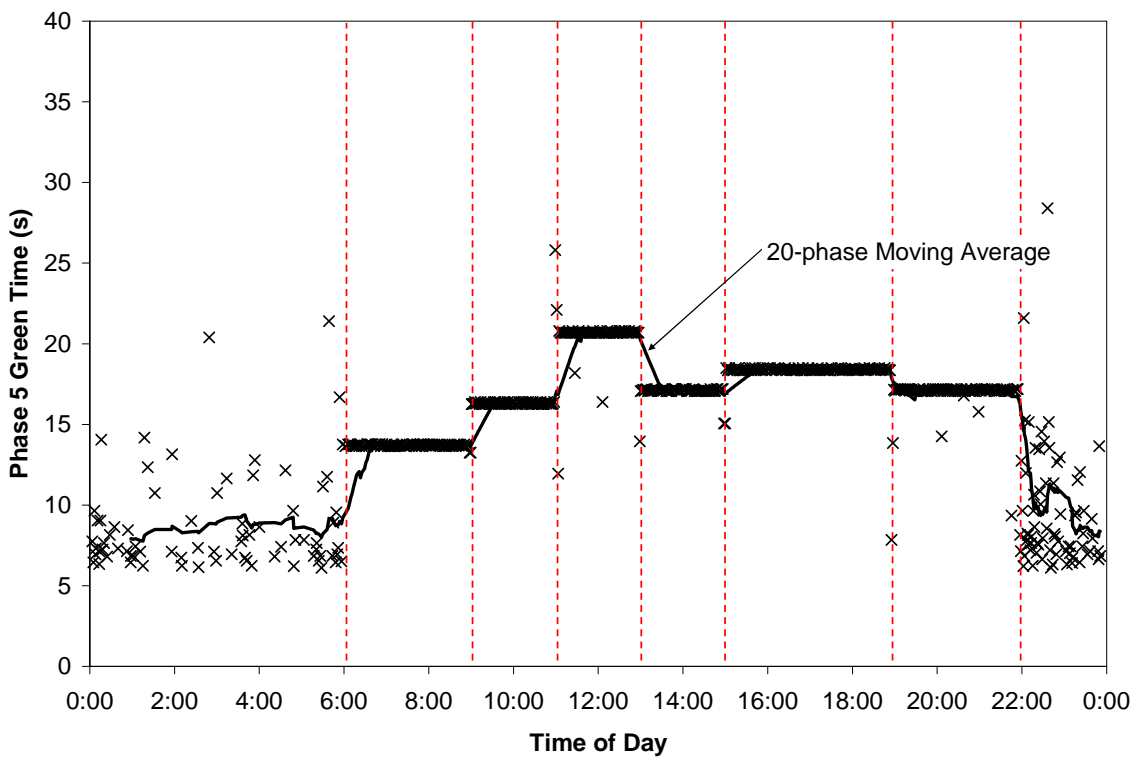

(b) Phase 5, Wednesday, March 14, 2007

Figure 4.4 Graph of green duration at Noblesville for a 24-hour period. 


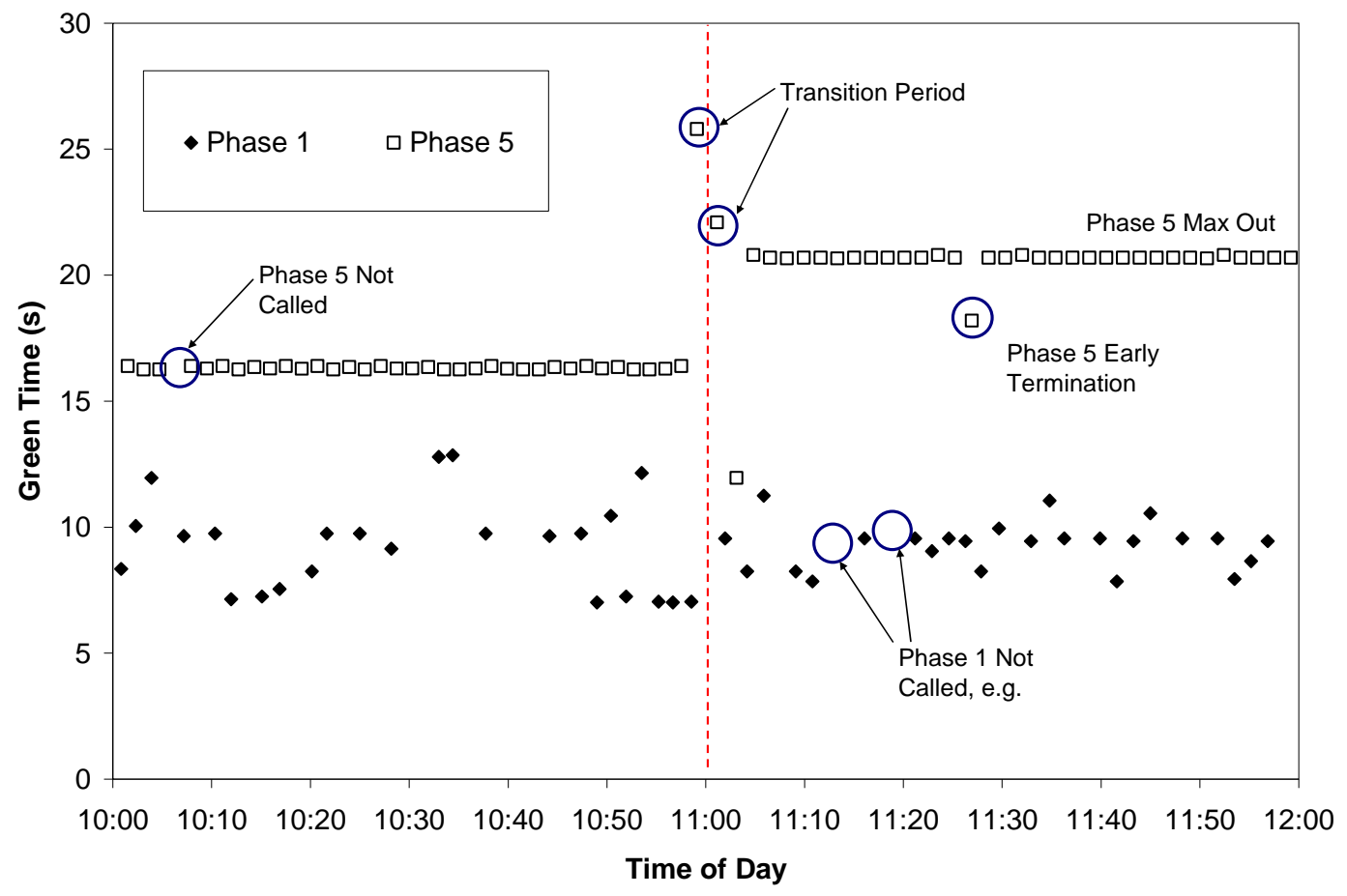

Figure 4.5 Graph of green duration at Noblesville on Wednesday, March 14, 2007, illustrating various events. 


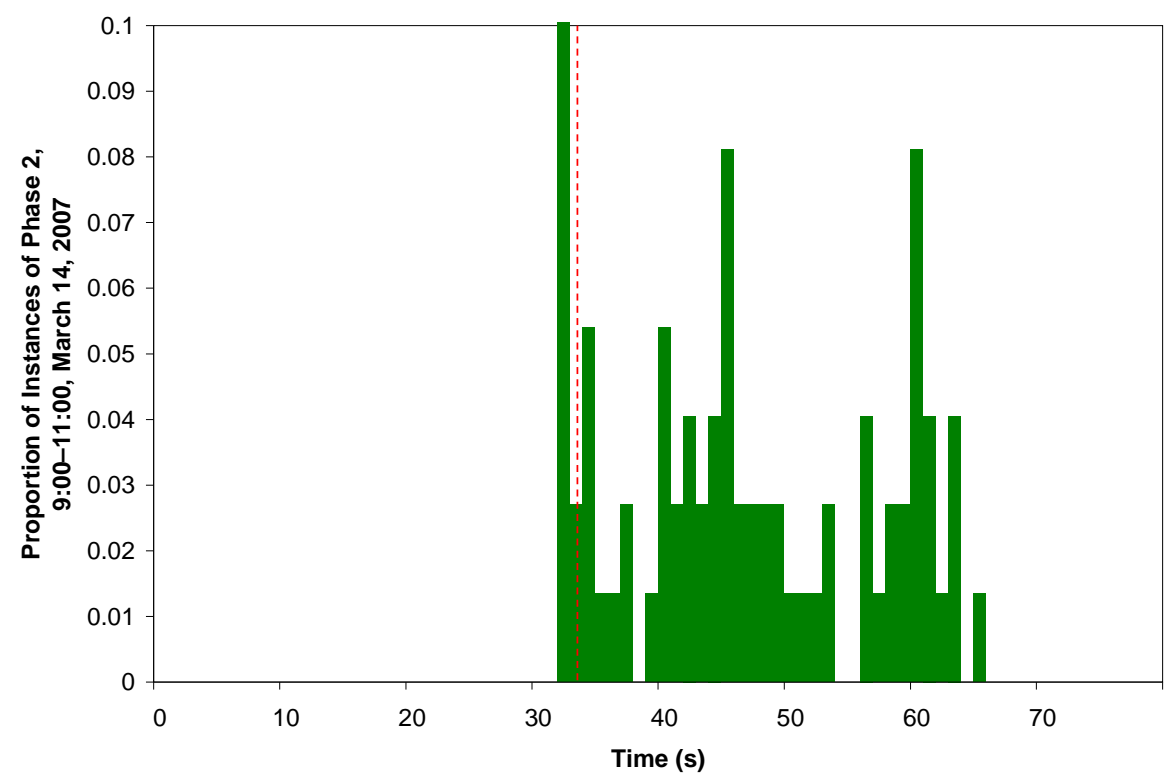

(a) Phase 2, 9:00-11:00, March 14, 2007

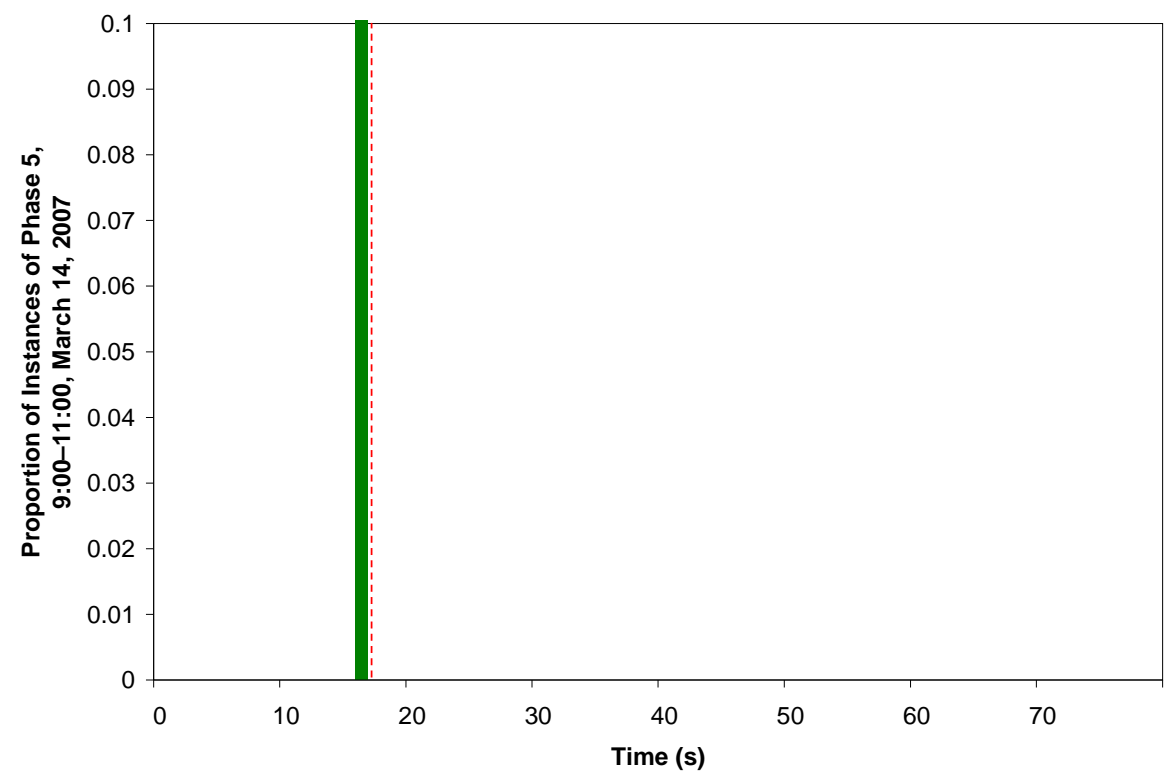

(b) Phase 5, 9:00-11:00, March 14, 2007

Figure 4.6 Distribution of green times for phases 2 and 5 during the midday time period at Noblesville on Wednesday, March 14, 2007. 


\subsubsection{Volume}

There are several ways to consider the number of vehicles serviced by each movement during a cycle. The simplest way is to simply count the number of vehicles and report the number. When comparing between cycles, we prefer to convert this to the equivalent hourly volume using the cycle length. Finally, to compare between different movements we normalize these flow rates with respect to the number of lanes for each movement.

Vehicles were counted in a two step process. The first step was to read through the phase state information and build a trace of the phase state through 24 hours. The rising edge marked the "on" state of the red indication, which was interpreted as the end of green. This was used to divide time between instances of a phase. The falling edge marked the beginning of green, and was used to determine whether vehicles were counted during the red or green indication. The use of this information will be discussed in Section 4.4.1. The division of time into cycles is illustrated in Figure 4.7. Here, phase 4 is the phase of interest, and cycles are defined by phase 2 .

Note that the phase covers two cycles. In Figure 4.7, the phase 4 interval that spans between points $t_{\mathrm{R} 1}$ and $t_{\mathrm{R} 2}$ is said to belong to cycle 2 , because the beginning of green $\left(t_{\mathrm{G} 1}\right)$ falls within the boundaries of cycle 2 . The reason for this is that vehicles arriving after a red indication is shown for a phase cannot move forward (without running the red light) until the next green indication. For example, in Figure 4.7, vehicle A arrives during the phase 4 green indication of cycle 1, and is consequently counted for phase 4 during cycle 1 . Vehicles $\mathrm{B}$ and $\mathrm{C}$ are counted for phase 4 during cycle 2 because they arrive after the cutoff point $t_{\mathrm{R} 1}$. Even though vehicle B technically arrives during cycle 1 , it cannot move until after $t_{\mathrm{G} 1}$, which takes place during cycle 2 . Likewise, although vehicle D arrives during cycle 2, it cannot move until the green indication of cycle 3 .

Figure 4.7 also illustrates how phases are mapped to cycles, as discussed earlier. Note that phase 4 comes near the beginning of the cycle rather than at the end, from the viewpoint of the signal controller. The implication for phase 4 is that it can obtain green time from phase 3, which can terminate early, but not from phases 1 and 2 as is implied by a typical ring diagram such as the one in Figure 2.3(b). In the next chapter, we will discuss an operating method by which time may be transferred across the cycle boundary.

With the cutoff points for carrying out vehicle counts defined, the second step was to process vehicle arrival data and assign them to cycles. Because vehicle arrivals were ordered by time, this could be done by counting arrivals for each interval in succession, changing to the next interval whenever an arrival was found to occur later than the ending 
cutoff point. The total number of vehicles arriving during the cycle was converted to the equivalent hourly volume using the formula

$$
v_{i}=\left(\frac{N}{C} \times 3600\right) \frac{1}{n_{L}},
$$

where $v_{i}$ represents the volume for phase $i$ in veh/h/ln, $N$ represents the number of vehicles for the movement, $C$ represents the cycle length in seconds, $n_{L}$ is the number of lanes, and 3600 is a factor that converts from veh/s to veh/h. To calculate total volume per approach instead of per lane, the $n_{L}$ factor is omitted.

This volume represents the flow rate of vehicles making a particular movement during a cycle. This quantity would be the flow rate if the signalized intersection did not exist, and vehicles arrived uniformly. We use this value to quantify the demand for each movement at an intersection during a particular cycle. In reality, the volume for each phase must be served in the green time provided by the signal. The actual flow rates that are taking place are much more close to saturation, because that is the rate at which queues disperse after the beginning of green. The service flow rate can be found from

$$
q_{i}=\left(\frac{N}{g_{i}} \times 3600\right) \frac{1}{n_{L}},
$$

which is the same as Equation 4.4 except that we use the green time $g_{i}$ rather than $C$. This quantity reflects the use of green. While it does not reflect the demand for a movement relative to the others, it can be interpreted as the demand for every movement if compared to the maximum flow rate. This will be discussed further in 4.3.3.

Sample calculations of volume for phases 2 and 6 at Noblesville on March 14, 2007 are shown in Table 4.3. For an interval of phase 2 that began at 10:56:38, 11 vehicles were counted. Dividing by $C$ and multiplying by 3600 , we find that this is the equivalent of $413 \mathrm{veh} / \mathrm{h}$ for the northbound movement. Because there are 2 lanes serving this movement, the normalized volume is equal to $206 \mathrm{veh} / \mathrm{h} / \mathrm{ln}$. Graphs of volumes for phase 2 and phase 6 over 24 hours are shown in Figure 4.8. The two graphs illustrate the nature of traffic flow along this arterial. Phase 6 , the southbound movement, has a narrow peak in the morning hours, while phase 2 , the northbound movement, has a more broad peak in the evening. This reflects the function of the intersection as part of an arterial serving commuters who work in Indianapolis and dwell in northern suburbs. 
The choice of a 20-point moving average is somewhat arbitrary, but is meant to provide a graph that shows the trends apparent in the data with a minimal amount of noise. In Figure 4.9, six different plots of phase 2 volumes are shown with various choices of moving average. The choice of 20 points over 10 is rather subjective, but was felt to express the trend of the curve without excessive smoothing. 20 points represents approximately 40 minutes ( 20 minutes in either direction at each point).

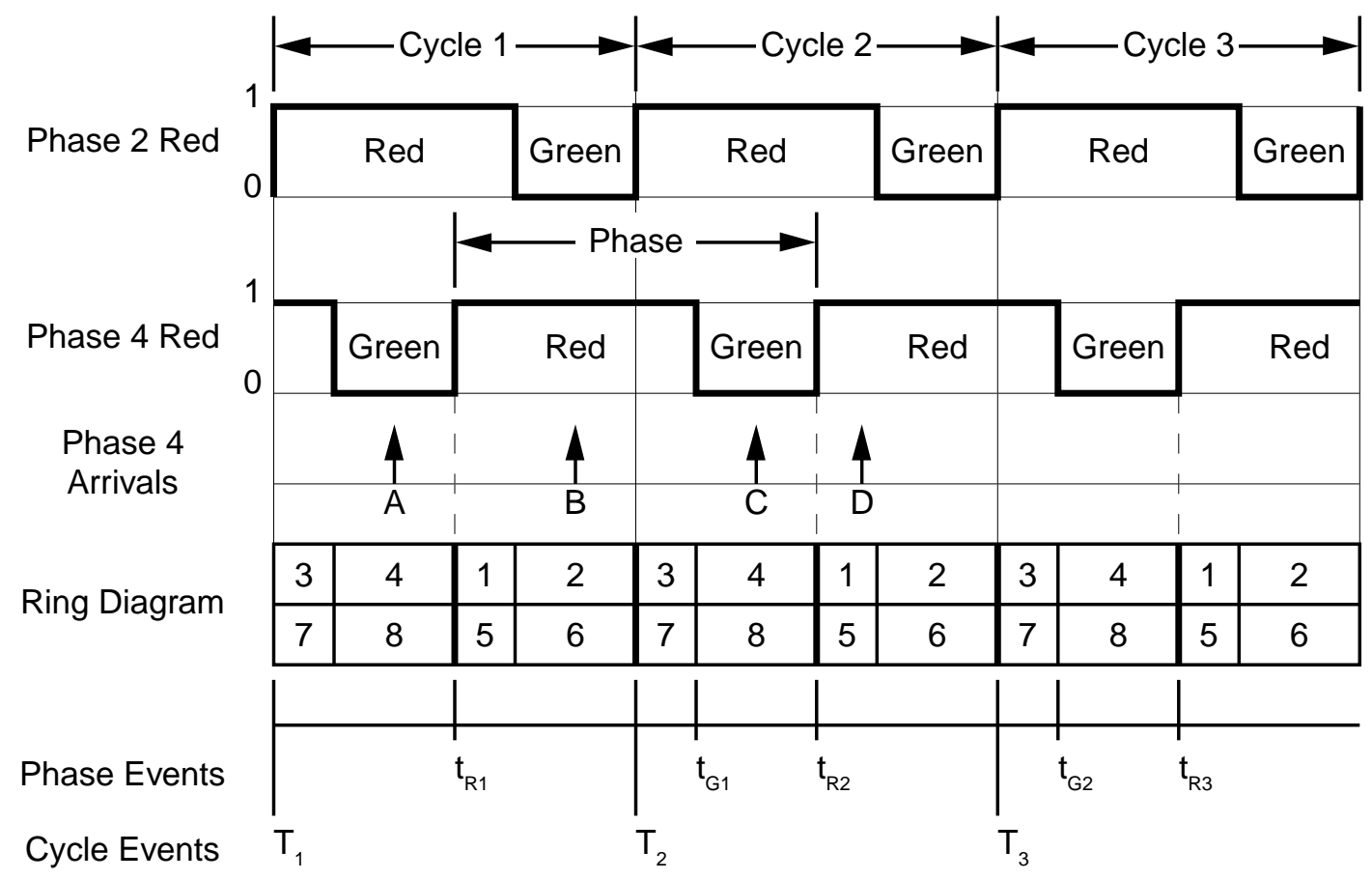

Figure 4.7 Vehicle counting based on phase state and cycle state events. 
Table 4.3 Calculation of volumes at Noblesville.

(a) Phase 2, Wednesday, March 14, 2007

\begin{tabular}{|c|r|r|r|r|r|}
\hline Time & \multicolumn{1}{|c|}{$\mathrm{C}(\mathrm{s})$} & $\mathrm{N}$ & $\mathrm{n}_{\mathrm{L}}(\ln )$ & $\mathrm{V}_{2}(\mathrm{veh} / \mathrm{h})$ & $\mathrm{v}_{2}(\mathrm{veh} / \mathrm{h} / \mathrm{ln})$ \\
\hline $10: 53: 30$ & 96.1 & 21 & 2 & 787 & 394 \\
\hline $10: 55: 13$ & 96.0 & 13 & 2 & 488 & 244 \\
\hline $10: 56: 38$ & 96.0 & 11 & 2 & 413 & 206 \\
\hline $10: 58: 32$ & 96.1 & 19 & 2 & 712 & 356 \\
\hline $10: 59: 46$ & 121.5 & 16 & 2 & 474 & 237 \\
\hline $11: 01: 57$ & 118.7 & 10 & 2 & 303 & 152 \\
\hline $11: 04: 10$ & 102.0 & 7 & 2 & 247 & 124 \\
\hline $11: 05: 51$ & 102.0 & 12 & 2 & 424 & 212 \\
\hline $11: 07: 06$ & 102.1 & 12 & 2 & 423 & 212 \\
\hline $11: 09: 05$ & 102.0 & 18 & 2 & 635 & 318 \\
\hline
\end{tabular}

(b) Phase 6, Wednesday, March 14, 2007

\begin{tabular}{|c|r|r|r|r|r|}
\hline Time & $\mathrm{C}(\mathrm{s})$ & $\mathrm{N}$ & $\mathrm{n}_{\mathrm{L}}(\ln )$ & $\mathrm{v}_{6}(\mathrm{veh} / \mathrm{h})$ & $\mathrm{v}_{6}(\mathrm{veh} / \mathrm{h} / \mathrm{ln})$ \\
\hline $10: 53: 21$ & 96.1 & 11 & 2 & 412 & 206 \\
\hline $10: 55: 00$ & 96.0 & 9 & 2 & 338 & 169 \\
\hline $10: 56: 30$ & 96.0 & 21 & 2 & 788 & 394 \\
\hline $10: 58: 24$ & 96.1 & 9 & 2 & 337 & 169 \\
\hline $10: 59: 49$ & 121.5 & 18 & 2 & 533 & 267 \\
\hline $11: 01: 49$ & 118.7 & 8 & 2 & 243 & 121 \\
\hline $11: 04: 00$ & 102.0 & 14 & 2 & 494 & 247 \\
\hline $11: 05: 42$ & 102.0 & 9 & 2 & 318 & 159 \\
\hline $11: 07: 09$ & 102.1 & 12 & 2 & 423 & 212 \\
\hline $11: 08: 53$ & 102.0 & 15 & 2 & 529 & 265 \\
\hline
\end{tabular}




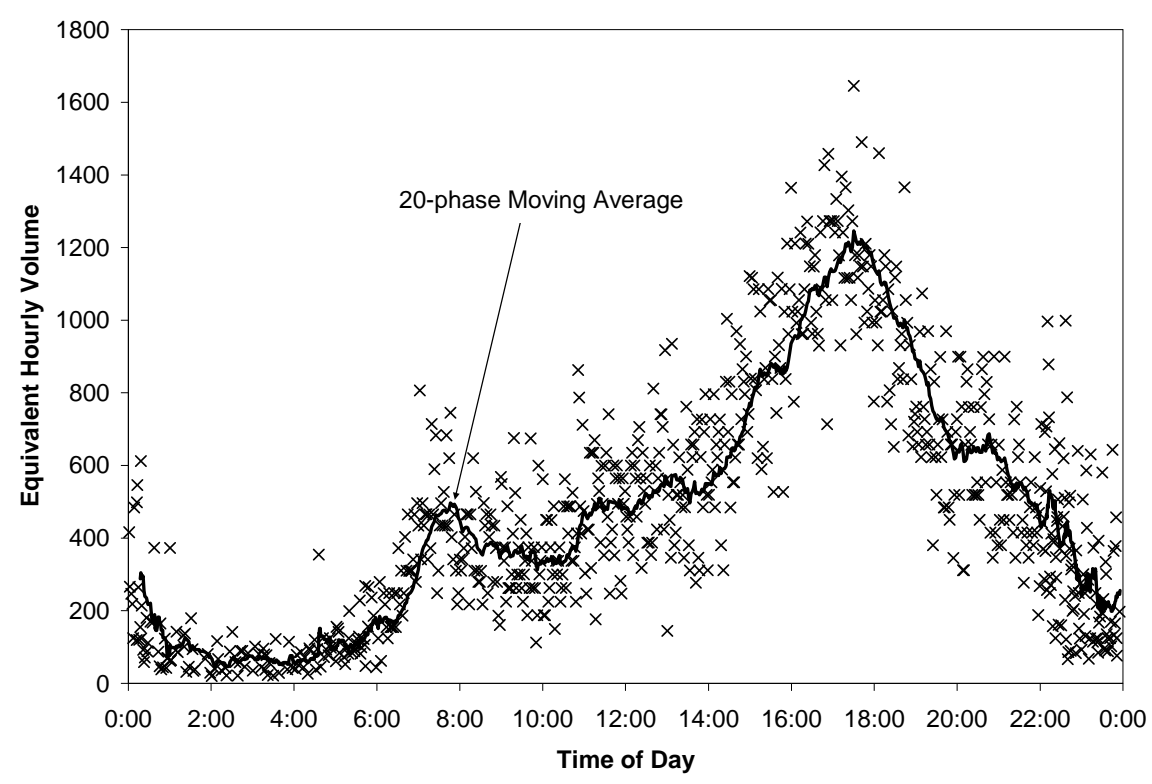

(a) Phase 2, Wednesday, March 14, 2007

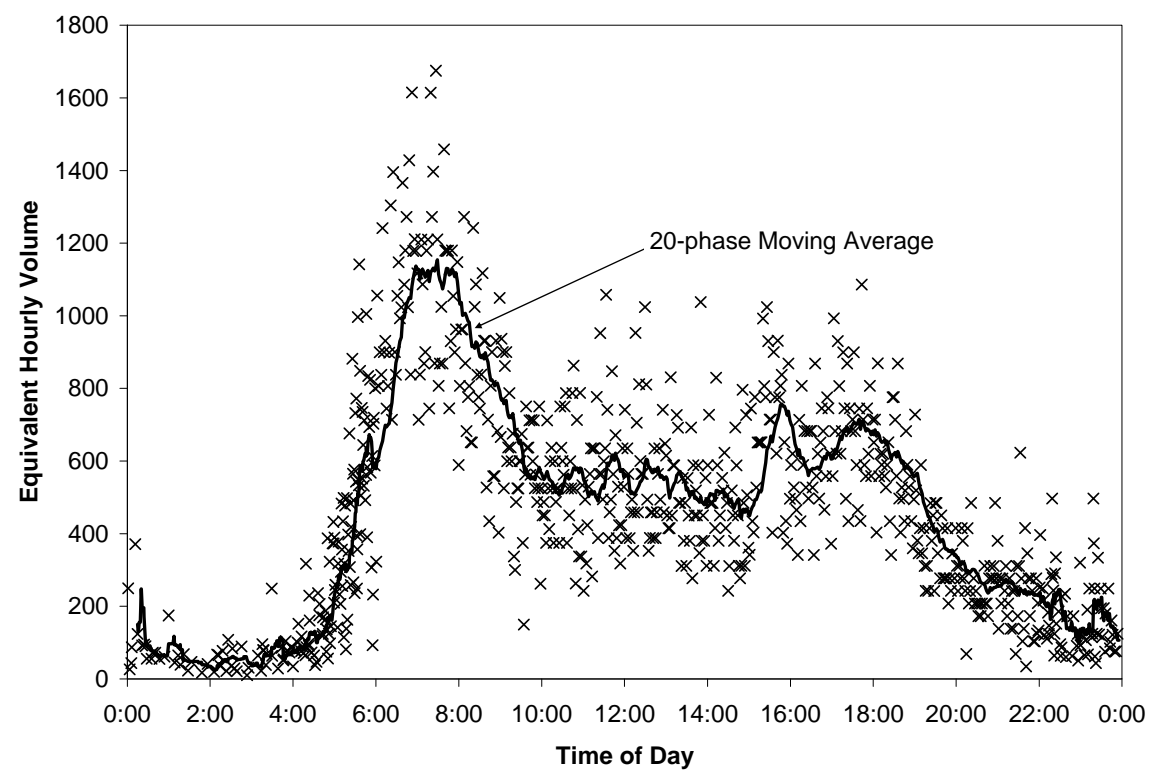

(b) Phase 6, Wednesday, March 14, 2007

Figure 4.8 Graphs of volumes at Noblesville. 


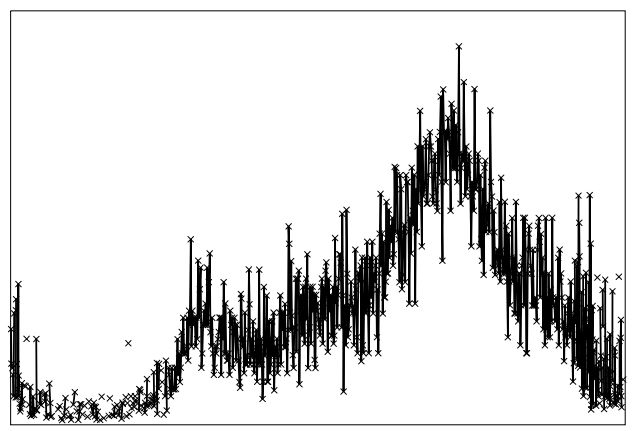

(a) No average

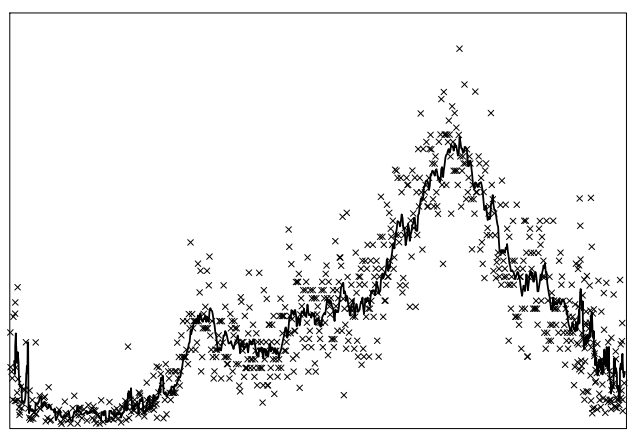

(c) 10-phase moving average

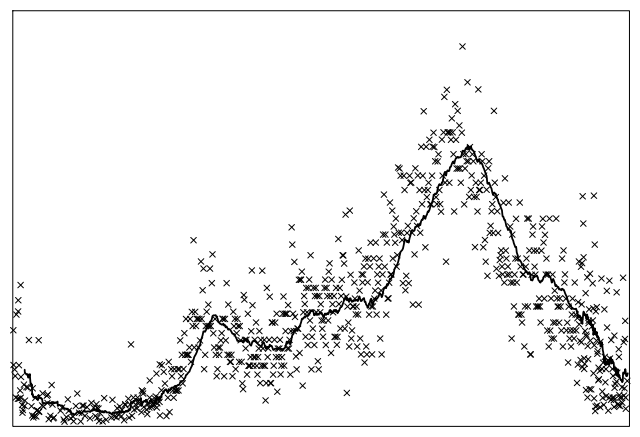

(e) 30-phase moving average

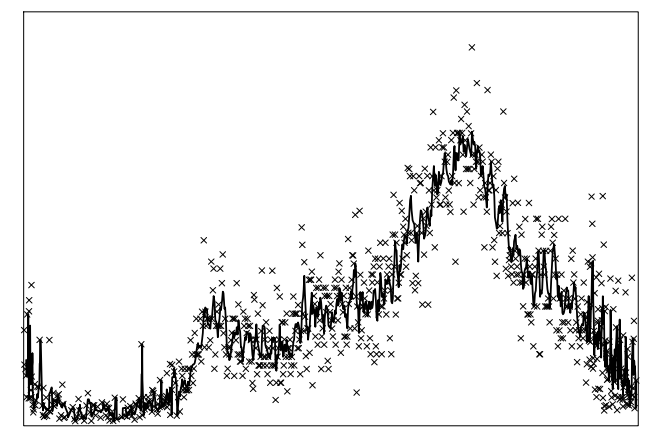

(b) 5-phase moving average

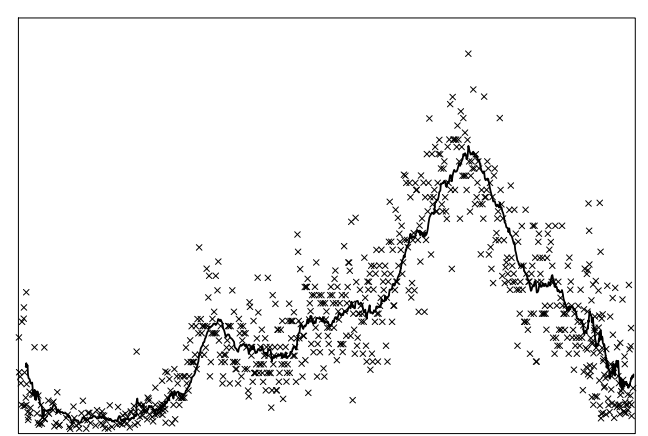

(d) 20-phase moving average

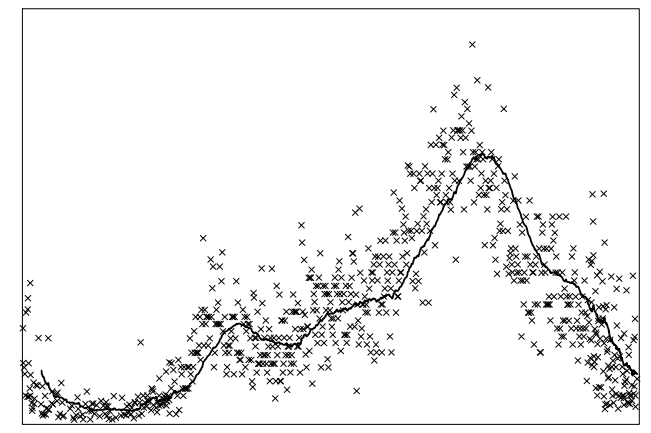

(f) 50-phase moving average

Figure 4.9 Choice of moving average. 


\subsection{Capacity Analysis}

This section describes methods of performance analysis using performance measures that use the perspective of intersection capacity. The measures discussed here include estimated capacity, observed capacity, volume-to-capacity ratio, number of split failures, and critical volume-to-capacity ratio.

\subsubsection{Estimated Capacity}

The estimated capacity of each phase is the maximum number of vehicles that would be serviced by that phase if it ran green times as allocated by the controller splits, with no early terminations or skipped phases. This situation would exist for a pretimed signal, or an actuated signal where every phase was called and each ran to its force-off point.

The estimated capacity of phase $i$ based on the split is given in $v e h / h / l n$ by

$\hat{C}_{\text {split }, i}=\frac{S_{i}}{\hat{C}} \times S$.

Equation 4.6

Here, $S_{i}$ is the programmed split of phase $i$ (in s); $\hat{C}$ is the estimated cycle length (in s), and $s$ is the saturation flow rate (in veh/h/ln). In this study, we have used a saturation flow rate of $1900 \mathrm{veh} / \mathrm{h} / \mathrm{ln}$. The value of $s$ is dependent on driver behavior at the intersection, and the choice of its magnitude has implications for calculated values of capacity. The estimated cycle length, $\hat{C}$, is the programmed value, which should be equal to the measured value for operating modes with fixed cycle length. For free operation, the measured value should be used instead, because the cycle length will vary from cycle to cycle.

For a dual ring controller, phases in the upper ring run concurrently with phases in the lower ring. Phases 1, 2, 3, and 4 in the upper ring each take a share of the total capacity of $1900 \mathrm{veh} / \mathrm{h} / \mathrm{ln}$ (including lost time, which each phase contains); while phases $5,6,7$, and 8 in the lower ring divide up their own $1900 \mathrm{veh} / \mathrm{h} / \mathrm{ln}$. The total capacity of the intersection would still be $1900 \mathrm{veh} / \mathrm{h} / \mathrm{ln}$, since the arithmetic total of both rings' capacities would be divided by the number of paths to maintain units of veh/h/ln. The actual maximum capacity of an intersection would depend on the number of lanes that can serve vehicles and the division of a cycle between the various phases. 
The overall capacity of each ring is apportioned to each phase according to the relative proportions of the splits. However, not all of that capacity is useable by moving vehicles. Some is used by the red clearance interval. A further portion is consumed by lost time. The remainder would be the split-based estimation of green time, given (in s) by

$\hat{g}_{i}=S_{i}-t_{L}-R_{i}$

Equation 4.7

where $S_{i}$ is the programmed split in seconds, $t_{L}$ is the lost time (given by Equation 2.5), and $R_{i}$ is the red clearance interval, all in units of $\mathrm{s}$. The amount of capacity taken away from moving vehicles is referred to here as the lost capacity. The amount of lost capacity estimated for phase $i$ is calculated by

$\hat{C}_{\text {lost }, i}=\frac{t_{L}+R_{i}}{C} \times S$,

Equation 4.8

where each variable is the same as defined in Equation 4.6 and Equation 4.7. The HCM suggests approximating lost time by using the controller yellow time. In this paper, we have used $2 \mathrm{~s}$ for $t_{L}$. The use of this value is discussed in more detail in Section 4.3.3.

The amount of capacity estimated to serve vehicles is found by subtracting the lost capacity from the split capacity, by

$\hat{c}_{i}=\left(\hat{c}_{\mathrm{split}, i}-\hat{c}_{\mathrm{lost}, i}\right)$,

Equation 4.9

which, when we expand quantities according to the three previous equations, gives the estimated capacity $\hat{c}_{i}$ for phase $i($ in veh/h/ln) as

$\hat{c}_{i}=\frac{\hat{g}_{i}}{C} \times s$.

Equation 4.10

Example calculations of estimated capacity at Noblesville are shown in Table 4.4. In Table 4.4(a), the calculations are for the 9:00-11:00 time period, when $\mathrm{C}=96 \mathrm{~s}$; Table 4.4(b) shows calculations for the 6:00-9:00 time period, when $\mathrm{C}=116 \mathrm{~s}$. In the case where $\mathrm{C}=96 \mathrm{~s}$, phase 2 has a split of $37 \%$, which gives us a split time $\mathrm{s}_{2}=35.5$. Subtracting $t_{L}=2 \mathrm{~s}$ and $R_{2}=1 \mathrm{~s}$, we obtain $\hat{g}_{2}=32.5 \mathrm{~s}$. The estimated capacity $\hat{c}_{\text {split }, i}=$ $(35.5 / 96) \times 1900=703 \mathrm{veh} / \mathrm{h} / \mathrm{ln}$. The lost capacity $\hat{c}_{\text {lost }, i}$ is equal to $(4 / 96) \times 1900=59$ 
$\mathrm{veh} / \mathrm{h} / \mathrm{ln}$. The estimated capacity for phase 2 is $\hat{c}_{i}=\left(\hat{c}_{\text {split }, i}-\hat{c}_{\text {lost }, i}\right)=703-59=644$ $\mathrm{veh} / \mathrm{h} / \mathrm{ln}$. The total capacity of either ring for all 4 phases plus lost capacity is equal to $1900 \mathrm{veh} / \mathrm{h} / \mathrm{ln}$.

As $C$ increases, the proportion of total green time for a ring increases. When $C=$ $96 \mathrm{~s}$, the total green time for either ring is $81.2 \mathrm{~s}$, which is $84.5 \%$ of the cycle. When $C$ increases to $116 \mathrm{~s}$, total green time becomes 101.2 , which represents $87.2 \%$. The totals for ring 1 and ring 2 need not be equal. This equality occurs because lost times are the same for each geometrically opposing phase pair, a consequence of symmetric intersection design. 
Table 4.4 Calculation of estimated capacities for Noblesville.

(a) 09:00-11:00, C = $96 \mathrm{~s}$

\begin{tabular}{|c|c|c|c|c|c|c|c|c|c|}
\hline & \multirow{2}{*}{ Phase } & \multirow{2}{*}{$\begin{array}{c}\text { Split } \\
(\%)\end{array}$} & \multirow[b]{2}{*}{$S_{i}(\mathrm{~s})$} & \multicolumn{2}{|c|}{ Clearance (s) } & \multirow{2}{*}{$\hat{g}_{i}(\mathrm{~s})$} & \multicolumn{3}{|c|}{ Capacity (veh/h/ln) } \\
\hline & & & & $t_{L}$ & $R_{i}$ & & $\hat{C}_{\text {split }, i}$ & $\hat{C}_{\text {lost }, i}$ & $\hat{c}_{i}$ \\
\hline \multirow{4}{*}{ 夰 } & 1 & 14 & 13.4 & 2.0 & 1.8 & 9.6 & 266 & 75 & 191 \\
\hline & 2 & 37 & 35.5 & 2.0 & 1.0 & 32.5 & 703 & 59 & 644 \\
\hline & 3 & 15 & 14.4 & 2.0 & 2.0 & 10.4 & 285 & 79 & 206 \\
\hline & 4 & 34 & 32.6 & 2.0 & 2.0 & 28.6 & 646 & 79 & 567 \\
\hline \multirow{4}{*}{$\begin{array}{l}\text { No } \\
.00 \\
.0\end{array}$} & 5 & 22 & 21.1 & 2.0 & 1.8 & 17.3 & 418 & 75 & 343 \\
\hline & 6 & 29 & 27.8 & 2.0 & 1.0 & 24.8 & 551 & 59 & 492 \\
\hline & 7 & 14 & 13.4 & 2.0 & 2.0 & 9.4 & 266 & 79 & 187 \\
\hline & 8 & 35 & 33.6 & 2.0 & 2.0 & 29.6 & 665 & 79 & 586 \\
\hline
\end{tabular}

\begin{tabular}{|r|r|r|r|} 
Totals, Ring $1 \quad 81.2$ & 1900 & 293 & 1607 \\
\hline
\end{tabular}

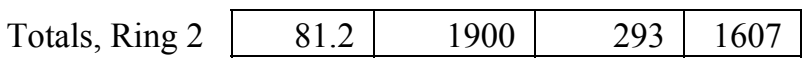

(b) 06:00-09:00, C = 116 s

\begin{tabular}{|c|c|c|c|c|c|c|c|c|c|}
\hline & \multirow{2}{*}{ Phase } & \multirow{2}{*}{$\begin{array}{c}\text { Split } \\
(\%)\end{array}$} & \multirow{2}{*}{$s_{i}(\mathrm{~s})$} & \multicolumn{2}{|c|}{ Clearance (s) } & \multirow{2}{*}{$\hat{g}_{i}(\mathrm{~s})$} & \multicolumn{3}{|c|}{ Capacity (veh/h/ln) } \\
\hline & & & & $t_{L}$ & $R_{i}$ & & $\hat{c}_{\text {split }, i}$ & $\hat{c}_{\text {lost }, i}$ & $\hat{C}_{i}$ \\
\hline \multirow{4}{*}{$\overrightarrow{0.0}$} & 1 & 12 & 13.9 & 2.0 & 1.8 & 10.1 & 228 & 62 & 166 \\
\hline & 2 & 41 & 47.6 & 2.0 & 1.0 & 44.6 & 779 & 49 & 730 \\
\hline & 3 & 22 & 25.5 & 2.0 & 2.0 & 21.5 & 418 & 66 & 352 \\
\hline & 4 & 25 & 29.0 & 2.0 & 2.0 & 25.0 & 475 & 66 & 409 \\
\hline \multirow{4}{*}{ 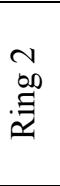 } & 5 & 16 & 18.6 & 2.0 & 1.8 & 14.8 & 304 & 62 & 242 \\
\hline & 6 & 37 & 42.9 & 2.0 & 1.0 & 39.9 & 703 & 49 & 654 \\
\hline & 7 & 11 & 12.8 & 2.0 & 2.0 & 8.8 & 209 & 66 & 143 \\
\hline & 8 & 36 & 41.8 & 2.0 & 2.0 & 37.8 & 684 & 66 & 618 \\
\hline & & & & \multicolumn{2}{|c|}{ Totals, Ring 1} & 101.2 & 1900 & 242 & 1658 \\
\hline & & & & \multicolumn{2}{|c|}{ Totals, Ring 2} & 101.2 & 1900 & 242 & 1658 \\
\hline
\end{tabular}




\subsubsection{Observed Capacity}

The observed capacity of each phase is calculated similarly to the estimated capacity. However, instead of using green times estimated from controller splits, observed capacity is based on measured green times. The equation for the observed capacity of phase $i$ is (5)

$c_{i}=\frac{g_{i}}{C} \times s$,

Equation 4.11

where $g_{i}$ is the measured effective green time in seconds as given by Equation 4.3, $C$ is the measured cycle length as given by Equation 4.1, and $s$ is the saturation flow rate in $\mathrm{veh} / \mathrm{h} / \mathrm{ln}$, which as before is equal to $1900 \mathrm{veh} / \mathrm{h} / \mathrm{ln}$.

This equation simply divides $s$ among fractions of $C$ based on $g_{i}$. A remaining proportion of time is the amount of capacity eaten up by lost time during clearance intervals. This may be calculated using Equation 4.8. When all of the fractions of $s$ are added together they should equal $s$. It is completely independent of the assumed value of $s$. Therefore, $s$ could be adjusted to reflect conditions at the intersection. Since $C$ and $g_{i}$ are measured from detector events, and all green indications and clearance intervals take place between the two cycle end points, any large divergence from $s$ in the summation reveals an error in the accounting of time. Miscalculated green times, incorrect clearance intervals, or mismatched phases and cycles are possible causes. Small divergences may result from rounding errors.

For actuated signal operation, observed capacities of individual phases are likely to be considerably different from estimated capacities determined by split times. This arises from the allocation of green time by actuation. If each phase times to its force-off point, the controller split proportions will be achieved; however, if this is not the case, then some phases will terminate early, or may not be called at all. The capacity surrendered by those phases is reallocated to succeeding phases.

Table 4.5(a) shows calculations of observed green time for phase 4 at Noblesville on March 14, 2007. For example, an instance of Phase 4 that began at 11:04:00 has an observed green time of $26.6 \mathrm{~s}$. The cycle length at this time was $102 \mathrm{~s}$, from which we calculate an observed capacity of $(26.6 / 102) \times 1900=496 \mathrm{veh} / \mathrm{h} / \mathrm{ln}$. This is the volume that could be served by this phase under the capacity flow rate of $1900 \mathrm{veh} / \mathrm{h} / \mathrm{ln}$. Table 4.5(b) compares observed capacity for all eight phases for ten cycles, verifying that the sum of observed capacity and lost capacity for eight phases adds up to approximately 1900. 
Figure 4.10 shows 24 hour plots of observed capacity for phases 6 and 3 at Noblesville on March 14, 2007. A plot of estimated capacity is shown by the green line. The trace of phase 6 shows that for many cycles, phase 6 achieves no more than its observed capacity. However, for other cycles, phase 6 is able to obtain additional green time from the early termination of preceding phases, thus increasing its capacity beyond what would be supplied by the split. Phase 3, in contrast, occurs at the very beginning of the cycle and cannot obtain any additional green time. Therefore, it never provides any more capacity than what it is provided by the split, while occasionally providing less when it terminates early.

Figure 4.11 shows a comparison between estimated and observed capacity in terms of the splits, division of cycle time, and capacity. The data here relate to a cycle beginning at 10:55:57 at Noblesville. The cycle length was 96 s. Only ring 1 is shown here. Phase 3 is assigned a split of $15 \%$ while phase 4 is assigned $34 \%$. From the splits, we estimated green times of $10.4 \mathrm{~s}$ and $28.6 \mathrm{~s}$ for phases 3 and 4 respectively. This corresponds to estimated capacities of $206 \mathrm{veh} / \mathrm{h} / \mathrm{ln}$ for phase 3 and $567 \mathrm{veh} / \mathrm{h} / \mathrm{ln}$ for phase 4 . In the actual cycle, phase 3 was not called, so it received no green time and therefore no capacity. Phase 4 terminated a few seconds early, so its observed capacity was somewhat less than its estimated capacity. The same thing happened to phase 1. Phase 2 inherited the extra green time.

Notice that because phase 3 was not called, the lost time and clearance interval associated with it were left out of this cycle. Thus, the total lost time was $4 \mathrm{~s}$ less than the estimated lost time, and the amount of lost capacity was reduced. This must be taken into account when calculating average capacities for a block of cycles in a TOD plan. When phases are actuated, the average amount of observed lost capacity will always be less than the estimated lost capacity. 
Table 4.5 Calculations of observed capacity at Noblesville.

(a) Phase 4, Wednesday, March 14, 2007

\begin{tabular}{|r|r|r|r|r|r|}
\cline { 5 - 6 } & \multicolumn{2}{c|}{ Capacities $(\mathrm{veh} / \mathrm{h} / \mathrm{ln})$} \\
\hline $\begin{array}{r}\text { Phase } 4 \\
\text { Begin Phase }\end{array}$ & $\begin{array}{c}\text { Phase } 4 \\
\text { Begin Green }\end{array}$ & $g_{4}(\mathrm{~s})$ & \multicolumn{1}{c|}{$C(\mathrm{~s})$} & \multicolumn{1}{c|}{$s$} & \multicolumn{1}{c|}{$c_{4}$} \\
\hline $10: 53: 22$ & $10: 54: 41$ & 16.8 & 96.0 & 1900 & 332 \\
\hline $10: 55: 00$ & $10: 56: 03$ & 24.8 & 96.0 & 1900 & 491 \\
\hline $10: 56: 30$ & $10: 57: 53$ & 28.6 & 96.1 & 1900 & 566 \\
\hline $10: 58: 24$ & $10: 59: 34$ & 13.6 & 121.5 & 1900 & 212 \\
\hline $10: 59: 49$ & $11: 01: 36$ & 11.5 & 118.7 & 1900 & 183 \\
\hline $11: 01: 50$ & $11: 03: 31$ & 26.6 & 102.0 & 1900 & 496 \\
\hline $11: 04: 00$ & $11: 05: 13$ & 26.6 & 102.0 & 1900 & 496 \\
\hline $11: 05: 42$ & $11: 06: 56$ & 12.0 & 102.1 & 1900 & 222 \\
\hline $11: 07: 09$ & $11: 08: 38$ & 13.4 & 102.0 & 1900 & 249 \\
\hline $11: 08: 53$ & $11: 10: 20$ & 16.0 & 102.0 & 1900 & 297 \\
\hline
\end{tabular}

(b) Phases 1-8, Wednesday, March 14, 2007

\begin{tabular}{|c|c|c|c|c|c|c|c|c|c|c|c|c|}
\hline & \multicolumn{6}{|c|}{ Ring 1, Capacity (veh/h/ln) } & \multicolumn{6}{|c|}{ Ring 2 Capacity (veh/h/ln) } \\
\hline $\begin{array}{l}\text { Cycle } \\
\text { Time }\end{array}$ & $c_{1}$ & $c_{2}$ & $c_{3}$ & $c_{4}$ & Lost & Total & $c_{5}$ & $c_{6}$ & $c_{7}$ & $c_{8}$ & Lost & Total \\
\hline $10: 54: 21$ & 241 & 827 & 207 & 332 & 293 & 1899 & 322 & 725 & 163 & 375 & 313 & 1898 \\
\hline 10:55:57 & 139 & 1056 & 0 & 491 & 214 & 1900 & 322 & 853 & 0 & 491 & 234 & 1900 \\
\hline $10: 57: 33$ & 139 & 697 & 207 & 566 & 293 & 1901 & 324 & 492 & 162 & 610 & 312 & 1901 \\
\hline 10:59:09 & 0 & 1285 & 230 & 212 & 172 & 1898 & 404 & 808 & 170 & 275 & 247 & 1903 \\
\hline 11:01:10 & 113 & 1117 & 251 & 183 & 237 & 1900 & 354 & 860 & 0 & 500 & 189 & 1902 \\
\hline 11:03:09 & 178 & 722 & 228 & 496 & 276 & 1900 & 223 & 658 & 0 & 799 & 220 & 1899 \\
\hline 11:04:51 & 154 & 746 & 230 & 496 & 276 & 1901 & 387 & 496 & 0 & 801 & 220 & 1904 \\
\hline $11: 06: 33$ & 0 & 1242 & 228 & 222 & 205 & 1897 & 385 & 768 & 173 & 278 & 294 & 1899 \\
\hline 11:08:15 & 209 & 937 & 228 & 249 & 276 & 1899 & 385 & 742 & 0 & 554 & 220 & 1900 \\
\hline 11:09:57 & 154 & 944 & 228 & 297 & 276 & 1899 & 386 & 694 & 172 & 356 & 294 & 1902 \\
\hline
\end{tabular}




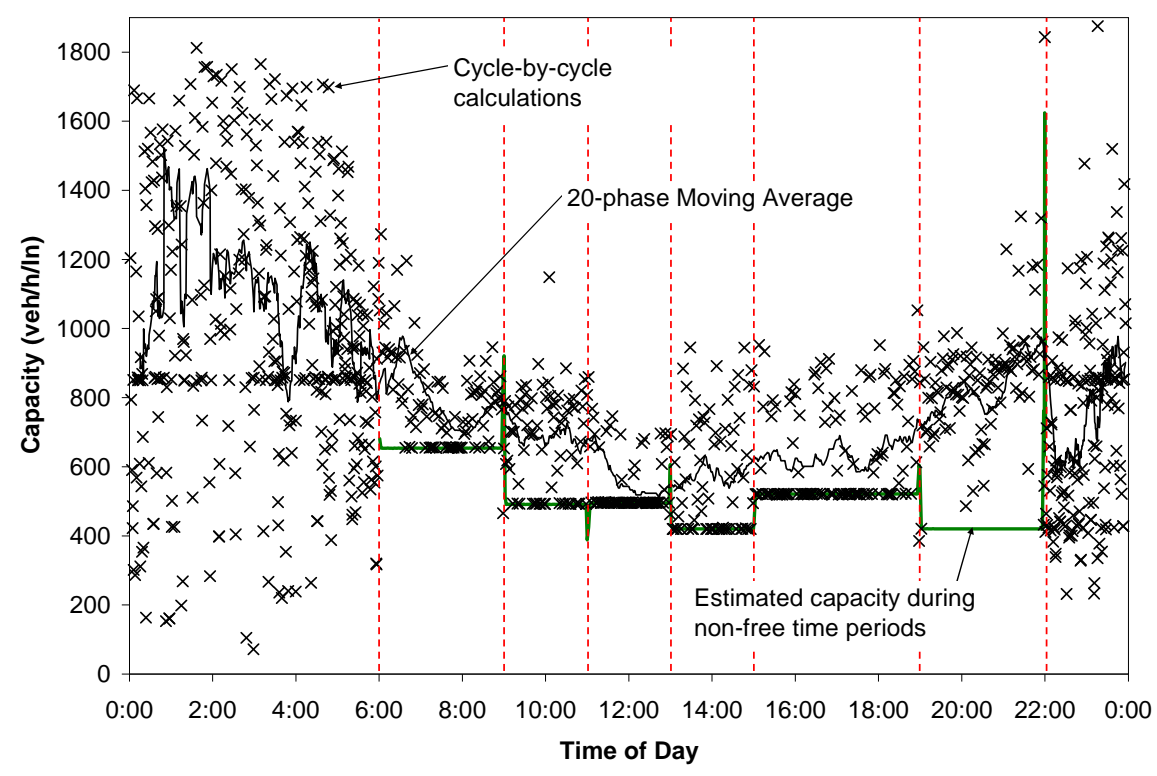

(a) Phase 6, Wednesday, March 14, 2007

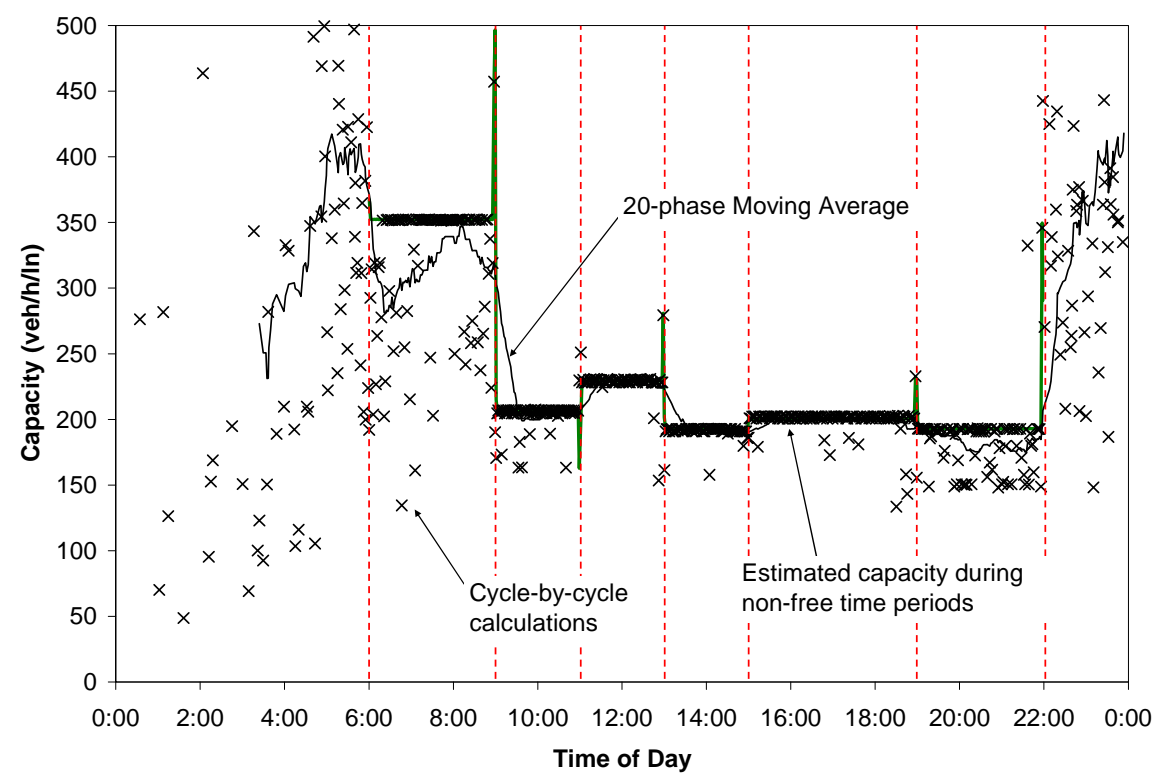

(b) Phase 3, Wednesday, March 14, 2007

Figure 4.10 Graphs of observed vs. estimated capacity at Noblesville. 


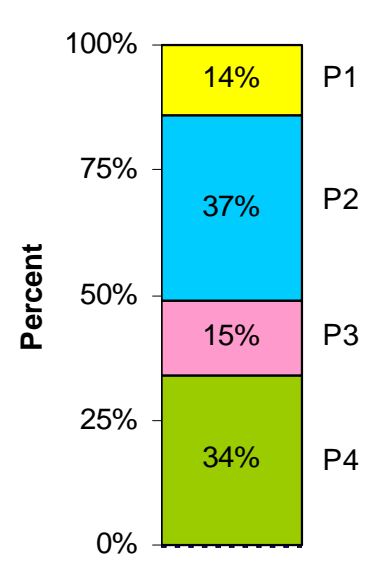

(a) Controller Split (\%)

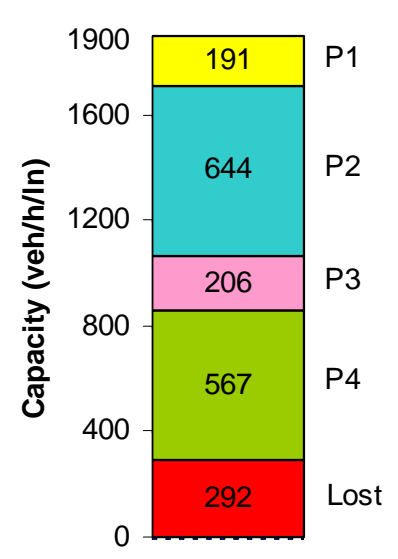

(d) Estimated Capacity $(\mathrm{veh} / \mathrm{h} / \mathrm{ln})$

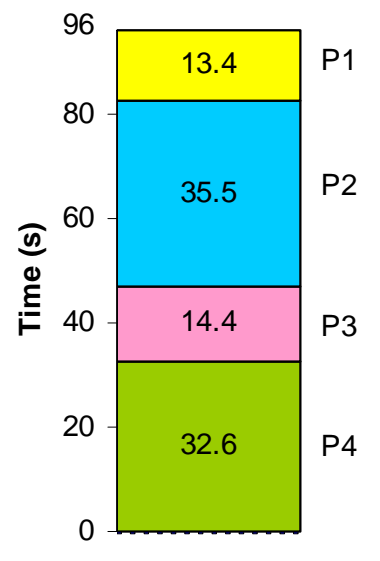

(b) Controller Split (s)

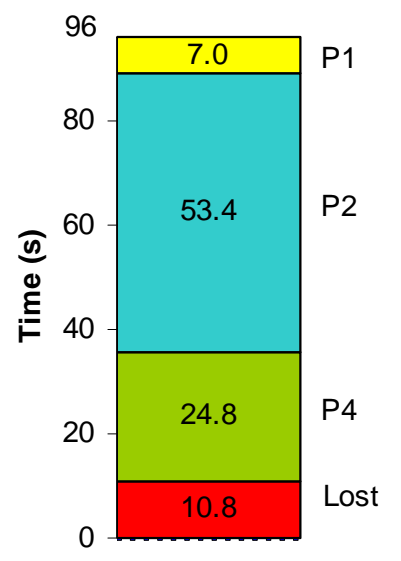

(e) Measured Green (s)

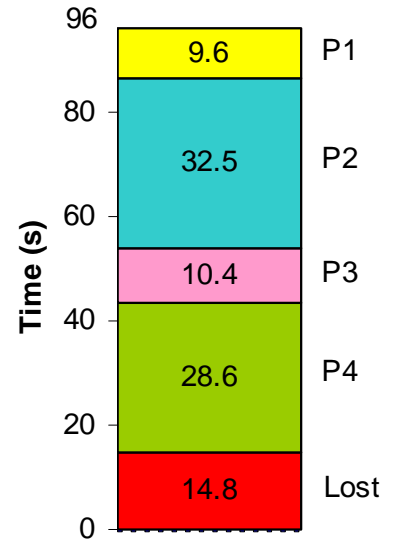

(c) Estimated Green (s)

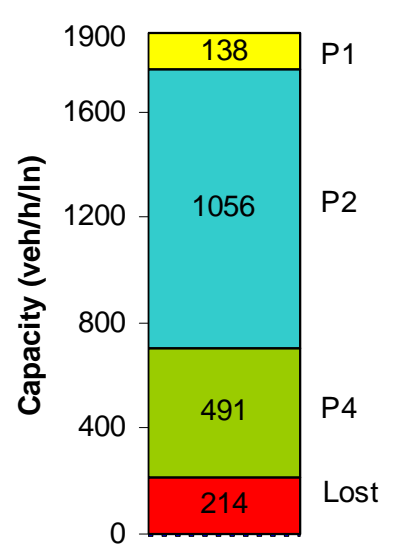

(f) Observed Capacity (veh/h/ln)

Figure 4.11 A comparison of estimated capacity to observed capacity for ring 1 (phases 1-4) for data collected at Noblesville at 10:55:37 on March 14, 2007. 


\subsubsection{Volume-to-Capacity (v/c) Ratio}

The volume-to-capacity ratio, also known as the degree of saturation, is a commonly used measure to describe the performance of a phase at an intersection. The number reflects the proportion of capacity that is utilized by vehicles at an intersection. When defined as the maximum amount of volume that may be served, then the $\mathrm{v} / \mathrm{c}$ ratio should never be higher than 1 . However, capacity is dependent on assumptions about saturation flow rate $s$ and lost time $t_{L}$ (given by Equation 2.5). Values of the $\mathrm{v} / \mathrm{c}$ ratio are scaled by those assumptions. The choice of $s$ and $t_{L}$ should reflect a compromise between what is acceptable and what is practical for operation at a signal. In this work, we have used values of $t_{L}=2 \mathrm{~s}$ and $s=1900 \mathrm{veh} / \mathrm{h} / \mathrm{ln}$. In reality, many more vehicles may make use of the intersection than even liberal assumptions of $s$ and $t_{\mathrm{L}}$ may provide for, leading to $\mathrm{v} / \mathrm{c}$ ratios greater than 1 .

The $\mathrm{v} / \mathrm{c}$ ratio $X_{i}$ may be found, simply enough, by taking the ratio of the volume to the observed capacity, thus:

$$
X_{i}=\frac{v_{i}}{c_{i}},
$$

Equation 4.12

where $v_{i}$ is the volume served by phase $i$ (in veh/h/ln), as discussed in Section 4.2.3; and $c_{i}$ is the observed capacity (in veh/h/ln) described in Equation 4.11. Substituting for $c_{i}$, this equation becomes

$$
X_{i}=\frac{v_{i}}{s} \frac{C}{g_{i}},
$$

Equation 4.13

where $v_{i}$ is the volume as above (in veh/h/ln), $s$ is the saturation flow rate (in veh/h/ln), $g_{i}$ is the effective green time (in s), and $C$ is the cycle length (in s).

One additional way to calculate the $\mathrm{v} / \mathrm{c}$ ratio is to consider the service flow rate defined $q_{i}$ by Equation 4.5 and divide by the maximum flow rate:

$$
X_{i}=\frac{q_{i}}{s} .
$$

Equation 4.14

All of the equations presented for $X_{i}$ in this section are equivalent. 
Table 4.6 shows calculation of the $\mathrm{v} / \mathrm{c}$ ratio for phases 6 and 5 at Noblesville on March 14, 2007. For an instance of phase 5 at 11:07:51, 13 vehicles were served in a cycle length of $102.1 \mathrm{~s}$. We calculate an equivalent hourly volume of $458 \mathrm{veh} / \mathrm{h} / \mathrm{ln}$ for this movement during this cycle. Given a green time of $20.7 \mathrm{~s}$, we calculate $\mathrm{v} / \mathrm{c}=1.19$ for phase 5 during this cycle. Alternately, we divide $458 \mathrm{veh} / \mathrm{h} / \mathrm{ln}$ by the observed capacity of 385 to obtain the same number, or we calculate the service flow rate $q=(13 / 20.7) \times$ $3600=2261 \mathrm{veh} / \mathrm{h} / \mathrm{ln}$ and divide by $s=1900 \mathrm{veh} / \mathrm{h} / \mathrm{ln}$ to obtain $\mathrm{v} / \mathrm{c}=1.19$.

The meaning of $\mathrm{v} / \mathrm{c}=1.19$ is that, given a saturation flow rate $s=1900 \mathrm{veh} / \mathrm{h} / \mathrm{ln}$, assuming lost time $t_{L}=2 \mathrm{~s}$, this phase served $19 \%$ more vehicles than expected if the service flow rate was equal to $1900 \mathrm{veh} / \mathrm{h} / \mathrm{ln}$. What happened is that vehicles actually moved at a higher saturation flow rate. For our purposes, we assume this implies that some vehicles were not serviced during the green phase, and were forced to wait until the next cycle. For our purposes, we define the occurrence of a split failure when the $\mathrm{v} / \mathrm{c}$ ratio exceeds 1.0 (14). Although we treat this as a binary threshold, a more rigorous might be that the probability that a split failure occurred increases as the $\mathrm{v} / \mathrm{c}$ ratio increases. As we discussed before, values of $\mathrm{v} / \mathrm{c}$ are scaled by the assumptions of $s$ and $t_{L}$. In this work, for sake of simplicity, we have used the cutoff point $\mathrm{v} / \mathrm{c}>1$ to determine when a split failure occurred. A more detailed analysis would require the visual evaluation of cycles where $\mathrm{v} / \mathrm{c}$ was close to or above 1 , to determine the actual probability that a split failure occurred. Under other assumptions of $s$ and $t_{L}$, an appropriate cutoff point could be higher or lower than 1.

The interpretation of the traffic engineer is that this movement has no underutilized green time that could be transferred to other movements without substantially degrading service. Note that the immediately preceding phase, phase 6 , has a v/c ratio equal to 0.28 (i.e., the instance that began at 11:07:09). During this cycle at least, it seems reasonable that phase 6 could have given up some of its capacity to phase 5. Of course, phase 6 is also a through movement for the major street, and at some times of the day it also could not afford to give up its green time. It would be advantageous to be able to make the allocations on a cycle-by-cycle basis rather than relying on splits alone. In the following chapter, we will present a mode of operation in which this can occur.

Figure 4.12 shows plots of $\mathrm{v} / \mathrm{c}$ ratio for phases 6 and 5 at Noblesville on Wednesday, March 14, 2007. Phase 6 runs close to capacity during the morning peak hour, reflecting the strong southbound movement. For the rest of the day, phase 6 runs at about $50 \%$ of capacity, dropping off sharply at night, when phase 6 enjoys long green times while the controller operates in free mode. There are several points above the $\mathrm{v} / \mathrm{c}=$ 
1 line, representing split failures, but it is not enough to indicate a problem with this movement. Phase 5 operates near capacity for most of the day, and there are a large number of points above the $\mathrm{v} / \mathrm{c}=1$ line, particularly during the afternoon and evening peak hour.

The stratification of the $\mathrm{v} / \mathrm{c}$ ratio in phase 5 is a consequence of the consistency of green time between cycles, and the fact that the $\mathrm{v} / \mathrm{c}$ ratio is based on integer values of the number of vehicles. Each line represents a particular number of vehicles, as shown in Figure 4.13. Any point that diverges from those lines represents an early termination of green, as demonstrated by the singular point in that plot in which this took place. If phase 5 were able to acquire additional green time from phase 6 , it would break up the pattern by increasing green times. Values of the $\mathrm{v} / \mathrm{c}$ ratio corresponding to any particular number of vehicles increases as $C$ and $g$ decrease. For example, when $C=116 \mathrm{~s}, 7$ vehicles served by $18.4 \mathrm{~s}$ gives $\mathrm{v} / \mathrm{c}=0.72$, while when $C=104 \mathrm{~s}, 7$ vehicles served by $17.1 \mathrm{~s}$ gives $\mathrm{v} / \mathrm{c}=0.77$.

Figure 4.14 shows video stills of the northbound approach at Noblesville. The stills are taken at the beginning and end of green indications for phase 5 for two different cycles on March 14, 2007. The times indicated on the camera are one hour behind the actual times because of an inability of the data collection system to display daylight savings time outside of the traditional dates. Figure 4.14(a) shows the beginning of green for a cycle with $\mathrm{v} / \mathrm{c}=0.55$. There are three vehicles in queue at the beginning of green. During the green indication, which lasted $13.7 \mathrm{~s}$, one additional vehicle arrived, so four were served in total. At the end of green, seen in Figure 4.14(b), there are no vehicles remaining to be served. The next two pictures show the same events for a cycle with $\mathrm{v} / \mathrm{c}$ $=1.25$. Figure 4.14(c) shows the beginning of green. The queue is very long; it is impossible to determine from the picture how many vehicles are in queue. The green indication here was also $13.7 \mathrm{~s}$, and altogether 9 vehicles were served. This was the highest number of vehicles that managed to squeeze through $13.7 \mathrm{~s}$ of green during the 06:00-09:00 time period. Figure 4.14(d) is a snapshot at the very beginning of the all-red clearance interval; the van can be seen executing the left turn past the yellow. Also, note that there is still a queue remaining in the approach lane, indicating that a split failure took place. 
Table 4.6 Calculation of v/c ratio at Noblesville.

(a) Phase 6, Wednesday, March 14, 2007

\begin{tabular}{|c|r|r|r|r|r|r|r|r|}
\hline $\begin{array}{c}\text { Begin } \\
\text { Green }\end{array}$ & $C(\mathrm{~s})$ & $\begin{array}{c}s \\
(\mathrm{veh} / \mathrm{h} / \mathrm{ln})\end{array}$ & $\begin{array}{c}n_{L} \\
(\mathrm{ln})\end{array}$ & $\begin{array}{c}N \\
(\mathrm{veh})\end{array}$ & $\begin{array}{c}v_{6} \\
(\mathrm{veh} / \mathrm{h} / \mathrm{ln})\end{array}$ & $g_{6}(\mathrm{~s})$ & \multicolumn{1}{c|}{$X_{6}$} & $\begin{array}{c}C_{6} \\
(\mathrm{veh} / \mathrm{h} / \mathrm{ln})\end{array}$ \\
\hline $10: 53: 21$ & 96.1 & 1900 & 2 & 11 & 206 & 39.1 & 0.266 & 774 \\
\hline $10: 55: 00$ & 96.0 & 1900 & 2 & 9 & 169 & 36.6 & 0.233 & 725 \\
\hline $10: 56: 30$ & 96.0 & 1900 & 2 & 21 & 394 & 43.1 & 0.462 & 853 \\
\hline $10: 58: 24$ & 96.1 & 1900 & 2 & 9 & 169 & 24.9 & 0.342 & 492 \\
\hline $10: 59: 49$ & 121.5 & 1900 & 2 & 18 & 267 & 51.6 & 0.330 & 808 \\
\hline $11: 01: 49$ & 118.7 & 1900 & 2 & 8 & 121 & 53.7 & 0.141 & 860 \\
\hline $11: 04: 00$ & 102.0 & 1900 & 2 & 14 & 247 & 35.3 & 0.375 & 658 \\
\hline $11: 05: 42$ & 102.0 & 1900 & 2 & 9 & 159 & 26.6 & 0.320 & 496 \\
\hline $11: 07: 09$ & 102.1 & 1900 & 2 & 12 & 212 & 41.3 & 0.275 & 768 \\
\hline $11: 08: 53$ & 102.0 & 1900 & 2 & 15 & 265 & 39.8 & 0.357 & 742 \\
\hline
\end{tabular}

(b) Phase 5, Wednesday, March 14, 2007

\begin{tabular}{|c|r|r|r|r|r|r|r|r|}
\hline $\begin{array}{c}\text { Begin } \\
\text { Green }\end{array}$ & \multicolumn{1}{c|}{$C(\mathrm{~s})$} & $\begin{array}{c}s \\
(\mathrm{veh} / \mathrm{h} / \mathrm{ln})\end{array}$ & $\begin{array}{c}n_{L} \\
(\mathrm{ln})\end{array}$ & $\begin{array}{c}N \\
(\mathrm{veh})\end{array}$ & $\begin{array}{c}v_{5} \\
(\mathrm{veh} / \mathrm{h} / \mathrm{ln})\end{array}$ & $g_{5}(\mathrm{~s})$ & \multicolumn{1}{c|}{$X_{5}$} & $\begin{array}{c}C_{5} \\
(\mathrm{veh} / \mathrm{h} / \mathrm{ln})\end{array}$ \\
\hline 10:54:01 & 96.1 & 1900 & 1 & 6 & 225 & 16.3 & 0.699 & 322 \\
\hline $10: 55: 37$ & 96.0 & 1900 & 1 & 5 & 188 & 16.3 & 0.583 & 322 \\
\hline $10: 57: 13$ & 96.0 & 1900 & 1 & 7 & 263 & 16.3 & 0.814 & 322 \\
\hline $10: 58: 49$ & 96.1 & 1900 & 1 & 9 & 337 & 16.4 & 1.040 & 324 \\
\hline $11: 00: 41$ & 121.5 & 1900 & 1 & 7 & 207 & 25.8 & 0.514 & 404 \\
\hline $11: 02: 43$ & 118.7 & 1900 & 1 & 10 & 303 & 22.1 & 0.858 & 354 \\
\hline $11: 04: 35$ & 102.0 & 1900 & 1 & 6 & 212 & 12.0 & 0.951 & 223 \\
\hline $11: 06: 09$ & 102.0 & 1900 & 1 & 9 & 318 & 20.8 & 0.820 & 387 \\
\hline $11: 07: 51$ & 102.1 & 1900 & 1 & 13 & 458 & 20.7 & 1.190 & 385 \\
\hline $11: 09: 33$ & 102.0 & 1900 & 1 & 12 & 423 & 20.7 & 1.100 & 385 \\
\hline
\end{tabular}




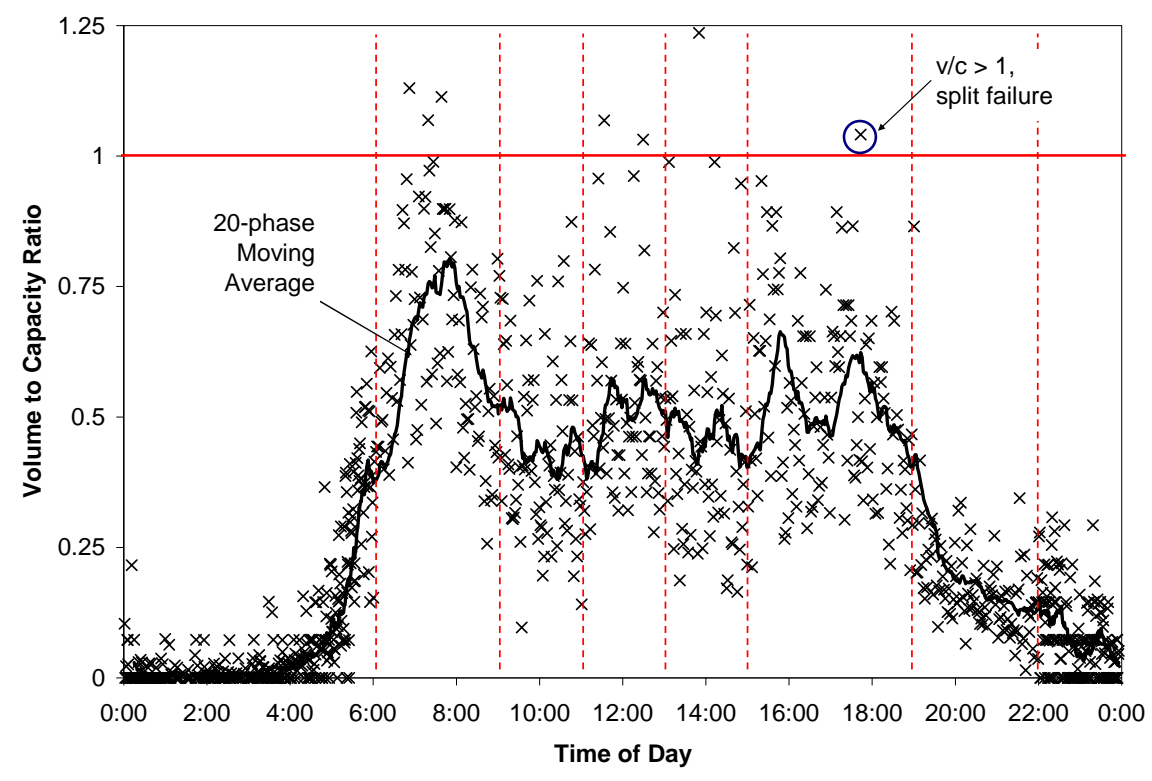

(a) Phase 6, Wednesday, March 14, 2007

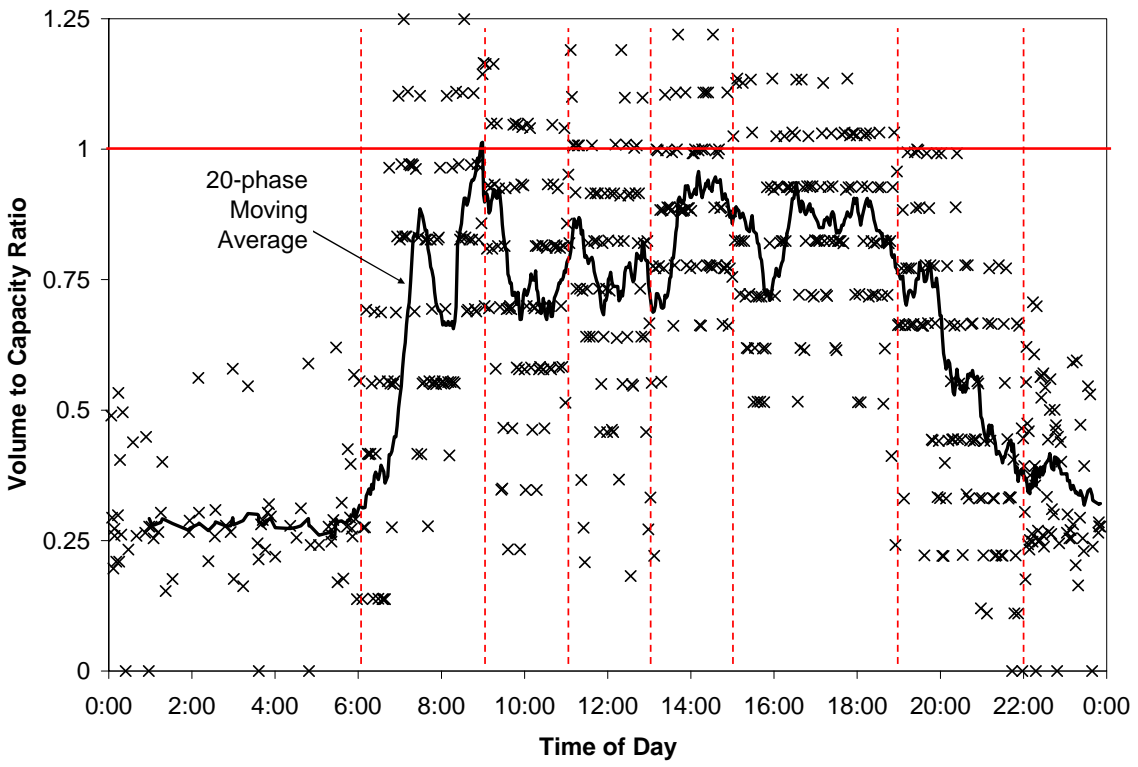

(b) Phase 5, Wednesday, March 14, 2007

Figure 4.12 Graphs of Volume-to-Capacity Ratio for Noblesville. 


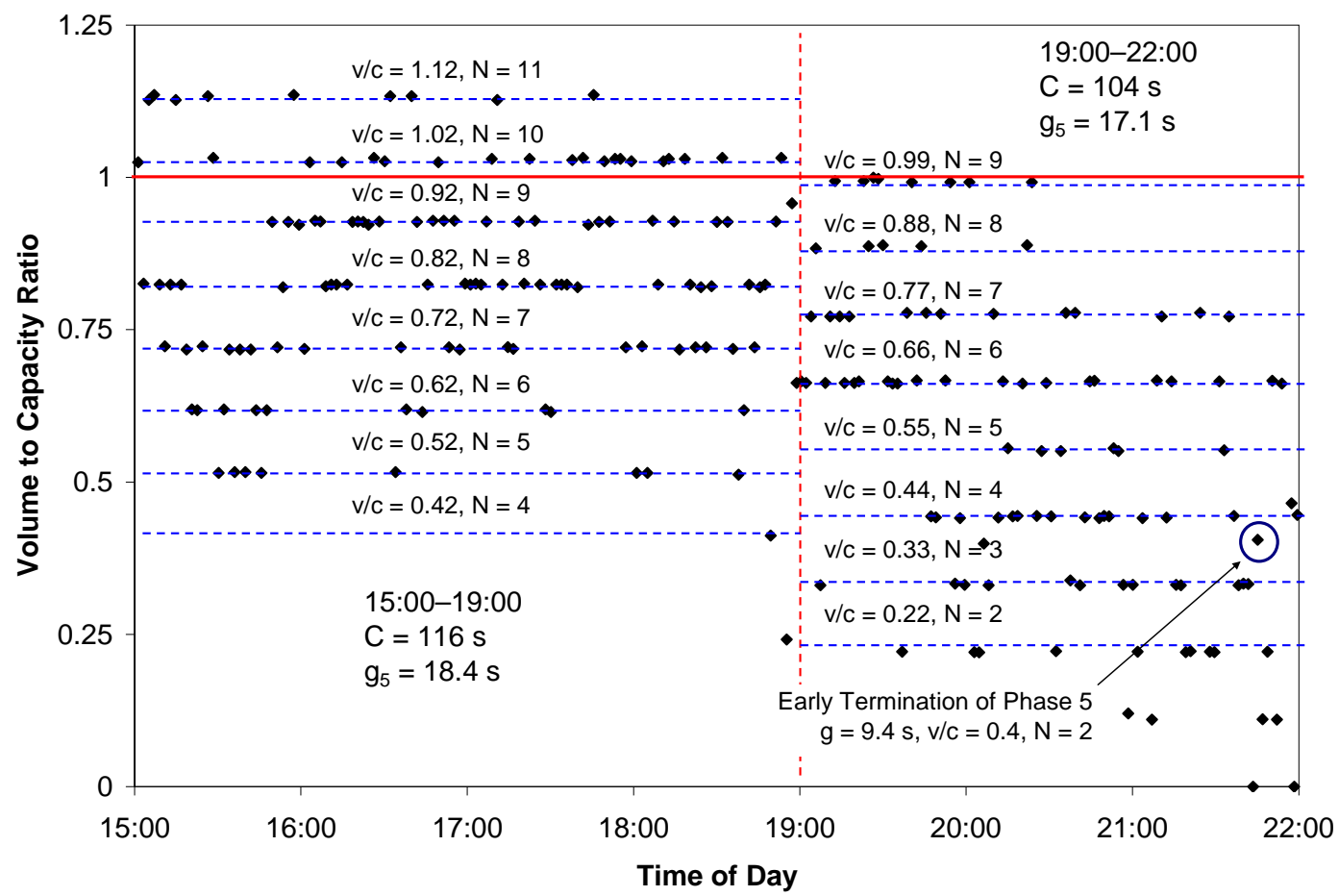

Figure 4.13 A graph of v/c ratio for phase 5 at Noblesville on Wednesday, March 14, 2007, showing stratification patterns. 


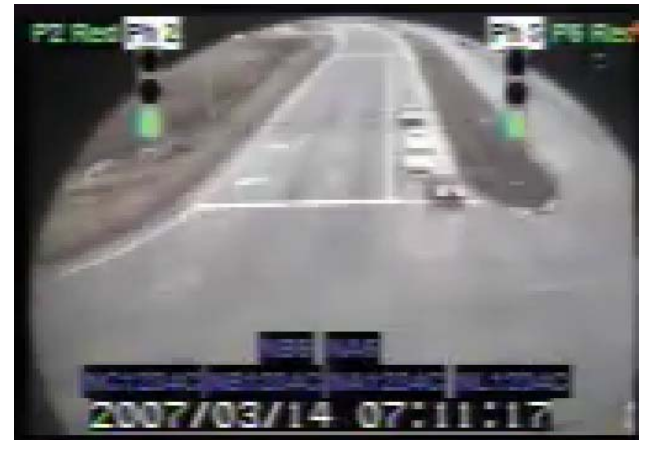

(a) Beginning of green, $\mathrm{v} / \mathrm{c}=0.55$

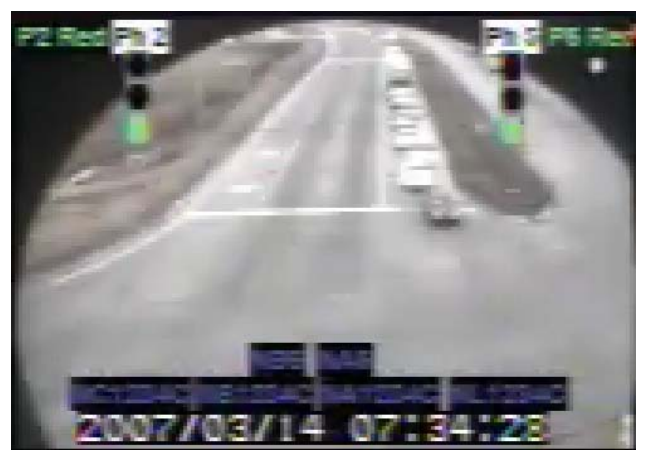

(c) Beginning of green, $\mathrm{v} / \mathrm{c}=1.25$

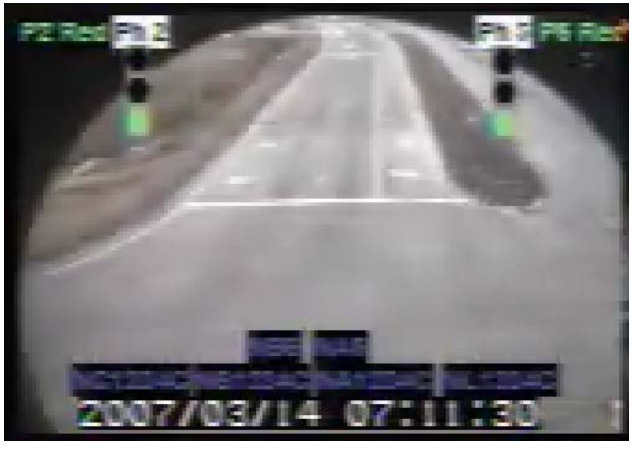

(b) End of green, v/c $=0.55$

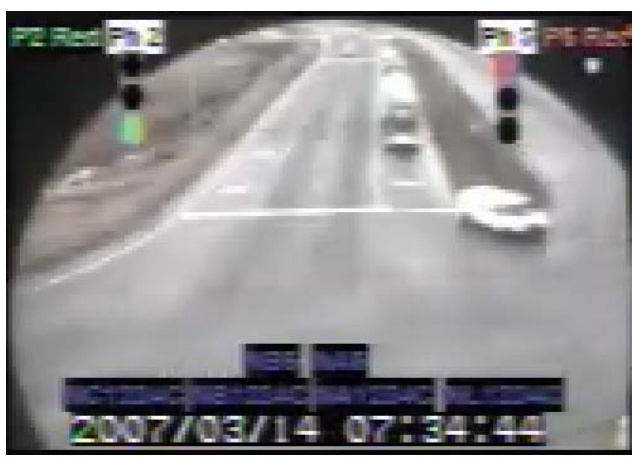

(d) End of green, v/c $=1.25$

Figure 4.14 Camera views of traffic conditions for phase 5 at Noblesville on March 14, 2007, with different $\mathrm{v} / \mathrm{c}$ ratios. 


\subsubsection{Number of Split Failures}

As discussed in the previous section, a split failure is defined as the termination of the green indication of a phase before it meets all of its demand. The higher the value of the $\mathrm{v} / \mathrm{c}$ ratio, the more likely it is that a split failure occurred. In this work, we have used a cutoff value of $\mathrm{v} / \mathrm{c}>1$. As discussed in the previous section, the scale of the $\mathrm{v} / \mathrm{c}$ ratio is dependent on assumptions of $s$ and $t_{L}$. An appropriate cutoff point for split failures is also dependent on these assumptions. Under different assumptions than the ones used here, a different cutoff point might be appropriate. A method for determining this would be a possible direction for future research.

Counts of split failures are an alternative means of scrutinizing the use of capacity by a movement. Average values of v/c ratio can potentially mask a large number of split failures. The number of split failures demonstrates how many times during a time period that the phase did not have enough green time to serve all of its demand. A high number of split failures for a phase indicates a need for additional capacity that might not be revealed by an examination of $\mathrm{v} / \mathrm{c}$ ratios alone.

Raw split failure counts are not directly comparable between each other because every TOD period has a different number of cycles taking place because of different cycle lengths as well as different lengths of TOD periods. However, the raw counts are useful to qualitatively describe the quality of service for each phase, and may be used for comparison between two different days that have the same TOD plan. This is how the quantity will be used in the following chapter to compare performance between different modes of operation. Split failure counts per TOD period may be compared by normalizing the values by the number of times the phase was served within each TOD. However, a percentage does not depict quite the same qualitative portrait than a count of the number of times that split failures happened.

Figure 4.15 shows a graph of $\mathrm{v} / \mathrm{c}$ by time of day for phase 1 at Noblesville on Wednesday, March 14, 2007. The black line shows the average value of v/c for each time period, while numbers at the bottom give the quantity of that average value. There are five split failures in total for the day, with three occurring during the AM peak hour (6:00-9:00) and two occurring during the PM peak hour (15:00-19:00). These two time periods also have the highest average v/c ratios. However, there is no particular reason to suppose from the average $\mathrm{v} / \mathrm{c}$ ratios alone that any split failures should have taken place. Additionally, the magnitude of the average v/c ratio does not give any information about the number of split failures that are likely to occur. The AM peak has a lower average value of v/c ratio than the PM peak, but has more split failures. 
Table 4.7(a) shows the total number of split failures for all eight phases at Noblesville between 6:00 and 22:00 on Wednesday, March 14, 2007. Table 4.7(b) gives the percentage of phases that the split failure counts represent within each TOD. Table 4.7(c) gives the average $\mathrm{v} / \mathrm{c}$ ratios for the same time periods. In general, there are more split failures reported for TOD periods with higher average values of $v / c$, but the two quantities give different information. For example, during the AM peak hour of 6:009:00, the average $\mathrm{v} / \mathrm{c}$ ratios of phases 3 and 5 are very similar. The ratio for Phase 5 is only slightly higher. The number of split failures provides a different perspective. Phase 3 had no split failures, whereas phase 5 had 11. Figure 4.16 shows a plot of the average v/c ratio of a TOD period versus the percentage of split failures reported for the same TOD. This graph shows that the percentage of phases in a TOD with $\mathrm{v} / \mathrm{c}$ ratios greater than 1 increases as the average $\mathrm{v} / \mathrm{c}$ ratio of all phases approaches 1 . The spread of points indicates that some phases sustain high average $\mathrm{v} / \mathrm{c}$ ratios with few or no split failures, whereas other phases sustain similar ratios with many.

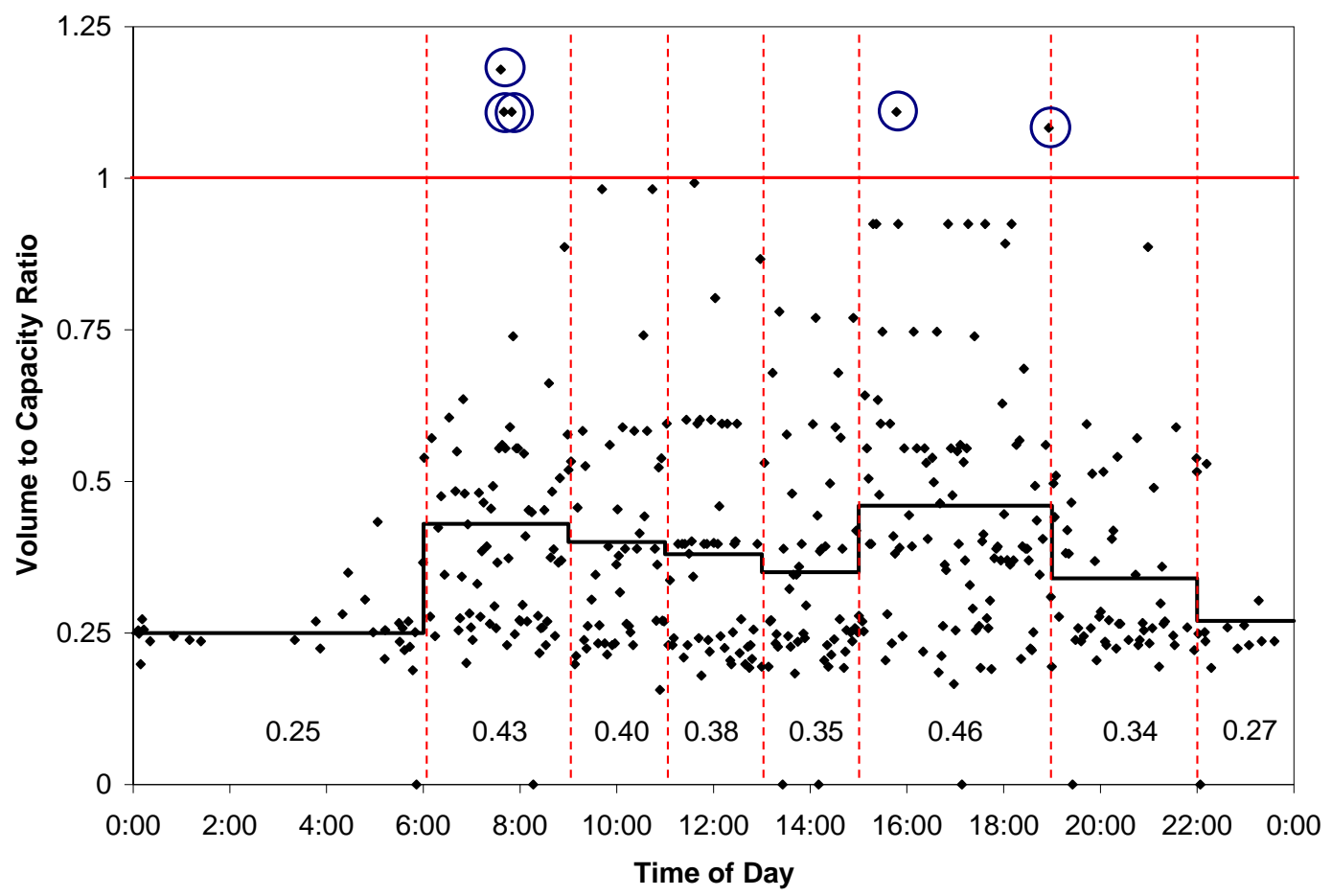

Figure 4.15 Graph of v/c ratio at Noblesville for phase 1, Wednesday, March 14, 2007, with five split failures indicated. 
Table 4.7 Counts of split failures and average v/c ratio values at Noblesville on March 14, 2007 by time of day for all eight phases.

(a) Number of split failures by TOD

\begin{tabular}{|c|r|r|r|r|r|r|r|}
\hline Phase & $\begin{array}{c}\text { 06:00- } \\
\text { 09:00 }\end{array}$ & $\begin{array}{c}\text { 09:00- } \\
11: 00\end{array}$ & $\begin{array}{c}\text { 11:00- } \\
13: 00\end{array}$ & $\begin{array}{c}\text { 13:00- } \\
15: 00\end{array}$ & $\begin{array}{c}\text { 15:00- } \\
19: 00\end{array}$ & $\begin{array}{c}\text { 19:00- } \\
\text { 22:00 }\end{array}$ & \multicolumn{1}{c|}{ Total } \\
\hline $\mathbf{1}$ & 3 & 0 & 0 & 0 & 2 & 0 & 5 \\
\hline $\mathbf{2}$ & 0 & 0 & 0 & 0 & 4 & 0 & 4 \\
\hline $\mathbf{3}$ & 0 & 0 & 0 & 0 & 0 & 0 & 0 \\
\hline $\mathbf{4}$ & 20 & 12 & 20 & 12 & 39 & 8 & 111 \\
\hline $\mathbf{5}$ & 11 & 13 & 14 & 10 & 29 & 0 & 77 \\
\hline $\mathbf{6}$ & 3 & 0 & 2 & 1 & 1 & 0 & 7 \\
\hline $\mathbf{7}$ & 0 & 0 & 0 & 1 & 0 & 0 & 1 \\
\hline $\mathbf{8}$ & 0 & 0 & 0 & 0 & 0 & 0 & 0 \\
\hline Total & 37 & 25 & 36 & 24 & 75 & 8 & 205 \\
\hline
\end{tabular}

(b) Percentage of phases with split failures by TOD

\begin{tabular}{|c|r|r|r|r|r|r|r|}
\hline Phase & $\begin{array}{c}\mathbf{0 6 : 0 0}- \\
\mathbf{0 9 : 0 0}\end{array}$ & $\begin{array}{c}\mathbf{0 9 : 0 0} \\
\mathbf{1 1 : 0 0}\end{array}$ & $\begin{array}{c}\mathbf{1 1 : 0 0} \\
\mathbf{1 3 : 0 0}\end{array}$ & $\begin{array}{c}\mathbf{1 3 : 0 0} \\
\mathbf{1 5 : 0 0}\end{array}$ & $\begin{array}{c}\mathbf{1 5 : 0 0} \\
\mathbf{1 9 : 0 0}\end{array}$ & $\begin{array}{c}\mathbf{1 9 : 0 0} \\
\mathbf{2 2 : 0 0}\end{array}$ & Average \\
\hline $\mathbf{1}$ & $4.2 \%$ & $0.0 \%$ & $0.0 \%$ & $0.0 \%$ & $2.0 \%$ & $0.0 \%$ & $1.0 \%$ \\
\hline $\mathbf{2}$ & $0.0 \%$ & $0.0 \%$ & $0.0 \%$ & $0.0 \%$ & $3.2 \%$ & $0.0 \%$ & $0.5 \%$ \\
\hline $\mathbf{3}$ & $0.0 \%$ & $0.0 \%$ & $0.0 \%$ & $0.0 \%$ & $0.0 \%$ & $0.0 \%$ & $0.0 \%$ \\
\hline $\mathbf{4}$ & $21.5 \%$ & $16.2 \%$ & $28.6 \%$ & $17.4 \%$ & $31.2 \%$ & $7.8 \%$ & $20.4 \%$ \\
\hline $\mathbf{5}$ & $12.1 \%$ & $17.3 \%$ & $20.0 \%$ & $14.5 \%$ & $23.4 \%$ & $0.0 \%$ & $14.6 \%$ \\
\hline $\mathbf{6}$ & $3.2 \%$ & $0.0 \%$ & $2.9 \%$ & $1.4 \%$ & $0.8 \%$ & $0.0 \%$ & $1.4 \%$ \\
\hline $\mathbf{7}$ & $0.0 \%$ & $0.0 \%$ & $0.0 \%$ & $1.7 \%$ & $0.0 \%$ & $0.0 \%$ & $0.3 \%$ \\
\hline $\mathbf{8}$ & $0.0 \%$ & $0.0 \%$ & $0.0 \%$ & $0.0 \%$ & $0.0 \%$ & $0.0 \%$ & $0.0 \%$ \\
\hline All & $5.4 \%$ & $4.7 \%$ & $6.9 \%$ & $4.6 \%$ & $7.9 \%$ & $1.1 \%$ & $5.1 \%$ \\
\hline
\end{tabular}

(c) Average v/c ratios by TOD.

\begin{tabular}{|c|c|c|c|c|c|r|r|}
\hline Phase & $\begin{array}{c}\text { 06:00- } \\
\text { 09:00 }\end{array}$ & $\begin{array}{c}\text { 09:00- } \\
\mathbf{1 1 : 0 0}\end{array}$ & $\begin{array}{c}\mathbf{1 1 : 0 0 -} \\
\mathbf{1 3 : 0 0}\end{array}$ & $\begin{array}{c}\mathbf{1 3 : 0 0 -} \\
\mathbf{1 5 : 0 0}\end{array}$ & $\begin{array}{c}\mathbf{1 5 : 0 0 -} \\
\mathbf{1 9 : 0 0}\end{array}$ & $\begin{array}{c}\mathbf{1 9 : 0 0} \\
\mathbf{2 2 : 0 0}\end{array}$ & Average \\
\hline $\mathbf{1}$ & 0.43 & 0.39 & 0.38 & 0.35 & 0.46 & 0.34 & 0.39 \\
\hline $\mathbf{2}$ & 0.21 & 0.21 & 0.31 & 0.42 & 0.64 & 0.28 & 0.35 \\
\hline $\mathbf{3}$ & 0.69 & 0.61 & 0.64 & 0.64 & 0.64 & 0.50 & 0.62 \\
\hline $\mathbf{4}$ & 0.79 & 0.68 & 0.86 & 0.74 & 0.89 & 0.69 & 0.78 \\
\hline $\mathbf{5}$ & 0.73 & 0.75 & 0.77 & 0.87 & 0.84 & 0.55 & 0.75 \\
\hline $\mathbf{6}$ & 0.63 & 0.44 & 0.51 & 0.46 & 0.52 & 0.17 & 0.46 \\
\hline $\mathbf{7}$ & 0.42 & 0.42 & 0.50 & 0.52 & 0.53 & 0.45 & 0.48 \\
\hline $\mathbf{8}$ & 0.34 & 0.31 & 0.28 & 0.31 & 0.29 & 0.26 & 0.30 \\
\hline
\end{tabular}




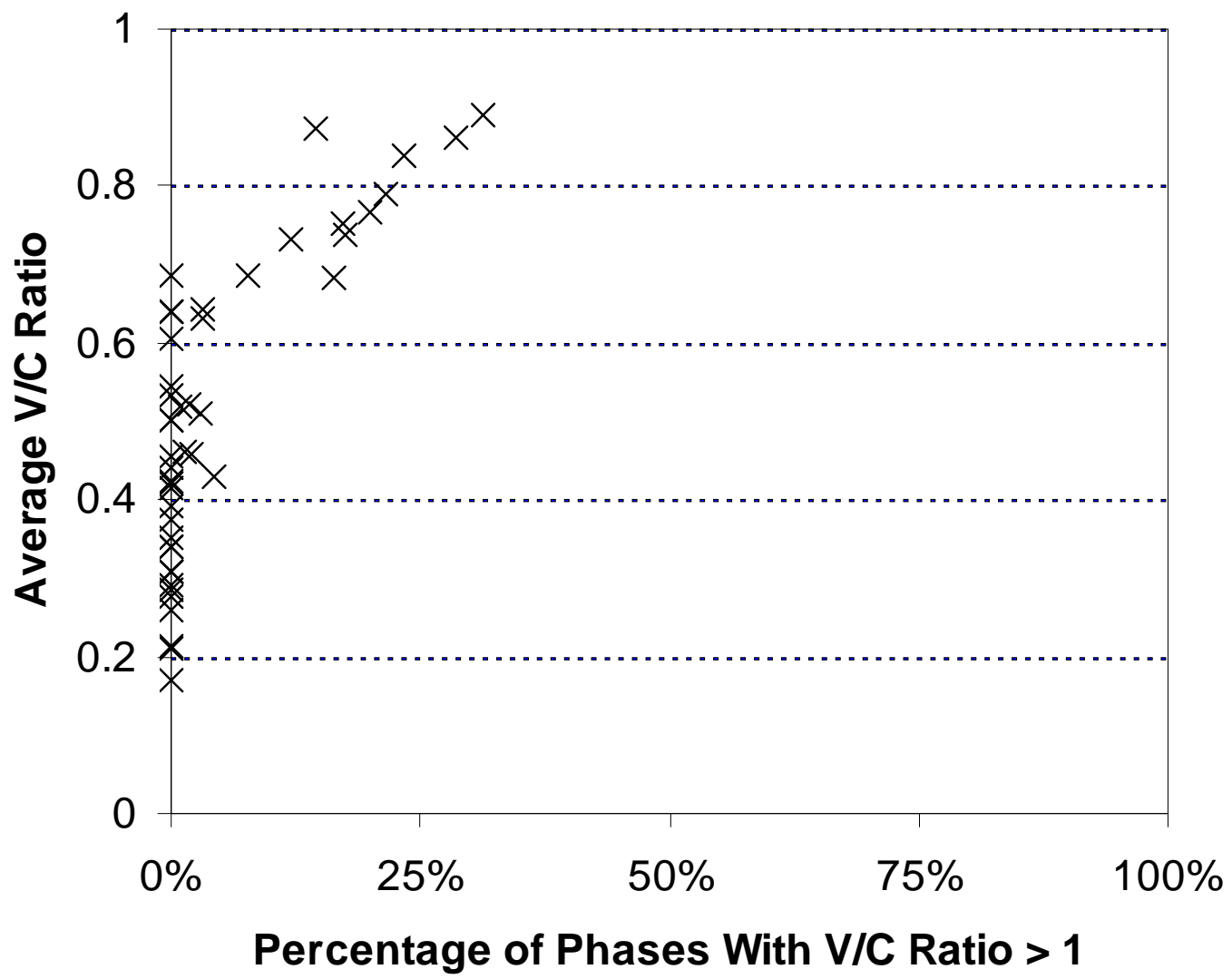

Figure 4.16 Average v/c ratio of a TOD plan period versus the proportion of phases within the same TOD plan period reporting split failures. 


\subsubsection{Critical v/c Ratio}

The critical Volume-to-Capacity Ratio $\left(X_{c}\right)$, also known as the degree of intersection saturation, is a cycle-based measure that describes the degree to which critical movements at an intersection are utilized. The HCM equation for this measure is given as $(5,6)$ :

$$
X_{c}=\sum_{i}\left(\frac{v}{s}\right)_{c i}\left(\frac{C}{C-L}\right) .
$$

Equation 4.15

where $C$ is the cycle length; $L$ is the lost time; and in the summation term over critical phases $c i, v$ represents the volume and $s$ is the saturation flow rate. The terminology "critical phase" refers to that phase having the greater volume in situations where more than one phase runs concurrently; the sequence of critical phases in a given cycle is referred to as the "critical path." The HCM describes rules for determining critical paths under various phase configurations, but in this report we will restrict our discussion to eight-phase dual-ring controllers. Figure 4.17 shows the four possible critical paths through a dual-ring eight phase controller. For such operating schemes, Equation 4.15 becomes

$$
X_{c}=\left[\max \left(Y_{12}, Y_{56}\right)+\max \left(Y_{34}, Y_{78}\right)\right] \times\left(\frac{C}{C-L}\right)
$$

where, for example, $Y_{12}=v_{1} / s+v_{2} / s$.

$X_{C}$ represents the degree to which the total intersection capacity is used by summing volumes moving through the critical path. The measure is not intended to determine whether or not capacity problems exist for individual phases. Individual phases in the critical path may have volumes approaching saturation. However, the calculated $\mathrm{X}_{\mathrm{C}}$ values will not necessarily reflect high intersection utilization, unless the volumes are high for all the movements in the critical path.

An example calculation of $\mathrm{X}_{\mathrm{C}}$ for eight cycles is presented in Table 4.8 and Table 4.9. First, we conduct a comparison between volumes of phase pairs $(1,2)$ and $(5,6)$ on the left side of the barrier, as shown in Table 4.8 (a) for eight midday cycles. Whichever phase pair has the greatest combined volume is said to be critical. For example, during the cycle beginning at 14:42:20, phases 5 and 6 have a higher combined volume than phases 1 and 2, therefore they are considered to be critical. We repeat this comparison for 
phases on the right side of the barrier, as shown in Table 4.8 (b). This gives us the critical path through the cycle as well as the needed volumes for the maximum terms in Table 4.9(c).

The total lost time $L$ is a summation of the lost times associated with each occurring critical phase during a cycle. Lost time calculations are shown in Table 4.9(a) and (b) for the left and right sides of the barrier respectively. When a phase is skipped, its lost time is equal to zero. When the traffic volumes and lost time amounts have been found, we can proceed with the $\mathrm{X}_{\mathrm{C}}$ calculation of Equation 4.16, as shown in Table 4.9 (c). In this case, the intersection is clearly under saturation during these cycles and has significant reserve capacity.

Figure 4.18 is a 24-hour graph of $\mathrm{X}_{\mathrm{C}}$ at the Noblesville test intersection on March 14,2007 . There is very low use during the overnight (0:00-6:00 and 22:00-24:00) free period, moderate use during most of the day, with large peaks during the AM and PM rush hours as well as a less prominent around noon. The critical path is shown by the symbol marking each data point. Typical of a weekday traffic pattern, phase group $(5,6)$ is critical in the morning whereas $(1,2)$ is critical in the evening. Phase group $(3,4)$ is dominant throughout most of the day. As $X_{C}$ approaches 1, less opportunity to transfer capacity from any particular group of movements to others. Lower $X_{C}$ values show where such opportunities exist.

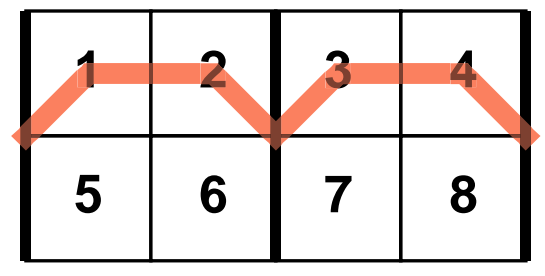

(a) Critical Path 1234

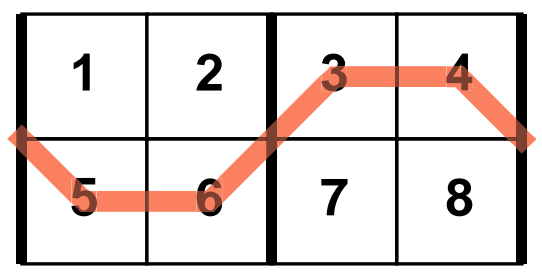

(c) Critical Path 5634

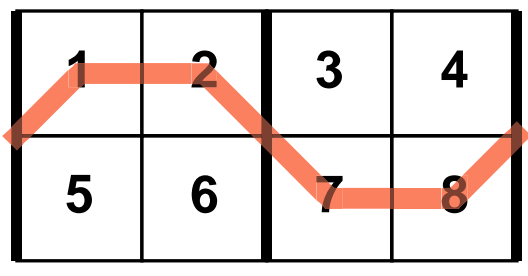

(b) Critical Path 1278

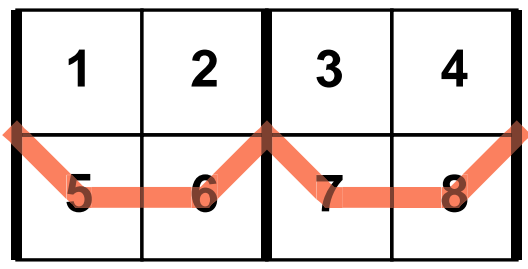

(d) Critical Path 5678

Figure 4.17 Possible critical paths through an eight-phase, dual ring controlled intersection. 
Table 4.8 Sample calculation of $\mathrm{X}_{\mathrm{C}}$ on a cycle-by-cycle basis.

(a) Volume-to-saturation ratios and determination of critical path (left side of barrier).

\begin{tabular}{|c|c|c|c|c|c|c|c|}
\hline \multirow{2}{*}{ Time } & \multicolumn{4}{|c|}{ Volumes (veh/h/ln) } & \multirow{2}{*}{$\mathrm{v}_{1+2}$} & \multirow{2}{*}{$\mathrm{v}_{5+6}$} & \multirow{2}{*}{$\mathrm{CP}_{\mathrm{L}}$} \\
\hline & $\mathrm{v}_{1}$ & $\mathrm{v}_{2}$ & $\mathrm{v}_{5}$ & $\mathrm{v}_{6}$ & & & \\
\hline $14: 42: 20$ & 35 & 329 & 277 & 294 & 363 & 571 & 56 \\
\hline $14: 44: 04$ & 35 & 329 & 277 & 225 & 363 & 502 & 56 \\
\hline $14: 45: 48$ & 0 & 467 & 208 & 156 & 467 & 363 & 12 \\
\hline $14: 47: 32$ & 0 & 363 & 242 & 138 & 363 & 381 & 56 \\
\hline $14: 49: 16$ & 35 & 433 & 242 & 156 & 467 & 398 & 12 \\
\hline $14: 51: 00$ & 35 & 415 & 242 & 398 & 450 & 640 & 56 \\
\hline $14: 52: 44$ & 35 & 398 & 346 & 242 & 433 & 588 & 56 \\
\hline $14: 54: 28$ & 138 & 450 & 208 & 156 & 589 & 363 & 12 \\
\hline
\end{tabular}

(b) Volume-to-saturation ratios and determination of critical path (right side of barrier).

\begin{tabular}{|c|c|c|c|c|c|c|c|}
\hline \multirow{2}{*}{ Time } & \multicolumn{4}{|c|}{ Volumes (veh/h/ln) } & \multirow{2}{*}{$\mathrm{V}_{3+4}$} & \multirow{2}{*}{$\mathrm{v}_{7+8}$} & \multirow{2}{*}{$\mathrm{CP}_{\mathrm{R}}$} \\
\hline & $\mathrm{V}_{3}$ & $\mathrm{~V}_{4}$ & $\mathrm{~V}_{7}$ & $\mathrm{~V}_{8}$ & & & \\
\hline $14: 42: 20$ & 69 & 415 & 104 & 294 & 484 & 398 & 34 \\
\hline $14: 44: 04$ & 173 & 208 & 69 & 208 & 381 & 277 & 34 \\
\hline $14: 45: 48$ & 0 & 484 & 242 & 104 & 484 & 346 & 34 \\
\hline $14: 47: 32$ & 138 & 381 & 69 & 190 & 519 & 260 & 34 \\
\hline $14: 49: 16$ & 138 & 415 & 138 & 138 & 554 & 277 & 34 \\
\hline $14: 51: 00$ & 173 & 277 & 104 & 190 & 450 & 294 & 34 \\
\hline $14: 52: 44$ & 69 & 311 & 0 & 173 & 381 & 173 & 34 \\
\hline $14: 54: 28$ & 173 & 485 & 104 & 173 & 658 & 277 & 34 \\
\hline
\end{tabular}


Table 4.9 Sample calculation of $\mathrm{X}_{\mathrm{C}}$ on a cycle-by-cycle basis.

(a) Calculation of total lost time (left side of barrier).

\begin{tabular}{|c|c|c|c|c|c|c|}
\hline \multirow{2}{*}{ Time } & \multicolumn{4}{|c|}{ Lost time by phase (s) } & \multirow{2}{*}{$\mathrm{CP}_{\mathrm{L}}$} & \multirow{2}{*}{$L_{\mathrm{L}}$} \\
\hline & 1 & 2 & 5 & 6 & & \\
\hline $14: 42: 20$ & 3.2 & 5.0 & 3.2 & 5.0 & 56 & 8.2 \\
\hline $14: 44: 04$ & 3.2 & 5.0 & 3.2 & 5.0 & 56 & 8.2 \\
\hline $14: 45: 48$ & 0.0 & 5.0 & 3.2 & 5.0 & 12 & 5 \\
\hline $14: 47: 32$ & 0.0 & 5.0 & 3.2 & 5.0 & 56 & 5 \\
\hline $14: 49: 16$ & 3.2 & 5.0 & 3.2 & 5.0 & 12 & 8.2 \\
\hline $14: 51: 00$ & 3.2 & 5.0 & 3.2 & 5.0 & 56 & 8.2 \\
\hline $14: 52: 44$ & 3.2 & 5.0 & 3.2 & 5.0 & 56 & 8.2 \\
\hline $14: 54: 28$ & 3.2 & 5.0 & 3.2 & 5.0 & 12 & 8.2 \\
\hline
\end{tabular}

(b) Calculation of total lost time (right side of barrier).

\begin{tabular}{|c|c|c|c|c|c|c|}
\hline \multirow{2}{*}{ Time } & \multicolumn{4}{|c|}{ Lost time by phase (s) } & \multirow{2}{*}{$\mathrm{CP}_{\mathrm{R}}$} & \multirow{2}{*}{$L_{\mathrm{R}}$} \\
\hline & 3 & 4 & 7 & 8 & & \\
\hline $14: 42: 20$ & 3.2 & 3.9 & 3.2 & 3.9 & 34 & 7.1 \\
\hline 14:44:04 & 3.2 & 3.9 & 3.2 & 3.9 & 34 & 7.1 \\
\hline $14: 45: 48$ & 0.0 & 3.9 & 3.2 & 3.9 & 34 & 7.1 \\
\hline $14: 47: 32$ & 3.2 & 3.9 & 3.2 & 3.9 & 34 & 7.1 \\
\hline $14: 49: 16$ & 3.2 & 3.9 & 3.2 & 3.9 & 34 & 7.1 \\
\hline 14:51:00 & 3.2 & 3.9 & 3.2 & 3.9 & 34 & 7.1 \\
\hline 14:52:44 & 3.2 & 3.9 & 0.0 & 3.9 & 34 & 3.9 \\
\hline $14: 54: 28$ & 3.2 & 3.9 & 3.2 & 3.9 & 34 & 7.1 \\
\hline
\end{tabular}

(c) Calculation of $\mathrm{X}_{\mathrm{C}}$ and $\mathrm{C}_{\mathrm{W}}$.

\begin{tabular}{|c|r|r|r|r|r|r|r|}
\hline Time & $\max \left(v_{12}, v_{56}\right)$ & $\max \left(v_{34}, v_{78}\right)$ & $\sum_{i}\left(\frac{v}{s}\right)_{c i}$ & $L(\mathrm{~s})$ & $C(\mathrm{~s})$ & $\frac{C}{C-L}$ & $X_{C}$ \\
\hline $14: 42: 20$ & 571 & 484 & 0.56 & 15.3 & 104.0 & 1.17 & 0.65 \\
\hline $14: 44: 04$ & 502 & 381 & 0.46 & 15.3 & 104.0 & 1.17 & 0.54 \\
\hline $14: 45: 48$ & 467 & 484 & 0.50 & 12.1 & 104.0 & 1.13 & 0.57 \\
\hline $14: 47: 32$ & 381 & 519 & 0.47 & 12.1 & 104.0 & 1.13 & 0.54 \\
\hline $14: 49: 16$ & 467 & 554 & 0.54 & 15.3 & 104.0 & 1.17 & 0.63 \\
\hline $14: 51: 00$ & 640 & 450 & 0.57 & 15.3 & 104.0 & 1.17 & 0.67 \\
\hline $14: 52: 44$ & 588 & 381 & 0.51 & 12.1 & 104.0 & 1.13 & 0.58 \\
\hline $14: 54: 28$ & 589 & 658 & 0.66 & 15.3 & 104.0 & 1.17 & 0.77 \\
\hline
\end{tabular}




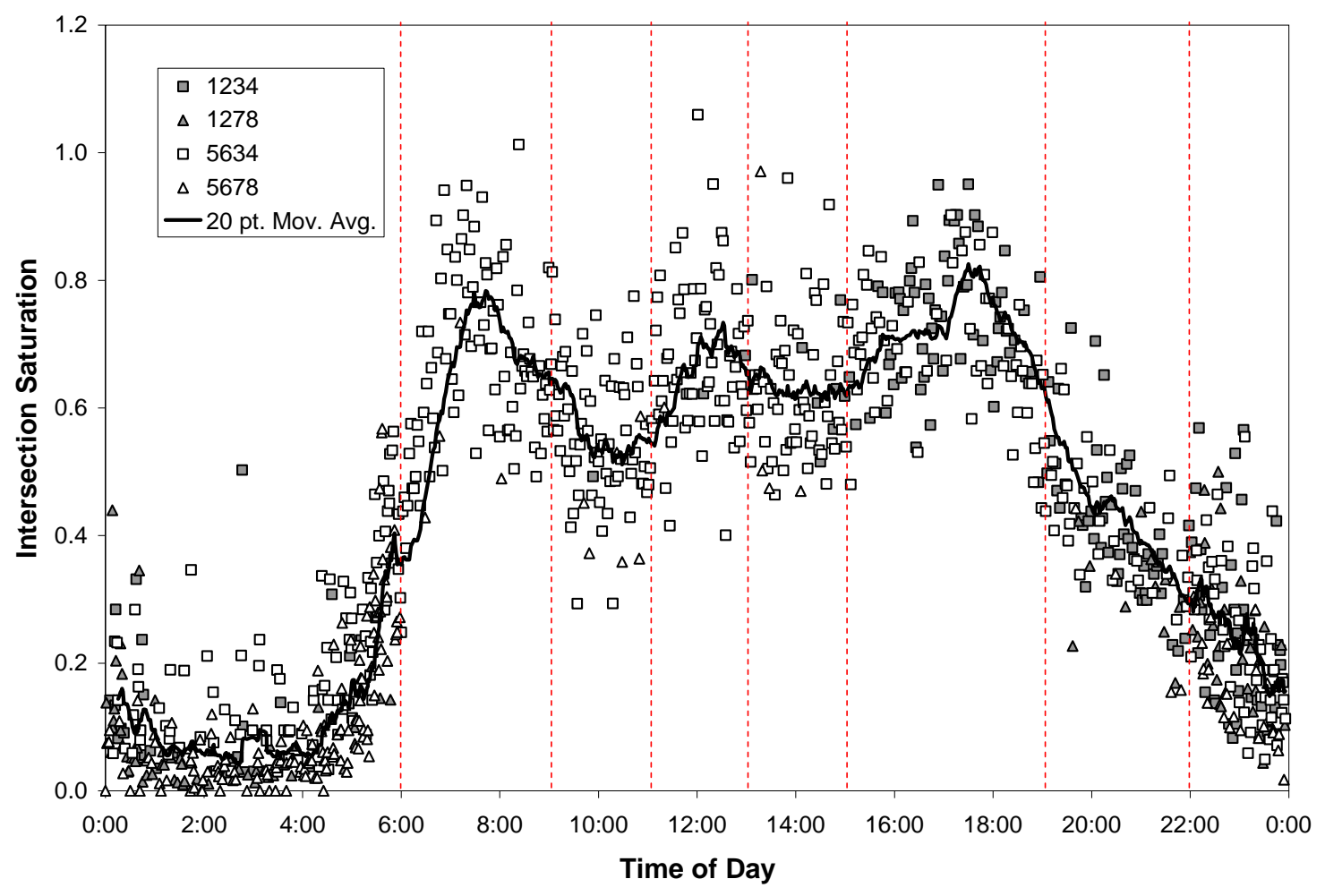

Figure 4.18 An example plot of critical v/c ratio at the Noblesville test intersection on Wednesday, March 14, 2007. 


\subsection{Progression Analysis}

Capacity analysis is useful when determining performance of an isolated intersection, but it does not reveal much about an intersection's performance in an arterial system.

The fewer the number of times a vehicle must stop as it travels along an arterial, the better its progression. If the vehicle passes through all of the intersections along the arterial during green, it would experiencing excellent progression. On the other hand, if the vehicle was forced to stop at every intersection, its progression would be poor. Signal coordination schemes are intended to provide the best possible progression, sometimes along multiple intersecting corridors. In this work, we present a few performance measures for evaluating progression at the standpoint of a signal in a coordinated system.

\subsubsection{Arrival Type}

Arrival type (AT) is a qualitative description of progression of vehicles through each phase of an intersection that is based in part by the proportion of vehicles that arrive during the green indication. There are six categories of AT based on a measure called the platoon ratio. The platoon ratio of phase $i$ is defined by (5)

$R_{p}=\frac{C}{g_{i}} \times P O G$

Equation 4.17

where $\mathrm{C}$ is the cycle length, $g_{i}$ is the green time for phase $i$, and POG is the proportion of vehicles arriving on green. This quantity is calculated from

$P O G=\frac{N_{g}}{N_{g}+N_{r}}$,

where $N_{g}$ is the total number of vehicles that arrive during the green indication and $N_{r}$ is the number that arrive on the red indication.

Table 4.10 shows the categories of AT defined by the HCM (5). Different integer values of AT correspond to ranges of the platoon ratio. An AT of 6 corresponds to ideal progression, with all vehicles passing through the signal on green, while an AT of 1 represents the worst case scenario, with all vehicles arriving on red. In this study, we 
have used an interpolation to assign values of AT based on the platoon ratio. The reason for doing this was to be able to calculate more accurate averages between cycles, and develop more descriptive curves. The equations of interpolation are shown in Table 4.10, and are illustrated in Figure 4.19, which shows both the interpolated and integer definitions of AT as functions of the platoon ratio. Note that the integer values act as the "floor" of the interpolation. AT values are held between the boundary values of 1 and 6 .

Table 4.11 contains example calculations of AT for phases 2 and 6 at Noblesville on Wednesday, March 14, 2007. The advance detectors were used to detect arrivals for these phases. A phase 2 cycle whose green phase began at 10:53:30 saw 21 arrivals total, with 8 on red and 13 on green. That gives a POG value equal to $13 / 21=0.619$. Because $C=96.1 \mathrm{~s}$ and $g_{2}=49.3 \mathrm{~s}, C / g_{2}=1.95$. When multiplied by POG this returns $R_{p}=1.21$. Applying the definitions of Table 4.10, this corresponds to an integer AT of 4, or an interpolated AT of 4.16. Although fewer than half of the total arrivals were counted on green, the high $C / g$ factor led to a high $\mathrm{R}_{\mathrm{p}}$ value, which caused AT to also be high. This is particularly illustrated by the phase 6 cycle at 10:58:24, where POG $=0.556$, but because $C / g=3.86$, AT was calculated as 6 .

Figure 4.20 shows plots of AT for phases 2 and 6 over 24 hours at Noblesville on Wednesday, March 14, 2007. As can be seen in these graphs, there is strong variation from cycle to cycle throughout the entire day. The 20-point moving average reveals some trends that roughly correspond to changes in the TOD plan. In general, better AT is reported for phase 2 , which makes sense because this is a coordinated movement. The packing of vehicles into platoons encourages higher POG values. Phase 6 is not coordinated, and arrivals are random. We obtain middle-range AT values. Slightly better than random AT values are calculated at different times of the day. This may be attributed to an unintended interaction with the upstream signal. We expect AT equal to 3 , but slightly more vehicles tend to be captured during green than in red. For perfectly uniform arrivals, we would expect increases in POG to be completely mitigated by proportional changes in the $\mathrm{C} / \mathrm{g}$ ratio, but this is not shown by our data.

AT cannot be calculated using stop bar detectors; advance detectors must be used, and they must be sufficiently far from the stop bar to prevent queueing over them. The effect of queueing over the advance detectors is to make it appear that more arrivals take place on green; when the queue disperses, the indication of the phase is green. Detector counts caused by vehicles in the dispersing queue are all considered to be green, whereas in reality those vehicles arrived on red. This causes AT to be artificially inflated.

Figure 4.21 shows an plot of AT calculated for the southbound "advance" detectors at West Lafayette on August 13, 2007. Recall from Chapter 3 that the 
"advance" detectors for phase 6 are actually the furthest in the set of four detectors at the stop bar (see Figure 3.11). Notice how many points rest on the line corresponding to AT $=6$. The only region where AT is likely to be accurate is during the early morning hours, when volumes are not high enough to cause queues to form over the detectors.

Table 4.10 Definition of arrival type (5) based on platoon ratio, including equations used for interpolated values.

\begin{tabular}{|c|c|c|c|c|}
\hline $\begin{array}{c}\text { Arrival } \\
\text { Type }\end{array}$ & $\begin{array}{c}\text { Range of Platoon } \\
\text { Ratio }\end{array}$ & $\begin{array}{c}\text { Default } \\
\text { Value } \\
\text { of } R_{p}\end{array}$ & $\begin{array}{c}\text { Progression } \\
\text { Quality }\end{array}$ & $\begin{array}{c}\text { Interpolated Arrival } \\
\text { Type Equation }\end{array}$ \\
\hline 1 & $R_{p} \leq 0.50$ & 0.333 & Very poor & $2 R_{p}+1$ \\
\hline 2 & $0.50<R_{p} \leq 0.85$ & 0.667 & Unfavorable & $\frac{R_{p}}{0.35}+\left(3-\frac{0.85}{0.35}\right)$ \\
\hline 3 & $0.85<R_{p} \leq 1.15$ & 1.000 & Random arrivals & $\frac{R_{p}}{0.3}+\left(4-\frac{1.15}{0.3}\right)$ \\
\hline 4 & $1.15<R_{p} \leq 1.50$ & 1.333 & Favorable & $\frac{R_{p}}{0.35}+\left(5-\frac{1.5}{0.35}\right)$ \\
\hline 5 & $1.50<R_{p} \leq 2.00$ & 1.667 & $\begin{array}{c}\text { Highly } \\
\text { favorable }\end{array}$ & $2 R_{p}+2$ \\
\hline 6 & $R_{p}>2.00$ & 2.000 & Exceptional & 6 \\
\hline
\end{tabular}




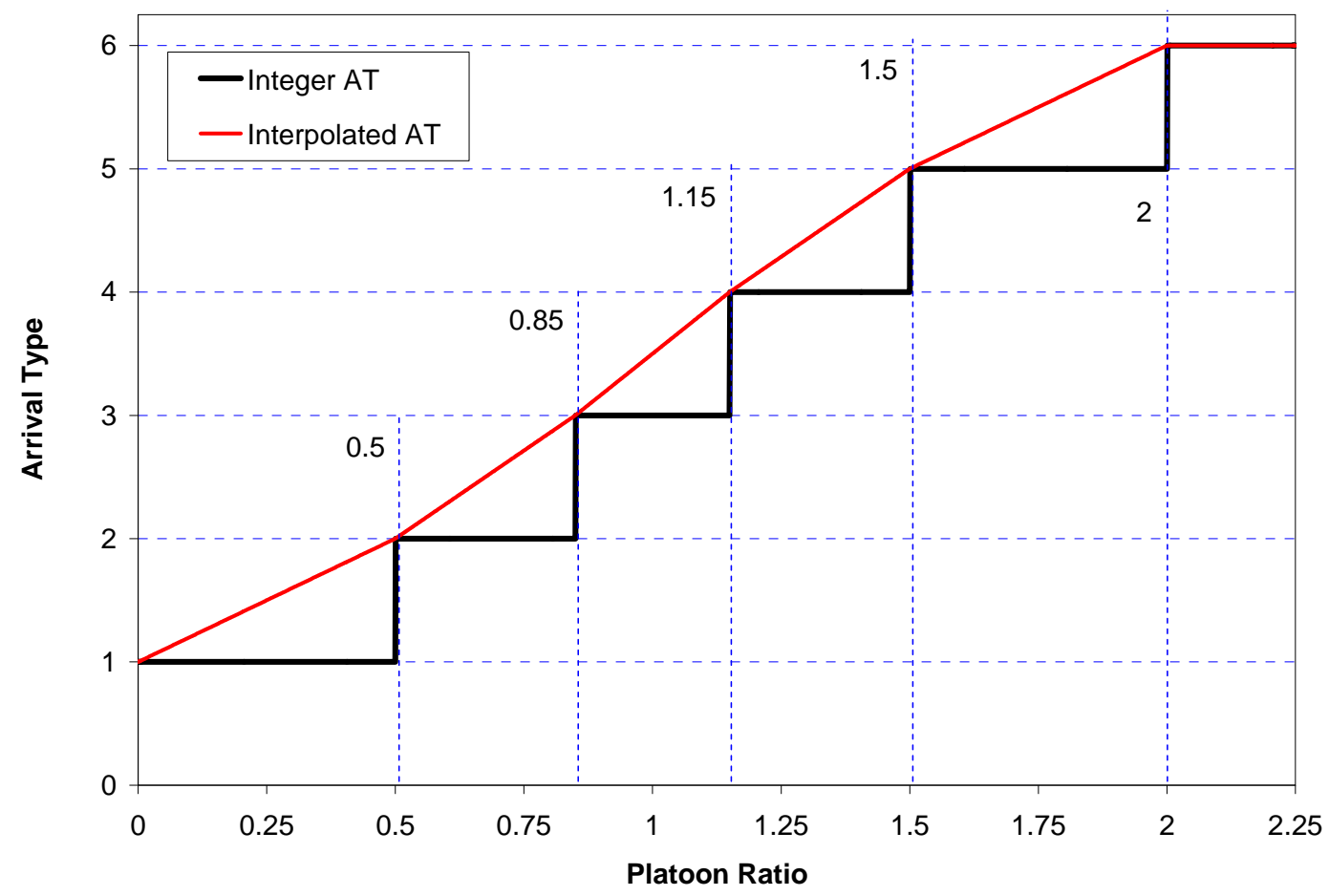

Figure 4.19 Interpolated versus integer arrival type plotted against platoon ratio. 
Table 4.11 Calculation of Arrival Type at Noblesville.

(a) Phase 2, Wednesday, March 14, 2007.

\begin{tabular}{|c|r|r|c|c|c|c|c|c|r|r|}
\hline Time & \multicolumn{1}{|c|}{$N_{r}$} & \multicolumn{1}{|c|}{$N_{g}$} & \multicolumn{1}{c}{$N$} & \multicolumn{1}{c}{ POG } & \multicolumn{1}{c|}{$C$} & $g_{2}$ & \multicolumn{1}{c|}{$C / g_{2}$} & $R_{p}$ & $\begin{array}{c}\text { Integer } \\
\text { AT }\end{array}$ & $\begin{array}{c}\text { Interpolated } \\
\text { AT }\end{array}$ \\
\hline $10: 53: 30$ & 8 & 13 & 21 & 0.619 & 96.1 & 49.3 & 1.95 & 1.21 & 4 & 4.16 \\
\hline $10: 55: 13$ & 2 & 11 & 13 & 0.846 & 96.0 & 41.8 & 2.30 & 1.95 & 5 & 5.89 \\
\hline $10: 56: 38$ & 1 & 10 & 11 & 0.909 & 96.0 & 53.3 & 1.80 & 1.64 & 5 & 5.27 \\
\hline $10: 58: 32$ & 10 & 9 & 19 & 0.474 & 96.1 & 35.2 & 2.73 & 1.29 & 3 & 4.40 \\
\hline $10: 59: 46$ & 3 & 13 & 16 & 0.813 & 121.5 & 82.1 & 1.48 & 1.20 & 3 & 4.15 \\
\hline $11: 01: 57$ & 2 & 8 & 10 & 0.800 & 118.7 & 69.8 & 1.70 & 1.36 & 4 & 4.60 \\
\hline $11: 04: 10$ & 2 & 5 & 7 & 0.714 & 102.0 & 38.8 & 2.63 & 1.88 & 5 & 5.76 \\
\hline $11: 05: 51$ & 4 & 8 & 12 & 0.667 & 102.0 & 40.1 & 2.55 & 1.70 & 5 & 5.40 \\
\hline $11: 07: 06$ & 1 & 11 & 12 & 0.917 & 102.1 & 66.7 & 1.53 & 1.40 & 4 & 4.72 \\
\hline $11: 09: 05$ & 4 & 14 & 18 & 0.778 & 102.0 & 50.3 & 2.03 & 1.58 & 5 & 5.15 \\
\hline
\end{tabular}

(b) Phase 6, Wednesday, March 14, 2007.

\begin{tabular}{|c|r|r|r|c|c|c|c|c|r|r|}
\hline Time & $N_{r}$ & $N_{g}$ & \multicolumn{1}{c|}{$N$} & \multicolumn{1}{c}{$P O G$} & $C$ & $g_{2}$ & $C / g_{2}$ & $R_{p}$ & $\begin{array}{c}\text { Integer } \\
\text { AT }\end{array}$ & $\begin{array}{c}\text { Interpolated } \\
\text { AT }\end{array}$ \\
\hline 10:53:21 & 5 & 6 & 11 & 0.545 & 96.1 & 39.1 & 2.45 & 1.34 & 4 & 4.54 \\
\hline $10: 55: 00$ & 3 & 6 & 9 & 0.667 & 96.0 & 36.6 & 2.62 & 1.75 & 5 & 5.49 \\
\hline $10: 56: 30$ & 7 & 14 & 21 & 0.667 & 96.0 & 43.1 & 2.23 & 1.49 & 4 & 4.96 \\
\hline $10: 58: 24$ & 4 & 5 & 9 & 0.556 & 96.1 & 24.9 & 3.86 & 2.14 & 6 & 6.00 \\
\hline $10: 59: 49$ & 7 & 11 & 18 & 0.611 & 121.5 & 51.6 & 2.35 & 1.44 & 4 & 4.82 \\
\hline $11: 01: 49$ & 3 & 5 & 8 & 0.625 & 118.7 & 53.7 & 2.21 & 1.38 & 4 & 4.66 \\
\hline $11: 04: 00$ & 9 & 5 & 14 & 0.357 & 102.0 & 35.3 & 2.89 & 1.03 & 3 & 3.60 \\
\hline $11: 05: 42$ & 6 & 3 & 9 & 0.333 & 102.0 & 26.6 & 3.83 & 1.28 & 4 & 4.36 \\
\hline $11: 07: 09$ & 4 & 8 & 12 & 0.667 & 102.1 & 41.3 & 2.47 & 1.65 & 5 & 5.30 \\
\hline $11: 08: 53$ & 12 & 3 & 15 & 0.200 & 102.0 & 39.8 & 2.56 & 0.51 & 2 & 2.03 \\
\hline
\end{tabular}




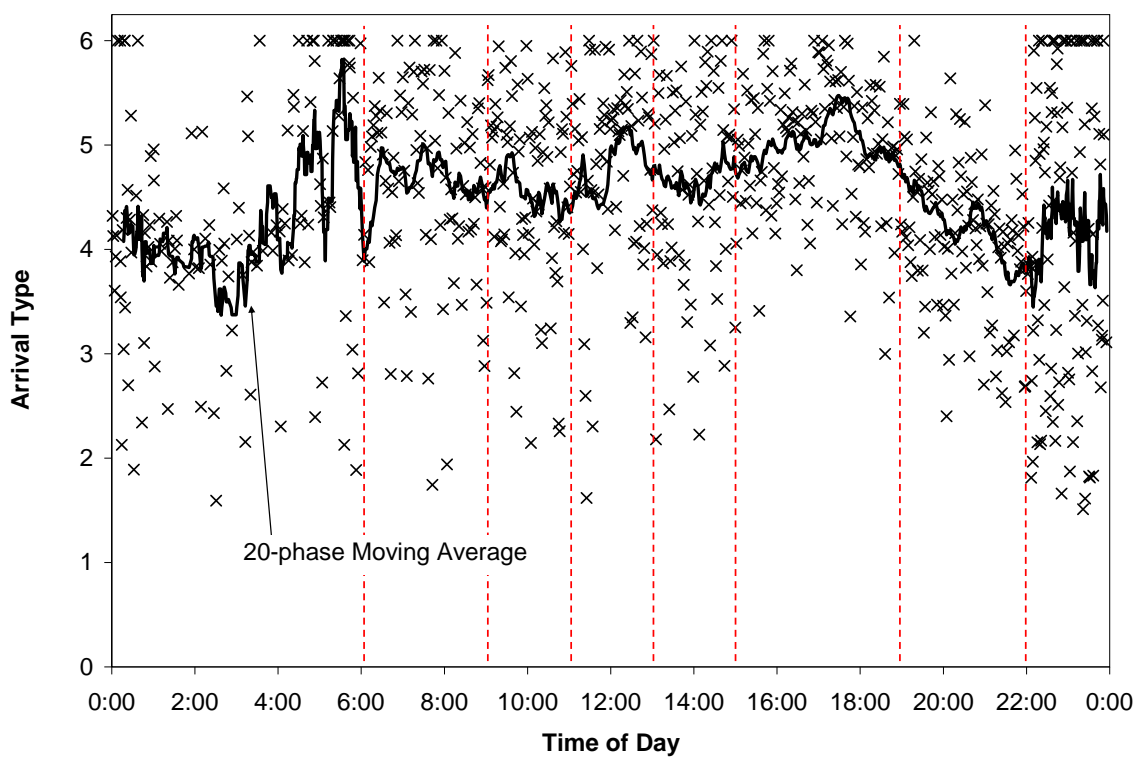

(a) Phase 2, Wednesday, March 14, 2007.

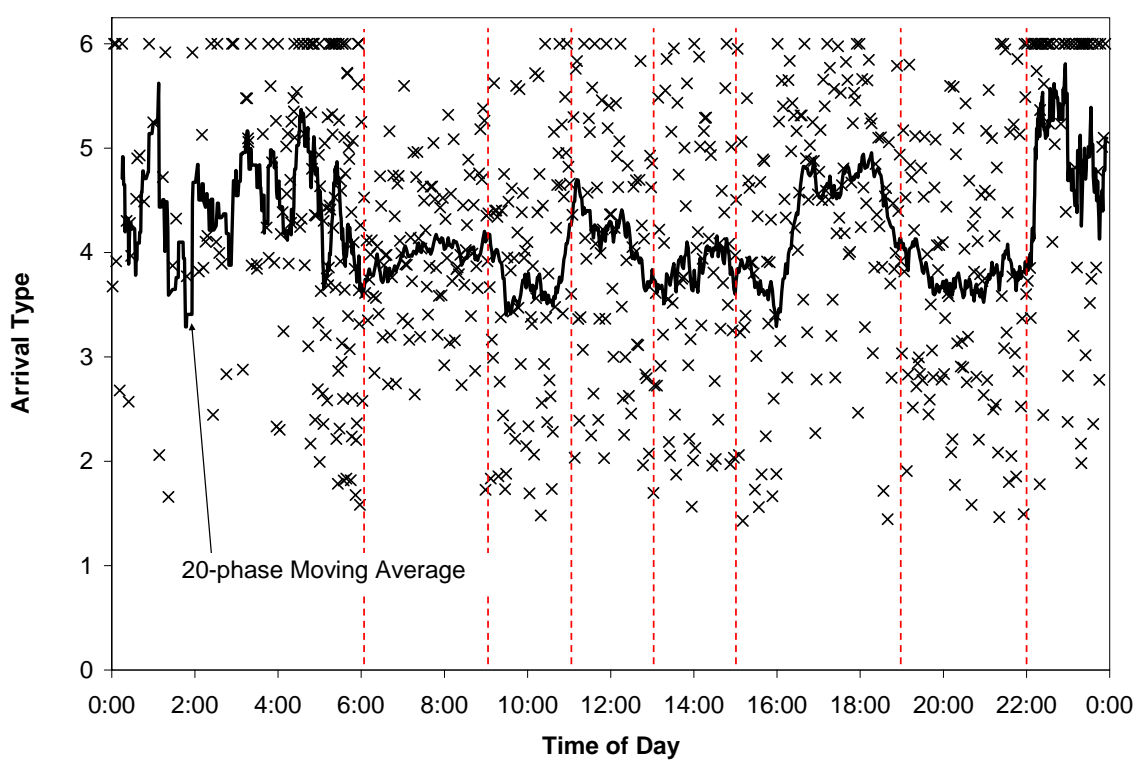

(b) Phase 6, Wednesday, March 14, 2007.

Figure 4.20 Graphs of Arrival Type at Noblesville. 


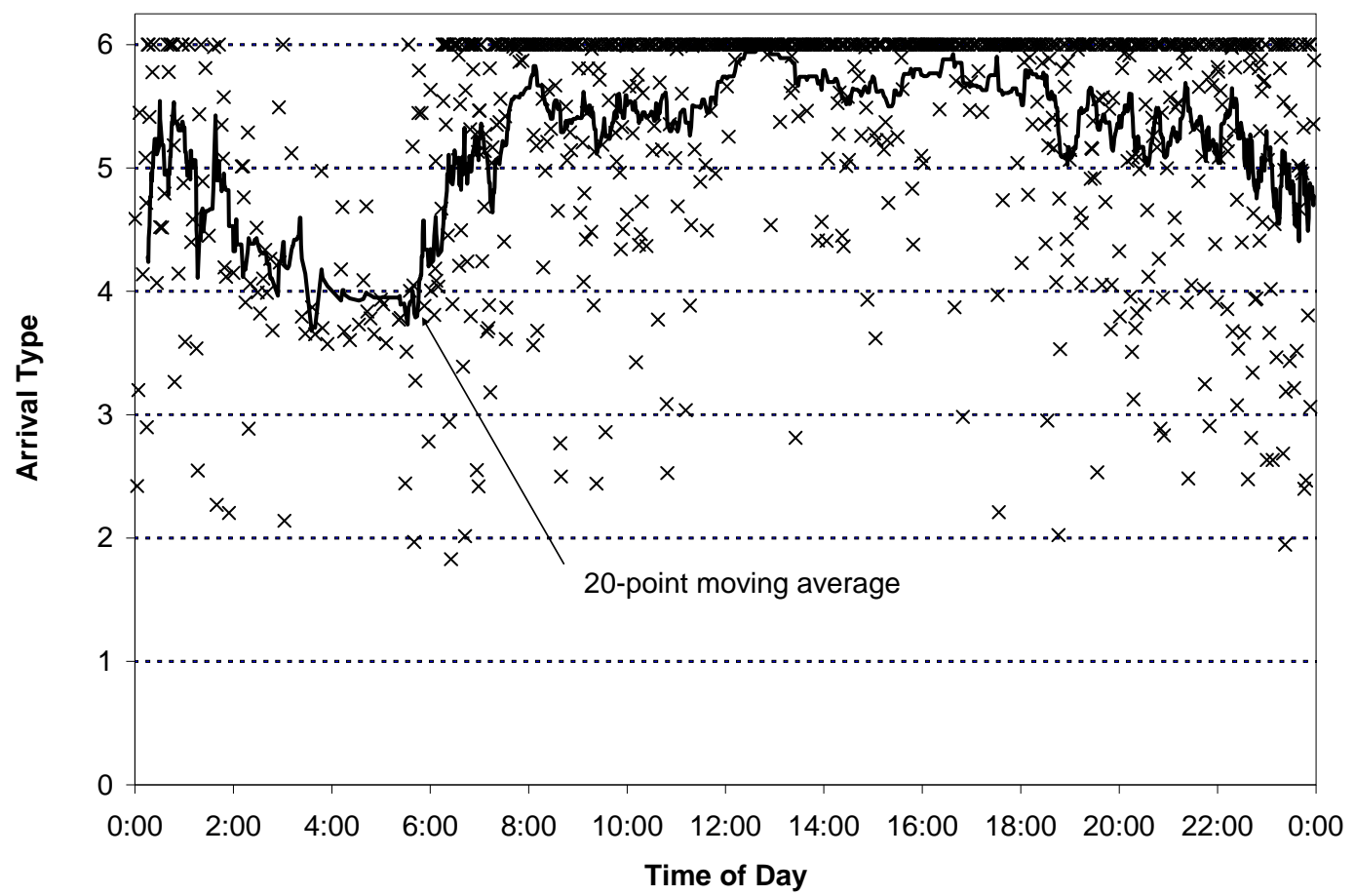

Figure 4.21 Arrival type calculated for phase 6 at West Lafayette on August 13, 2007. 


\subsubsection{Platoon Profile}

In addition to arrival type, which attempts to quantify the characteristics of progression, we may also construct platoon profiles in order to visualize what is taking place during a certain phase at an intersection. A platoon profile has two components: a distribution of vehicle arrivals during the cycle time; and a green profile that is a cumulative distribution aggregated from the two individual cumulative distributions for the beginning of green and end of green times. The platoon profiles are very similar to the flow profile plots generated by the TRANSYT simulation software package (15). The difference is that the beginning and end of the green indication are probability distributions, and departures are not indicated in the profiles shown in this work.

Table 4.12(a) shows calculations of time in cycle for phase 2 arrivals at Noblesville on Wednesday, March 14, 2007. These calculations were made to build a platoon profile for the 6:00-9:00 time period. The "time in cycle" for each arrival is the amount of time after the beginning of the cycle in which the arrival took place. For example, an arrival occurring at 6:27:57 has a ms time of 545500347; the cycle began at 545454158. Subtracting the latter number from the former, we obtain a time in cycle of $46.2 \mathrm{~s}$. This is thrown into the bin labeled as $46 \mathrm{~s}$. The cycle beginning time is actually the previous end of green for the phase for which the profile is being constructed. We cannot do calculations to the nearest ms with respect to the actual cycle beginning time because of clock drift. Of course, for phase 2, which is the leading phase, the two are equivalent.

Table 4.12(b) gives a sample of calculation results for bins for phase 2 arrivals. One-second bins are used. A total of 1,084 arrivals occurred for phase 2 during the 6:009:00 time period; the proportion of the total arrivals is calculated for each bin using the number of arrivals in each bin. For example, for the $4 \mathrm{~s}$ bin, 7 out of 1,084 arrivals are logged, which gives a proportion of $0.6 \%$.

Figure 4.22 shows a plot of time in cycle versus time of day. As time moves forward, the time in cycle increases. The red dashed lines represent the cycle boundaries, where time in cycle resets to zero. This plot verifies that our calculations are reasonable. Figure 4.23 shows vehicle arrival distributions for phases 2 and 6 at Noblesville on March 14, 2007, using $1 \mathrm{~s}$ bins. The packaging of phase 2 arrivals into platoons can be seen; there is a stronger probability for arrivals approximately $60-90 \mathrm{~s}$ after the beginning

of the cycle than at other times. Phase 6, on the other hand, exhibits an almost uniform distribution, which shows the random nature of phase 6 arrivals. 
Table 4.13(a) shows calculations of beginning of green and end of green for phase 2 cycles at Noblesville on Wednesday, March 14, 2007. Important timestamps are shown in the first six columns. The "last end of green" time represents the previous time when the green indication ended. This serves as the beginning of the cycle. The beginning of green and end of green times denote when the green indication was shown for the phase of interest. The times calculated in the last two columns are calculated from the ms times as follows: the "begin green" time was found by subtracting the beginning of green time from the last end of green; and the "end green" time was found by subtracting the end of green time from the beginning of green time. Bins for the distributions were based on $1 \mathrm{~s}$ intervals.

Table 4.13(b) shows the binning for green profiles. Each count of items represents the number of cycles in the bin; the proportions are calculated based on the total number of cycles for the 6:00-9:00 time period. The beginning of green and end of green cumulative distributions are calculated for each bin by summing all of the preceding bins. Bins 66-70 show the increase in the cumulative beginning of green distribution to $100 \%$ of cycles; bins 113-117 show the cumulative end of green distribution growing from 0 to $100 \%$. The difference of the two grows to $100 \%$ in bins $66-70$ and falls to $0 \%$ in bins 113-117. That number represents the overall probability that the phase is showing the green indication at that time in the cycle.

The difference between the cumulative beginning of green and the cumulative end of green is used to plot the shape of the green profile, as shown in Figure 4.24. Green profiles are shown for phases 2 and 6 . The leading edge of the profile represents the cumulative proportion of cycles beginning given the time in cycle plotted along the $\mathrm{x}$ axis. The falling edge represents the cumulative proportion of cycles ending at those times. The falling edge appears to be angled rather than vertical because that is the manner in which the spreadsheet program plotted the points.

The "bump" on the end of the phase 6 profile represents a cycle where phase 5 was not called, causing phase 6 to end later than it normally would have. Recall that the beginning of cycle is determined by the last end of green for the phase of interest. For phase 6, whenever phase 5 is not called, this pushes that end of green time about $15 \mathrm{~s}$ later in time, causing the end of green for that cycle to be measured as occurring $15 \mathrm{~s}$ later, and the beginning of green for the next cycle to be measured as occurring $15 \mathrm{~s}$ later. Fortunately, phase 5 is almost always called, so this did not cause a problem for phase 6 . It would be preferable to eliminate this problem by using the actual cycle endpoint, but the clock drift prevents this for phase 6 . If all data were collected on the same clock, this problem would be easily resolved. 
Figure 4.25 contains the superimposed graphs of Figure 4.22 and Figure 4.24. These are the platoon profile graphs for phases 2 and 6 at Noblesville for the 6:00-9:00 time period on Wednesday, March 14, 2007. The relative scale of the cycle and arrival profiles does not have any meaning; rather, these graphs are simply intended to show the distribution of arrivals related to the probability that the cycle is green. Most of the arrivals for phase 2 occur during a region of the time in cycle when there is a high probability that the indication is green. Note the dropoff in arrivals toward the end of the cycle. This indicates that there is an underutilized portion of the green indication that may be more efficiently used by other phases. Phase 6's arrivals are almost uniformly distributed throughout the cycle; the number of arrivals captured by the region inside the green profile is entirely a function of the length of green.

These graphs give some insight about the meaning of AT values calculated in the previous section. Before, we saw that AT values for phase 2 were generally higher than those of phase 6; the reason why is that the signals to the south that regulate northbound traffic are coordinated, whereas the nearest signal to the north is sufficiently far away that arrivals are almost totally random in the southbound direction. The high AT values for phase 2 are a result of more arrivals occurring during green. Phase 6 has middle range AT values, corresponding to the random arrivals occurring for this approach. AT is calculated slightly higher than 3 because slightly more arrivals occur during green than red. There may be a weak interaction with the $191^{\text {st }} \mathrm{St}$ signal that causes this.

A standard interpretation of platoon profiles is shown by Figure 4.26. For good progression, we would expect the arrival distribution and the green profile to be more or less coincident, as in Figure 4.26(a). This corresponds to an AT of 5 or 6. For random arrivals, we expect the situation shown in Figure 4.26(b). Medium-range AT values correspond to these kinds of profiles. Arrivals are uniformly distributed. The proportion of vehicles that arrive on green depends on the length of the green time. AT cannot become greater than 3, because any gains in $P$ in Equation 4.17 would be canceled out by proportional decreases in $C / g$. In the limit where $g \rightarrow C$ and $P \rightarrow 1$, the Platoon Ratio becomes 1.0, corresponding to $\mathrm{AT}=3$, which is representative of perfectly random arrivals. Figure 4.26 (c) shows a situation indicating poor progression, corresponding to an AT of 1 or 2 . In this case, the majority of arrivals occur when the indication is red. We would expect the green profile and the arrival distributions to peak in different regions of the cycle in this case. 
Table 4.12 Calculation of vehicle arrival distribution at Noblesville on Wednesday, March 14, 2007, 6:00 - 9:00.

(a) Sample calculations of time in cycle for Phase 2 arrivals.

\begin{tabular}{|c|c|r|r|r|}
\hline \multicolumn{2}{|c|}{ Arrival Time } & \multicolumn{1}{c|}{$\begin{array}{c}\text { Phase 2 Begin } \\
\text { Time }(\mathrm{ms})\end{array}$} & $\begin{array}{c}\text { Time in Cycle } \\
(\mathrm{s})\end{array}$ & \multicolumn{1}{c|}{ Bin } \\
\cline { 1 - 2 } $\mathrm{h}: \mathrm{m}: \mathrm{s}$ & \multicolumn{1}{c|}{$\mathrm{ms}$} & 545338084 & 70.9 & 70 \\
\hline $06: 26: 25$ & 545408970 & 545338084 & 90.7 & 90 \\
\hline $06: 26: 45$ & 545428760 & 545338084 & 111.5 & 111 \\
\hline $06: 27: 06$ & 545449552 & 545 & 46 \\
\hline $06: 27: 57$ & 545500347 & 545454158 & 46.2 & 48 \\
\hline $06: 27: 59$ & 545503150 & 545454158 & 49.0 & 48 \\
\hline $06: 27: 59$ & 545503150 & 545454158 & 49.0 & 63 \\
\hline $06: 28: 13$ & 545517200 & 545454158 & 63.0 & 64 \\
\hline $06: 28: 15$ & 545518602 & 545454158 & 64.4 & 49 \\
\hline $06: 29: 56$ & 545619690 & 545570097 & 49.6 & 49 \\
\hline $06: 29: 56$ & 545619791 & 545570097 & 49.7 & \\
\hline
\end{tabular}

(b) Sample of binning calculations for Phase 2 arrivals.

\begin{tabular}{|r|r|r|r|r|}
\hline $\begin{array}{c}\text { Bin time } \\
\text { (s) }\end{array}$ & \multicolumn{1}{c|}{$\begin{array}{c}\text { Arrivals } \\
\text { in Bin }\end{array}$} & \multicolumn{1}{c|}{$\begin{array}{c}\text { Total } \\
\text { Arrivals }\end{array}$} & $\begin{array}{c}\text { Proportion } \\
\text { (decimal) }\end{array}$ & $\begin{array}{c}\text { Proportion } \\
\text { (percent) }\end{array}$ \\
\hline 0 & 0 & 1084 & 0.00000 & $0.0 \%$ \\
\hline 1 & 6 & 1084 & 0.00554 & $0.6 \%$ \\
\hline 2 & 3 & 1084 & 0.00277 & $0.3 \%$ \\
\hline 3 & 6 & 1084 & 0.00554 & $0.6 \%$ \\
\hline 4 & 7 & 1084 & 0.00646 & $0.6 \%$ \\
\hline 5 & 7 & 1084 & 0.00646 & $0.6 \%$ \\
\hline 6 & 4 & 1084 & 0.00369 & $0.4 \%$ \\
\hline 7 & 0 & 1084 & 0.00000 & $0.0 \%$ \\
\hline 8 & 1 & 1084 & 0.00092 & $0.1 \%$ \\
\hline 9 & 6 & 1084 & 0.00554 & $0.6 \%$ \\
\hline 10 & 8 & 1084 & 0.00738 & $0.7 \%$ \\
\hline
\end{tabular}




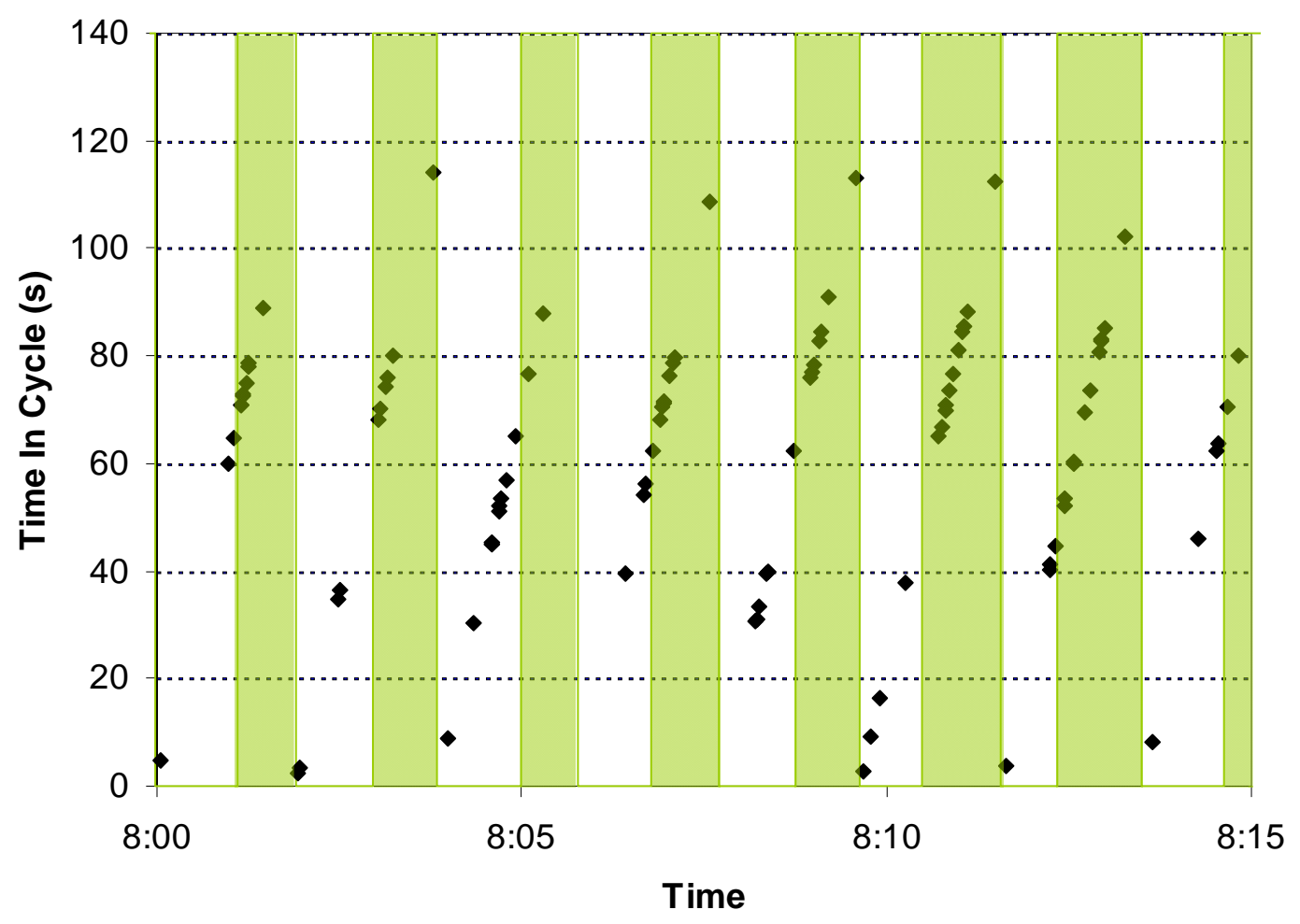

Figure 4.22 Graph of time in cycle for phase 2 arrivals over time of day at Noblesville on Wednesday, March 14, 2007, with green windows represented by the shaded regions. 


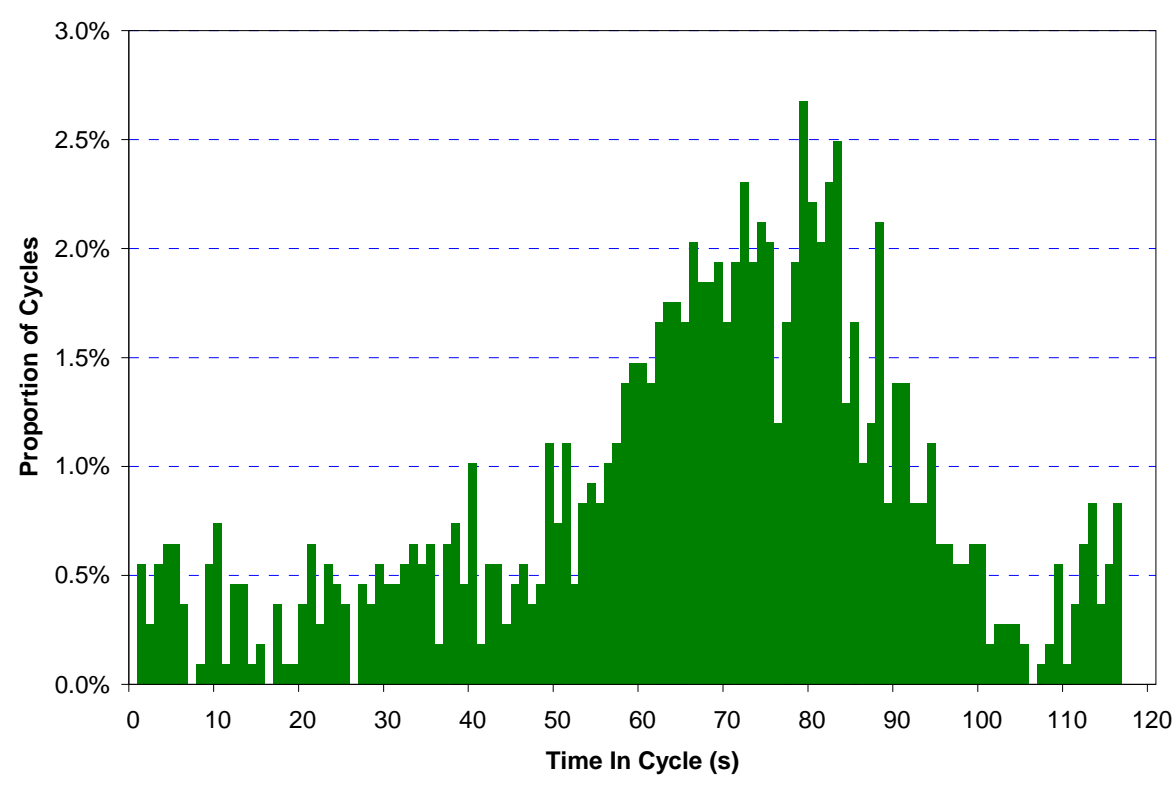

(a) Phase 2, Wednesday, March 14, 2007, 6:00 - 9:00

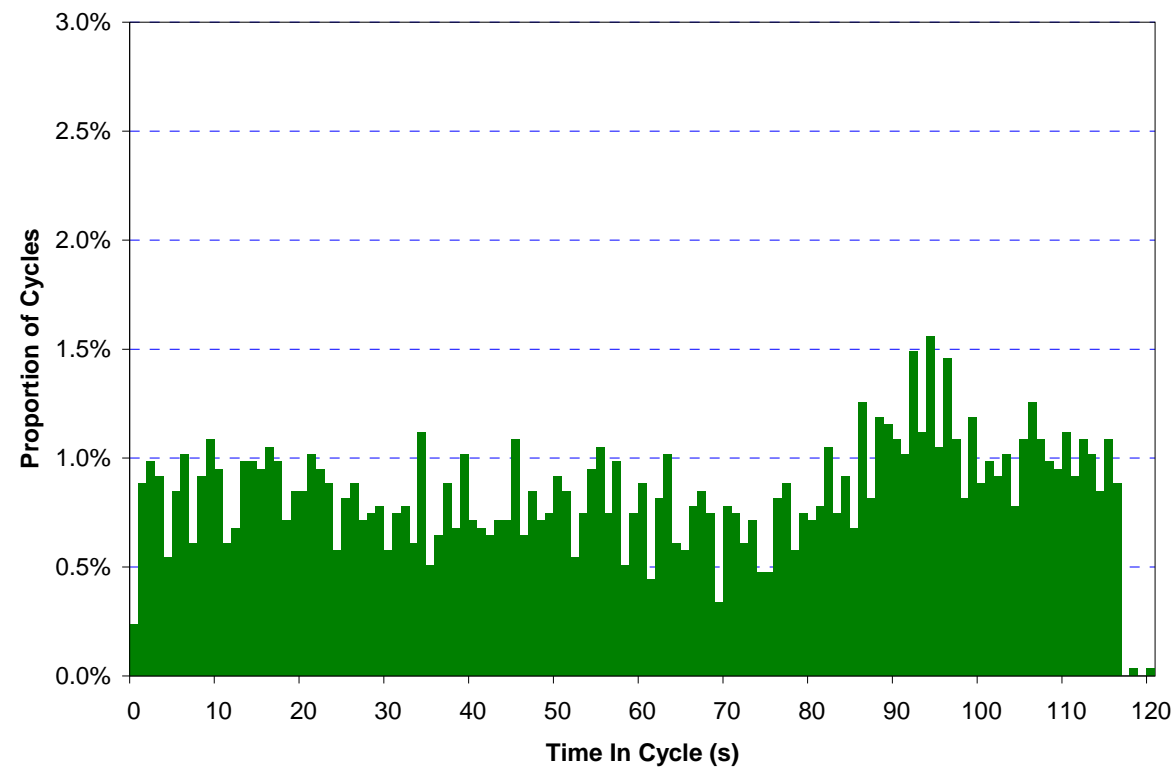

(b) Phase 6, Wednesday, March 14, 2007, 6:00 - 9:00

Figure 4.23 Graph of vehicle arrival distributions at Noblesville. 
Table 4.13 Calculations of green profile at Noblesville on Wednesday, March 14, 2007, 6:00 - 9:00.

(a) Sample calculations of time in cycle for phase 2 events.

\begin{tabular}{|c|c|c|c|c|c|c|c|}
\hline \multicolumn{3}{|c|}{ h:m:s time } & \multicolumn{3}{|c|}{ ms time } & \multirow[b]{2}{*}{$\begin{array}{l}\text { begin } \\
\text { green }\end{array}$} & \multirow[b]{2}{*}{$\begin{array}{l}\text { end } \\
\text { green }\end{array}$} \\
\hline $\begin{array}{c}\text { last end of } \\
\text { green }\end{array}$ & $\begin{array}{l}\text { begin } \\
\text { green }\end{array}$ & $\begin{array}{l}\text { end of } \\
\text { green }\end{array}$ & $\begin{array}{c}\text { last end of } \\
\text { green }\end{array}$ & begin green & end of green & & \\
\hline 06:17:30 & $06: 18: 21$ & $06: 19: 26$ & 544873892 & 544924285 & 544989931 & 50.4 & 116.0 \\
\hline $06: 19: 26$ & $06: 20: 12$ & $06: 21: 22$ & 544989931 & 545035219 & 545106105 & 45.3 & 116.2 \\
\hline $06: 21: 22$ & $06: 22: 06$ & $06: 23: 18$ & 545106105 & 545149357 & 545222044 & 43.3 & 115.9 \\
\hline $06: 23: 18$ & $06: 23: 58$ & $06: 25: 14$ & 545222044 & 545261592 & 545338084 & 39.5 & 116.0 \\
\hline $06: 25: 14$ & $06: 26: 13$ & $06: 27: 10$ & 545338084 & 545396421 & 545454158 & 58.3 & 116.1 \\
\hline $06: 27: 10$ & $06: 27: 55$ & 06:29:06 & 545454158 & 545498444 & 545570097 & 44.3 & 115.9 \\
\hline 06:29:06 & $06: 29: 46$ & 06:31:02 & 545570097 & 545609779 & 545686171 & 39.7 & 116.1 \\
\hline 06:31:02 & 06:32:04 & $06: 32: 58$ & 545686171 & 545747511 & 545802210 & 61.3 & 116.0 \\
\hline $06: 32: 58$ & $06: 33: 46$ & $06: 34: 54$ & 545802210 & 545849801 & 545918284 & 47.6 & 116.1 \\
\hline $06: 34: 54$ & $06: 35: 33$ & $06: 36: 50$ & 545918284 & 545956630 & 546034324 & 38.3 & 116.0 \\
\hline
\end{tabular}

(b) Sample of binning calculations for Phase 2 green profile.

\begin{tabular}{|r|r|r|r|r|r|r|r|}
\cline { 2 - 7 } \multicolumn{1}{c|}{} & \multicolumn{3}{c|}{ Beginning of Green } & \multicolumn{3}{c|}{ End of Green } & \multirow{2}{*}{ Difference } \\
\hline Bin & \# in Bin & Proportion & Cumulative & \# in Bin & Proportion & Cumulative & \\
\hline 66 & 2 & $2.1 \%$ & $66.0 \%$ & 0 & $0.0 \%$ & $0.0 \%$ & $66.0 \%$ \\
\hline 67 & 8 & $8.5 \%$ & $74.5 \%$ & 0 & $0.0 \%$ & $0.0 \%$ & $74.5 \%$ \\
\hline 68 & 5 & $5.3 \%$ & $79.8 \%$ & 0 & $0.0 \%$ & $0.0 \%$ & $79.8 \%$ \\
\hline 69 & 3 & $3.2 \%$ & $83.0 \%$ & 0 & $0.0 \%$ & $0.0 \%$ & $83.0 \%$ \\
\hline 70 & 16 & $17.0 \%$ & $100.0 \%$ & 0 & $0.0 \%$ & $0.0 \%$ & $100.0 \%$ \\
\hline$\ldots$ & & & & & & & \\
\hline 113 & 0 & $0.0 \%$ & $100.0 \%$ & 0 & $0.0 \%$ & $3.19 \%$ & $96.8 \%$ \\
\hline 114 & 0 & $0.0 \%$ & $100.0 \%$ & 0 & $0.0 \%$ & $3.19 \%$ & $96.8 \%$ \\
\hline 115 & 0 & $0.0 \%$ & $100.0 \%$ & 0 & $0.0 \%$ & $3.19 \%$ & $96.8 \%$ \\
\hline 116 & 0 & $0.0 \%$ & $100.0 \%$ & 38 & $40.4 \%$ & $43.62 \%$ & $56.4 \%$ \\
\hline 117 & 0 & $0.0 \%$ & $100.0 \%$ & 53 & $56.4 \%$ & $100.0 \%$ & $0.0 \%$ \\
\hline
\end{tabular}




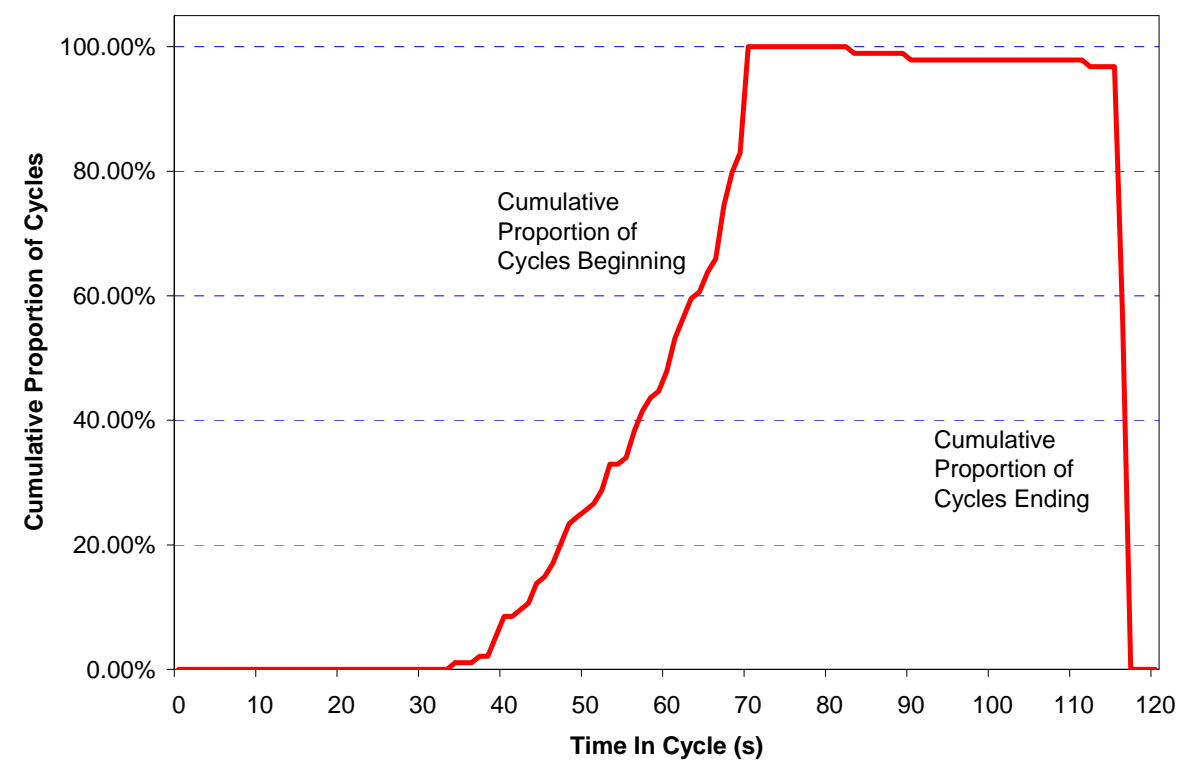

(a) Phase 2, Wednesday, March 14, 2007, 6:00 - 9:00

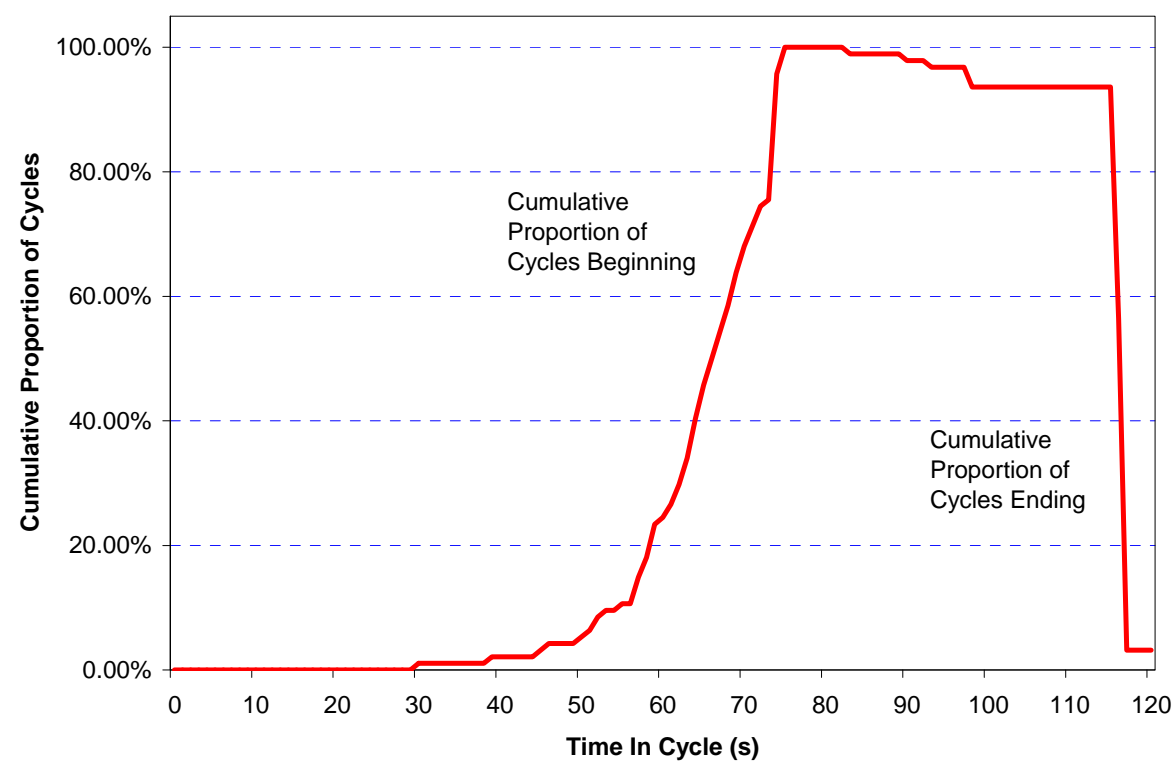

(b) Phase 6, Wednesday, March 14, 2007, 6:00 - 9:00

Figure 4.24 Graphs of cycle profiles at Noblesville on Wednesday, March 14, 2007. 


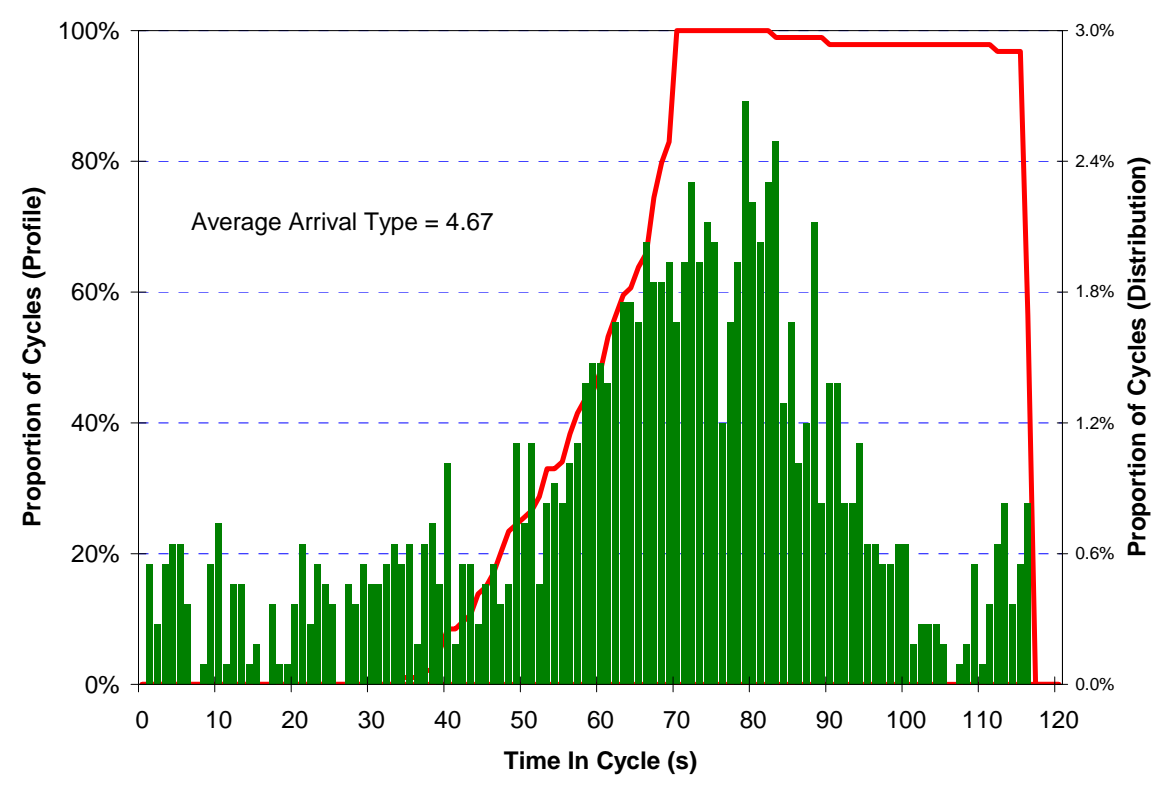

(a) Phase 2, Wednesday, March 14, 2007, 6:00 - 9:00

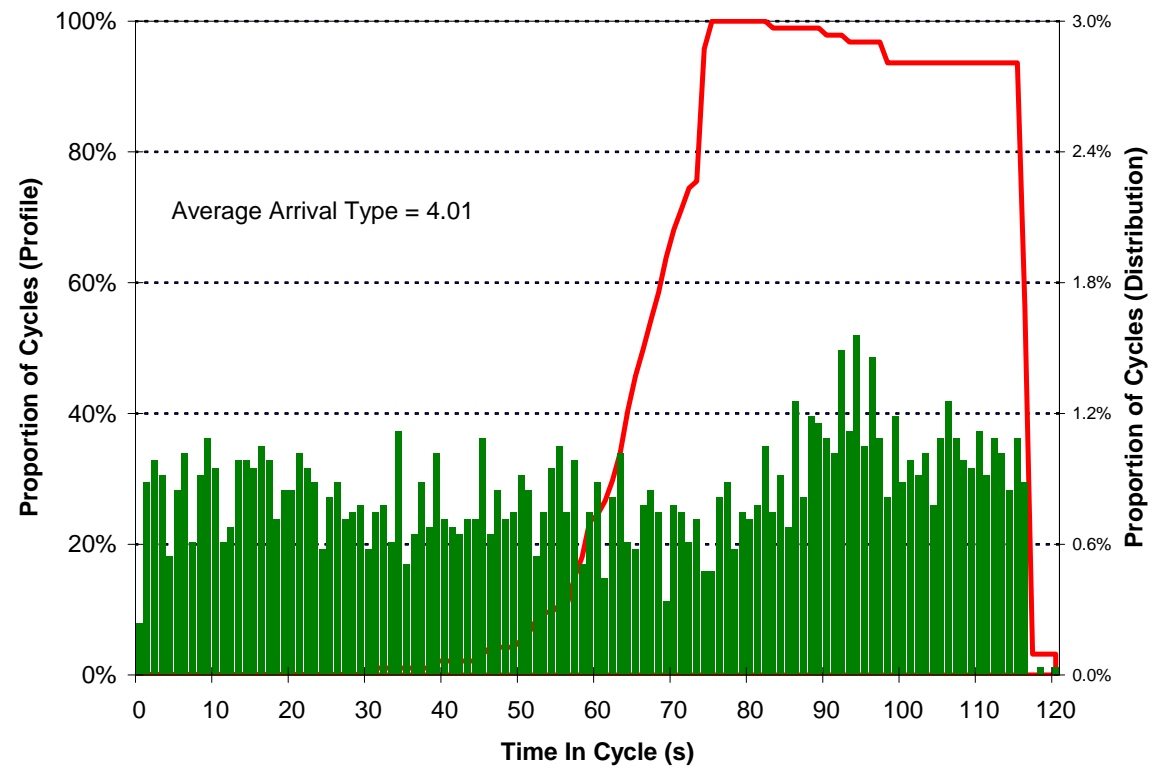

(b) Phase 6, Wednesday, March 14, 2007, 6:00 - 9:00

Figure 4.25 Synthesis of cycle profiles and arrival distribution graphs, for data collected at Noblesville on Wednesday, March 14, 2007. 


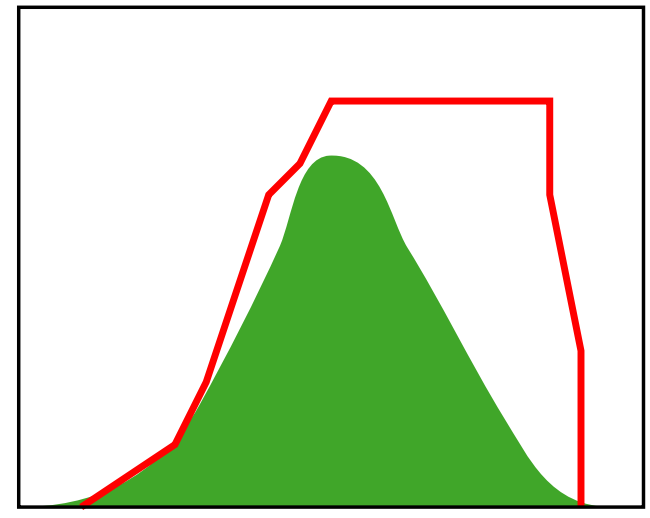

(a) good progression, $\mathrm{AT}=5$ or 6

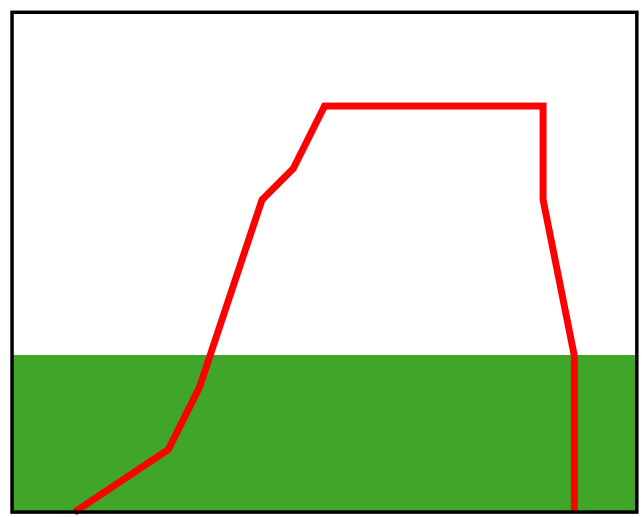

(b) random arrivals, $\mathrm{AT}=3$

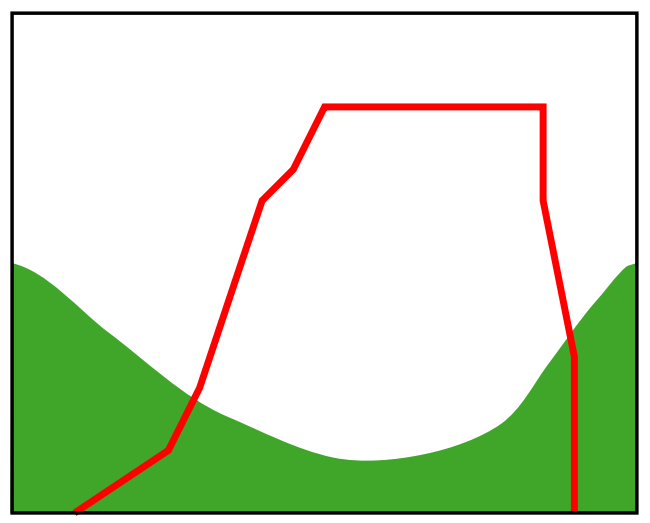

(c) poor progression, $\mathrm{AT}=1$ or 2

Figure 4.26 Interpretation of platoon profile graphs. 


\subsection{Statistical Comparisons}

\subsubsection{Introduction}

This section describes the statistical methods used in this report for comparison between different modes of operation. In the studies presented in Chapter 5, data were collected for cases before and after operational changes, and performance measures were calculated using that data. Statistical tests were carried out in order to verify whether the changes had a significant impact on the operation.

\subsubsection{Pairwise Statistical Comparison}

In comparing operation between a before and after case, it was desirable to determine whether changes could not have possibly taken place because of random variations in the data. For this purpose, a statistical t-test was carried out for data from two days with similar volume characteristics. This test assumes that our data were normally distributed.

Cycle-by-cycle performance measures were binned by time periods according to the TOD plan. The data were also separated by phase. Table 2.2 shows the TOD plan for the Noblesville test intersection. A pairwise comparison was then carried out between a 24-hour data set from before the operational change was implemented, and another 24hour data set from after the change was implemented. T-values were then calculated for each bin using the equation

$$
t=\frac{\bar{X}_{1}-\bar{X}_{2}}{s_{\bar{X}_{1}-\bar{X}_{2}}}
$$

where $\bar{X}_{1}$ and $\bar{X}_{2}$ were the mean values of a performance measure in the before and after case respectively, and

$$
s_{\bar{X}_{1}-\bar{X}_{2}}=\sqrt{\frac{\left(n_{1}-1\right) s_{1}^{2}+\left(n_{2}+1\right) s_{2}^{2}}{n_{1}+n_{2}+2}\left(\frac{1}{n_{1}}+\frac{1}{n_{2}}\right)},
$$


where $n_{1}$ and $n_{2}$ represented the number of observations in the before and after cases respectively, and $s_{1}$ and $s_{2}$ are the respective standard deviations of the observations in the before and after cases. For each TOD plan time period, for each of the eight phases, a $\mathrm{t}$-value was calculated. The magnitude of the number reflected the amount of difference between the two sets of observations (i.e., a particular performance measure).

From the t-values, P-values were calculated from the t-distribution. A significance level of $\alpha=0.05$ was used in this study to distinguish whether a difference in means could not have arisen as a result of random variation.

\subsubsection{Aggregate Statistical Comparison}

In the two studies mentioned in Chapter 5, three weeks of data were collected for operation in the time periods before and after an operational change. The pairwise statistical comparison described above was useful to identify the meaning of differences in plots of performance measures. In order to generalize the trends, data were aggregated between the 15 weekdays present in each data set (before and after). For each of the 15 weekdays in each data set, mean values were calculated by phase for each TOD plan period. To build the aggregate statistical comparison, each of the 15 mean values in the before case were compared to the 15 mean values of the after case. T-values were calculated according to Equation 4.19. This process was repeated for each performance measure, for each time period, for each phase. The aggregation methodology is illustrated in Figure 4.27. 


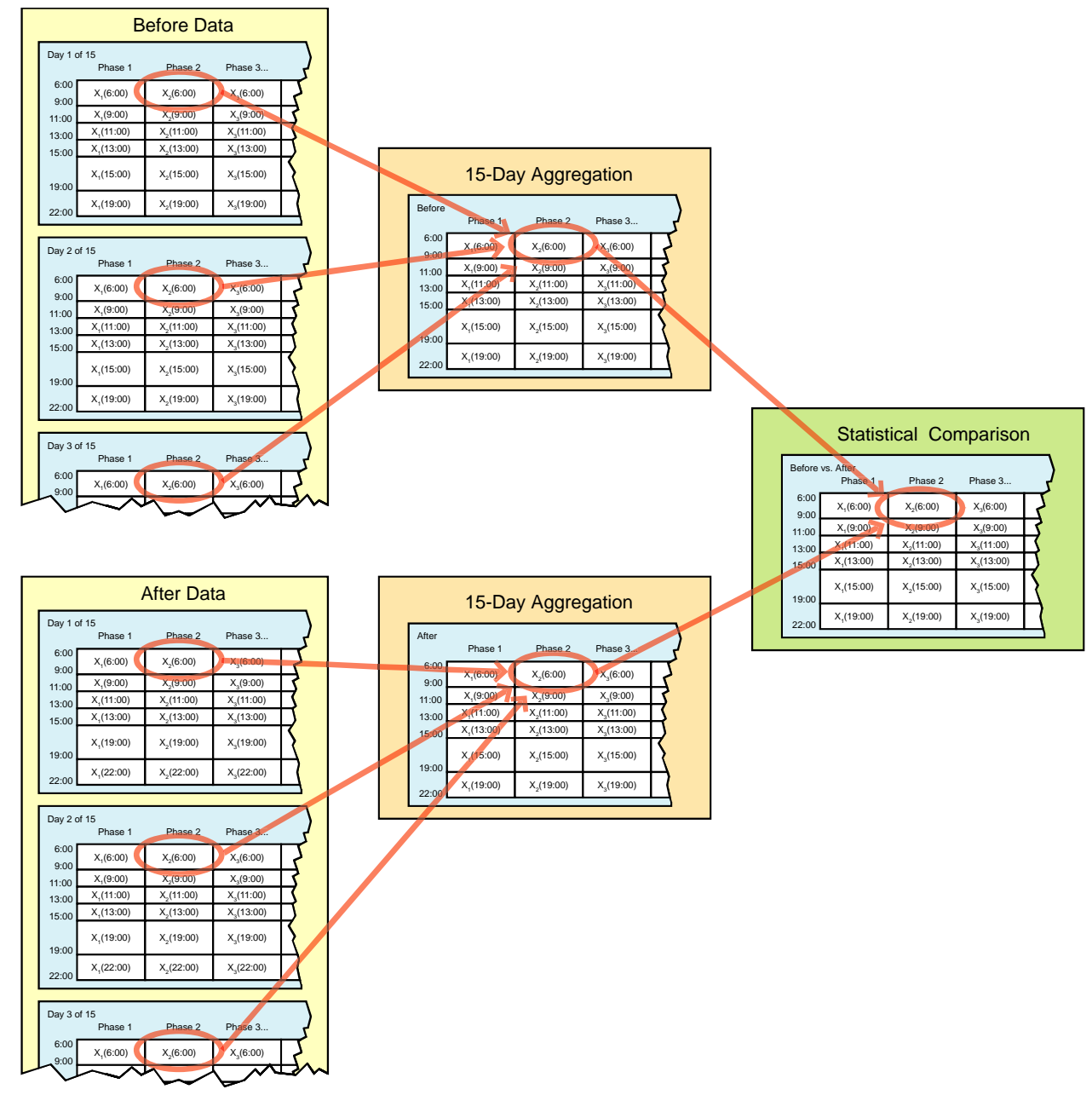

Figure 4.27 An illustration of the data aggregation methodology for statistical comparisons. 


\section{CHAPTER 5. APPLICATIONS}

\subsection{Introduction}

Chapter 3 presented methods of data collection employed for this report. Chapter 4 discussed the conversion of raw data into performance measures. This chapter presents two studies in which these methods of data collection and performance measure generation were employed to draw conclusions about changes in signal operation.

- The first study compares operation before and after the implementation of actuated coordinated phases at the Noblesville test intersection. This is a specific study of the impact of one modification to the operating scheme.

- The second study is a more general comparison of operation before and after numerous changes were made at the Noblesville test intersection. Among these changes include the use of a lagging left turn, adjustment of splits and cycle length, alteration of reference phases, and switching from non-coordinated, fullyactuated to coordinated semi-actuated during a particular time of day.

\subsection{Implementation of Actuated Coordinated Phases at Noblesville}

In March, 2007, an uncommon operating scheme was implemented at the Noblesville test intersection. The impacts of the operational change were measured using data collection techniques and performance measures described in previous chapters. This section presents an expanded version of that study (16).

\subsubsection{Introduction}

Signal coordination was discussed in Chapter 2. The purpose of coordinating signals is to provide acceptable quality traffic progression along arterials with close- to moderately-spaced intersections. Whereas in CBDs and other areas with short links between intersections, pretimed signal control is typically employed, on high-speed arterials, actuation is used at signals to allow access to crossing movements. Such intersections often operate in semi-actuated mode. The green indication is held by the 
coordinated through movements until there a call is placed for a crossing movement. The end of green for the coordinated phases is fixed to the reference point, with minor streets being given green after this point in the cycle has passed. This is in contrast to the end of green for the non-coordinated phases, which may gap out at any time after the minimum green is served.

A typical eight-phase ring diagram is shown in Figure 5.1(a). The coordinated movements are served by phases 2 and 6 . An example of a resultant timeline of serviced phases is shown in Figure 5.1(b). One advantage to semi-actuated operation is that detection is not required on the arterial through lanes. However, the main disadvantage to this type of operation is that it is insensitive to changes in demand at the intersection. On coordinated arterials with semi-actuated intersections, the coordinated phases are typically given the largest splits in order to facilitate progression. During some cycles, demand for the coordinated movements may drop off sharply, but the coordinated phases continue to be served while queues stand on the side streets. When long cycle lengths are used, these queues may stand for a minute or longer waiting for green. This situation is particularly undesirable when the demand for the coordinated phase has been served; vehicles on the minor movements are asked to wait for no reason.

Another problem with semi-actuated operation is that when the splits for the minor phases are not adequate to serve their demand, there is usually little chance for green time to be obtained by those phases. The coordinated phases typically absorbs green time that is released by other early-terminating phases. However, because the coordinated phases must always time to the reference point (as in Figure 5.1(b), $t_{1}$ ), there is no opportunity for green time to be redistributed to the minor phases in the event that coordinated demand is served. The minor phases can extend green only to the force-off points; during cycles with high demand, some vehicles are cut off and forced to wait for the next cycle to move; a few additional seconds of green time in these cases may be all that is needed to prevent this scenario.

One solution to these problems is to actuate a portion of the coordinated phases. Whereas the coordinated phases in a semi-actuated system always end at the same point in the cycle (in Figure 5.1(a), the termination of phases 2 and 6), actuated coordinated phases can gap out and release time to succeeding phases. Enough green time is retained by the coordinated phase to avoid disrupting progression. In this report, we refer to such phases as "fully actuated coordinated phases," reflecting that the signal operates in fully actuated mode, yet retains provisions for coordination. There is not a standard term for this mode of operation (17). 
When running in semi-actuated mode, the cycle length is fixed to the end of the coordinated phases(Figure 5.1(b), $t_{1}, t_{2}$ ). When fixed force-offs are used, this operating scheme is advantageous to coordinated phases 2 and 6 , because they inherit the green from any phases that terminate early. However, there is no opportunity for the minor phases to recover green time once phases 2 and 6 have met their demand. Actuating a portion of the coordinated phases, as shown in Figure 5.1(c), allows this to occur. The shaded sections represent portions of time that can be allocated to phases as needed. If the coordinated movements are saturated (Figure 5.1(d), $t_{2}$ ), this time will be retained by them; if no demand is present, the coordinated movements can gap out and pass the green to the following phases (Figure 5.1(d), $t_{1}$ ). Under semi-actuated operation, the controller would dwell in the coordinated phases until split times expire, even if demand drops to zero (Figure 5.1(c), $t_{1}, t_{2}$ ).

The primary advantage of using fully actuated coordinated phases is that it is a demand responsive operating scheme. The decision to redistribute green time is made on a cycle-by-cycle basis. Cycles where the coordinated phases experience high volumes will still be able to make use of the maximum split, but if demand is lower, the phases will gap out. When using fixed force-offs, fully actuated coordinated phases allow this extra green time from the coordinated phases to be available for successive phases in the cycle. For example, because of their location in the ring (Figure 5.1(a)), phases 3 and 7 do not have the opportunity to use excess green time from a preceding phase when operated in semi-actuated mode. However, if phases 2 and 6 are allowed to gap out, phases 3 and 7 may receive the green earlier, and they may also retain the green for a longer amount of time.

There are some disadvantages to using fully actuated coordinated phases. The green window for coordinated movements can be reduced significantly, requiring greater precision when establishing offsets. Additionally, detection is required on the coordinated movements. Many coordinated signals running in semi-actuated mode do not have detectors in the lanes serving the coordinated movements. In some cases, however, mainline detectors are available to facilitate free operation during certain time periods, and are left unused during time periods when semi-actuated mode is in effect. Additionally, the installation of detectors to enable fully actuated coordinated phases might be an attractive option where changing lane configurations is cost prohibitive.

It might be argued that, if the coordinated phases are given a sufficient minimum green, the signal could be placed in fully-actuated, free mode to accomplish the same purpose. However, this is not the case; in free mode, cycles are unpredictable. Unlike a minimum green time, an actuated coordinated phase forces the green window to begin 
and end at fixed points in time, with the option of starting earlier, if other phases terminate early; or ending later, if the coordinated demand requires it. The minimum green is timed relative to the beginning of green for the phase, whenever that occurs; but actuated coordinated phase timing is relative to the reference point, which is repeated during each cycle and is related to the coordinated offset.

This section quantitatively documents that using fully actuated coordinated phases can improve operations by reducing split failures and more effectively balancing v/c ratios for all phases without adversely impacting coordination. 


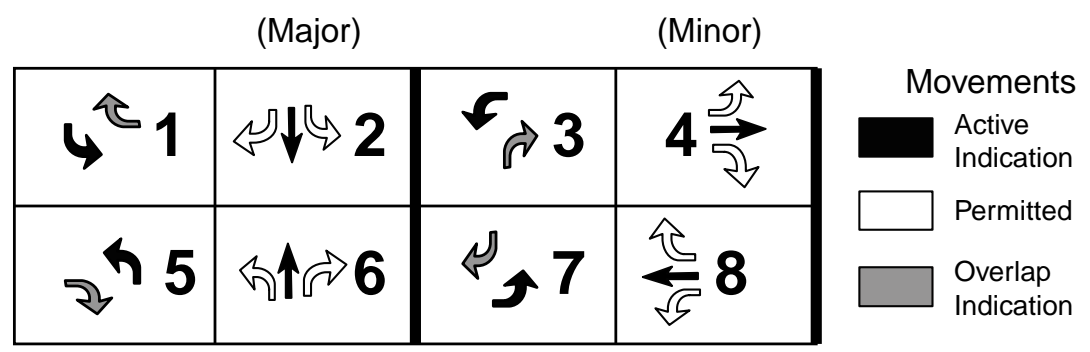

(a) Typical 8-phase ring diagram.

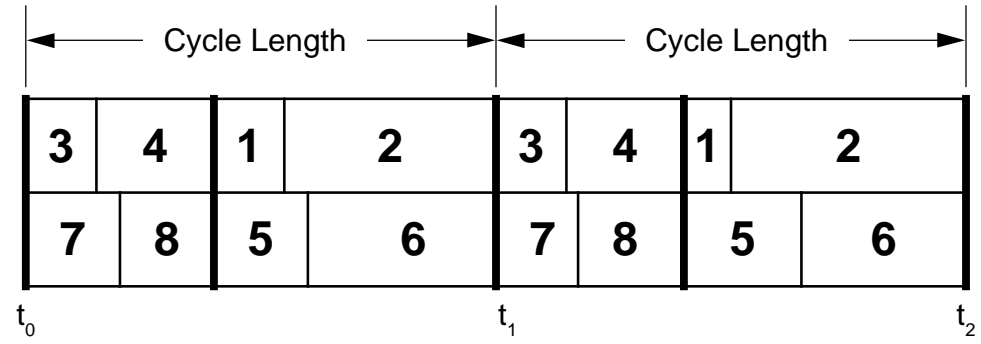

(b) Allocation of time by controller using a semi-actuated scheme.

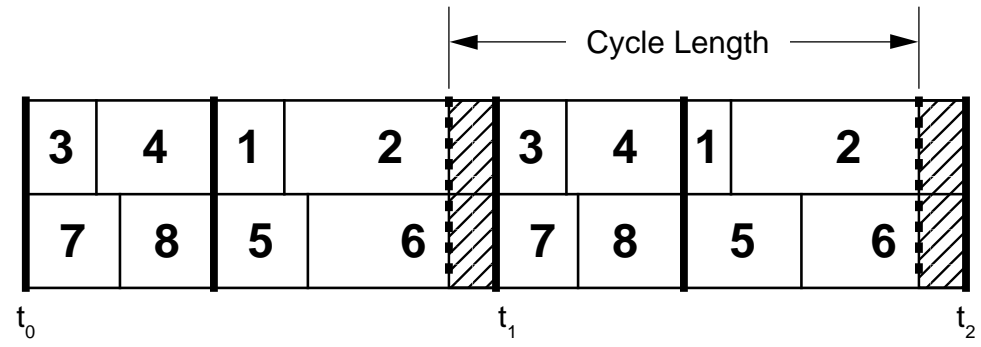

(c) Defining actuated portions of coordinated phases.

\begin{tabular}{|l|l|l|l|l|l|l|l|l|}
\hline 3 & 4 & 1 & 2 & $3:$ & 4 & 1 & 2 \\
\hline 7 & 8 & 5 & 6 & $7 !$ & 8 & 5 & $6 !$ \\
\hline
\end{tabular}

(d) Allocation of time by controller using actuated coordinated phases.

Figure 5.1 Actuated and non-actuated coordinated phases in a typical 8-phase dual ring operating scheme with coordinated phases 2 and 6. 


\subsubsection{Literature Review}

Fully actuated traffic signals have been evaluated in various field studies over the last half century $(18,19,20)$. Recent field studies $(21,22,23)$ and simulations $(24,17)$ have been conducted with regard to coordinated operation that have touched on fully actuated coordinated phases. Some guidelines $(23,25)$ have been written on the selection of control strategies for actuated signals in coordinated systems. System improvements have been reported from using fully actuated coordinated phases in terms of performance measures such as floating-car travel times (21), queues and number of stops(22), and delay $(21,22,23,24,17)$. However, there is no published work comparing the performance of actuated coordinated phases versus non-actuated coordinated phases. Additionally, this study will attempt to investigate this operating mode using data that could be automatically collected at an intersection.

\subsubsection{Field Studies}

Berry (18) carried out a field study of delay at signalized intersections, comparing the amount of delay between fully actuated, semi-actuated, and pretimed control schemes. The effects of coordination on actuated signals was also studied. Contemporary (late-1950s) methods of delay and travel time measurement included analysis of photographs taken at regular intervals. Fully actuated control was found to be superior to both semi-actuated and pretimed control schemes in terms of stopped-time delay. The coordination of signals was found to reduce the amount of delay by half; however, in Berry's study, pretimed operation was used to facilitate coordination.

Ficklin (19) compared fully-actuated to semi-actuated control, and observed that semi-actuated control was effective when signal spacing was tight, whereas fullyactuated control was superior for isolated intersections. This comparison did not make use of fully actuated coordinated phases; instead, this study reported that an operating scheme favoring coordination was not viable when the segment lengths between signals was great.

A study by Jovanis and Gregor (20) acknowledged that the use of actuation signals in coordinated systems made it possible to vary green times on a cycle by cycle basis. A method was demonstrated that set force-off and reference points to accommodate the coordinated movement. An actuated coordinated timing plan was developed for a six-signal system. Evaluation of the operating plan was done in 
simulation. The simulation results, however, revealed that pretimed coordinated control produced better results than actuated coordinated control.

Chang and Koothrappally (21) produced a field study that confirmed the effectiveness of using coordinated control with actuated signals (termed "coordinated actuated" signal operation). The measures of effectiveness in this study were delay and the number of stops. This study reported that semi-actuated control provided the best results compared to pretimed or fully-actuated control. However, this study did not evaluate the situation of using fully actuated coordinated phases, which would modify the semi-actuated control scheme to enable early return to the crossing movements. Chaudhary and Balke (22) investigated coordinated actuated control further, confirming that the coordination of actuated signals reduced delays, number of stops, and lengths of queues.

\subsubsection{Simulations of Actuated Signals in Coordinated Systems}

In 2001, Khatib et al. (24) documented results of a hardware-in-the-loop simulation in which a control algorithm was used that was able to change signal timing parameters on a cycle-by-cycle basis. The proposed control system was intended to eliminate spillbacks. In this control method, splits were optimized on a cycle-by-cycle basis using vehicle arrival volumes, resulting in significant decreases in delays, particularly for minor street movements that were constrained by spillbacks on the major arterial. The control system proposed in this study allowed more flexible adjustments than the use of fully actuated coordinated phases makes possible. However, this study demonstrated that changing signal timing parameters on a cycle-by-cycle basis allowed the system to be operated more efficiently.

A 2006 article by de Castro Neto et al. (17) reviews the performance of actuated

phases in coordinated systems. Hardware-in-the-loop simulations were carried out to compare operation under pretimed and semi-actuated operations. The operating parameters under comparison were variations of semi-actuated timing strategies, and relative performance of the modes was dependent on the amount of traffic volume. Fully actuated coordinated operation was not touched upon in the simulation, but it was presented as a subset of coordinated operation in which the end of the coordinated phase is actuated. It was commented that fully actuated coordinated operation was "not common."

This article also commented on the lack of consistency in terminology related to actuated coordinated signal systems. By the terminology of this article, the mode of fully 
actuated coordinated operation investigated in the present section of this report would be characterized as "coordinated semi-actuated" with actuated termination.

\subsubsection{Guidelines for Actuated Signals in Coordinated Systems}

Chang (25) proposed guidelines for actuated signals in coordinated systems in a 1996 article. This article focused mainly on a procedure for selecting a signal control strategy. Fully-actuated, non-coordinated control was recommended for light traffic operation; pretimed control was recommended when cross street saturation occurred; and semi-actuated control was recommended in cases where patterns were unpredictable and cross street volumes were undersaturated. This study did not mention a coordinated system control strategy in which coordinated phase termination was actuated.

Skabardonis et al. (23) studied various operation schemes for coordinated signal systems that were applied to signals in Los Angeles, CA. The authors note that previous guidelines for signal control are applicable to isolated intersections, not to intersections in a coordinated system. The authors propose recommendations to determine whether to use pretimed, semi-actuated, or fully actuated control based on v/c ratios, and the geometry of the network. In general, semi-actuated control was selected for arterial intersections in coordinated systems, while fully actuated control was selected for isolated intersections. This study considered only fully-actuated control without coordination, and semiactuated control with fixed phase termination.

\subsubsection{Study Location and Duration}

The location used in this study is the intersection of State Roads 37 and 32/38 in Noblesville, Indiana, which is described in Chapter 3. Data for this study were collected on all four approaches. Lead-lag phasing was in use for the entire duration of this study, and lane-by-lane detection was used for the through movements on all four approaches. As noted in Chapter 3, the Noblesville test intersection is the northernmost signal in a coordinated system; Northbound arrivals are coordinated while southbound arrivals are not. The distances to the nearest signal are $1448 \mathrm{~m}$ (4750') to the north and $732 \mathrm{~m}\left(2400^{\prime}\right)$ to the south.

To compare between operation with and without actuated coordinated phases, data for three weeks of each case were collected. From March 4, 2007 through March 24, 2007, the signal operated in semi-actuated mode, with the coordinated phases ending at fixed points in time. Data logging was successfully undertaken at the same time. On 
March 25, 2007, the signal was operation was changed to use actuated coordinated phases. Data was collected for three additional weeks in the period spanning March 25, 2007 and May 5, 2007. Some of the weeks in that period were rejected because of traffic incidents, adjacent construction, or data loss due to computer downtime. In total, three weeks of data from before implementation and three weeks of data from after implementation of actuated-coordinated phases were obtained. Figure 5.2 shows the dates used for data collection.

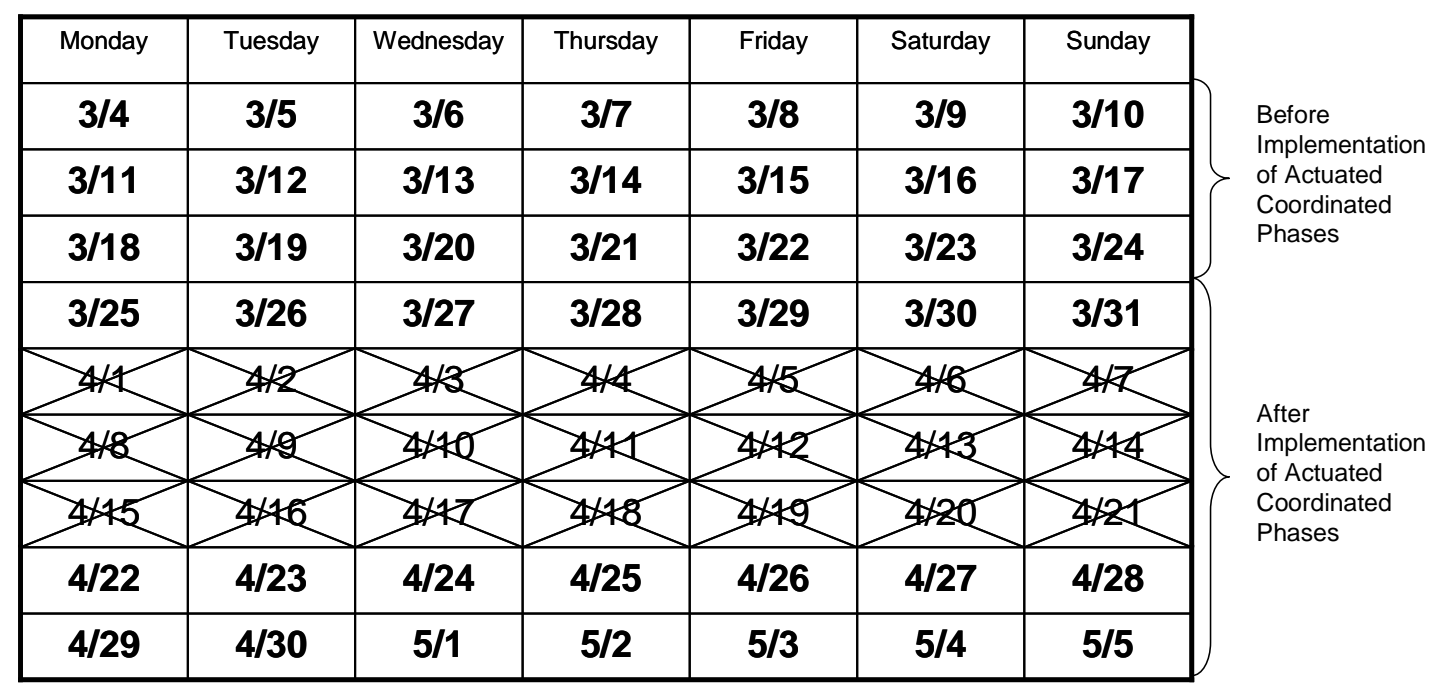

Figure 5.2 Calendar showing dates of data collection used in the study of fully actuatedcoordinated phases. 


\subsubsection{Experiment Design and Expectations}

Figure 5.3(a) shows the ring diagram for operation at the Noblesville test intersection. As discussed before, the northbound left turn lags the southbound through movement, allowing the northbound left turn to be coordinated with the upstream signal. The actuated portion of the coordinated phases is shown in Figure 5.3(b). The length of the actuated portion is equal to $10 \%$ of the total cycle length, ranging from $9.6 \mathrm{sec}$ to 11.6 sec, depending on the time of day. This is an arbitrary (but reasonable) amount of time hard-coded by the controller.

Because phase 5 lags phase 6 , the endpoint of the actuated portion of phase 6 does not come at the reference point (the barrier). This makes the situation slightly more complex than the general case described earlier. Phase 6 is able to release green time to phase 5 as soon as it gaps out during its actuated period. Time cannot be reallocated across the barrier until both phases 2 and 5 gap out; phase 2 during the actuated portion of the phase, and phase 5 after the minimum green interval has expired and the detection zone has emptied. Once this has occurred, any additional time is then available to the phases immediately after the barrier, 3 and 7 in this case. Figure 5.3(c) shows an example where the entire actuated portion of each coordinated phase has been released to following phases. Because phase 6 terminates early, phase 5 is allowed to discharge vehicles at an earlier point in time; the amount of green time available to phase 5 also increases. Because phases 2 and 5 terminate at an earlier point in time, the phases on the other side of the barrier are able to begin earlier as well as to extend their green times.

Fully actuated coordinated phases are particularly attractive with lead/lag phasing, ensuring the lagging left turn is not too long or too short. There is a possibility that phase 5 will absorb most, if not all, of the benefit of using actuated coordinated phases, as it will remain green whenever demand for either phase 2 or 5 is high. However, this should not take place during every cycle. When demand for phases 2 and 5 drops off, phases 3 and 7 will also receive additional green time. Whenever phases 3 and 7 are skipped, or whenever they do not time to their force-off points, then phases 4 and 8 would benefit. 


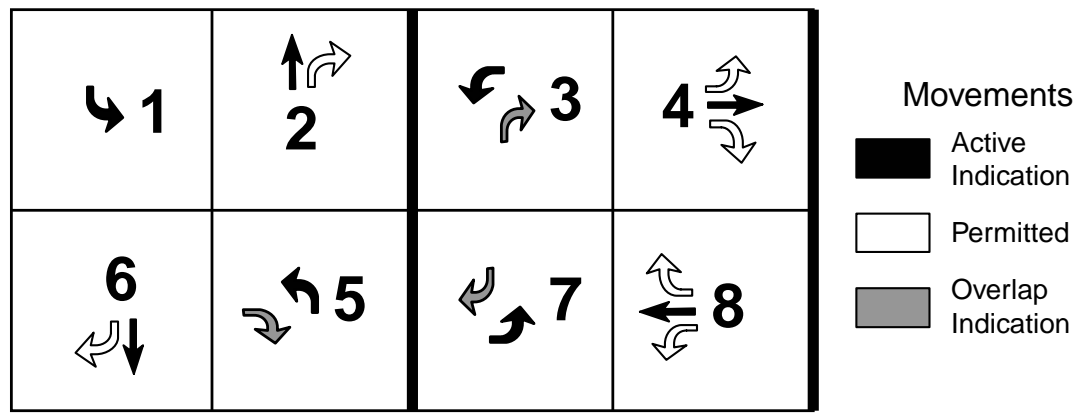

(a) Noblesville test intersection phase diagram.
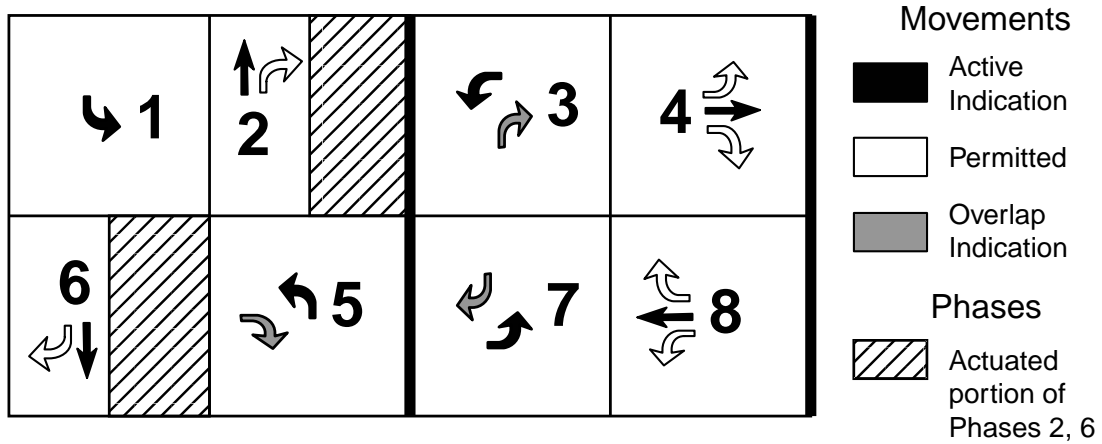

(b) Actuated portion of coordinated phases.

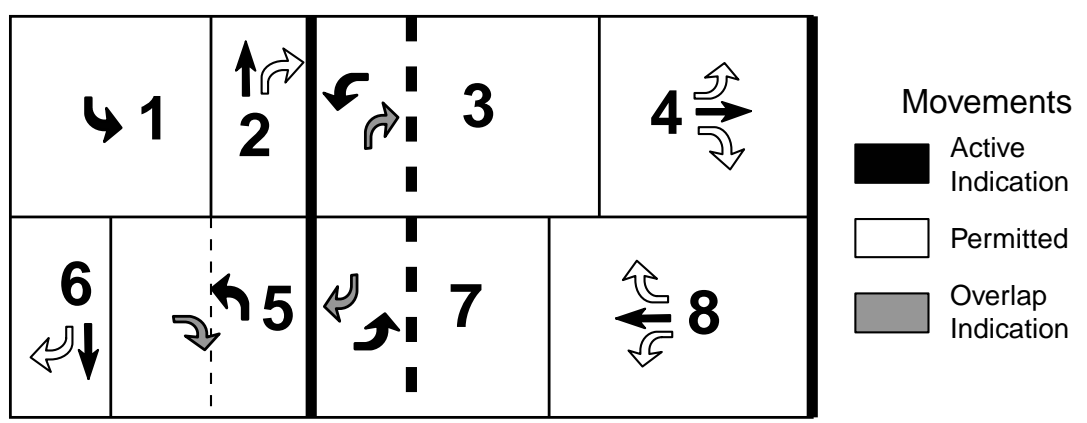

(c) Example reallocation of split time.

Figure 5.3 Experimental expectations of implementing actuated coordinated phases at the Noblesville test intersection. 


\subsubsection{Selection of Performance Measures}

To compare operation with non-actuated coordinated phases to operation with actuated coordinated phases, several performance measures were selected:

- Equivalent hourly volumes were measured to identify the demand for all movements at the intersection.

- Duration of green time was measured to characterize signal operation, including the response of the signal to demand.

- v/c Ratios revealed the degree to which capacity for every phase at the intersection was utilized, indicating signal performance with regard to how efficiently demand was met.

- The number of split failures were tabulated.

- The critical v/c ratio was calculated to evaluate overall signal performance.

- Arrival type (AT) was used to evaluate the quality of progression through the coordinated phases of the signal.

- Platoon profiles were created to visualize the affect of changing operation on progression in qualitative analysis.

The methods for compiling these performance measures were discussed in Chapter 4. The performance measures were calculated for all eight phases, for every cycle during the day, with the exception of AT, which was calculated only for phases 2 and 6.

\subsubsection{Qualitative Analysis}

To investigate the impact of actuated coordinated phases on the values of the performance metrics, a pair of days with similar traffic volumes was selected for detailed evaluation. The two days compared were Wednesday, March 7, 2007, and Wednesday, March 28, 2007.

Figure 5.4 shows a graph of the equivalent hourly volume of each phase (numbered at the upper left corner of each of the eight graphs) over 24 hours for March 7 and March 28, using a 20-point moving average. The coordinated phases, 2 and 6, have the highest volumes. The peaking characteristics of these phases are apparent in the 
graphs, with phase 6 peaking in the morning (revealing strong demand for southbound traffic heading to Indianapolis during the morning rush hour) and phase 2 peaking in the afternoon (revealing northbound traffic leaving Indianapolis during the evening rush hour).

Figure 5.5 shows histograms of green durations for all eight phases during the AM and PM peak periods. The top graphs for each phase show the case without actuated coordinated phases, while the bottom graphs were obtained with actuated coordinated phases. For example, the histogram in the extreme upper left corner is for phase 1 (P1b) in the before case, where the coordinated phases are not actuated (March 7); the histogram immediately below is for phase 1 in the after case (P1a), when the coordinated phases were actuated (March 28). The vertical dashed lines indicate controller split times, while the large arrows show the difference in mean green times between the before and after cases. The impact of using actuated coordinated phases is demonstrated by changes in the shapes of the distributions; in the case of phase 1, little impact is observed.

However, other phases clearly show changes in the distribution of green. For example, according to our expectations as illustrated by Figure 5.3, we anticipated a reduction in phase 2 green times and an increase in phase 3 green times, and similar shifts for phases 6 and 5. In Figure 5.5(a) and (b), the distributions of phases 3 and 5 shift to the right, while those of phases 2 and 6 shift to the left. This indicates that phases 2 and 6 frequently had shorter green times, while phases 3 and 5 had longer green times. Additionally, we observed an increase in green time for phase 7. Phases 4 and 8 captured additional green time in the AM peak hour, but did not in the PM peak.

Plots of green time for eight phases over 24 hours are shown by Figure 5.6. These graphs convey the same information as the green time histograms of Figure 5.5, but here we can see the uniformity of the changes throughout the day. Green time can easily be converted into capacity, as shown in Figure 5.7. The shapes of the graphs are the same; only the scaling is different. As can be seen in Figure 5.7, a small amount of capacity is shaved away from phases 2 and 6 , and is given to the following phases. As we saw earlier, phase 5 benefits the most from this arrangement. However, phases 3 and 7 can also be seen to acquire additional capacity. The other phases do not appear to be affected.

Figure 5.8 shows the cycle-by-cycle v/c ratios for all eight phases. Graphs for the before and after cases are stacked on top of each other, as in Figure 5.5. For example, in the upper left corner, we find plots of the before $(\mathrm{P} 1 \mathrm{~b})$ and after $(\mathrm{P} 1 \mathrm{~b}) \mathrm{v} / \mathrm{c}$ ratios for phase 1. The thin black lines show 20-point moving averages. Cumulative frequencies of $\mathrm{v} / \mathrm{c}$ ratios for the AM and PM peak periods are plotted in Figure 5.9, with arrows indicating the difference in means between the before and the after cases. The general trend 
throughout the day was a reduction in $\mathrm{v} / \mathrm{c}$ ratios for phases $3,5,7$, and 8 ; and increases in $\mathrm{v} / \mathrm{c}$ ratios for phases 2 and 6 . Phase 5 in particular receives the most benefit, as shown in Figure 5.9 by the leftward movement of the cumulative frequency plots. The reduction in $\mathrm{v} / \mathrm{c}$ ratio for phase 3 in the AM peak period is attributed to lower demand for that phase on March 28 compared to March 7. The average green time was increased by only $0.1 \mathrm{~s}$ [see Figure 5.5(a)]. However, PM peak period volumes for both days were similar and the average green time increased, leading to a decrease in the $\mathrm{v} / \mathrm{c}$ ratio for phase 3 . These changes are attributed to actuating the coordinated phase. Phases 7 and 8 see reductions in the AM peak period, but not in the PM period. Phase 2 is virtually unaffected, whereas phase 6 shows an increased $\mathrm{v} / \mathrm{c}$ ratio.

Counts of reported split failures are given in Table 5.1 for all 8 phases under both types of operation. Figure 5.10 shows the 24 hour totals of split failures by phase graphically; Figure 5.11 shows the 24-hour intersection totals for all eight phases. As explained in Chapter 4, a split failure is defined as termination of a phase with a v/c ratio greater than 1.0. For phases 3 and 5, the number of split failures was reduced for nearly every time period in the day. Phase 7 also had fewer split failures. Not many split failures occurred for phases 2 and 6 under either mode of operation, giving evidence that adequate green time is provided for the coordinated phases both with and without the use of actuated coordinated phases. The number of split failures increased for phases 1 and 4 . For phase 4, the increase mainly occurred during the PM peak hour, where demand was the same for both days, but the phase had less green time in the after case. This was probably due to the fact that phase 3 had a higher volumes in the after case, causing it to be called more often, reducing the average green time for phase 4 and hence leading to more split failures. As for phase 1, the cause is unclear. Because volumes, green times, and $\mathrm{v} / \mathrm{c}$ ratios were similar for phase 1 in the before and after cases, the increase in split failures is attributed to random variation, whereby a handful of cycles occurred for which vehicle arrivals were clustered more densely than others.

By reallocating time from phase to phase within the same ring and across the barrier, $\mathrm{v} / \mathrm{c}$ ratios changed between the before and after cases. The amount of green time for phases 2 and 6 was reduced because those phases were allowed to gap out in the after case, whereas they could not do so before. The v/c ratios for phases 2 and 6 are slightly higher, yet remain less than 1.0; few split failures occurred for those phases. This indicates that green time is being used more efficiently by those phases. A portion of the green time given up by phases 2 and 6 was used by phases 3 and 5 . As a result, the v/c ratios for these values dropped. Phase 5 in particular was operating many cycles with $\mathrm{v} / \mathrm{c}$ ratios above 1 in the before case. The additional green time for this movement had a 
sizeable impact on the v/c ratio throughout the day. Similar effects were seen in phase 3, particularly in the morning. Phases 7 and 8 appeared to benefit during the AM peak hour, but were unaffected in the evening. Phase 4 does not appear to be affected by the use of actuated coordinated phases.

Plots of critical v/c ratio $\left(\mathrm{X}_{\mathrm{C}}\right)$ for March 7 and March 28 are shown in Figure 5.12. The critical $\mathrm{v} / \mathrm{c}$ ratio represents the overall performance of the intersection. The two graphs are similar; $\mathrm{X}_{\mathrm{C}}$ is higher during the peak periods and lower during the off-peak periods. There is a slight overall increase in $X_{C}$ in the after case. The proportions of critical paths used for calculating $X_{C}$ are shown in Figure 5.13(a) for March 7 and Figure 5.13(b) for March 28. There is little difference between the two days. The critical path consists of those phases that have higher individual v/c ratios. Note that phases 5 and 6 are a component of the critical path for most of the day, except after 3:00 PM, when phases 1 and 2 become more prevalent. Additionally, phases 3 and 4 are more critical than phases 7 and 8 . Given the prevalence of phase pairs $(5,6)$ and $(3,4)$, the increases in $\mathrm{v} / \mathrm{c}$ ratios for those phases is driving the upward shift in $X_{C}$. The increase in $X_{C}$ shows that the intersection as a whole operates a somewhat closer to saturation. The implication is that there is less flexibility to accommodate additional changes to the splits.

For coordinated movements, changes in AT quantify changes in progression through the signal. One possible negative impact of using actuated coordinated phases is that a late arriving platoon may be cut off by early termination of the coordinated phases. To determine whether this would occur at the Noblesville test site, AT was measured for the coordinated phases. Graphs of AT are shown in Figure 5.14. Phase 2 is virtually unaffected by the implementation of actuated coordinated operation. There is very little difference between the two graphs; overall AT is between 4 and 5, indicating that favorable progression took place on both March 7 and March 28 .

Interestingly, the phase 6 AT became slightly lower during the PM peak hour. The upstream intersection for phase 6 was not coordinated with the test intersection. With random arrivals, an AT value of 3 is expected, which was obtained in the after case. Values of AT greater than 3 obtained in the before case reveal an unexpected interaction with the upstream signal, which was lost when actuated-coordinated phases were implemented. One possible explanation for the interaction may be harmonics created by the rhythmic release of vehicles by the Noblesville test intersection, influencing the cycle patterns of the upstream signal. The upstream signal in turn regularly releases vehicles in the same rhythm; those vehicles arrive in the southbound lanes of the test intersection; arrivals are therefore slightly better than random. However, it is unclear why this 
interaction should be lost because of the implementation of actuated coordinated phases. Further investigation of this phenomenon would extend beyond the scope of this study.

Platoon profile graphs are shown for phase 2 in Figure 5.15, and for phase 6 in Figure 5.16. In both figures, the profiles are plotted for the AM and PM peaks for both March 7 and March 28. The volume of traffic for phases 2 and 6 were very similar on both days. The change in the shape of the cycle profile, shown by the red line in these graphs, indicates the increased variability in the cycle end times due to actuating the coordinated phases. The cycle profile for phase 6 can be seen to narrow markedly in the after case, showing where time has been released to phase 5 . There appears to be no change in the shape of the arrival profile for phase 2. However, phase 6 arrivals can be seen to change slightly. Slightly more arrivals occur during the green indication in the before case, in both the AM and PM peak hours. As mentioned earlier, this is attributed to an unintended interaction with the upstream signal. This interaction was lost in the after case, with the result that arrivals have become more random, and AT has decreased to values closer to 3 . 

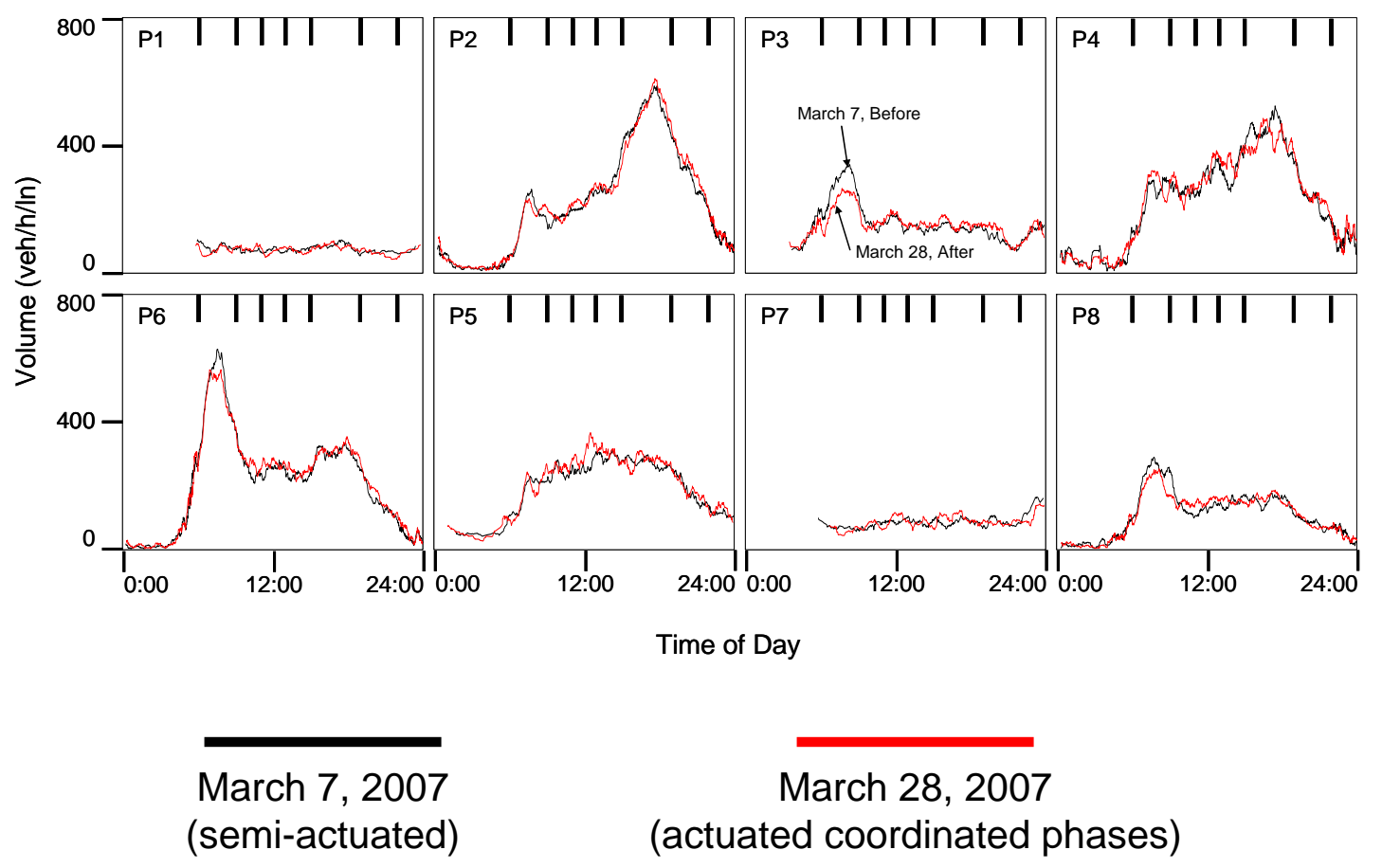

Figure 5.4 Equivalent hourly volumes by phase (20 pt. average), for Wednesday, March 7, 2007 (with non-actuated coordinated phases) and Wednesday, March 28, 2007 (with actuated coordinated phases). 


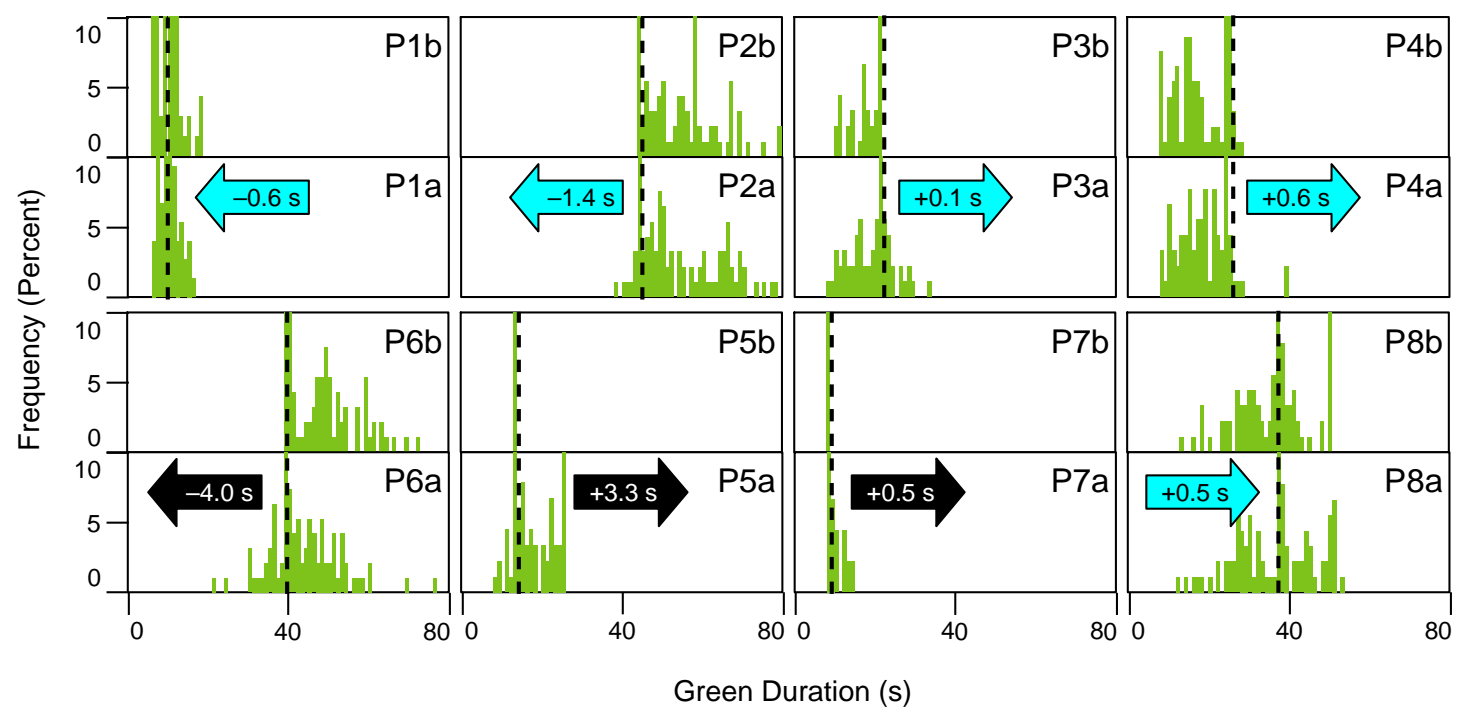

(a) 6:00 - 9:00 AM Morning Peak $(\mathrm{C}=116 \mathrm{sec})$

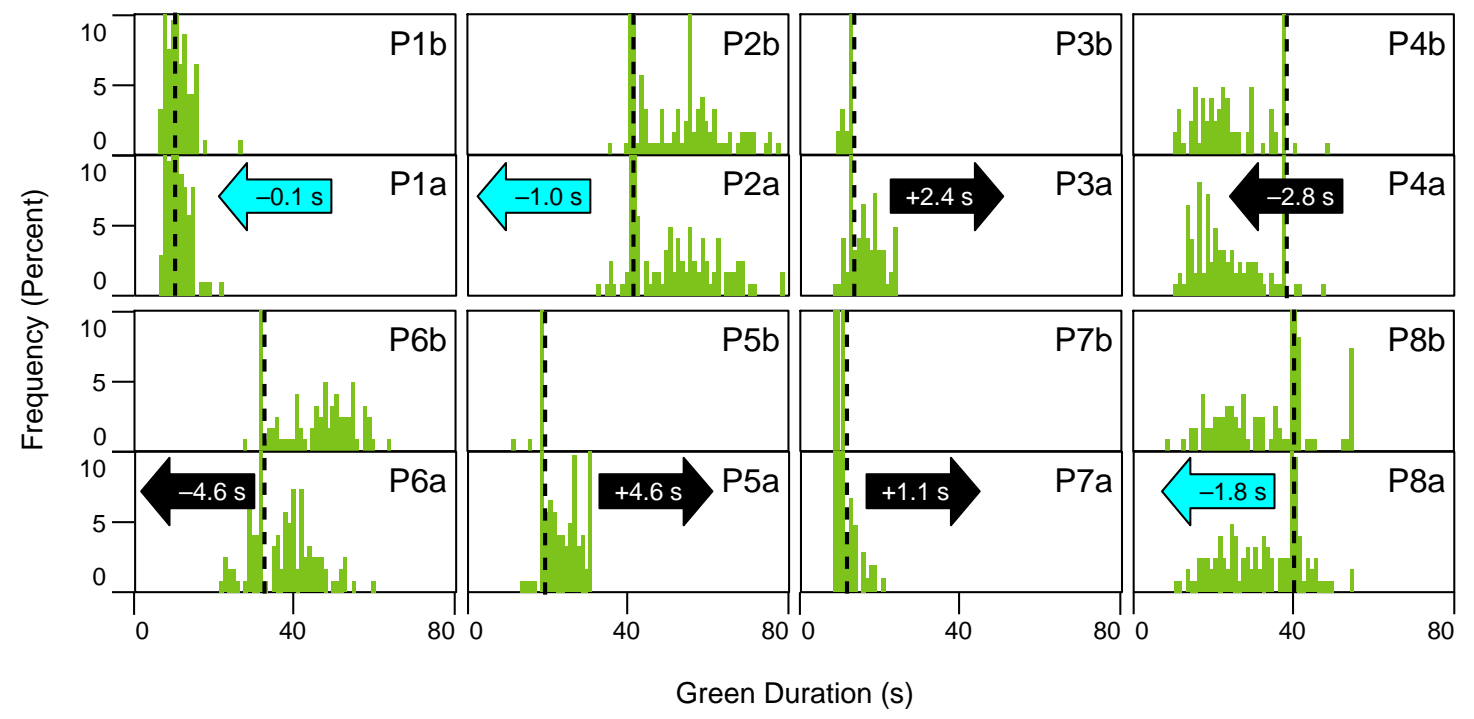

(b) 3:00 - 7:00 PM Evening Peak $(\mathrm{C}=116 \mathrm{sec})$
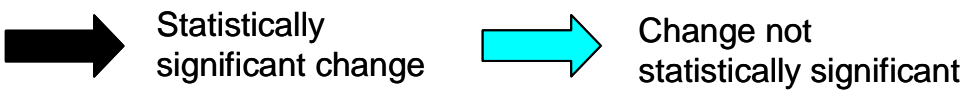

Figure 5.5 Green time histograms for all 8 phases for cycles. Arrows indicate changes in mean values. 

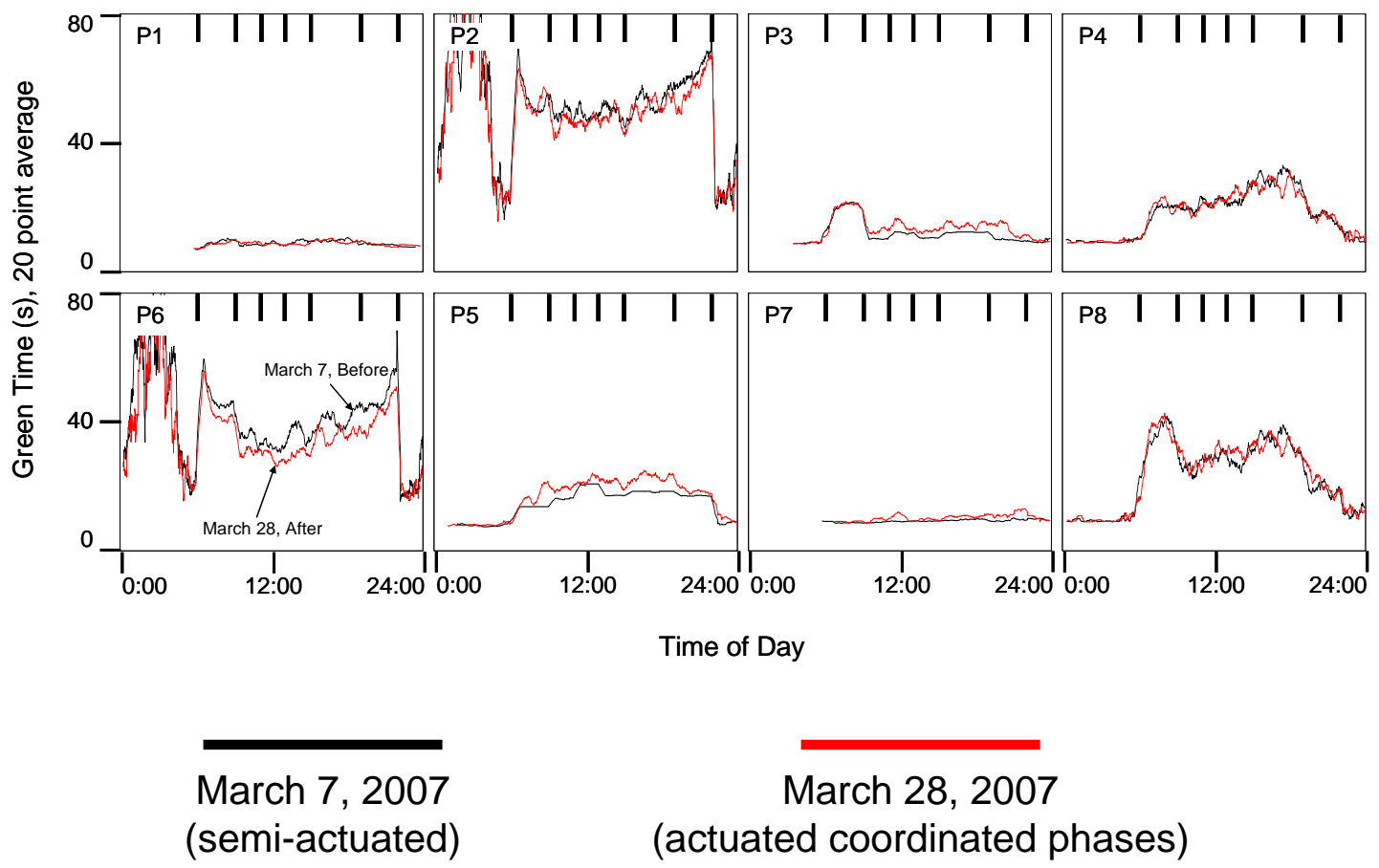

Figure 5.6 Plots of green duration by phase (20 pt. average), for Wednesday, March 7 (with non-actuated coordinated phases) and Wednesday, March 28, 2007 (with actuated coordinated phases). 

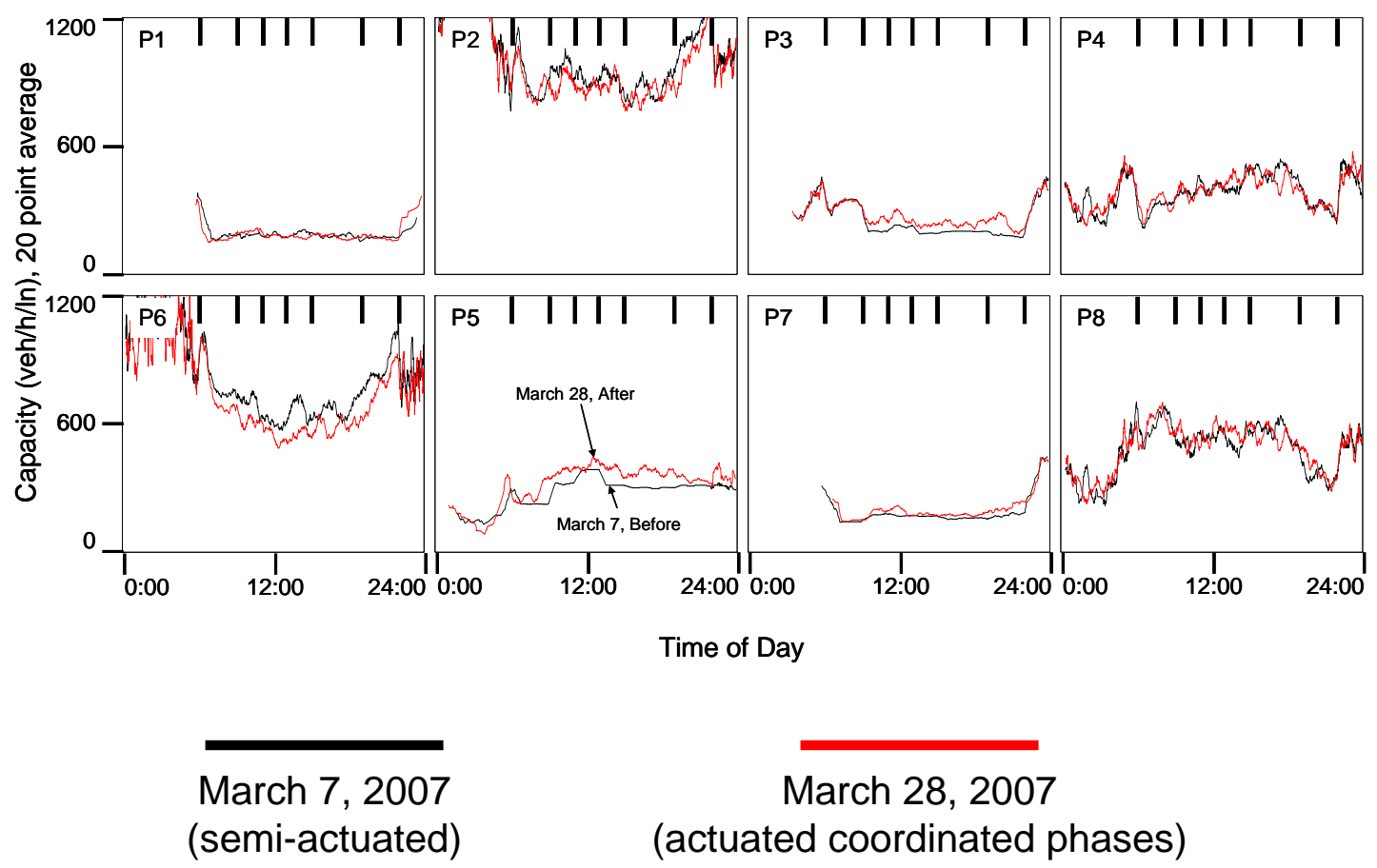

Figure 5.7 Plots of capacity by phase (20 pt. average), for Wednesday, March 7, 2007 (with non-actuated coordinated phases) and Wednesday, March 28, 2007 (with actuated coordinated phases). 


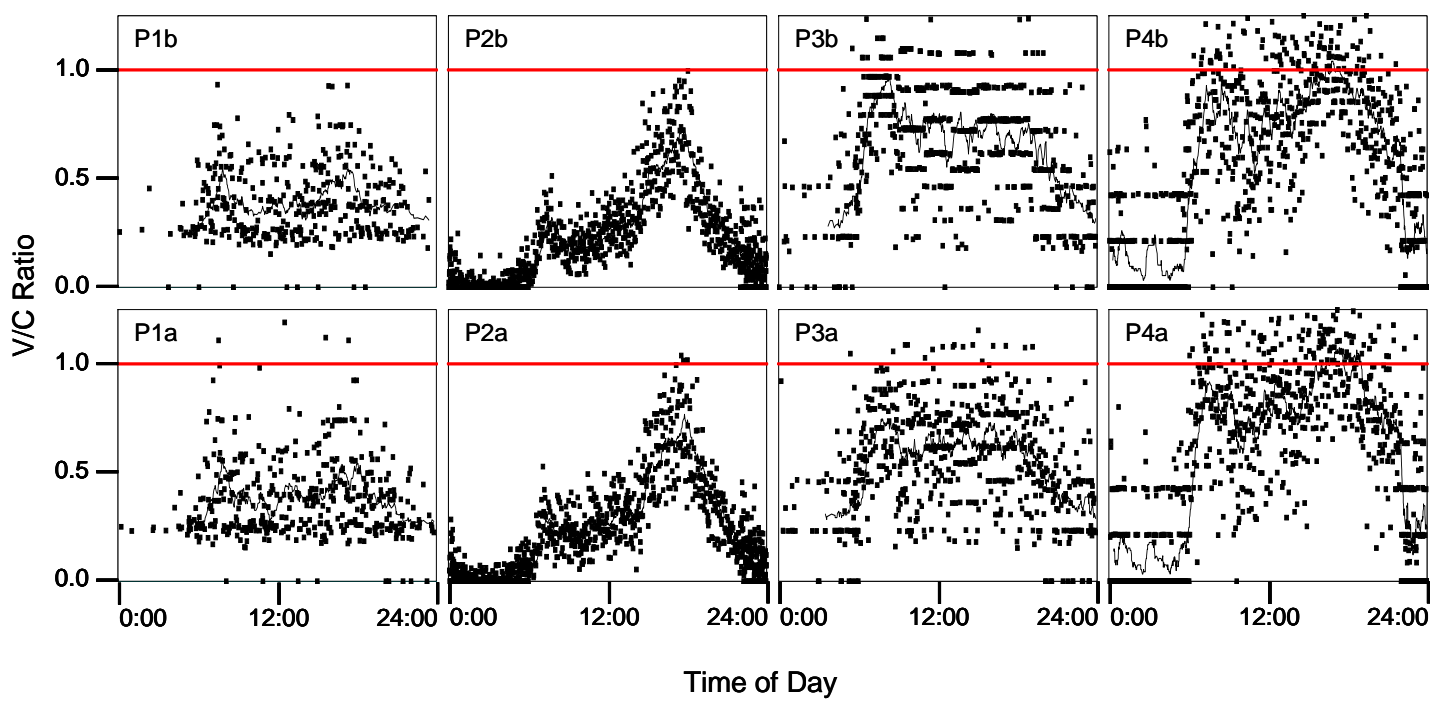

(a) Phases 1, 2, 3, and 4 .

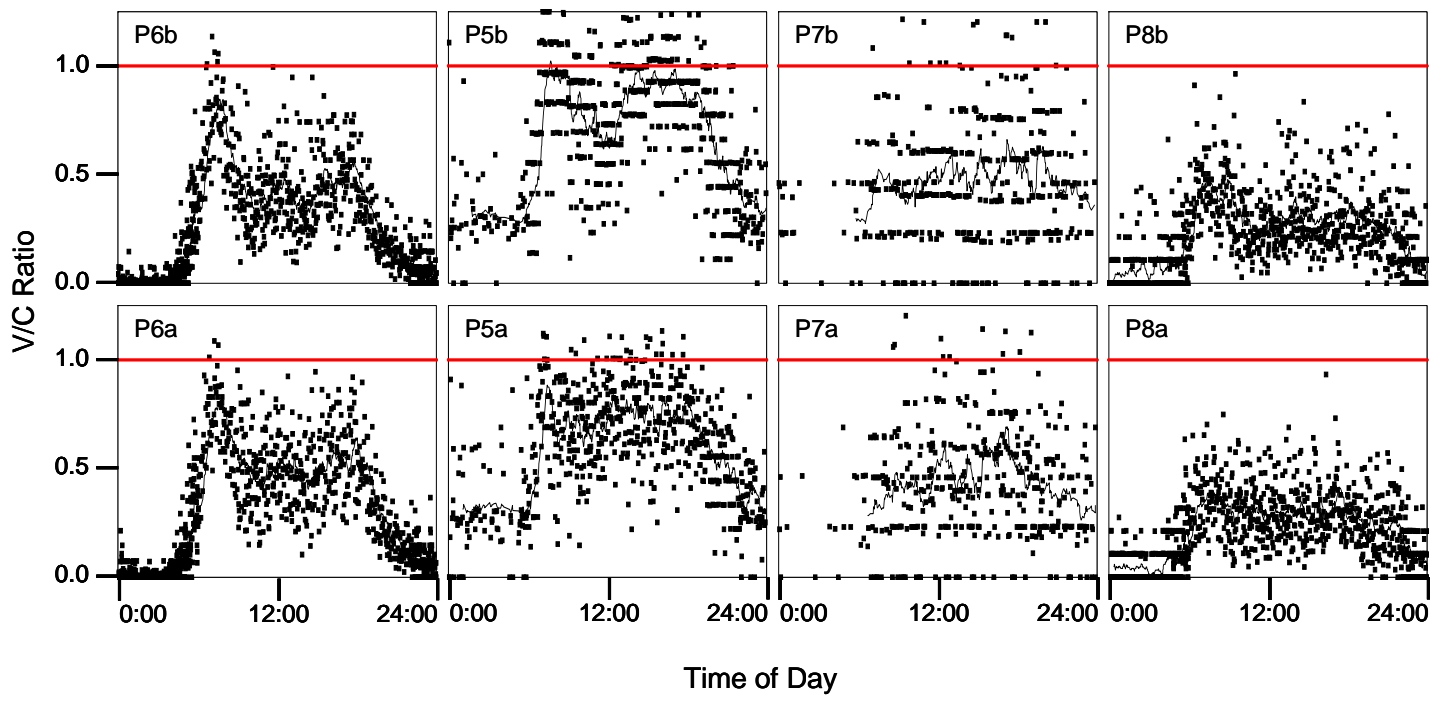

(b) Phases 6, 5, 7, and 8 .

Figure 5.8 Cycle-by-cycle calculations of volume-to-capacity ratio. 


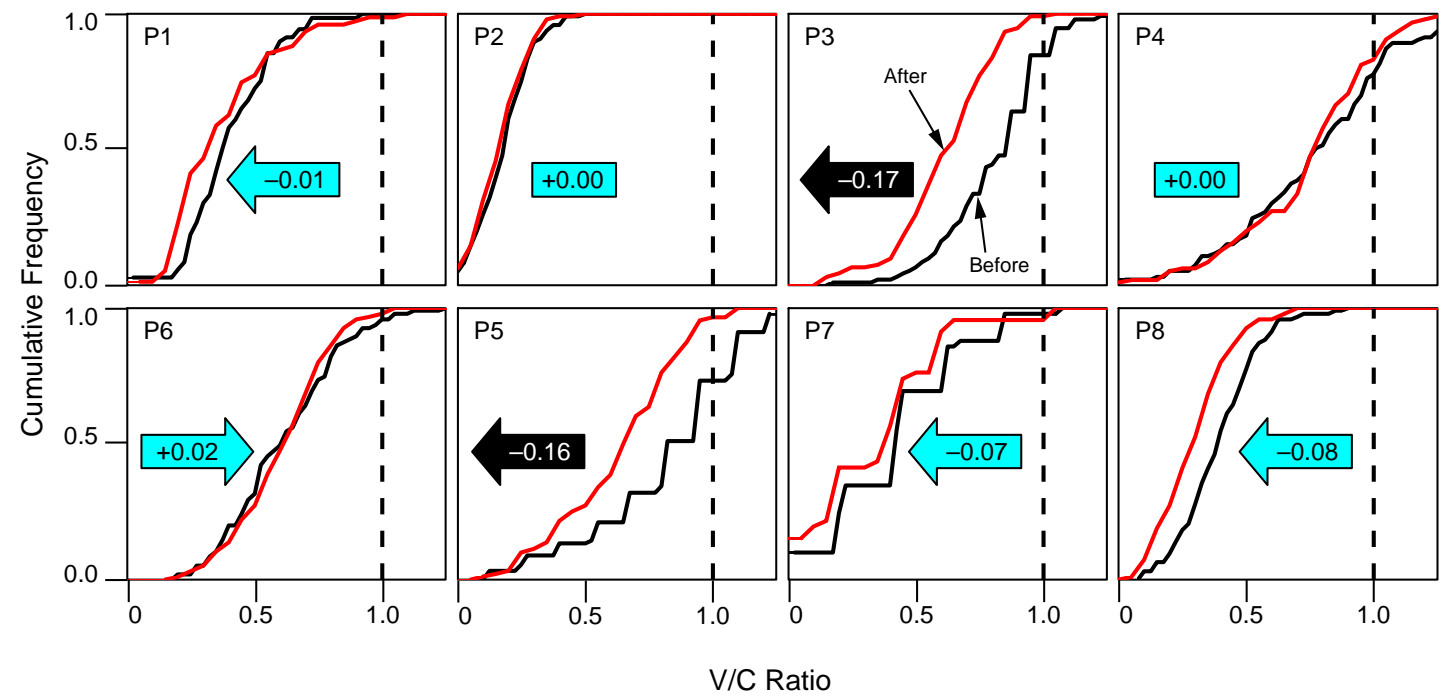

(a) 6:00 - 9:00 AM Morning Peak

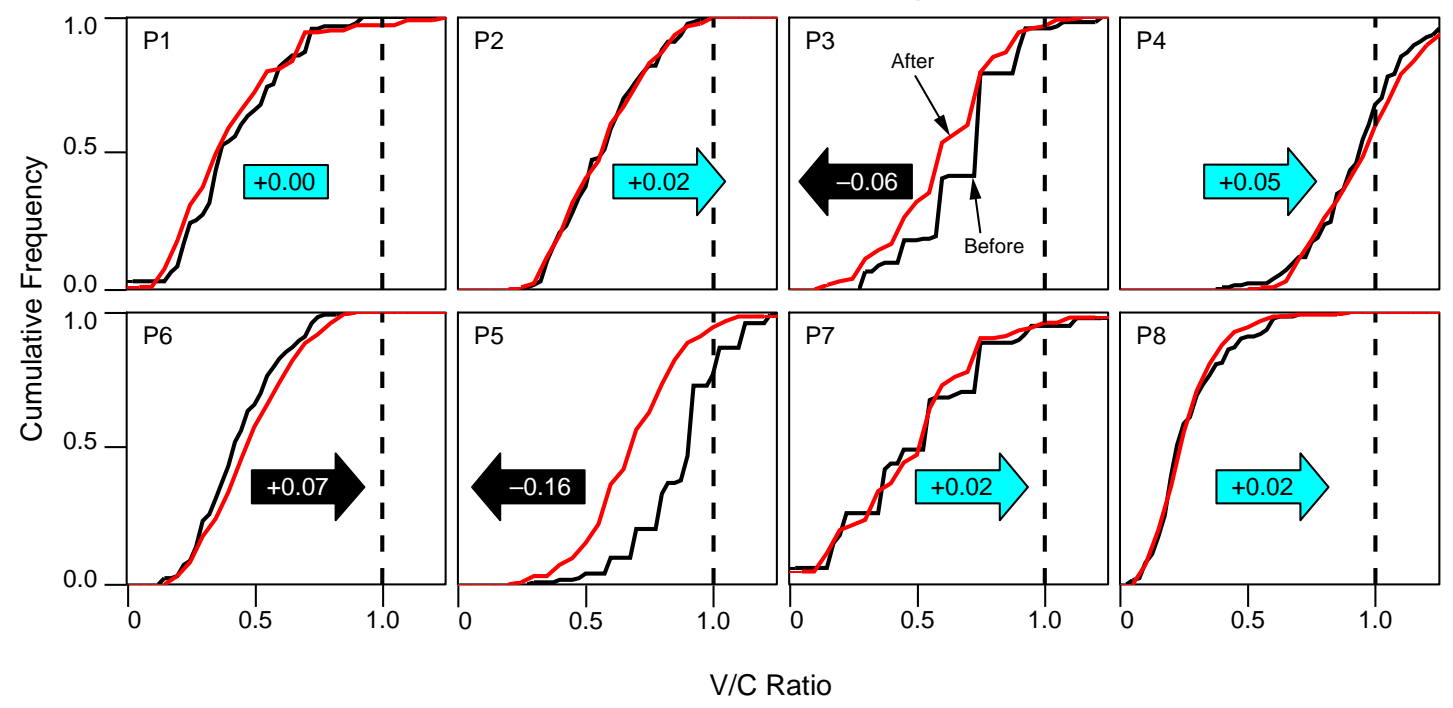

(b) 3:00 - 7:00 PM Evening Peak

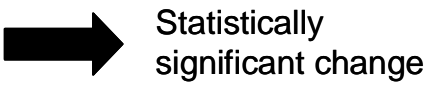

Change not

statistically significant

Figure 5.9 Graphs of cumulative frequency of v/c ratios. Arrows indicate change in average value of $v / c$ ratio. 
Table 5.1 Number of split failures reported for Wednesday, March 7, 2007 (without actuated coordinated phases) and for Wednesday, March 28, 2007 (with actuated coordinated phases) by phase and by TOD plan time period.

\begin{tabular}{|c|c|c|c|c|c|c|c|c|c|}
\hline Phase & Date & Operation & $\begin{array}{c}\text { 06:00- } \\
\text { 09:00 } \\
\end{array}$ & $\begin{array}{c}\text { 09:00- } \\
\text { 11:00 } \\
\end{array}$ & $\begin{array}{c}11: 00- \\
13: 00\end{array}$ & $\begin{array}{c}13: 00- \\
15: 00 \\
\end{array}$ & $\begin{array}{c}15: 00- \\
19: 00 \\
\end{array}$ & $\begin{array}{c}19: 00- \\
22: 00 \\
\end{array}$ & Total \\
\hline \multirow{2}{*}{1} & $3 / 07$ & non-act. & 0 & 0 & 0 & 0 & 0 & 0 & 0 \\
\hline & $3 / 28$ & act.-coord. & 1 & 0 & 1 & 0 & 3 & 0 & 5 \\
\hline \multirow{2}{*}{2} & $3 / 07$ & non-act. & 0 & 0 & 0 & 0 & 0 & 0 & 0 \\
\hline & $3 / 28$ & act.-coord. & 0 & 0 & 0 & 0 & 3 & 0 & 3 \\
\hline \multirow{2}{*}{3} & $3 / 07$ & non-act. & 14 & 12 & 7 & 7 & 5 & 4 & 49 \\
\hline & $3 / 28$ & act.-coord. & 1 & 2 & 3 & 4 & 5 & 1 & 16 \\
\hline \multirow{2}{*}{4} & $3 / 07$ & non-act. & 22 & 9 & 15 & 19 & 49 & 9 & 123 \\
\hline & $3 / 28$ & act.-coord. & 18 & 7 & 16 & 13 & 64 & 12 & 130 \\
\hline \multirow{2}{*}{5} & $3 / 07$ & non-act. & 24 & 10 & 9 & 13 & 33 & 2 & 91 \\
\hline & $3 / 28$ & act.-coord. & 4 & 4 & 7 & 4 & 11 & 0 & 30 \\
\hline \multirow{2}{*}{6} & $3 / 07$ & non-act. & 6 & 0 & 0 & 0 & 0 & 0 & 6 \\
\hline & $3 / 28$ & act.-coord. & 3 & 0 & 0 & 1 & 0 & 0 & 4 \\
\hline \multirow{2}{*}{7} & $3 / 07$ & non-act. & 1 & 4 & 4 & 4 & 5 & 3 & 21 \\
\hline & $3 / 28$ & act.-coord. & 2 & 1 & 3 & 1 & 6 & 1 & 14 \\
\hline \multirow{2}{*}{8} & $3 / 07$ & non-act. & 0 & 0 & 0 & 0 & 0 & 0 & 0 \\
\hline & $3 / 28$ & act.-coord. & 0 & 0 & 0 & 0 & 0 & 0 & 0 \\
\hline \multirow{2}{*}{\multicolumn{2}{|c|}{ Total }} & non-act. & 67 & 35 & 35 & 43 & 92 & 18 & 290 \\
\hline & & act.-coord. & 29 & 14 & 30 & 23 & 92 & 14 & 202 \\
\hline
\end{tabular}




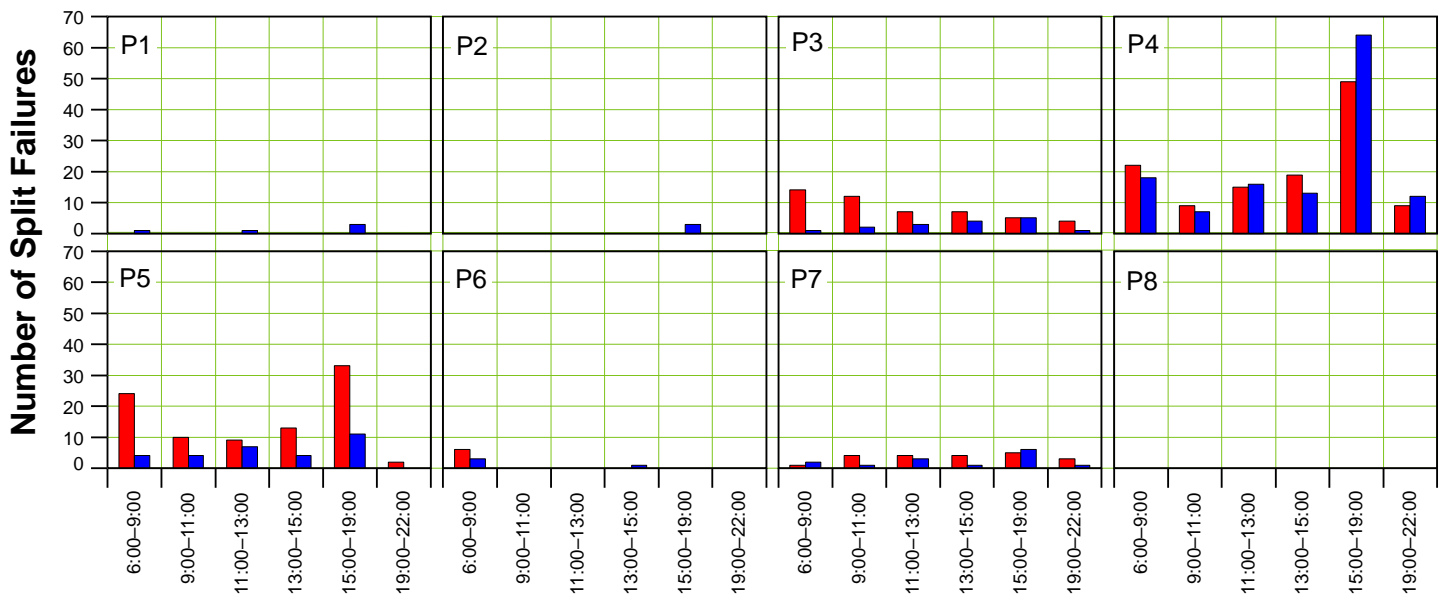

Time of Day Plan Time Period

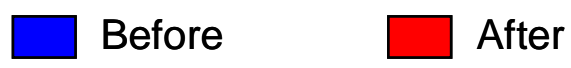

Figure 5.10 Total number of split failures by phase at Noblesville on Wednesday, March 7, 2008 (Before, without actuated coordinated phases) and Wednesday, March 28, 2007 (After, with actuated coordinated phases). 


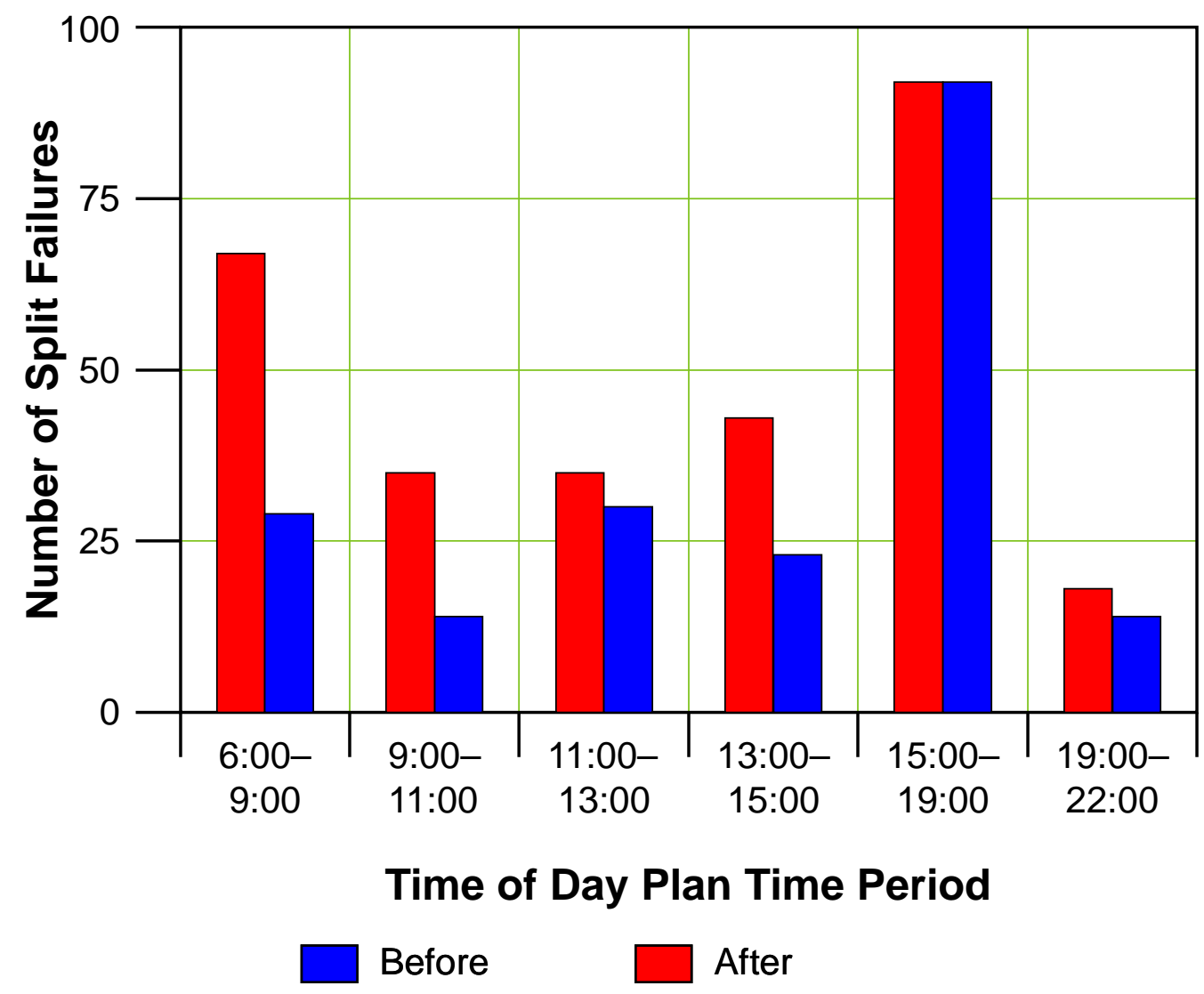

Figure 5.11 Total number of split failures for all eight phases at Noblesville on Wednesday, March 7, 2008 (Before, without actuated coordinated phases) and Wednesday, March 28, 2007 (After, with actuated coordinated phases). 


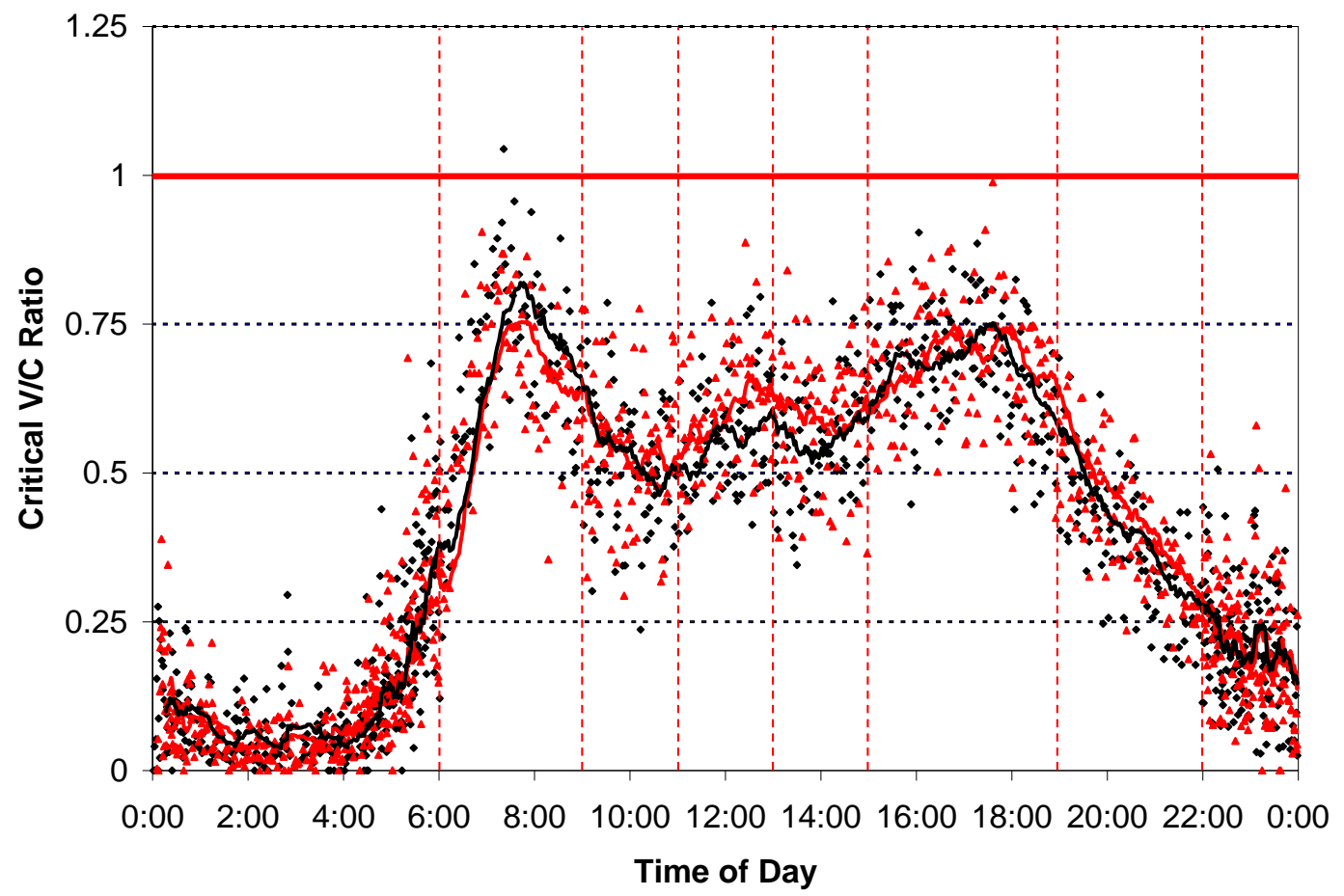

$\longrightarrow \begin{aligned} & \text { March 7, 2007, } \\ & \text { semiactuated }\end{aligned}-\begin{aligned} & \text { March 28, 2007, } \\ & \text { actuated coordinated phases }\end{aligned}$

Figure 5.12 Comparison of critical v/c ratio between Wednesday, March 7, 2007 (without actuated coordinated phases) and Wednesday, March 28, 2007 (with actuated coordinated phases). 


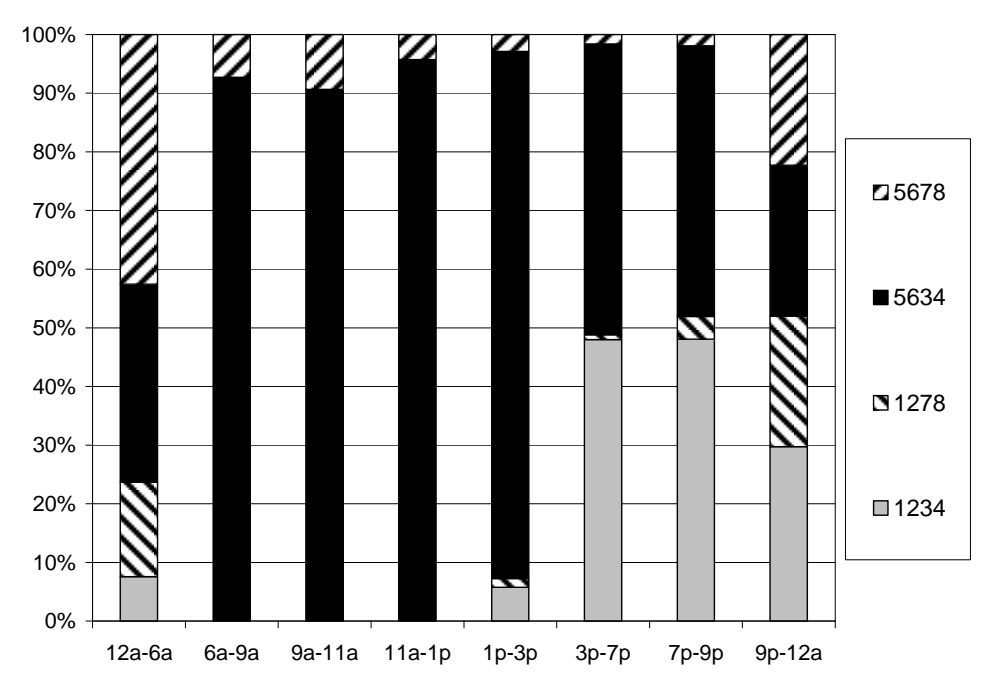

(a) Wednesday, March 7, 2007

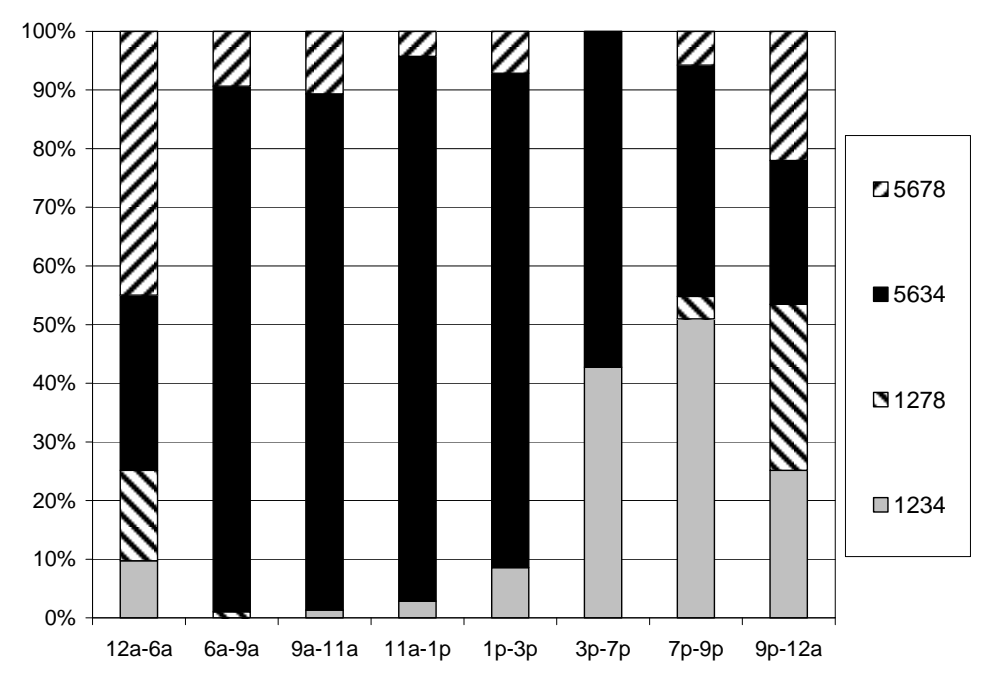

(b) Wednesday, March 28, 2007

Figure 5.13 Distribution of critical path for calculation of critical v/c ratio at Noblesville, showing the prevalence of phase pairs $(5,6)$ and $(3,4)$. 


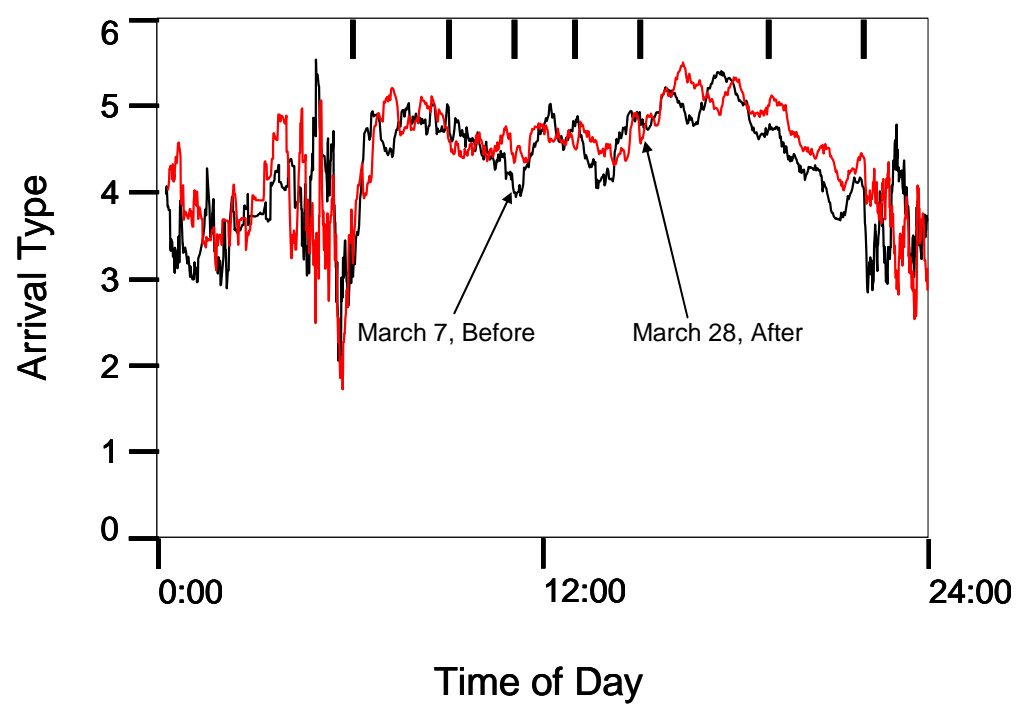

(a) Phase 2 Arrival Type

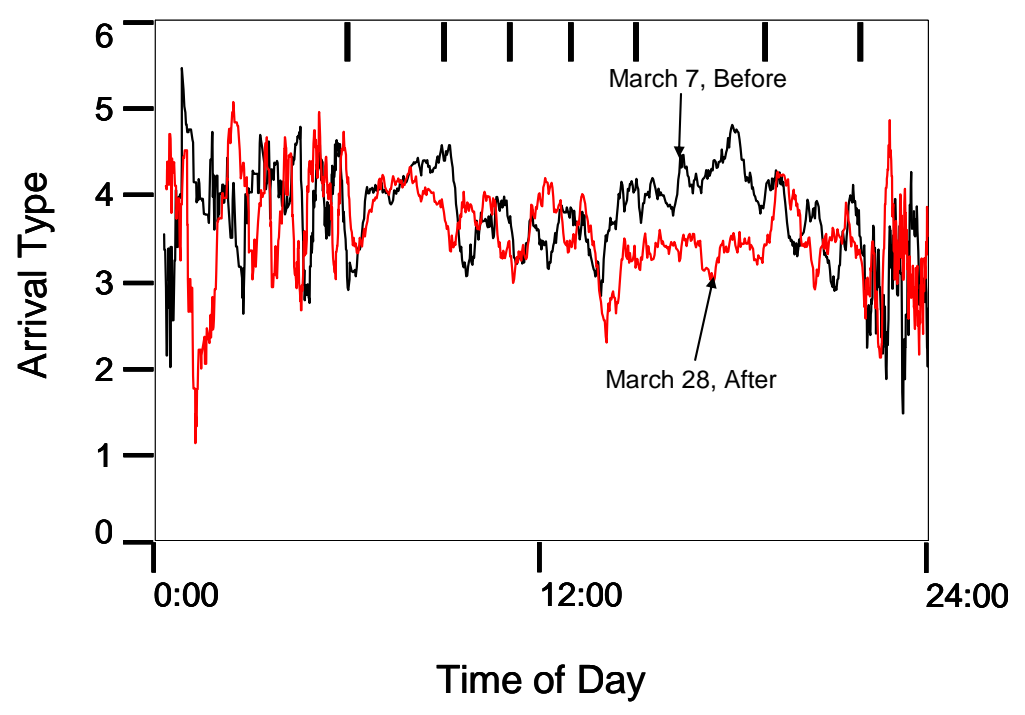

(b) Phase 6 Arrival Type

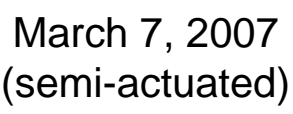

March 28, 2007 (actuated coordinated phases)

Figure 5.14 Arrival type by time of day, for (a) phase 2 and (b) phase 6 for Wednesday, March 7 (with non-actuated coordinated phases) and Wednesday, March 28, 2007 (with actuated coordinated phases). 


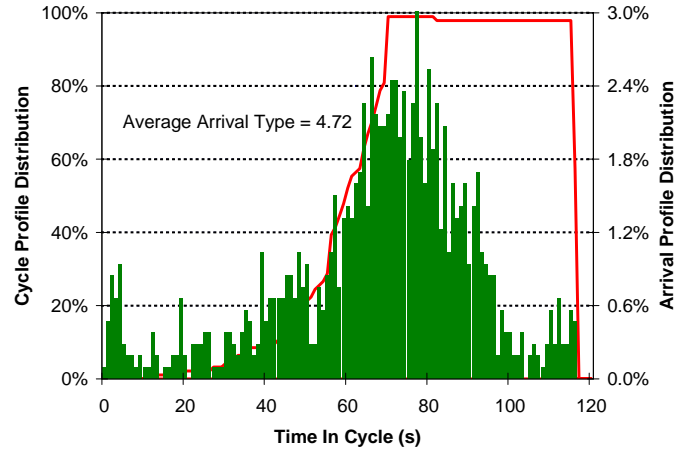

(a) March 7, 06:00-09:00 AM Peak (without actuated coordinated phases)

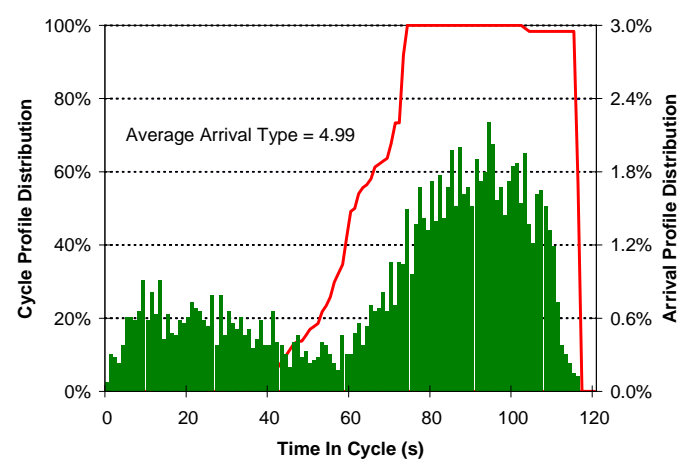

(c) March 7, 15:00-19:00 PM Peak (without actuated coordinated phases)

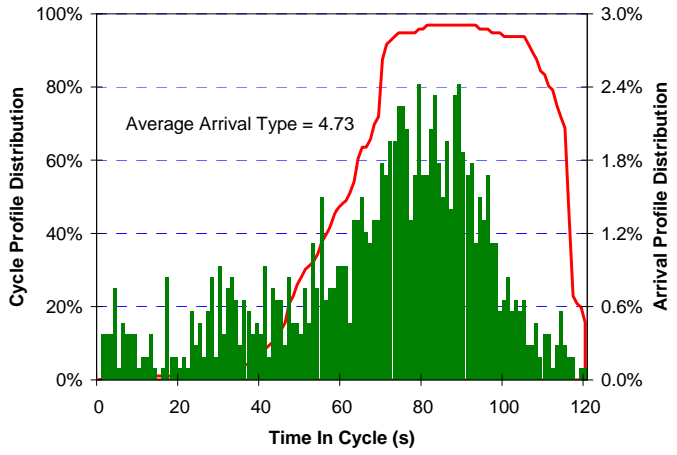

(b) March 28, 06:00-09:00 AM Peak (with actuated coordinated phases)

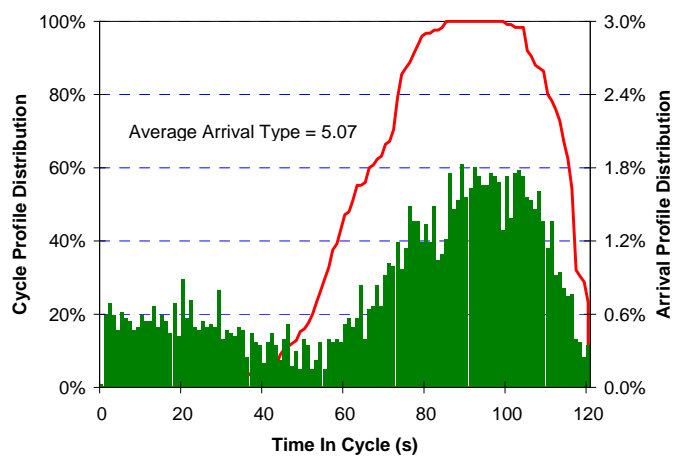

(d) March 28, 15:00-19:00 PM Peak (with actuated coordinated phases)

Figure 5.15 Platoon profile charts for Phase 2 at Noblesville on Wednesday, March 7 and Wednesday, March 28, 2007. 


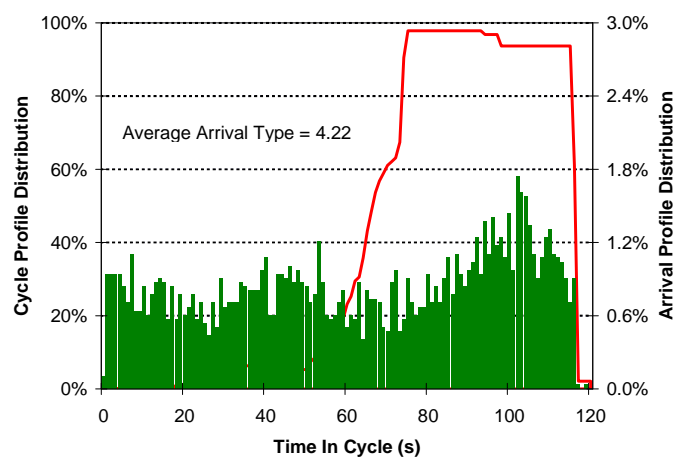

(a) March 7, 06:00-09:00 AM Peak (without actuated coordinated phases)

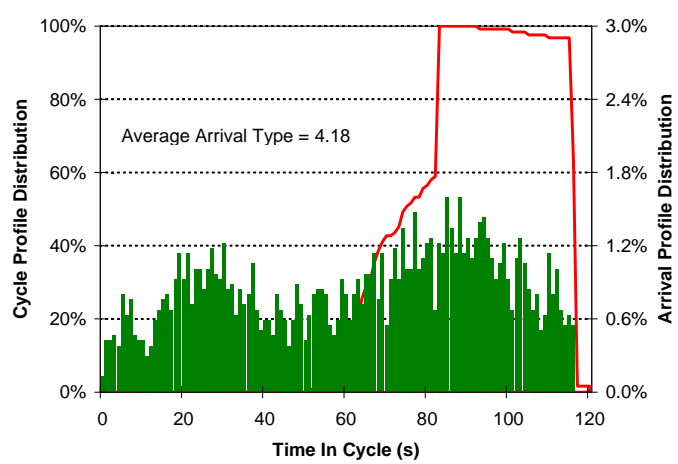

(c) March 7, 15:00-19:00 PM Peak (without actuated coordinated phases)

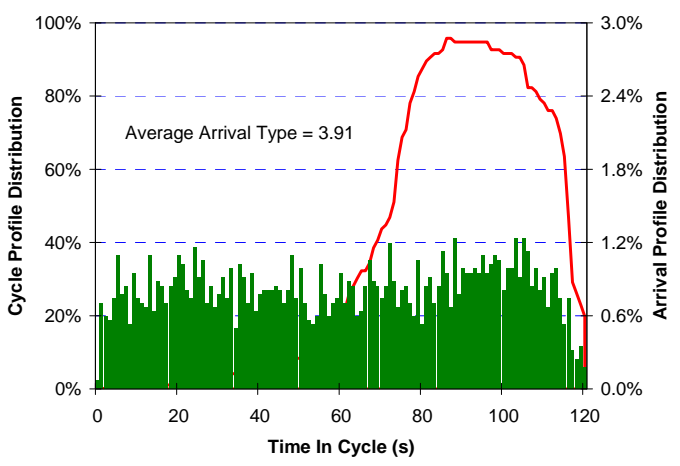

(b) March 28, 06:00-09:00 AM Peak (with actuated coordinated phases)

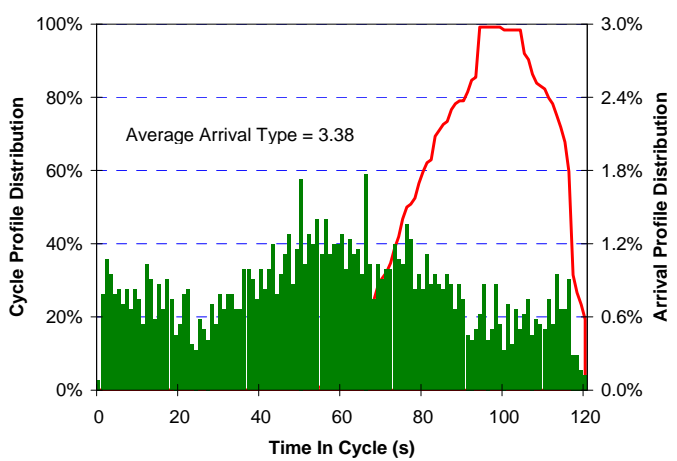

(d) March 28, 15:00-19:00 PM Peak (with actuated coordinated phases)

Figure 5.16 Platoon profile charts for Phase 6 at Noblesville on Wednesday, March 7 and Wednesday, March 28, 2007. 


\subsubsection{Statistical Analysis}

To evaluate the changes that took place at the Noblesville test intersection, a statistical analysis was carried out that compared the states of performance measures in the before and after cases. Statistical t-tests were used, which were explained in Chapter 4. A pairwise comparison was conducted for the pair of days examined in the previous section. A comparison of data aggregated over the two three-week before and after periods was executed to reveal the overall trend.

\subsubsection{Pairwise Statistical Comparison}

Statistical t-tests were carried out for the pair of days (March 7 and March 28, 2007) that was selected for qualitative analysis. Differences in mean volumes are shown in Table 5.2(a); corresponding statistical t-values and P-values are given in Table 5.3(a) and Table 5.4(b) respectively. In these tables, values are shown for all eight phases for each time period defined by the TOD plan (see Table 2.2). Statistically significant values are shown in boldface. Volumes on March 7 and March 28 were not significantly different in 43 out of 48 cases. Because of this, we do not attribute differences in green time, v/c ratio, and AT to differences in volume for those cases; instead, we attribute those differences to changes in operation.

Changes in mean green time that were observed in Figure 5.5 are confirmed by the differences of means in Table 5.2(b), with statistical t-values in

Table 5.3(b) and P-values in Table 5.4(b). Phase 6 saw a significant amount of reduction throughout the entire day. Phase 2 also saw a reduction, but it was only significant during the 19:00-22:00 time period. Significant increases were observed for phases 3, 5, and 7 . Phase 8 also saw an increase in green time, but it was not statistically significant. These changes correspond with our expectations, with phases 2 and 6 shifting green time to following phases.

Mean values of v/c ratio also changed for various phases, as indicated by the difference in cycle based mean $\mathrm{v} / \mathrm{c}$ ratios given in Table 5.2(c). The t-statistics are presented in Table 5.3(c) and the P-values are given in Table 5.4(c). As we observed earlier, with similar volumes, the differences in mean $\mathrm{v} / \mathrm{c}$ ratios occurred inverse to changes in mean green time. Shorter green durations on phases 2 and 6 led to increased $\mathrm{v} / \mathrm{c}$ ratios. This had a greater impact on phase 6 . Meanwhile, phases 3, 5, and 7 gained 
green time, leading to decreases of their $\mathrm{v} / \mathrm{c}$ ratios. These changes were more significant for phases 3 and 5 .

The difference in mean values of AT are given in Table 5.2(d), with t-statistics in Table 5.3(d) and P-values in Table 5.4(d). Phase 2 arrival types do not show any negative impact. Rather, a slight increase is seen, due to increasing values of $\mathrm{C} / \mathrm{g}$ that result from decreasing green times with consistent proportions of arrivals on green (P). The impact on Phase 6 AT varied by time of day, but significant decreases were seen during in the AM and PM peak hours. As stated earlier, these decreases are attributed to an unforeseen interaction with the upstream signal occurring in the before case that was not present in the after case. The attributes of the interaction are unclear; it only appears to have an impact during the peak hours. For random arrivals, AT would be expected to be approximately equal to 3 in both cases. This is seen in the after case.

\subsubsection{Aggregate Statistical Comparison}

To verify whether the trends of the pairwise comparison may be generalized across the entire analysis period, we compared aggregate performance measures for three weeks of operation with non-actuated coordinated phases to three weeks with actuated coordinated phases. The method of aggregation was described in Chapter 4 .

Table 5.5(a) shows differences in mean volumes by phase and time of day, with corresponding t-values appearing in Table 5.6(a), and P-values in Table 5.7(a). As is evident in both tables, volumes in the 3 weeks with actuated coordinated phases were significantly higher than volumes in 3 weeks with non-actuated coordinated phases in 22 out of 48 cases. Whether or not the volume changes have a significant impact on the data is unclear. However, this is not believed this to be the case, as very similar trends occurred in both statistical analyses.

Differences in mean green times are shown in Table 5.5(b), with t-statistics in Table 5.6(b) and P-values in Table 5.7(b). The differences are similar to those seen in the pairwise comparison of similar days in Table 5.2(b). Despite increases in volume, green durations of phases 2 and 6 decreased, while those of phases 3, 5, 7, and 8 increased. These changes are consistently significant across virtually all time periods. Phase 4 saw significant increases in green time in 3 out of 6 time periods; this phase was not seen to benefit at all in the pairwise comparison. Similarly, phase 1 reports increases in green time, something that was not seen in the pairwise comparison. When the data is aggregated across three week comparison periods, increases in green time for all noncoordinated phases are observed. 
The changes in $\mathrm{v} / \mathrm{c}$ ratios are presented in Table 5.5(c); t-statistics are in Table 5.6(c) while P-values are in Table 5.7(c). Even though traffic volumes significantly increased for many phases and time periods, $\mathrm{v} / \mathrm{c}$ ratio changes remained consistent with our expectations from the reallocation of green time. Phases 2 and 6 saw increases in v/c ratios throughout most time periods, while phases 3, 5, 7, and 8 decreased. Volumes for phases 7 and 8 were similar, yet $\mathrm{v} / \mathrm{c}$ ratios for those phases decreased, confirming the impact of using actuated coordinated phases. Even though volumes for phases 3 and 5 increased, $v / c$ ratios decreased, further confirming that these phases benefit from using actuated coordinated phases. Unfortunately, the benefits for phases 1 and 4 were totally obscured by increased volumes. Phases 1 and 4 both report higher v/c ratios; this is attributed to higher volumes, as increased green time should have decreased the $\mathrm{v} / \mathrm{c}$ ratios.

The coordinated phases ( 2 and 6) sustained increased v/c ratios due to decreased green time. However, the resulting $\mathrm{v} / \mathrm{c}$ ratios remain safely far from saturation. 15weekday average $\mathrm{v} / \mathrm{c}$ ratios for all eight phases by TOD plan time are shown in Table 5.8. The $\mathrm{v} / \mathrm{c}$ ratio for phase 2, for example, across 15 weekdays for all time periods was 0.34 , without actuated coordinated phases. After actuated coordinated phases were implemented, the overall $\mathrm{v} / \mathrm{c}$ ratio increased to 0.375 . The 15 -day average $\mathrm{v} / \mathrm{c}$ ratios for phase 2 in the PM peak period (15:00-19:00) were 0.65 and 0.69 in the before and after cases respectively. Phase 6 increased similarly from 0.44 to 0.51 across all time periods, and from 0.62 to 0.69 during the AM peak period (06:00-09:00). The v/c ratios of all the other phases decreased, except for phases 1 and 4. These phases sustained increased volumes that increased their $\mathrm{v} / \mathrm{c}$ ratios.

Counts of split failures for each 15-weekday analysis period are shown in Table 5.9; the aggregate totals for the eight phases are shown in Figure 5.17, while the intersection totals are shown in Figure 5.18. The percentage of cycles ending in split failure are given in Table 5.10; Figure 5.19 shows the averages for each of the eight phases, while the intersection average percentages of split failures are shown in Figure 5.20. These numbers are tabulated for all 15 days of data for the before case (without actuated coordinated phases) and for the after case (with actuated coordinated phases). The reduction in the number of split failures is similar to what was found in the pairwise comparison of similar days. The total number of split failures was reduced from 4,111 to 3,367, a decrease of $18 \%$. Decreases for phases 5, 3, and 7 were considerable. Phase 5, for example, reported a reduction of about $50 \%$ altogether. During the PM peak period (15:00-19:00), phase 5 went from having nearly $30 \%$ of its cycles ending with split failures to $14 \%$. The weakest improvement for phase 5 took place during the AM peak 
period, showing that phase 6 retained green time according to high demand for the southbound movement. Phases 3 and 7 also report significant improvements. An increase is seen in the total number of split failures for phases 2 and 6 , mainly during the peak periods. This is due to the reduction of green time for those phases, combined with slightly higher demand. The additional split failures occurred mainly during the peak periods. The percentage of cycles ending with split failures for phases 2 and 6 is still very low.

Phases 1 and 4 show increases in the number of split failures, as in the pairwise comparison. In the pairwise comparison, the increases for phase 4 were due to a loss of green time caused by an increase in demand for phase 3, while the increases for phase 1 were attributed to random variation. For the aggregate case, there are several competing trends involving these phases. Volumes increased for phases 1, 3, and 4. The amount of green time available to all of these phases also increased, overall. However, while phase 3 saw decreases in $\mathrm{v} / \mathrm{c}$ ratios, the $\mathrm{v} / \mathrm{c}$ ratios for phases 1 and 4 increasesd. In the case of phase 1, it would appear that the higher volume of demand was large enough to eliminate any benefit gained from added green time. Phase 4 had more split failures for the same reason; also, the increase in volume for phase 3 prevented phase 4 from obtaining enough green time to relieve its high $\mathrm{v} / \mathrm{c}$ ratios.

Changes in AT are given in Table 5.5(d), with t-statistics in Table 5.6(d) and Pvalues in Table 5.7(d). Phase 6 saw decreases in AT during the AM and PM peak time periods. These results are consistent with those observed in the pairwise comparison. As discussed earlier, the changes in AT for phase 6 are attributed to an interaction taking place during the peak periods that disappeared when operation was changed. Phase 2 AT, on the other hand, improved across all six time periods, significantly in three of them, revealing more efficient service of the coordinated phase. The same value of $P$ is achieved using less green time in the same cycle length as before. If progression was adversely affected, AT would not have increased. 
Table 5.2 Difference in means (significant in boldface) for the pairwise comparison of operation on March 7 (without actuated coordinated phases) and March 28, 2007 (with actuated coordinated phases) at Noblesville, IN.

(a) Volume $(\mathrm{veh} / \mathrm{h} / \mathrm{ln})$

\begin{tabular}{|c|r|r|r|r|r|r|r|r|}
\hline Time & \multicolumn{1}{|c|}{1} & \multicolumn{1}{c|}{2} & \multicolumn{1}{c|}{3} & \multicolumn{1}{c|}{4} & \multicolumn{1}{c|}{5} & \multicolumn{1}{c|}{6} & \multicolumn{1}{c|}{7} & \multicolumn{1}{c|}{8} \\
\hline 06:00-09:00 & -6 & +7 & -53 & +13 & +4 & -45 & -7 & $-\mathbf{7 8}$ \\
\hline 09:00-11:00 & +7 & +12 & -8 & +9 & +14 & +43 & -4 & +29 \\
\hline 11:00-13:00 & -3 & +16 & +13 & +9 & $+\mathbf{4 5}$ & +25 & +8 & +38 \\
\hline 13:00-15:00 & -4 & -36 & +9 & +21 & +7 & +28 & -4 & +7 \\
\hline 15:00-19:00 & -1 & +17 & $+\mathbf{1 4}$ & -21 & +5 & +16 & +14 & +9 \\
\hline 19:00-22:00 & -8 & +35 & +14 & +16 & +2 & +35 & -7 & -2 \\
\hline
\end{tabular}

(b) Green Time (s)

\begin{tabular}{|c|c|c|c|c|c|c|c|c|}
\hline Time & 1 & 2 & 3 & 4 & 5 & 6 & 7 & 8 \\
\hline 06:00-09:00 & -0.6 & -1.4 & +0.1 & +0.6 & +3.3 & -4.0 & +0.5 & +0.5 \\
\hline 09:00-11:00 & +0.7 & -3.2 & +2.3 & -0.3 & +3.1 & -4.2 & +0.8 & +1.1 \\
\hline 11:00-13:00 & -0.3 & -2.4 & +2.6 & +0.0 & +1.5 & -3.8 & +1.3 & +1.0 \\
\hline 13:00-15:00 & -0.9 & -2.2 & +2.2 & +2.2 & +3.7 & -7.0 & +0.6 & +3.1 \\
\hline 15:00-19:00 & -0.1 & -1.0 & +2.4 & -2.8 & +4.6 & -4.6 & +1.1 & -1.8 \\
\hline 19:00-22:00 & -0.3 & -5.8 & +3.1 & +0.5 & +1.6 & -6.5 & +1.9 & +2.0 \\
\hline
\end{tabular}

(c) $\mathrm{v} / \mathrm{c}$ Ratio

\begin{tabular}{|c|c|c|c|c|c|c|c|c|}
\hline Time & 1 & 2 & 3 & 4 & 5 & 6 & 7 & 8 \\
\hline 06:00-09:00 & -0.01 & +0.00 & $\mathbf{- 0 . 1 7}$ & +0.00 & $\mathbf{- 0 . 1 6}$ & +0.02 & -0.07 & $\mathbf{- 0 . 0 8}$ \\
\hline 09:00-11:00 & +0.01 & +0.03 & $\mathbf{- 0 . 1 7}$ & +0.02 & $\mathbf{- 0 . 0 8}$ & $+\mathbf{0 . 0 9}$ & -0.06 & +0.01 \\
\hline 11:00-13:00 & -0.01 & +0.02 & -0.07 & +0.03 & +0.06 & $+\mathbf{0 . 0 7}$ & -0.02 & +0.03 \\
\hline 13:00-15:00 & +0.02 & -0.01 & $\mathbf{- 0 . 0 9}$ & -0.03 & $\mathbf{- 0 . 1 5}$ & $\mathbf{+ 0 . 1 0}$ & -0.05 & -0.03 \\
\hline 15:00-19:00 & +0.00 & +0.02 & $\mathbf{- 0 . 0 6}$ & $+\mathbf{0 . 0 5}$ & $\mathbf{- 0 . 1 6}$ & $+\mathbf{0 . 0 7}$ & +0.02 & +0.02 \\
\hline 19:00-22:00 & -0.04 & $\mathbf{+ 0 . 0 4}$ & $\mathbf{- 0 . 0 9}$ & +0.04 & -0.05 & $\mathbf{+ 0 . 0 5}$ & $\mathbf{- 0 . 1 2}$ & -0.02 \\
\hline
\end{tabular}

(d) Arrival Type

\begin{tabular}{|c|c|c|c|c|c|c|c|c|}
\hline Time & 1 & 2 & 3 & 4 & 5 & 6 & 7 & 8 \\
\hline $06: 00-09: 00$ & & +0.01 & & & & $-\mathbf{0 . 3 1}$ & & \\
\hline $09: 00-11: 00$ & & +0.09 & & & & +0.09 & & \\
\hline $11: 00-13: 00$ & & -0.04 & & & & +0.10 & & \\
\hline $13: 00-15: 00$ & & +0.11 & & & & -0.51 & & \\
\hline $15: 00-19: 00$ & & +0.08 & & & & $\mathbf{- 0 . 8 0}$ & & \\
\hline $19: 00-22: 00$ & & $+\mathbf{0 . 3 7}$ & & & & +0.00 & & \\
\hline
\end{tabular}


Table 5.3 Statistical t-values (significant in boldface) for the pairwise comparison of operation on March 7 (without actuated coordinated phases) and March 28, 2007 (with actuated coordinated phases) at Noblesville, IN.

(a) Volume

\begin{tabular}{|c|r|r|r|r|r|r|r|r|}
\hline Time & \multicolumn{1}{|c|}{1} & \multicolumn{1}{c|}{2} & \multicolumn{1}{c|}{3} & \multicolumn{1}{c|}{4} & \multicolumn{1}{c|}{5} & 6 & \multicolumn{1}{c|}{7} & \multicolumn{1}{c|}{8} \\
\hline 06:00-09:00 & -0.8 & +0.3 & $\mathbf{- 4 . 0}$ & +0.8 & +0.4 & -1.2 & -0.8 & $-\mathbf{3 . 1}$ \\
\hline 09:00-11:00 & +0.7 & +0.6 & -0.8 & +0.5 & +1.0 & +1.5 & -0.3 & +1.4 \\
\hline 11:00-13:00 & -0.4 & +0.7 & +1.3 & +0.5 & +3.0 & +0.9 & +0.7 & +2.0 \\
\hline 13:00-15:00 & -0.4 & -1.2 & +0.9 & +1.0 & +0.5 & +1.0 & -0.3 & +0.3 \\
\hline 15:00-19:00 & -0.1 & +0.6 & $+\mathbf{2 . 0}$ & -1.2 & +0.6 & +0.8 & +1.8 & +0.6 \\
\hline 19:00-22:00 & -1.2 & +1.3 & +1.4 & +1.1 & +0.2 & +1.9 & -0.8 & -0.1 \\
\hline
\end{tabular}

(b) Green Time

\begin{tabular}{|c|c|c|c|c|c|c|c|c|}
\hline Time & 1 & 2 & 3 & 4 & 5 & 6 & 7 & 8 \\
\hline 06:00-09:00 & -1.3 & -0.8 & +0.1 & +0.7 & +6.3 & -2.7 & +2.2 & +0.4 \\
\hline 09:00-11:00 & +1.3 & -1.8 & +5.6 & -0.3 & +6.4 & -3.5 & +2.1 & +0.6 \\
\hline 11:00-13:00 & -0.6 & -1.5 & +5.3 & -0.0 & +2.8 & -4.0 & +3.3 & +0.7 \\
\hline $13: 00-15: 00$ & -1.4 & -1.1 & +4.7 & +1.4 & +6.3 & -5.0 & +2.5 & +1.9 \\
\hline 15:00-19:00 & -0.2 & -0.7 & +7.1 & -2.3 & +11.1 & -4.1 & +4.3 & -1.3 \\
\hline $19: 00-22: 00$ & -0.8 & -2.7 & +6.6 & +0.6 & +5.3 & -2.4 & +3.7 & +1.7 \\
\hline
\end{tabular}

(c) $\mathrm{v} / \mathrm{c}$ Ratio

\begin{tabular}{|c|c|c|c|c|c|c|c|c|}
\hline Time & 1 & 2 & 3 & 4 & 5 & 6 & 7 & 8 \\
\hline 06:00-09:00 & -0.3 & +0.3 & -6.1 & +0.1 & -4.1 & +0.6 & -1.2 & -3.5 \\
\hline 09:00-11:00 & +0.3 & +1.8 & -4.0 & +0.5 & -2.3 & +3.1 & -0.9 & +0.5 \\
\hline 11:00-13:00 & -0.3 & +1.6 & -2.0 & +0.7 & +1.8 & +2.8 & -0.4 & +1.4 \\
\hline 13:00-15:00 & +0.7 & -0.3 & -2.2 & -0.8 & -4.5 & +3.0 & -0.7 & -1.0 \\
\hline 15:00-19:00 & +0.1 & +1.0 & -2.4 & +2.1 & -7.0 & +3.1 & +0.5 & +0.9 \\
\hline 19:00-22:00 & -1.6 & +2.6 & -2.4 & +1.2 & -1.5 & +3.2 & -2.7 & -0.9 \\
\hline
\end{tabular}

(d) Arrival Type

\begin{tabular}{|l|r|r|r|r|r|r|r|c|}
\hline \multicolumn{1}{|c|}{ Time } & 1 & 2 & 3 & 4 & 5 & 6 & 7 & 8 \\
\hline $06: 00-09: 00$ & & +0.0 & & & & -2.5 & & \\
\hline $09: 00-11: 00$ & & +0.5 & & & & +0.4 & & \\
\hline $11: 00-13: 00$ & & -0.3 & & & & +0.5 & & \\
\hline $13: 00-15: 00$ & & +0.7 & & & & -2.0 & & \\
\hline $15: 00-19: 00$ & & +0.9 & & & & -5.6 & & \\
\hline $19: 00-22: 00$ & & +3.8 & & & & -0.0 & & \\
\hline
\end{tabular}


Table 5.4 Statistical P-values (significant in boldface) for the pairwise comparison of operation on March 7 (without actuated coordinated phases) and March 28, 2007 (with actuated coordinated phases) at Noblesville, IN.

(a) Volume

\begin{tabular}{|c|c|c|r|r|r|r|r|r|}
\hline Time & 1 & 2 & \multicolumn{1}{|c|}{3} & 4 & \multicolumn{1}{c|}{5} & 6 & 7 & \multicolumn{1}{c|}{8} \\
\hline 06:00-09:00 & $41.6 \%$ & $77.2 \%$ & $\mathbf{0 . 0} \%$ & $43.4 \%$ & $72.0 \%$ & $24.7 \%$ & $40.5 \%$ & $\mathbf{0 . 3 \%}$ \\
\hline 09:00-11:00 & $50.3 \%$ & $58.0 \%$ & $45.3 \%$ & $64.7 \%$ & $33.4 \%$ & $13.9 \%$ & $74.4 \%$ & $17.8 \%$ \\
\hline 11:00-13:00 & $72.0 \%$ & $48.8 \%$ & $20.5 \%$ & $63.2 \%$ & $\mathbf{0 . 3 \%}$ & $35.7 \%$ & $46.0 \%$ & $\mathbf{4 . 5 \%}$ \\
\hline 13:00-15:00 & $65.9 \%$ & $22.7 \%$ & $38.6 \%$ & $32.4 \%$ & $60.6 \%$ & $30.1 \%$ & $76.5 \%$ & $73.0 \%$ \\
\hline 15:00-19:00 & $88.8 \%$ & $53.1 \%$ & $\mathbf{4 . 8 \%}$ & $21.8 \%$ & $56.3 \%$ & $45.4 \%$ & $7.8 \%$ & $55.3 \%$ \\
\hline 19:00-22:00 & $23.3 \%$ & $19.0 \%$ & $16.3 \%$ & $26.2 \%$ & $85.3 \%$ & $6.4 \%$ & $41.9 \%$ & $89.8 \%$ \\
\hline
\end{tabular}

(b) Green Time

\begin{tabular}{|c|c|c|c|c|c|c|c|c|}
\hline Time & 1 & 2 & 3 & 4 & 5 & 6 & 7 & 8 \\
\hline 06:00-09:00 & $20.1 \%$ & $43.4 \%$ & $91.5 \%$ & $49.3 \%$ & $0.0 \%$ & $0.8 \%$ & $3.2 \%$ & $72.6 \%$ \\
\hline 09:00-11:00 & $20.1 \%$ & $8.2 \%$ & $0.0 \%$ & $80.0 \%$ & $0.0 \%$ & $0.1 \%$ & $3.4 \%$ & $52.2 \%$ \\
\hline 11:00-13:00 & $53.6 \%$ & $14.0 \%$ & $0.0 \%$ & $99.9 \%$ & $0.6 \%$ & $0.0 \%$ & $0.1 \%$ & $48.7 \%$ \\
\hline $13: 00-15: 00$ & $16.1 \%$ & $28.6 \%$ & $0.0 \%$ & $17.2 \%$ & $0.0 \%$ & $0.0 \%$ & $1.5 \%$ & $6.5 \%$ \\
\hline $15: 00-19: 00$ & $80.4 \%$ & $49.9 \%$ & $0.0 \%$ & $2.1 \%$ & $0.0 \%$ & $0.0 \%$ & $0.0 \%$ & $18.2 \%$ \\
\hline 19:00-22:00 & $41.6 \%$ & $0.8 \%$ & $0.0 \%$ & $53.5 \%$ & $0.0 \%$ & $1.8 \%$ & $0.0 \%$ & $8.3 \%$ \\
\hline
\end{tabular}

(c) $\mathrm{v} / \mathrm{c}$ Ratio

\begin{tabular}{|c|c|r|r|r|r|r|c|c|}
\hline Time & 1 & \multicolumn{1}{|c|}{2} & \multicolumn{1}{c|}{3} & 4 & 5 & 6 & 7 & 8 \\
\hline 06:00-09:00 & $73.8 \%$ & $74.6 \%$ & $\mathbf{0 . 0 \%}$ & $95.7 \%$ & $\mathbf{0 . 0} \%$ & $56.7 \%$ & $22.4 \%$ & $\mathbf{0 . 1 \%}$ \\
\hline 09:00-11:00 & $77.3 \%$ & $7.6 \%$ & $\mathbf{0 . 0 \%}$ & $62.7 \%$ & $\mathbf{2 . 6 \%}$ & $\mathbf{0 . 3 \%}$ & $37.9 \%$ & $60.6 \%$ \\
\hline 11:00-13:00 & $76.2 \%$ & $11.3 \%$ & $5.0 \%$ & $48.8 \%$ & $7.7 \%$ & $\mathbf{0 . 6 \%}$ & $69.2 \%$ & $16.0 \%$ \\
\hline 13:00-15:00 & $47.9 \%$ & $78.6 \%$ & $\mathbf{2 . 7 \%}$ & $44.4 \%$ & $\mathbf{0 . 0 \%}$ & $\mathbf{0 . 4 \%}$ & $46.5 \%$ & $31.7 \%$ \\
\hline 15:00-19:00 & $92.7 \%$ & $32.9 \%$ & $\mathbf{1 . 7 \%}$ & $\mathbf{4 . 1 \%}$ & $\mathbf{0 . 0 \%}$ & $\mathbf{0 . 2 \%}$ & $60.7 \%$ & $34.6 \%$ \\
\hline 19:00-22:00 & $11.9 \%$ & $\mathbf{0 . 9 \%}$ & $\mathbf{1 . 8 \%}$ & $21.9 \%$ & $12.6 \%$ & $\mathbf{0 . 2 \%}$ & $\mathbf{0 . 9 \%}$ & $37.5 \%$ \\
\hline
\end{tabular}

(d) Arrival Type

\begin{tabular}{|c|c|c|c|c|c|c|c|c|}
\hline Time & 1 & 2 & 3 & 4 & 5 & 6 & 7 & 8 \\
\hline $06: 00-09: 00$ & & $97.5 \%$ & & & & $\mathbf{1 . 2 \%}$ & & \\
\hline $09: 00-11: 00$ & & $59.4 \%$ & & & & $67.5 \%$ & & \\
\hline $11: 00-13: 00$ & & $77.9 \%$ & & & & $61.8 \%$ & & \\
\hline $13: 00-15: 00$ & & $46.9 \%$ & & & & $5.1 \%$ & & \\
\hline $15: 00-19: 00$ & & $34.7 \%$ & & & & $\mathbf{0 . 0 \%}$ & & \\
\hline $19: 00-22: 00$ & & $\mathbf{0 . 0 \%}$ & & & & $98.7 \%$ & & \\
\hline
\end{tabular}


Table 5.5 Difference in means (significant in boldface) for the 15-weekday aggregate comparison of operation with and without actuated coordinated phases at Noblesville, IN.

(a) Volume (veh/h/ln)

\begin{tabular}{|c|r|r|r|r|r|r|r|r|}
\hline Time & \multicolumn{1}{|c|}{1} & \multicolumn{1}{c|}{2} & \multicolumn{1}{c|}{3} & \multicolumn{1}{c|}{4} & \multicolumn{1}{c|}{5} & \multicolumn{1}{c|}{6} & \multicolumn{1}{c|}{7} & \multicolumn{1}{c|}{8} \\
\hline 06:00-09:00 & $\mathbf{+ 6}$ & $\mathbf{+ 2 2}$ & +9 & $\mathbf{+ 1 7}$ & $\mathbf{+ 2 0}$ & +6 & +1 & -3 \\
\hline 09:00-11:00 & $\mathbf{+ 1 0}$ & $\mathbf{+ 2 8}$ & $\mathbf{+ 1 3}$ & $\mathbf{+ 3 0}$ & $\mathbf{+ 1 2}$ & $\mathbf{+ 3 1}$ & -5 & +12 \\
\hline $11: 00-13: 00$ & $\mathbf{+ 9}$ & $\mathbf{+ 3 4}$ & $\mathbf{+ 1 6}$ & +13 & $\mathbf{+ 1 5}$ & +6 & -1 & +7 \\
\hline $13: 00-15: 00$ & $\mathbf{+ 1 0}$ & +30 & $\mathbf{+ 1 4}$ & $+\mathbf{3 1}$ & $\mathbf{+ 1 1}$ & +25 & -4 & +5 \\
\hline $15: 00-19: 00$ & $+\mathbf{+}$ & +16 & $\mathbf{+ 1 2}$ & -1 & +6 & +22 & +4 & +2 \\
\hline $19: 00-22: 00$ & +4 & +30 & $\mathbf{+ 1 8}$ & $\mathbf{+ 1 5}$ & +3 & +35 & 0 & +10 \\
\hline
\end{tabular}

(b) Green Time (s)

\begin{tabular}{|c|c|c|c|c|c|c|c|c|}
\hline Time & 1 & 2 & 3 & 4 & 5 & 6 & 7 & 8 \\
\hline 06:00-09:00 & +0.2 & -2.1 & +1.3 & +0.6 & +2.8 & -4.6 & +0.4 & +2.0 \\
\hline 09:00-11:00 & +0.4 & -4.1 & +2.2 & +1.0 & +2.2 & -5.2 & +0.5 & +2.5 \\
\hline $11: 00-13: 00$ & +0.4 & -2.6 & +2.1 & -0.3 & +1.1 & -3.3 & +0.6 & +1.7 \\
\hline $13: 00-15: 00$ & +0.4 & -3.5 & +1.8 & +1.1 & +3.2 & -5.9 & +0.7 & +2.8 \\
\hline $15: 00-19: 00$ & +0.4 & -2.3 & +1.7 & -0.3 & +3.2 & -4.6 & +0.7 & +0.8 \\
\hline $19: 00-22: 00$ & -0.1 & -5.1 & +2.6 & +0.8 & +1.7 & -5.9 & +1.4 & +3.0 \\
\hline
\end{tabular}

(c) $\mathrm{v} / \mathrm{c}$ Ratio

\begin{tabular}{|c|c|c|c|c|c|c|c|c|}
\hline Time & 1 & 2 & 3 & 4 & 5 & 6 & 7 & 8 \\
\hline 06:00-09:00 & +0.02 & +0.02 & -0.02 & +0.03 & -0.07 & +0.07 & -0.02 & -0.02 \\
\hline 09:00-11:00 & +0.03 & +0.04 & -0.07 & +0.04 & -0.07 & +0.09 & -0.05 & -0.02 \\
\hline 11:00-13:00 & +0.03 & +0.04 & -0.05 & +0.04 & -0.01 & +0.05 & -0.05 & -0.01 \\
\hline $13: 00-15: 00$ & +0.04 & +0.05 & -0.04 & +0.03 & -0.11 & $\begin{array}{r}+0.09 \\
\end{array}$ & -0.06 & -0.03 \\
\hline 15:00-19:00 & +0.03 & +0.04 & -0.03 & +0.01 & -0.11 & +0.07 & -0.01 & -0.01 \\
\hline 19:00-22:00 & +0.01 & +0.04 & -0.06 & +0.01 & -0.06 & +0.06 & -0.07 & -0.02 \\
\hline
\end{tabular}

(d) Arrival Type

\begin{tabular}{|l|c|c|c|c|c|c|c|c|}
\hline Time & 1 & 2 & 3 & 4 & 5 & 6 & 7 & 8 \\
\hline $06: 00-09: 00$ & & $+\mathbf{0 . 1 0}$ & & & & $\mathbf{- 0 . 1 3}$ & & \\
\hline $09: 00-11: 00$ & & +0.04 & & & & +0.04 & & \\
\hline $11: 00-13: 00$ & & +0.02 & & & & -0.06 & & \\
\hline $13: 00-15: 00$ & & $+\mathbf{0 . 1 1}$ & & & & +0.14 & & \\
\hline $15: 00-19: 00$ & & +0.07 & & & & $\mathbf{- 0 . 7 3}$ & & \\
\hline $19: 00-22: 00$ & & $+\mathbf{0 . 2 4}$ & & & & +0.03 & & \\
\hline
\end{tabular}


Table 5.6 Statistical t-values (significant in boldface) for the 15-weekday aggregate comparison of operation with and without actuated coordinated phases at Noblesville, IN.

(a) Volume

\begin{tabular}{|c|c|c|c|c|c|c|c|c|}
\hline Time & 1 & 2 & 3 & 4 & 5 & 6 & 7 & 8 \\
\hline $06: 00-09: 00$ & $+\mathbf{2 . 6}$ & $\mathbf{+ 2 . 3}$ & +1.9 & $\mathbf{+ 3 . 0}$ & $\mathbf{+ 5 . 3}$ & +0.6 & +0.3 & -0.3 \\
\hline 09:00-11:00 & $\mathbf{+ 4 . 3}$ & $\mathbf{+ 3 . 0}$ & $\mathbf{+ 3 . 4}$ & $\mathbf{+ 3 . 4}$ & $\mathbf{+ 2 . 8}$ & $\mathbf{+ 3 . 0}$ & -1.8 & +1.6 \\
\hline $11: 00-13: 00$ & $\mathbf{+ 4 . 4}$ & $\mathbf{+ 2 . 8}$ & $\mathbf{+ 4 . 8}$ & +1.6 & $\mathbf{+ 2 . 2}$ & +0.4 & -0.3 & +0.9 \\
\hline 13:00-15:00 & $\mathbf{+ 3 . 9}$ & +2.0 & $\mathbf{+ 4 . 0}$ & $\mathbf{+ 3 . 6}$ & $\mathbf{+ 2 . 1}$ & +1.8 & -1.3 & +0.7 \\
\hline 15:00-19:00 & $+\mathbf{2 . 7}$ & +1.9 & $\mathbf{+ 4 . 0}$ & -0.1 & +1.7 & +1.0 & +2.0 & +0.2 \\
\hline 19:00-22:00 & +1.6 & +1.4 & $\mathbf{+ 4 . 4}$ & $\mathbf{+ 2 . 1}$ & +0.6 & +1.9 & -0.1 & +1.9 \\
\hline
\end{tabular}

(b) Green Time

\begin{tabular}{|c|c|c|c|c|c|c|c|c|}
\hline Time & 1 & 2 & 3 & 4 & 5 & 6 & 7 & 8 \\
\hline 06:00-09:00 & +2.1 & -5.3 & +8.6 & +2.2 & +23.3 & -12.2 & +2.8 & +4.1 \\
\hline 09:00-11:00 & +2.9 & -7.1 & +19.6 & +2.4 & +15.4 & -14.0 & +3.9 & +5.0 \\
\hline 11:00-13:00 & +2.7 & -4.3 & +13 & -0.6 & +6.6 & -8.4 & +3.9 & +3.9 \\
\hline $13: 00-15: 00$ & +2.5 & -4.3 & +11.7 & +1.7 & +19.1 & -10.6 & +7.4 & +4.2 \\
\hline $15: 00-19: 00$ & +3.5 & -2.8 & +13.3 & -0.3 & +12.6 & -8.1 & +10 & +1.1 \\
\hline $19: 00-22: 00$ & -0.3 & -5.5 & +13.4 & +2.5 & +13.3 & -7.5 & +9.6 & +5.7 \\
\hline
\end{tabular}

(c) $\mathrm{v} / \mathrm{c}$ Ratio

\begin{tabular}{|c|c|c|c|c|c|c|c|c|}
\hline Time & 1 & 2 & 3 & 4 & 5 & 6 & 7 & 8 \\
\hline $06: 00-09: 00$ & +2.2 & +3.5 & -1.6 & +2.6 & -4.6 & +7.0 & -1.0 & -2.3 \\
\hline $09: 00-11: 00$ & +3.5 & +5.4 & -3.9 & +2.6 & -5.9 & +8.2 & -4.8 & -2.2 \\
\hline $11: 00-13: 00$ & +3.7 & +3.9 & -3.3 & +3.0 & -0.4 & +2.6 & -3.5 & -1.0 \\
\hline $13: 00-15: 00$ & +3.4 & +3.1 & -2.2 & +2.8 & -6.8 & +4.9 & -3.3 & -3.9 \\
\hline $15: 00-19: 00$ & +1.8 & +2.5 & -1.9 & +0.7 & -9.3 & +2.9 & -1.3 & -1.4 \\
\hline 19:00-22:00 & +1.2 & +3.0 & -4.2 & +0.8 & -3.6 & +3.5 & -6.0 & -4.4 \\
\hline
\end{tabular}

(d) Arrival Type

\begin{tabular}{|l|r|r|c|c|c|c|c|c|}
\hline Time & 1 & 2 & 3 & 4 & 5 & 6 & 7 & 8 \\
\hline $06: 00-09: 00$ & & +2.3 & & & & -2.6 & & \\
\hline $09: 00-11: 00$ & & +1.0 & & & & +0.7 & & \\
\hline $11: 00-13: 00$ & & +0.4 & & & & -1.3 & & \\
\hline $13: 00-15: 00$ & & +2.4 & & & & +2.0 & & \\
\hline $15: 00-19: 00$ & & +1.8 & & & & $-\mathbf{6 . 8}$ & & \\
\hline $19: 00-22: 00$ & & $+\mathbf{6 . 7}$ & & & & +0.8 & & \\
\hline
\end{tabular}


Table 5.7 Statistical P-values (significant in boldface) for the 15-weekday aggregate comparison of operation with and without actuated coordinated phases at Noblesville, IN.

(a) Volume

\begin{tabular}{|c|c|c|r|r|r|r|r|r|}
\hline Time & 1 & 2 & 3 & 4 & \multicolumn{1}{c|}{5} & \multicolumn{1}{c|}{6} & \multicolumn{1}{c|}{7} & \multicolumn{1}{c|}{8} \\
\hline $06: 00-09: 00$ & $\mathbf{1 . 6 \%}$ & $\mathbf{3 . 0 \%}$ & $7.1 \%$ & $\mathbf{0 . 6 \%}$ & $\mathbf{0 . 0 \%}$ & $55.2 \%$ & $78.6 \%$ & $74.2 \%$ \\
\hline $09: 00-11: 00$ & $\mathbf{0 . 0 \%}$ & $\mathbf{0 . 5 \%}$ & $\mathbf{0 . 2 \%}$ & $\mathbf{0 . 2 \%}$ & $\mathbf{0 . 8 \%}$ & $\mathbf{0 . 6 \%}$ & $9.1 \%$ & $12.6 \%$ \\
\hline $11: 00-13: 00$ & $\mathbf{0 . 0 \%}$ & $\mathbf{0 . 8} \%$ & $\mathbf{0 . 0 \%}$ & $12.8 \%$ & $\mathbf{3 . 5 \%}$ & $72.0 \%$ & $75.2 \%$ & $35.7 \%$ \\
\hline $13: 00-15: 00$ & $\mathbf{0 . 1 \%}$ & $6.0 \%$ & $\mathbf{0 . 0} \%$ & $\mathbf{0 . 1 \%}$ & $\mathbf{4 . 8 \%}$ & $8.1 \%$ & $20.4 \%$ & $49.5 \%$ \\
\hline $15: 00-19: 00$ & $\mathbf{1 . 1 \%}$ & $7.3 \%$ & $\mathbf{0 . 0} \%$ & $92.4 \%$ & $9.7 \%$ & $34.4 \%$ & $5.0 \%$ & $85.8 \%$ \\
\hline $19: 00-22: 00$ & $12.6 \%$ & $16.1 \%$ & $\mathbf{0 . 0} \%$ & $\mathbf{4 . 4 \%}$ & $53.3 \%$ & $7.0 \%$ & $90.6 \%$ & $6.7 \%$ \\
\hline
\end{tabular}

(b) Green Time

\begin{tabular}{|c|c|c|c|c|c|c|c|c|}
\hline Time & 1 & 2 & 3 & 4 & 5 & 6 & 7 & 8 \\
\hline 06:00-09:00 & $4.0 \%$ & $0.0 \%$ & $0.0 \%$ & $4.0 \%$ & $0.0 \%$ & $0.0 \%$ & $0.9 \%$ & $0.0 \%$ \\
\hline 09:00-11:00 & $0.7 \%$ & $0.0 \%$ & $0.0 \%$ & $2.2 \%$ & $0.0 \%$ & $0.0 \%$ & $0.1 \%$ & $0.0 \%$ \\
\hline 11:00-13:00 & $1.3 \%$ & $0.0 \%$ & $0.0 \%$ & $56.2 \%$ & $0.0 \%$ & $0.0 \%$ & $0.1 \%$ & $0.1 \%$ \\
\hline $13: 00-15: 00$ & $1.9 \%$ & $0.0 \%$ & $0.0 \%$ & $10.7 \%$ & $0.0 \%$ & $0.0 \%$ & $0.0 \%$ & $0.0 \%$ \\
\hline 15:00-19:00 & $0.2 \%$ & $0.8 \%$ & $0.0 \%$ & $73.2 \%$ & $0.0 \%$ & $0.0 \%$ & $0.0 \%$ & $27.4 \%$ \\
\hline 19:00-22:00 & $77.9 \%$ & $0.0 \%$ & $0.0 \%$ & $2.0 \%$ & $0.0 \%$ & $0.0 \%$ & $0.0 \%$ & $0.0 \%$ \\
\hline
\end{tabular}

$\mathrm{v} / \mathrm{c}$ Ratio

\begin{tabular}{|c|r|r|r|r|r|r|r|r|}
\hline Time & 1 & 2 & 3 & 4 & 5 & 6 & 7 & \multicolumn{1}{c|}{8} \\
\hline $06: 00-09: 00$ & $\mathbf{3 . 7 \%}$ & $\mathbf{0 . 2 \%}$ & $11.8 \%$ & $\mathbf{1 . 3 \%}$ & $\mathbf{0 . 0 \%}$ & $\mathbf{0 . 0 \%}$ & $31.0 \%$ & $\mathbf{3 . 1 \%}$ \\
\hline $09: 00-11: 00$ & $\mathbf{0 . 2 \%}$ & $\mathbf{0 . 0 \%}$ & $\mathbf{0 . 1 \%}$ & $\mathbf{1 . 6 \%}$ & $\mathbf{0 . 0 \%}$ & $\mathbf{0 . 0 \%}$ & $\mathbf{0 . 0 \%}$ & $\mathbf{3 . 4 \%}$ \\
\hline $11: 00-13: 00$ & $\mathbf{0 . 1 \%}$ & $\mathbf{0 . 1 \%}$ & $\mathbf{0 . 3 \%}$ & $\mathbf{0 . 6 \%}$ & $70.0 \%$ & $\mathbf{1 . 4 \%}$ & $\mathbf{0 . 2 \%}$ & $34.6 \%$ \\
\hline $13: 00-15: 00$ & $\mathbf{0 . 2 \%}$ & $\mathbf{0 . 4 \%}$ & $\mathbf{3 . 3 \%}$ & $\mathbf{0 . 8 \%}$ & $\mathbf{0 . 0 \%}$ & $\mathbf{0 . 0 \%}$ & $\mathbf{0 . 3 \%}$ & $\mathbf{0 . 1 \%}$ \\
\hline $15: 00-19: 00$ & $8.6 \%$ & $\mathbf{1 . 7 \%}$ & $7.2 \%$ & $47.8 \%$ & $\mathbf{0 . 0 \%}$ & $\mathbf{0 . 8 \%}$ & $19.6 \%$ & $18.7 \%$ \\
\hline $19: 00-22: 00$ & $25.4 \%$ & $\mathbf{0 . 5 \%}$ & $\mathbf{0 . 0 \%}$ & $44.0 \%$ & $\mathbf{0 . 1 \%}$ & $\mathbf{0 . 1 \%}$ & $\mathbf{0 . 0 \%}$ & $\mathbf{0 . 0 \%}$ \\
\hline
\end{tabular}

(d) Arrival Type

\begin{tabular}{|c|r|r|r|r|r|r|c|c|}
\hline Time & 1 & 2 & 3 & 4 & 5 & 6 & 7 & 8 \\
\hline $06: 00-09: 00$ & & $\mathbf{2 . 6 \%}$ & & & & $\mathbf{1 . 5 \%}$ & & \\
\hline $09: 00-11: 00$ & & $34.4 \%$ & & & & $46.3 \%$ & & \\
\hline $11: 00-13: 00$ & & $70.4 \%$ & & & & $20.9 \%$ & & \\
\hline $13: 00-15: 00$ & & $\mathbf{2 . 6 \%}$ & & & & $5.0 \%$ & & \\
\hline $15: 00-19: 00$ & & $8.7 \%$ & & & & $\mathbf{0 . 0 \%}$ & & \\
\hline $19: 00-22: 00$ & & $\mathbf{0 . 0 \%}$ & & & & $44.6 \%$ & & \\
\hline
\end{tabular}


Table 5.8 Mean values of v/c Ratio for all eight phases at Noblesville, IN by time of day, aggregated over 15 weekdays of operation with and without actuated-coordinated phases.

\begin{tabular}{|c|r|r|r|r|r|r|r|r|}
\cline { 2 - 10 } \multicolumn{1}{c|}{} & \multicolumn{2}{c|}{ Phase 1 } & \multicolumn{2}{c|}{ Phase 2 } & \multicolumn{2}{c|}{ Phase 3 } & \multicolumn{2}{c|}{ Phase 4 } \\
\hline Time & Before & \multicolumn{1}{c|}{ After } & Before & \multicolumn{1}{c|}{ After } & Before & \multicolumn{1}{c|}{ After } & Before & \multicolumn{1}{c|}{ After } \\
\hline 06:00-09:00 & 0.42 & 0.44 & 0.20 & 0.22 & 0.73 & 0.71 & 0.80 & 0.83 \\
\hline 09:00-11:00 & 0.36 & 0.39 & 0.21 & 0.24 & 0.71 & 0.64 & 0.68 & 0.72 \\
\hline 11:00-13:00 & 0.38 & 0.41 & 0.29 & 0.33 & 0.69 & 0.65 & 0.80 & 0.84 \\
\hline 13:00-15:00 & 0.36 & 0.40 & 0.39 & 0.43 & 0.72 & 0.69 & 0.75 & 0.79 \\
\hline 15:00-19:00 & 0.47 & 0.50 & 0.65 & 0.69 & 0.71 & 0.68 & 0.91 & 0.92 \\
\hline 19:00-22:00 & 0.33 & 0.34 & 0.29 & 0.33 & 0.55 & 0.50 & 0.74 & 0.75 \\
\hline $\mathbf{0 6 : 0 0 - 2 2 : 0 0}$ & $\mathbf{0 . 3 9}$ & $\mathbf{0 . 4 2}$ & $\mathbf{0 . 3 4}$ & $\mathbf{0 . 3 8}$ & $\mathbf{0 . 6 9}$ & $\mathbf{0 . 6 4}$ & $\mathbf{0 . 7 8}$ & $\mathbf{0 . 8 1}$ \\
\hline
\end{tabular}

\begin{tabular}{|c|c|r|r|r|r|r|r|r|}
\cline { 2 - 10 } \multicolumn{1}{c|}{} & \multicolumn{2}{c|}{ Phase 5} & \multicolumn{2}{c|}{ Phase 6} & \multicolumn{2}{c|}{ Phase 7 } & \multicolumn{2}{c|}{ Phase 8 } \\
\hline Time & Before & \multicolumn{1}{c|}{ After } & Before & \multicolumn{1}{c|}{ After } & Before & After & Before & After \\
\hline 06:00-09:00 & 0.76 & 0.69 & 0.62 & 0.69 & 0.43 & 0.41 & 0.36 & 0.34 \\
\hline 09:00-11:00 & 0.75 & 0.69 & 0.41 & 0.50 & 0.44 & 0.38 & 0.30 & 0.28 \\
\hline 11:00-13:00 & 0.73 & 0.73 & 0.48 & 0.53 & 0.52 & 0.47 & 0.28 & 0.28 \\
\hline 13:00-15:00 & 0.89 & 0.78 & 0.42 & 0.51 & 0.52 & 0.46 & 0.32 & 0.29 \\
\hline 15:00-19:00 & 0.88 & 0.77 & 0.53 & 0.61 & 0.54 & 0.53 & 0.30 & 0.30 \\
\hline 19:00-22:00 & 0.57 & 0.51 & 0.20 & 0.25 & 0.48 & 0.41 & 0.26 & 0.24 \\
\hline $\mathbf{0 6 : 0 0 - 2 2 : 0 0}$ & $\mathbf{0 . 7 7}$ & $\mathbf{0 . 7 0}$ & $\mathbf{0 . 4 4}$ & $\mathbf{0 . 5 1}$ & $\mathbf{0 . 4 9}$ & $\mathbf{0 . 4 4}$ & $\mathbf{0 . 3 0}$ & $\mathbf{0 . 2 9}$ \\
\hline
\end{tabular}


Table 5.9 Number of split failures reported for 15 weekdays without actuated coordinated phases, and 15 weekdays with actuated coordinated phases, by phase and by TOD plan time period.

\begin{tabular}{|c|l|r|r|r|r|r|r|r|}
\hline \multirow{2}{*}{ Phase } & Operation & $\begin{array}{r}06: 00- \\
09: 00\end{array}$ & $\begin{array}{r}09: 00- \\
11: 00\end{array}$ & $\begin{array}{r}11: 00- \\
13: 00\end{array}$ & $\begin{array}{r}13: 00- \\
15: 00\end{array}$ & $\begin{array}{c}15: 00- \\
19: 00\end{array}$ & $\begin{array}{c}19: 00- \\
22: 00\end{array}$ & Total \\
\hline \multirow{3}{*}{1} & non-act. & 8 & 2 & 7 & 0 & 28 & 1 & 46 \\
\cline { 2 - 9 } & act.-coord. & 16 & 3 & 6 & 7 & 46 & 0 & 78 \\
\hline \multirow{2}{*}{2} & non-act. & 0 & 0 & 0 & 5 & 53 & 0 & 58 \\
\cline { 2 - 9 } & act.-coord. & 0 & 0 & 0 & 3 & 84 & 1 & 88 \\
\hline \multirow{2}{*}{3} & non-act. & 73 & 133 & 74 & 111 & 137 & 91 & 619 \\
\cline { 2 - 9 } & act.-coord. & 45 & 56 & 37 & 85 & 86 & 31 & 340 \\
\hline \multirow{3}{*}{4} & non-act. & 324 & 117 & 206 & 168 & 600 & 261 & 1676 \\
\cline { 2 - 9 } & act.-coord. & 356 & 146 & 244 & 220 & 637 & 278 & 1881 \\
\hline \multirow{2}{*}{5} & non-act. & 230 & 172 & 158 & 180 & 556 & 35 & 1331 \\
\cline { 2 - 9 } & act.-coord. & 144 & 76 & 85 & 92 & 260 & 12 & 669 \\
\hline \multirow{2}{*}{6} & non-act. & 60 & 2 & 3 & 6 & 23 & 2 & 96 \\
\cline { 2 - 9 } & act.-coord. & 73 & 9 & 4 & 15 & 38 & 0 & 139 \\
\hline \multirow{2}{*}{7} & non-act. & 28 & 32 & 53 & 51 & 72 & 44 & 280 \\
\cline { 2 - 9 } & act.-coord. & 23 & 16 & 32 & 29 & 56 & 16 & 172 \\
\hline \multirow{2}{*}{8} & non-act. & 3 & 1 & 1 & 0 & 0 & 0 & 5 \\
\cline { 2 - 9 } & act.-coord. & 0 & 0 & 0 & 0 & 0 & 0 & 0 \\
\hline \multirow{2}{*}{ Total } & non-act. & 726 & 459 & 502 & 521 & 1469 & 434 & 4111 \\
\cline { 2 - 9 } & act.-coord. & 657 & 306 & 408 & 451 & 1207 & 338 & 3367 \\
\hline
\end{tabular}




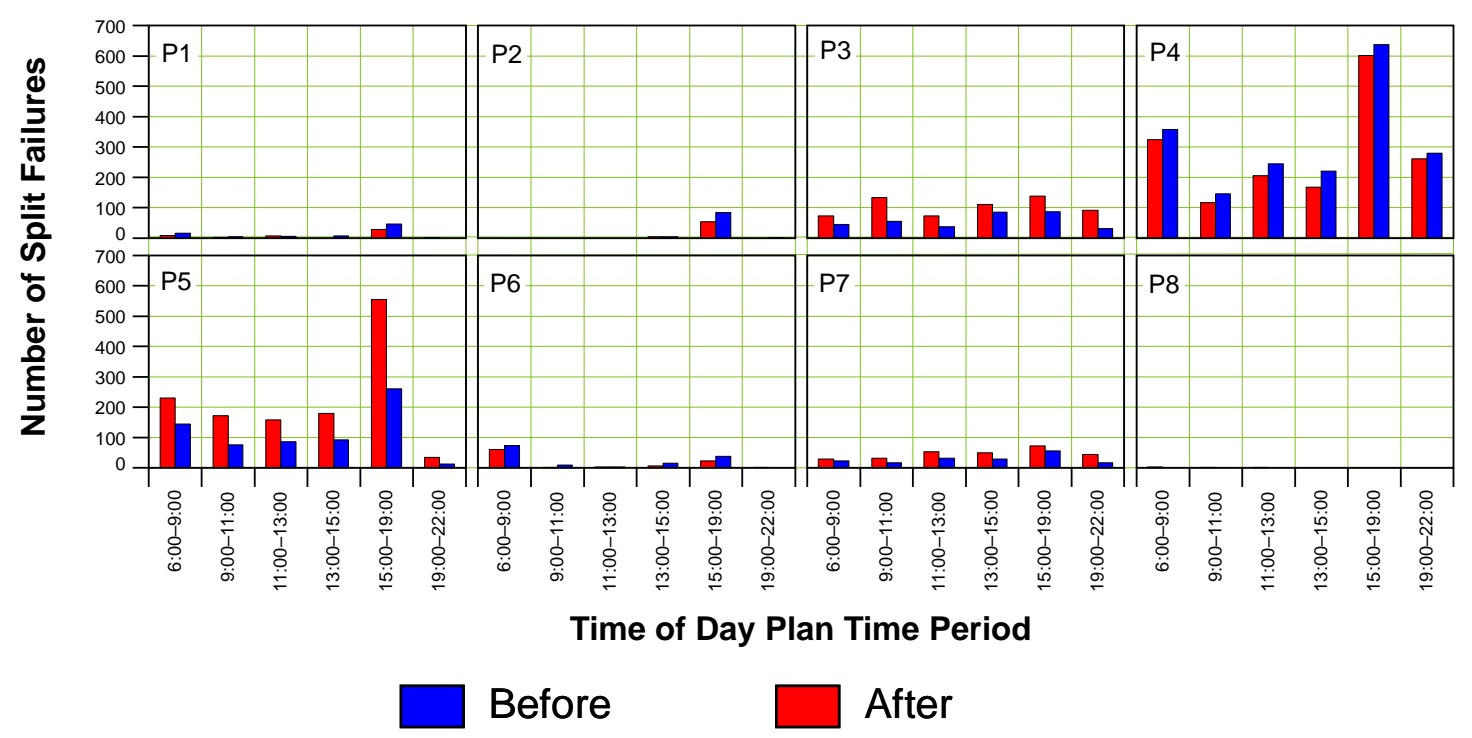

Figure 5.17 Total number of split failures by phase at Noblesville in all 15 weekdays without actuated coordinated phases (Before), and 15 weekdays with actuated coordinated phases (After). 


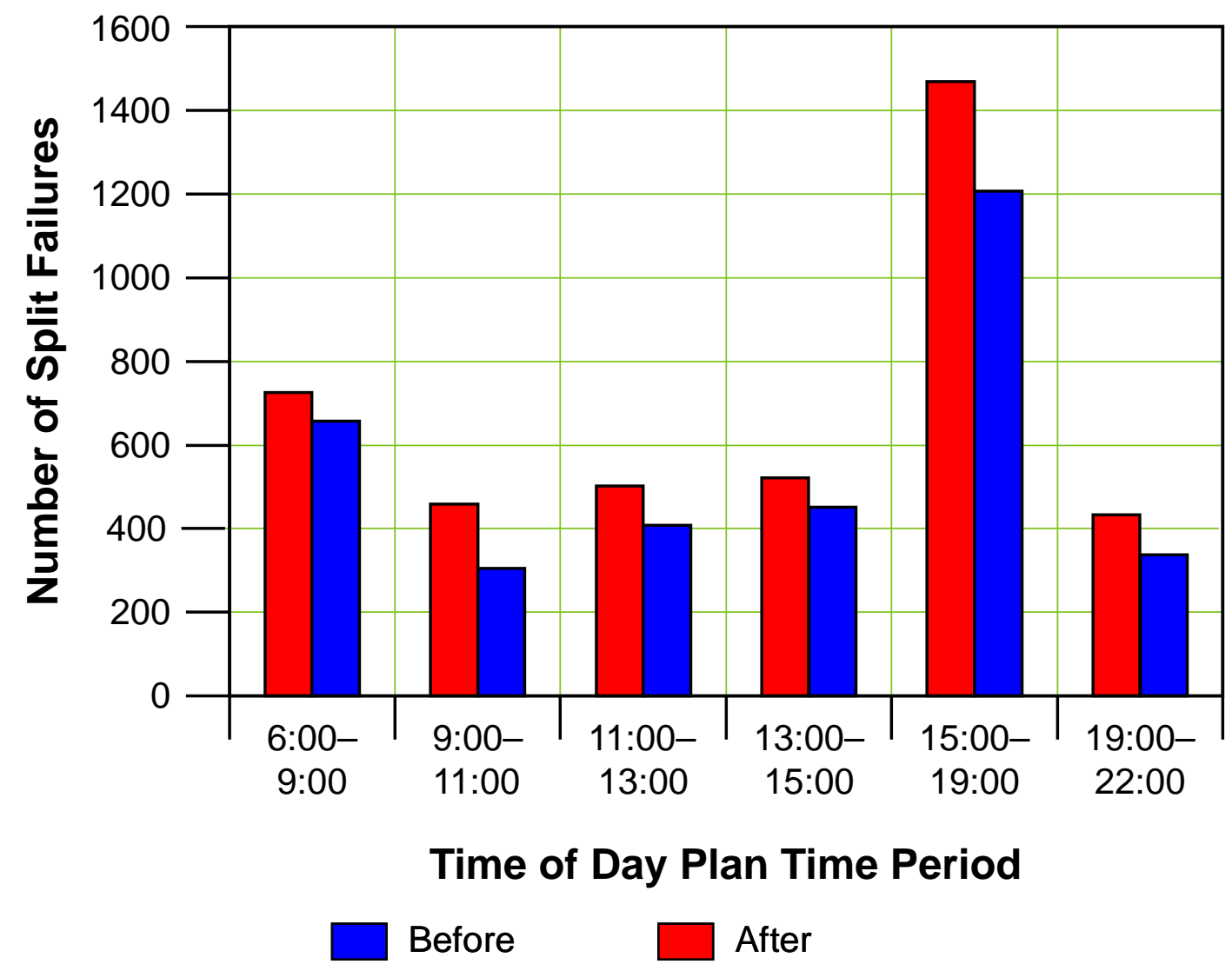

Figure 5.18 Total number of split failures in all eight phases at Noblesville in all 15 weekdays without actuated coordinated phases (Before), and 15 weekdays with actuated coordinated phases (After). 
Table 5.10 Percentage of cycles having split failures in all 15 weekdays without actuated coordinated phases, and 15 weekdays with actuated coordinated phases, by phase and by TOD plan time period.

\begin{tabular}{|c|l|r|r|r|r|r|r|r|}
\hline \multirow{2}{*}{ Phase } & Operation & $\begin{array}{r}06: 00- \\
09: 00\end{array}$ & $\begin{array}{c}09: 00- \\
11: 00\end{array}$ & $\begin{array}{c}11: 00- \\
13: 00\end{array}$ & $\begin{array}{c}13: 00- \\
15: 00\end{array}$ & $\begin{array}{c}15: 00- \\
19: 00\end{array}$ & $\begin{array}{c}19: 00- \\
22: 00\end{array}$ & \multicolumn{2}{c|}{ Total } \\
\hline \multirow{2}{*}{1} & non-act. & 0.8 & 0.3 & 0.9 & 0.0 & 1.8 & 0.1 & 0.8 \\
\cline { 2 - 9 } & act.-coord. & 1.4 & 0.3 & 0.8 & 1.0 & 2.8 & 0.0 & 1.3 \\
\hline \multirow{2}{*}{2} & non-act. & 0.0 & 0.0 & 0.0 & 0.5 & 2.8 & 0.0 & 0.7 \\
\cline { 2 - 9 } & act.-coord. & 0.0 & 0.0 & 0.0 & 0.3 & 4.5 & 0.1 & 1.1 \\
\hline \multirow{2}{*}{3} & non-act. & 5.3 & 12.3 & 7.1 & 10.8 & 7.4 & 6.6 & 8.0 \\
\cline { 2 - 9 } & act.-coord. & 3.2 & 5.1 & 3.6 & 8.4 & 4.6 & 2.2 & 4.4 \\
\hline \multirow{2}{*}{4} & non-act. & 23.1 & 10.5 & 19.7 & 16.2 & 32.0 & 16.8 & 20.9 \\
\cline { 2 - 9 } & act.-coord. & 25.0 & 13.0 & 23.2 & 21.0 & 34.2 & 17.9 & 23.3 \\
\hline \multirow{2}{*}{5} & non-act. & 16.9 & 15.3 & 15.0 & 17.4 & 29.9 & 2.3 & 16.8 \\
\cline { 2 - 9 } & act.-coord. & 10.6 & 6.8 & 8.1 & 8.9 & 14.0 & 0.8 & 8.4 \\
\hline \multirow{2}{*}{6} & non-act. & 4.2 & 0.2 & 0.3 & 0.6 & 1.2 & 0.1 & 1.2 \\
\cline { 2 - 9 } & act.-coord. & 5.1 & 0.8 & 0.4 & 1.4 & 2.0 & 0.0 & 1.7 \\
\hline \multirow{2}{*}{7} & non-act. & 4.1 & 5.1 & 7.2 & 6.5 & 4.6 & 3.8 & 5.1 \\
\cline { 2 - 9 } & act.-coord. & 3.5 & 2.5 & 4.3 & 4.0 & 3.6 & 1.5 & 3.2 \\
\hline \multirow{2}{*}{8} & non-act. & 0.2 & 0.1 & 0.1 & 0.0 & 0.0 & 0.0 & 0.1 \\
\cline { 2 - 9 } & act.-coord. & 0.0 & 0.0 & 0.0 & 0.0 & 0.0 & 0.0 & 0.0 \\
\hline \multirow{2}{*}{ Total } & non-act. & 7.2 & 5.7 & 6.5 & 6.8 & 10.3 & 3.9 & 7.0 \\
\cline { 2 - 9 } & act.-coord. & 6.4 & 3.7 & 5.2 & 5.9 & 8.4 & 3.0 & 5.7 \\
\hline
\end{tabular}



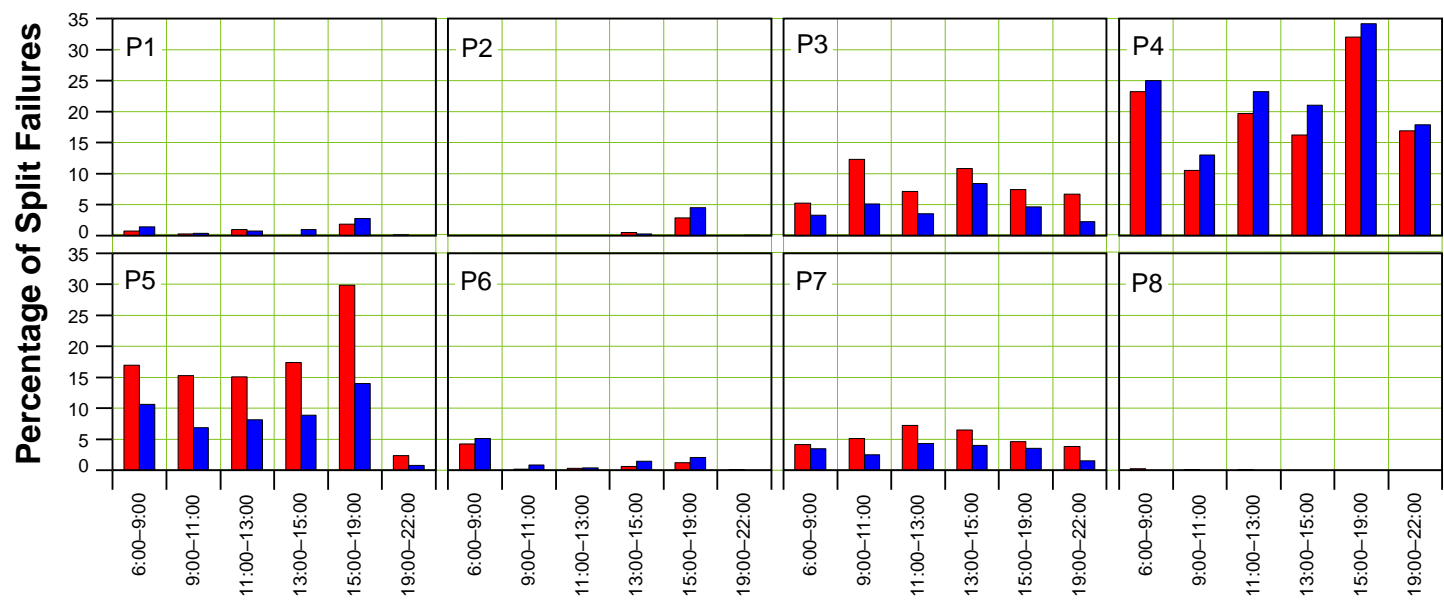

Time of Day Plan Time Period

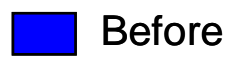

$\square$ After

Figure 5.19 Percentage of all cycles by phase at Noblesville having split failures in all 15 weekdays without actuated coordinated phases (Before), and 15 weekdays with actuated coordinated phases (After). 


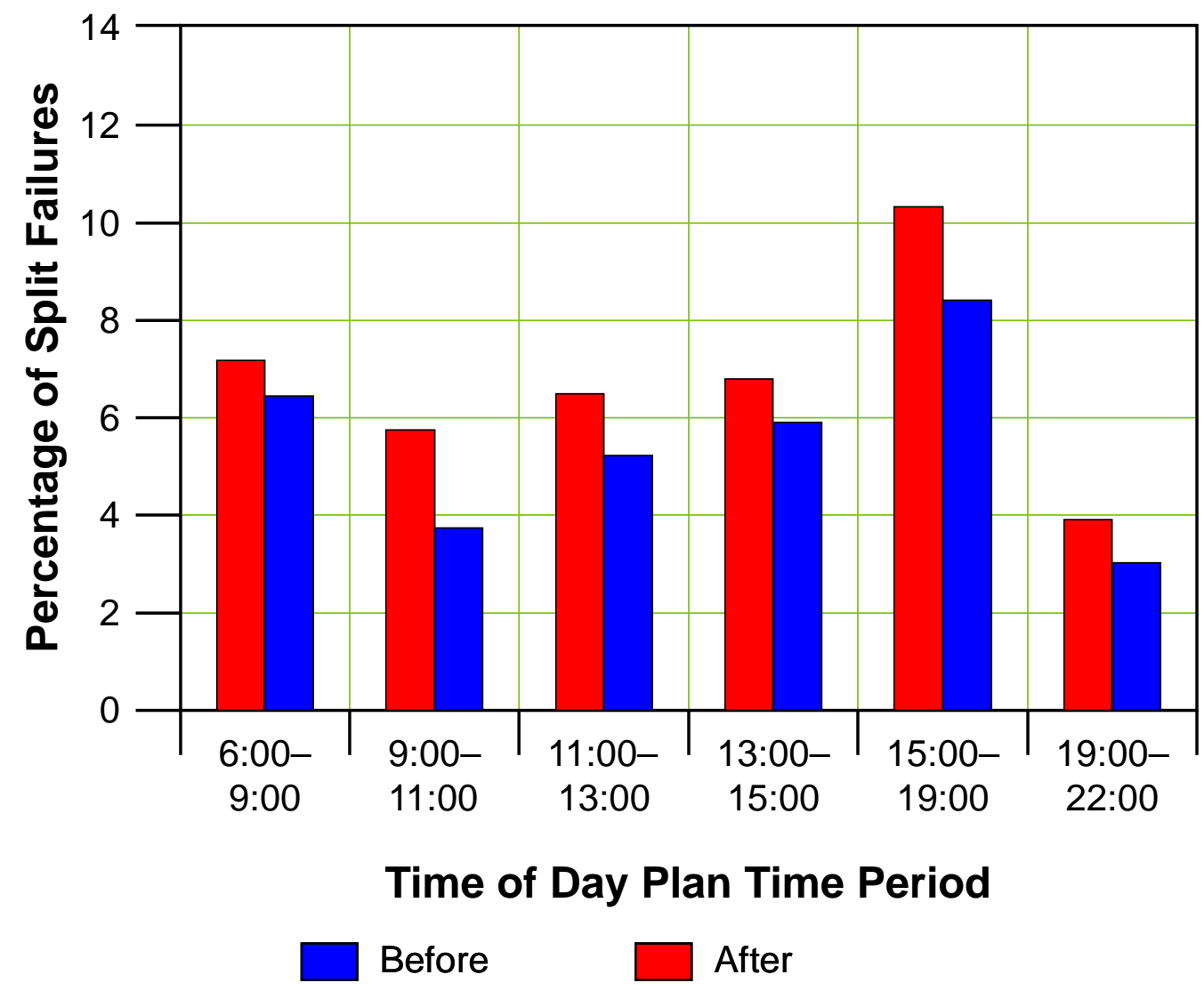

Figure 5.20 Percentage of all cycles in all eight phases at Noblesville having split failures in all 15 weekdays without actuated coordinated phases (Before), and 15 weekdays with actuated coordinated phases (After). 


\subsubsection{Conclusion}

The impact of implementing actuated coordinated phases is demonstrated by the movement of green time across the barrier and the resulting changes in $\mathrm{v} / \mathrm{c}$ ratios. The reallocation of green time from undersaturated actuated portions of coordinated phases allows latter phases to access additional green time. The removal of underutilized green time from the coordinated phases had minor impacts on arterial progression, evidenced by the comparison of AT values between the two modes of operation. The addition of green time to the non-coordinated phases provided significant benefits, which is observable in the decrease of $\mathrm{v} / \mathrm{c}$ ratios and by the reduction in the occurrence of split failures. The use of actuated coordinated phases is recommended to traffic engineers to provide robust control that can more effectively respond to varying traffic conditions. This study shows that this mode of operation increases intersection throughput with relatively inexpensive modifications, especially when detection on the coordinated movements is available. 


\subsection{Operational Impacts of Noblesville Signal Retiming}

\subsubsection{Introduction}

The previous study addressed a specific change in operation. Using real time data collection, performance measures were calculated, making it possible to determine the impacts of actuating the coordinated phase at an intersection. In this section, the effects of a full scale signal retiming action are investigated. The effects of changes in operating modes and timing parameters are investigated with regard to the efficiency with which the capacity is used at the signal, and the quality of progression along the arterial on which the signal operates.

\subsubsection{Operational Changes}

In late 2006, the timing plans of signals along the SR 37 corridor to the northeast of Indianapolis, Indiana were assessed, and new timing plans were implemented, including a new plan for the intersection of SR 37 and SR 32, the Noblesville test intersection. Three separate types of changes were made to the operating plan, which will be discussed in more detail in this section:

- The northbound left turn was programmed to lag the southbound through movement instead of coming before it.

- During the early evening, the cycle mode of operation was changed from noncoordinated, fully-actuated to semi-actuated coordinated.

- The splits and cycle lengths were changed, as well as the reference phase during certain time periods.

\subsubsection{Lagging Left Turn}

Figure 5.21(a) shows the ring diagram for operation at Noblesville under a traditional eight-phase control scheme. This is virtually the same diagram as seen in Figure 2.3(b), with the only difference being in the assignment of overlaps. Prior to retiming, the Noblesville test intersection ran under this scheme, with all left turns leading the through movements. One of the changes to operation was that the northbound left turn, previously a leading left turn, became a lagging left, as shown in Figure 5.21(b). The position of phases 5 and 6 have been swapped. 
Lagging left turns can be used to improve progression. At the Noblesville test intersection, southbound vehicles arrive at random; it does not matter whether phase 5 leads or lags phase 6 for the purpose of progression. However, if phase 5 is lagging, the movement is essentially coordinated with the upstream signals to the south. That is, approaching northbound left turning vehicles arrive at the intersection before their desired movement is given the green indication. If phase 5 comes at the beginning of the phase, it may not even be called for the approaching vehicles, with the result that left turning vehicles will have to wait for the next cycle to move.

Another reason that lagging left turns were implemented at Noblesville was that northbound left turns feed into a relatively short segment of roadway, forming queues with Cumberland Road. Although there are two lanes on this segment, motorists favor the right lane, because there is a merging section to the east of the intersection of Cumberland and SR 32, where the left lane ends. Because of this, there is a potential for queues to spill into the intersection of SR 32 and 37, being fed by the northbound left turns there. This spillback would block phase 6 , if phase 5 came first. By moving phase 5 after phase 6 , this situation is avoided.

\subsubsection{Implementation of Semi-Actuated Operation}

Fully-actuated and semi-actuated operation were defined in Chapter 2, and discussed in detail in the previous study. The Noblesville test intersection has the ability to operate in either fully-actuated or semi-actuated modes. During the time in which the signal retiming took place, the intersection was operated in semi-actuated mode during the daytime hours, and in fully-actuated ("free") mode during low-volume evening hours. Signals along SR 37 were therefore coordinated during the daytime hours, and not coordinated during evening hours. Before signal retiming, the overnight fully-actuated period began at 19:00 and ended at 6:00 the next morning. A new pattern was added for the 19:00-22:00 time period, and implemented during retiming.

Figure 5.22 shows a plot of cycle length at Noblesville for Wednesday, June 28, 2006 and Wednesday, March 21, 2007. The vertical dashed lines indicate the boundaries of TOD plan periods. These boundaries were used both before and after retiming. Notice the difference in behavior during the 19:00-22:00 time period. Under fully-actuated operation, resulting cycle lengths are fairly short. There is enough volume during this time period to cause most phases to be called; but the volume is low enough for phases to gap out early. There is obviously no opportunity for coordination under this scheme, as there is no reference point for coordination to utilize. The beginning and end of green are randomly interspaced. 


\subsubsection{Timing Changes}

In addition to the two changes described above, the splits and cycle length were also altered at the Noblesville test intersection. Changes in cycle length are visible in Figure 5.22. As mentioned before, the same TOD boundaries applied both before and after retiming. The cycle length was decreased during peak periods, and increased during the off-peak periods. Before retiming, a cycle length of $124 \mathrm{~s}$ was used during the AM and PM peaks. This was decreased to $116 \mathrm{~s}$ after retiming. The cycle lengths for the 9:00-11:00 and 11:00-13:00 time periods were respectively increased from 78 to $96 \mathrm{~s}$ and from 98 to $102 \mathrm{~s}$. The PM peak period originally began at 13:00 and ran through 19:00. After retiming, this period was broken into a 13:00-15:00 and 15:00-19:00 segment. Additionally, a fixed cycle length was imposed during the 19:00-22:00 time period, whereas before the signal ran in free mode.

For comparison purposes, the 13:00-19:00 afternoon peak period in the before case was split up into 13:00-15:00 and 15:00-19:00 segments to be congruous with the TOD plan after retiming.

Table 5.11(a) gives the observed splits for June 28, 2006 (before retiming), while Table 5.11(b) shows this information for March 21, 2007 (after retiming). At the time of writing, the actual operating plan in use during June 2006 was not available. It was possible to approximate the splits by calculating them based on the measured green times and cycle lengths. The observed split of phase $i$ was found by

$S_{i, \text { observed }}=\frac{G_{i}+R_{i}}{C}$,

Equation 5.1

where $G_{i}$ is the measured length of the green indication, $R_{i}$ is the all red clearance interval, and $C$ is the cycle length. The values shown in Table 5.11 are averaged over all of the cycles in the associated time period. The observed splits in Table 5.11(b) may be compared to the actual splits in Table 2.2. The observed splits of the coordinated phases are higher than the actual splits. However, the difference in the observed splits allows us to obtain a sense of how signal timing was changed. Generally, the coordinated phases ( 2 and 6) were given a greater share of the total green time, while the splits of the noncoordinated phases were reduced. Note that for free (fully-actuated) periods, the splits are not defined.

In addition to these adjustments, the reference phase changed for the midday TOD periods. Before retiming, during the 9:00-11:00 and 11:00-13:00 time periods, the reference phase at Noblesville was phase 4 . After retiming, phase 2 was used as the 
reference phase during all time periods with semi-actuated operation. Phase 4 was coordinated with the upstream signal to the west of the intersection; the beginning of green for phase 2 was not guaranteed to occur at a deterministic point from 9:00-13:00 before retiming.

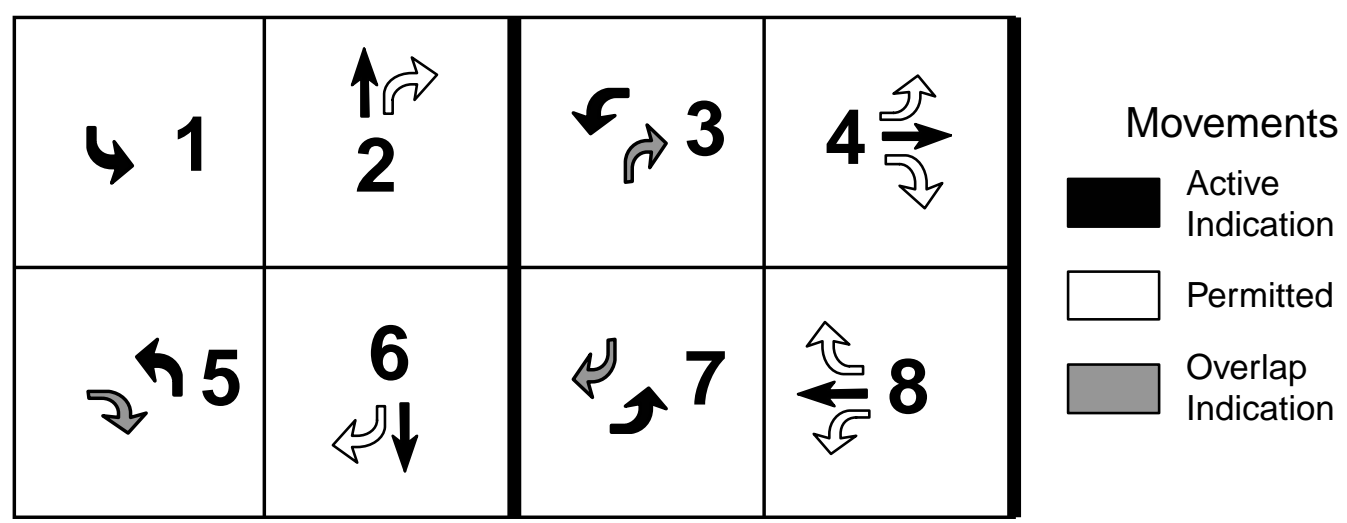

(a) Conventional phasing

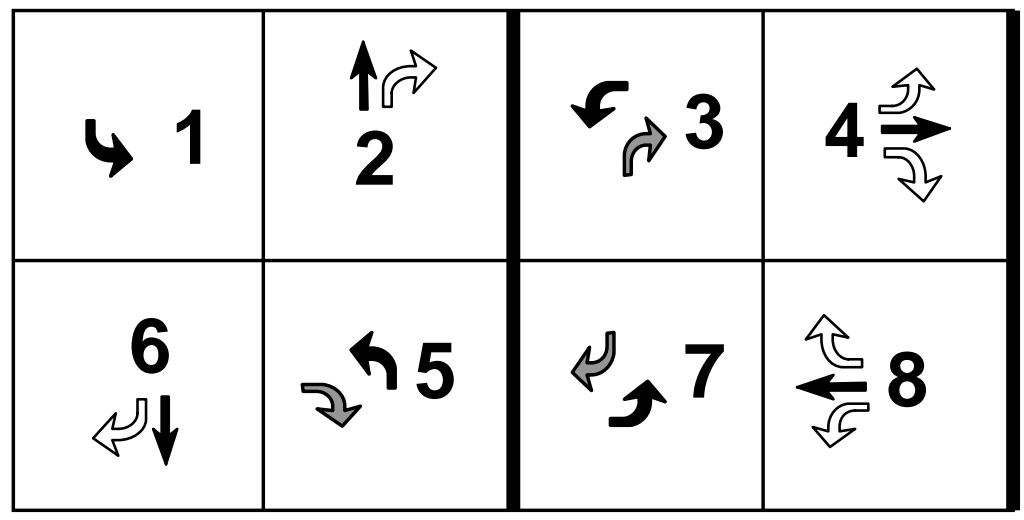

Movements

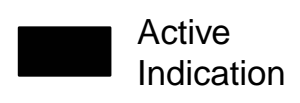

$\square$ Permitted

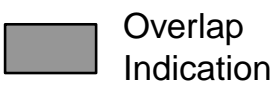

(b) Lead/lag phasing

Figure 5.21 Ring diagrams showing operation at the Noblesville test intersection (a) with conventional phasing and (b) with lead/lag phasing. 


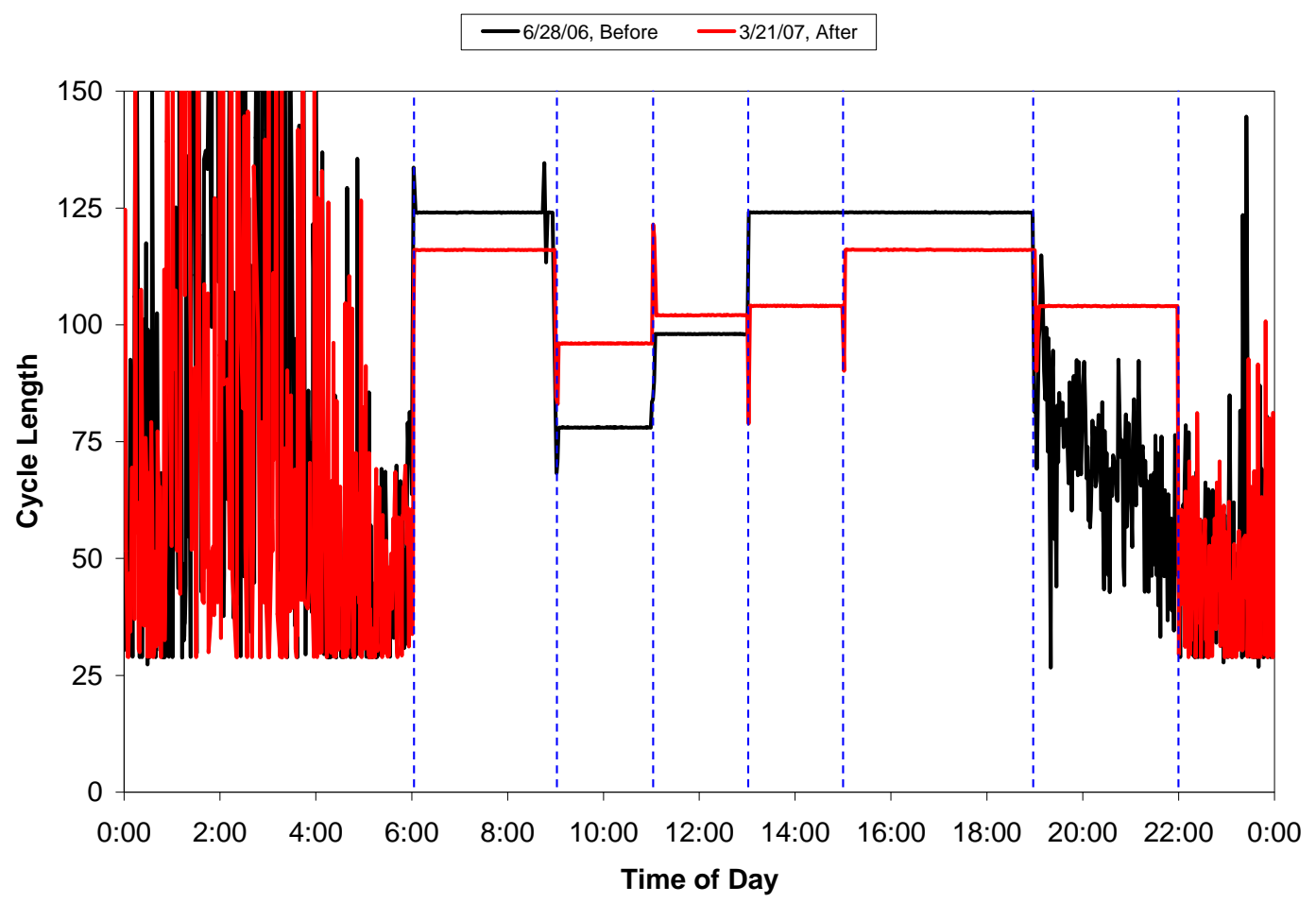

Figure 5.22 Cycle length. 
Table 5.11 Observed splits.

(a) Before, $6 / 28$

\begin{tabular}{|c|c|c|c|c|c|c|c|c|c|c|}
\hline \multirow[b]{2}{*}{$\begin{array}{l}\text { Begin } \\
\text { Time }\end{array}$} & \multirow[b]{2}{*}{$\begin{array}{l}\text { Cycle } \\
\text { Length } \\
\text { (s) }\end{array}$} & \multirow[b]{2}{*}{$\begin{array}{c}\text { Reference } \\
\text { Phase }\end{array}$} & \multicolumn{4}{|c|}{ Ring 1} & \multicolumn{4}{|c|}{ Ring 2} \\
\hline & & & 1 & 2 & 3 & 4 & 5 & 6 & 7 & 8 \\
\hline 0:00 & Free & Free & Free & Free & Free & Free & Free & Free & Free & Free \\
\hline 6:00 & 124 & 2 & 0.09 & 0.47 & 0.19 & 0.25 & 0.18 & 0.38 & 0.06 & 0.38 \\
\hline $9: 00$ & 78 & 4 & 0.12 & 0.47 & 0.16 & 0.25 & 0.24 & 0.35 & 0.09 & 0.32 \\
\hline $11: 00$ & 98 & 4 & 0.1 & 0.48 & 0.18 & 0.24 & 0.28 & 0.3 & 0.09 & 0.33 \\
\hline 13:00 & 124 & 2 & 0.1 & 0.43 & 0.15 & 0.32 & 0.23 & 0.3 & 0.12 & 0.35 \\
\hline 19:00 & Free & Free & Free & Free & Free & Free & Free & Free & Free & Free \\
\hline 22:00 & Free & Free & Free & Free & Free & Free & Free & Free & Free & Free \\
\hline
\end{tabular}

(b) After, $3 / 21$

\begin{tabular}{|c|c|c|c|c|c|c|c|c|c|c|}
\hline \multirow[b]{2}{*}{$\begin{array}{l}\text { Begin } \\
\text { Time }\end{array}$} & \multirow[b]{2}{*}{$\begin{array}{l}\text { Cycle } \\
\text { Length } \\
\text { (s) }\end{array}$} & \multirow[b]{2}{*}{$\begin{array}{c}\text { Reference } \\
\text { Phase }\end{array}$} & \multicolumn{4}{|c|}{ Ring 1} & \multicolumn{4}{|c|}{ Ring 2} \\
\hline & & & 1 & 2 & 3 & 4 & 5 & 6 & 7 & 8 \\
\hline 0:00 & Free & Free & Free & Free & Free & Free & Free & Free & Free & Free \\
\hline 6:00 & 116 & 2 & 0.09 & 0.52 & 0.2 & 0.19 & 0.14 & 0.47 & 0.05 & 0.34 \\
\hline 9:00 & 96 & 2 & 0.09 & 0.52 & 0.15 & 0.24 & 0.2 & 0.41 & 0.08 & 0.31 \\
\hline 11:00 & 102 & 2 & 0.09 & 0.5 & 0.16 & 0.25 & 0.24 & 0.35 & 0.08 & 0.33 \\
\hline 13:00 & 104 & 2 & 0.08 & 0.5 & 0.14 & 0.28 & 0.2 & 0.38 & 0.1 & 0.32 \\
\hline 15:00 & 116 & 2 & 0.1 & 0.46 & 0.14 & 0.3 & 0.19 & 0.37 & 0.1 & 0.34 \\
\hline 19:00 & 104 & 2 & 0.08 & 0.59 & 0.12 & 0.21 & 0.19 & 0.48 & 0.1 & 0.23 \\
\hline $22: 00$ & Free & Free & Free & Free & Free & Free & Free & Free & Free & Free \\
\hline
\end{tabular}




\subsubsection{Methodology}

The location of this study was the Noblesville test intersection. The data volumes compared in this study consisted of six weeks of data. Three weeks from June 2006 were collected, representing the state of the signal before retiming. Three weeks from March 2007 were used to analyze operation after retiming. The data from June 2006 was collected for the purpose of evaluating lane-by-lane detection in a study carried out by Smaglik et al. (9), while the data from March 2007 was collected to compare actuatedcoordinated operation, which was discussed in the previous section. Data from the three weeks without fully actuated coordinated phases were used to study the effects of retiming. Lane-by-lane detection was in use in both data volumes.

An approach similar to that of the previous study was used for analysis. To qualitatively analyze changes in operation, days with similar volume were selected for which performance measures would be directly compared. There were no pairs of days from both data sets that had similar volumes for all TOD plan time periods. Instead, two pairs of days were selected:

- Monday, June 19, 2006 and Monday, March 12, 2007 had similar volumes for most phases during the midday (11:00-13:00) and early evening (19:00-22:00) time periods.

- Wednesday, June 28, 2006 and Wednesday, March 21, 2007 had similar volumes for most phases during the AM and PM peak hours.

Data from 15 weekdays was aggregated using the methods described in Section 4.5.3, repeating the process that was carried out in the previous study. The pairwise and aggregate statistical tests substantiated the observations made in the qualitative analysis.

In the following sections, impacts are examined for each of three types of TOD plan scenarios which were subject to different kinds of operational changes during retiming.

- In the midday time periods, 9:00-11:00 and 11:00-13:00, the reference phase changed from phase 4 to phase 2. Additionally, the cycle length was increased, the splits were revised, and lagging left turns were put into operation. The 11:0013:00 time period is examined in the qualitative analysis, as there were no pairs of data sets with similar volumes in the 9:00-11:00 time period. 
- The early evening (19:00-22:00) time period operated in free, fully-actuated mode before retiming. A fixed cycle length was implemented with phase 6 as the reference phase. Lagging left turns also had an impact.

- The AM (6:00-9:00) and PM (15:00-19:00) peak periods experienced changes due to the implementation of lead-lag operation and the readjustment of splits and reduction of cycle length. Coordination along the arterial was in place both before and after retiming.

\subsubsection{Qualitative Analysis}

\subsubsection{Volume}

Equivalent hourly volumes calculated by cycle over 24 hours are shown in Figure 5.23 and Figure 5.24. The pair of days for comparison of off-peak operation (June 19 and March 12) is shown in Figure 5.23. The time periods outside of the 11:00-13:00 and 19:00-22:00 time periods are shaded blue. Midday volumes are roughly the same, except for phases 5 and 6, which had lower volumes in the after case. Evening volumes are also similar, except for phases 5 and 7, which were again lower. Volumes on the dates used for pairwise comparison of peak hour operation (June 28 and March 21) are shown in Figure 5.24. Again, the time periods other than peak hours are shaded blue. AM peak hour volumes were similar, except for phase 3 , which had an increase. PM peak hour volumes were different for phases 3,4 , and 7 . Phases 3 and 7 saw decreases in volume whereas phase 4 saw an increase. Phases 2, 5, and 6 had similar volumes in the AM and PM peak periods, allowing us to rule out volume changes as causes for operational changes.

\subsubsection{Capacity Analysis}

Green time histograms are shown in Figure 5.25 (off-peak) and Figure 5.26 (peak). The arrangement of the histograms is the same as in Figure 5.5, with the before case stacked above the after case. "P1b," for example, denotes Phase 1 in the before case, while "P1a" denotes Phase 1 in the after case. The arrows show the difference in means between the two cases, with black arrows signifying when the change was statistically significant. Histograms for the 11:00-13:00 midday time period are shown in Figure 5.25(a); those for the 19:00-22:00 early evening period are shown in Figure 5.25(b); and 
the AM and PM peak periods are shown in Figure 5.26(a) and Figure 5.26(b) respectively.

For the midday period, significant increases in green time are observable for phases 1, 4, and 6; decreases occurred for phases 3 and 5. The forward movement of the distributions for phases 2 and 6 are notable, even though the increase for phase 2 did not turn out to be statistically significant. Phase 6 clearly benefited the most from the change, at the expense of Phase 5. Having been pushed to the end of the effective cycle, phase 5 can no longer obtain additional green time from earlier phases. Its distribution became very narrow after retiming, nearly always maxing out. Interestingly, phase 4 saw an increase in green time, despite the reference phase being shifted to phase 2. Even though phase 4 was the reference phase before retiming, there was probably little opportunity for it to absorb any green time from early terminations of phases 2 and 6 , because of equally high demand for arterial movement.

The early evening period shows some drastic changes in green time distribution. Six phases had a significant change in green time, with phases 2, 4, 5, and 6 increasing, and phases 3 and 7 decreasing. Phases 1 and 8 were unaffected. Green time for phases 2 and 6 increased dramatically, with phase 2 gaining $30.3 \mathrm{~s}$ on average, and phase 6 gaining 2 gaining $27.4 \mathrm{~s}$. The reason for this is that these were the coordinated phases in the after case. Before retiming, the signal operated in free mode, and the two phases tended to gap out well under $40 \mathrm{~s}$. In semi-actuated mode, phases 2 and 6 held the green for much longer intervals. The average green time for phase 5 increased, although its distribution can be seen to narrow significantly. Phase 5 maxed out during nearly every single cycle in the after case, despite a decrease in volume. The reason for this is that demand was delivered to phase 5 by northbound coordination; While phase 6 was being served, a queue was building for phase 5 , fed by a strong northbound movement released by upstream signals. Thus, the northbound left turn is used more efficiently, although the fact that it maxes out during each cycle suggests that it may not receive enough green time. Analysis of v/c ratios will investigate this further.

Because the cycle length varies under free operation, histograms of average green time per cycle only tell a small part of the story. Cumulative green times for phase 2 and 5 are shown in Figure 5.27 and Figure 5.28 respectively. In both graphs, the black line shows cumulative green time before retiming while the red line shows the data from after retiming. In general, phase 2 gained green time, while phase 5 lost it. There are many more points along the black lines, showing that under free operation, the controller services all phases in a shorter amount of time. The steady slopes of the lines indicate that demand was fairly constant for both phases. 
The changes in peak period green times are shown in Figure 5.26. Many significant decreases in green time are seen: phases 4, 5, 7, and 8 in the AM peak, and phases $1,3,4,5,7$, and 8 in the PM peak. The reason for this is that the cycle length decreased, thereby causing green times to decrease overall. However, note that the coordinated phases, 2 and 6, do not share in the loss. Green time for phase 2 has stayed the same, while phase 6 gained green time. Because the cycle length decreased, this means that the coordinated phases are obtaining more cumulative green time as phases of the same duration are repeated more frequently. This is illustrated in Figure 5.29, which shows the cumulative green plotted over the time of day in the 6:00-9:00 time period. Over this three-hour period, phase 2 has acquired $340 \mathrm{~s}(5 \mathrm{~m} 40 \mathrm{~s})$ of green that it did not have in the before case.

Because capacity is calculated from green time, plots of the observed capacity give another way of visualizing the meaning of changes in green time. Figure 5.30 and Figure 5.31 show plots of observed capacity for all eight phases between 6:00 and 22:00. Figure 5.30 contains plots for June 19 and March 12, which were used for comparison of off-peak periods. Figure 5.31 contain the plots for June 28 and March 21, meant to show changes for peak periods. The same trends are apparent in both graphs. The coordinated phases have obtained additional capacity in every single time period, while noncoordinated phases have lost capacity in most time periods. These changes are more pronounced in the 19:00-22:00 time period, where the coordinated phases saw large increases in green time on a cycle-by-cycle basis, because cycle lengths were longer.

So far, we have discussed volume and capacity; the ratio of the two is the next topic. Cumulative frequency graphs of v/c ratio are shown in Figure 5.32 and Figure 5.33, with midday (11:00-13:00) plots in Figure 5.32(a); early evening (19:00-22:00) plots in Figure 5.32(b); AM peak period plots in Figure 5.33(a); and PM peak period plots in Figure 5.33(b).

The midday cumulative frequency plots of v/c ratio show relatively little change, compared to the changes in green time histograms in Figure 5.25(a). The only significant changes are for phase 3, which saw an increase in average $\mathrm{v} / \mathrm{c}$ ratio; and for phase 6 , which saw a substantial decrease in average v/c ratio. Part of this decrease may be attributed to the decrease in volume that took place for phase 6 , but that decrease was enhanced by the addition of $6.7 \mathrm{~s}$ to the average green time per cycle for this phase. None of the average $\mathrm{v} / \mathrm{c}$ ratios that changed were due to volume changes. Only phase 5 saw a different volume. Phase 5, which also saw a decrease in volume and a decrease in green time, had no change in v/c. Phase 3 , the only other phase to have lost green time, 
sustained an increase in v/c, as its volume did not change. The other phases seem to have been unaffected.

The story is considerably different for the early evening period. Here, every phase except for phase 5 as reported a significant change in average $\mathrm{v} / \mathrm{c}$ ratio. The coordinated phases return substantially lower v/c ratios, while the non-coordinated phases had increases in their $\mathrm{v} / \mathrm{c}$ ratios. The increase was acute for phase 3 , with some cycles having split failures, as seen by the extension of the plot into the region to the right of the $\mathrm{v} / \mathrm{c}=$ 1.0 line. The same thing occurred for phase 7, although its volume decreased. Clearly, the coordinated phases receive the most benefit from the use of semi-actuated operation. It would appear that phase 5 is served equally well under both modes; however, the increase in green time and decrease in volume that took place prevent us from making a solid conclusion.

As we discussed earlier, the peak periods saw a decrease in cycle length, but the change in green time for the coordinated phases was zero or positive. The impact on the $\mathrm{v} / \mathrm{c}$ ratios is shown in Figure 5.33. In both the AM and PM peak periods, phase 6 sees an decrease in the average v/c ratio, while phase 2's average $\mathrm{v} / \mathrm{c}$ ratio is unchanged. The minor phases withstand increases in $\mathrm{v} / \mathrm{c}$ ratio to varying degrees. The increase for phase 4 is acute in the morning, which may be explained by the large loss of green time sustained by that phase. The left turns are unaffected in the morning, except for phase 5 . All of the non-coordinated phases see increases in v/c ratio in the evening. This is because the coordinated phases are obtaining a greater share of the green time in each cycle. The v/c ratio for Phase 5 increases substantially in both the morning and evening peak hours. Whereas when phase 5 was a leading phase, it could obtain additional green time when preceding phases 4 and 8 terminated early, as a lagging phase, any additional time that phase 5 might have received was consumed by phase 6 .

Table 5.12 shows the percentage of cycles ending in split failures for all eight phases by TOD plan. This percentage is calculated from all phases taking place in all 15 weekdays in each of the before and after data sets. Counts of split failures are not given here, because since the number of cycles is considerably different, the numbers are not comparable. Note that average percentages are given for coordinated and noncoordinated phases in addition to the averages for all phases. The aggregated average percentages of split failures are shown for all eight phases graphically in Figure 5.34. The percentages averaged over all coordinated phases are shown in Figure 5.35; averaged over non-coordinated phases, in Figure 5.36; and averaged over all eight phases, in Figure 5.37. 
The changes in percentages of split failures is consistent with the changes occurring in average green duration and $\mathrm{v} / \mathrm{c}$ ratios. The coordinated phases ( 2 and 6 ) see a decrease in the number of split failures from 2.5 to $1 \%$ of all cycles, while the noncoordinated phases see an increase from 5 to $9 \%$. The percentage of all phases for all cycles taking place at the intersection rises from 4.3 to $6.9 \%$ after retiming.

When broken down by time of day, the coordinated phases benefit particularly well during the midday TOD periods (9:00-11:00 and 11:00-13:00). In both cases, the percentage of coordinated phases ending in split failure fell from $3.5 \%$ or higher to $0.1 \%$. Interestingly, during these time periods, the percentage of split failures also fell for phase 4. This seems to be due to the increase in the cycle length, allowing the green time for phase 4 to increase. During the peak hours, the coordinated phases reduced their percentage of split failures because of retiming, although the results are not as pronounced. Phase 6 benefited strongly in the AM peak, and phase 2 in the PM peak. There were virtually no split failures during the early evening hours for phases 2 and 6 .

Of all the phases, phase 5 suffers the most in terms of the increase in the number of split failures. The percentage for across all TOD periods increased from 3 to $16.6 \%$. During the PM peak hour, the percentage of cycles ending in split failure rose from 3.7 to $29.9 \%$, averaged across the 15 weekdays in the before and after cases. Phase 6 experiences the most benefit, with the total percentage of split failures falling from 3.7 to $1.2 \%$. The transposition of phases 5 and 6 in the ring diagram works to the advantage of phase 6 , allowing phase 6 additional green time to serve vehicles. At the same time, the building of queues for the northbound left turn by the adjacent coordinated through movement, in conjunction with reduced green time for phase 5 , drives up the $\mathrm{v} / \mathrm{c}$ ratio for phase 5 , leading to more split failures.

\subsubsection{Progression Analysis}

In the capacity analysis presented above, it was determined that retiming the signal reduced the $\mathrm{v} / \mathrm{c}$ ratios of the coordinated phases, while increasing those ratios for the non-coordinated phases. However, only a portion of the benefits for phases 2 and 6 is captured in capacity analysis. Because these phases belong to the through movements along a coordinated arterial, the quality of progression is also important to effective signal operation.

Figure 5.38 shows a plot of arrival type (20-point moving average) for June 19 and March 12, the pair of days selected for comparing midday and early evening operation. Figure 5.39 shows arrival type for June 28 and March 21, which are intended to illustrate operation during peak periods. The other periods are shaded in the graphs, but 
the trends for both pairs of days are similar. The arrival type for phase 6 hovers around the value of $\mathrm{AT}=3$ in both the before and after cases, for both days. This is true of all time periods, except where AT is slightly above 3 during the PM peak period in the before case (Figure 5.39(b)). In the actuated-coordinated study, slightly better than random results were occasionally obtained for phase 6 . This was attributed to an unforeseen interaction, as the upstream signal for phase 6 is not coordinated with the test intersection.

Northbound progression, indicated by the Phase 2 arrival type, shows considerable improvement during nearly all of the time periods under consideration. An increase in AT is apparent in the midday and evening periods (Figure 5.38(a)). In the midday, this is because of the change of the reference phase to phase 2 , thereby making coordination possible. Before, we can see that AT hovered between 2 and 3, indicating poor progression. In the early evening, coordination was made possible by the change from free to semi-actuated operation. As for the peak periods (Figure 5.39(a)), phase 2 AT improved during the AM but showed no change during the PM peak. The improvement in the AM reflects that the new signal timing plan is more appropriate to coordination along the arterial than the old plan. The lack of improvement in the PM is a result of coordination having been adequate in the old plan; it was not harmed by the new timing plan.

The platoon profiles reveal the same information in a different way. Platoon profiles for phases 2 and 6 are shown in Figure 5.40 for the 11:00-13:00 midday period; Figure 5.41 for the 19:00-22:00 early evening period; Figure 5.42 for the 6:00-9:00 AM peak period; and Figure 5.43 for the 15:00-19:00 PM peak period.

In the midday period, the green window is clearly missing the arrivals before retiming (Figure 5.40(a)). The peak of the arrivals occurs during the red indication for phase 2. After retiming (Figure 5.40(b)), many more arrivals are captured during the green. The arrival type improved from 3.45 to 4.47 , reflecting this improvement. There was apparently no coordination going on along the arterial in the before case. Recall that the reference phase was phase 4 before retiming. There is not much change in the platoon profiles for Phase 6 (Figure 5.40(c) and (d)). The lump on the end of the cycle profile for Phase 6 in Figure 5.40(d) is the result of a few cycles where phase 5 was not actuated; this is an artifact of the way that the cycle profile is calculated, as discussed in Chapter 4.

The early evening period profiles are shown in Figure 5.41. In the profiles for the before case (Figure 5.41(a) and (c)), the shapes of both the cycle and arrival distributions are influenced by the randomness of cycle length under fully actuated operation. That is, the shape of the arrival distribution shows more arrivals occurring at lower values of time 
in cycle, because there were fewer cycles that lasted long enough for arrivals to have taken place at higher values. Under fully actuated operation, there is no way to shift the distributions to the right or to the left. The profiles for Figure 5.41(b) and (d) were found under semi-actuated operation, with constant cycle length. The impacts on the coordinated phases are clear. Phase 2 sees a marked improvement, with most arrivals occurring during green. Although the distribution appears different for phase 6, it is merely because the "before" case in Figure 5.41(c) does not accurately show the random nature of the arrivals. There was not much difference between the before and after cases for phase 6 . This reflects that, when arrivals are random, progression is the same under both fully actuated and semi-actuated operation.

The platoon profiles for the AM peak in Figure 5.42 are similar to those for the midday peak. However, the improvement in progression for phase 2 (Figure 5.42(a) and (b)) is the result of improved signal timing; coordination was taking place in the before case, but it was not well timed. In the before case (Figure 5.42(a)), the band of arrivals is not captured by the green distribution. Most of the distribution was captured after retiming (Figure 5.42(b)). The arrival type improved from 4.21 to 4.53 . There was not much difference for phase 6 (Figure 5.42(c) and (d)).

Finally, platoon profiles for the PM peak are shown in Figure 5.43. There is little difference in the profiles for phase 2 (Figure 5.43(a) and (b)), which reveals that coordination had been sufficient before retiming, and that coordination was not disrupted by retiming. Phase 6 sees a slight decrease in arrival type, the cause of which is subtly revealed in the profiles (Figure 5.43(c) and (d)). In the arrival distribution, there seems to be a slight peak. The cause of the peak reveals an interaction with the upstream signal, which is not coordinated with the test intersection. For whatever reason, during this peak period, southbound arrivals have a slight distribution. The peak in the southbound arrival distribution was captured more adequately before retiming (Figure 5.43(c)) than after retiming (Figure 5.43(d)). Because of the distance of the upstream signal, coordination was not considered in the southbound direction with regards to the timing plan. Although the peak in the arrival distribution does not appear to be significant in the shape of the distribution, it was sufficient to decrease AT from 4.10 to 3.44. 


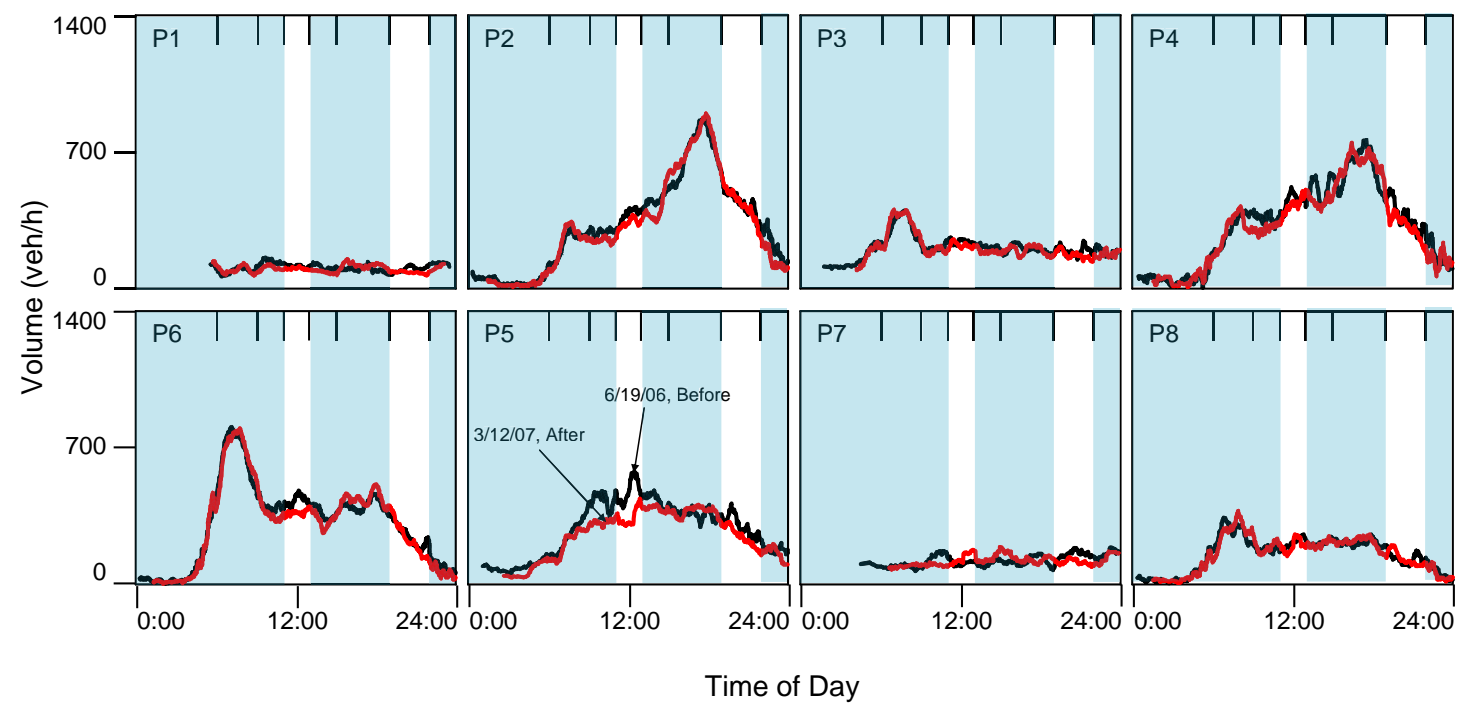

June 19, 2006, before changes

March 12, 2007, after changes

Figure 5.23 Cycle-by-cycle volumes (20 point average) at Noblesville for Monday, June 19, 2006 and Monday, March 12, 2007, with the midday (11:00-13:00) and early evening (19:00-22:00) time periods highlighted. 


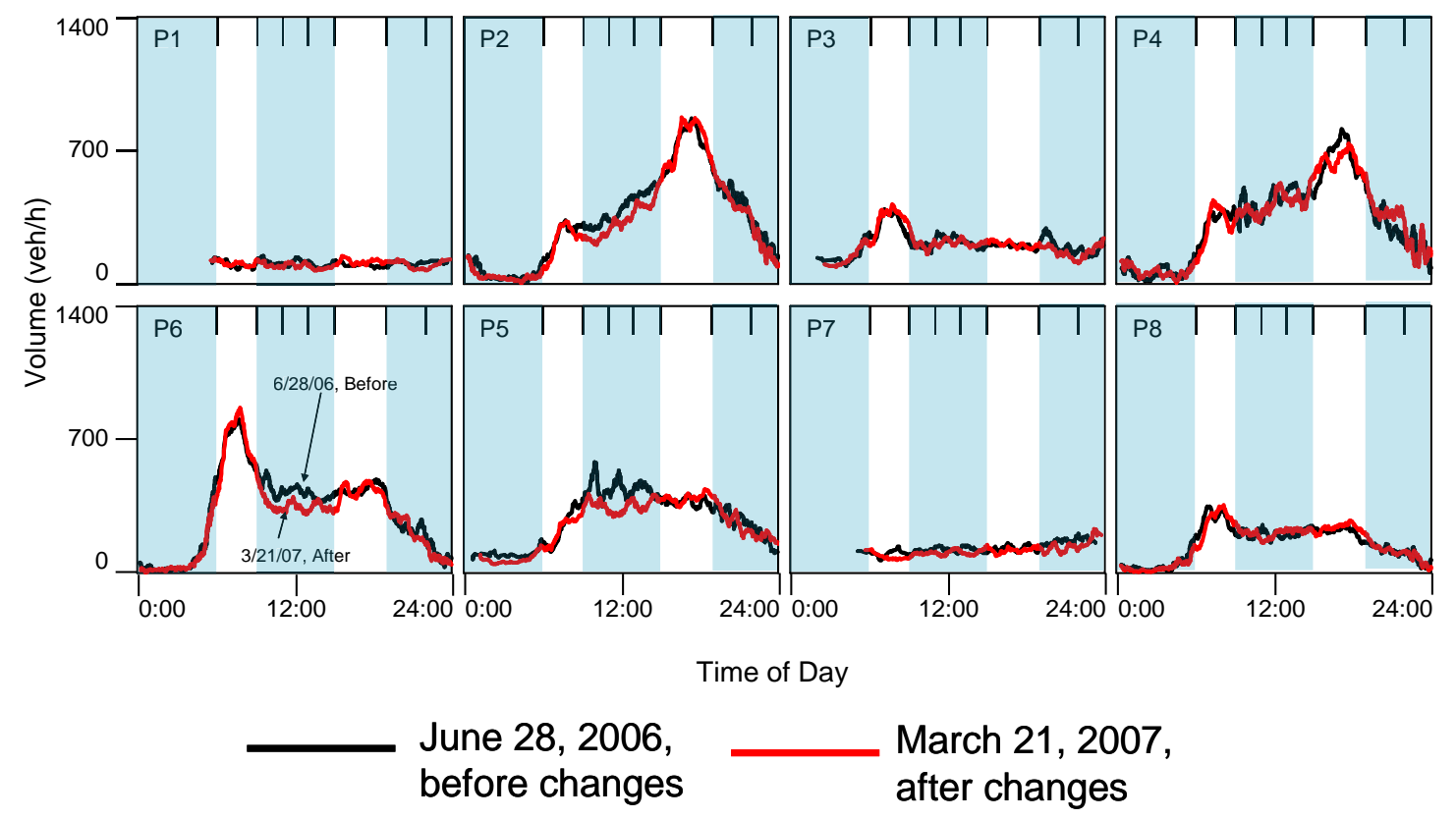

Figure 5.24 Cycle-by-cycle volumes (20 point average) at Noblesville for Wednesday, June 28, 2006 and Wednesday, March 21, 2007, with the AM (6:00-9:00) and PM (15:00-19:00) peak periods highlighted. 

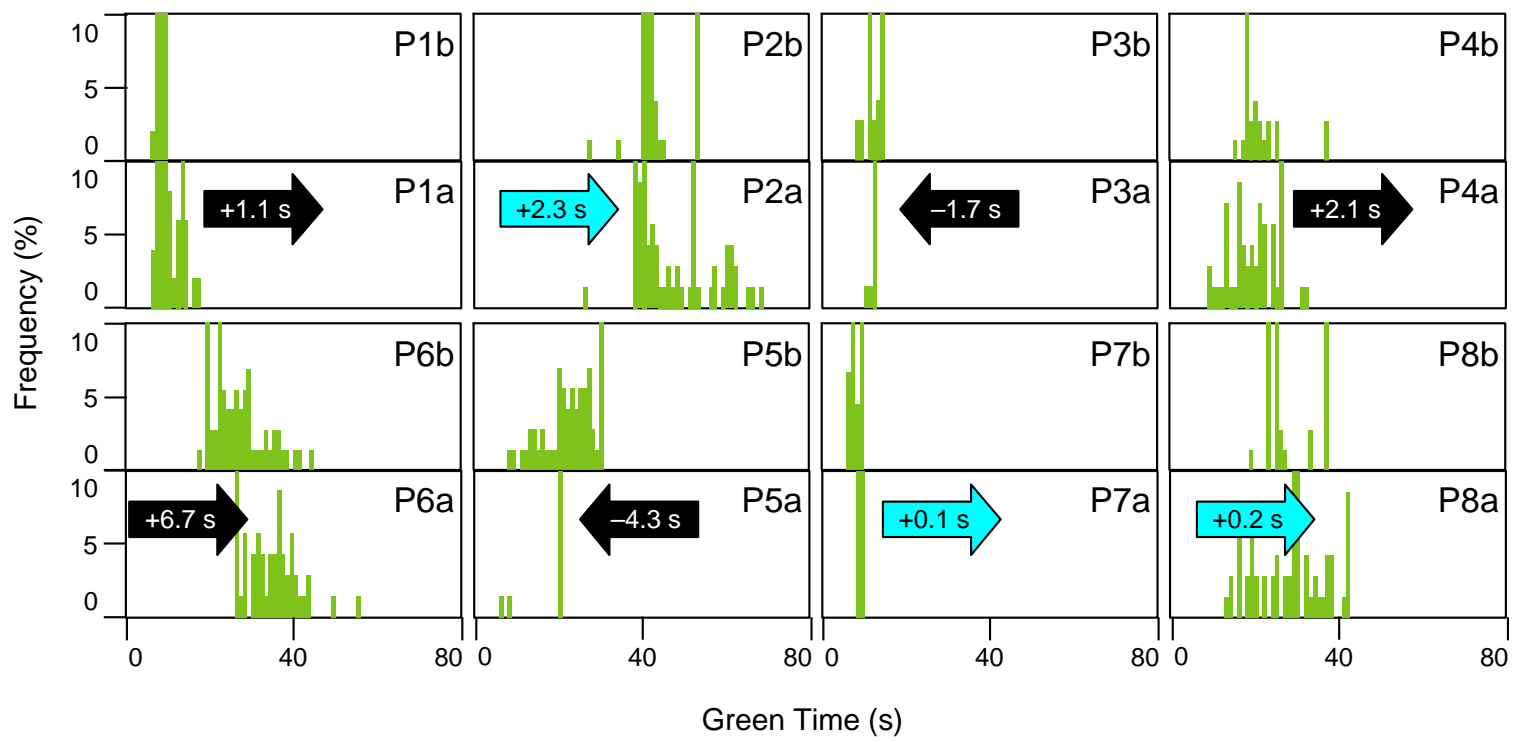

(a) Midday, 11:00-13:00

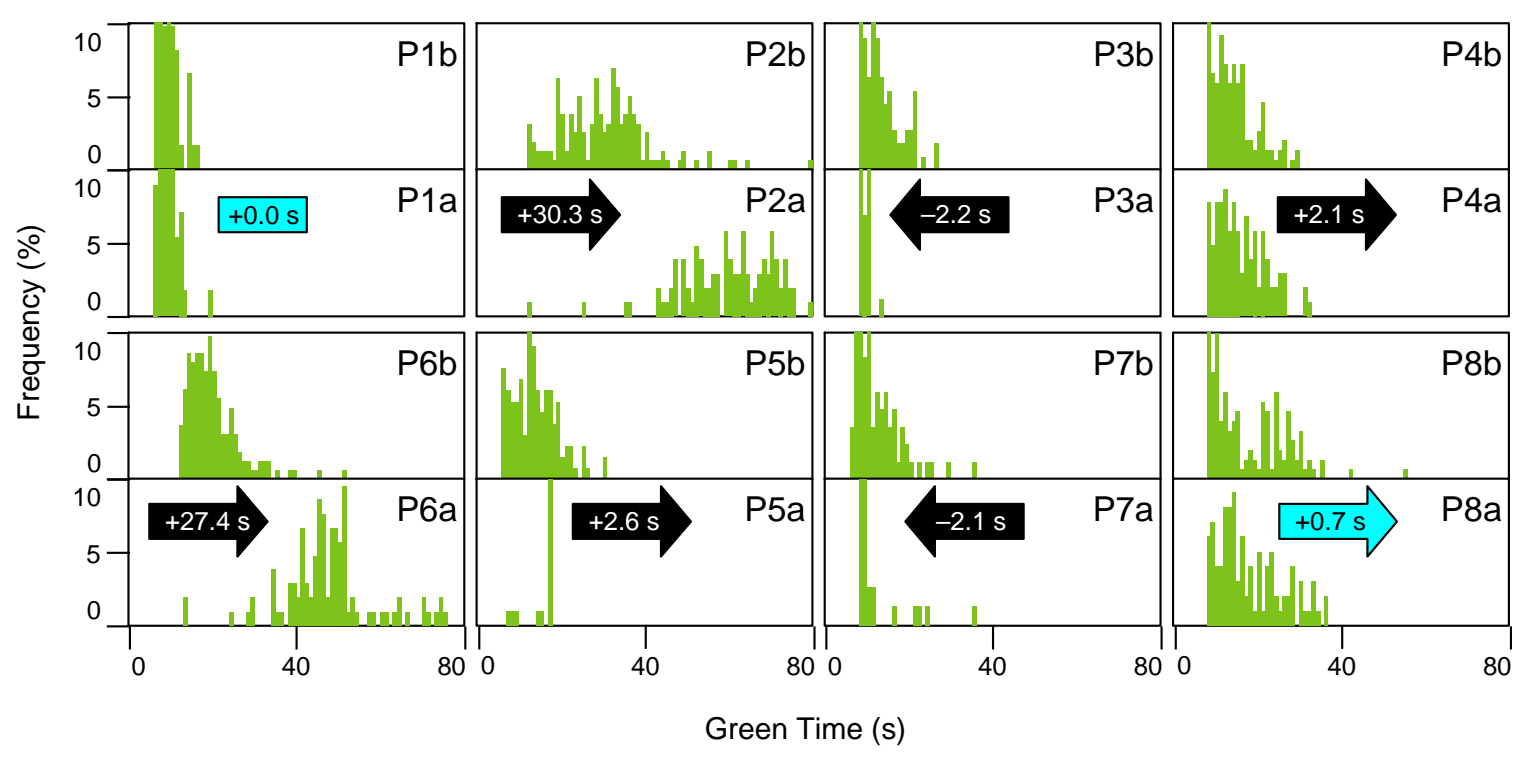

(b) Early evening, 19:00-22:00

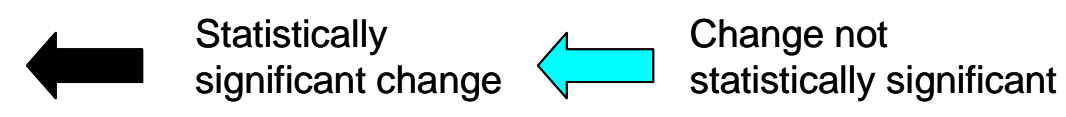

Figure 5.25 Green time distributions at Noblesville for Monday, June 19, 2006 (before retiming) and Monday, March 12, 2006 (after retiming). 


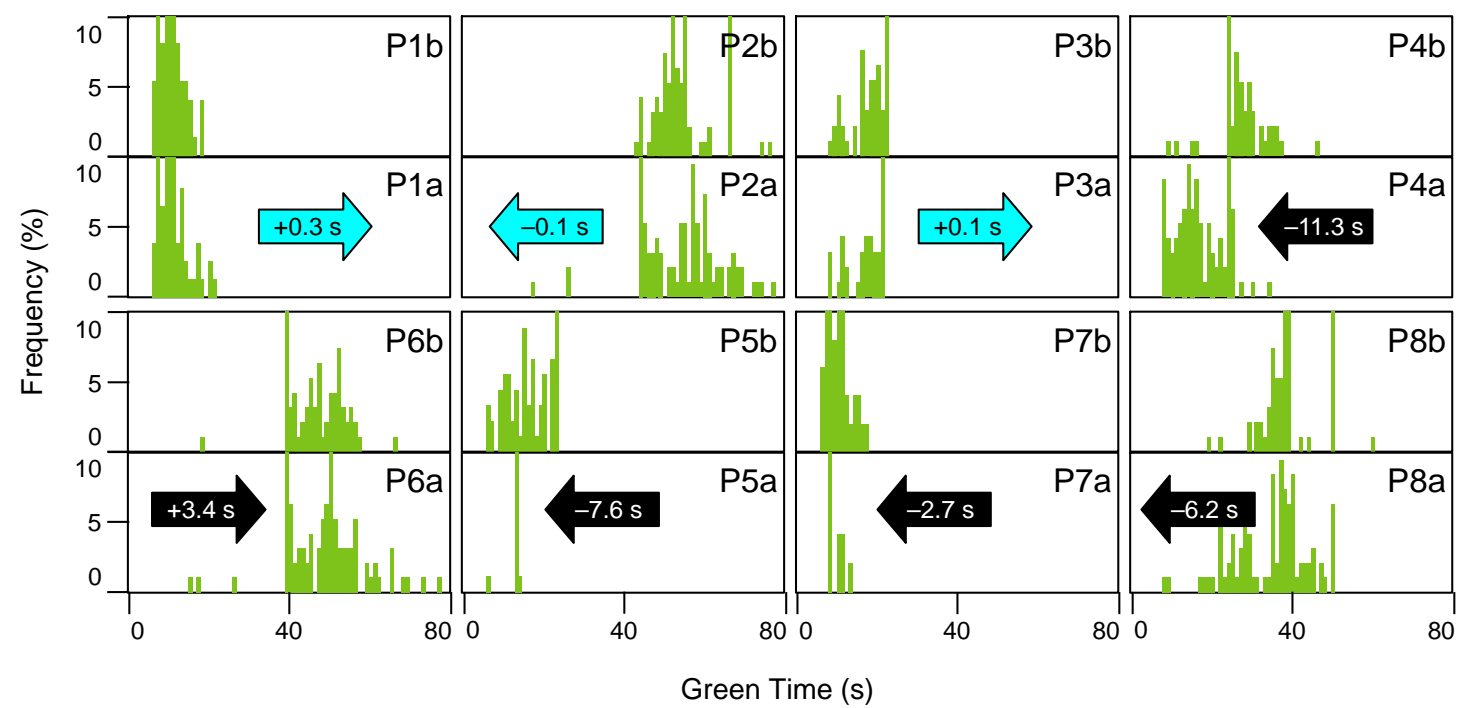

(a) AM peak period, 6:00-9:00
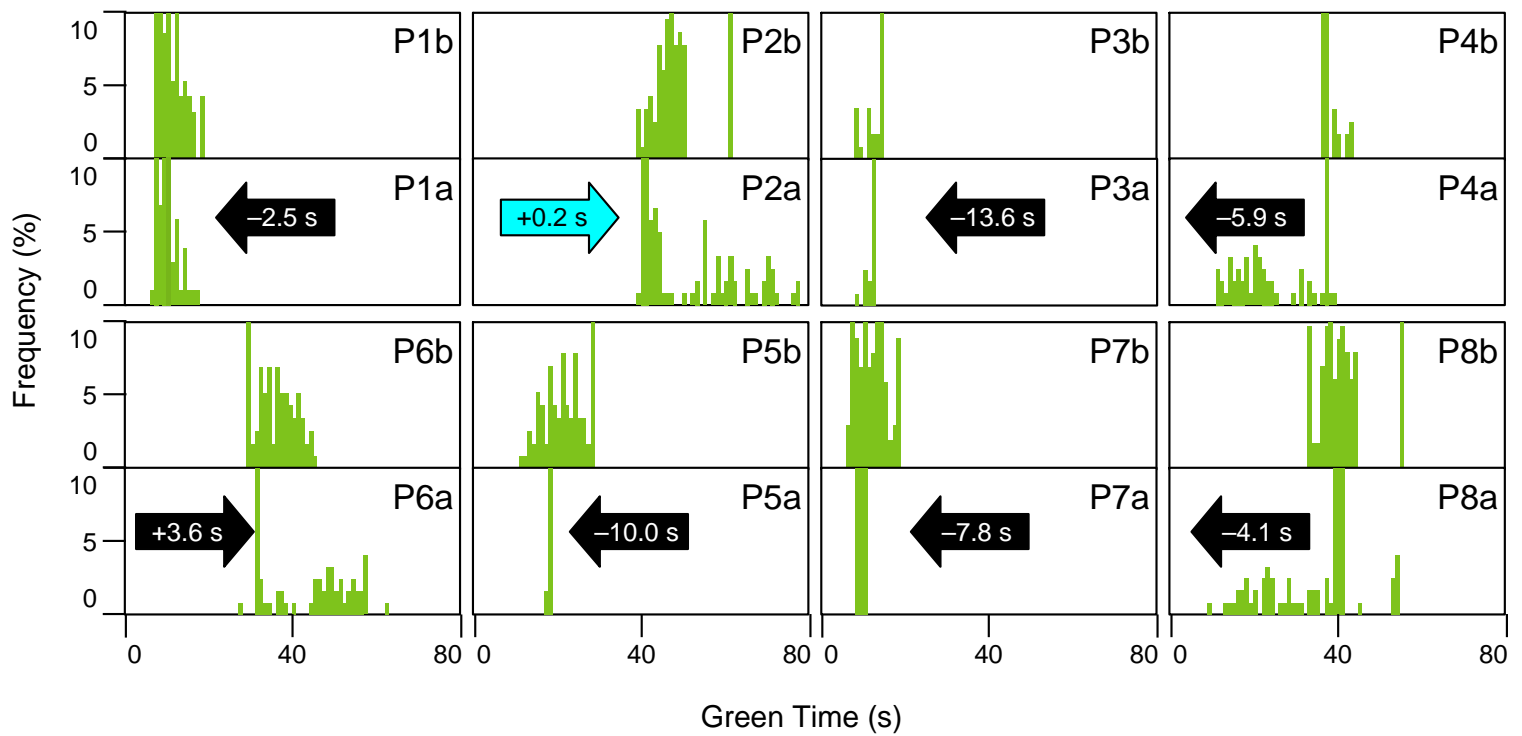

(b) PM peak period, 11:00-13:00

Statistically significant change

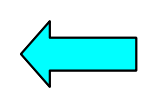

Change not statistically significant

Figure 5.26 Green time distributions at Noblesville for Wednesday, June 28, 2006 (before retiming) and Wednesday, March 21, 2006 (after retiming). 


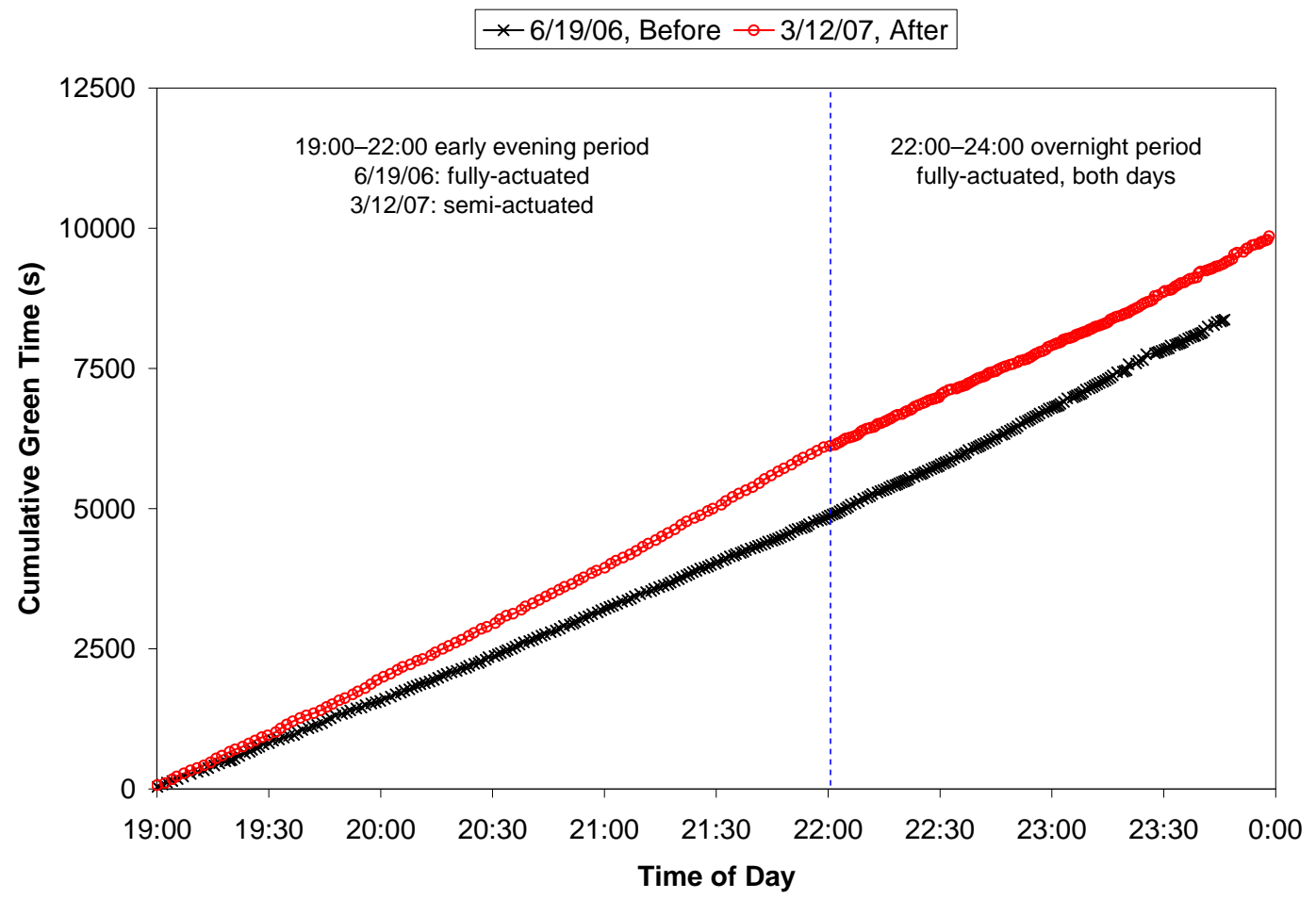

Figure 5.27 Cumulative green time for phase 2 at Noblesville during evening hours for Monday, June 19, 2006 (before retiming) and Monday, March 12, 2007 (after retiming). 


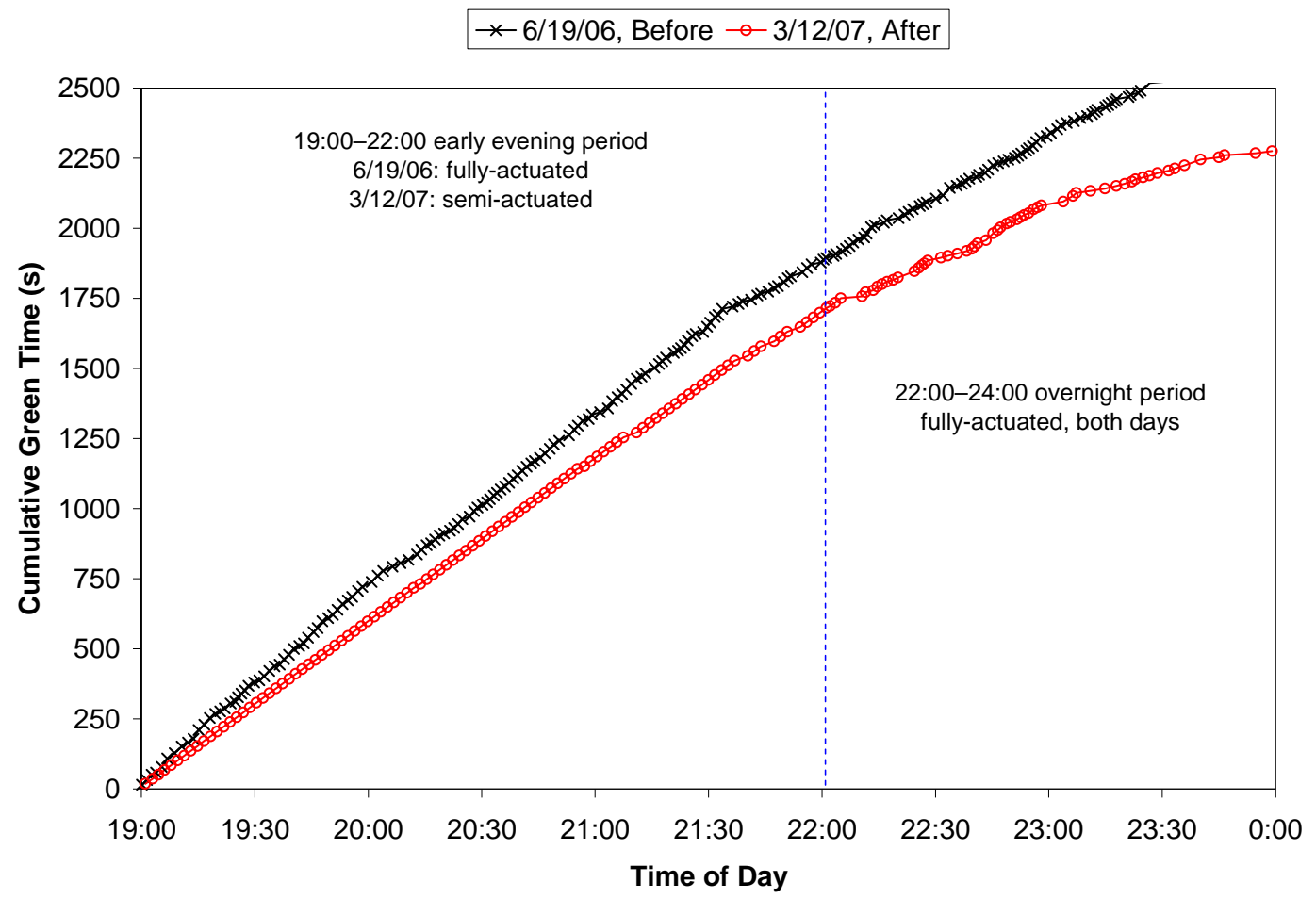

Figure 5.28 Cumulative green time for phase 5 at Noblesville during evening hours for Monday, June 19, 2006 (before retiming) and Monday, March 12, 2007 (after retiming). 


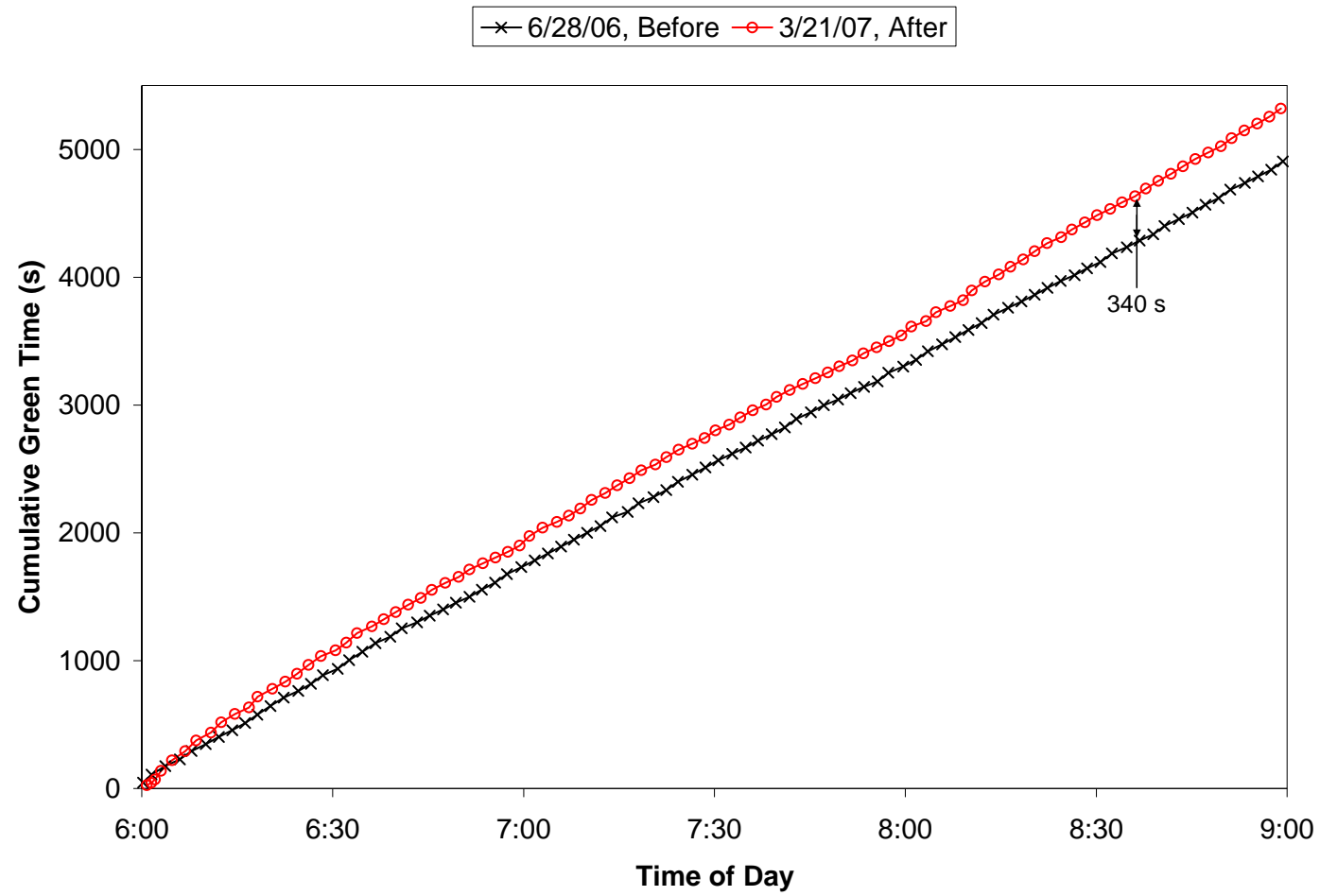

Figure 5.29 Cumulative green time for phase 2 at Noblesville during the AM peak period for Wednesday, June 28, 2006 (before retiming) and Wednesday, March 21, 2007 (after retiming). 

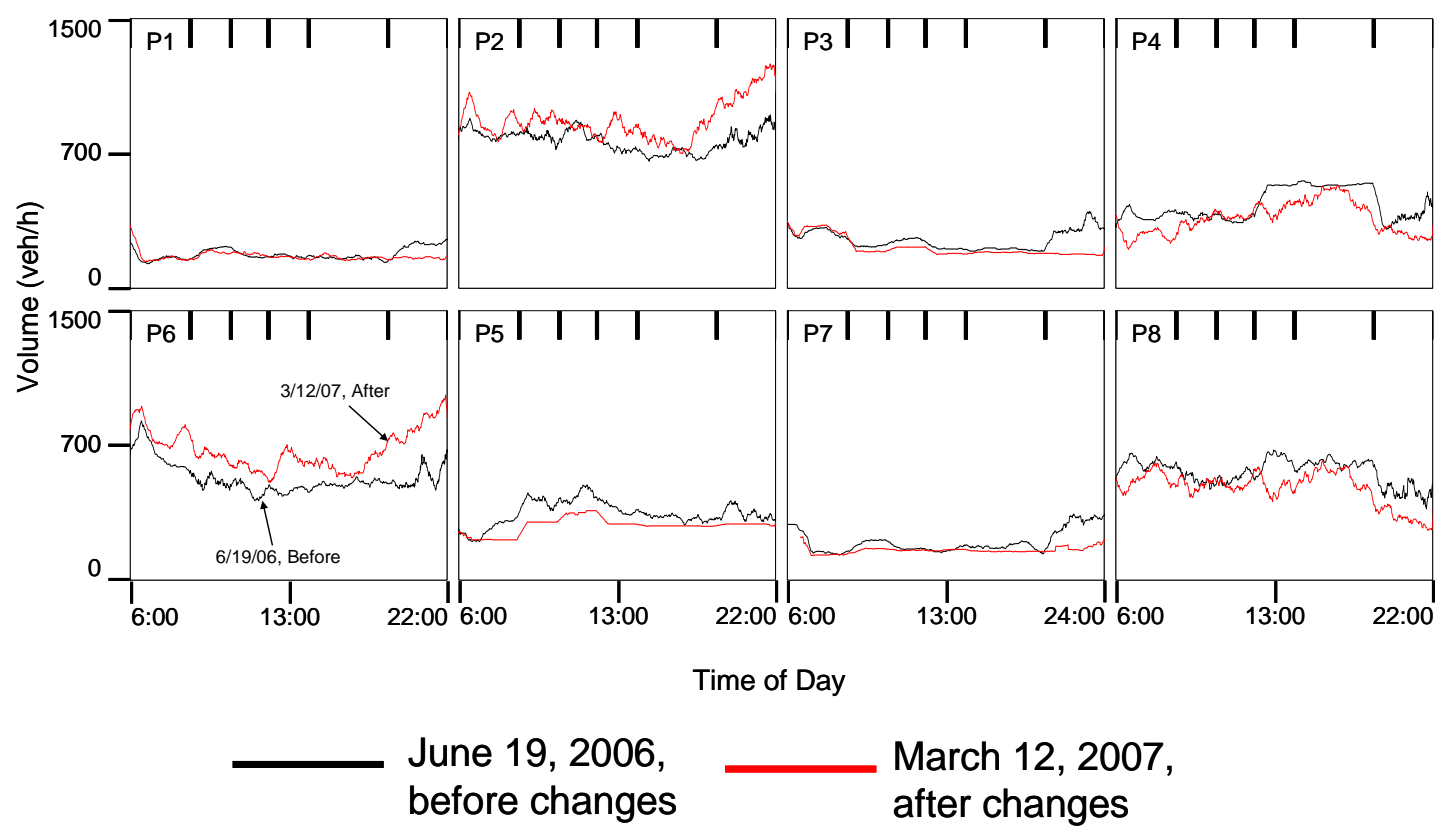

Figure 5.30 Cycle-by-cycle observed capacity (20 point average) at Noblesville for Monday, June 19, 2006 and Monday, March 12, 2007, with the midday (11:00-13:00) and early evening (19:00-22:00) time periods highlighted. 

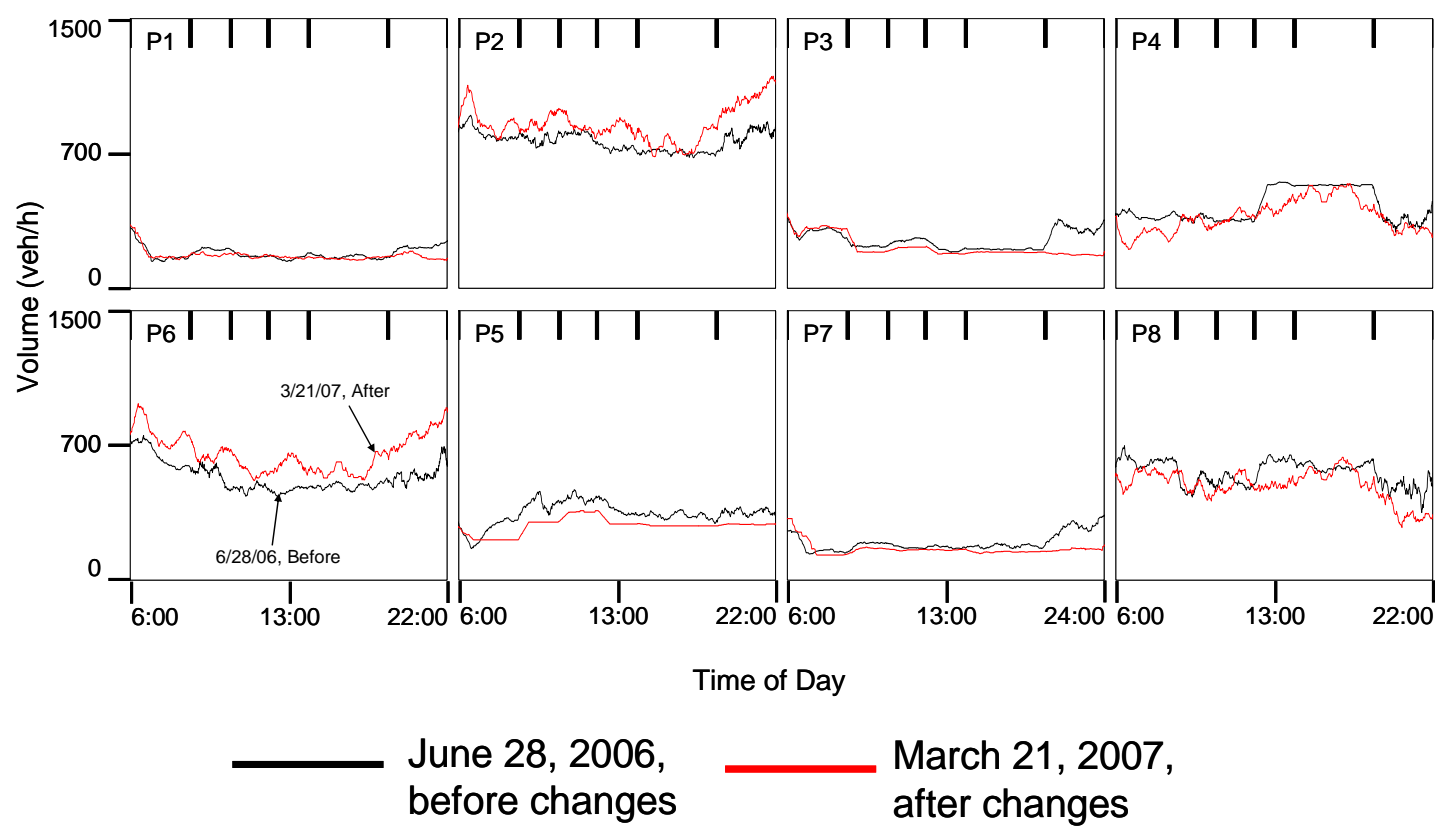

Figure 5.31 Cycle-by-cycle observed capacity ( 20 point average) at Noblesville for Wednesday, June 28, 2006 and Wednesday, March 21, 2007, with the AM (6:00-9:00) and PM (15:00-19:00) peak periods highlighted. 


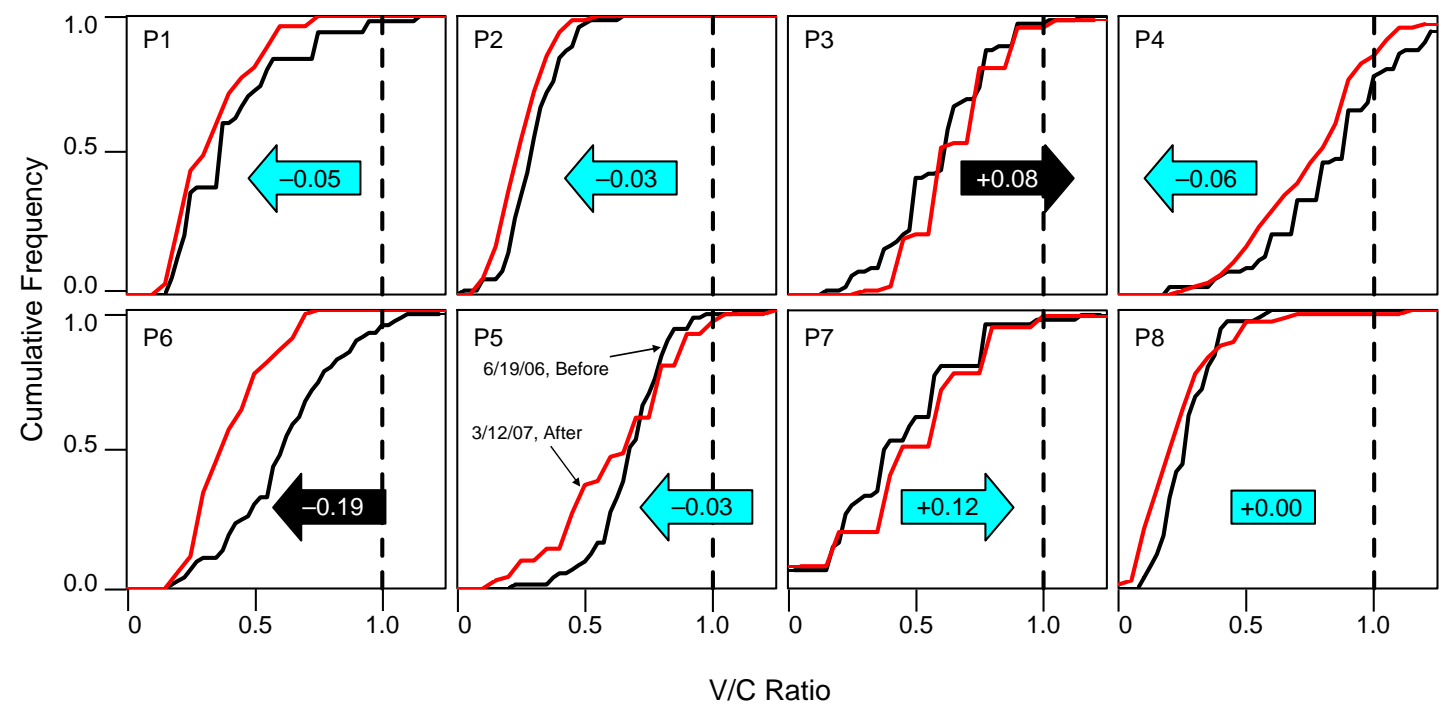

(a) Midday, 11:00-13:00

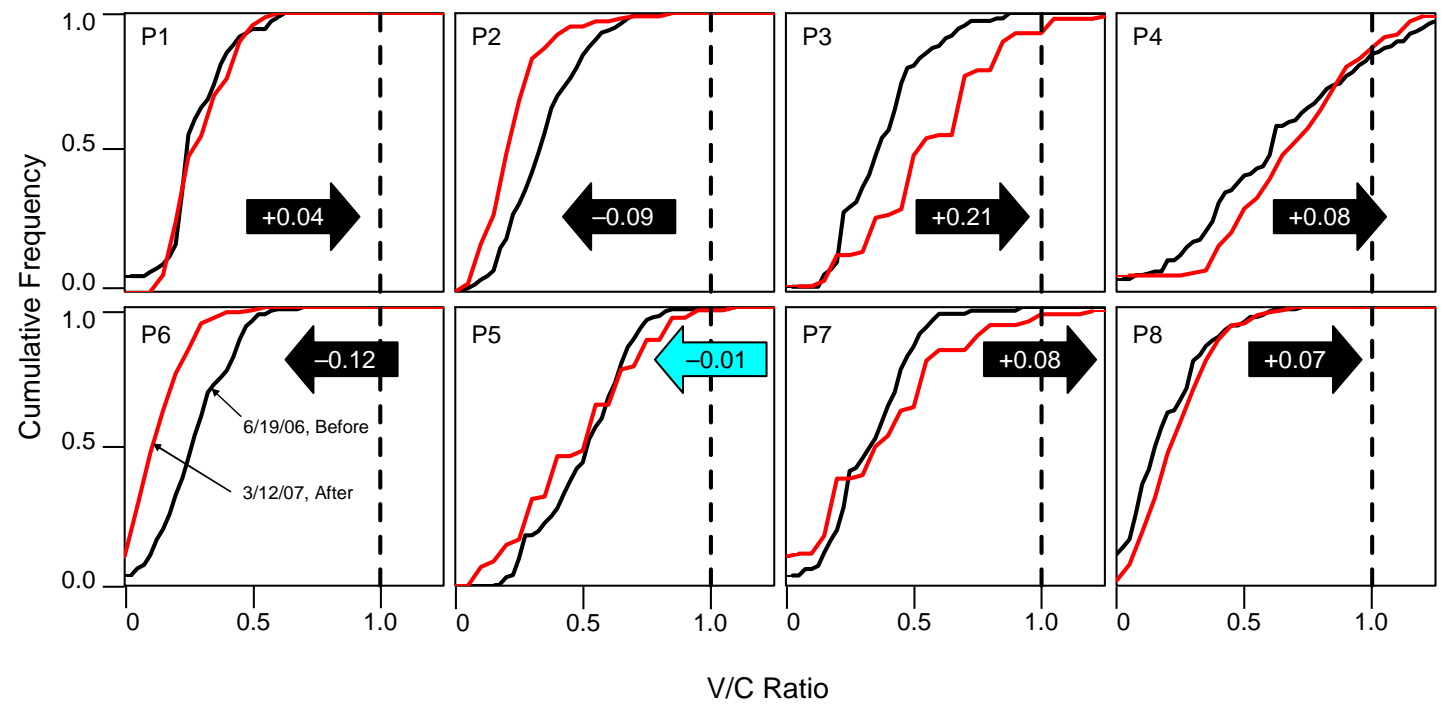

(b) Early evening, 19:00-22:00

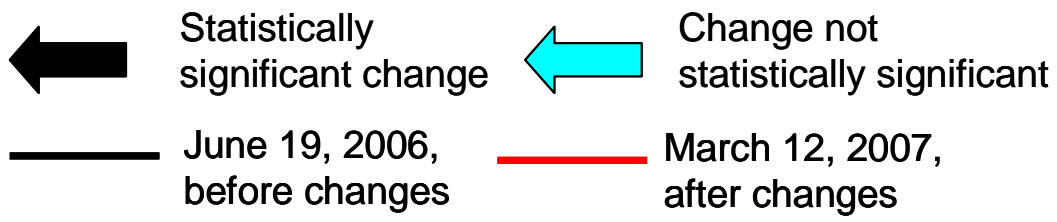

Figure 5.32 Plots of v/c ratio cumulative frequencies at Noblesville for Monday, June 19, 2006 (before retiming) and Monday, March 12, 2006 (after retiming). 


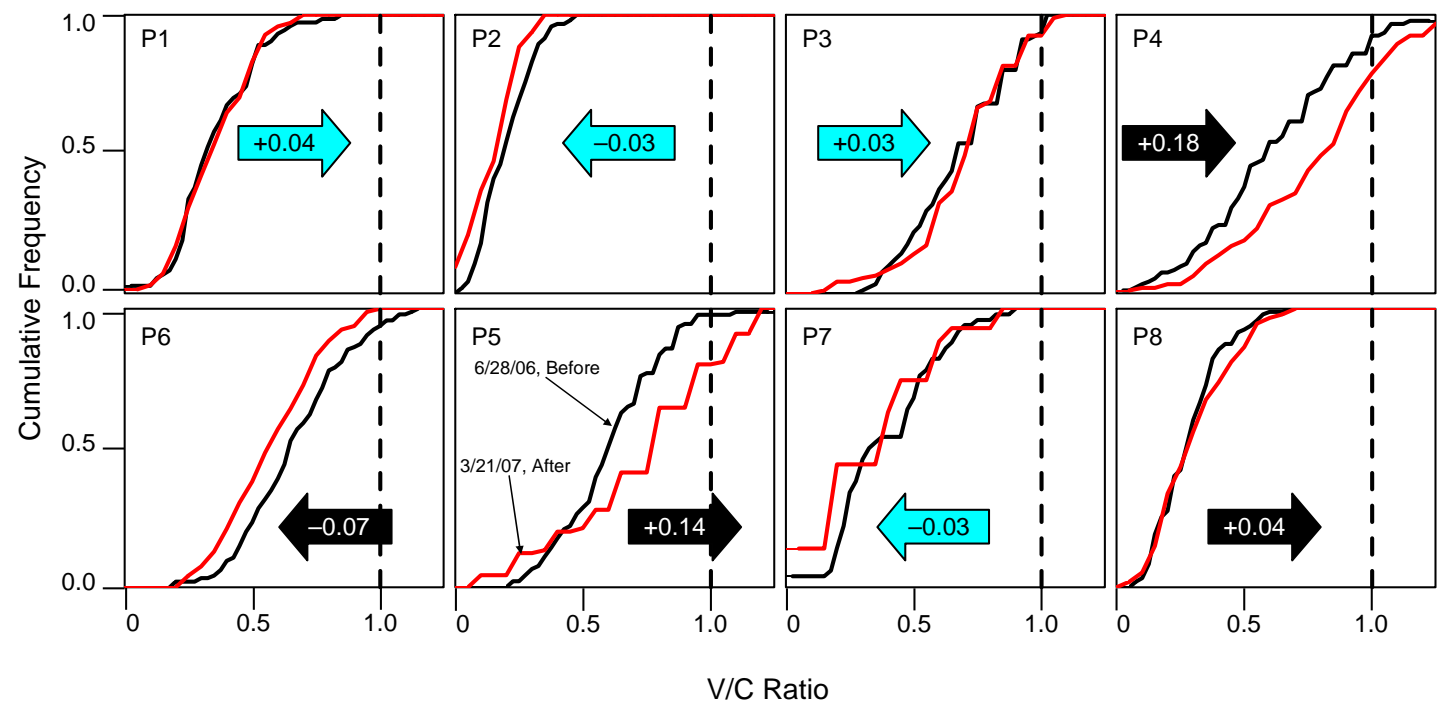

(a) AM peak period, 6:00-9:00

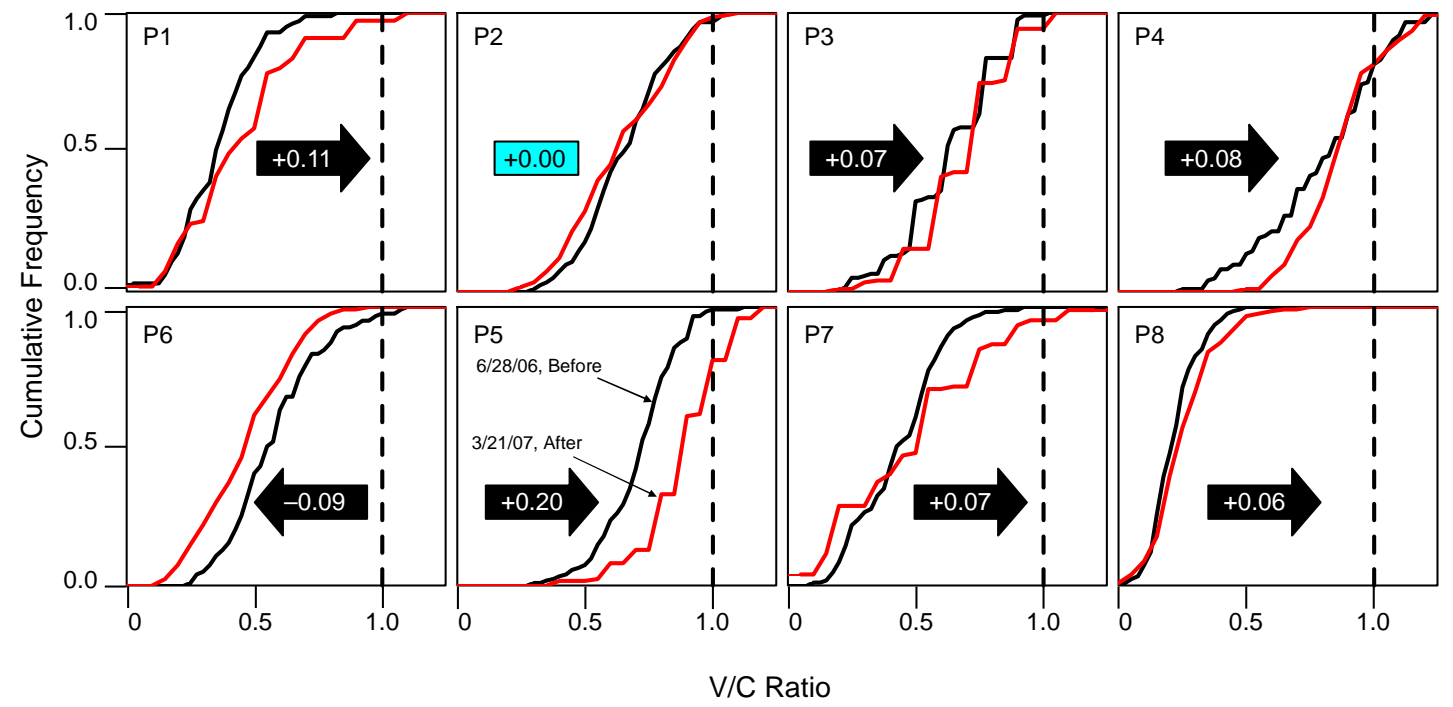

(b) PM peak period, 15:00-19:00

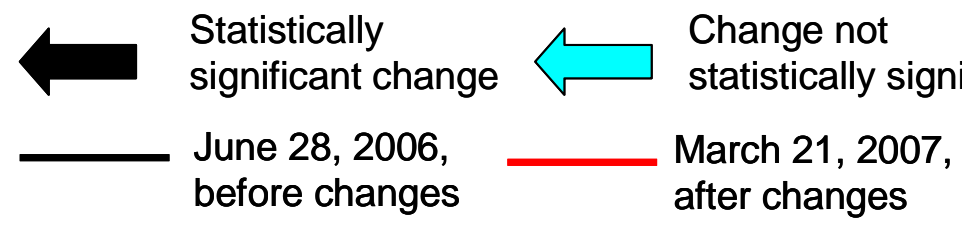

Figure 5.33 Plots of v/c ratio cumulative frequencies at Noblesville for Wednesday, June 28, 2006 (before retiming) and Wednesday, March 21, 2006 (after retiming). 
Table 5.12 Percentage of cycles ending in split failure at Noblesville, tabulated by phase and by TOD plan time period, averaged over 15 days before retiming and 15 days after retiming.

\begin{tabular}{|c|c|c|c|c|c|c|c|c|}
\hline Phase & Period & 6:00 & 9:00 & 11:00 & 13:00 & 15:00 & 19:00 & Avg. \\
\hline \multirow{2}{*}{1} & before & 0.2 & 0.5 & 1.1 & 0.1 & 0.1 & 0.0 & 0.3 \\
\hline & after & 0.7 & 0.2 & 0.8 & 0.0 & 1.6 & 0.1 & 0.7 \\
\hline \multirow{2}{*}{2} & before & 0.0 & 0.0 & 0.0 & 1.5 & 5.7 & 0.0 & 1.4 \\
\hline & after & 0.0 & 0.0 & 0.0 & 0.5 & 2.8 & 0.0 & 0.7 \\
\hline \multirow{2}{*}{3} & before & 6.2 & 5.5 & 2.9 & 8.5 & 4.0 & 0.2 & 4.2 \\
\hline & after & 5.2 & 12.0 & 7.0 & 12.3 & 7.6 & 6.2 & 8.1 \\
\hline \multirow{2}{*}{4} & before & 10.0 & 12.2 & 34.1 & 5.1 & 29.2 & 16.8 & 18.4 \\
\hline & after & 23.7 & 10.4 & 19.8 & 17.4 & 31.4 & 16.7 & 21.0 \\
\hline \multirow{2}{*}{5} & before & 4.5 & 4.7 & 0.5 & 5.5 & 3.7 & 0.4 & 3.0 \\
\hline & after & 16.5 & 15.3 & 15.0 & 17.3 & 29.9 & 2.3 & 16.6 \\
\hline \multirow{2}{*}{6} & before & 6.9 & 7.3 & 6.9 & 0.5 & 2.9 & 0.0 & 3.7 \\
\hline & after & 4.2 & 0.2 & 0.3 & 0.6 & 1.2 & 0.1 & 1.2 \\
\hline \multirow{2}{*}{7} & before & 0.1 & 1.2 & 4.4 & 0.4 & 0.4 & 0.3 & 1.1 \\
\hline & after & 4.1 & 4.5 & 6.9 & 6.1 & 4.5 & 3.9 & 4.9 \\
\hline \multirow{2}{*}{8} & before & 0.0 & 0.0 & 0.0 & 0.1 & 0.0 & 0.0 & 0.0 \\
\hline & after & 0.2 & 0.1 & 0.1 & 0.0 & 0.0 & 0.0 & 0.1 \\
\hline \multicolumn{9}{|c|}{ Coordinated Phases $(2,6)$} \\
\hline \multirow{2}{*}{ Avg. } & before & 3.5 & 3.7 & 3.5 & 1.0 & 4.3 & 0.0 & 2.5 \\
\hline & after & 2.1 & 0.1 & 0.1 & 0.5 & 2.0 & 0.1 & 1.0 \\
\hline \multicolumn{9}{|c|}{ Non-coordinated Phases $(1,3,4,5,7,8)$} \\
\hline \multirow{2}{*}{ Avg. } & before & 3.9 & 4.5 & 7.6 & 3.6 & 6.6 & 3.6 & 5.0 \\
\hline & after & 9.1 & 7.5 & 8.6 & 9.3 & 12.9 & 5.2 & 9.0 \\
\hline \multicolumn{9}{|c|}{ All Phases } \\
\hline \multirow{2}{*}{ Avg. } & before & 3.8 & 4.3 & 6.5 & 2.9 & 6.0 & 2.5 & 4.3 \\
\hline & after & 7.2 & 5.5 & 6.4 & 7.0 & 10.1 & 3.8 & 6.9 \\
\hline
\end{tabular}




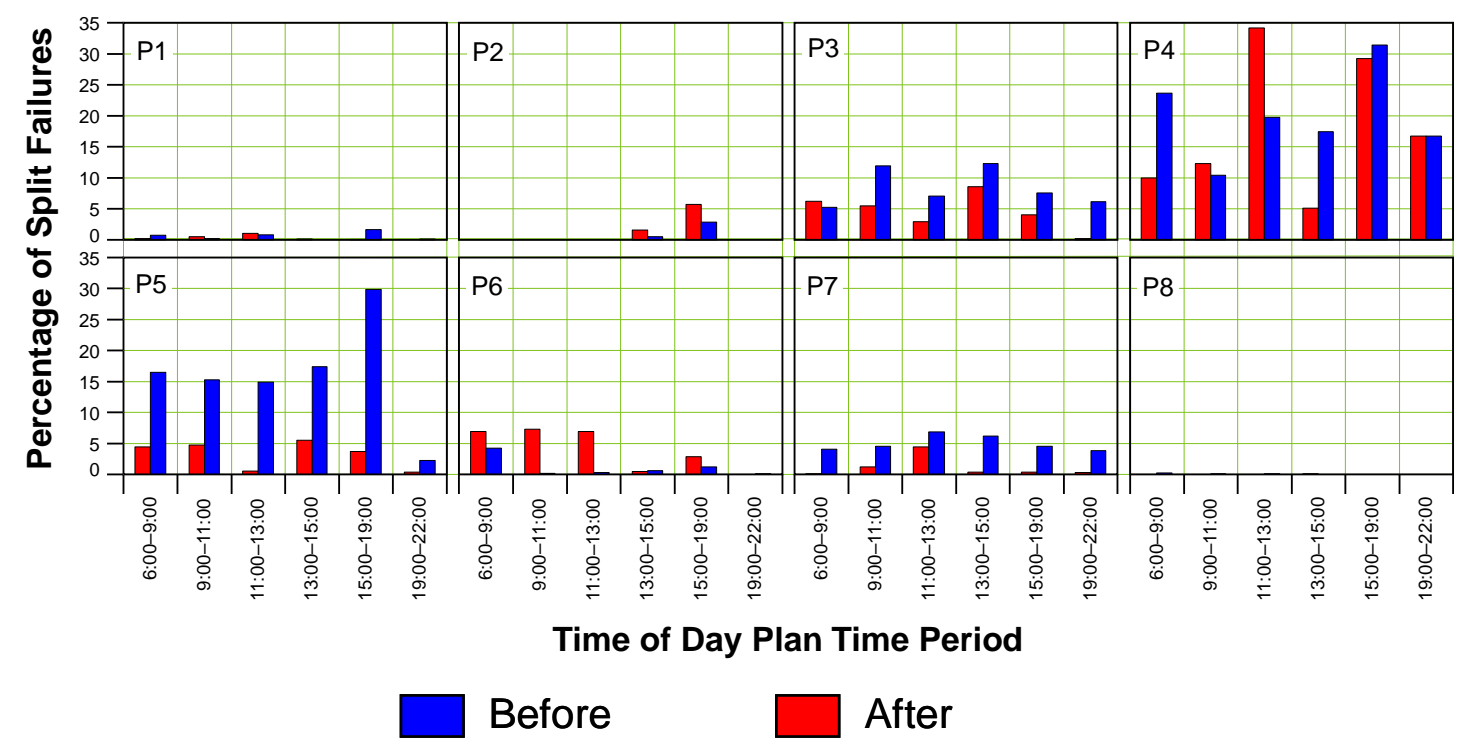

Figure 5.34 Percentage of cycles at the Noblesville test intersection ending in split failure, shown by phase and by TOD plan time period, averaged over 15 days before retiming and 15 days after retiming. 


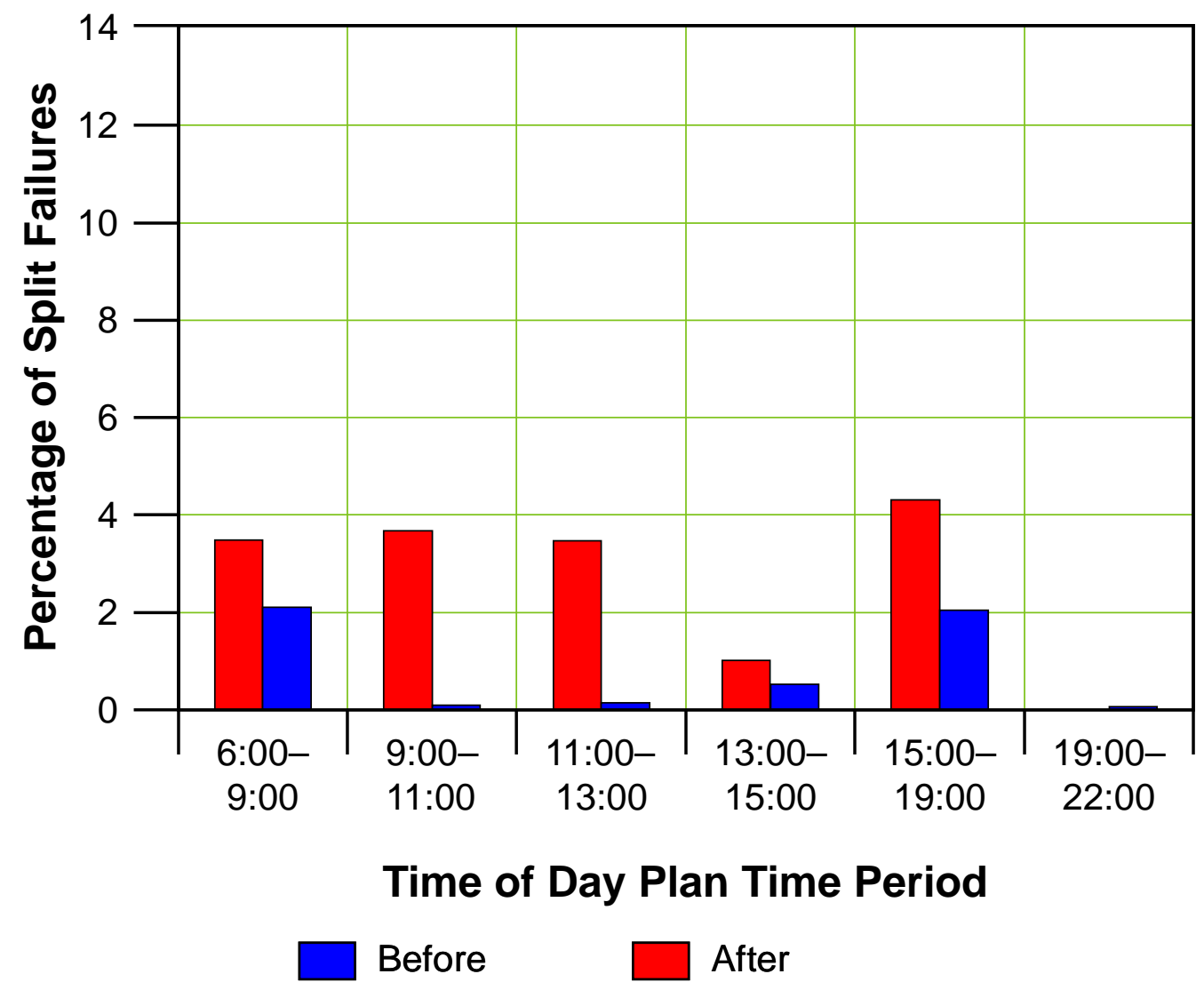

Figure 5.35 Percentage of cycles ending in split failure for coordinated phases 2 and 6 at Noblesville, averaged over 15 days before retiming and 15 days after retiming. 


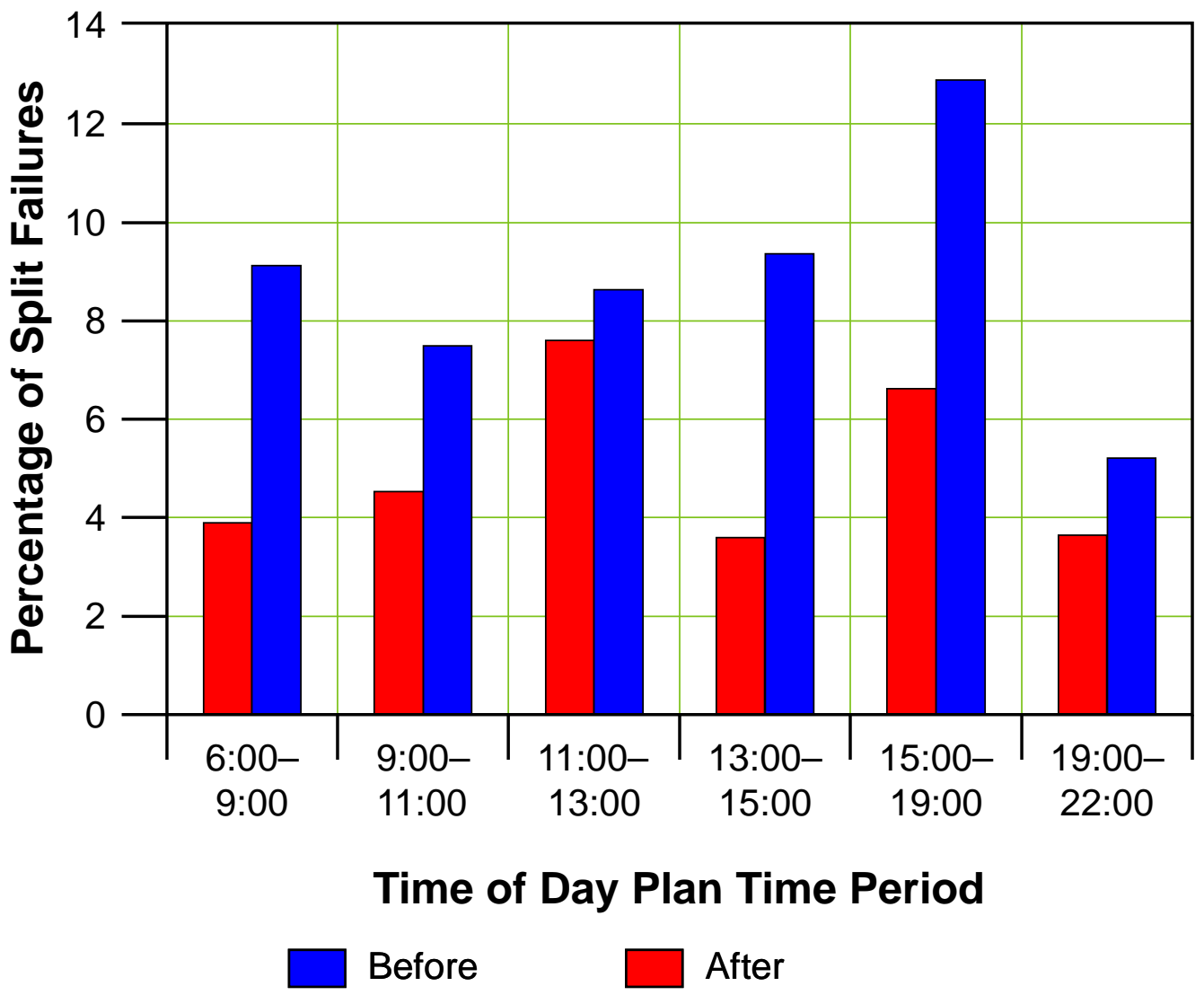

Figure 5.36 Percentage of cycles ending in split failure for non-coordinated phases 1, 3, $4,5,7$, and 8 at Noblesville, averaged over 15 days before retiming and 15 days after retiming. 


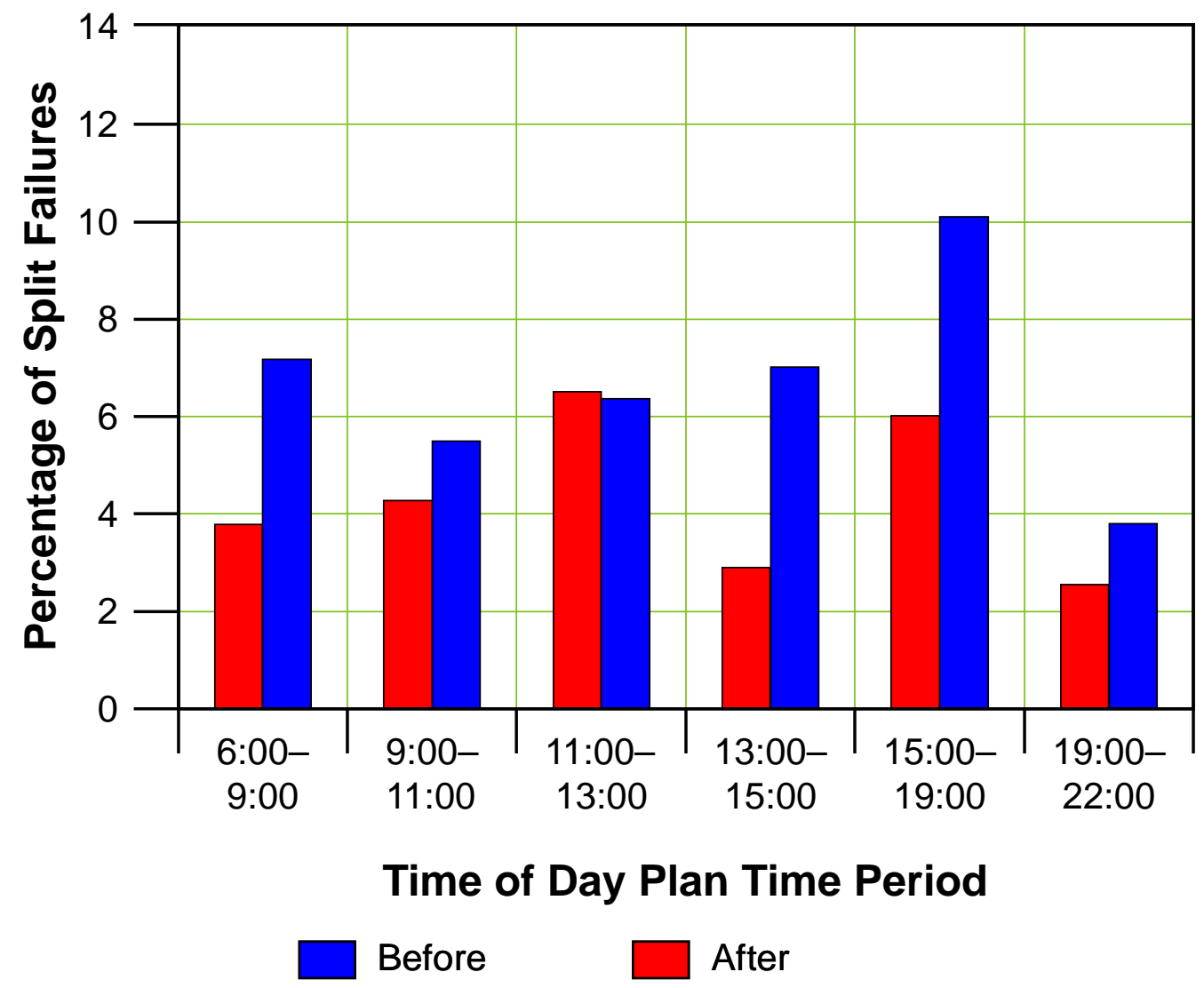

Figure 5.37 Percentage of cycles ending in split failure for all eight phases at Noblesville, averaged over 15 days before retiming and 15 days after retiming. 


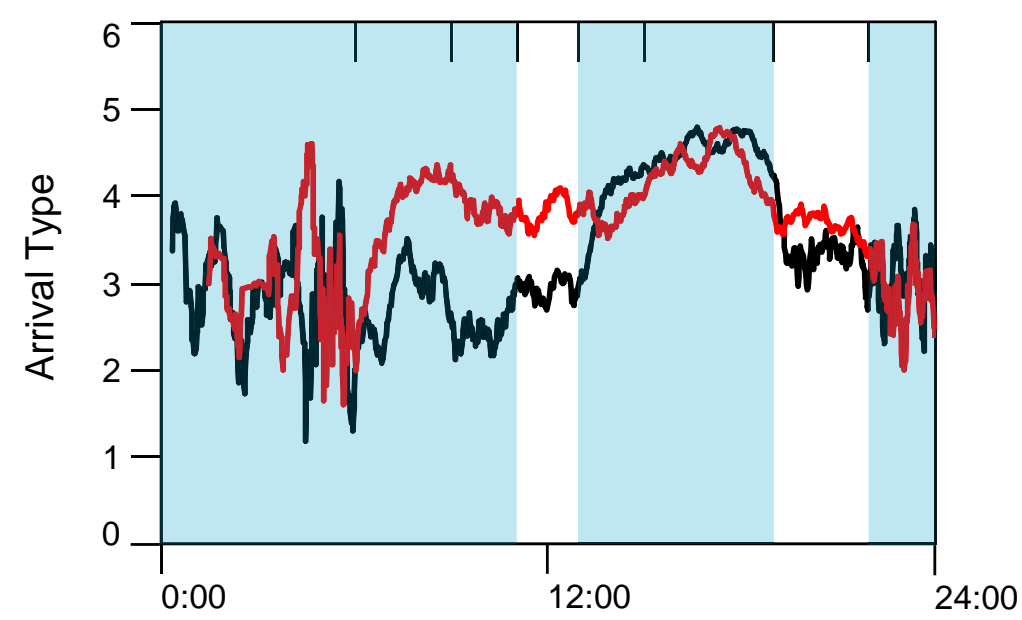

Time of Day

(a) Phase 2

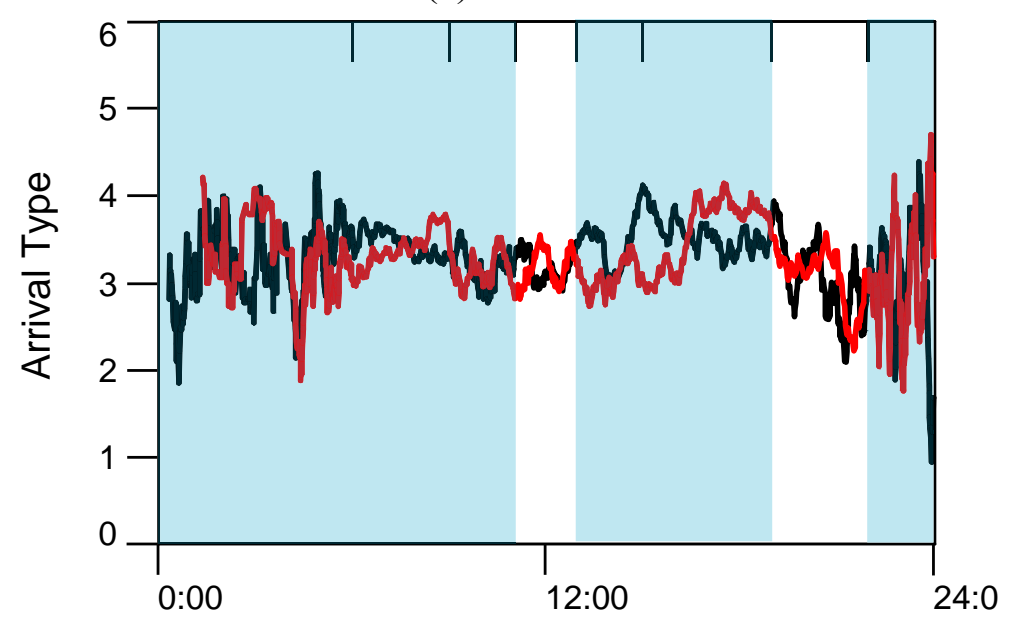

Time of Day

(b) Phase 6

June 19, 2006, March 12, 2007, before changes after changes

Figure 5.38 Arrival Type (20 point average) at Noblesville, for Monday, June 19, 2006 (before retiming) and Monday, March 12, 2007 (after retiming), with the 11:00-13:00 midday and 19:00-22:00 early evening time periods highlighted. 


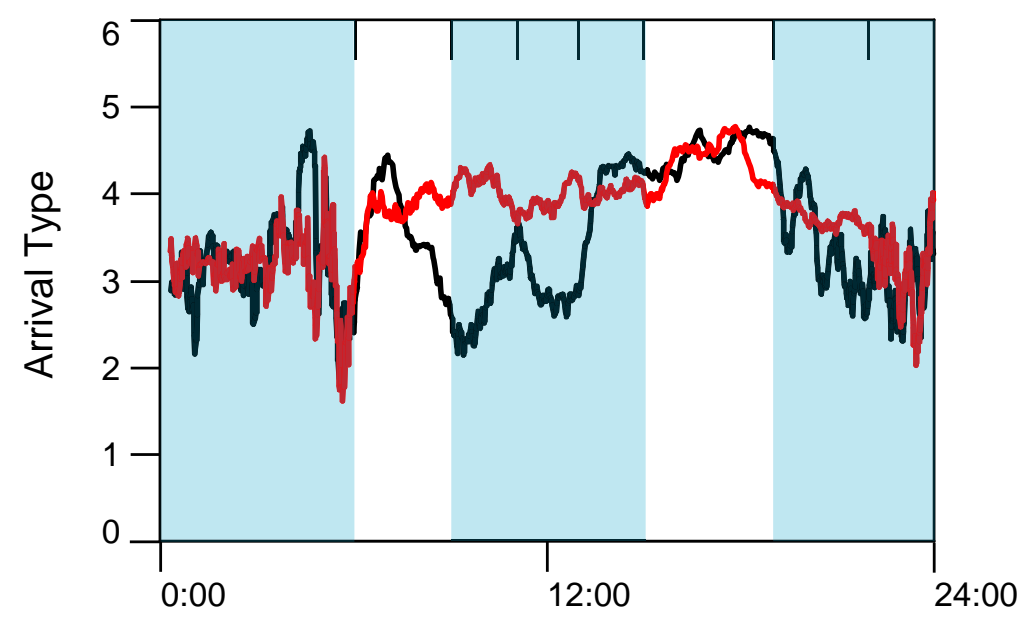

Time of Day

(a) Phase 2

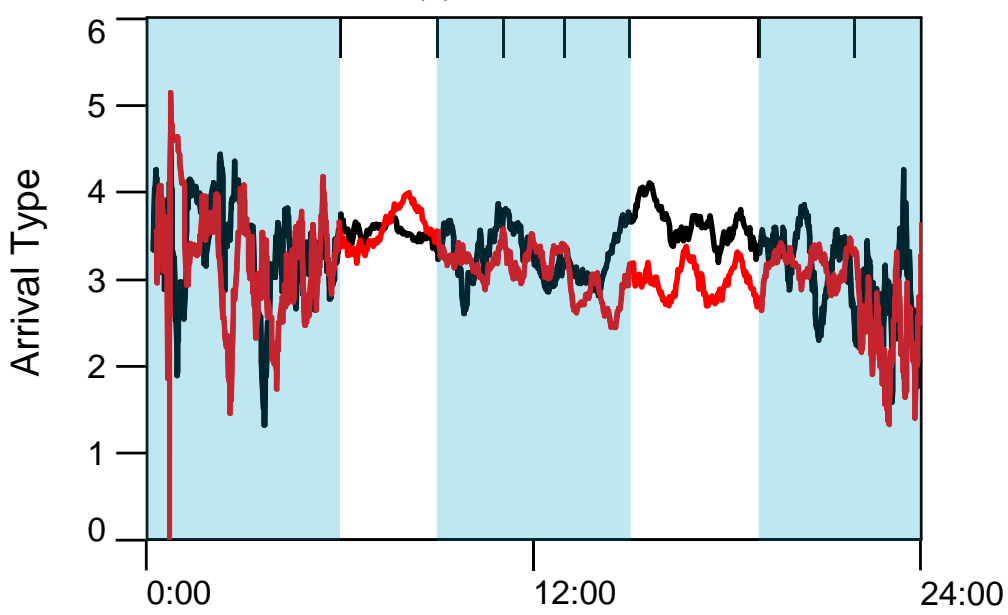

Time of Day

(b) Phase 6

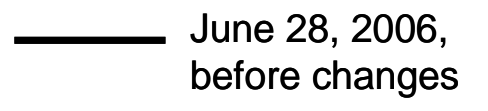
March 21, 2007, after changes

Figure 5.39 Arrival Type (20 point average) at Noblesville, for Wednesday, June 28, 2006 (before retiming) and Wednesday, March 21, 2007 (after retiming), with the 6:009:00 AM and 15:00-19:00 PM peak periods highlighted. 


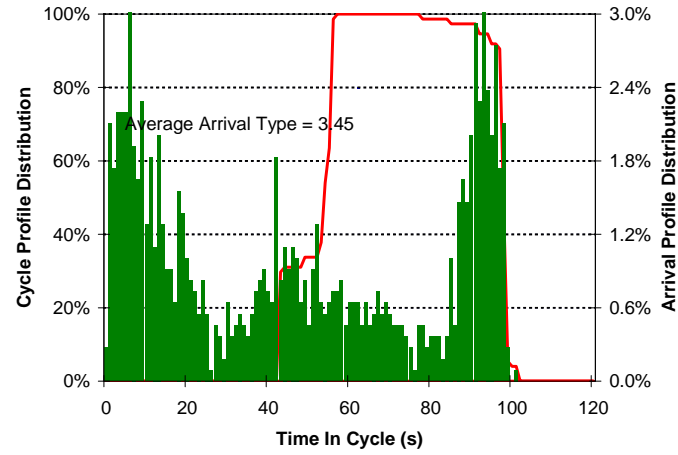

(a) Phase 2, Before (6/19/06)

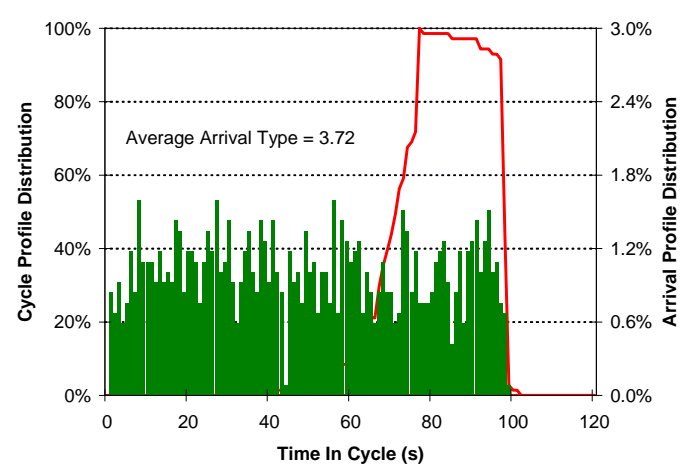

(c) Phase 6, Before $(6 / 19 / 06)$

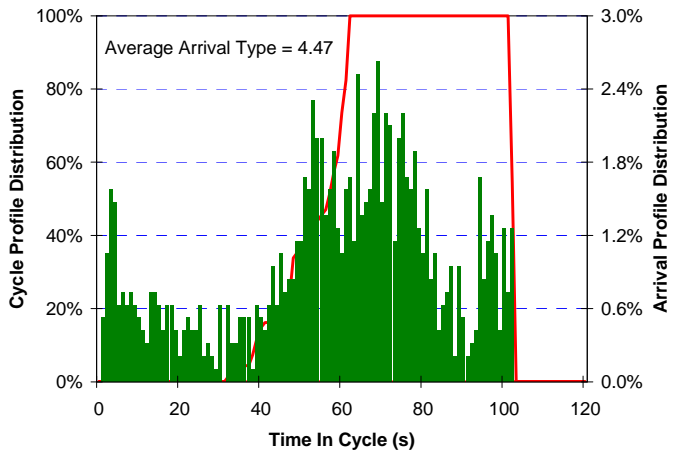

(b) Phase 2, After (3/12/07)

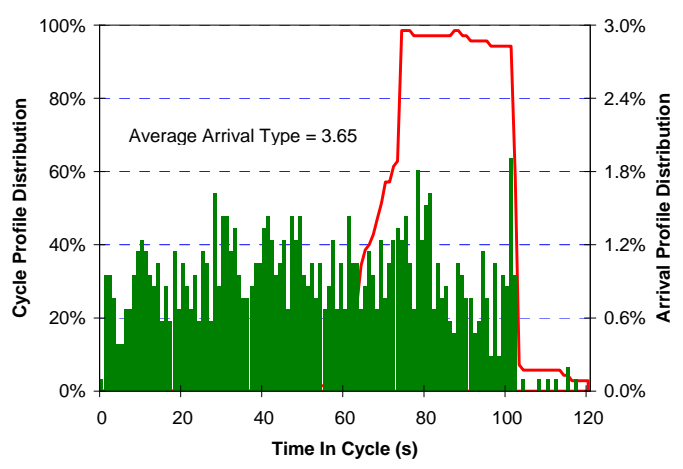

(d) Phase 6, After (3/12/07)

Figure 5.40 Platoon profiles for phases 2 and 6 at Noblesville, before and after retiming, during the 11:00-13:00 midday time period. 


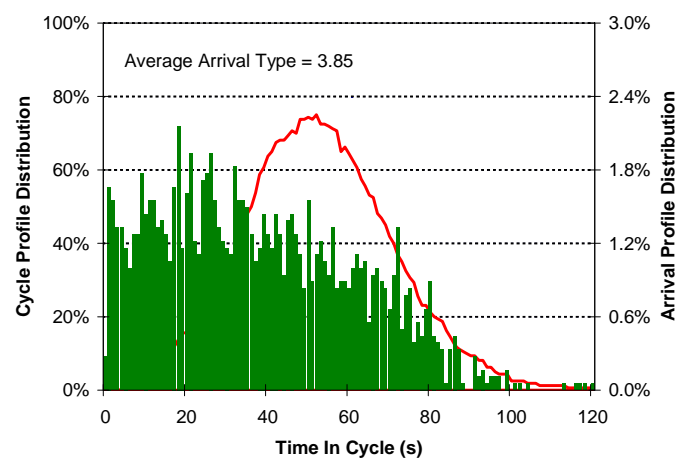

(a) Phase 2, Before (6/19/06)

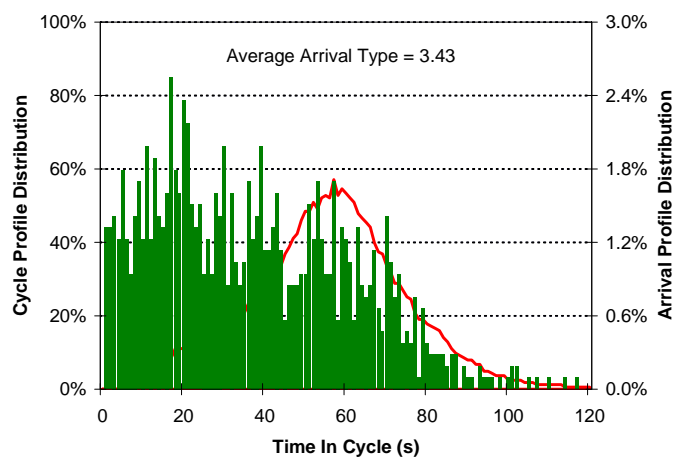

(c) Phase 6, Before (6/19/06)

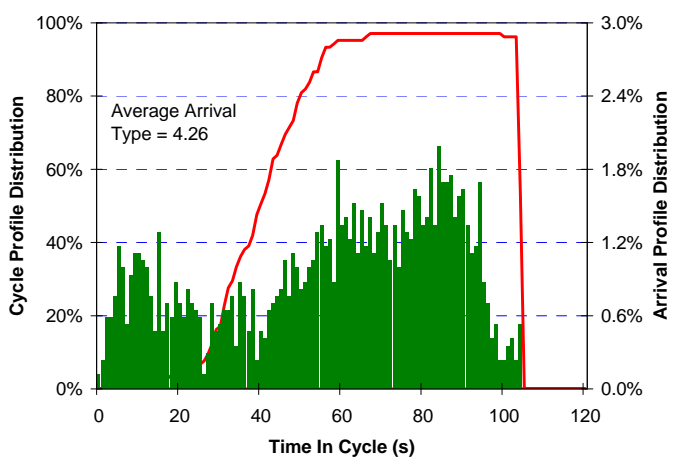

(b) Phase 2, After (3/12/07)

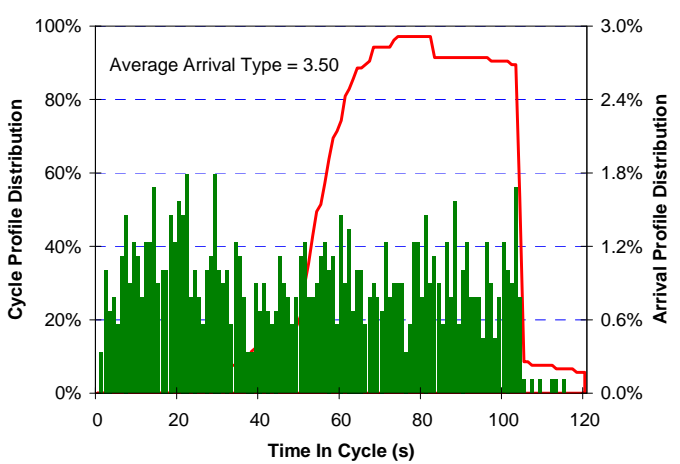

(d) Phase 6, After (3/12/07)

Figure 5.41 Platoon profiles for phases 2 and 6 at Noblesville, before and after retiming, during the 15:00-19:00 early evening time period. 


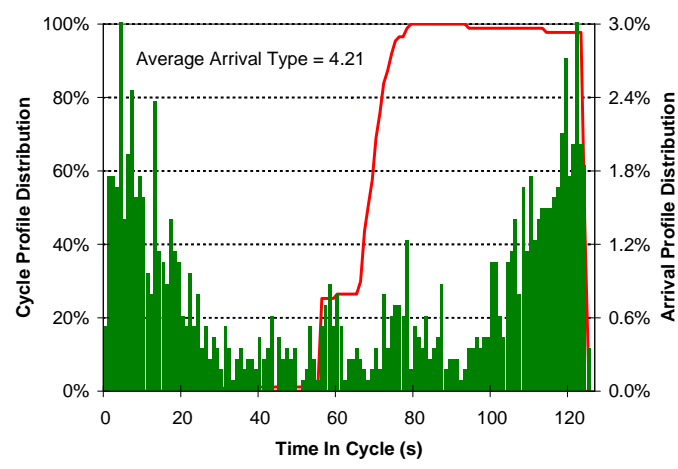

(a) Phase 2, Before (6/28/06)

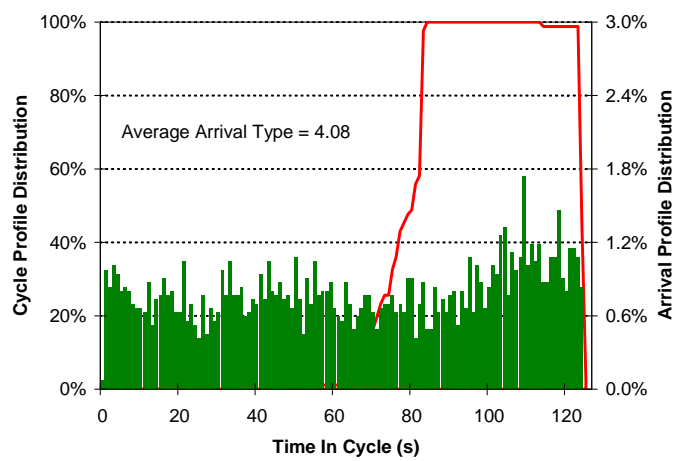

(c) Phase 6, Before (6/28/06)

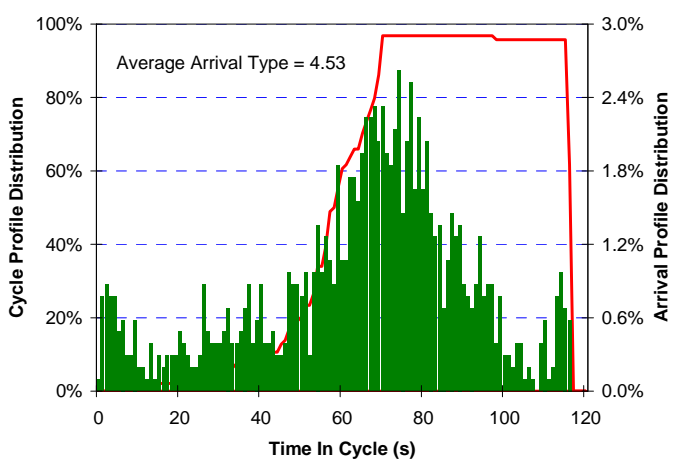

(b) Phase 2, After (3/21/07)

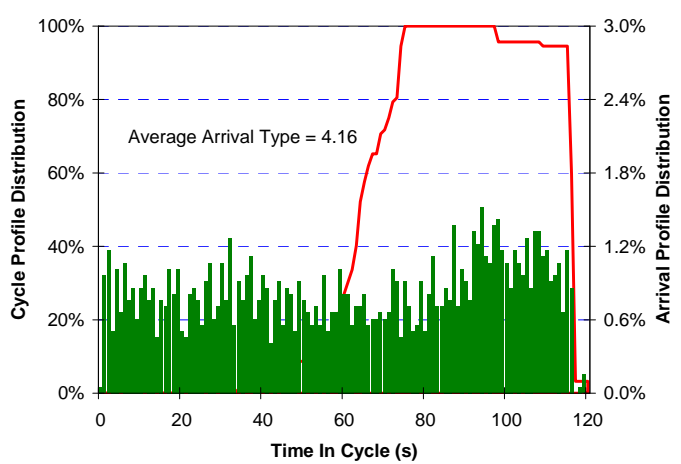

(d) Phase 6, After (3/21/07)

Figure 5.42 Platoon profiles for phases 2 and 6 at Noblesville, before and after retiming, during the 6:00-9:00 AM peak period. 


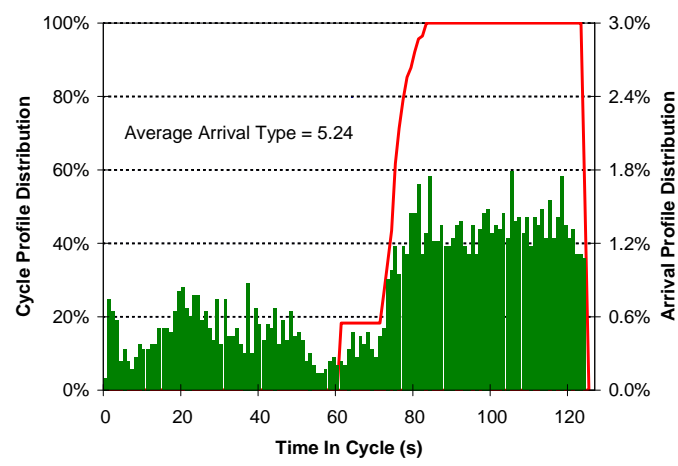

(a) Phase 2, Before (6/28/06)

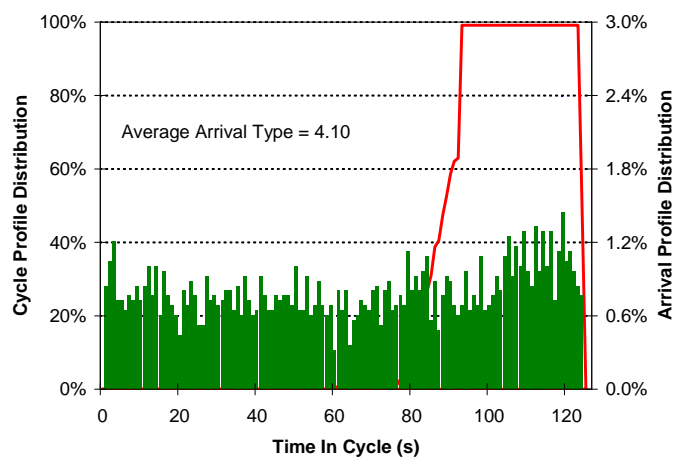

(c) Phase 6, Before (6/28/06)

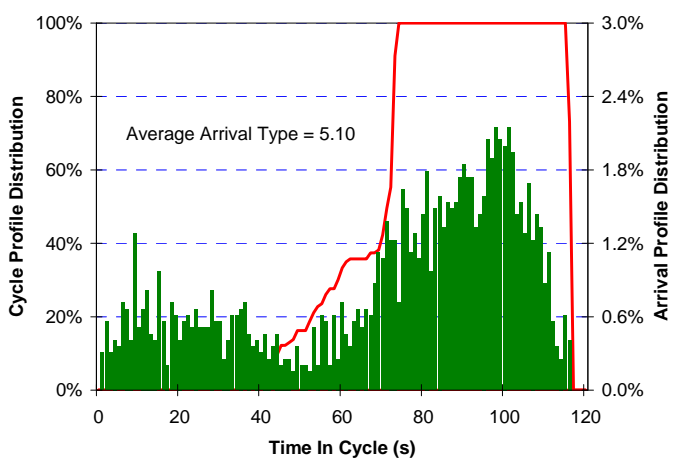

(b) Phase 2, After (3/21/07)

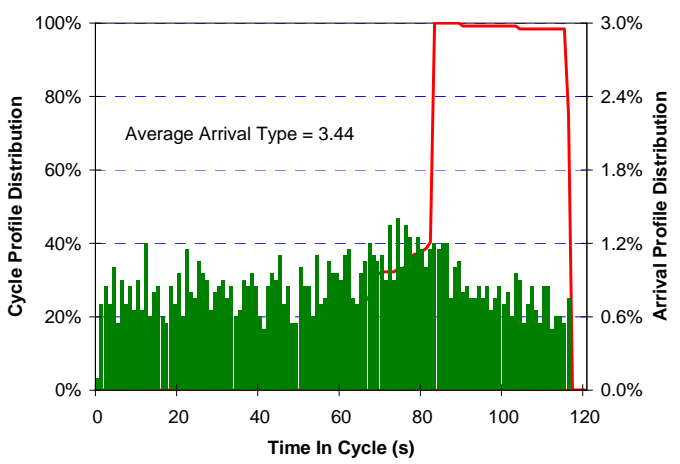

(d) Phase 6, After (3/21/07)

Figure 5.43 Platoon profiles for phases 2 and 6 at Noblesville, before and after retiming, during the 15:00-19:00 PM peak period. 


\subsubsection{Statistical Analysis}

This section presents the statistical tables for the two pairwise comparisons described in the previous section, as well as an aggregate comparison between 15 days before and 15 days after signal retiming. The methodology for aggregation was described in Section 4.5.3. In the tables shown in this section, each item is shown for each of the eight phases by the TOD plan time period. Statistically significant (at the $95 \%$ confidence level) figures are shown in boldface.

\subsubsection{Pairwise Statistical Comparisons}

The changes in green time, v/c ratio, and arrival type were described thoroughly in the previous section for the two pairs of days considered. The associated statistical tables are included here for the sake of completeness.

Statistical data from the pairwise comparison between Monday, June 19, 2006 and Monday, March 12, 2007 is presented in Tables 5.13-5.15. The difference in mean values of volume are shown in Table 5.13(a), green time in Table 5.13(b), v/c ratio in Table 5.13(c), and arrival type in Table 5.13(d). The corresponding parts of Table 5.14 and Table 5.15 present the corresponding statistical t-values and P-values respectively. This pair of days was intended to be used for comparison of the midday (11:00-13:00) and early evening (19:00-22:00) time periods, because the volumes were very similar between the two days during those time periods, as shown in Table 5.13(a). Among the 15 days before and 15 days after retiming, a pair with more similar volumes during those time periods could not be found.

The pair of days selected for comparison of performance measures for peak periods consisted of Wednesday, June 28, 2006 and Wednesday, March 21, 2007. The data are shown in Table 5.16 (difference in means), Table 5.17 (t-values), and Table 5.18 (P-values). In each table, part (a) gives the relevant information for volume; part (b) for green time; part (c) for v/c ratios; and part (d) for the arrival types. The difference in means for the volumes for this pair of days is shown in Table 5.16(a), revealing that volumes were mostly similar between the two days. Again, among the 15 days in each of the before and after data sets, a pair of days with more similar volumes did not exist.

These sets of statistical tables confirm the observations made in the previous section. It is not necessary to repeat all of those observations again. Rather, a summary will suffice. The trend in most time periods was to increase the green time for 
coordinated phases 2 and 6 , thereby lowering the $\mathrm{v} / \mathrm{c}$ ratios for those phases. The cost was suffered by the non-coordinated phases, which generally saw their $\mathrm{v} / \mathrm{c}$ ratios increasing. The new timing plan improved coordination, resulting in higher AT values for phase 2 for most time periods.

\subsubsection{Aggregate Statistical Comparison}

The two pairwise comparisons described in the previous section and in the above mentioned statistical tables characterized the changes in performance measures observed in days when demand was roughly the same. The aggregate comparison examines all of those changes along with the changes in volume in order to ascertain the overall movement within the average performance measures for each phase, for each time period.

The differences in mean values of volume, averaged over the 15 days in each of the before and after data sets, are shown in Table 5.19(a), with statistical t-values in Table 5.20(a) and P-values in Table 5.21(a). Volumes were lower for most phases during most time periods in March 2007 compared to June 2006, with the exception of the AM peak period, where volume increased for most phases. The impact on the resulting performance measures cannot be separated by this analysis. However, when changes in a performance measure are observed that contradict an expected change due to volume, it is possible to conclude that the impact was due to the operation change rather than the volume change.

The difference in mean values of green time are shown in Table 5.19(b), with tvalues given in Table 5.20(b) and P-values in Table 5.21(b). The green time for the coordinated phases increased in most time periods, and there were no decreases. Even when cycle lengths decreased, as during the AM and PM peak periods, the green time of the coordinated phases nevertheless increased. For phase 6, this was partly the result of using the lagging left turn. Phase 5 could no longer obtain additional green time from phase 6 , whereas before retiming, it was possible for phase 5 to time to its force-off point, depriving phase 6 of green time. The other reason for phase 6 to increase its green time was that its splits were greater. This was the main reason for phase 2 to see an increase in green time.

The non-coordinated phases, in the meanwhile, generally saw decreases in green time, except for those times of day in which the cycle length increased, as during the midday periods. When this occurred, the green time for the non-coordinated phases increased as the entire cycle length increased. This is especially true of the 9:00-11:00 time period, when cycle length went from $78 \mathrm{~s}$ to $96 \mathrm{~s}$. During the peak periods, when 
cycle lengths decreased from $124 \mathrm{~s}$ to $116 \mathrm{~s}$, the coordinated phases did not lose green time at all, so the shorter cycle length resulted from taking time away from the noncoordinated phases, as is evident in the decrease in green time for those phases. This is particularly noticeable during the 13:00-15:00 time period, when cycle length decreased from $124 \mathrm{~s}$ to $104 \mathrm{~s}$. The $20 \mathrm{~s}$ difference is reflected in losses of over $10 \mathrm{~s}$ in phases 4 and 8 , and other sizeable losses for the left turn phases.

During the early evening time period, phases $2,4,5$, and 6 see increases in green time, while phases 1,3 , and 7 see decreases. The increases are very large for the coordinated phases, a result of the implementation of semi-actuated operation, forcing those phases to hold the green when they would have gapped out under free operation. Under semi-actuated operation, phases 4 and 5 see more green time, whereas phases 3 and 7 see less green time. These changes are due to the difference between the way that the green time is distributed under the two kinds of operation. Under free operation, the time of each phase was based entirely on whatever demand was present, whereas under semi-actuated operation, the time was influenced by the splits. The splits for phases 4 and 5 are larger than those of phases 3 and 7. So, when semi-actuated operation was implemented, phases 4 and 5 saw more green time while phases 3 and 7 saw less of it.

The differences in mean $\mathrm{v} / \mathrm{c}$ ratios are given in Table 5.19(c), with t-values in Table 5.20(c) and P-values in Table 5.21(c). These trends are somewhat more uniform than the changes in green time. The average $\mathrm{v} / \mathrm{c}$ ratio decreased for the coordinated phases during every time period. This may be attributed in part to lower volumes, but also is a result of more green time being received by these phases. The cost for improving $\mathrm{v} / \mathrm{c}$ of the coordinated phases was that $\mathrm{v} / \mathrm{c}$ ratios increased for all of the non-coordinated phases. Despite decreased volumes, v/c ratios nevertheless increased, affirming the impact of the new timing plan.

The $\mathrm{v} / \mathrm{c}$ ratios of the non-coordinated phases decreased during the midday periods (9:00-11:00 and 11:00-13:00), when increased cycle lengths led to increased green time for each phase during a cycle. This may lead one to conclude that we can further improve $\mathrm{v} / \mathrm{c}$ by continuing to increase the cycle length. However, this is not a complete view of the signal operation. For one, the cycle length must be maintained at a certain interval in order to facilitate coordination, which requires that a reference point be repeated at a regular interval. The other reason is that as cycle length increases, the amount of delay increases for the minor movements.

During the early evening period, when operation changed from fully-actuated to semi-actuated, $\mathrm{v} / \mathrm{c}$ ratios again decreased for the coordinated phases while increasing for all of the other phases, including phases 4 and 5, which also had increased green time. 
The $\mathrm{v} / \mathrm{c}$ increases took place despite decreased volumes for all phases. This reflects the different ways that phases were served in the two types of operation. Under free operation, each phase was served within a short time after a call was made, and a smaller number of vehicles was served. Under semi-actuated operation, the non-coordinated phases were served only after phases 2 and 6 timed to the reference point. Thus, queues formed on the approaches for the non-coordinated phases, and their green times were used more closely to saturation. This can be seen in phases 3 and 7, which lost green time, as well as in phases 4 and 5, which gained green time. All of these phases had lower volumes; but the change in operation caused them to serve vehicles with higher $\mathrm{v} / \mathrm{c}$ ratios. For phase 5, the placement of the phase in the cycle was also influential. Since it became a lagging green, and its arrivals were fed by the coordinated northbound movement along the arterial, this phase saw an increase in $\mathrm{v} / \mathrm{c}$ despite a decrease in volume and an increase in green time.

Finally, the difference in mean values of arrival type of phases 2 and 6 are shown in Table 5.19(d), with t-values in Table 5.20(d) and P-values in Table 5.21(d). The results are different for the two phases. Phase 6 sees little impact for most time periods, which is what we expect, since it is not coordinated with the upstream signal. There is a statistically significant decrease in AT during the AM peak (6:00-9:00) and the afternoon (13:00-15:00) time periods. As mentioned previously, the decrease in AT is likely due to a slight peak in the distribution of arrivals during the cycle caused by an interaction with the upstream signal that was captured better before retiming. It should be noted that the differences in AT are not great.

As for phase 2, the results are quite different. Large increases in AT were reported for the first three time periods as well as the early evening time period. For the AM peak period, and the two midday time periods, AT increased by a value of approximately 1 , which is equivalent to an entire category (as detailed in Table 4.10). The improvement during the early evening was not as pronounced. Decreases in AT were observed during the afternoon period and the PM peak period, indicating that progression was slightly better before retiming. Although statistically significant, the magnitudes of these changes are not large. The degradation of AT implies that is an opportunity to improve coordination during those time periods. For the two time periods in question, traffic volumes were either the same as before retiming, or they had decreased. Changing conditions at downstream signals that reduced the quality of progression may also have caused AT to decrease. 
Table 5.13 Difference in means (significant in boldface) for the pairwise comparison of operation on June 19, 2006 (before changes) and March 12, 2007 (after changes) at Noblesville, IN.

(a) Volume $(\mathrm{veh} / \mathrm{h} / \mathrm{ln})$

\begin{tabular}{|c|c|c|c|c|c|c|c|c|}
\hline Time & 1 & 2 & 3 & 4 & 5 & 6 & 7 & 8 \\
\hline 06:00-09:00 & +1 & +19 & +16 & -6 & -39 & +33 & +3 & +24 \\
\hline 09:00-11:00 & -14 & -62 & -7 & -32 & -82 & -59 & -33 & +17 \\
\hline $11: 00-13: 00$ & -3 & -52 & -10 & -5 & -88 & -86 & +19 & -25 \\
\hline $13: 00-15: 00$ & -17 & -83 & -13 & -47 & -24 & -44 & +26 & -13 \\
\hline $15: 00-19: 00$ & +14 & +45 & -5 & +17 & +7 & +46 & +7 & +5 \\
\hline $19: 00-22: 00$ & -12 & -22 & -18 & -33 & -39 & -37 & -31 & +2 \\
\hline
\end{tabular}

(b) Green Time (s)

\begin{tabular}{|c|c|c|c|c|c|c|c|c|}
\hline Time & 1 & 2 & 3 & 4 & 5 & 6 & 7 & 8 \\
\hline 06:00-09:00 & -0.7 & +0.5 & -0.1 & -9.4 & -4.9 & +3.7 & -1.1 & -7.7 \\
\hline 09:00-11:00 & +1.2 & +13.3 & +1.1 & +3.0 & -1.7 & +12.5 & -0.0 & +4.3 \\
\hline 11:00-13:00 & +1.1 & +2.3 & -1.7 & +2.1 & -4.3 & +6.7 & +0.1 & +0.2 \\
\hline 13:00-15:00 & -2.6 & -0.3 & -3.2 & -11.7 & -7.1 & +5.0 & -2.1 & -14.2 \\
\hline 15:00-19:00 & -0.5 & +2.8 & -1.8 & -6.8 & -3.7 & +4.1 & -2.3 & -7.1 \\
\hline 19:00-22:00 & +0.0 & +30.3 & -2.2 & +2.1 & +2.6 & +27.4 & -2.1 & +0.7 \\
\hline
\end{tabular}

(c) $\mathrm{v} / \mathrm{c}$ Ratio

\begin{tabular}{|c|c|c|c|c|c|c|c|c|}
\hline Time & 1 & 2 & 3 & 4 & 5 & 6 & 7 & 8 \\
\hline $06: 00-09: 00$ & +0.01 & -0.00 & +0.01 & $+\mathbf{0 . 2 0}$ & +0.03 & $\mathbf{- 0 . 0 6}$ & +0.08 & $+\mathbf{0 . 0 8}$ \\
\hline $09: 00-11: 00$ & -0.04 & $\mathbf{- 0 . 0 6}$ & +0.05 & -0.05 & +0.04 & $\mathbf{- 0 . 1 9}$ & -0.07 & +0.03 \\
\hline $11: 00-13: 00$ & -0.05 & -0.03 & $+\mathbf{0 . 0 8}$ & -0.06 & -0.03 & $\mathbf{- 0 . 1 9}$ & +0.12 & -0.00 \\
\hline $13: 00-15: 00$ & -0.04 & $\mathbf{- 0 . 0 9}$ & +0.02 & $+\mathbf{0 . 1 3}$ & $+\mathbf{0 . 1 1}$ & $\mathbf{- 0 . 1 5}$ & $+\mathbf{0 . 1 8}$ & $+\mathbf{0 . 0 6}$ \\
\hline $15: 00-19: 00$ & $+\mathbf{0 . 0 9}$ & -0.02 & +0.04 & $+\mathbf{0 . 1 7}$ & $+\mathbf{0 . 1 4}$ & -0.04 & $+\mathbf{0 . 1 3}$ & $+\mathbf{0 . 0 4}$ \\
\hline $19: 00-22: 00$ & $\mathbf{+ 0 . 0 4}$ & $\mathbf{- 0 . 0 9}$ & $+\mathbf{0 . 2 1}$ & $\mathbf{+ 0 . 0 8}$ & -0.01 & $\mathbf{- 0 . 1 2}$ & $+\mathbf{0 . 0 8}$ & $+\mathbf{0 . 0 7}$ \\
\hline
\end{tabular}

(d) Arrival Type

\begin{tabular}{|c|c|c|c|c|c|c|c|c|}
\hline Time & 1 & 2 & 3 & 4 & 5 & 6 & 7 & 8 \\
\hline $06: 00-09: 00$ & & $+\mathbf{1 . 2 1}$ & & & & -0.05 & & \\
\hline $09: 00-11: 00$ & & $\mathbf{+ 1 . 5 4}$ & & & & -0.02 & & \\
\hline $11: 00-13: 00$ & & $\mathbf{+ 1 . 0 1}$ & & & & -0.08 & & \\
\hline $13: 00-15: 00$ & & $\mathbf{- 0 . 3 7}$ & & & & $-\mathbf{0 . 4 8}$ & & \\
\hline $15: 00-19: 00$ & & $\mathbf{- 0 . 2 1}$ & & & & +0.21 & & \\
\hline $19: 00-22: 00$ & & $\mathbf{+ 0 . 4 0}$ & & & & +0.07 & & \\
\hline
\end{tabular}


Table 5.14 Statistical t-values (significant in boldface) for the pairwise comparison of operation on June 19, 2006 (before changes) and March 12, 2007 (after changes) at Noblesville, IN.

(a) Volume

\begin{tabular}{|c|c|c|c|c|c|c|c|c|}
\hline Time & 1 & 2 & 3 & 4 & 5 & 6 & 7 & 8 \\
\hline 06:00-09:00 & +0.2 & +0.8 & +1.4 & -0.4 & -3.4 & +1.0 & +0.3 & +1.0 \\
\hline 09:00-11:00 & -1.2 & -3.1 & -0.7 & -1.7 & -4.5 & -2.2 & -2.7 & +0.9 \\
\hline 11:00-13:00 & -0.4 & -2.0 & -1.1 & -0.3 & -4.8 & -3.1 & +1.2 & -1.1 \\
\hline $13: 00-15: 00$ & -2.3 & -2.6 & -1.4 & -2.2 & -1.8 & -1.8 & +2.1 & -0.6 \\
\hline $15: 00-19: 00$ & +2.3 & +1.6 & -0.8 & +1.0 & +0.7 & +2.3 & +1.0 & +0.3 \\
\hline 19:00-22:00 & -1.9 & -0.8 & -1.8 & -1.9 & -3.1 & -1.8 & -3.4 & +0.1 \\
\hline
\end{tabular}

(b) Green Time

\begin{tabular}{|c|c|c|c|c|c|c|c|c|}
\hline Time & 1 & 2 & 3 & 4 & 5 & 6 & 7 & 8 \\
\hline 06:00-09:00 & -1.4 & +0.4 & -0.2 & -11.2 & -9.3 & +3.1 & -2.7 & -5.8 \\
\hline 09:00-11:00 & +2.9 & +10.1 & +5.7 & +3.7 & -3.2 & +10.7 & -0.1 & +3.6 \\
\hline 11:00-13:00 & +2.8 & +1.8 & -8.2 & +2.7 & -5.5 & +6.2 & +0.7 & +0.2 \\
\hline $13: 00-15: 00$ & -4.3 & -0.2 & -15 & -9.0 & -11.1 & +3.8 & -4.9 & -8.2 \\
\hline 15:00-19:00 & -1.2 & +2.3 & -11.4 & -7.4 & -7.6 & +4.1 & -6.3 & -6.2 \\
\hline 19:00-22:00 & +0.1 & +21 & -4.7 & +3.1 & $\begin{array}{l}+4.8 \\
\end{array}$ & +26 & -2.8 & +0.7 \\
\hline
\end{tabular}

(c) v/c Ratio

\begin{tabular}{|r|r|r|r|r|r|r|r|r|}
\hline Time & \multicolumn{1}{|c|}{1} & \multicolumn{1}{c|}{2} & 3 & \multicolumn{1}{c|}{4} & \multicolumn{1}{c|}{5} & 6 & 7 & 8 \\
\hline 06:00-09:00 & +0.4 & -0.0 & +0.2 & +5.2 & +0.8 & -2.4 & +1.7 & +3.9 \\
\hline 09:00-11:00 & -0.9 & -4.0 & +1.1 & -1.2 & +1.1 & -5.7 & -1.3 & +1.4 \\
\hline 11:00-13:00 & -1.2 & -1.7 & +2.4 & -1.5 & -0.9 & -5.9 & +1.4 & -0.0 \\
\hline 13:00-15:00 & -1.4 & -3.1 & +0.4 & +3.0 & +3.8 & -5.1 & +2.6 & +3.2 \\
\hline 15:00-19:00 & +3.4 & -0.9 & +1.4 & +5.8 & +6.3 & -1.9 & +3.8 & +2.7 \\
\hline 19:00-22:00 & +2.1 & $-\mathbf{5 . 1}$ & $+\mathbf{6 . 8}$ & $+\mathbf{+ 1 . 9}$ & -0.2 & $-\mathbf{7 . 2}$ & +2.2 & +3.6 \\
\hline
\end{tabular}

(d) Arrival Type

\begin{tabular}{|l|r|r|r|r|r|r|r|c|}
\hline Time & 1 & 2 & 3 & 4 & 5 & 6 & 7 & 8 \\
\hline $06: 00-09: 00$ & & $+\mathbf{6 . 4}$ & & & & -0.4 & & \\
\hline $09: 00-11: 00$ & & $\mathbf{+ 8 . 0}$ & & & & -0.1 & & \\
\hline $11: 00-13: 00$ & & $\mathbf{+ 6 . 2}$ & & & & -0.4 & & \\
\hline $13: 00-15: 00$ & & $-\mathbf{2 . 6}$ & & & & -2.1 & & \\
\hline $15: 00-19: 00$ & & $-\mathbf{2 . 5}$ & & & & +1.5 & & \\
\hline $19: 00-22: 00$ & & +2.8 & & & & +0.3 & & \\
\hline
\end{tabular}


Table 5.15 Statistical P-values (significant in boldface) for the pairwise comparison of operation on June 19, 2006 (before changes) and March 12, 2007 (after changes) at Noblesville, IN.

(a) Volume

\begin{tabular}{|c|r|r|r|r|r|r|r|c|}
\hline Time & \multicolumn{1}{|c|}{1} & \multicolumn{1}{c|}{2} & \multicolumn{1}{c|}{3} & \multicolumn{1}{c|}{4} & \multicolumn{1}{c|}{5} & \multicolumn{1}{c|}{6} & \multicolumn{1}{c|}{7} & 8 \\
\hline 06:00-09:00 & $84.6 \%$ & $41.1 \%$ & $17.6 \%$ & $66.1 \%$ & $\mathbf{0 . 1 \%}$ & $32.5 \%$ & $77.7 \%$ & $31.1 \%$ \\
\hline 09:00-11:00 & $22.2 \%$ & $\mathbf{0 . 2 \%}$ & $48.7 \%$ & $8.3 \%$ & $\mathbf{0 . 0 \%}$ & $\mathbf{2 . 9 \%}$ & $\mathbf{0 . 9 \%}$ & $36.8 \%$ \\
\hline 11:00-13:00 & $71.7 \%$ & $5.0 \%$ & $28.5 \%$ & $78.2 \%$ & $\mathbf{0 . 0} \%$ & $\mathbf{0 . 3 \%}$ & $23.6 \%$ & $29.4 \%$ \\
\hline 13:00-15:00 & $\mathbf{2 . 6 \%}$ & $\mathbf{1 . 1 \%}$ & $16.3 \%$ & $\mathbf{2 . 8 \%}$ & $7.1 \%$ & $7.7 \%$ & $3.5 \%$ & $52.5 \%$ \\
\hline 15:00-19:00 & $\mathbf{2 . 5 \%}$ & $10.7 \%$ & $42.7 \%$ & $33.5 \%$ & $47.7 \%$ & $\mathbf{2 . 0 \%}$ & $32.9 \%$ & $76.6 \%$ \\
\hline 19:00-22:00 & $6.0 \%$ & $43.0 \%$ & $7.9 \%$ & $5.6 \%$ & $\mathbf{0 . 2 \%}$ & $6.6 \%$ & $\mathbf{0 . 1 \%}$ & $89.9 \%$ \\
\hline
\end{tabular}

(b) Green Time

\begin{tabular}{|c|c|c|c|c|c|c|c|c|}
\hline Time & 1 & 2 & 3 & 4 & 5 & 6 & 7 & 8 \\
\hline 06:00-09:00 & $15.0 \%$ & $66.4 \%$ & $87.1 \%$ & $0.0 \%$ & $0.0 \%$ & $0.3 \%$ & $0.9 \%$ & $0.0 \%$ \\
\hline 09:00-11:00 & $0.5 \%$ & $0.0 \%$ & $0.0 \%$ & $0.0 \%$ & $0.2 \%$ & $0.0 \%$ & $89.5 \%$ & $0.0 \%$ \\
\hline 11:00-13:00 & $0.6 \%$ & $7.8 \%$ & $0.0 \%$ & $0.7 \%$ & $0.0 \%$ & $0.0 \%$ & $49.5 \%$ & $84.5 \%$ \\
\hline $13: 00-15: 00$ & $0.0 \%$ & $87.2 \%$ & $0.0 \%$ & $0.0 \%$ & $0.0 \%$ & $0.0 \%$ & $0.0 \%$ & $0.0 \%$ \\
\hline 15:00-19:00 & $22.6 \%$ & $2.5 \%$ & $0.0 \%$ & $0.0 \%$ & $0.0 \%$ & $0.0 \%$ & $0.0 \%$ & $0.0 \%$ \\
\hline 19:00-22:00 & $90.2 \%$ & $0.0 \%$ & $0.0 \%$ & $0.2 \%$ & $0.0 \%$ & $0.0 \%$ & $0.6 \%$ & $48.7 \%$ \\
\hline
\end{tabular}

(c) $\mathrm{v} / \mathrm{c}$ Ratio

\begin{tabular}{|c|c|c|c|c|c|c|c|c|}
\hline Time & 1 & 2 & 3 & 4 & 5 & 6 & 7 & 8 \\
\hline 06:00-09:00 & $67.8 \%$ & $97.5 \%$ & $82.2 \%$ & $0.0 \%$ & $40.5 \%$ & $1.8 \%$ & $9.7 \%$ & $0.0 \%$ \\
\hline 09:00-11:00 & $37.4 \%$ & $0.0 \%$ & $28.4 \%$ & $24.1 \%$ & $29.0 \%$ & $0.0 \%$ & $20.8 \%$ & $16.8 \%$ \\
\hline 11:00-13:00 & $21.9 \%$ & $10.0 \%$ & $1.6 \%$ & $14.8 \%$ & $35.7 \%$ & $0.0 \%$ & $15.6 \%$ & $96.9 \%$ \\
\hline $13: 00-15: 00$ & $15.7 \%$ & $0.2 \%$ & $68.7 \%$ & $0.3 \%$ & $0.0 \%$ & $0.0 \%$ & $1.0 \%$ & $0.2 \%$ \\
\hline $15: 00-19: 00$ & $0.1 \%$ & $37.8 \%$ & $16.6 \%$ & $0.0 \%$ & $0.0 \%$ & $5.6 \%$ & $0.0 \%$ & $0.8 \%$ \\
\hline $19: 00-22: 00$ & $3.6 \%$ & $0.0 \%$ & $0.0 \%$ & $6.3 \%$ & $82.4 \%$ & $0.0 \%$ & $2.8 \%$ & $0.0 \%$ \\
\hline
\end{tabular}

(d) Arrival Type

\begin{tabular}{|c|c|c|c|c|c|c|c|c|}
\hline Time & 1 & 2 & 3 & 4 & 5 & 6 & 7 & 8 \\
\hline $06: 00-09: 00$ & & $\mathbf{0 . 0 \%}$ & & & & $69.2 \%$ & & \\
\hline $09: 00-11: 00$ & & $\mathbf{0 . 0 \%}$ & & & & $92.5 \%$ & & \\
\hline $11: 00-13: 00$ & & $\mathbf{0 . 0 \%}$ & & & & $70.4 \%$ & & \\
\hline $13: 00-15: 00$ & & $\mathbf{0 . 9 \%}$ & & & & $\mathbf{4 . 2 \%}$ & & \\
\hline $15: 00-19: 00$ & & $\mathbf{1 . 2 \%}$ & & & & $14.2 \%$ & & \\
\hline $19: 00-22: 00$ & & $\mathbf{0 . 5 \%}$ & & & & $75.9 \%$ & & \\
\hline
\end{tabular}


Table 5.16 Difference in means (significant in boldface) for the pairwise comparison of operation on June 28, 2006 (before changes) and March 21, 2007 (after changes) at Noblesville, IN.

(a) Volume $(\mathrm{veh} / \mathrm{h} / \mathrm{ln})$

\begin{tabular}{|c|r|r|r|r|r|r|r|r|}
\hline Time & \multicolumn{1}{|c|}{1} & \multicolumn{1}{c|}{2} & \multicolumn{1}{c|}{3} & \multicolumn{1}{c|}{4} & \multicolumn{1}{c|}{5} & \multicolumn{1}{c|}{6} & \multicolumn{1}{c|}{7} & \multicolumn{1}{c|}{8} \\
\hline 06:00-09:00 & +10 & -31 & $+\mathbf{2 7}$ & -14 & -12 & +49 & -13 & +0 \\
\hline 09:00-11:00 & -8 & $\mathbf{- 1 0 3}$ & -2 & -7 & $\mathbf{- 5 6}$ & $\mathbf{- 1 3 5}$ & -7 & +2 \\
\hline 11:00-13:00 & -8 & $\mathbf{- 8 6}$ & $\mathbf{- 1 8}$ & +1 & $\mathbf{- 7 7}$ & $\mathbf{- 1 2 3}$ & -7 & +6 \\
\hline 13:00-15:00 & -8 & $\mathbf{- 1 0 3}$ & +9 & -8 & $-\mathbf{3 0}$ & $\mathbf{- 6 9}$ & -2 & +2 \\
\hline 15:00-19:00 & $+\mathbf{1 5}$ & +44 & +1 & -8 & $\mathbf{+ 1 8}$ & -20 & -7 & $+\mathbf{3 8}$ \\
\hline 19:00-22:00 & -11 & -34 & $\mathbf{- 3 1}$ & $\mathbf{+ 4 1}$ & -19 & -8 & $\mathbf{- 2 9}$ & -2 \\
\hline
\end{tabular}

(b) Green Time (s)

\begin{tabular}{|c|c|c|c|c|c|c|c|c|}
\hline Time & 1 & 2 & 3 & 4 & 5 & 6 & 7 & 8 \\
\hline 06:00-09:00 & +0.2 & -0.1 & +0.1 & -8.9 & -4.5 & +4.2 & -1.2 & -7.7 \\
\hline 09:00-11:00 & +0.7 & +13.1 & +0.8 & +2.9 & -1.3 & +10.9 & +0.8 & +3.8 \\
\hline 11:00-13:00 & +0.6 & +4.6 & -1.7 & +2.3 & -3.1 & +6.2 & +0.1 & +0.8 \\
\hline $13: 00-15: 00$ & -2.0 & -0.7 & -3.0 & -11.0 & -6.3 & +2.5 & -2.7 & -12.5 \\
\hline 15:00-19:00 & -0.8 & +0.2 & -1.9 & -5.3 & -4.5 & +3.6 & -2.7 & -4.5 \\
\hline 19:00-22:00 & +0.6 & +27.6 & -2.2 & +4.4 & +2.6 & +24.7 & -1.4 & +1.8 \\
\hline
\end{tabular}

(c) $\mathrm{v} / \mathrm{c}$ Ratio

\begin{tabular}{|c|c|c|c|c|c|c|c|c|}
\hline Time & 1 & 2 & 3 & 4 & 5 & 6 & 7 & 8 \\
\hline $06: 00-09: 00$ & +0.04 & -0.03 & +0.03 & $+\mathbf{0 . 1 8}$ & $+\mathbf{0 . 1 4}$ & $\mathbf{- 0 . 0 7}$ & -0.03 & $+\mathbf{0 . 0 4}$ \\
\hline $09: 00-11: 00$ & +0.01 & $\mathbf{- 0 . 0 9}$ & +0.07 & +0.04 & $+\mathbf{0 . 1 0}$ & $\mathbf{- 0 . 2 2}$ & +0.01 & +0.01 \\
\hline $11: 00-13: 00$ & -0.05 & $\mathbf{- 0 . 0 6}$ & +0.05 & -0.04 & -0.04 & $\mathbf{- 0 . 2 1}$ & -0.02 & +0.01 \\
\hline $13: 00-15: 00$ & -0.03 & $\mathbf{- 0 . 1 1}$ & $+\mathbf{0 . 1 1}$ & $+\mathbf{0 . 1 6}$ & +0.06 & $\mathbf{- 0 . 1 5}$ & +0.05 & $+\mathbf{0 . 0 7}$ \\
\hline $15: 00-19: 00$ & $+\mathbf{0 . 1 1}$ & +0.00 & $+\mathbf{0 . 0 7}$ & $+\mathbf{0 . 0 8}$ & $+\mathbf{0 . 2 0}$ & $\mathbf{- 0 . 0 9}$ & $+\mathbf{0 . 0 7}$ & $+\mathbf{0 . 0 6}$ \\
\hline $19: 00-22: 00$ & +0.03 & $\mathbf{- 0 . 0 9}$ & $+\mathbf{0 . 1 6}$ & $+\mathbf{0 . 1 9}$ & $\mathbf{+ 0 . 0 8}$ & $\mathbf{- 0 . 0 8}$ & $+\mathbf{0 . 1 3}$ & $+\mathbf{0 . 0 6}$ \\
\hline
\end{tabular}

(d) Arrival Type

\begin{tabular}{|c|r|r|r|r|r|r|r|c|}
\hline Time & 1 & 2 & 3 & 4 & 5 & 6 & 7 & 8 \\
\hline $06: 00-09: 00$ & & $+\mathbf{0 . 3 3}$ & & & & +0.09 & & \\
\hline $09: 00-11: 00$ & & $\mathbf{+ 1 . 4 8}$ & & & & -0.16 & & \\
\hline $11: 00-13: 00$ & & $\mathbf{+ 1 . 2 9}$ & & & & +0.00 & & \\
\hline $13: 00-15: 00$ & & $\mathbf{- 0 . 3 0}$ & & & & $\mathbf{- 0 . 4 9}$ & & \\
\hline $15: 00-19: 00$ & & -0.15 & & & & $-\mathbf{0 . 6 6}$ & & \\
\hline $19: 00-22: 00$ & & $+\mathbf{0 . 4 2}$ & & & & +0.04 & & \\
\hline
\end{tabular}


Table 5.17 Statistical t-values (significant in boldface) for the pairwise comparison of operation on June 28, 2006 (before changes) and March 21, 2007 (after changes) at Noblesville, IN.

(a) Volume

\begin{tabular}{|c|c|c|c|c|c|c|c|c|}
\hline Time & 1 & 2 & 3 & 4 & 5 & 6 & 7 & 8 \\
\hline 06:00-09:00 & +1.4 & -1.3 & +2.1 & -0.9 & -1.0 & +1.3 & -1.5 & +0.0 \\
\hline 09:00-11:00 & -0.9 & -5.0 & -0.2 & -0.2 & -3.0 & -4.4 & -0.7 & +0.1 \\
\hline 11:00-13:00 & -0.9 & -3.3 & -2.0 & +0.1 & -4.4 & -4.7 & -0.7 & +0.3 \\
\hline $13: 00-15: 00$ & -1.1 & -3.6 & +1.0 & -0.4 & -2.2 & -3.1 & -0.2 & +0.1 \\
\hline $15: 00-19: 00$ & +2.7 & +1.6 & +0.2 & -0.4 & +2.0 & -0.9 & -1.1 & +2.6 \\
\hline 19:00-22:00 & -1.4 & -1.3 & -3.1 & +2.4 & -1.6 & -0.4 & -2.4 & -0.2 \\
\hline
\end{tabular}

(b) Green Time

\begin{tabular}{|c|c|c|c|c|c|c|c|c|}
\hline Time & 1 & 2 & 3 & 4 & 5 & 6 & 7 & 8 \\
\hline 06:00-09:00 & +0.3 & -0.1 & +0.1 & -11.3 & -7.6 & +3.4 & -2.7 & -6.2 \\
\hline 09:00-11:00 & +1.9 & +10.7 & +5.6 & +3.7 & -2.1 & +8.9 & +3.1 & +3.4 \\
\hline 11:00-13:00 & +1.8 & +3.8 & -7.8 & +3.3 & -4.1 & +6.0 & +0.4 & +0.7 \\
\hline 13:00-15:00 & -3.8 & -0.4 & -12.2 & -8.7 & -9.8 & +1.9 & -6.0 & -7.6 \\
\hline 15:00-19:00 & -2.5 & +0.2 & -13.6 & -5.9 & -10 & +3.6 & -7.8 & -4.1 \\
\hline 19:00-22:00 & +1.6 & +22.6 & -5.4 & +5.8 & +5.2 & +25.3 & -3.0 & +1.6 \\
\hline
\end{tabular}

(c) $\mathrm{v} / \mathrm{c}$ Ratio

\begin{tabular}{|r|r|r|r|r|r|r|r|r|}
\hline Time & 1 & \multicolumn{1}{|c|}{2} & 3 & 4 & \multicolumn{1}{c|}{5} & \multicolumn{1}{c|}{6} & \multicolumn{1}{c|}{7} & 8 \\
\hline 06:00-09:00 & +1.4 & -1.9 & +1.2 & $+\mathbf{4 . 2}$ & +3.8 & -2.3 & -0.6 & +2.2 \\
\hline 09:00-11:00 & +0.2 & $-\mathbf{6 . 7}$ & +1.7 & +0.5 & +3.0 & $-\mathbf{6 . 3}$ & +0.2 & +0.2 \\
\hline 11:00-13:00 & -1.2 & -3.5 & +1.5 & -0.9 & -1.3 & $-\mathbf{6 . 8}$ & -0.3 & +0.5 \\
\hline 13:00-15:00 & -1.0 & -4.7 & +2.6 & +3.7 & +1.9 & -6.1 & +1.0 & +3.2 \\
\hline 15:00-19:00 & +4.0 & +0.1 & +2.9 & +3.0 & +10 & -3.8 & +2.1 & +4.1 \\
\hline 19:00-22:00 & +1.3 & -5.2 & +4.9 & +4.6 & +2.8 & -5.2 & +3.0 & +3.6 \\
\hline
\end{tabular}

(d) Arrival Type

\begin{tabular}{|l|r|r|r|r|r|r|r|r|}
\hline Time & 1 & \multicolumn{1}{|c|}{2} & 3 & 4 & 5 & 6 & 7 & 8 \\
\hline $06: 00-09: 00$ & & +2.1 & & & & +0.8 & & \\
\hline $09: 00-11: 00$ & & $+\mathbf{+ 9}$ & & & & -0.8 & & \\
\hline $11: 00-13: 00$ & & $+\mathbf{8 . 3}$ & & & & +0.0 & & \\
\hline $13: 00-15: 00$ & & -2.6 & & & & -2.4 & & \\
\hline $15: 00-19: 00$ & & -1.8 & & & & -4.6 & & \\
\hline $19: 00-22: 00$ & & +2.6 & & & & +0.2 & & \\
\hline
\end{tabular}


Table 5.18 Statistical P-values (significant in boldface) for the pairwise comparison of operation on June 28, 2006 (before changes) and March 21, 2007 (after changes) at Noblesville, IN.

(a) Volume

\begin{tabular}{|c|r|r|r|r|r|r|r|c|}
\hline Time & \multicolumn{1}{|c|}{1} & \multicolumn{1}{c|}{2} & \multicolumn{1}{c|}{3} & \multicolumn{1}{c|}{4} & \multicolumn{1}{c|}{5} & 6 & 7 & \multicolumn{1}{c|}{8} \\
\hline 06:00-09:00 & $17.4 \%$ & $18.4 \%$ & $\mathbf{3 . 4 \%}$ & $38.8 \%$ & $31.7 \%$ & $19.1 \%$ & $13.1 \%$ & $99.6 \%$ \\
\hline 09:00-11:00 & $38.1 \%$ & $\mathbf{0 . 0 \%}$ & $80.5 \%$ & $80.4 \%$ & $\mathbf{0 . 3 \%}$ & $\mathbf{0 . 0 \%}$ & $48.8 \%$ & $91.6 \%$ \\
\hline 11:00-13:00 & $36.0 \%$ & $\mathbf{0 . 1 \%}$ & $\mathbf{4 . 5 \%}$ & $93.5 \%$ & $\mathbf{0 . 0 \%}$ & $\mathbf{0 . 0 \%}$ & $47.1 \%$ & $76.6 \%$ \\
\hline 13:00-15:00 & $26.0 \%$ & $\mathbf{0 . 1 \%}$ & $33.0 \%$ & $71.4 \%$ & $\mathbf{3 . 1 \%}$ & $\mathbf{0 . 3 \%}$ & $84.5 \%$ & $93.1 \%$ \\
\hline 15:00-19:00 & $\mathbf{0 . 7 \%}$ & $11.5 \%$ & $80.9 \%$ & $65.7 \%$ & $\mathbf{5 . 1 \%}$ & $36.1 \%$ & $28.8 \%$ & $\mathbf{0 . 9 \%}$ \\
\hline 19:00-22:00 & $15.9 \%$ & $21.0 \%$ & $\mathbf{0 . 2 \%}$ & $\mathbf{1 . 5 \%}$ & $11.2 \%$ & $67.8 \%$ & $\mathbf{1 . 6 \%}$ & $87.9 \%$ \\
\hline
\end{tabular}

(b) Green Time

\begin{tabular}{|c|c|c|c|c|c|c|c|c|}
\hline Time & 1 & 2 & 3 & 4 & 5 & 6 & 7 & 8 \\
\hline 06:00-09:00 & $74.6 \%$ & $93.0 \%$ & $89.8 \%$ & $0.0 \%$ & $0.0 \%$ & $0.1 \%$ & $0.8 \%$ & $0.0 \%$ \\
\hline 09:00-11:00 & $6.4 \%$ & $0.0 \%$ & $0.0 \%$ & $0.0 \%$ & $3.4 \%$ & $0.0 \%$ & $0.2 \%$ & $0.1 \%$ \\
\hline 11:00-13:00 & $7.3 \%$ & $0.0 \%$ & $0.0 \%$ & $0.1 \%$ & $0.0 \%$ & $0.0 \%$ & $70.8 \%$ & $48.0 \%$ \\
\hline $13: 00-15: 00$ & $0.0 \%$ & $66.1 \%$ & $0.0 \%$ & $0.0 \%$ & $0.0 \%$ & $5.6 \%$ & $0.0 \%$ & $0.0 \%$ \\
\hline 15:00-19:00 & $1.3 \%$ & $84.9 \%$ & $0.0 \%$ & $0.0 \%$ & $0.0 \%$ & $0.0 \%$ & $0.0 \%$ & $0.0 \%$ \\
\hline 19:00-22:00 & $11.7 \%$ & $0.0 \%$ & $0.0 \%$ & $0.0 \%$ & $0.0 \%$ & $0.0 \%$ & $0.3 \%$ & $11.5 \%$ \\
\hline
\end{tabular}

(c) $\mathrm{v} / \mathrm{c}$ Ratio

\begin{tabular}{|c|c|c|c|c|c|c|c|c|}
\hline Time & 1 & 2 & 3 & 4 & 5 & 6 & 7 & 8 \\
\hline 06:00-09:00 & $17.8 \%$ & $5.5 \%$ & $23.8 \%$ & $0.0 \%$ & $0.0 \%$ & $2.2 \%$ & $53.1 \%$ & $3.1 \%$ \\
\hline 09:00-11:00 & $83.5 \%$ & $0.0 \%$ & $9.6 \%$ & $59.7 \%$ & $0.3 \%$ & $0.0 \%$ & $86.7 \%$ & $81.2 \%$ \\
\hline 11:00-13:00 & $23.8 \%$ & $0.1 \%$ & $12.9 \%$ & $37.9 \%$ & $20.6 \%$ & $0.0 \%$ & $72.9 \%$ & $62.8 \%$ \\
\hline $13: 00-15: 00$ & $34.2 \%$ & $0.0 \%$ & $0.9 \%$ & $0.0 \%$ & $6.6 \%$ & $0.0 \%$ & $32.0 \%$ & $0.2 \%$ \\
\hline $15: 00-19: 00$ & $0.0 \%$ & $88.5 \%$ & $0.4 \%$ & $0.3 \%$ & $0.0 \%$ & $0.0 \%$ & $3.9 \%$ & $0.0 \%$ \\
\hline $19: 00-22: 00$ & $18.6 \%$ & $0.0 \%$ & $0.0 \%$ & $0.0 \%$ & $0.6 \%$ & $0.0 \%$ & $0.3 \%$ & $0.0 \%$ \\
\hline
\end{tabular}

(d) Arrival Type

\begin{tabular}{|c|c|c|c|c|c|c|c|c|}
\hline Time & 1 & 2 & 3 & 4 & 5 & 6 & 7 & 8 \\
\hline $06: 00-09: 00$ & & $\mathbf{3 . 7 \%}$ & & & & $43.9 \%$ & & \\
\hline $09: 00-11: 00$ & & $\mathbf{0 . 0 \%}$ & & & & $44.1 \%$ & & \\
\hline $11: 00-13: 00$ & & $\mathbf{0 . 0 \%}$ & & & & $98.4 \%$ & & \\
\hline $13: 00-15: 00$ & & $\mathbf{1 . 2 \%}$ & & & & $\mathbf{1 . 7 \%}$ & & \\
\hline $15: 00-19: 00$ & & $7.1 \%$ & & & & $\mathbf{0 . 0 \%}$ & & \\
\hline $19: 00-22: 00$ & & $\mathbf{1 . 0 \%}$ & & & & $83.5 \%$ & & \\
\hline
\end{tabular}


Table 5.19 Difference in means (significant in boldface) for the 15-weekday aggregate comparison of operation before and after operational changes at Noblesville, IN.

(a) Volume $(\mathrm{veh} / \mathrm{h} / \mathrm{ln})$

\begin{tabular}{|c|c|c|c|c|c|c|c|c|}
\hline Time & 1 & 2 & 3 & 4 & 5 & 6 & 7 & 8 \\
\hline 06:00-09:00 & +10 & +9 & +25 & +9 & -22 & +26 & -4 & +18 \\
\hline 09:00-11:00 & -8 & -73 & 0 & -15 & -64 & -77 & -11 & -1 \\
\hline 11:00-13:00 & -10 & -81 & +3 & -13 & -51 & -67 & -4 & -17 \\
\hline 13:00-15:00 & -1 & -99 & -7 & -24 & -18 & -69 & +2 & -5 \\
\hline $15: 00-19: 00$ & +3 & +2 & -4 & -30 & -2 & +11 & -6 & +18 \\
\hline 19:00-22:00 & -17 & -55 & -32 & -31 & -31 & -75 & -29 & -22 \\
\hline
\end{tabular}

(b) Green Time (s)

\begin{tabular}{|c|c|c|c|c|c|c|c|c|}
\hline Time & 1 & 2 & 3 & 4 & 5 & 6 & 7 & 8 \\
\hline 06:00-09:00 & -0.4 & +0.2 & +0.2 & -8.4 & -4.6 & +3.1 & -1.5 & -6.3 \\
\hline 09:00-11:00 & +0.9 & +13.5 & +1.0 & +3.7 & -1.7 & +11.8 & +0.5 & +4.5 \\
\hline 11:00-13:00 & +0.3 & +3.9 & -0.4 & +1.7 & -3.8 & +6.0 & +0.1 & +1.2 \\
\hline 13:00-15:00 & -1.7 & -0.7 & -3.2 & -10.4 & -6.9 & +2.8 & -2.5 & -11.1 \\
\hline 15:00-19:00 & -1.4 & +2.0 & -1.9 & -6.0 & -4.8 & +4.5 & -2.8 & -5.2 \\
\hline 19:00-22:00 & -0.4 & +27.4 & -2.6 & +1.5 & +1.6 & +24.4 & -2.2 & -0.1 \\
\hline
\end{tabular}

(c) $\mathrm{v} / \mathrm{c}$ Ratio

\begin{tabular}{|c|c|c|c|c|c|c|c|c|}
\hline Time & 1 & 2 & 3 & 4 & 5 & 6 & 7 & 8 \\
\hline 06:00-09:00 & +0.05 & +0.00 & +0.03 & +0.21 & +0.1 & -0.06 & +0.04 & +0.05 \\
\hline 09:00-11:00 & +0.01 & -0.07 & +0.08 & -0.03 & +0.08 & -0.19 & +0.01 & +0.01 \\
\hline $11: 00-13: 00$ & -0.05 & -0.05 & +0.06 & -0.06 & +0.05 & -0.16 & +0.00 & -0.01 \\
\hline $13: 00-15: 00$ & +0.00 & -0.10 & +0.04 & +0.11 & +0.11 & -0.15 & +0.06 & +0.05 \\
\hline 15:00-19:00 & +0.06 & -0.05 & +0.05 & +0.06 & +0.15 & -0.08 & +0.08 & $\begin{array}{r}+0.04 \\
\end{array}$ \\
\hline 19:00-22:00 & +0.02 & -0.11 & +0.13 & +0.06 & +0.03 & -0.14 & +0.09 & +0.04 \\
\hline
\end{tabular}

(d) Arrival Type

\begin{tabular}{|c|r|r|r|r|r|r|c|c|}
\hline Time & 1 & 2 & 3 & 4 & 5 & 6 & 7 & 8 \\
\hline $06: 00-09: 00$ & & $\mathbf{+ 0 . 9 9}$ & & & & $-\mathbf{0 . 0 9}$ & & \\
\hline $09: 00-11: 00$ & & $\mathbf{+ 1 . 3 5}$ & & & & -0.10 & & \\
\hline $11: 00-13: 00$ & & $\mathbf{+ 1 . 2 1}$ & & & & +0.02 & & \\
\hline $13: 00-15: 00$ & & $\mathbf{- 0 . 3 7}$ & & & & $-\mathbf{0 . 3 7}$ & & \\
\hline $15: 00-19: 00$ & & $\mathbf{- 0 . 1 8}$ & & & & +0.05 & & \\
\hline $19: 00-22: 00$ & & $\mathbf{+ 0 . 4 5}$ & & & & +0.00 & & \\
\hline
\end{tabular}


Table 5.20 Statistical t-values (significant in boldface) for the 15-weekday aggregate comparison of operation before and after operational changes at Noblesville, IN.

(a) Volume

\begin{tabular}{|c|c|c|c|c|c|c|c|c|}
\hline Time & 1 & 2 & 3 & 4 & 5 & 6 & 7 & 8 \\
\hline 06:00-09:00 & +6.4 & +1.6 & +6.2 & +2.1 & -7 & +3.4 & -1.5 & +2.3 \\
\hline 09:00-11:00 & -3.3 & -8.8 & -0.1 & -2.2 & -15.4 & -5.8 & -4.5 & -0.1 \\
\hline 11:00-13:00 & -5.3 & -6.5 & +0.3 & -1.5 & -6.9 & -4.3 & -1.5 & -2.0 \\
\hline 13:00-15:00 & -0.2 & -6.8 & -2.5 & -2.9 & -4.2 & -6.5 & +0.8 & -0.8 \\
\hline $15: 00-19: 00$ & +0.7 & +0.2 & -1.3 & -3.1 & -0.5 & +0.5 & -2.7 & +1.9 \\
\hline 19:00-22:00 & -8.2 & -2.4 & -9.1 & -3.1 & -5.6 & -3.9 & -11.4 & -3.6 \\
\hline
\end{tabular}

(b) Green Time

\begin{tabular}{|c|c|c|c|c|c|c|c|c|}
\hline Time & 1 & 2 & 3 & 4 & 5 & 6 & 7 & 8 \\
\hline 06:00-09:00 & -2.9 & +0.7 & +1.7 & -38.8 & -36.7 & +9.7 & -9.8 & -18.8 \\
\hline 09:00-11:00 & +9.3 & +49.9 & +28.5 & +13.3 & -12.5 & +36.3 & +6.7 & +11.5 \\
\hline 11:00-13:00 & +3.0 & +8.7 & -0.7 & +2.7 & -14.5 & +14.8 & +1.9 & +3 \\
\hline $13: 00-15: 00$ & -10.3 & -1.3 & -70 & -22.7 & -37.3 & +5.6 & -21.9 & -22.2 \\
\hline 15:00-19:00 & -5.7 & +3.3 & -41.6 & -13.5 & -23.3 & +8.7 & -23.6 & -10.6 \\
\hline 19:00-22:00 & -1.9 & +32.3 & -10.6 & +3.6 & +5.5 & +32.8 & -8.9 & -0.1 \\
\hline
\end{tabular}

(c) $\mathrm{v} / \mathrm{c}$ Ratio

\begin{tabular}{|c|c|c|c|c|c|c|c|c|}
\hline Time & 1 & 2 & 3 & 4 & 5 & 6 & 7 & 8 \\
\hline 06:00-09:00 & +7.5 & -0.8 & +2.6 & +21.9 & +9.2 & -8.3 & +2.6 & +5.9 \\
\hline 09:00-11:00 & +0.6 & -12.5 & +4.9 & -1.5 & +8.2 & -15.2 & +1.0 & +0.9 \\
\hline $11: 00-13: 00$ & -6.1 & -6.2 & +3.6 & -2.4 & +3.5 & -8.6 & -0.2 & -1.1 \\
\hline 13:00-15:00 & +0.4 & -8.3 & +3.2 & +7.9 & +11.4 & -10.5 & +3.9 & $\begin{array}{r}+8.8 \\
\end{array}$ \\
\hline 15:00-19:00 & +5.0 & -4.2 & +3.0 & +4.0 & +15.9 & -3.4 & +7.8 & +6.1 \\
\hline 19:00-22:00 & +2.5 & -7.5 & +9.5 & +2.4 & +2.1 & -8.7 & +7.9 & +5.3 \\
\hline
\end{tabular}

(d) Arrival Type

\begin{tabular}{|c|r|r|r|r|r|r|r|r|}
\hline Time & 1 & \multicolumn{1}{|c|}{2} & 3 & 4 & 5 & 6 & 7 & 8 \\
\hline $06: 00-09: 00$ & & $\mathbf{+ 1 3 . 8}$ & & & & -2.2 & & \\
\hline $09: 00-11: 00$ & & $+\mathbf{2 5 . 7}$ & & & & -1.6 & & \\
\hline $11: 00-13: 00$ & & $\mathbf{+ 2 3 . 4}$ & & & & +0.2 & & \\
\hline $13: 00-15: 00$ & & $\mathbf{- 9 . 6}$ & & & & $\mathbf{- 6 . 8}$ & & \\
\hline $15: 00-19: 00$ & & $\mathbf{- 6 . 0}$ & & & & +0.4 & & \\
\hline $19: 00-22: 00$ & & $+\mathbf{+ 7 . 4}$ & & & & +0.1 & & \\
\hline
\end{tabular}


Table 5.21 Statistical P-values (significant in boldface) for the 15-weekday aggregate comparison of operation before and after operational changes at Noblesville, IN.

(a) Volume

\begin{tabular}{|c|c|c|c|c|c|c|c|c|}
\hline Time & 1 & 2 & 3 & 4 & 5 & 6 & 7 & 8 \\
\hline 06:00-09:00 & $0.0 \%$ & $13.0 \%$ & $0.0 \%$ & $4.2 \%$ & $0.0 \%$ & $0.2 \%$ & $14.0 \%$ & $2.7 \%$ \\
\hline 09:00-11:00 & $0.2 \%$ & $0.0 \%$ & $92.8 \%$ & $4.0 \%$ & $0.0 \%$ & $0.0 \%$ & $0.0 \%$ & $92.8 \%$ \\
\hline 11:00-13:00 & $0.0 \%$ & $0.0 \%$ & $75.1 \%$ & $14.1 \%$ & $0.0 \%$ & $0.0 \%$ & $14.3 \%$ & $5.8 \%$ \\
\hline $13: 00-15: 00$ & $80.9 \%$ & $0.0 \%$ & $1.7 \%$ & $0.8 \%$ & $0.0 \%$ & $0.0 \%$ & $43.4 \%$ & $44.5 \%$ \\
\hline $15: 00-19: 00$ & $51.2 \%$ & $88.1 \%$ & $21.7 \%$ & $0.5 \%$ & $65.6 \%$ & $60.2 \%$ & $1.1 \%$ & $6.4 \%$ \\
\hline $19: 00-22: 00$ & $0.0 \%$ & $2.1 \%$ & $0.0 \%$ & $0.5 \%$ & $0.0 \%$ & $0.1 \%$ & $0.0 \%$ & $0.1 \%$ \\
\hline
\end{tabular}

(b) Green Time

\begin{tabular}{|c|c|c|c|c|c|c|c|c|}
\hline Time & 1 & 2 & 3 & 4 & 5 & 6 & 7 & 8 \\
\hline 06:00-09:00 & $0.7 \%$ & $46.8 \%$ & $10.0 \%$ & $0.0 \%$ & $0.0 \%$ & $0.0 \%$ & $0.0 \%$ & $0.0 \%$ \\
\hline 09:00-11:00 & $0.0 \%$ & $0.0 \%$ & $0.0 \%$ & $0.0 \%$ & $0.0 \%$ & $0.0 \%$ & $0.0 \%$ & $0.0 \%$ \\
\hline 11:00-13:00 & $0.5 \%$ & $0.0 \%$ & $47.3 \%$ & $1.0 \%$ & $0.0 \%$ & $0.0 \%$ & $7.2 \%$ & $0.5 \%$ \\
\hline 13:00-15:00 & $0.0 \%$ & $19.7 \%$ & $0.0 \%$ & $0.0 \%$ & $0.0 \%$ & $0.0 \%$ & $0.0 \%$ & $0.0 \%$ \\
\hline $15: 00-19: 00$ & $0.0 \%$ & $0.3 \%$ & $0.0 \%$ & $0.0 \%$ & $0.0 \%$ & $0.0 \%$ & $0.0 \%$ & $0.0 \%$ \\
\hline 19:00-22:00 & $7.1 \%$ & $0.0 \%$ & $0.0 \%$ & $0.1 \%$ & $0.0 \%$ & $0.0 \%$ & $0.0 \%$ & $90.4 \%$ \\
\hline
\end{tabular}

$\mathrm{v} / \mathrm{c}$ Ratio

\begin{tabular}{|c|c|c|c|c|c|c|c|c|}
\hline Time & 1 & 2 & 3 & 4 & 5 & 6 & 7 & 8 \\
\hline 06:00-09:00 & $0.0 \%$ & $44.8 \%$ & $1.6 \%$ & $0.0 \%$ & $0.0 \%$ & $0.0 \%$ & $1.3 \%$ & $0.0 \%$ \\
\hline 09:00-11:00 & $52.9 \%$ & $0.0 \%$ & $0.0 \%$ & $15.1 \%$ & $0.0 \%$ & $0.0 \%$ & $34.9 \%$ & $37.4 \%$ \\
\hline 11:00-13:00 & $0.0 \%$ & $0.0 \%$ & $0.1 \%$ & $2.3 \%$ & $0.2 \%$ & $0.0 \%$ & $87.3 \%$ & $28.0 \%$ \\
\hline 13:00-15:00 & $67.9 \%$ & $0.0 \%$ & $0.3 \%$ & $0.0 \%$ & $0.0 \%$ & $0.0 \%$ & $0.1 \%$ & $0.0 \%$ \\
\hline 15:00-19:00 & $0.0 \%$ & $0.0 \%$ & $0.5 \%$ & $0.0 \%$ & $0.0 \%$ & $0.2 \%$ & $0.0 \%$ & $0.0 \%$ \\
\hline 19:00-22:00 & $2.1 \%$ & $0.0 \%$ & $0.0 \%$ & $2.4 \%$ & $4.9 \%$ & $0.0 \%$ & $0.0 \%$ & $0.0 \%$ \\
\hline
\end{tabular}

(d) Arrival Type

\begin{tabular}{|c|c|c|c|c|c|c|c|c|}
\hline Time & 1 & 2 & 3 & 4 & 5 & 6 & 7 & 8 \\
\hline $06: 00-09: 00$ & & $\mathbf{0 . 0} \%$ & & & & $3.7 \%$ & & \\
\hline $09: 00-11: 00$ & & $\mathbf{0 . 0} \%$ & & & & $11.8 \%$ & & \\
\hline $11: 00-13: 00$ & & $\mathbf{0 . 0 \%}$ & & & & $81.8 \%$ & & \\
\hline $13: 00-15: 00$ & & $\mathbf{0 . 0 \%}$ & & & & $\mathbf{0 . 0 \%}$ & & \\
\hline $15: 00-19: 00$ & & $\mathbf{0 . 0 \%}$ & & & & $66.2 \%$ & & \\
\hline $19: 00-22: 00$ & & $\mathbf{0 . 0 \%}$ & & & & $94.6 \%$ & & \\
\hline
\end{tabular}




\subsubsection{Conclusion}

The signal retiming plan had impacts on operation as seen in the changes in $\mathrm{v} / \mathrm{c}$ ratios and in arrival types. Northbound progression along the arterial was improved, as seen by the higher values of AT for phase 2. The improvements were of enough magnitude to categorically improve the AT. Additionally, the v/c ratios of the coordinated phases decreased, and the number of split failures for phases 2 and 6 decreased. For phase 6, these were more meaningful performance measures, since the arrivals were random. The impacts on the non-coordinated phases were mostly negative, with increasing $\mathrm{v} / \mathrm{c}$ ratios occurring for most time periods, and more split failures taking place.

Phase 5, the northbound left turn, saw a dramatic increase in the number of split failures. Part of this was due to the implementation of the lagging left turn, which caused phase 5 vehicles to arrive prior to the green indication, but also prevented phase 5 from obtaining additional green time from phase 6 . The high number of split failures indicates that this phase may not be receiving enough green time.

Phase 4 lost a significant amount of green time during some portions of the day, and saw increases in its $\mathrm{v} / \mathrm{c}$ ratios as well as increases in the number of split failures. There were already many split failures before retiming, and phase 4 was not relieved by the new operating plan. This phase would probably benefit by receiving more green time. Given the nearness of the upstream signal with Cumberland Road (see Figure 3.5), this phase would probably benefit from coordination in the east-west direction in addition to the north-south direction. Lagging left turns for the eastbound and westbound approaches might also aid phase 4 , by preventing phase 3 from using cycle time that would otherwise be given to phase 4 . 


\section{CHAPTER 6. SUMMARY OF RESULTS}

\subsection{Methodology}

Chapter 2 presented a review of fundamental traffic operation concepts, including detection, actuation, and coordination. The methods of data collection used in this study were presented in Chapter 3, and the procedures by which this data was translated into performance measures was detailed in Chapter 4. The methods of data collection and performance measure extraction were limited to what could reasonably be accomplished by a signal controller in a traffic cabinet, using data that could be collected by commonly available signal equipment such as loop detectors.

\subsection{Applications}

In Chapter 5, two studies were presented that made use of the data collection methods described by Chapter 3 and the performance measures described in Chapter 4 . These studies compared signal operation before and after changes made by the controlling agency. The impacts of implementing fully actuated coordinated phases were investigated. The adjustment of green time on a cycle-by-cycle basis was observed, and the effects of these adjustments were seen in the changes in $\mathrm{v} / \mathrm{c}$ ratios and numbers of split failures. A more general before/after study investigated the impacts of various changes. The implementation of a lagging left turn was seen to improve progression along the arterial corridor, while causing some minor phases to become more saturated. Switching from fully-actuated, non-coordinated to semi-actuated, coordinated operation also benefited progression. The impacts of changing the splits could also be observed in the performance measures.

\subsection{Moving Toward Real-Time Data Collection}

This study demonstrates the usefulness of cycle-by-cycle data collected in real time. All of the relevant information in Chapter 5 was gathered by data collection techniques that could have been carried out by a traffic signal controller with a data collection module, and downloaded to a computer for higher level analysis. The latter part of that task was essentially accomplished in this study; minimal field time was 
required in gathering that data, and relatively little time went into processing the raw data. The process of reducing the data to performance measures was automated using macro spreadsheets. The entire process could be packaged into a component of a traffic signal controller, giving it the capability to report on signal operation in real time.

As discussed in Chapter 2, the infrastructure for data collection is already available at many intersections. In some cases, the detectors and controllers may be used in modes of operation that are incompatible with the methods presented in this study. However, with further development, it should be possible to fit data collection methods within the limitations of the operating plans of a particular intersection. For example, in this study, counts obtained from pulse mode detection were used. There may be other data that could be obtained from presence mode detection methods. If it were readily available as a component of signal controllers, data collection could be a non-intrusive element, available for the traffic engineer to take advantage of as needed.

\subsection{Directions For Future Research}

Before manufacturers will begin to consider addition of performance measure calculation to signal controllers, these capabilities will have to be included in specifications. Before that can happen, first of all, it must be shown that currently available technology is capable of supporting the added features; secondly, the reliability of the performance measures must be validated; and finally, the benefits of automatic data collection must be fully demonstrated. This report has presented data that could be generated with technology that is currently available, but not yet implemented in traffic signal controllers. The utility of this data was demonstrated in the two traffic operations studies presented herein.

Future research should include a verification of the performance measures collected by an automatic data collection system, to characterize the reliability of those methods, in comparison with other established methods. The set of performance measures presented in this report could be expanded to include measures such as delay, HCM level of service, and so on. The performance of networks of traffic signals could be studied with placement of data collection modules in multiple intersections along a corridor. An in-depth review of the disparities between detector technology and data collection capability could be carried out to identify what performance measures could feasibly be obtained using what currently lies in the field. Finally, a cost-benefit analysis should be conducted to substantiate the hypothesis that the effort spent in automating the data collection process provides a multiplicity of returns. 


\section{BIBLIOGRAPHY}

1. Sessions, G.M., Traffic Devices: Historical Aspects Thereof. Institute of Traffic Engineers (Washington, DC), 1971.

2. Household Vehicles Energy Use: Latest Data and Trends. Energy Information Administration (Washington, DC), 2005. Retrieved from http://www.eia.doe.gov/emeu/rtecs/nhts survey/2001/index.html

3. Benefits of Retiming Traffic Signals: An ITE Informational Report. Institute of Transportation Engineers (Washington, DC), 2005.

4. 2007 National Traffic Signal Report Card Technical Report. National Transportation Operations Coalition (Washington, DC), 2005.

5. Transportation Research Board. Highway Capacity Manual, National Research Council, Washington, D.C., 2000.

6. Day, C.M., D.M. Bullock, and J.R. Sturdevant, "Cycle Length Performance Measures: Revisiting and Extending Fundamentals," Transportation Research Record Paper No. 09-0061, under review.

7. Kell, J.H. and I.J. Fullteron, Manual of Traffic Signal Design, Institute of Transportation Engineers, Washington, DC, 1991.

8. Orcutt, F.L., The Traffic Signal Book, Prentice Hall, Englewood Cliffs, NJ, 1993.

9. Smaglik, E., D.M. Bullock, and T. Urbanik, "An Evaluation of Lane by Lane Vehicle Detection for Actuated Controllers Serving Multi-Lane Approaches," Transportation Research Record \#1925, TRB, National Research Council, Washington, DC, pp. 123-133, 2005.

10. Shoup, G.E. and D.M. Bullock, "A Dynamic Offset Tuning Procedure Using Travel Time Data," Transportation Research Record \#1683, TRB, National Research Council, Washington, DC, p. 94, 1999.

11. Smaglik, E.J., A. Sharma, D.M. Bullock, J.R. Sturdevant, and G. Duncan, "Event Based Data Collection for Generating Actuated Controller Performance Measures." Transportation Research Record, Paper No. 07-1094, TRB, National Research Council, Washington, DC, in press, 2007.

12. Milazzo, J., Foyle, R. S., et al. "Overcoming Obstacles to the Use of Traffic Signal Detector Data for Traffic Forecasting." North Carolina DOT, May 2001.

13. Balke, KN, Charara, H and Parker, R. Development of a Traffic Signal Performance Measurement System. Report \# 0-04422-2, TTI May, 2005.

14. Smaglik, E.J., D.M. Bullock, and T. Urbanik, "Adaptive Split Control Using Enhanced Detector Data." Proceedings of the 2005 ITE International Annual 
Meeting and Exhibit, Paper AB05H-11, Melbourne, Australia, August 7-10, 2005.

15. Robertson, D. I. TRANSYT: A Traffic Network Study Tool. Report \# LR 253, Road Research Laboratory, Crowthorne, Berkshire, UK, 1969.

16. Day, C.M., E.J. Smaglik, D.M. Bullock, and J.R. Sturdevant, "Quantitative Evaluation of Fully Actuated Versus Non-Actuated Coordinated Phases," Transportation Research Record, Paper No. 08-0383, TRB, National Research Council, Washington, DC, in press, 2008.

17. de Castro-Neto, M.M., T. Urbanik, and L.D. Han, "Noncoordinated Phases in Coordinated Traffic Signal System: Evaluation of Alternative Permissive Periods on Performance," Transportation Research Record \#1978, TRB, National Research Council, Washington, DC, pp. 8-15, 2006.

18. Berry, D.S., "Field Measurement of Delay at Signalized Intersections," HRB Proceedings, Vol. 35, pp. 505-522, 1956.

19. Ficklin, N.C., "For and Against Semi-Actuated Traffic Signal Control," Traffic Engineering, Vol. 43, No. 6, pp. 20-25, 1973.

20. Jovanis, P.P. and J.A. Gregor, "Coordination of Actuated Arterial Traffic Signal Systems," Journal of Transportation Engineering, ASCE, Vol. 112, No. 4, pp. 416-432, 1986.

21. Chang, E.C-P. and J. Koothrappally, "Field Verification of Coordinated Actuated Control," Transportation Research Record \#1456, TRB, National Research Council, Washington, DC, pp.83-90, 1994.

22. Chaudhary, N.A. and K.N. Balke, "Real-Time Coordinated-Actuated Traffic Control During Congested Conditions," Texas Transportation Institute, Austin, TX, Study \#0-1288, 1997.

23. Skabardonis, A., R.L. Bertini, and B.R. Gallagher, "Development and Application of Control Strategies for Signalized Intersections in Coordinated Systems," Transportation Research Record \#1634, TRB, National Research Council, Washington, DC, pp. 110-117, 1998.

24. Khatib, Z., A. Abdel-Rahim, G. Judd, and K. Jagarapu, "Actuated Coordinated Signalized System," National Institute for Advanced Transportation Technology, University of Idaho, Report \# N01-20, 2001.

25. Chang, E.C-P., "Guidelines for Actuated Controllers in Coordinated Systems," Transportation Research Record \#1554, TRB, National Research Council, Washington, DC, pp. 61-73, 1996. 